\title{
Catalogue of Topological Electronic Materials
}

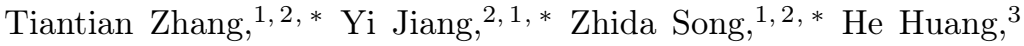 \\ Yuqing He, ${ }^{3,2}$ Zhong Fang, ${ }^{1}$ Hongming Weng, ${ }^{1},{ }^{\dagger}$ and Chen Fang ${ }^{1,4, \text { 周 }}$ \\ ${ }^{1}$ Beijing National Labratory for Condensed Matter Physics, \\ and Institute of Physics, Chinese Academy of Sciences, Beijing 100190, China \\ ${ }^{2}$ University of Chinese Academy of Sciences, Beijing 100049, China \\ ${ }^{3}$ Computer Network Information Center, Chinese Academy of Sciences, China \\ ${ }^{4}$ CAS Centre for Excellence in Topological Quantum Computation, Beijing, China
}

Topological electronic materials are new quantum states of matter hosting novel linear responses in the bulk and anomalous gapless states at the boundary, and are for scientific and applied reasons under intensive research in physics and in materials sciences[1-4]. The detection for such materials has so far been hindered by the level of complication involved in the calculation of the so-called topological invariants, and is hence considered a specialized task that requires both experience with materials and expertise with advanced theoretical tools. Here we introduce an effective, efficient and fully automated algorithm in obtaining the topological invariants for all non-magnetic materials that are known to human, based on recently developed principles [5] that allow for exhaustive mappings between the symmetry representation of occupied bands and the topological invariants 6 - 8]. Our algorithm requires as input only the occupied-band information (energy and wavefunction) at a handful (up to eight) of high-symmetry points in the Brillouin zone, which is readily calculable with any firstprinciples software. In return, it is capable of providing a detailed topological classification of all non-magnetic materials. Equipped with this method we have scanned through a total of 39519 materials available in structural databases [9-11, and found that as many as $\mathbf{8 0 5 6}$ of them are actually topological (8889 if spin-orbital coupling is neglected). These are further catalogued into classes of 5005 topological semimetals[12, 13, 1814 topological insulators [14-16] and 1237 topological crystalline insulators[17], most of which are new to human knowledge. All the results are available and searchable at http://materiae .iphy . ac.cn/; and for each topological material, we have plotted the band structure as well as the local density of states, shown on the same website.

\footnotetext{
* These authors contributed to this work equally

$\dagger$ hmweng@iphy.ac.cn

$\ddagger$ cfang@iphy.ac.cn
}

\section{CONTEXT}

We begin by giving further context of the research by briefly introducing the recent development in the field. In the past decade, great progress has been made to understand the nontrivial topology in a band structure. In any band structure having direct gap at all momentum, new quantum numbers known as topological invariants can be defined in terms of the wavefunctions of all valence bands. Topological invariants are the defining properties of all topological materials. The type and form of the invariants depend on the dimensionality and the symmetry of the system. In chronological order, timereversal symmetry was first found to protect a $Z_{2}$ invariant in two [14, 15] and three dimensions [16, soon followed by crystalline symmetries such as inversion 18, 19 (parity), mirror planes 20, 21, and others, each of niwhich brings in some new and independent topological invariant [7, 17, 22 29]. A full characterization of the topology of a given crystal hence amounts to listing all these invariants protected by all elements in the corresponding space group (SG).

Parallel to this line of progression is the emergence of the field of topological semimetals 4, 30, where the conduction and the valence bands have band crossings, i. e., topological nodes, robust against symmetry-preserving perturbations. Depending on the degeneracy and dimensionality of the nodes, topological semimetals are further classified into nodal-point and nodal-line semimetals. A topological semimetal is characterized by the number and the type of all its band crossings.

Numerical prediction of topological materials thus requires the evaluation of all topological invariants or the identification of all topological nodes, both of which require lengthy and involved calculation. The challenge have prevented people from doing a thorough scanning for topological materials, and successful examples have been mostly ascribed to experience and intuition of experienced researchers.

A series of recent theoretical works have greatly improved the situation by finding the complete mapping from the irreducible representations of valence bands to topological invariants and topological nodes [5] $8,29,31$. By recognizing that these theories can be fused together with first principles numerical methods, the latter of which provide the input for the former, here we develop a fully automated search algorithm that can readily be 
used to scan through large materials databases.

\section{THE ALGORITHM}

We now describe the full diagnosis algorithm for an arbitrary crystalline material, which is summarized in a flow chart in Fig. 1. For each material that is shared in both the Materials Project[9, 10] and the International Crystal Structure Database 11, we load its basic information from the Materials Project. The established mapping between symmetry data and topology data only applies to non-magnetic materials, therefore as first step we check against record if the total magnetization in one unit cell is less than $10^{-1} \mu_{B}$. If not, we label it as "magnetic" and load the next material; if yes, we proceed to the second step. In the second step we check if the number of electrons in one unit cell is even. If it is odd, this material is labeled as "conventional metal", but if even, we proceed to loading its structural data and atomic positions from the Materials Project. The third step is symmetrizing and standardizing the atomic positions using PHONOPY [32] [33]. This concludes the preparation phase of the process, after which out of 39519 scanned materials, 10348 materials are labeled "magnetic" and 2483 labeled "conventional metal", and 26688 materials go to the next phase.

We load the atomic positions and pseudo potential information of each remaining material into Vienna $\mathrm{Ab}$ initio Simulation Package (VASP 34 37, which may be replaced with any other software of choice). The first principles calculation is done with two settings, called "soc-setting" and "nsoc-setting" where we turn on and off the spin-orbital coupling, respectively. The two settings, mathematically, correspond to two symmetry classes, the symplectic and the orthogonal classes 38, the mappings between symmetry data and topology data drastically differ in these two classes 8, 27, 29. In realistic electronic materials, spin-orbital coupling is never exactly zero, yet for systems consisting of small atoms, the absence of spinorbital coupling is a relevant approximation [39 41]. For materials with high atomic numbers and high orbital electrons, where large spin-orbital coupling exists, the results obtained in the nsoc-setting should be used with caution. The output of the calculation is a list of energy levels and corresponding Bloch wavefunctions at each highsymmetry point. A high-symmetry point (HSP) is a momentum in the Brillouin zone, the invariant subgroup of which is larger than that of any point in its neighborhood. For example, $\Gamma$ point is always a HSP, as it is the only point in the neighborhood that is invariant under timereversal symmetry. With such information collected for all 26688 materials, we proceed to the analysis phase.

In the analysis phase, we first determine the irreducible representations for each valence band, or in the case of higher than one dimensional irreducible representations, for each degenerate multiplet of valence bands. The valence bands are defined as the lowest $N$ bands in the band structure, where $N$ is the number of electrons per unit cell. Here the "irreducible representations" include the degenerate irreducible representations that are pinned at the same energy due to time-reversal symmetry 42 . To this end, we calculate the character for each symmetry operation, and, by comparing these characters to character tables on the Bilbao Crystallographic Server (BCS), recently available thanks to decent efforts [31], we identify the irreducible representation for each (multiplet of) valence band(s). Then, we check at each HSP if the top (multiplet of) valence band(s) is partially filled. For example, if the top valence bands belong to a four dimensional irreducible representation, and if the filling number ( $N$ minus the number of bands below the top valence bands) is less than four, then we have a partially filled HSP. Each partially filled HSP is a robust band crossing point, making the material a topological semimetal, with at least one topological node at the partially filled HSP. We then denote the corresponding material "high-symmetry point semimetal" (HSPSM). If none of the HSP is partially filled, we generate for the material a series of integers we call the "symmetry data". Each entry in the data is the number of the appearances of a given irreducible representation in the valence bands at each HSP. We proceed to check if the symmetry data satisfy all "compatibility relations". Compatibility relations are the necessary conditions for a band structure to have direct gap along certain high-symmetry lines in the Brillouin zone. These relations have been derived explicitly and available on the BCS [5, 31]. Each group of compatibility relations correspond to a certain high-symmetry line joining two high-symmetry points; and if any one relation in the group is unsatisfied, there is, at least, one topological node along this line between the conduction and the valence bands. When that happens, we denote the material "high-symmetry line semimetal" (HSLSM) and also output the line on which the node should appear. For the nsoc-setting, 5508 materials are labeled as HSPSM and 3269 as HSLSM, and for the soc-setting, 2713 materials belong to HSPSM and 2292 to HSLSM. If all compatibility relations are satisfied (17157 materials in the nsoc-setting and 20745 in the soc-setting), the material may have direct gap along all high-symmetry lines. For these materials, we proceed to compute the symmetry-based indicators.

The group structures of indicators are derived in Ref. [6, and their explicit expressions in Ref. [7, 8, and 29. The indicators for each space group, if exist, are a set of several $\mathbb{Z}_{n}$ numbers, and they roughly speaking quantify how any given symmetry data differs from that of an atomic insulator having the same crystal structure. The symmetry indicators have the following properties: (i) if any indicator is nonzero, the material is not an atomic insulator, i. e., topologically nontrivial, (ii) two materials with different indicators are topologically distinct and (iii) the topological distinction between two materials having the same indicators cannot be diagnosed using symmetry data. We remark that point (iii) above 


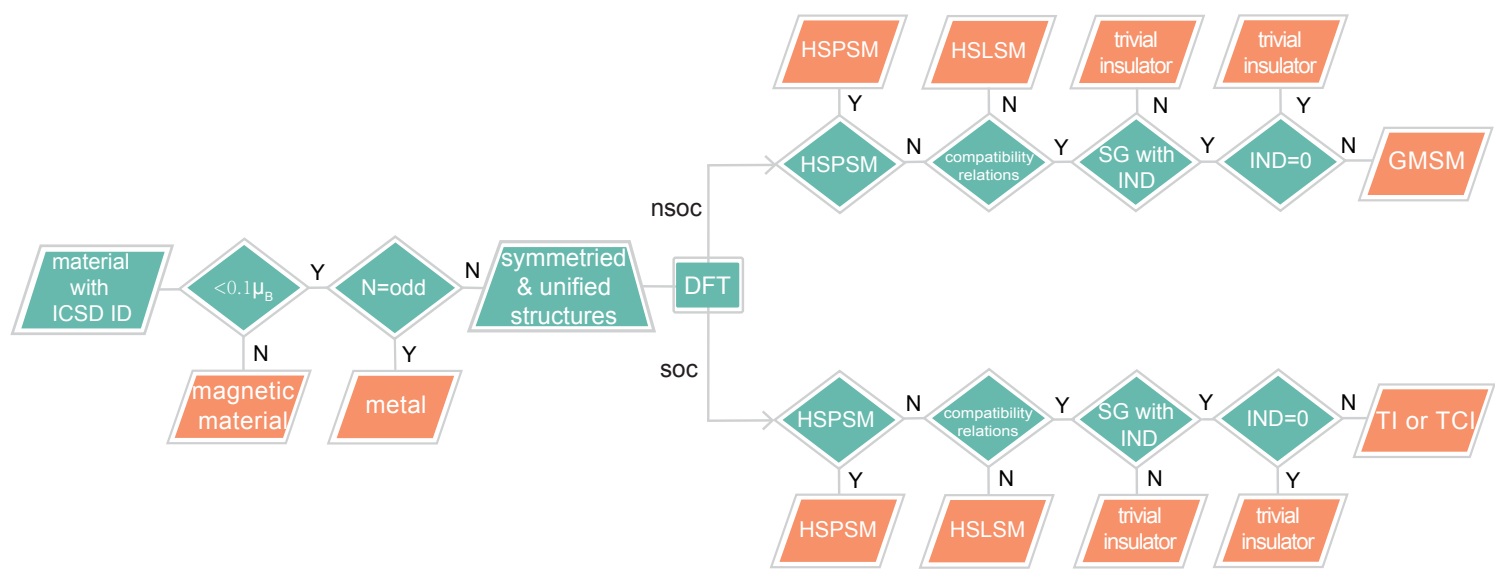

Figure 1. The flowchart shows how a material is processed in our diagnosis algorithm, starting from the left. We first check against the record at the Materials Project if the material has ferromagnetism, and check if there are even number of electrons in one primitive unit cell. If yes for both, we feed the material into the first-principles calculation for the band structure and compute the symmetry data, before checking if there be partially filled irreducible representation at high-symmetry points. If not, the symmetry data is checked against all compatibility relations, and, should all relations be satisfied, is fed into the calculator for symmetry-based indicators (IND). At each checkpoint, a material either goes on to the next step, or is labeled as magnetic, conventional metal, high-symmetry point semimetal or high-symmetry line semimetal. At the final step, depending on the values of indicators, a material is labeled as generic momentum semimetal, topological insulator, topological crystalline insulator, or trivial insulator. From the first-principles calculation step, all steps are taken for two settings: non-spin-orbitalcoupling setting and spin-orbital-coupling setting.

means that all information on topology that may be extracted from symmetry data has been contained in the the values of indicators. In Ref. 77 and 29], it is shown that each nonzero combination of indicators in the socsetting correspond to some topological (crystalline) insulator, and that in the nsoc-setting correspond to a topological semimetal with nodes at generic momenta. In the soc-setting, the topological invariants corresponding to each nonzero set of indicators are found in Ref.[7], and the topological surface states in Ref. 29]; in nsoc-setting, Ref. 8 gives for each nonzero set of indicators the number, type and topological charge (if any) of the topological nodes. This concludes the final analysis phase and with it our topological diagnosis of all materials.

\section{RESULTS}

Each material is now labeled with one of the following: HSPSM = high-symmetry point semimetal (both settings), HSLSM = high-symmetry line semimetal (both settings), GMSM = generic momenta semimetal (nsocsetting only), $\mathrm{TI}=$ topological insulator (soc-setting only), TCI = topological crystalline insulator (soc-setting only), magnetic, conventional metal, and trivial insulator. Out of these, the first five classes are considered topological materials and listed by the respective class in Tables II, III, IV, V, VI in the Supplementary Materials. In Table II, each material in HSPSM is shown together with HSP where partial fillings occur and the irreducible representations that are partially filled; and each material in Table III is shown together with the high-symmetry line(s) where compatibility relations are unsatisfied. Each material in GMSM, TI and TCI is shown together with the values of its symmetry-based indicators, two types of which deserve separate notes. Indicators displayed in blue in Table IV signify nodal lines that have $\mathrm{Z}_{2}$-monopole topological charges 41], which are yet to be found in electronic materials [43, 44]. Indicators displayed in blue in Table $\mathrm{V}$ have their likelihood of corresponding to topological crystalline insulators having only one-dimensional helical edge states on the boundary but not having surface states [27, 45 48, also known as "high order topological insulators" in recent studies 49 . The exhaustive scan, naturally, not only yields almost all (nonmagnetic) topological materials that have ever been so far predicted in theory or verified in experiments, but also predicts many more topological materials that have not appeared in literature. Out of these new candidates, we pick one material in each class for display. BaPPt in Fig. 2(a) is found a HSPSM where the conduction and the valence bands meet at $\Gamma$ and $R$. The degeneracy at $R$ is sixfold stabilized by nonsymmorphic space group symmetries, and importantly, there is an electron pocket near R. These facts qualify BaPPt as a good candidate for study of "new fermions" beyond Weyl and Dirac fermions in real materials [50]. In the HSLSM class (nsoc-setting), we pick $\mathrm{YCoC}_{2}$, the band structure of which is shown in Fig. 2(b). The band structure shows that band crossings are along Z- $\Gamma, \mathrm{Z}-\mathrm{T}, \mathrm{Z}-\mathrm{Y}$, and Z-R lines, and a more detailed analysis (in Sec. IV B) shows that the band crossings in this materials are nodal lines protected by mirror planes, and the nodal rings touch each other. $\mathrm{Sr}_{2} \mathrm{NiOsO}_{6}$ (nsoc-setting) in Fig. 2(c) has no band crossing along any 
(a) BaPPt HSPSM (soc) (b) $\mathrm{YCoC}_{2} \operatorname{HSLSM}$ (nsoc) (c) $\mathrm{Sr}_{2} \mathrm{NiOsO}_{6} \mathrm{GMSM}_{\text {(nsoc) }}$
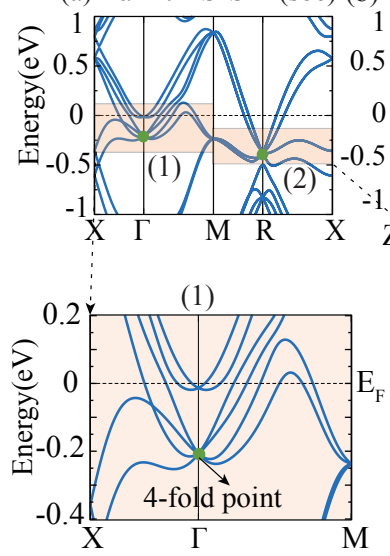

(f) SG 198

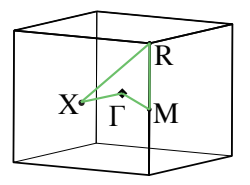

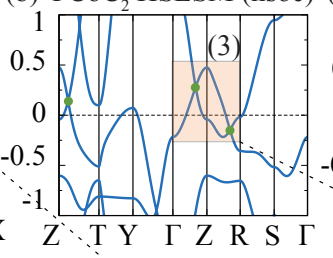
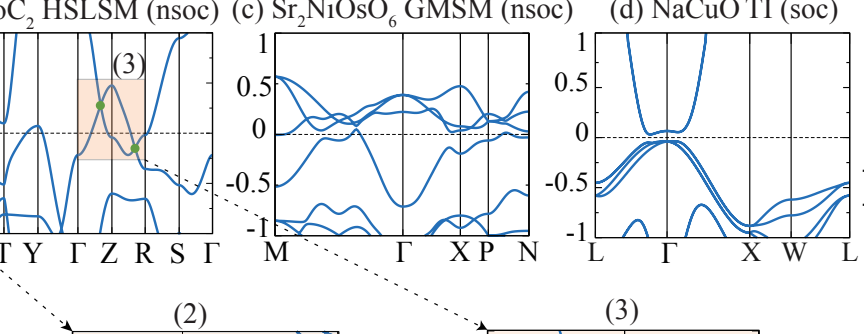

$(2)$
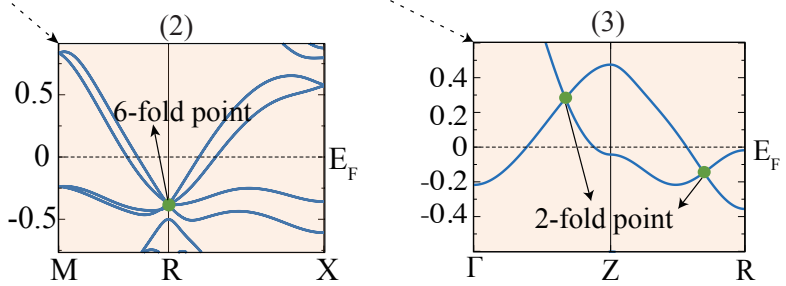

(g)

(h)

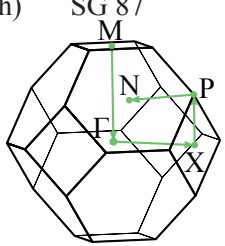

(e) $\mathrm{ZrTi}_{2} \mathrm{H}_{4} \mathrm{TCI}$ (soc)

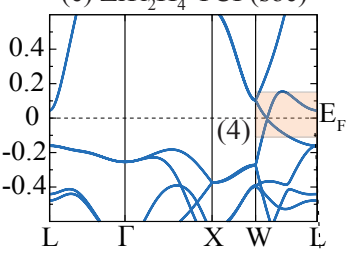

(4)

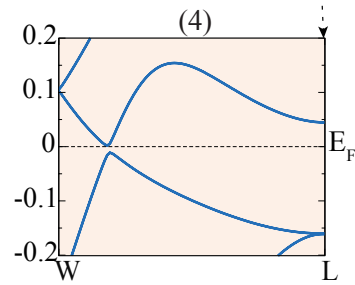

(j) $\quad$ SG 227

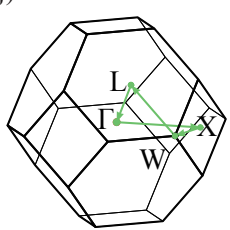

Figure 2. The five candidates for the five classes of topological materials: (a) high-symmetry point semimetal BaPPt having a sixfold degeneracy near the Fermi energy at $\mathrm{R}$, (b) high-symmetry line semimetal $\mathrm{YCoC}_{2}$ which has two connected nodal rings (nsoc-setting), (c) generic momenta semimetal $\mathrm{Sr}_{2} \mathrm{NiOsO}_{6}$ having nodal rings that have $\mathbb{Z}_{2}$ monopole charge (nsoc-setting), (d) topological insulator $\mathrm{NaCuO}$ and (e) topological crystalline insulator $\mathrm{ZrTi}_{2} \mathrm{H}_{4}$. For each candidate material, we plot the band structure in (a-e), the Brilluoin zone with high-symmetry points marked in ( $\mathrm{f}-\mathrm{j})$, and if necessary, zoomed-in regions of the band structure (1-4).

of the high-symmetry lines, but the indicators of (0002) imply that at generic momentum there must be $2 \bmod 4$ nodal rings where the conduction and the valence bands cross. Each of the ring has $\mathbb{Z}_{2}$ topological charge, making $\mathrm{Sr}_{2} \mathrm{NiOsO} 6$ the first candidate electronic material (with small spin-orbital coupling) hosting $\mathbb{Z}_{2}$ nontrivial nodal rings. $\mathrm{NaCuO}[51$ is a new noncentrosymmetric topological insulator [Fig. 2(d)], featuring three band inversion between the $d$ orbital and the $s$ orbital at $\Gamma$, with the band gap $\sim 0.1 \mathrm{eV}$. For noncentrosymmetric systems, the classic Fu-Kane formula[16 does not apply, so that an eigenvalue diagnosis would be impossible without our new method. $\mathrm{ZrTi}_{2} \mathrm{H}_{4}$ in Fig. 2(e) has band crossings along L-W lines without spin-orbital coupling, but as the coupling turns on opens a full gap of $\sim 10 \mathrm{meV}$ at all momenta, making the material a topological crystalline insulator. The indicators of (0002) pin down the topological invariants of the TCI to two possible sets [7]. Eigenvalue diagnosis cannot distinguish them further, but a detailed calculation (see Sec. IV C) of the mirror Chern number at $k_{z}=0$-plane helps choose the correct set. In this set, all nonzero invariants are protected by screw rotation symmetries or glide plane symmetries, so that $\mathrm{ZrTi}_{2} \mathrm{H}_{4}$ is a material candidate for a screw-axis $\mathbb{Z}_{2}$ TCI[7, 29], having 1D helical edge states on its surface without 2D surface states.

All the above results are available at http:// materiae.iphy.ac.cn/, which features an interactive user interface that facilitates search in the vast data. At the same website, we have also shown the band structures 52 and density of states for each material diagnosed as topological.

In summary, the exhaustive search using our new method yields a huge number of new materials predictions. Since a candidate topological material may at the same time superconducts (such as $\mathrm{SrSn}_{4}[53$ and $\left.\mathrm{LiTi}_{2} \mathrm{O}_{4}[54]\right)$, has negative coefficient of thermal expansion (such as $\mathrm{ScF}_{3}[55]$ ), or possesses other unusual properties, the complete list of these materials connects the field of topological physics to many other areas of solid state physics and materials sciences.

Note added: In the finalizing stage of the current paper, we became aware of the three recent preprints that use symmetry-based indicators for diagnosis of topological materials 56 58, which have covered one, five and eight out of 230 space groups in their searches, respectively. 
(2011)

[3] C.-K. Chiu, J. C. Y. Teo, A. P. Schnyder, and S. Ryu, Rev. Mod. Phys. 88, 035005 (2016).

[4] N. P. Armitage, E. J. Mele, and A. Vishwanath, Rev. Mod. Phys. 90, 015001 (2018).

[5] B. Bradlyn, L. Elcoro, J. Cano, M. G. Vergniory, Z. Wang, C. Felser, M. I. Aroyo, and B. A. Bernevig, Nature 547, 298 EP (2017)

[6] H. C. Po, A. Vishwanath, and H. Watanabe, Nature Communications 8, 50 (2017)

[7] Z. Song, T. Zhang, Z. Fang, and C. Fang, ArXiv e-prints (2017), arXiv:1711.11049 [cond-mat.mes-hall].

[8] C. Fang, Z. Song, and T. Zhang, ArXiv e-prints (2017), arXiv:1711.11050 [cond-mat.mes-hall]

[9] A. Jain, S. P. Ong, G. Hautier, W. Chen, W. D. Richards, S. Dacek, S. Cholia, D. Gunter, D. Skinner, G. Ceder, and K. a. Persson, APL Materials 1, 011002 (2013)

[10] S. P. Ong, W. D. Richards, A. Jain, G. Hautier, M. Kocher, S. Cholia, D. Gunter, V. L. Chevrier, K. A. Persson, and G. Ceder, Computational Materials Science 68, 314 (2013).

[11] M. Hellenbrandt, Crystallography Reviews 10, 17 (2004)

[12] Z. Fang, N. Nagaosa, K. S. Takahashi, A. Asamitsu, R. Mathieu, T. Ogasawara, H. Yamada, M. Kawasaki, Y. Tokura, and K. Terakura, Science 302, 92 (2003).

[13] S. Murakami, New Journal of Physics 9, 356 (2007)

[14] C. L. Kane and E. J. Mele, Phys. Rev. Lett. 95, 146802 (2005)

[15] B. A. Bernevig, T. L. Hughes, and S.-C. Zhang, Science 314, 1757 (2006).

[16] L. Fu and C. L. Kane, Phys. Rev. B 76, 045302 (2007).

[17] L. Fu, Phys. Rev. Lett. 106, 106802 (2011).

[18] A. M. Turner, Y. Zhang, and A. Vishwanath, Phys. Rev. B 82, 241102 (2010)

[19] T. L. Hughes, E. Prodan, and B. A. Bernevig, Phys. Rev. B 83, 245132 (2011).

[20] T. H. Hsieh, H. Lin, J. Liu, W. Duan, A. Bansil, and L. Fu, Nature Communications 3, 982 EP (2012)

[21] C.-K. Chiu, H. Yao, and S. Ryu, Phys. Rev. B 88, $075142(2013)$

[22] A. Alexandradinata, C. Fang, M. J. Gilbert, and B. A. Bernevig, Phys. Rev. Lett. 113, 116403 (2014)

[23] C. Fang and L. Fu, Phys. Rev. B 91, 161105 (2015)]

[24] K. Shiozaki, M. Sato, and K. Gomi, Phys. Rev. B 91, $155120(2015)$

[25] Z. Wang, A. Alexandradinata, R. J. Cava, and B. A. Bernevig, Nature 532, 189 EP (2016)

[26] B. J. Wieder, B. Bradlyn, Z. Wang, J. Cano, Y. Kim, H.-S. D. Kim, A. M. Rappe, C. L. Kane, and B. A. Bernevig, ArXiv e-prints (2017), arXiv:1705.01617 [cond-mat.mes-hall].

[27] Z. Song, Z. Fang, and C. Fang, Phys. Rev. Lett. 119, 246402 (2017)

[28] C. Fang and L. Fu, ArXiv e-prints (2017), arXiv:1709.01929 [cond-mat.mes-hall]

[29] E. Khalaf, H. C. Po, A. Vishwanath, and H. Watanabe, ArXiv e-prints (2017), arXiv:1711.11589 [cond-mat.strel].

[30] A. A. Burkov, Nature Materials 15, 1145 EP (2016)

[31] L. Elcoro, B. Bradlyn, Z. Wang, M. G. Vergniory, J. Cano, C. Felser, B. A. Bernevig, D. Orobengoa, G. de la Flor, and M. I. Aroyo, Journal of Applied Crystallography 50, 1457 (2017).

[32] A. Togo and I. Tanaka, Scr. Mater. 108, 1 (2015).
[33] 166 materials are discarded at this step for discrepancy in the space groups identified in PHONOPY and those given on the databases as we decrease the error tolerance in atomic position from default $10^{-5} \AA$ to $0.1 \AA$.

[34] G. Kresse and J. Hafner, Phys. Rev. B 47, 558 (1993).

[35] G. Kresse and J. Hafner, Phys. Rev. B 49, 14251 (1994)

[36] G. Kresse and J. Furthmuller, Computational Materials Science 6, 15 (1996).

[37| G. Kresse and J. Furthmuller, Phys. Rev. B 54, 11169 (1996)

[38] A. Altland and M. R. Zirnbauer, Phys. Rev. B 55, 1142 (1997)

[39] A. A. Burkov, M. D. Hook, and L. Balents, Phys. Rev. B 84, 235126 (2011)

[40] H. Weng, Y. Liang, Q. Xu, R. Yu, Z. Fang, X. Dai, and Y. Kawazoe, Phys. Rev. B 92, 045108 (2015).

[41] C. Fang, Y. Chen, H.-Y. Kee, and L. Fu, Phys. Rev. B 92, 081201 (2015).

[42] C. J. Bradley and A. P. Cracknell, The Mathematical Theory of Symmetry in Solids: Representation Theory for Point Groups and Space Groups (Oxford University Press, 2010).

[43] K. Li, C. Li, J. Hu, Y. Li, and C. Fang, Phys. Rev. Lett. 119, 247202 (2017)

[44] W. Yao, C. Li, L. Wang, S. Xue, Y. Dan, K. Iida, K. Kamazawa, K. Li, C. Fang, and Y. Li, ArXiv e-prints (to appear in Nature Physics) (2017), arXiv:1711.00632 [cond-mat.mes-hall].

[45] W. A. Benalcazar, B. A. Bernevig, and T. L. Hughes, Science 357, 61 (2017)

[46] J. Langbehn, Y. Peng, L. Trifunovic, F. von Oppen, and P. W. Brouwer, Phys. Rev. Lett. 119, 246401 (2017)

[47] W. A. Benalcazar, B. A. Bernevig, and T. L. Hughes, Phys. Rev. B 96, 245115 (2017).

[48] F. Schindler, Z. Wang, M. G. Vergniory, A. M. Cook, A. Murani, S. Sengupta, A. Y. Kasumov, R. Deblock, S. Jeon, I. Drozdov, H. Bouchiat, S. Guéron, A. Yazdani, B. A. Bernevig, and T. Neupert, ArXiv e-prints (2018), arXiv:1802.02585 [cond-mat.mtrl-sci]

[49] F. Schindler, A. M. Cook, M. G. Vergniory, Z. Wang, S. S. P. Parkin, B. A. Bernevig, and T. Neupert, Science Advances 4 (2018), 10.1126/sciadv.aat0346.

[50] B. Bradlyn, J. Cano, Z. Wang, M. G. Vergniory, C. Felser, R. J. Cava, and B. A. Bernevig, Science 353 (2016), 10.1126/science.aaf5037.

[51] A related material, NaAgO, was mentioned in Ref.[57].

[52] Y. Hinuma, G. Pizzi, Y. Kumagai, F. Oba, and I. Tanaka, Computational Materials Science 128, 140 (2017).

[53] X. Lin, S. L. Budako, G. D. Samolyuk, M. S. Torikachvili, and P. C. Canfield, Journal of Physics: Condensed Matter 23, 455703 (2011)

[54] E. G. Moshopoulou, Journal of the American Ceramic Society 82, 3317 (1999).

[55] C. W. Li, X. Tang, J. A. Muñoz, J. B. Keith, S. J. Tracy, D. L. Abernathy, and B. Fultz, Phys. Rev. Lett. 107, 195504 (2011)

[56] X. Zhou, C.-H. Hsu, T.-R. Chang, H.-J. Tien, Q. Ma, P. Jarillo-Herrero, N. Gedik, A. Bansil, V. M. Pereira, S.-Y. Xu, H. Lin, and L. Fu, ArXiv e-prints (2018), arXiv:1805.05215 [cond-mat.mtrl-sci].

[57] F. Tang, H. C. Po, A. Vishwanath, and X. Wan, ArXiv e-prints (2018), arXiv:1805.07314 [cond-mat.mes-hall]

[58] F. Tang, H. C. Po, A. Vishwanath, and X. Wan, ArXiv 
e-prints (2018), arXiv:1806.04128 [cond-mat.mes-hall]

[59] H. J. Monkhorst and J. D. Pack, Phys. Rev. B 13, 5188 (1976)

[60] About 400 materials are not converged or converge to wrong states in the nsoc-setting and 600 in the socsetting.

[61] A. Alexandradinata and B. A. Bernevig, Phys. Rev. B 93, 205104 (2016).

62] Y. Wang, H. Lin, T. Das, M. Hasan, and A. Bansil, New Journal of Physics 13, 085017 (2011).

[63] Z. Wang, H. Weng, Q. Wu, X. Dai, and Z. Fang, Physical Review B 88, 125427 (2013).

[64] M. Hirayama, R. Okugawa, S. Ishibashi, S. Murakami, and T. Miyake, Phys. Rev. Lett. 114, 206401 (2015).

[65] R. Li, Y. Xu, J. He, S. Ullah, J. Li, J.-M. Liu, D. Li, C. Franchini, H. Weng, and X.-Q. Chen, ArXiv e-prints (2016), arXiv:1610.07142 [cond-mat.mtrl-sci].

[66] P. Tang, B. Yan, W. Cao, S.-C. Wu, C. Felser, and W. Duan, Phys. Rev. B 89, 041409 (2014)

[67] C. Le, X. Wu, S. Qin, Y. Li, R. Thomale, F. Zhang, and J. Hu, ArXiv e-prints (2018), arXiv:1801.05719 [condmat.mes-hall]

[68] W. Wu, Y. Liu, S. Li, C. Zhong, Z.-M. Yu, X.-L. Sheng, Y. X. Zhao, and S. A. Yang, Phys. Rev. B 97, 115125 (2018)

[69] C. Chen, S.-S. Wang, L. Liu, Z.-M. Yu, X.-L. Sheng, Z. Chen, and S. A. Yang, Physical Review Materials 1, 044201 (2017).

[70] S.-D. Guo and B.-G. Liu, Journal of Physics: Condensed Matter 30, 105701 (2018)

[71] H. Weng, C. Fang, Z. Fang, and X. Dai, Physical Review B 94, 165201 (2016)

[72] S. Ullah, J. Li, R. Li, Q. Xie, H. Ma, D. Li, Y. Li, and X.-Q. Chen, arXiv preprint arXiv:1706.06876 (2017).

[73] J.-P. Sun, D. Zhang, et al., Chinese Physics Letters 34, 027102 (2017).

[74] Q. Gibson, L. Schoop, L. Muechler, L. Xie, M. Hirschberger, N. Ong, R. Car, and R. Cava, Physical Review B 91, 205128 (2015).

[75] X. Feng, C. Yue, Z. Song, Q. Wu, and B. Wen, Phys. Rev. Materials 2, 014202 (2018).

[76] C. Park, S. W. Kim, and M. Yoon, Phys. Rev. Lett. 120, 026401 (2018).

[77] R. Li, H. Ma, X. Cheng, S. Wang, D. Li, Z. Zhang, Y. Li, and X.-Q. Chen, Physical review letters 117, 096401 (2016).

[78] Z. Wang and Y. Sun, Phys. Rev. B 85, 195320 (2012).

[79] Z. Liu, B. Zhou, Y. Zhang, Z. Wang, H. Weng, D. Prabhakaran, S.-K. Mo, Z. Shen, Z. Fang, X. Dai, et al., Science 343, 864 (2014).

[80] Z. Wang, A. Alexandradinata, R. J. Cava, and B. A. Bernevig, Nature 532, 189 EP (2016)

[81] H.-J. Zhang, S. Chadov, L. Müchler, B. Yan, X.-L. Qi, J. Kübler, S.-C. Zhang, and C. Felser, Physical review letters 106, 156402 (2011).

[82] P. Tang, Q. Zhou, and S.-C. Zhang, Phys. Rev. Lett. 119, $206402(2017)$

833 M. Yang and W.-M. Liu, Scientific Reports 4, 5131 (2014), arXiv:1310.7413 [cond-mat.mtrl-sci].

[84] H. Huang, J. Liu, and W. Duan, Physical Review B 90, 195105 (2014).

[85] J. Ruan, S.-K. Jian, H. Yao, H. Zhang, S.-C. Zhang, and D. Xing, Nature communications 7, 11136 (2016).

[86] F. Virot, R. Hayn, M. Richter, and J. van den Brink,
Physical review letters 111, 146803 (2013).

[87] X.-L. Sheng, Z. Wang, R. Yu, H. Weng, Z. Fang, and X. Dai, Physical Review B 90, 245308 (2014).

[88] H. Lin, L. A. Wray, Y. Xia, S. . Xu, S. Jia, R. J. Cava, A. Bansil, and M. Z. Hasan, ArXiv e-prints (2010), arXiv:1004.0999 [cond-mat.mes-hall].

[89] H. Huang, J. Liu, and W. Duan, Phys. Rev. B 90, 195105 (2014)

[90] T. H. Hsieh, J. Liu, and L. Fu, Phys. Rev. B 90, 081112 (2014)

[91] H. Weng, Y. Liang, Q. Xu, R. Yu, Z. Fang, X. Dai, and Y. Kawazoe, Physical Review B 92, 045108 (2015).

[92] M. Zeng, C. Fang, G. Chang, Y.-A. Chen, T. Hsieh, A. Bansil, H. Lin, and L. Fu, arXiv preprint arXiv:1504.03492 (2015).

[93] X.-L. Sheng, Z.-M. Yu, R. Yu, H. Weng, and S. A. Yang, The journal of physical chemistry letters 8, 3506 (2017).

[94] Q. Xu, R. Yu, Z. Fang, X. Dai, and H. Weng, Physical Review B 95, 045136 (2017).

[95] C. Xu, J. Chen, G.-X. Zhi, Y. Li, J. Dai, and C. Cao, Phys. Rev. B 93, 195106 (2016).

[96] D. Wu, J. Liao, W. Yi, X. Wang, P. Li, H. Weng, Y. Shi, Y. Li, J. Luo, X. Dai, et al., Applied Physics Letters 108, 042105 (2016).

[97] W. Zhang, R. Yu, W. Feng, Y. Yao, H. Weng, X. Dai, and Z. Fang, Physical review letters 106, 156808 (2011).

[98] A. Narayan, Phys. Rev. B 92, 220101 (2015).

[99] S. F. Weber, R. Chen, Q. Yan, and J. B. Neaton, Phys. Rev. B 96, 235145 (2017)

[100] Y. Chen, Y. Xie, S. A. Yang, H. Pan, F. Zhang, M. L. Cohen, and S. Zhang, Nano letters 15, 6974 (2015).

[101] H. Weng, X. Dai, and Z. Fang, Physical review X 4, $011002(2014)$.

[102] W. Feng, D. Xiao, J. Ding, and Y. Yao, Physical review letters 106, 016402 (2011).

[103] W. Feng, D. Xiao, J. Ding, and Y. Yao, Phys. Rev. Lett. 106, 016402 (2011)

[104] Q. Xu, Z. Song, S. Nie, H. Weng, Z. Fang, and X. Dai, Physical Review B 92, 205310 (2015).

[105] L. M. Schoop, M. N. Ali, C. Straßer, A. Topp, A. Varykhalov, D. Marchenko, V. Duppel, S. S. P. Parkin, B. V. Lotsch, and C. R. Ast, Nature Communications.

[106] C. Le, S. Qin, X. Wu, X. Dai, P. Fu, C. Fang, and J. Hu, Physical Review B 96, 115121 (2017).

[107] Z. Wang and G. Wang, Journal of the Physical Society of Japan 86, 124714 (2017).

[108] H. Huang, J. Liu, D. Vanderbilt, and W. Duan, Phys. Rev. B 93, 201114 (2016).

[109] T.-R. Chang, I. Pletikosic, T. Kong, G. Bian, A. Huang, J. Denlinger, S. K. Kushwaha, B. Sinkovic, H.-T. Jeng, T. Valla, W. Xie, and R. J. Cava, ArXiv e-prints (2017), arXiv:1711.09167 [cond-mat.mtrl-sci]

[110] Y. Chen, J. G. Analytis, J.-H. Chu, Z. Liu, S.-K. Mo, X.-L. Qi, H. Zhang, D. Lu, X. Dai, Z. Fang, et al., science 325, 178 (2009).

[111] H. Zhang, C.-X. Liu, X.-L. Qi, X. Dai, Z. Fang, and S.-C. Zhang, Nature Physics.

[112] Y. Xia, D. Qian, D. Hsieh, L. Wray, A. Pal, H. Lin, A. Bansil, D. Grauer, Y. S. Hor, R. J. Cava, and M. Z. Hasan, Nature Physics.

[113] H. Zhang, C.-X. Liu, X.-L. Qi, X. Dai, Z. Fang, and S.-C. Zhang, Nature physics 5, 438 (2009).

[114] F. Schindler, Z. Wang, M. G. Vergniory, A. M. Cook, 
A. Murani, S. Sengupta, A. Y. Kasumov, R. Deblock, S. Jeon, I. Drozdov, H. Bouchiat, S. Guéron, A. Yazdani, B. A. Bernevig, and T. Neupert, ArXiv e-prints (2018), arXiv:1802.02585 [cond-mat.mtrl-sci]

[115] P. Zhang, J.-Z. Ma, Y. Ishida, L.-X. Zhao, Q.-N. Xu, B.Q. Lv, K. Yaji, G.-F. Chen, H.-M. Weng, X. Dai, et al., Physical review letters 118, 046802 (2017).

[116] H. Gao, Y. Kim, J. W. F. Venderbos, C. L. Kane, E. J. Mele, A. M. Rappe, and W. Ren, ArXiv e-prints (2018), arXiv:1802.04815 [cond-mat.mtrl-sci]

[117] A. Yamakage, Y. Yamakawa, Y. Tanaka, and Y. Okamoto, Journal of the Physical Society of Japan 85, 013708 (2016)

[118] E. Emmanouilidou, B. Shen, X. Deng, T.-R. Chang, A. Shi, G. Kotliar, S.-Y. Xu, and N. Ni, Physical Review B 95, 245113 (2017).

[119] Y. Du, B. Wan, D. Wang, L. Sheng, C.-G. Duan, and X. Wan, Scientific reports 5, 14423 (2015).

[120] C. Chen, S.-S. Wang, L. Liu, Z.-M. Yu, X.-L. Sheng, Z. Chen, and S. A. Yang, Phys. Rev. Materials 1, 044201 (2017)

[121] Z. Wang, G. Wang, X. Shi, D. Wang, and X. Tian, Journal of Physics D: Applied Physics 50, 465304 (2017)

[122] Y. Du, F. Tang, D. Wang, L. Sheng, E.-j. Kan, C.G. Duan, S. Y. Savrasov, and X. Wan, npj Quantum Materials 2, 3 (2017).

[123] Y. Sun, Z. Zhong, T. Shirakawa, C. Franchini, D. Li, Y. Li, S. Yunoki, and X.-Q. Chen, Phys. Rev. B 88, $235122(2013)$

[124] Y. Sun, X.-Q. Chen, C. Franchini, D. Li, S. Yunoki, Y. Li, and Z. Fang, Physical Review B 84, 165127 (2011).

[125] B. Yan, C.-X. Liu, H.-J. Zhang, C.-Y. Yam, X.-L. Qi, T. Frauenheim, and S.-C. Zhang, EPL (Europhysics Letters) 90, 37002 (2010).

[126] H. Lin, R. Markiewicz, L. Wray, L. Fu, M. Hasan, and A. Bansil, Physical review letters 105, 036404 (2010).

[127] L.-L. Wang and D. D. Johnson, Physical Review B 83, 241309 (2011).

[128] H. Lin, T. Das, L. Wray, S.-Y. Xu, M. Hasan, and A. Bansil, New Journal of Physics 13, 095005 (2011).

[129] F. Pielnhofer, T. V. Menshchikova, I. P. Rusinov, A. Zeugner, I. Y. Sklyadneva, R. Heid, K.-P. Bohnen, P. Golub, A. I. Baranov, E. V. Chulkov, et al., Journal of Materials Chemistry C 5, 4752 (2017).

[130] T. V. Menshchikova, S. V. Eremeev, and E. V. Chulkov, Applied Surface Science 267, 1 (2013).

[131] R. Yu, Q. Wu, Z. Fang, and H. Weng, Phys. Rev. Lett. 119, 036401 (2017).

[132] Y. Sun, Q.-Z. Wang, S.-C. Wu, C. Felser, C.-X. Liu, and B. Yan, Phys. Rev. B 93, 205303 (2016)

\section{METHODS}

\section{A. Setting up the first principles numerics}

All the calculations in this work are performed by Vienna abinitio Simulation Package (VASP) with the generalized gradient approximation (GGA) of PerdewBurke-Ernzerhof (PBE) type exchange-correlation potential [34 37]. The psudopotential files we used are from
VASP software package and they are listed in the website http://materiae.iphy.ac.cn/. The cut-off energy of plane wave basis set is set to be the ENMAX value in the pseudo-potential file plus 25\%. A $\Gamma$-centered MonkhorstPack grid with $30 \mathrm{k}$-points per $\AA^{-1}$ is used for the selfconsistent calculations [59]. A maximum number of electronic self-consistency steps is given in our calculations, such that a material for which the calculation does not converge within 300 self-consistency loop steps is labeled and discarded. Two itinerant schemes are used in this process: the special Davidson block iteration scheme and Residual minimization method direct inversion in the iterative subspace (RMM-DIIS) 60.

This diagnosis scheme is supposed to find all topological materials that may be diagnosed using symmetry eigenvalues. It is emphasized that some topological materials have the same symmetry data as atomic insulators, and so cannot be found using the scheme. In other words, materials not included in Tables II, III, IV, V] VI may still be topological, but they cannot be identified without using more complicated diagnosis such as Wilson loops. While the mapping from symmetry data to topology data is mathematically rigorous, the validity of generalized gradient approximation depends on the actual material, so that if a material has significant correlation effect at the Fermi energy, the results are likely to be inaccurate. For example, for compounds containing rare-earth elements with possibly partial-filling $f$-orbitals, the strong correlation effect is dominant and we have left them for further detailed study. From experience, we marked out a few elements with color that often induce strong correlation effects in compounds due to partially filled $d$ - or $f$-shell in Tables II, III, IV, V, VI as warning (blue for $d$ and red for $f$ ). Here $\mathrm{Ti}, \mathrm{Y}, \mathrm{Zr}, \mathrm{Nb}, \mathrm{Mo}, \mathrm{La}, \mathrm{Lu}, \mathrm{Hf}, \mathrm{Ta}$, $\mathrm{W}$ and $\mathrm{Pt}$, however, are not marked out because, while having partially filled $d$-shells, they in many known cases do not bring about strong correlation effects.

\section{B. Proof that band crossings in $\mathrm{YCoC}_{2}$ are nodal lines}

Table III shows that compatibility relations for $\mathrm{YCoC}_{2}$ are not satisfied along Z- $\Gamma$, Z-T, Z-Y, and Z-R lines, where the conduction bands and valence bands touch. $\mathrm{Z}-\Gamma$ and $\mathrm{Z}-\mathrm{Y}$ are on the green mirror plane, and Z-R and $\mathrm{Z}-\mathrm{T}$ are on the yellow mirror plane in Fig. 2(g). It is then found that the two intersecting bands have opposite mirror eigenvalues for both mirror planes, implying that the band crossings are in fact nodal lines which lying on the two mirror invariant planes. Specially, since the band crossing point along Z-T is shared by the two mirror planes, the two nodal lines intersect each other at this point. Therefore, there are two nodal rings, on the green and the yellow planes respectively, that are centered at $\mathrm{Z}$, and the two intersect each other at two points along $\mathrm{Z}-\mathrm{T}$ and minus $\mathrm{Z}-\mathrm{T}$, respectively. 


\section{Determine all topological invariants of $\mathrm{ZrTi}_{2} \mathbf{H}_{4}$}

In order to determine the topological invariants of $\mathrm{ZrTi}_{2} \mathrm{H}_{4}$ (space group $F d \overline{3} m$ ), we look up its SI set, which is $\left(z_{2 w, 1}, z_{2 w, 2}, z_{2 w, 3}, z_{4}\right)=(0002)$, in table I of Ref. [7] and find that there are only two possibilities for the invariants for this SI set. In the first case, the mirror Chern number on the $1 \overline{1} 0$ plane in the Brillouin zone (the yellow plane in Fig. 30 is $2(\bmod 4)$, whereas in the second case this mirror Chern number is $0(\bmod 4)$. By ab-initio calculation, as decribed in the next paragraph, we find that this mirror Chern number is 0 and thus $\mathrm{ZrTi}_{2} \mathrm{H}_{4}$ belongs to the second case. In this case, nontrivial TCI invariants include: $(\mathrm{d}, \mathrm{i})$ hourglass invariant protected by glide plane $\left\{m_{001} \mid \frac{1}{4} \frac{1}{4} 0\right\}$, (ii) rotation-invariant protected by rotation $\left\{2_{1 \overline{1} 0} \mid 000\right\}$, (iii) inversion-invariant, (iv) screw-invariant protected by $\left\{4_{001} \mid 0 \frac{1}{4} \frac{1}{4}\right\}$, as well as those invariants protected by symmetries equivalent with above symmetries. All of these invariants are $\mathbb{Z}_{2}$ type and correspond to either two-dimensional or one-dimensional anomalous surface states. Here we propose two real space configurations to detect such surface states. In Fig. 3, we show the one-dimensional helical mode protected by screws and/or inversion. The cubic sample is cut out along 100, 010, and 001 surfaces, all of which are fully gapped. As long as the cubic preserves inversion symmetry there must be an inversion-symmetric one-dimensional helical mode on the boundary, whose shape depends on the experimental situation. However, in presence of the four-fold screw symmetries, which protect nontrivial screw-invariants, the shape of helical mode is further constrained. We consider the sample is large enough such that the four-fold screw symmetry, $\left\{4_{001} \mid 0 \frac{1}{4} \frac{1}{4}\right\}$, is preserved on the side surfaces far away from the top and bottom surfaces. Then, due to discussion in Ref. [7, 29], four one-dimensional helical modes run along the screw axis and transform to each other in turn under the screw operation. Similarly, along any equivalent screw axis there also exist four onedimensional helical modes. The helical mode shown in Fig. 3 is a configuration satisfying all the above symmetry conditions. In Fig. 3, we show the two-dimensional surface states protected by glide and/or two-fold rotation symmetries. The sample is cut out along $110,1 \overline{1} 0$, and 001 surfaces, wherein the 001 surface is fully gapped whereas the $1 \overline{1} 0$ and 110 surfaces are gapless. Due to the hourglass-invariant protected by $\left\{m_{001} \mid \frac{1}{4} \frac{1}{4} 0\right\}$ symmetry the $1 \overline{1} 0$ surface must have a hourglass mode, and due to the rotation-invariant protected by $\left\{2_{1 \overline{1} 0} \mid 000\right\}$ the $1 \overline{1} 0$ surface must have $2(\bmod 4)$ Dirac nodes. In fact, the two kinds of surface states are consistent with each other: at an even filling number, which is necessary for insulator in presence of time-reversal, the two hourglass crossings protected by glide symmetry also play the role of Dirac nodes for the rotation-invariant. Therefore the $1 \overline{1} 0$ surface has a $C_{2}$-symmetric hourglass mode. The 110 surface has a similar surface state since it is equivalent with $1 \overline{1} 0$ surface.

Now let us briefly describe how we calculate the mir-

\begin{tabular}{c|ccccccccccccc}
\hline $\mathbb{Z}_{2,2,2,4}$ & weak & $m_{(2)}^{101}$ & $g_{\frac{1}{4} \frac{1}{4} 0}^{001}$ & $g_{\frac{1}{4} \frac{1}{4} 0}^{001}$ & $g_{\frac{1}{4} \frac{1}{2} \frac{1}{4}}^{101}$ & $2^{001}$ & $2^{011}$ & $i$ & $2_{1}^{001}$ & $2_{1}^{011}$ & $4_{1}^{001}$ & $4_{3}^{001}$ & $\overline{4}^{001}$ \\
\hline 0000 & 000 & 0 & 0 & 0 & 0 & 0 & 0 & 0 & 0 & 0 & 0 & 0 & 0 \\
0000 & 000 & 2 & 0 & 0 & $\mathbb{1}$ & 0 & $\mathbb{1}$ & 0 & 0 & $\mathbb{1}$ & $\mathbb{1}$ & $\mathbb{1}$ & $\mathbb{1}$ \\
0002 & 000 & 0 & $\mathbb{1}$ & $\mathbb{1}$ & 0 & 0 & $\mathbb{1}$ & $\mathbb{1}$ & 0 & $\mathbb{1}$ & $\mathbb{1}$ & $\mathbb{1}$ & 0 \\
0002 & 000 & 2 & $\mathbb{1}$ & $\mathbb{1}$ & $\mathbb{1}$ & 0 & 0 & $\mathbb{1}$ & 0 & 0 & 0 & 0 & $\mathbb{1}$ \\
\hline
\end{tabular}

Table I. Possible invariants for SG 227.

ror Chern number ab-initioly. First, the parallelogram spaned by $\mathbf{G}_{1}$ and $\mathbf{G}_{2}$ shown in Fig. 3 is recognized as the minimal periodic cell in the mirror plane, wherein $\mathbf{G}_{1}$ is along 110-direction and $\mathbf{G}_{2}$ is along 111-direction. We therefore calculate the mirror Chern number within this parallelogram. For each point along the $\Gamma \mathbf{G}_{1}$-line, $k \mathbf{G}_{1}$, we define a Wilson loop matrix as

$$
\begin{aligned}
W_{n n^{\prime}}(k)= & \sum_{n_{1} \cdots n_{N-1} \in o c c}\left\langle u_{n, k \mathbf{G}_{1}} \mid u_{n_{1}, k \mathbf{G}_{1}+\frac{\mathbf{G}_{2}}{N}}\right\rangle\left\langle u_{n_{1}, k \mathbf{G}_{1}+\frac{\mathbf{G}_{2}}{N} \mid \cdots}\right. \\
& \times\left|u_{n_{N-1}, k \mathbf{G}_{1}+(N-1) \frac{\mathbf{G}_{2}}{N}}\right\rangle\left\langle u_{n_{N-1}, k \mathbf{G}_{1}+(N-1) \frac{\mathbf{G}_{2}}{N} \mid}\right. \\
& \hat{V}^{\mathbf{G}_{2}}\left|u_{n_{N-1}, k \mathbf{G}_{1}}\right\rangle
\end{aligned}
$$

where $N$ is a large enough integer to describe the infinite limit $N \rightarrow \infty,\left|u_{n, \mathbf{k}}\right\rangle$ the periodic part of the Bloch wave-function, $n, n^{\prime}, n_{i}(i=1 \cdots N-1)$ the occupied band indices, and $\hat{V}^{\mathbf{G}_{2}}$ the embedding operator 61. On the other hand, for each $k \mathbf{G}_{1}$ we can define the mirror representation matrix as

$$
M_{n n^{\prime}}(k)=\left\langle u_{n, k \mathbf{G}_{1}}|\hat{M}| u_{n^{\prime}, k \mathbf{G}_{1}}\right\rangle,
$$

where $\hat{M}$ is the operator of symmetry operation $\left\{m_{1 \overline{1} 0} \mid 000\right\}$. Since each $k$-point in Eq. (1) is mirrorinvariant, one can prove that $M(k)$ always commutes with $W(k)$. Therefore, we can project the Wilson loop matrix into the subspace having mirror eigenvalue $+i$, then the mirror Chern number is just given by the winding number of the projected Wilson loop. In Fig. 3, we plot the eigenvalues of the projected Wilson loop matrix as a function of $k$, from which one can find that the winding number is 0 . 


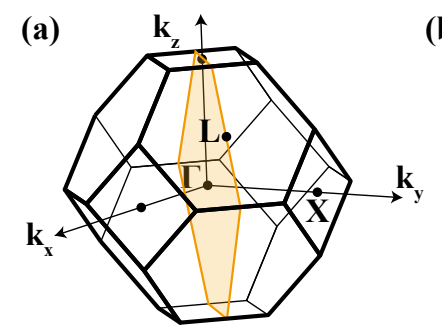

(c)

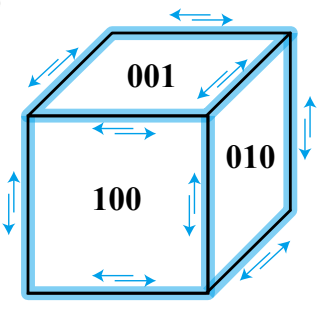

(b)

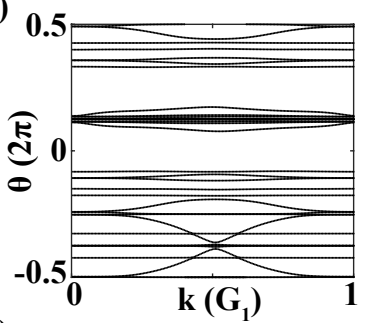

(d)

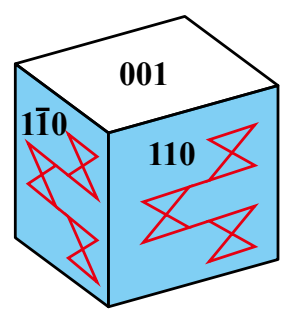

Figure 3. (a) Brillouin zone for $\mathrm{ZrTi}_{2} \mathrm{H}_{4}$, in which the yellow plane is $m_{1 \overline{1} 0}$. (b) Wilson loop for $\mathrm{ZrTi}_{2} \mathrm{H}_{4}$ in the $m_{1 \overline{1} 0}$ plane. (c) One-dimensional helical modes in a cubic $\mathrm{ZrTi}_{2} \mathrm{H}_{4}$ sample. (d) Two-dimensional surface states on each surface of a cubic $\mathrm{ZrTi}_{2} \mathrm{H}_{4}$ sample. 


\section{SUPPLEMENTARY MATERIALS: TABLES OF TOPOLOGICAL MATERIALS}

Here we have listed all topological materials identified in our scanning of the database, with different types of materials given in different tables. We note that the definition of indicators in Tables IV, V], VI follows Ref [7, 8, and the notations of irreducible representations of double space groups are the same as those on the BCS. We make a description for the symbols in the tables below:

- In Table II symbols in the second column represent irreducible representations. For example, in symbol $\bar{C}_{2} \bar{C}_{2}(4)$, $C$ is the name of the high symmetry point, $\bar{C}_{2}$ is the name of the irreducible representation defined in the BCS, the overbar means that this is a double group irreducible representation, and 4 in parenthesis denotes the dimension of the irreducible representation. Two irreducible representations written together indicate that the time-reversal symmetry enforces them to be degenerate with doubled dimension.

- In Table III the second column lists all the high symmetry lines that violate compatibility relationships, which reveal the existance of band crossing. The band crossings can be isolated points, parts of nodal lines and nodal surface, which correspond to Weyl/Dirac semimetals, nodal line semimetals and nodal surface semimetals, respectively.

- In Table IV, V, VI, some indicator groups are different from those in Ref [7, 8]. For example, indicator group for SG 13 is $\mathbb{Z}_{2} \times \mathbb{Z}_{2}$ in the nsoc-setting in Ref [8], but is $\mathbb{Z}_{2} \times \mathbb{Z}_{2} \times \mathbb{Z}_{2} \times \mathbb{Z}_{4}$ in our table, which is the indicator of SG 2 in Ref [8]. This is because SG 13, which is a supergroup of SG 2, has additional symmetries that annihilate two of the three $\mathbb{Z}_{2}$ generators, forcing them to vanish. This annihilation of weak generators occur in many other space groups, but it is far from obvious which generators are killed, because it depends on the specific space group considered: some times one of the three must vanish and some times two must be equal, etc.. Therefore, we choose to list all three indices for any centrosymmetric space group, and remind the readers that they are not all independent.

For topological semimetals, including high-symmetry point semimetals, high-symmetry line semimetals and generic momenta semimetals, the ideal Fermi surfaces only consist of nodal points. However, in reality these materials are also compensate semimetals, having electron and hole pockets that are non-topological. Due to the linear crossings at topological nodes, the density of states of topological Fermi surface is expected to be smaller compared with that of a normal Fermi surface. Hence, topological semimetals are considered "good" when the density of states at the Fermi energy is small. For readers to find good materials more easily, we order the topological semimetals in the following ways:

- In Table [I] materials having the same space group and the same partially filled high-symmetry points are ordered, from low to high, by their densities of states at the Fermi energy, and we insert "||" between materials having density of states lower and higher than $0.5 \mathrm{eV}^{-1}$ per unit cell.

- In Table III materials having the same space group and the same high-symmetry lines where band crossings appear are ordered, from low to high, by their densities of states at the Fermi energy, and we insert "|l" in each entry between materials having density of states lower and higher than $0.5 \mathrm{eV}^{-1}$ per unit cell.

- In Table IV, materials having the same space group and the same symmetry-based indicators are ordered, from low to high, by their densities of states at the Fermi energy, and we insert "|" between materials having density of states lower and higher than $0.5 \mathrm{eV}^{-1}$ per unit cell.

Ideal topological insulators and topological crystalline insulators have a charge gap, but well-defined band topology only requires a full direct gap at each momentum. In fact, many materials discovered in this class have vanishing indirect gaps. In order to give an idea how "ideal" one candidate material is, for each material in Table V and Table VI. we have computed the density of states at the Fermi level (not explicitly shown), and by that information order the materials as follows:

- In Table $\mathrm{V}$, materials having the same space group and the same symmetry-based indicators are ordered, from low to high, by their densities of states at the Fermi energy, and we insert "||" between materials having zero density of states and the rest.

- In Table VI, materials having the same space group and the same symmetry-based indicators are ordered, from low to high, by their densities of states at the Fermi energy, and we insert "||" between materials having zero density of states and the rest. 
In the Tables, if one material has been predicted as topological in a previous publication, the paper is cited immediately after the material formula. Also, if a material formula corresponds to more than one crystal structures in the same space group, we append its ICSD number after it for clarity. Some partially filled $d$ - or $f$-shell elements are colored in blue and red respectively in the tables below, as mentioned in Sec. IV A.

Table II: List of High Symmetry Point Semimetal

\begin{tabular}{|c|c|c|}
\hline \multicolumn{3}{|c|}{$\begin{array}{c}\text { List of High Symmetry Point Semimetal } \\
\end{array}$} \\
\hline $\begin{array}{l}\text { SOC or } \\
\text { NSOC }\end{array}$ & $\begin{array}{c}\text { Degenerate Irreducible } \\
\text { Representations }\end{array}$ & High Symmetry Point Semimetals \\
\hline \multicolumn{3}{|r|}{ Space Group 7: $P c$} \\
\hline NSOC & $\begin{array}{l}\mathrm{A}_{1} \mathrm{~A}_{2}(2), \mathrm{B}_{1} \mathrm{~B}_{2}(2), \\
\mathrm{D}_{1} \mathrm{D}_{2}(2), \mathrm{E}_{1} \mathrm{E}_{2}(2)\end{array}$ & $\| \mathrm{ReN}_{2}, \mathrm{Ga}_{9} \mathrm{Rh}_{2}, \mathrm{Ga}_{9} \mathrm{Ir}_{2}, \mathrm{La}_{14} \mathrm{C}_{14} \mathrm{Cl}_{9}$ \\
\hline \multicolumn{3}{|r|}{ Space Group 9: $C c$} \\
\hline NSOC & $\mathrm{A}_{1} \mathrm{~A}_{2}(2), \mathrm{M}_{1} \mathrm{M}_{2}(2)$ & $\mathrm{CeCoC}_{2}$ \\
\hline \multicolumn{3}{|r|}{ Space Group 11: $P 2_{1} / m$} \\
\hline NSOC & $\mathrm{C}_{1}(2), \mathrm{D}_{1}(2), \mathrm{E}_{1}(2), \mathrm{Z}_{1}(2)$ & $\begin{array}{c}\mathrm{TlCuHSeO}_{5}, \| \mathrm{LiBe}, \mathrm{H}_{3} \mathrm{~W}(\mathrm{ICSD}: 247596), \mathrm{H}_{3} \mathrm{~W}(\mathrm{ICSD}: 247600), \\
\mathrm{ReN}_{2}, \mathrm{TaS}_{3}, \mathrm{Li}_{7} \mathrm{Sn}_{3}, \mathrm{ZrCu}, \mathrm{Sb}(\mathrm{MoS})_{2}, \mathrm{LaSi}_{2} \mathrm{Ru}, \mathrm{NbSe}_{3}, \mathrm{NdSi}_{2} \mathrm{Ru}, \\
\mathrm{TaNi}_{2} \mathrm{Te}_{3}, \mathrm{PrSi}_{2} \mathrm{Ru}, \mathrm{ZrRh}_{\mathrm{H}} \mathrm{Hi} \mathrm{HiP}_{2} \mathrm{Ni} \mathrm{Zr}_{2} \mathrm{NiP}_{3} \mathrm{Y}_{3} \mathrm{IrI}_{3}, \mathrm{KYbSiS}_{4}, \mathrm{Bi}_{9} \mathrm{I}_{2}, \\
\mathrm{Y}_{3}\left(\mathrm{Ge}_{3} \mathrm{Pt}_{2}\right)_{2}, \mathrm{La}_{3} \mathrm{BCCl}_{3}, \mathrm{CaCu}, \mathrm{HfIr}, \mathrm{Sm}_{3} \mathrm{Ti}_{3}\left(\mathrm{SeO}_{4}\right)_{2}, \mathrm{Rb}_{9} \mathrm{O}_{2}, \mathrm{YbP}_{5}, \\
\mathrm{TaFeTe}_{3}, \mathrm{HgSBr}_{\mathrm{H}} \mathrm{Cu}_{6} \mathrm{AgBi}_{12} \mathrm{PbS}_{22}, \mathrm{La}_{2} \mathrm{Ni}_{12} \mathrm{P}_{5}, \mathrm{Ta}_{3} \mathrm{Te}_{14} \mathrm{Pd}_{3}, \\
\mathrm{Ca}_{4} \mathrm{P}_{7} \mathrm{Ir}_{8}, \mathrm{Ca}_{4} \mathrm{As}_{7} \mathrm{Ir}_{8}, \mathrm{ZrCr}_{5} \mathrm{P}_{3}, \mathrm{UCr}_{5} \mathrm{P}_{3}\end{array}$ \\
\hline $\mathrm{SOC}$ & $\begin{array}{l}\overline{\mathrm{C}}_{2} \overline{\mathrm{C}}_{2}(4), \overline{\mathrm{D}}_{2} \overline{\mathrm{D}}_{2}(4) \\
\overline{\mathrm{E}}_{2} \overline{\mathrm{E}}_{2}(4), \overline{\mathrm{Z}}_{2} \overline{\mathrm{Z}}_{2}(4)\end{array}$ & $\begin{array}{c}\| \mathrm{TlCuHSeO}_{5}, \mathrm{LiBe}, \mathrm{H}_{3} \mathrm{~W}(\mathrm{ICSD}: 247596), \mathrm{H}_{3} \mathrm{~W}(\mathrm{ICSD}: 247600) \\
\mathrm{ReN}_{2}, \mathrm{TaS}_{3}, \mathrm{Li}_{7} \mathrm{Sn}_{3}, \mathrm{ZrCu}, \mathrm{Sb}(\mathrm{MoS})_{2}, \mathrm{NbSe}, \mathrm{LaSi}_{2} \mathrm{Ru}, \mathrm{NdSi}_{2} \mathrm{Ru}, \\
\mathrm{PrSi}_{2} \mathrm{Ru}, \mathrm{ZrRh}_{2} \mathrm{TaNi}_{2} \mathrm{Te}_{3}, \mathrm{Zr}_{2} \mathrm{NiP}, \mathrm{Hf}_{2} \mathrm{NiP}, \mathrm{KYbSiS}_{4}, \mathrm{Bi}_{9} \mathrm{I}_{2}, \mathrm{Y}_{3} \mathrm{IrI}_{3}, \\
\mathrm{Y}_{3}\left(\mathrm{Ge}_{3} \mathrm{Pt}_{2}\right)_{2}, \mathrm{La}_{3} \mathrm{BCCl}_{3}, \mathrm{CaCu}, \mathrm{YbP}_{5}, \mathrm{TaFeTe}_{3}, \mathrm{La}_{2} \mathrm{Ni}_{12} \mathrm{P}_{5} \\
\mathrm{Ta}_{3} \mathrm{Te}_{14} \mathrm{Pd}_{3}, \mathrm{Ca}_{4} \mathrm{P}_{7} \mathrm{Ir}_{8}, \mathrm{Ca}_{4} \mathrm{As}_{7} \mathrm{Ir}_{8}, \mathrm{HgSBr}, \mathrm{ZrCr}_{5} \mathrm{P}_{3}\end{array}$ \\
\hline \multicolumn{3}{|r|}{ Space Group 13: $P 2 / c$} \\
\hline NSOC & $\mathrm{A}_{1}(2), \mathrm{B}_{1}(2), \mathrm{D}_{1}(2), \mathrm{E}_{1}(2)$ & $\| \mathrm{ReN}_{2}, \mathrm{YbTaO}_{4}, \mathrm{~K}_{2} \mathrm{Mn}_{3} \mathrm{~S}_{4}, \mathrm{~K}\left(\mathrm{~V}_{5} \mathrm{~S}_{8}\right)_{2}$ \\
\hline $\mathrm{SOC}$ & $\begin{array}{l}\overline{\mathrm{A}}_{2} \overline{\mathrm{A}}_{2}(4), \overline{\mathrm{B}}_{2} \overline{\mathrm{B}}_{2}(4) \\
\overline{\mathrm{D}}_{2} \overline{\mathrm{D}}_{2}(4), \overline{\mathrm{E}}_{2} \overline{\mathrm{E}}_{2}(4)\end{array}$ & $\| \mathrm{ReN}_{2}, \mathrm{YbTaO}_{4}$ \\
\hline \multicolumn{3}{|r|}{ Space Group 14: $P 2_{1} / c$} \\
\hline \multirow{5}{*}{ NSOC } & $\begin{array}{c}\mathrm{A}_{1}(2), \mathrm{B}_{1}(2), \mathrm{C}_{1}(2) \\
\mathrm{D}_{1}^{-} \mathrm{D}_{2}^{-}(2), \mathrm{E}_{1}^{-} \mathrm{E}_{2}^{-}(2), \mathrm{Z}_{1}(2)\end{array}$ & $\begin{array}{c}\| \mathrm{Al}_{9} \mathrm{Ir}_{2}, \mathrm{Al}_{9} \mathrm{Rh}_{2}, \mathrm{Al}_{9} \mathrm{Co}_{2}, \mathrm{Sr}_{2} \mathrm{CeIrO}_{6}, \mathrm{La}_{2} \mathrm{ZnRhO}_{6}, \mathrm{Sr}_{2} \mathrm{HoRuO}_{6}, \\
\mathrm{La}_{2} \mathrm{MgIrO}_{6}, \mathrm{La}_{2} \mathrm{MgRhO}_{6}, \mathrm{Sr}_{2} \mathrm{CaIrO}_{6}, \mathrm{Pu}_{2} \mathrm{Si}_{4} \mathrm{Tc}_{3}, \mathrm{Sr}_{2} \mathrm{LuRuO}_{6}, \\
\mathrm{Ca}_{3} \mathrm{ReO}_{6}\end{array}$ \\
\hline & $\begin{array}{c}\mathrm{A}_{1}(2), \mathrm{B}_{1}(2), \mathrm{C}_{1}(2) \\
\mathrm{D}_{1}^{-} \mathrm{D}_{2}^{-}(2), \mathrm{E}_{1}^{+} \mathrm{E}_{2}^{+}(2), \mathrm{Z}_{1}(2)\end{array}$ & $\| \mathrm{IrC}_{4}, \mathrm{UCuO}_{4}, \mathrm{Lu}_{5}\left(\mathrm{Co}_{2} \mathrm{Si}_{7}\right)_{2}, \mathrm{Y}_{5}\left(\mathrm{Si}_{7} \mathrm{Os}_{2}\right)_{2}, \mathrm{La}_{15} \mathrm{~B}_{14} \mathrm{C}_{19}, \operatorname{Ir}\left(\mathrm{Cl}_{2} \mathrm{~F}_{3}\right)_{2}$ \\
\hline & $\begin{array}{c}\mathrm{A}_{1}(2), \mathrm{B}_{1}(2), \mathrm{C}_{1}(2) \\
\mathrm{D}_{1}^{+} \mathrm{D}_{2}^{+}(2), \mathrm{E}_{1}^{-} \mathrm{E}_{2}^{-}(2), \mathrm{Z}_{1}(2)\end{array}$ & $\begin{array}{c}\mathrm{Ag}_{3} \mathrm{O}_{4}, \mathrm{Cu}_{5} \mathrm{Se}_{2}\left(\mathrm{ClO}_{4}\right)_{2}, \mathrm{La}_{8} \mathrm{C}_{8} \mathrm{Cl}_{5}, \mathrm{~Tb}_{12} \mathrm{Co}_{7}, \mathrm{Er}_{12} \mathrm{Co}_{7}, \mathrm{Dy}_{12} \mathrm{Co}_{7} \\
\mathrm{Ho}_{12} \mathrm{Co}_{7}, \mathrm{Sr}_{2} \mathrm{ZnReO}_{6} \\
\end{array}$ \\
\hline & $\begin{array}{c}\mathrm{A}_{1}(2), \mathrm{B}_{1}(2), \mathrm{C}_{1}(2), \\
\mathrm{D}_{1}^{+} \mathrm{D}_{2}^{+}(2), \mathrm{E}_{1}^{+} \mathrm{E}_{2}^{+}(2), \mathrm{Z}_{1}(2)\end{array}$ & $\mathrm{CuH}_{2}\left(\mathrm{SeO}_{3}\right)_{2}, \| \mathrm{AgF}_{2}, \mathrm{CuF}_{2}, \mathrm{Sc}\left(\mathrm{ReO}_{3}\right)_{2}, \mathrm{La}_{20} \mathrm{C}_{20} \mathrm{Cl}_{13}$ \\
\hline & $\mathrm{A}_{1}(2), \mathrm{C}_{1}(2)$ & $\| \mathrm{Eu}_{2} \mathrm{SiO}_{4}$ \\
\hline $\mathrm{SOC}$ & $\begin{array}{l}\overline{\mathrm{A}}_{2} \overline{\mathrm{A}}_{2}(4), \overline{\mathrm{B}}_{2} \overline{\mathrm{B}}_{2}(4) \\
\overline{\mathrm{C}}_{2} \overline{\mathrm{C}}_{2}(4), \overline{\mathrm{Z}}_{2} \overline{\mathrm{Z}}_{2}(4)\end{array}$ & $\begin{array}{c}\| \mathrm{CuF}_{2}, \mathrm{IrC}_{4}, \mathrm{Al}_{9} \mathrm{Ir}_{2}, \mathrm{Al}_{9} \mathrm{Rh}_{2}, \mathrm{Al}_{9} \mathrm{Co}_{2}, \mathrm{Sc}\left(\mathrm{ReO}_{3}\right)_{2}, \mathrm{Ag}_{3} \mathrm{O}_{4}, \\
\mathrm{Y}_{5}\left(\mathrm{Si}_{7} \mathrm{Os}_{2}\right)_{2}, \mathrm{Cu}_{5} \mathrm{Se}_{2}\left(\mathrm{ClO}_{4}\right)_{2}, \mathrm{La}_{8} \mathrm{C}_{8} \mathrm{Cl}_{5}, \mathrm{Pu}_{2} \mathrm{Si}_{4} \mathrm{Tc}_{3}, \mathrm{Ir}\left(\mathrm{Cl}_{2} \mathrm{~F}_{3}\right)_{2}, \\
\mathrm{La}_{15} \mathrm{~B}_{14} \mathrm{C}_{19}, \mathrm{La}_{20} \mathrm{C}_{20} \mathrm{Cl}_{13}, \mathrm{La}_{2} \mathrm{ZnRhO}_{6}, \mathrm{Sr}_{2} \mathrm{CeIrO}_{6}, \mathrm{La}_{2} \mathrm{MgIrO}_{6}, \\
\mathrm{La}_{2} \mathrm{MgRhO}_{6}, \mathrm{CuH}_{2}\left(\mathrm{SeO}_{3}\right)_{2}, \mathrm{Sr}_{2} \mathrm{HoRuO}_{6}, \mathrm{Sr}_{2} \mathrm{ZnReO}_{6}\end{array}$ \\
\hline \multicolumn{3}{|r|}{ Space Group 15: $C 2 / c$} \\
\hline NSOC & $\mathrm{A}_{1}(2), \mathrm{M}_{1}(2)$ & 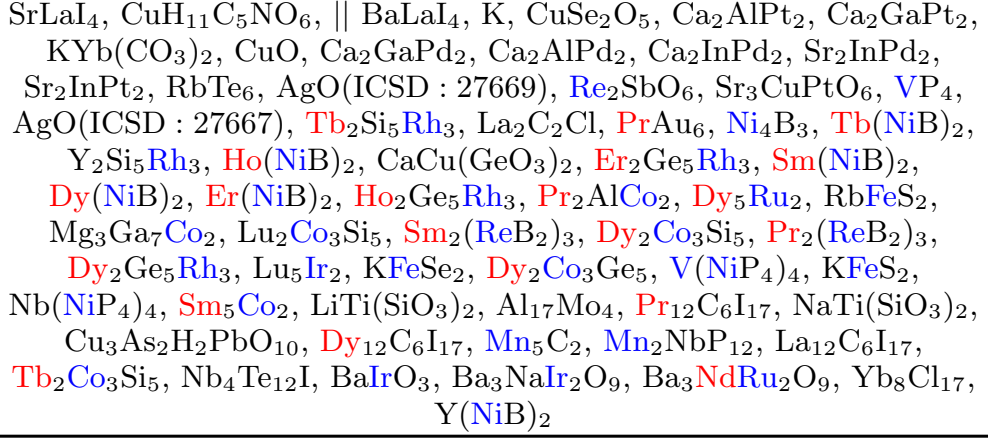 \\
\hline
\end{tabular}


Table II - continued

\begin{tabular}{|c|c|c|}
\hline $\begin{array}{l}\text { SOC or } \\
\text { NSOC }\end{array}$ & $\begin{array}{l}\text { Degenerate Irreducible } \\
\text { Representations }\end{array}$ & High Symmetry Point Semimetals \\
\hline $\mathrm{SOC}$ & $\overline{\mathrm{A}}_{2} \overline{\mathrm{A}}_{2}(4), \overline{\mathrm{M}}_{2} \overline{\mathrm{M}}_{2}(4)$ & 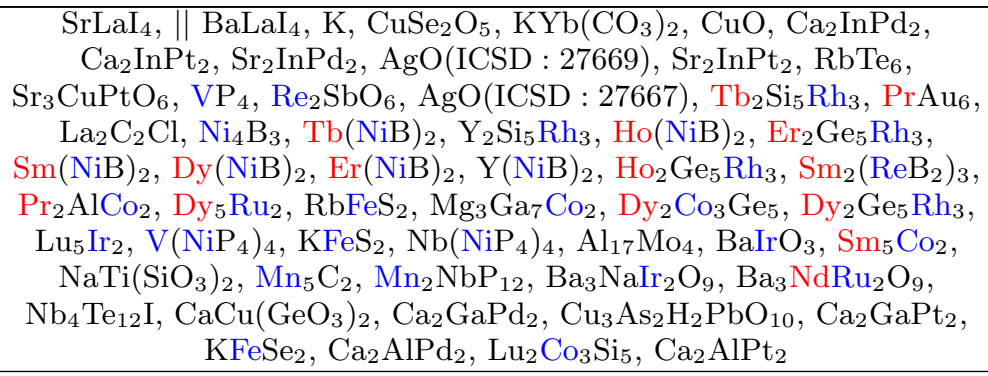 \\
\hline \multicolumn{3}{|r|}{ Space Group 18: $P 2_{1} 2_{1} 2$} \\
\hline NSOC & $\begin{array}{l}\mathrm{R}_{1} \mathrm{R}_{2}(2), \mathrm{S}_{1} \mathrm{~S}_{2}(2), \mathrm{T}_{1}(2) \\
\quad \mathrm{U}_{1}(2), \mathrm{X}_{1}(2), \mathrm{Y}_{1}(2)\end{array}$ & $\| \mathrm{Na}_{4} \mathrm{Nb}_{9}\left(\mathrm{P}_{2} \mathrm{O}_{13}\right)_{3}$ \\
\hline \multicolumn{3}{|r|}{ Space Group 19: $P 2_{1} 2_{1} 2_{1}$} \\
\hline NSOC & $\mathrm{R}_{1} \mathrm{R}_{1}(4)$ & $\mathrm{CuP}(\mathrm{HO})_{5}, \| \mathrm{ThP}_{7}$ \\
\hline \multicolumn{3}{|r|}{ Space Group 20: $C 222_{1}$} \\
\hline NSOC & $\mathrm{R}_{1} \mathrm{R}_{2}(2), \mathrm{T}_{1}(2), \mathrm{Z}_{1}(2)$ & (2) \\
\hline \multicolumn{3}{|r|}{ Space Group 26: $P m c 2_{1}$} \\
\hline NSOC & $\begin{array}{l}\mathrm{R}_{1} \mathrm{R}_{3}(2), \mathrm{T}_{1} \mathrm{~T}_{3}(2) \\
\mathrm{U}_{1} \mathrm{U}_{3}(2), \mathrm{Z}_{1} \mathrm{Z}_{3}(2)\end{array}$ & $\| \mathrm{KCuCO}_{3} \mathrm{~F}$ \\
\hline $\mathrm{SOC}$ & $\begin{array}{l}\overline{\mathrm{R}}_{5} \overline{\mathrm{R}}_{5}(4), \overline{\mathrm{T}}_{5} \overline{\mathrm{T}}_{5}(4) \\
\overline{\mathrm{U}}_{5} \overline{\mathrm{U}}_{5}(4), \overline{\mathrm{Z}}_{5} \overline{\mathrm{Z}}_{5}(4)\end{array}$ & \multirow[t]{2}{*}{$\| \mathrm{KCuCO}_{3} \mathrm{~F}$} \\
\hline \multicolumn{2}{|c|}{ Space Group 28: Pma2 } & \\
\hline NSOC & $\mathrm{R}_{1}(2), \mathrm{S}_{1}(2), \mathrm{U}_{1}(2), \mathrm{X}_{1}(2)$ & $\mathrm{H}_{5} \mathrm{~W}$ \\
\hline \multicolumn{3}{|r|}{ Space Group 31: Pmn2 } \\
\hline \multirow[t]{2}{*}{ NSOC } & $\begin{array}{l}\mathrm{R}_{1}(2), \mathrm{S}_{1}(2), \mathrm{T}_{2} \mathrm{~T}_{4}(2) \\
\mathrm{U}_{1}(2), \mathrm{X}_{1}(2), \mathrm{Z}_{2} \mathrm{Z}_{4}(2)\end{array}$ & $\|$ OsN, $\mathrm{Sr}_{5} \mathrm{Nb}_{5} \mathrm{O}_{16}, \mathrm{La}_{5} \mathrm{Ti}_{5} \mathrm{O}_{17}$ \\
\hline & $\begin{array}{l}\mathrm{R}_{1}(2), \mathrm{S}_{1}(2), \mathrm{T}_{1} \mathrm{~T}_{3}(2) \\
\mathrm{U}_{1}(2), \mathrm{X}_{1}(2), \mathrm{Z}_{1} \mathrm{Z}_{3}(2)\end{array}$ & $\| \mathrm{Y}(\mathrm{GePt})_{2}, \mathrm{Nd}(\mathrm{GePt})_{2}, \operatorname{Pr}(\mathrm{GePt})_{2}, \mathrm{La}(\mathrm{GePt})_{2}$ \\
\hline $\mathrm{SOC}$ & $\overline{\mathrm{T}}_{5} \overline{\mathrm{T}}_{5}(4), \overline{\mathrm{Z}}_{5} \overline{\mathrm{Z}}_{5}(4)$ & $\begin{array}{c}\text { OsN, Y(GePt })_{2}, \mathrm{Nd}(\mathrm{GePt})_{2}, \mathrm{Pr}(\mathrm{GePt})_{2}, \mathrm{La}(\mathrm{GePt})_{2}, \mathrm{Sr}_{5} \mathrm{Nb}_{5} \mathrm{O}_{16}, \\
\mathrm{La}_{5} \mathrm{Ti}_{5} \mathrm{O}_{17}, \mathrm{~Tb}(\mathrm{GePt})_{2}\end{array}$ \\
\hline \multicolumn{3}{|r|}{ Space Group 33: Pna2 $2_{1}$} \\
\hline NSOC & $\mathrm{U}_{1} \mathrm{U}_{1}(4)$ & $\| \mathrm{KCu}_{3} \mathrm{~S}_{2} \mathrm{ClO}_{9}, \mathrm{LaYbO}_{3}$ \\
\hline \multicolumn{3}{|r|}{ Space Group 36: $C m c 2_{1}$} \\
\hline \multirow{3}{*}{ NSOC } & $\mathrm{R}_{1} \mathrm{R}_{2}(2), \mathrm{T}_{2} \mathrm{~T}_{4}(2), \mathrm{Z}_{2} \mathrm{Z}_{4}(2)$ & $\| \mathrm{Cu}(\mathrm{HO})_{2}, \mathrm{La}\left(\mathrm{Ni}_{2} \mathrm{~B}\right)_{6}, \mathrm{Cs}_{2} \mathrm{PrO}_{3}$ \\
\hline & $\mathrm{R}_{1} \mathrm{R}_{2}(2), \mathrm{T}_{1} \mathrm{~T}_{3}(2), \mathrm{Z}_{2} \mathrm{Z}_{4}(2)$ & $\| \mathrm{AsPd}_{3} \mathrm{~Pb}_{2}, \mathrm{~Tb}\left(\mathrm{Ni}_{2} \mathrm{~B}\right)_{6}$ \\
\hline & $\mathrm{R}_{1} \mathrm{R}_{2}(2), \mathrm{T}_{1} \mathrm{~T}_{3}(2), \mathrm{Z}_{1} \mathrm{Z}_{3}(2)$ & $\| \mathrm{KN}_{2}, \mathrm{BaCuF}_{4}, \mathrm{NiAs}, \mathrm{Y}_{2} \mathrm{Sn}_{5} \mathrm{Rh}_{3}, \mathrm{Ba}_{2} \operatorname{In}_{13} \operatorname{Ir}_{4}$ \\
\hline $\mathrm{SOC}$ & $\overline{\mathrm{T}}_{5} \overline{\mathrm{T}}_{5}(4), \overline{\mathrm{Z}}_{5} \overline{\mathrm{Z}}_{5}(4)$ & $\begin{array}{c}\| \mathrm{AsPd}_{3} \mathrm{~Pb}_{2}, \mathrm{BaCuF}_{4}, \mathrm{NiAs}, \mathrm{Y}_{2} \mathrm{Sn}_{5} \mathrm{Rh}_{3}, \mathrm{Ba}_{2} \mathrm{In}_{13} \mathrm{Ir}_{4}, \mathrm{La}\left(\mathrm{Ni}_{2} \mathrm{~B}\right)_{6} \\
\mathrm{Cs}_{2} \mathrm{PrO}_{3}, \mathrm{~Tb}\left(\mathrm{Ni}_{2} \mathrm{~B}\right)_{6}\end{array}$ \\
\hline \multicolumn{3}{|r|}{ Space Group 37: Ccc2 } \\
\hline NSOC & $\mathrm{T}_{1} \mathrm{~T}_{2}(2), \mathrm{Z}_{1} \mathrm{Z}_{2}(2)$ & $\mathrm{La}_{8} \mathrm{Nb}_{7} \mathrm{~S}_{22}$ \\
\hline $\mathrm{SOC}$ & $\overline{\mathrm{T}}_{5} \overline{\mathrm{T}}_{5}(4), \overline{\mathrm{Z}}_{5} \overline{\mathrm{Z}}_{5}(4)$ & $\| \mathrm{La}_{8} \mathrm{Nb}_{7} \mathrm{~S}_{22}$ \\
\hline \multicolumn{3}{|r|}{ Space Group 40: $A m a 2$} \\
\hline NSOC & $\mathrm{T}_{1}(2), \mathrm{Z}_{1}(2)$ & $\mathrm{PrTe}_{3}$ \\
\hline \multicolumn{3}{|r|}{ Space Group 43: $F d d 2$} \\
\hline NSOC & $\mathrm{T}_{1}(2), \mathrm{Y}_{1}(2), \mathrm{Z}_{3} \mathrm{Z}_{4}(2)$ & $\begin{array}{c}\| \mathrm{Ba}_{5} \mathrm{P}_{9}, \mathrm{Hf}_{2} \mathrm{Ga}_{3}, \mathrm{Zr}_{2} \mathrm{Al}_{3}, \mathrm{Ho}_{3} \mathrm{Ge}_{5}, \mathrm{Sm}_{3} \mathrm{Ge}_{5}, \mathrm{~Tb}_{3} \mathrm{Ge}_{5}, \mathrm{Dy}_{3} \mathrm{Ge}_{5} \\
\mathrm{Zr}_{2} \mathrm{Ga}_{3}, \mathrm{Y}_{3} \mathrm{Ge}_{5}\end{array}$ \\
\hline $\mathrm{SOC}$ & $\overline{\mathrm{Z}}_{5} \overline{\mathrm{Z}}_{5}(4)$ & $\begin{array}{c}\| \mathrm{Ba}_{5} \mathrm{P}_{9}, \mathrm{Hf}_{2} \mathrm{Al}_{3}, \mathrm{Hf}_{2} \mathrm{Ga}_{3}, \mathrm{Zr}_{2} \mathrm{Al}_{3}, \mathrm{Ho}_{3} \mathrm{Ge}_{5}, \mathrm{Sm}_{3} \mathrm{Ge}_{5}, \mathrm{~Tb}_{3} \mathrm{Ge}_{5}, \mathrm{Y}_{3} \mathrm{Ge}_{5}, \\
\mathrm{Zr}_{2} \mathrm{Ga}_{3}\end{array}$ \\
\hline \multicolumn{3}{|r|}{ Space Group 51: Pmma } \\
\hline \multirow{5}{*}{ NSOC } & $\mathrm{R}_{2}(2), \mathrm{S}_{2}(2), \mathrm{U}_{2}(2), \mathrm{X}_{2}(2)$ & $\| \mathrm{B}_{2} \mathrm{CN}$ \\
\hline & $\mathrm{R}_{2}(2), \mathrm{S}_{2}(2), \mathrm{U}_{1}(2), \mathrm{X}_{1}(2)$ & $\mathrm{Y}_{7} \mathrm{C}_{3} \mathrm{I}_{6} \mathrm{O}$ \\
\hline & $\mathrm{R}_{2}(2), \mathrm{S}_{1}(2), \mathrm{U}_{2}(2), \mathrm{X}_{1}(2)$ & $\|$ MoRh \\
\hline & $\mathrm{R}_{2}(2), \mathrm{S}_{1}(2), \mathrm{U}_{1}(2), \mathrm{X}_{1}(2)$ & $\| \mathrm{NbPt}$ \\
\hline & $\mathrm{R}_{1}(2), \mathrm{S}_{2}(2), \mathrm{U}_{2}(2), \mathrm{X}_{1}(2)$ & $\mathrm{CaIn}_{4} \mathrm{Rh}, \mathrm{YbIn}_{4} \mathrm{Rh}, \mathrm{CaIn}_{4} \mathrm{Ir}$ \\
\hline
\end{tabular}


Table II - continued

\begin{tabular}{|c|c|c|}
\hline $\begin{array}{l}\text { SOC or } \\
\text { NSOC }\end{array}$ & $\begin{array}{c}\text { Degenerate Irreducible } \\
\text { Representations }\end{array}$ & High Symmetry Point Semimetals \\
\hline & $\mathrm{R}_{1}(2), \mathrm{S}_{2}(2), \mathrm{U}_{1}(2), \mathrm{X}_{1}(2)$ & \begin{tabular}{l||l|l} 
& HfIr \\
\end{tabular} \\
\hline & $\mathrm{R}_{1}(2), \mathrm{S}_{1}(2), \mathrm{U}_{2}(2), \mathrm{X}_{1}(2)$ & $\|$ IrW, MoIr, $\mathrm{SrIn}_{4} \mathrm{Ir}, \mathrm{BaIn}_{4} \mathrm{Ir}, \mathrm{TiFeH}$ \\
\hline & $\mathrm{R}_{1}(2), \mathrm{S}_{1}(2), \mathrm{U}_{1}(2), \mathrm{X}_{1}(2)$ & $\| \mathrm{CdAu}, \mathrm{VPt}, \mathrm{Nb}_{2} \mathrm{P}$ \\
\hline $\mathrm{SOC}$ & $\overline{\mathrm{R}}_{3} \overline{\mathrm{R}}_{4}(4), \overline{\mathrm{S}}_{3} \overline{\mathrm{S}}_{4}(4), \overline{\mathrm{U}}_{3} \overline{\mathrm{U}}_{4}(4)$, & $\begin{array}{l}\mathrm{B}_{2} \mathrm{CN}, \mathrm{CdAu}, \mathrm{HfIr}, \mathrm{IrW}, \mathrm{YbIn}_{4} \mathrm{Rh}, \mathrm{CaIn}_{4} \mathrm{Rh}, \mathrm{MoIr}, \mathrm{NbPt}, \mathrm{CaIn}_{4} \mathrm{Ir}, \\
\quad \mathrm{MoRh}, \mathrm{SrIn}_{4} \operatorname{Ir}, \mathrm{BaIn}_{4} \mathrm{Ir}, \mathrm{VPt}, \mathrm{TiFeH}, \mathrm{Y}_{7} \mathrm{C}_{3} \mathrm{I}_{6} \mathrm{O}, \mathrm{Nb}_{2} \mathrm{P} \\
\end{array}$ \\
\hline & & Space Group 52: Pnna \\
\hline NSOC & $\mathrm{S}_{1} \mathrm{~S}_{2}(4)$ & $\mathrm{Ag}_{2} \mathrm{BiO}_{3}, \| \mathrm{Sr}_{2} \mathrm{Bi}_{3}$ \\
\hline \multirow{21}{*}{ NSOC } & & Space Group 55: Pbam \\
\hline & $\begin{array}{c}\mathrm{R}_{3}^{-} \mathrm{R}_{4}^{-}(2), \mathrm{S}_{3}^{-} \mathrm{S}_{4}^{-}(2), \mathrm{T}_{2}(2) \\
\mathrm{U}_{1}(2), \mathrm{X}_{2}(2), \mathrm{Y}_{2}(2)\end{array}$ & $\| \mathrm{Ge}_{3} \mathrm{Rh}_{5}, \mathrm{Si}_{3} \mathrm{Rh}_{5}$ \\
\hline & $\begin{array}{c}\mathrm{R}_{3}^{-} \mathrm{R}_{4}^{-}(2), \mathrm{S}_{3}^{-} \mathrm{S}_{4}^{-}(2), \mathrm{T}_{1}(2) \\
\mathrm{U}_{2}(2), \mathrm{X}_{1}(2), \mathrm{Y}_{2}(2)\end{array}$ & $\| \mathrm{Nd}_{2} \mathrm{InNi}_{2}$ \\
\hline & $\begin{array}{c}\mathrm{R}_{3}^{-} \mathrm{R}_{4}^{-}(2), \mathrm{S}_{1}^{-} \mathrm{S}_{2}^{-}(2), \mathrm{T}_{2}(2) \\
\mathrm{U}_{1}(2), \mathrm{X}_{2}(2), \mathrm{Y}_{2}(2)\end{array}$ & $\| \mathrm{Ti}_{3} \mathrm{Rh}_{5}$ \\
\hline & $\begin{array}{c}\mathrm{R}_{3}^{-} \mathrm{R}_{4}^{-}(2), \mathrm{S}_{1}^{-} \mathrm{S}_{2}^{-}(2), \mathrm{T}_{1}(2) \\
\mathrm{U}_{1}(2), \mathrm{X}_{2}(2), \mathrm{Y}_{2}(2)\end{array}$ & $\| \mathrm{Ca}_{9} \mathrm{Zn}_{4} \mathrm{Sb}_{9}, \mathrm{Ca}_{9} \mathrm{Cd}_{4} \mathrm{Bi}_{9}, \mathrm{Sr}_{9} \mathrm{Cd}_{4} \mathrm{Bi}_{9}$ \\
\hline & $\begin{array}{c}\mathrm{R}_{3}^{-} \mathrm{R}_{4}^{-}(2), \mathrm{S}_{1}^{+} \mathrm{S}_{2}^{+}(2), \mathrm{T}_{2}(2) \\
\mathrm{U}_{1}(2), \mathrm{X}_{2}(2), \mathrm{Y}_{2}(2)\end{array}$ & $\| \mathrm{Ta}_{4} \mathrm{BTe}_{8}$ \\
\hline & $\begin{array}{c}\mathrm{R}_{3}^{-} \mathrm{R}_{4}^{-}(2), \mathrm{S}_{1}^{+} \mathrm{S}_{2}^{+}(2), \mathrm{T}_{2}(2) \\
\mathrm{U}_{1}(2), \mathrm{X}_{1}(2), \mathrm{Y}_{2}(2) \\
\end{array}$ & $\| \mathrm{Th}_{2} \mathrm{CoB}_{10}, \mathrm{Hf}_{3} \mathrm{Rh}_{5}, \mathrm{Sc}_{4} \mathrm{BCl}_{6}$ \\
\hline & $\begin{array}{c}\mathrm{R}_{3}^{+} \mathrm{R}_{4}^{+}(2), \mathrm{S}_{1}^{-} \mathrm{S}_{2}^{-}(2), \mathrm{T}_{2}(2) \\
\mathrm{U}_{2}(2), \mathrm{X}_{1}(2), \mathrm{Y}_{2}(2)\end{array}$ & $\| \operatorname{In}_{3} \mathrm{Pd}_{5}, \mathrm{Al}_{3} \mathrm{Pd}_{5}$ \\
\hline & $\begin{array}{c}\mathrm{R}_{3}^{+} \mathrm{R}_{4}^{+}(2), \mathrm{S}_{1}^{+} \mathrm{S}_{2}^{+}(2), \mathrm{T}_{1}(2) \\
\mathrm{U}_{2}(2), \mathrm{X}_{1}(2), \mathrm{Y}_{2}(2) \\
\end{array}$ & $\| \mathrm{Al}_{3} \mathrm{Pt}_{5}$ \\
\hline & $\begin{array}{l}\mathrm{R}_{3}^{+} \mathrm{R}_{4}^{+}(2), \mathrm{S}_{1}^{+} \mathrm{S}_{2}^{+}(2), \mathrm{T}_{1}(2) \\
\mathrm{U}_{1}(2), \mathrm{X}_{1}(2), \mathrm{Y}_{2}(2)\end{array}$ & $\| \mathrm{Ga}_{3} \mathrm{Pd}_{5}$ \\
\hline & $\begin{array}{c}\mathrm{R}_{1}^{-} \mathrm{R}_{2}^{-}(2), \mathrm{S}_{3}^{-} \mathrm{S}_{4}^{-}(2), \mathrm{T}_{1}(2) \\
\mathrm{U}_{2}(2), \mathrm{X}_{1}(2), \mathrm{Y}_{2}(2)\end{array}$ & $\| \mathrm{Nd}_{4} \mathrm{In}_{5} \mathrm{~S}_{13}, \mathrm{Sc}_{4} \mathrm{NCl}_{6}, \mathrm{La}_{4} \mathrm{In}_{5} \mathrm{~S}_{13}, \mathrm{Dy}_{5}\left(\mathrm{In}_{2} \mathrm{Ni}_{2}, \mathrm{Lu}_{5}\left(\mathrm{In}_{2} \mathrm{Ni}\right)_{2}\right.$ \\
\hline & $\begin{array}{c}\mathrm{R}_{1}^{-} \mathrm{R}_{2}^{-}(2), \mathrm{S}_{3}^{+} \mathrm{S}_{4}^{+}(2), \mathrm{T}_{2}(2) \\
\mathrm{U}_{2}(2), \mathrm{X}_{2}(2), \mathrm{Y}_{1}(2)\end{array}$ & $\| \mathrm{Li}_{13} \mathrm{Si}_{4}$ \\
\hline & $\begin{array}{c}\mathrm{R}_{1}^{+} \mathrm{R}_{2}^{+}(2), \mathrm{S}_{3}^{-} \mathrm{S}_{4}^{-}(2), \mathrm{T}_{2}(2) \\
\mathrm{U}_{1}(2), \mathrm{X}_{2}(2), \mathrm{Y}_{1}(2)\end{array}$ & $\| \mathrm{KNb}_{8} \mathrm{O}_{14}$ \\
\hline & $\begin{array}{c}\mathrm{R}_{1}^{+} \mathrm{R}_{2}^{+}(2), \mathrm{S}_{3}^{-} \mathrm{S}_{4}^{-}(2), \mathrm{T}_{2}(2) \\
\mathrm{U}_{1}(2), \mathrm{X}_{1}(2), \mathrm{Y}_{2}(2)\end{array}$ & $\| \mathrm{In}_{9} \mathrm{~Pb}_{4} \mathrm{~S}_{17}$ \\
\hline & $\begin{array}{l}\mathrm{R}_{1}^{+} \mathrm{R}_{2}^{+}(2), \mathrm{S}_{3}^{-} \mathrm{S}_{4}^{-}(2), \mathrm{T}_{1}(2) \\
\mathrm{U}_{2}(2), \mathrm{X}_{1}(2), \mathrm{Y}_{2}(2)\end{array}$ & $\| \mathrm{La}_{2} \mathrm{InNi}_{2}$ \\
\hline & $\begin{array}{c}\mathrm{R}_{1}^{+} \mathrm{R}_{2}^{+}(2), \mathrm{S}_{3}^{-} \mathrm{S}_{4}^{-}(2), \mathrm{T}_{1}(2) \\
\mathrm{U}_{1}(2), \mathrm{X}_{1}(2), \mathrm{Y}_{2}(2)\end{array}$ & $\| \mathrm{Y}_{5}\left(\operatorname{In}_{2} \mathrm{Pd}\right)_{2}$ \\
\hline & $\begin{array}{l}\mathrm{R}_{1}^{+} \mathrm{R}_{2}^{+}(2), \mathrm{S}_{3}^{+} \mathrm{S}_{4}^{+}(2), \mathrm{T}_{2}(2) \\
\mathrm{U}_{2}(2), \mathrm{X}_{2}(2), \mathrm{Y}_{1}(2)\end{array}$ & $\| \mathrm{Li}_{2} \mathrm{~B}_{2} \mathrm{Rh}_{3}$ \\
\hline & $\begin{array}{c}\mathrm{R}_{1}^{+} \mathrm{R}_{2}^{+}(2), \mathrm{S}_{3}^{+} \mathrm{S}_{4}^{+}(2), \mathrm{T}_{2}(2) \\
\mathrm{U}_{1}(2), \mathrm{X}_{2}(2), \mathrm{Y}_{1}(2)\end{array}$ & $\| \mathrm{Na}_{3}(\mathrm{CuS})_{4}$ \\
\hline & $\begin{array}{c}\mathrm{R}_{1}^{+} \mathrm{R}_{2}^{+}(2), \mathrm{S}_{3}^{+} \mathrm{S}_{4}^{+}(2), \mathrm{T}_{1}(2) \\
\mathrm{U}_{1}(2), \mathrm{X}_{2}(2), \mathrm{Y}_{2}(2) \\
\end{array}$ & $\| \mathrm{Ca}_{9} \mathrm{Zn}_{4} \mathrm{Bi}_{9}$ \\
\hline & $\begin{array}{c}\mathrm{R}_{1}^{+} \mathrm{R}_{2}^{+}(2), \mathrm{S}_{1}^{+} \mathrm{S}_{2}^{+}(2), \mathrm{T}_{2}(2) \\
\mathrm{U}_{1}(2), \mathrm{X}_{1}(2), \mathrm{Y}_{2}(2)\end{array}$ & $\| \mathrm{Sc}_{5}\left(\mathrm{In}_{2} \mathrm{Rh}\right)_{2}$ \\
\hline & $\begin{array}{c}\mathrm{R}_{1}^{+} \mathrm{R}_{2}^{+}(2), \mathrm{S}_{1}^{+} \mathrm{S}_{2}^{+}(2), \mathrm{T}_{1}(2) \\
\mathrm{U}_{1}(2), \mathrm{X}_{1}(2), \mathrm{Y}_{1}(2) \\
\end{array}$ & $\| \mathrm{Th}_{2} \mathrm{Al}_{7}$ \\
\hline \multirow{4}{*}{$\mathrm{SOC}$} & $\begin{array}{l}\overline{\mathrm{R}}_{6} \overline{\mathrm{R}}_{6}(4), \overline{\mathrm{S}}_{6} \overline{\mathrm{S}}_{6}(4), \overline{\mathrm{T}}_{3} \overline{\mathrm{T}}_{4}(4) \\
\overline{\mathrm{U}}_{3} \overline{\mathrm{U}}_{4}(4), \overline{\mathrm{X}}_{3} \overline{\mathrm{X}}_{4}(4), \overline{\mathrm{Y}}_{3} \overline{\mathrm{Y}}_{4}(4)\end{array}$ & $\begin{array}{c}\| \mathrm{Ta}_{4} \mathrm{BTe}_{8}, \mathrm{Nd}_{2} \mathrm{InNi}_{2}, \mathrm{Ca}_{9} \mathrm{Zn}_{4} \mathrm{Sb}_{9}, \mathrm{Nd}_{4} \mathrm{In}_{5} \mathrm{~S}_{13}, \mathrm{Si}_{3} \mathrm{Rh}_{5}, \mathrm{Ge}_{3} \mathrm{Rh}_{5}, \\
\mathrm{La}_{4} \mathrm{In}_{5} \mathrm{~S}_{13}, \mathrm{Lu}_{5}\left(\mathrm{In}_{2} \mathrm{Ni}_{2}\right.\end{array}$ \\
\hline & $\begin{array}{l}\overline{\mathrm{R}}_{6} \overline{\mathrm{R}}_{6}(4), \overline{\mathrm{S}}_{5} \overline{\mathrm{S}}_{5}(4), \overline{\mathrm{T}}_{3} \overline{\mathrm{T}}_{4}(4) \\
\mathrm{U}_{3} \overline{\mathrm{U}}_{4}(4), \overline{\mathrm{X}}_{3} \overline{\mathrm{X}}_{4}(4), \overline{\mathrm{Y}}_{3} \overline{\mathrm{Y}}_{4}(4)\end{array}$ & $\| \mathrm{Th}_{2} \mathrm{CoB}_{10}, \mathrm{Hf}_{3} \mathrm{Rh}_{5}, \mathrm{Ca}_{9} \mathrm{Cd}_{4} \mathrm{Bi}_{9}, \mathrm{Sr}_{9} \mathrm{Cd}_{4} \mathrm{Bi}_{9}$ \\
\hline & $\begin{array}{l}\overline{\mathrm{R}}_{5} \overline{\mathrm{R}}_{5}(4), \overline{\mathrm{S}}_{6} \overline{\mathrm{S}}_{6}(4), \overline{\mathrm{T}}_{3} \overline{\mathrm{T}}_{4}(4) \\
\overline{\mathrm{U}}_{3} \overline{\mathrm{U}}_{4}(4), \overline{\mathrm{X}}_{3} \overline{\mathrm{X}}_{4}(4), \overline{\mathrm{Y}}_{3} \overline{\mathrm{Y}}_{4}(4)\end{array}$ & $\| \mathrm{In}_{3} \mathrm{Pd}_{5}, \mathrm{Al}_{3} \mathrm{Pd}_{5}, \mathrm{Ca}_{9} \mathrm{Zn}_{4} \mathrm{Bi}_{9}, \mathrm{La}_{2} \mathrm{InNi}_{2}, \mathrm{In}_{9} \mathrm{~Pb}_{4} \mathrm{~S}_{17}$ \\
\hline & $\begin{array}{l}\overline{\mathrm{R}}_{5} \overline{\mathrm{R}}_{5}(4), \overline{\mathrm{S}}_{5} \overline{\mathrm{S}}_{5}(4), \overline{\mathrm{T}}_{3} \overline{\mathrm{T}}_{4}(4) \\
\mathrm{U}_{3} \overline{\mathrm{U}}_{4}(4), \overline{\mathrm{X}}_{3} \overline{\mathrm{X}}_{4}(4), \overline{\mathrm{Y}}_{3} \overline{\mathrm{Y}}_{4}(4)\end{array}$ & $\| \mathrm{Li}_{2} \mathrm{~B}_{2} \mathrm{Rh}_{3}, \mathrm{Al}_{3} \mathrm{Pt}_{5}, \mathrm{Na}_{3}(\mathrm{CuS})_{4}, \mathrm{Ga}_{3} \mathrm{Pd}_{5}, \mathrm{Th}_{2} \mathrm{Al}_{7}$ \\
\hline \multicolumn{3}{|r|}{ Space Group 56: Pccn } \\
\hline NSOC & $\mathrm{T}_{1} \mathrm{~T}_{2}(4), \mathrm{U}_{1} \mathrm{U}_{2}(4)$ & $\mathrm{Nd}_{5}(\mathrm{FeB})_{18}$ \\
\hline
\end{tabular}


Table II - continued

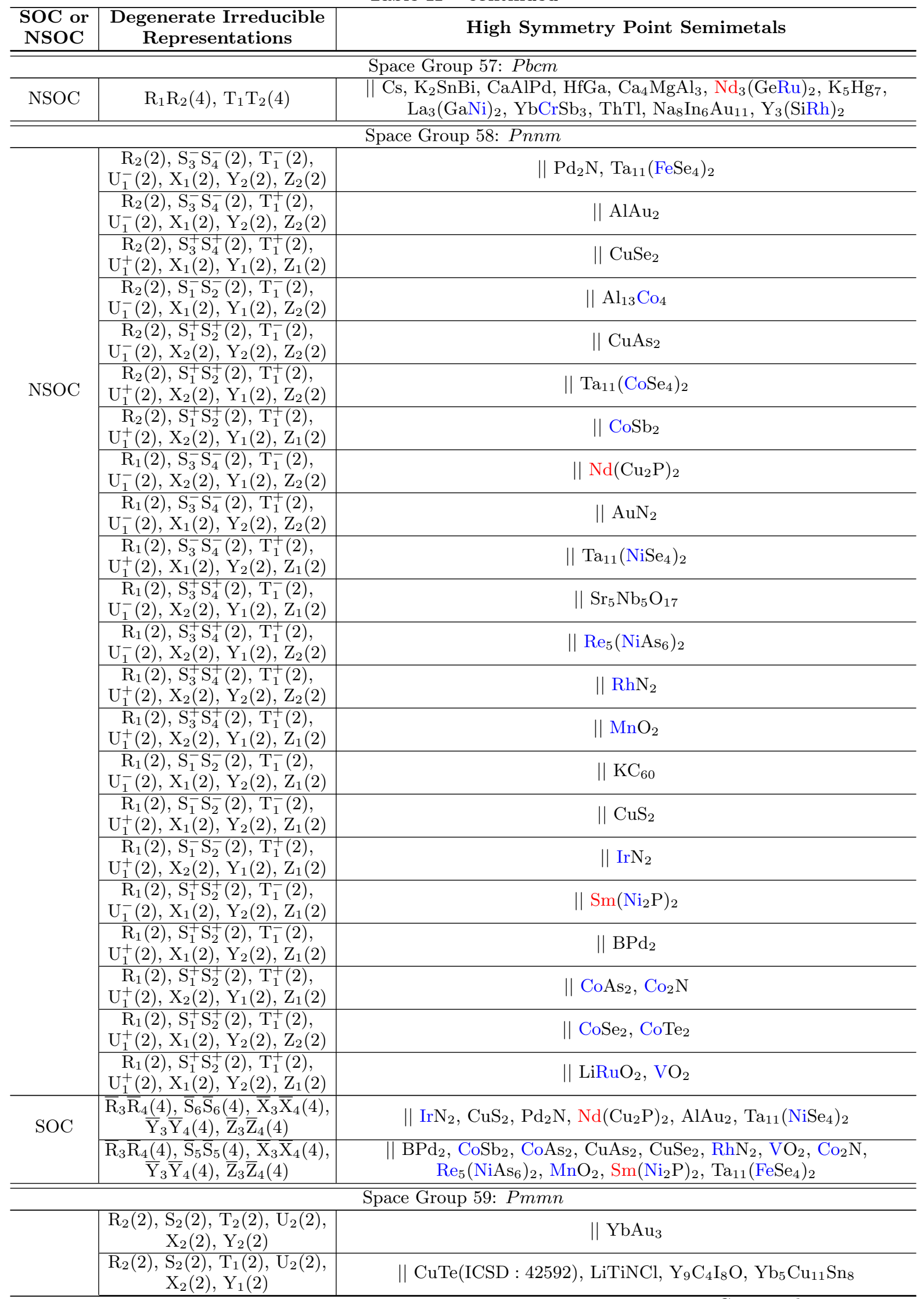


Table II - continued

\begin{tabular}{|c|c|c|}
\hline $\begin{array}{l}\text { SOC or } \\
\text { NSOC }\end{array}$ & $\begin{array}{c}\text { Degenerate Irreducible } \\
\text { Representations }\end{array}$ & High Symmetry Point Semimetals \\
\hline & $\begin{array}{c}\mathrm{R}_{2}(2), \mathrm{S}_{2}(2), \mathrm{T}_{1}(2), \mathrm{U}_{2}(2), \\
\mathrm{X}_{1}(2), \mathrm{Y}_{1}(2)\end{array}$ & $\| \mathrm{Ba}_{7} \mathrm{Ga}_{4} \mathrm{Sb}_{9}, \mathrm{La}_{9} \mathrm{~B}_{3} \mathrm{C}_{6} \mathrm{Br}_{5}, \mathrm{La}_{9} \mathrm{~B}_{3} \mathrm{C}_{6} \mathrm{I}_{5}$ \\
\hline & $\begin{array}{c}\mathrm{R}_{2}(2), \mathrm{S}_{2}(2), \mathrm{T}_{1}(2), \mathrm{U}_{1}(2) \\
\mathrm{X}_{2}(2), \mathrm{Y}_{2}(2) \\
\end{array}$ & $\| \mathrm{Cu}_{3} \mathrm{Sn}$ \\
\hline & $\begin{array}{c}\mathrm{R}_{2}(2), \mathrm{S}_{1}(2), \mathrm{T}_{2}(2), \mathrm{U}_{2}(2), \\
\mathrm{X}_{1}(2), \mathrm{Y}_{1}(2) \\
\end{array}$ & $\| \mathrm{Cu}_{3} \mathrm{Ge}, \mathrm{La}\left(\mathrm{In}_{2} \mathrm{Ni}\right)_{3}$ \\
\hline & $\begin{aligned} & \mathrm{R}_{2}(2), \mathrm{S}_{1}(2), \mathrm{T}_{2}(2), \mathrm{U}_{1}(2) \\
& \mathrm{X}_{1}(2), \mathrm{Y}_{1}(2) \\
&\end{aligned}$ & $\| \mathrm{HfAu}_{3}$ \\
\hline & $\begin{array}{c}\mathrm{R}_{2}(2), \mathrm{S}_{1}(2), \mathrm{T}_{1}(2), \mathrm{U}_{2}(2) \\
\mathrm{X}_{2}(2), \mathrm{Y}_{2}(2)\end{array}$ & || CuTe(ICSD : 44993) \\
\hline & $\begin{array}{c}\mathrm{R}_{2}(2), \mathrm{S}_{1}(2), \mathrm{T}_{1}(2), \mathrm{U}_{2}(2) \\
\mathrm{X}_{1}(2), \mathrm{Y}_{2}(2)\end{array}$ & $\| \mathrm{NbNi}_{3}$ \\
\hline & $\begin{aligned} & \mathrm{R}_{2}(2), \mathrm{S}_{1}(2), \mathrm{T}_{1}(2), \mathrm{U}_{1}(2) \\
& \mathrm{X}_{1}(2), \mathrm{Y}_{1}(2)\end{aligned}$ & $\| \mathrm{ZrAu}_{3}, \mathrm{TiCu}_{3}$ \\
\hline & $\begin{array}{c}\mathrm{R}_{1}(2), \mathrm{S}_{2}(2), \mathrm{T}_{2}(2), \mathrm{U}_{2}(2) \\
\mathrm{X}_{2}(2), \mathrm{Y}_{2}(2)\end{array}$ & $\| \mathrm{TiCuNi}_{2}$ \\
\hline & $\begin{array}{c}\mathrm{R}_{1}(2), \mathrm{S}_{2}(2), \mathrm{T}_{2}(2), \mathrm{U}_{2}(2) \\
\mathrm{X}_{1}(2), \mathrm{Y}_{2}(2)\end{array}$ & $\| \mathrm{TaNi}_{3}$ \\
\hline & $\begin{array}{c}\mathrm{R}_{1}(2), \mathrm{S}_{2}(2), \mathrm{T}_{2}(2), \mathrm{U}_{1}(2) \\
\mathrm{X}_{2}(2), \mathrm{Y}_{2}(2)\end{array}$ & $\| \mathrm{Ag}_{3} \mathrm{Sn}$ \\
\hline & $\begin{array}{c}\mathrm{R}_{1}(2), \mathrm{S}_{2}(2), \mathrm{T}_{1}(2), \mathrm{U}_{2}(2) \\
\mathrm{X}_{2}(2), \mathrm{Y}_{1}(2)\end{array}$ & $\| \mathrm{Ni}_{8} \mathrm{Ge}_{2} \mathrm{~B}_{3}, \mathrm{Pr}_{3} \mathrm{Sn}_{5} \mathrm{Au}_{6}$ \\
\hline & $\begin{array}{c}\mathrm{R}_{1}(2), \mathrm{S}_{2}(2), \mathrm{T}_{1}(2), \mathrm{U}_{1}(2) \\
\mathrm{X}_{2}(2), \mathrm{Y}_{1}(2)\end{array}$ & $\| \mathrm{Ni}_{6} \mathrm{Mo}_{2} \mathrm{P}_{3}$ \\
\hline & $\begin{aligned} & \mathrm{R}_{1}(2), \mathrm{S}_{2}(2), \mathrm{T}_{1}(2), \mathrm{U}_{1}(2) \\
& \mathrm{X}_{1}(2), \mathrm{Y}_{1}(2)\end{aligned}$ & $\| \mathrm{NbPt}_{3}, \mathrm{TaPt}_{3}$ \\
\hline & $\begin{array}{c}\mathrm{R}_{1}(2), \mathrm{S}_{1}(2), \mathrm{T}_{2}(2), \mathrm{U}_{2}(2) \\
\mathrm{X}_{2}(2), \mathrm{Y}_{2}(2)\end{array}$ & $\| \mathrm{Cu}_{3} \mathrm{BiSe}_{2} \mathrm{ClO}_{8}$ \\
\hline & $\begin{aligned} \mathrm{R}_{1}(2), & \mathrm{S}_{1}(2), \mathrm{T}_{2}(2), \mathrm{U}_{2}(2) \\
& \mathrm{X}_{1}(2), \mathrm{Y}_{1}(2)\end{aligned}$ & $\| \mathrm{NbPd}_{3}$ \\
\hline & $\begin{array}{c}\mathrm{R}_{1}(2), \mathrm{S}_{1}(2), \mathrm{T}_{2}(2), \mathrm{U}_{1}(2) \\
\mathrm{X}_{1}(2), \mathrm{Y}_{2}(2)\end{array}$ & $\| \mathrm{CrBrO}$ \\
\hline & $\begin{aligned} \mathrm{R}_{1}(2), & \mathrm{S}_{1}(2), \mathrm{T}_{1}(2), \mathrm{U}_{2}(2) \\
& \mathrm{X}_{2}(2), \mathrm{Y}_{1}(2)\end{aligned}$ & $\| \mathrm{TiClO}, \mathrm{TiBrO}$ \\
\hline & $\begin{array}{c}\mathrm{R}_{1}(2), \mathrm{S}_{1}(2), \mathrm{T}_{1}(2), \mathrm{U}_{2}(2), \\
\mathrm{X}_{1}(2), \mathrm{Y}_{2}(2)\end{array}$ & $\| \mathrm{Ni}_{3} \mathrm{Sb}$ \\
\hline & $\begin{aligned} \mathrm{R}_{1}(2), & \mathrm{S}_{1}(2), \mathrm{T}_{1}(2), \mathrm{U}_{1}(2) \\
& \mathrm{X}_{2}(2), \mathrm{Y}_{1}(2)\end{aligned}$ & $\| \mathrm{Pr}_{4} \mathrm{NiI}_{5}, \mathrm{Er}_{4} \mathrm{Si}_{9} \operatorname{Ir}_{13}$ \\
\hline & $\begin{array}{c}\mathrm{R}_{1}(2), \mathrm{S}_{1}(2), \mathrm{T}_{1}(2), \mathrm{U}_{1}(2), \\
\mathrm{X}_{1}(2), \mathrm{Y}_{1}(2)\end{array}$ & $\| \mathrm{ZrAsPd}, \mathrm{Ho}_{4} \mathrm{Ge}_{9} \mathrm{Ir}_{13}$ \\
\hline $\mathrm{SOC}$ & $\begin{array}{l}\overline{\mathrm{R}}_{3} \overline{\mathrm{R}}_{4}(4), \overline{\mathrm{S}}_{3} \overline{\mathrm{S}}_{4}(4), \overline{\mathrm{T}}_{3} \overline{\mathrm{T}}_{4}(4) \\
\mathrm{U}_{3} \overline{\mathrm{U}}_{4}(4), \overline{\mathrm{X}}_{3} \overline{\mathrm{X}}_{4}(4), \overline{\mathrm{Y}}_{3} \overline{\mathrm{Y}}_{4}(4)\end{array}$ & 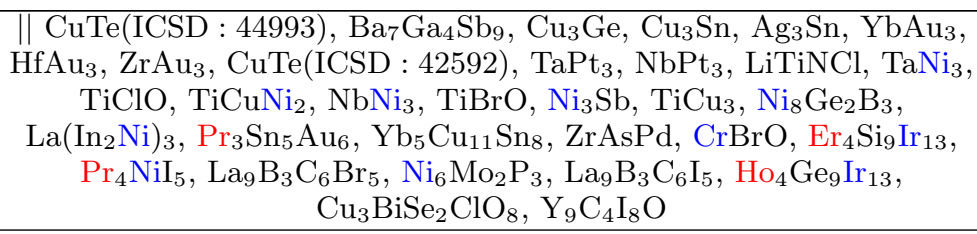 \\
\hline \multicolumn{3}{|r|}{ Space Group 60: Pbcn } \\
\hline NSOC & $\mathrm{R}_{1} \mathrm{R}_{2}(4), \mathrm{T}_{1} \mathrm{~T}_{2}(4), \mathrm{U}_{1} \mathrm{U}_{2}(4)$ & $\| \mathrm{ReO}_{2}, \mathrm{Al}_{3} \mathrm{FeSi}_{2}, \mathrm{Si}_{2} \mathrm{Ni}_{7} \mathrm{P}_{5}, \mathrm{Hf}_{2} \mathrm{Co}_{3} \mathrm{Si}_{4}, \mathrm{Mn}_{2} \mathrm{~N}, \mathrm{RbCuCl}_{3}, \mathrm{Fe}_{2} \mathrm{~N}$ \\
\hline \multicolumn{3}{|r|}{ Space Group 61: Pbca } \\
\hline NSOC & $\begin{array}{c}\mathrm{R}_{1}^{+} \mathrm{R}_{1}^{+}(4), \mathrm{S}_{1} \mathrm{~S}_{2}(4) \\
\mathrm{T}_{1} \mathrm{~T}_{2}(4), \mathrm{U}_{1} \mathrm{U}_{2}(4)\end{array}$ & $\| \mathrm{AgF}_{2}$ \\
\hline
\end{tabular}


Table II - continued

\begin{tabular}{|c|c|c|}
\hline $\begin{array}{l}\text { SOC or } \\
\text { NSOC }\end{array}$ & $\begin{array}{c}\text { Degenerate Irreducible } \\
\text { Representations }\end{array}$ & High Symmetry Point Semimetals \\
\hline NSOC & $\mathrm{R}_{1} \mathrm{R}_{2}(4), \mathrm{S}_{1} \mathrm{~S}_{2}(4)$ & 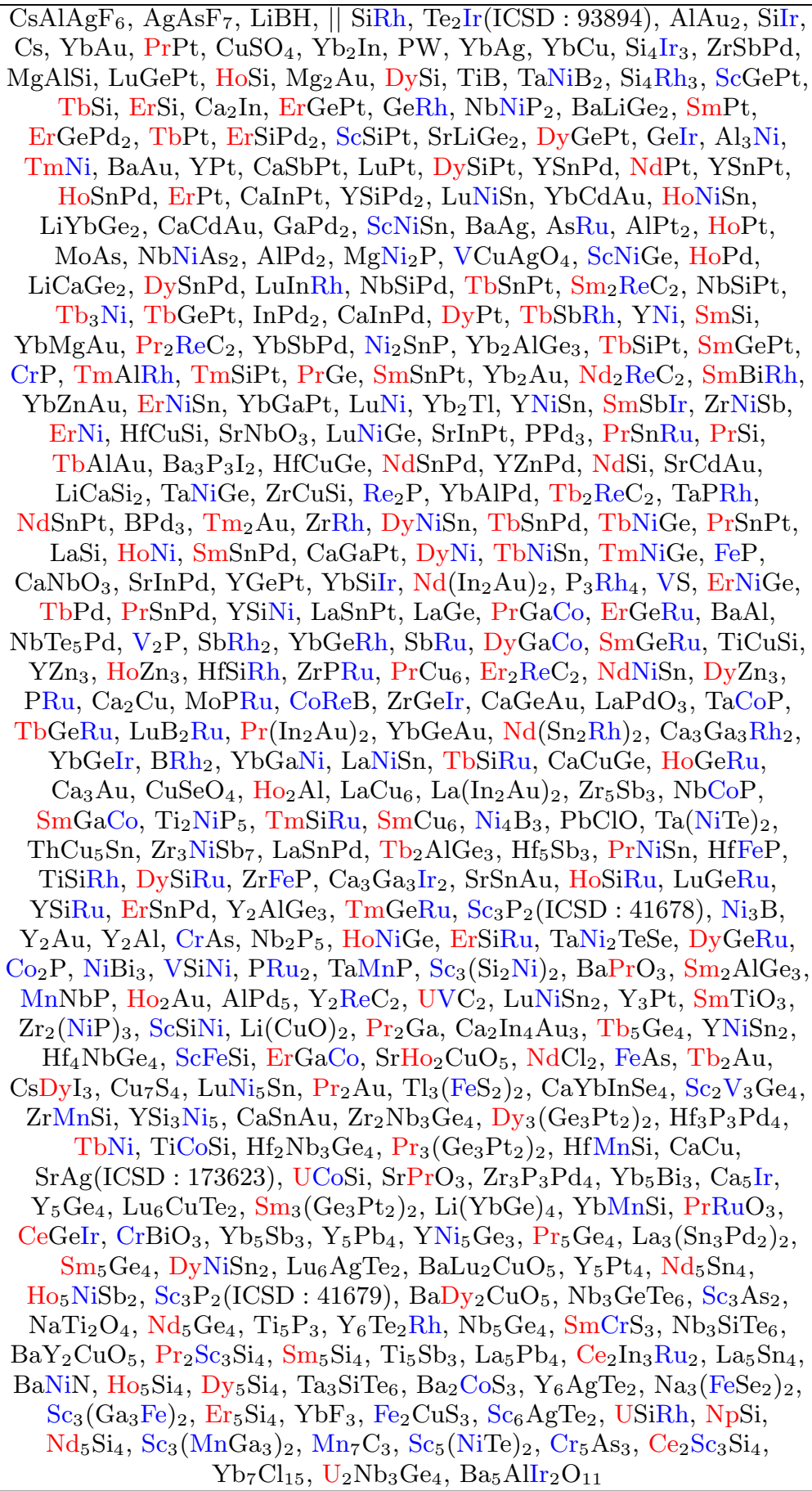 \\
\hline \multicolumn{3}{|r|}{ Space Group 63: $\mathrm{Cmcm}$} \\
\hline NSOC & $\mathrm{R}_{1}(2), \mathrm{T}_{2}(2), \mathrm{Z}_{2}(2)$ & 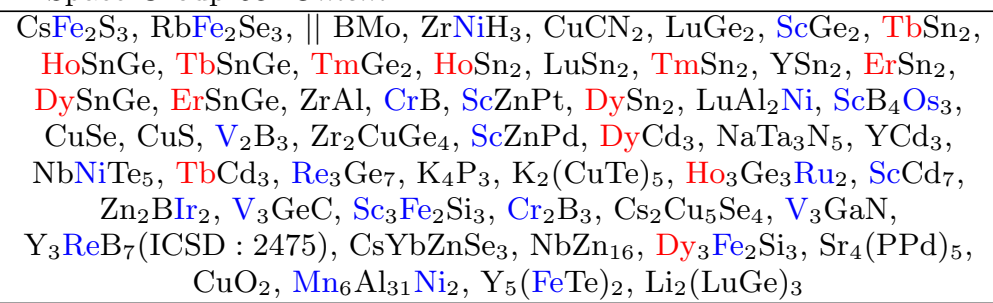 \\
\hline
\end{tabular}


Table II - continued

\begin{tabular}{|c|c|c|}
\hline $\begin{array}{l}\text { SOC or } \\
\text { NSOC }\end{array}$ & $\begin{array}{c}\text { Degenerate Irreducible } \\
\text { Representations }\end{array}$ & High Symmetry Point Semimetals \\
\hline & $\mathrm{R}_{1}(2), \mathrm{T}_{2}(2), \mathrm{Z}_{1}(2)$ & 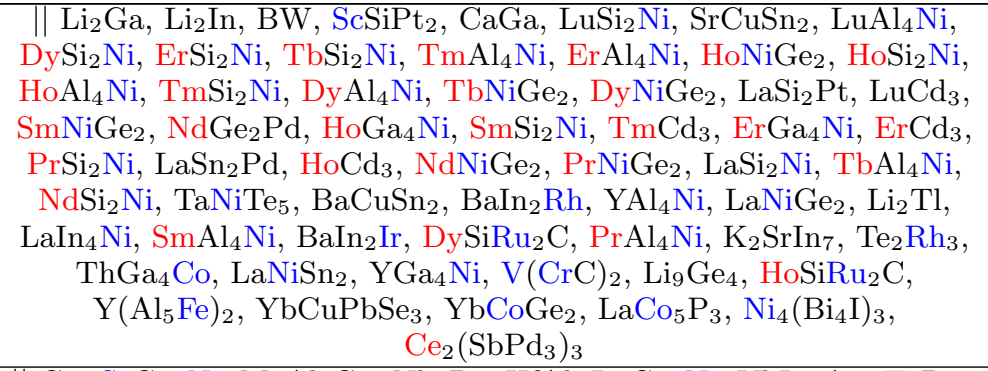 \\
\hline & $\mathrm{R}_{1}(2), \mathrm{T}_{1}(2), \mathrm{Z}_{2}(2)$ & 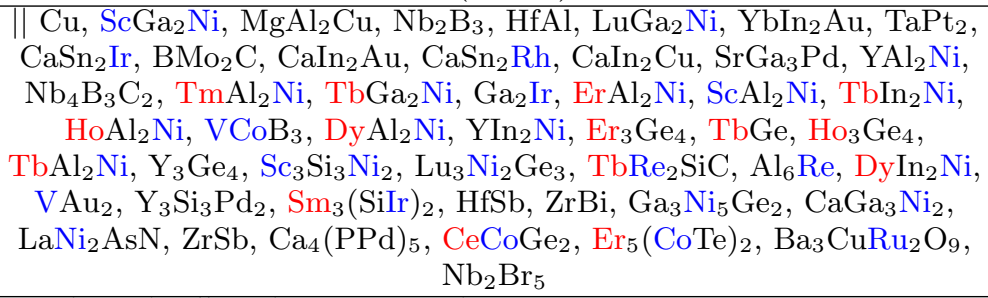 \\
\hline & $\mathrm{R}_{1}(2), \mathrm{T}_{1}(2), \mathrm{Z}_{1}(2)$ & 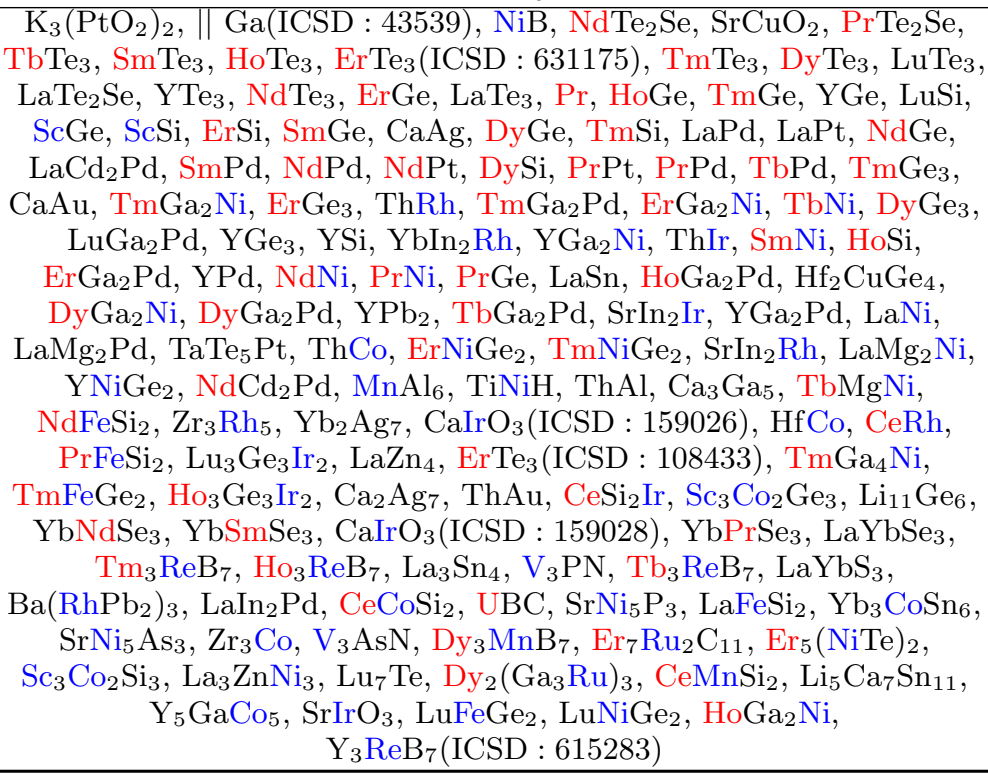 \\
\hline
\end{tabular}


Table II - continued

\begin{tabular}{|c|c|c|}
\hline $\begin{array}{l}\text { SOC or } \\
\text { NSOC }\end{array}$ & $\begin{array}{l}\text { Degenerate Irreducible } \\
\text { Representations }\end{array}$ & High Symmetry Point Semimetals \\
\hline $\mathrm{SOC}$ & $\overline{\mathrm{R}}_{2} \overline{\mathrm{R}}_{2}(4), \overline{\mathrm{T}}_{3} \overline{\mathrm{T}}_{4}(4), \overline{\mathrm{Z}}_{3} \overline{\mathrm{Z}}_{4}(4)$ & 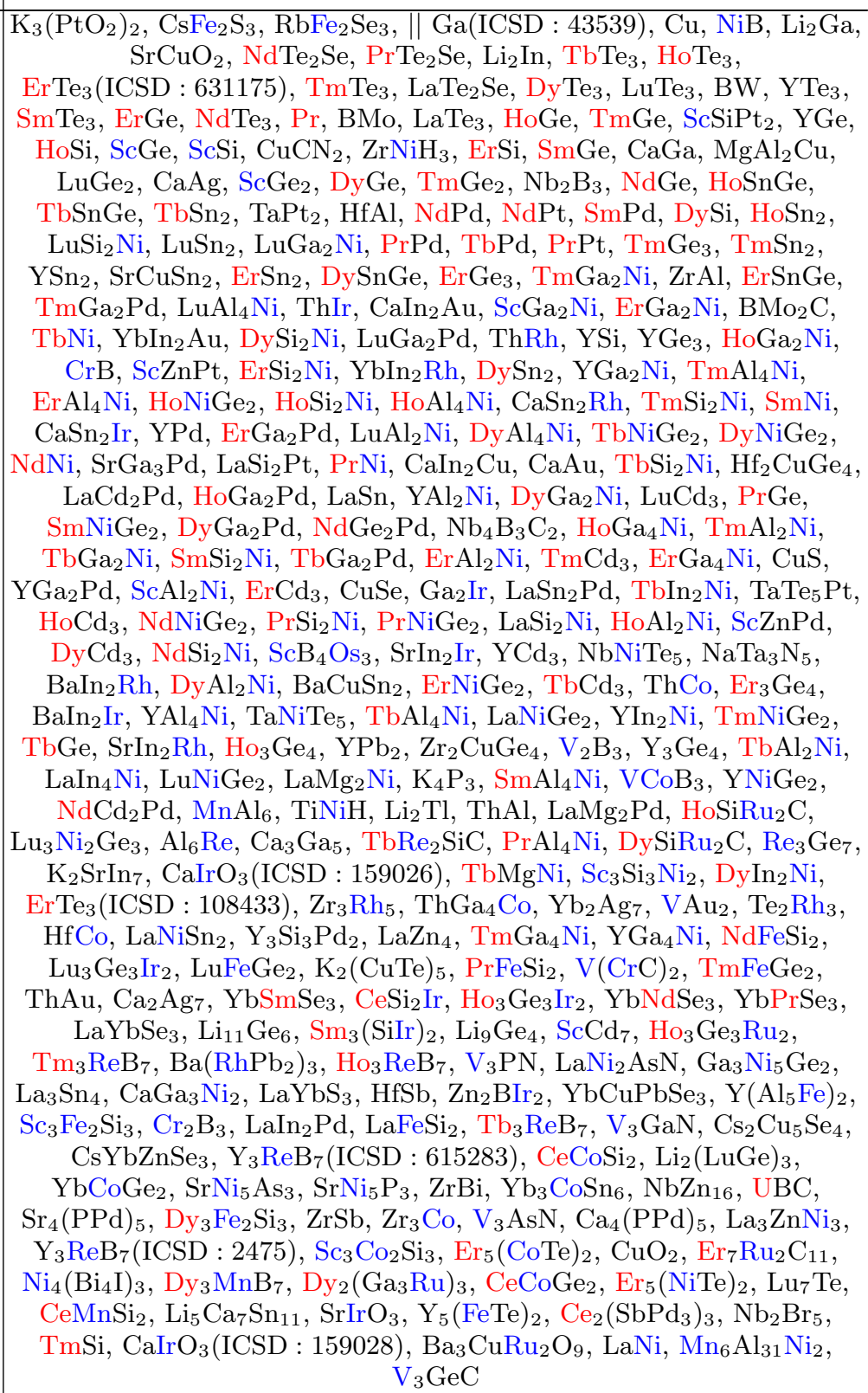 \\
\hline \multirow{8}{*}{ NSOC } & \multicolumn{2}{|r|}{ Space Group 64: Cmce } \\
\hline & $\begin{array}{c}\mathrm{R}_{1}^{-} \mathrm{R}_{2}^{-}(2), \mathrm{S}_{1}(2), \mathrm{T}_{2}(2) \\
\mathrm{Z}_{2}(2)\end{array}$ & $\| \mathrm{Ba}_{2} \mathrm{Tl}_{2} \mathrm{CuO}_{6}$ \\
\hline & $\begin{array}{c}\mathrm{R}_{1}^{-} \mathrm{R}_{2}^{-}(2), \mathrm{S}_{1}(2), \mathrm{T}_{2}(2) \\
\mathrm{Z}_{1}(2)\end{array}$ & $\| \mathrm{Er}_{3}\left(\mathrm{Si}_{3} \mathrm{Ru}\right)_{4}, \mathrm{Al}\left(\mathrm{Ni}_{3} \mathrm{~B}_{2}\right)_{4}$ \\
\hline & $\begin{array}{c}\mathrm{R}_{1}^{-} \mathrm{R}_{2}^{-}(2), \mathrm{S}_{1}(2), \mathrm{T}_{1}(2) \\
\mathrm{Z}_{2}(2)\end{array}$ & $\| \mathrm{Ce}_{2} \mathrm{Al}_{3} \mathrm{Ge}_{4}$ \\
\hline & $\begin{array}{c}\mathrm{R}_{1}^{-} \mathrm{R}_{2}^{-}(2), \mathrm{S}_{1}(2), \mathrm{T}_{1}(2) \\
\mathrm{Z}_{1}(2)\end{array}$ & $\| \mathrm{La}_{2} \mathrm{Al}_{3} \mathrm{Ge}_{4}$ \\
\hline & $\begin{array}{c}\mathrm{R}_{1}^{+} \mathrm{R}_{2}^{+}(2), \mathrm{S}_{1}(2), \mathrm{T}_{2}(2) \\
\mathrm{Z}_{2}(2)\end{array}$ & $\| \mathrm{Nd}_{2} \mathrm{CuO}_{4}, \mathrm{Ca}_{4} \mathrm{In}_{3} \mathrm{Au}_{10}$ \\
\hline & $\begin{array}{c}\mathrm{R}_{1}^{+} \mathrm{R}_{2}^{+}(2), \mathrm{S}_{1}(2), \mathrm{T}_{2}(2) \\
\mathrm{Z}_{1}(2)\end{array}$ & $\| \mathrm{Y}_{3}\left(\mathrm{Si}_{3} \mathrm{Os}\right)_{4}, \mathrm{Ba}_{4} \mathrm{Ir}_{3} \mathrm{O}_{10}$ \\
\hline & $\begin{array}{c}\mathrm{R}_{1}^{+} \mathrm{R}_{2}^{+}(2), \mathrm{S}_{1}(2), \mathrm{T}_{1}(2) \\
\mathrm{Z}_{2}(2)\end{array}$ & $\| \mathrm{Pr}_{2} \mathrm{CuO}_{4}, \mathrm{Tm}_{5}(\mathrm{SiSb})_{2}$ \\
\hline
\end{tabular}


Table II - continued

\begin{tabular}{|c|c|c|}
\hline $\begin{array}{l}\text { SOC or } \\
\text { NSOC }\end{array}$ & $\begin{array}{l}\text { Degenerate Irreducible } \\
\text { Representations }\end{array}$ & High Symmetry Point Semimetals \\
\hline $\mathrm{SOC}$ & $\overline{\mathrm{S}}_{2} \overline{\mathrm{S}}_{2}(4), \overline{\mathrm{T}}_{3} \overline{\mathrm{T}}_{4}(4), \overline{\mathrm{Z}}_{3} \overline{\mathrm{Z}}_{4}(4)$ & $\begin{array}{c}\| \mathrm{La}_{2} \mathrm{Al}_{3} \mathrm{Ge}_{4}, \mathrm{Y}_{3}\left(\mathrm{Si}_{3} \mathrm{Os}\right)_{4}, \mathrm{Er}_{3}\left(\mathrm{Si}_{3} \mathrm{Ru}\right)_{4}, \mathrm{Pr}_{2} \mathrm{CuO}_{4}, \mathrm{Ca}_{4} \mathrm{In}_{3} \mathrm{Au}_{10} \\
\mathrm{Tm}_{5}(\mathrm{SiSb})_{2}, \mathrm{Ba}_{4} \mathrm{Ir}_{3} \mathrm{O}_{10}, \mathrm{Ce}_{2} \mathrm{Al}_{3} \mathrm{Ge}_{4}, \mathrm{Al}\left(\mathrm{Ni}_{3} \mathrm{~B}_{2}\right)_{4} \\
\end{array}$ \\
\hline \multicolumn{3}{|r|}{ Space Group 65: $\mathrm{Cmmm}$} \\
\hline $\mathrm{SOC}$ & $\bar{\Gamma}_{5}(2), \overline{\mathrm{S}}_{5} \overline{\mathrm{S}}_{6}(2)$ & $\mathrm{Ce}\left(\mathrm{Re}_{2} \mathrm{Si}\right)_{2}$ \\
\hline \multicolumn{3}{|r|}{ Space Group 66: $\mathrm{Cccm}$} \\
\hline NSOC & $\mathrm{T}_{1}(2), \mathrm{Z}_{2}(2)$ & $\mathrm{Sr}_{2} \mathrm{Cu}\left(\mathrm{BiO}_{3}\right)_{2}$ \\
\hline \multicolumn{3}{|r|}{ Space Group 67: Cmme } \\
\hline NSOC & $\mathrm{R}_{1}(2), \mathrm{S}_{1}(2)$ & $\mathrm{Ba}_{2} \mathrm{NaO}$ \\
\hline $\mathrm{SOC}$ & $\overline{\mathrm{R}}_{2} \overline{\mathrm{R}}_{2}(4), \overline{\mathrm{S}}_{2} \overline{\mathrm{S}}_{2}(4)$ & $\mathrm{Ba}_{2} \mathrm{NaO}$ \\
\hline \multicolumn{3}{|r|}{ Space Group 68: Ccce } \\
\hline \multirow{3}{*}{ NSOC } & $\mathrm{R}_{1}(2), \mathrm{S}_{1}(2), \mathrm{T}_{2}(2), \mathrm{Z}_{1}(2)$ & $\| \mathrm{Sn}_{4} \mathrm{Au}$ \\
\hline & $\mathrm{R}_{1}(2), \mathrm{S}_{1}(2), \mathrm{T}_{1}(2), \mathrm{Z}_{2}(2)$ & $\mathrm{Lu}(\mathrm{BRh})_{4}$ \\
\hline & $\mathrm{R}_{1}(2), \mathrm{S}_{1}(2), \mathrm{T}_{1}(2), \mathrm{Z}_{1}(2)$ & $\mathrm{IrC}_{4}, \mathrm{Sn}_{4} \mathrm{Ir}$ \\
\hline $\mathrm{SOC}$ & $\overline{\mathrm{R}}_{2} \overline{\mathrm{R}}_{2}(4), \overline{\mathrm{S}}_{2} \overline{\mathrm{S}}_{2}(4), \overline{\mathrm{T}}_{3} \overline{\mathrm{T}}_{4}(4)$ & $\| \mathrm{IrC}_{4}, \mathrm{Sn}_{4} \mathrm{Au}, \mathrm{Sn}_{4} \mathrm{Ir}, \mathrm{Lu}(\mathrm{BRh})_{4}$ \\
\hline \multicolumn{3}{|r|}{ Space Group 70: Fddd } \\
\hline \multirow[t]{2}{*}{ NSOC } & $\mathrm{T}_{2}(2), \mathrm{Y}_{1}(2), \mathrm{Z}_{1}(2)$ & $\begin{array}{c}\mathrm{Nd}(\mathrm{BOs})_{2}, \mathrm{Sm}(\mathrm{BOs})_{2}, \mathrm{La}(\mathrm{BOs})_{2}, \mathrm{Pr}(\mathrm{BOs})_{2}, \mathrm{Nd}(\mathrm{BRu})_{2}, \mathrm{Sm}(\mathrm{BRu})_{2}, \\
\operatorname{Pr}(\mathrm{BRu})_{2}\end{array}$ \\
\hline & $\mathrm{T}_{1}(2), \mathrm{Y}_{1}(2), \mathrm{Z}_{1}(2)$ & \multirow{2}{*}{ 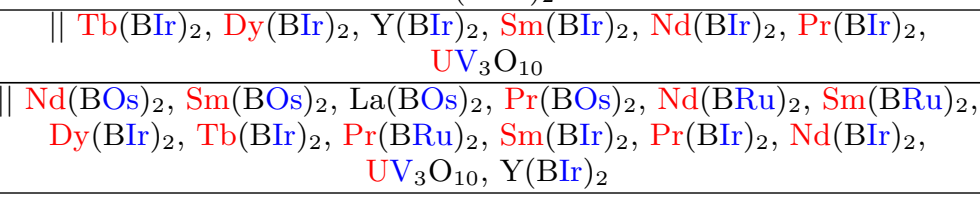 } \\
\hline SOC & $\overline{\mathrm{T}}_{3} \overline{\mathrm{T}}_{4}(4), \overline{\mathrm{Y}}_{3} \overline{\mathrm{Y}}_{4}(4), \overline{\mathrm{Z}}_{3} \overline{\mathrm{Z}}_{4}(4)$ & \\
\hline \multicolumn{3}{|r|}{ Space Group 72: Ibam } \\
\hline \multirow[t]{2}{*}{ NSOC } & $\mathrm{R}_{1}(2), \mathrm{S}_{1}(2), \mathrm{W}_{3} \mathrm{~W}_{4}(2)$ & \multirow{2}{*}{$\begin{array}{r}\| \mathrm{Cu}_{5} \mathrm{As}_{2}, \mathrm{Ba}_{2} \mathrm{Cu}_{3} \mathrm{P}_{4}, \mathrm{La}_{2} \mathrm{Ge}_{5} \mathrm{Ir}_{3}, \mathrm{Y}_{2} \mathrm{Ge}_{5} \mathrm{Ir}_{3}, \mathrm{Lu}_{2} \mathrm{Si}_{5} \mathrm{Ir}_{3}, \mathrm{La}_{2} \mathrm{Ge}_{5} \mathrm{Rh}_{3}, \\
\mathrm{Li}_{3} \mathrm{FeN}_{2}, \mathrm{La}_{2} \mathrm{Si}_{5} \mathrm{Rh}_{3}, \mathrm{Tm}_{2} \mathrm{Co}_{3} \mathrm{Si}_{5}, \mathrm{La}_{2} \mathrm{Sn}_{5} \mathrm{Rh}_{3}, \mathrm{CePu}_{4} \mathrm{Co}_{3} \\
\mathrm{Cb}_{2} \mathrm{CoSe}_{2}, \mathrm{Cs}_{2} \mathrm{CoSe}_{2}, \mathrm{Co}(\mathrm{TePd})_{2}, \mathrm{Co}\left(\mathrm{PdSe}_{2}, \mathrm{Nd}_{2} \mathrm{Ge}_{5} \mathrm{Rh}_{3},\right. \\
\mathrm{Sm}_{2} \mathrm{Ge}_{5} \mathrm{Ir}_{3}, \mathrm{Dy}_{2} \mathrm{Si}_{5} \mathrm{Rh}_{3}, \mathrm{Pr}_{2} \mathrm{Ge}_{5} \mathrm{Rh}_{3}, \mathrm{Pr}_{2} \mathrm{Co}_{3} \mathrm{Ge}_{5}, \mathrm{~K}_{2} \mathrm{CoSe}_{2}, \mathrm{Y}_{2} \mathrm{Co}_{3} \mathrm{Si}_{5}, \\
\mathrm{~K}_{2} \mathrm{CoS}_{2}\end{array}$} \\
\hline & $\mathrm{R}_{1}(2), \mathrm{S}_{1}(2), \mathrm{W}_{1} \mathrm{~W}_{2}(2)$ & \\
\hline $\mathrm{SOC}$ & $\begin{array}{c}\overline{\mathrm{R}}_{2} \overline{\mathrm{R}}_{2}(4), \overline{\mathrm{S}}_{2} \overline{\mathrm{S}}_{2}(4) \\
\overline{\mathrm{W}}_{5} \overline{\mathrm{W}}_{5}(4)\end{array}$ & $\begin{array}{c}\| \mathrm{Co}(\mathrm{TePd})_{2}, \mathrm{Cu}_{5} \mathrm{As}_{2}, \mathrm{Sm}_{2} \mathrm{Ge}_{5} \mathrm{Ir}_{3}, \mathrm{Co}(\mathrm{PdSe})_{2}, \mathrm{Ba}_{2} \mathrm{Cu}_{3} \mathrm{P}_{4}, \\
\mathrm{Nd}_{2} \mathrm{Ge}_{5} \mathrm{Rh}_{3}, \mathrm{Y}_{2} \mathrm{Ge}_{5} \mathrm{Ir}_{3}, \mathrm{Lu}_{2} \mathrm{Si}_{5} \mathrm{Ir}_{3}, \mathrm{La}_{2} \mathrm{Ge}_{5} \mathrm{Ir}_{3}, \mathrm{Dy}_{2} \mathrm{Si}_{5} \mathrm{Rh}_{3}, \mathrm{~K}_{2} \mathrm{CoS}_{2}, \\
\mathrm{Pr}_{2} \mathrm{Ge}_{5} \mathrm{Rh}_{3}, \mathrm{La}_{2} \mathrm{Ge}_{5} \mathrm{Rh}_{3}, \mathrm{Li}_{3} \mathrm{FeN}_{2}, \mathrm{Y}_{2} \mathrm{Co}_{3} \mathrm{Si}_{5}, \mathrm{La}_{2} \mathrm{Si}_{5} \mathrm{Rh}_{3}, \mathrm{Tm}_{2} \mathrm{Co}_{3} \mathrm{Si}_{5}, \\
\mathrm{La}_{2} \mathrm{Sn}_{5} \mathrm{Rh}_{3}, \mathrm{CePu}_{4} \mathrm{Co}_{3}, \mathrm{Cs}_{2} \mathrm{CoSe}_{2}, \mathrm{~K}_{2} \mathrm{CoSe}_{2}, \mathrm{Rb}_{2} \mathrm{CoSe}_{2}, \mathrm{Pr}_{2} \mathrm{Co}_{3} \mathrm{Ge}_{5}\end{array}$ \\
\hline \multicolumn{3}{|r|}{ Space Group 74: Imma } \\
\hline NSOC & $\mathrm{T}_{1}(2), \mathrm{W}_{1}(2)$ & $\begin{array}{c}\| \mathrm{N}_{2}, \mathrm{P}, \mathrm{Na}_{2} \mathrm{Cl}, \mathrm{SmAl}_{4}, \mathrm{PrGe}_{2}, \mathrm{TbAl}_{4}, \mathrm{GaCu}_{2}, \mathrm{PrSiGe}, \mathrm{LuGa}_{2}, \\
\mathrm{TmGa}_{2}, \mathrm{HoCu}_{2}, \mathrm{La}\left(\mathrm{ReSi}_{2}, \mathrm{NdSiGe}_{2} \mathrm{ErCu}_{2}, \mathrm{DyCu}_{2}, \mathrm{LuCu}_{2}, \mathrm{YCu}_{2},\right. \\
\mathrm{TbCu}_{2}, \mathrm{TmCu}_{2}, \mathrm{TmZn}_{2}, \mathrm{KHg}_{2}, \mathrm{ScGa}_{2}, \mathrm{SmCu}_{2}, \mathrm{RbHg}_{2}, \mathrm{NdCu}_{2}, \\
\mathrm{MgGaMo}_{4} \mathrm{O}_{7}, \mathrm{PrCu}_{2}, \mathrm{LuZn}_{2}, \mathrm{HoZn}_{2}, \mathrm{ErZn}_{2}, \mathrm{DyZn}_{2}, \mathrm{CsHg}_{2}, \mathrm{MoN}_{2}, \\
\mathrm{TbZn}_{2}, \mathrm{LaZn}_{2}, \mathrm{PrZn}_{2}, \mathrm{PrAu}_{2}, \mathrm{BaPrO}_{3}, \mathrm{LaAg}_{2}, \mathrm{LaAu}_{2}, \mathrm{NdAg}_{2}, \\
\mathrm{LaIn}_{2}, \mathrm{SmZn}_{2}, \mathrm{NdZn}_{2}, \mathrm{PrAg}_{2}, \mathrm{~K}_{3} \mathrm{TlAu}_{5}, \mathrm{Tl}(\mathrm{CuO})_{2}, \mathrm{~K}_{3} \mathrm{InAu}_{5}, \\
\mathrm{BaTbO}_{3}, \mathrm{La}_{3} \mathrm{SiPd}_{5}, \mathrm{NpAl}_{4}\end{array}$ \\
\hline SOC & $\overline{\mathrm{T}}_{2} \overline{\mathrm{T}}_{2}(4)$ & $\begin{array}{l}\| \mathrm{N}_{2}, \mathrm{P}, \mathrm{SmAl}_{4}, \mathrm{TbAl}_{4}, \mathrm{PrGe}_{2}, \mathrm{GaCu}_{2}, \mathrm{PrSiGe}_{\mathrm{LuGa}}, \mathrm{LmGa}_{2}, \\
\mathrm{NdSiGe} \mathrm{ErCu}_{2}, \mathrm{DyCu}_{2}, \mathrm{La}\left(\mathrm{ReSi}_{2}, \mathrm{LuCu}_{2}, \mathrm{KHg}_{2}, \mathrm{TbCu}_{2}, \mathrm{TmCu}_{2},\right. \\
\mathrm{TmZn}_{2}, \mathrm{ScGa}_{2}, \mathrm{RbHg}_{2}, \mathrm{SmCu}_{2}, \mathrm{NdCu}_{2}, \mathrm{MgGaMo}_{4} \mathrm{O}_{7}, \mathrm{CsHg}_{2}, \\
\mathrm{PrCu}_{2}, \mathrm{LuZn}_{2}, \mathrm{H}_{\mathrm{ZZ}}, \mathrm{ErZn}_{2}, \mathrm{DyZn}_{2}, \mathrm{LaZn}_{2}, \mathrm{PrZn}_{2}, \mathrm{MoN}_{2} \mathrm{TbZn}_{2}, \\
\mathrm{BaPrO}_{3}, \mathrm{PrAu}_{2}, \mathrm{LaAg}_{2}, \mathrm{LaAu}_{2}, \mathrm{NdAg}_{2}, \mathrm{LaIn}_{2}, \mathrm{SmZn}_{2}, \mathrm{NdZn}_{2}, \\
\mathrm{PrAg}_{2}, \mathrm{Tl}(\mathrm{CuO})_{2}, \mathrm{~K}_{3} \mathrm{TlAu}_{5}, \mathrm{~K}_{3} \mathrm{InAu}_{5}, \mathrm{BaTbO}_{3}, \mathrm{La}_{3} \mathrm{SiPd}_{5}, \mathrm{NpAl}_{4}\end{array}$ \\
\hline \multicolumn{3}{|r|}{ Space Group 81: $P \overline{4}$} \\
\hline NSOC & $\mathrm{A}_{3} \mathrm{~A}_{4}(2), \mathrm{M}_{3} \mathrm{M}_{4}(2)$ & $\mathrm{Cu}_{2} \mathrm{Te}_{2} \mathrm{Br}_{2} \mathrm{O}_{5}$ \\
\hline \multicolumn{3}{|r|}{ Space Group 82: $I \overline{4}$} \\
\hline \multirow{2}{*}{ NSOC } & $\mathrm{M}_{3} \mathrm{M}_{4}(2)$ & $\mathrm{Fe}_{2} \mathrm{NiP}, \mathrm{Fe}_{3} \mathrm{P}$ \\
\hline & $\Gamma_{3} \Gamma_{4}(2)$ & $\| \mathrm{Ta}_{3} \mathrm{Ge}, \mathrm{CdHgS}_{4}$ \\
\hline \multicolumn{3}{|r|}{ Space Group 83: $P 4 / m$} \\
\hline \multirow[b]{3}{*}{ NSOC } & $\mathrm{M}_{3}^{-} \mathrm{M}_{4}^{-}(2), \mathrm{Z}_{3}^{+} \mathrm{Z}_{4}^{+}(2)$ & $\| \mathrm{Ti}_{2} \mathrm{Ga}_{3}$ \\
\hline & $\mathrm{M}_{3}^{+} \mathrm{M}_{4}^{+}(2), \mathrm{Z}_{3}^{-} \mathrm{Z}_{4}^{-}(2)$ & $\| \mathrm{Ca}_{7} \mathrm{Ni}_{4} \mathrm{Sn}_{13}$ \\
\hline & $\mathrm{M}_{3}^{+} \mathrm{M}_{4}^{+}(2), \mathrm{Z}_{3}^{+} \mathrm{Z}_{4}^{+}(2)$ & $\| \mathrm{Ho}_{7} \operatorname{In}\left(\mathrm{CoGe}_{3}\right)_{4}, \mathrm{Dy}_{7} \mathrm{In}\left(\mathrm{CoGe}_{3}\right)_{4}$ \\
\hline
\end{tabular}


Table II - continued

\begin{tabular}{|c|c|c|}
\hline $\begin{array}{l}\text { SOC or } \\
\text { NSOC }\end{array}$ & $\begin{array}{l}\text { Degenerate Irreducible } \\
\text { Representations }\end{array}$ & High Symmetry Point Semimetals \\
\hline & $\Gamma_{3}^{-} \Gamma_{4}^{-}(2), \mathrm{Z}_{3}^{-} \mathrm{Z}_{4}^{-}(2)$ & $\mathrm{Nd}_{5} \mathrm{Cu}_{5} \mathrm{O}_{13}$ \\
\hline & $\mathrm{M}_{3}^{+} \mathrm{M}_{4}^{+}(2)$ & $\| \mathrm{V}_{3} \mathrm{As}_{2}$ \\
\hline & & Space Group 84: $P 4_{2} / m$ \\
\hline NSOC & $\mathrm{A}_{2}(2), \mathrm{M}_{3}^{+} \mathrm{M}_{4}^{+}(2), \mathrm{Z}_{2}(2)$ & $\mathrm{TbCdF}_{6}$ \\
\hline NSUC & $\mathrm{A}_{1}(2), \Gamma_{3}^{-} \Gamma_{4}^{-}(2), \mathrm{Z}_{1}(2)$ & $\| \mathrm{Zr}_{5} \mathrm{AlNi}_{4}$ \\
\hline $\mathrm{SOC}$ & $\overline{\mathrm{A}}_{3} \overline{\mathrm{A}}_{4}(4), \overline{\mathrm{Z}}_{3} \overline{\mathrm{Z}}_{4}(4)$ & $\| \mathrm{Zr}_{5} \mathrm{AlNi}_{4}, \mathrm{TbCdF}_{6}$ \\
\hline & & Space Group 85: $P 4 / n$ \\
\hline & $\begin{array}{c}\mathrm{A}_{1}(2), \mathrm{M}_{1}(2), \mathrm{R}_{1}(2), \mathrm{X}_{1}(2) \\
\mathrm{Z}_{3}^{+} \mathrm{Z}_{4}^{+}(2)\end{array}$ & $\| \mathrm{Hf}_{5} \mathrm{Sb}_{9}$ \\
\hline NSUC & $\mathrm{A}_{2}(2), \mathrm{M}_{2}(2), \mathrm{R}_{1}(2), \mathrm{X}_{1}(2)$ & $\mathrm{AgBF}_{5}$ \\
\hline & $\mathrm{Z}_{3}^{+} \mathrm{Z}_{4}^{+}(2)$ & $\mathrm{SrBr}_{2}$ \\
\hline $\mathrm{SOC}$ & $\begin{array}{c}\overline{\mathrm{A}}_{3} \overline{\mathrm{A}}_{4}(4), \overline{\mathrm{M}}_{3} \overline{\mathrm{M}}_{4}(4) \\
\overline{\mathrm{R}}_{2} \overline{\mathrm{R}}_{2}(4), \overline{\mathrm{X}}_{2} \overline{\mathrm{X}}_{2}(4)\end{array}$ & $\| \mathrm{AgBF}_{5}, \mathrm{Hf}_{5} \mathrm{Sb}_{9}$ \\
\hline & & Space Group 86: $P 4_{2} / n$ \\
\hline & $\begin{array}{l}\Gamma_{3}^{-} \Gamma_{4}^{-}(2), \mathrm{M}_{1}(2), \mathrm{R}_{1}(2) \\
\mathrm{X}_{1}(2), \mathrm{Z}_{2}(2) \\
\end{array}$ & $\| \mathrm{Tb}(\mathrm{BIr})_{4}, \mathrm{Y}(\mathrm{BIr})_{4}, \mathrm{Nd}(\mathrm{BIr})_{4}, \mathrm{La}(\mathrm{BIr})_{4}, \mathrm{Nd}(\mathrm{CoB})_{4}, \operatorname{Pr}(\mathrm{CoB})_{4}$ \\
\hline $\mathrm{NSOC}$ & $\begin{array}{c}\mathrm{A}_{3}^{-} \mathrm{A}_{4}^{-}(2), \mathrm{M}_{2}(2), \mathrm{R}_{1}(2) \\
\mathrm{X}_{1}(2), \mathrm{Z}_{1}(2)\end{array}$ & $\| \mathrm{Nd}(\mathrm{BOs})_{4}$ \\
\hline & $\mathrm{M}_{2}(2), \mathrm{R}_{1}(2), \mathrm{X}_{1}(2), \mathrm{Z}_{1}(2)$ & $\mathrm{La}(\mathrm{BRu})_{4}$ \\
\hline & $\mathrm{A}_{3}^{-} \mathrm{A}_{4}^{-}(2), \Gamma_{3}^{+} \Gamma_{4}^{+}(2)$ & $\| \mathrm{Ta}_{3} \mathrm{P}$ \\
\hline & $\Gamma_{3}^{-} \Gamma_{4}^{-}(2)$ & $\| \mathrm{Nb}_{3} \mathrm{Si}$ \\
\hline & $\Gamma_{3}^{+} \Gamma_{4}^{+}(2)$ & $\| \mathrm{Ta}_{3} \mathrm{Si}, \mathrm{Nb}_{3} \mathrm{As}, \mathrm{Nb}_{3} \mathrm{P}$ \\
\hline & $\mathrm{A}_{3}^{+} \mathrm{A}_{4}^{+}(2)$ & $\| \mathrm{Ti}_{3} \mathrm{P}, \mathrm{Ti}_{3} \mathrm{Si}$ \\
\hline $\mathrm{SOC}$ & $\begin{array}{c}\overline{\mathrm{M}}_{3} \overline{\mathrm{M}}_{4}(4), \overline{\mathrm{R}}_{2} \overline{\mathrm{R}}_{2}(4) \\
\overline{\mathrm{X}}_{2} \overline{\mathrm{X}}_{2}(4), \overline{\mathrm{Z}}_{3} \overline{\mathrm{Z}}_{4}(4)\end{array}$ & $\| \mathrm{Tb}(\mathrm{BIr})_{4}, \mathrm{Y}(\mathrm{BIr})_{4}, \mathrm{Nd}(\mathrm{BIr})_{4}, \mathrm{La}(\mathrm{BIr})_{4}, \mathrm{Nd}(\mathrm{BOs})_{4}, \mathrm{La}(\mathrm{BRu})_{4}$ \\
\hline & & Space Group 87: $I 4 / m$ \\
\hline & $\Gamma_{3}^{+} \Gamma_{4}^{+}(2), \mathrm{P}_{3} \mathrm{P}_{4}(2)$ & $\| \mathrm{Zr}_{5} \mathrm{Te}_{4}, \mathrm{Ti}_{5} \mathrm{Se}_{4}, \mathrm{Ti}_{5} \mathrm{Te}_{4}, \mathrm{Hf}_{5} \mathrm{Te}_{4}$ \\
\hline & $\Gamma_{3}^{+} \Gamma_{4}^{+}(2), \mathrm{M}_{3}^{-} \mathrm{M}_{4}^{-}(2)$ & $\| \mathrm{Sr}_{2} \mathrm{CuCO}_{5}$ \\
\hline NSOC & $\mathrm{P}_{3} \mathrm{P}_{4}(2)$ & $\| \mathrm{Ba}_{6} \mathrm{Fe}_{8} \mathrm{~S}_{15}$ \\
\hline IVNOC & $\mathrm{M}_{3}^{-} \mathrm{M}_{4}^{-}(2)$ & $\begin{array}{c}\| \mathrm{YbAu}_{4}, \mathrm{Mo}_{5} \mathrm{As}_{4}(\mathrm{ICSD}: 43186), \mathrm{Nd}\left(\mathrm{MoO}_{2}\right)_{6}, \mathrm{Pr}\left(\mathrm{MoO}_{2}\right)_{6}, \mathrm{Ni}_{4} \mathrm{~W}, \\
\mathrm{Ni}_{4} \mathrm{Mo}, \mathrm{Mo}_{5} \mathrm{As}_{4}(\mathrm{ICSD}: 610956), \mathrm{U}(\mathrm{CrC})_{4}\end{array}$ \\
\hline & $\Gamma_{3}^{-} \Gamma_{4}^{-}(2)$ & $\| \mathrm{Ba}\left(\mathrm{Cu}_{2} \mathrm{As}\right)_{4}, \mathrm{Ba}\left(\mathrm{Cu}_{2} \mathrm{P}\right)_{4}, \mathrm{Li}_{4} \mathrm{Yb}_{5} \mathrm{Ge}_{4}$ \\
\hline & $\Gamma_{3}^{+} \Gamma_{4}^{+}(2)$ & $\mathrm{Sr}_{2} \mathrm{CaWO}_{6}, \| \mathrm{Te}(\mathrm{HO})_{6}, \mathrm{Fe}_{4} \mathrm{C}, \mathrm{Ba}_{5} \mathrm{Yb}_{8} \mathrm{Zn}_{4} \mathrm{O}_{21}$ \\
\hline & & Space Group 88: $I 4_{1} / a$ \\
\hline & $\begin{aligned} \Gamma_{3}^{+} \Gamma_{4}^{+}(2), & \mathrm{M}_{2}(2), \mathrm{P}_{2} \mathrm{P}_{3}(2) \\
& \mathrm{X}_{1}(2)\end{aligned}$ & $\| \mathrm{Y}\left(\mathrm{PdO}_{2}\right)_{2}, \mathrm{Nd}\left(\mathrm{PdO}_{2}\right)_{2}, \operatorname{Pr}\left(\mathrm{PdO}_{2}\right)_{2}, \mathrm{La}\left(\mathrm{PdO}_{2}\right)_{2}$ \\
\hline & $\mathrm{M}_{2}(2), \mathrm{P}_{2} \mathrm{P}_{3}(2), \mathrm{X}_{1}(2)$ & $\| \mathrm{YbPO}_{4}$ \\
\hline NSOC & $\mathrm{M}_{2}(2), \mathrm{P}_{1} \mathrm{P}_{4}(2), \mathrm{X}_{1}(2)$ & $\| \mathrm{Al}_{17}\left(\mathrm{SiPd}_{2}\right)_{4}$ \\
\hline & $\mathrm{M}_{1}(2), \mathrm{P}_{2} \mathrm{P}_{3}(2), \mathrm{X}_{1}(2)$ & $\mathrm{Al}_{21} \mathrm{Pt}_{8}, \mathrm{Al}_{21} \mathrm{Pd}_{8}, \mathrm{Dy}\left(\mathrm{CuO}_{2}\right)_{2}, \mathrm{Y}\left(\mathrm{CuO}_{2}\right)_{2}$ \\
\hline & $\mathrm{M}_{1}(2), \mathrm{P}_{1} \mathrm{P}_{4}(2), \mathrm{X}_{1}(2)$ & $\| \mathrm{La}\left(\mathrm{CuO}_{2}\right)_{2}, \mathrm{Rb}_{15} \mathrm{Hg}_{16}$ \\
\hline & $\Gamma_{3}^{-} \Gamma_{4}^{-}(2)$ & || $\mathrm{DyNbO}_{4}(\mathrm{ICSD}: 239198)$ \\
\hline $\mathrm{SOC}$ & $\overline{\mathrm{M}}_{3} \overline{\mathrm{M}}_{4}(4), \overline{\mathrm{X}}_{2} \overline{\mathrm{X}}_{2}(4)$ & $\begin{array}{c}\| \mathrm{Y}\left(\mathrm{PdO}_{2}\right)_{2}, \mathrm{YbPO}_{4}, \mathrm{Al}_{21} \mathrm{Pt}_{8}, \mathrm{Al}_{21} \mathrm{Pd}_{8}, \mathrm{Al}_{17}\left(\mathrm{SiPd}_{2}\right)_{4}, \mathrm{Dy}\left(\mathrm{CuO}_{2}\right)_{2}, \\
\mathrm{Nd}\left(\mathrm{PdO}_{2}\right)_{2}, \operatorname{Pr}\left(\mathrm{PdO}_{2}\right)_{2}, \mathrm{Y}\left(\mathrm{CuO}_{2}\right)_{2}, \mathrm{Rb}_{15} \mathrm{Hg}_{16}, \mathrm{La}\left(\mathrm{CuO}_{2}\right)_{2}, \\
\mathrm{La}\left(\mathrm{PdO}_{2}\right)_{2}\end{array}$ \\
\hline & & Space Group 91: $P 4_{1} 22$ \\
\hline NSOC & $\Gamma_{5}(2)$ & $\| \mathrm{ThBC}, \mathrm{Ag}_{3} \mathrm{RuO}_{4}$ \\
\hline & & Space Group 92: $P 4_{1} 2_{1} 2$ \\
\hline & $\mathrm{A}_{1} \mathrm{~A}_{2}(4), \Gamma_{5}(2)$ & $\| \mathrm{Nd}_{5} \mathrm{Si}_{4}$ \\
\hline NSOC & $\Gamma_{5}(2)$ & $\| \mathrm{Ti}_{5} \mathrm{Si}_{4}, \mathrm{Y}_{3} \mathrm{Ni}_{2}$ \\
\hline & $\mathrm{A}_{1} \mathrm{~A}_{2}(4)$ & $\begin{array}{c}\mathrm{Sc}_{2} \mathrm{Re}_{3} \mathrm{Si}_{4}, \| \mathrm{Sc}_{2} \mathrm{~V}_{3} \mathrm{Si}_{4}, \mathrm{Pr}_{5} \mathrm{Si}_{4}, \mathrm{Nd}_{10}\left(\mathrm{~B}_{3} \mathrm{C}_{4}\right)_{3}, \mathrm{Pr}_{10}\left(\mathrm{~B}_{3} \mathrm{C}_{4}\right)_{3} \\
\mathrm{La}_{10}\left(\mathrm{~B}_{3} \mathrm{C}_{4}\right)_{3}, \mathrm{La}_{5} \mathrm{Si}_{4}\end{array}$ \\
\hline $\mathrm{SOC}$ & $\overline{\mathrm{A}}_{7} \overline{\mathrm{A}}_{7}(4)$ & $\mathrm{Sc}_{2} \mathrm{~V}_{3} \mathrm{Si}_{4}, \mathrm{Nd}_{10}\left(\mathrm{~B}_{3} \mathrm{C}_{4}\right)_{3}, \operatorname{Pr}_{10}\left(\mathrm{~B}_{3} \mathrm{C}_{4}\right)_{3}, \mathrm{Nd}_{5} \mathrm{Si}_{4}, \mathrm{La}_{10}\left(\mathrm{~B}_{3} \mathrm{C}_{4}\right)_{3}, \mathrm{La}_{5} \mathrm{Si}_{4}$ \\
\hline & & Space Group 97: I422 \\
\hline NSOC & $\mathrm{P}_{3} \mathrm{P}_{4}(2)$ & $\mathrm{Ta}_{2} \mathrm{Se}_{8} \mathrm{I} \|$ \\
\hline & & Space Group 98: $I 4_{1} 22$ \\
\hline NSOC & $\mathrm{M}_{5}(2)$ & $\mathrm{La}_{3} \mathrm{NiBr}_{3}$ \\
\hline
\end{tabular}


Table II - continued

\begin{tabular}{|c|c|c|}
\hline $\begin{array}{l}\text { SOC or } \\
\text { NSOC }\end{array}$ & $\begin{array}{c}\text { Degenerate Irreducible } \\
\text { Representations } \\
\end{array}$ & High Symmetry Point Semimetals \\
\hline \multicolumn{3}{|r|}{ Space Group 99: $P 4 m m$} \\
\hline NSOC & $\mathrm{A}_{5}(2)$ & $\| \mathrm{PrBPt}_{3}, \mathrm{NdBPt}_{3}$ \\
\hline \multicolumn{3}{|r|}{ Space Group 100: $P 4 b m$} \\
\hline NSOC & $\begin{array}{c}\mathrm{A}_{1} \mathrm{~A}_{3}(2), \mathrm{M}_{1} \mathrm{M}_{3}(2), \mathrm{R}_{1}(2) \\
\mathrm{X}_{1}(2)\end{array}$ & $\| \mathrm{BaLaCuBO}_{5}, \mathrm{BaNdCuBO}_{5}$ \\
\hline $\mathrm{SOC}$ & $\overline{\mathrm{A}}_{6} \overline{\mathrm{A}}_{7}(4), \overline{\mathrm{M}}_{6} \overline{\mathrm{M}}_{7}(4)$ & $\| \mathrm{BaLaCuBO}_{5}, \mathrm{BaNdCuBO}_{5}$ \\
\hline \multicolumn{3}{|r|}{ Space Group 104: P4nc } \\
\hline NSOC & $\begin{array}{c}\mathrm{A}_{5}(2), \mathrm{M}_{5}(2), \mathrm{R}_{1}(2), \mathrm{X}_{1}(2) \\
\mathrm{Z}_{5} \mathrm{Z}_{5}(4)\end{array}$ & $\| \mathrm{Ba}_{5} \mathrm{In}_{4} \mathrm{Bi}_{5}$ \\
\hline $\mathrm{SOC}$ & $\overline{\mathrm{A}}_{6} \overline{\mathrm{A}}_{7}(4), \overline{\mathrm{M}}_{6} \overline{\mathrm{M}}_{7}(4), \overline{\mathrm{Z}}_{6} \overline{\mathrm{Z}}_{6}(4)$ & $\mathrm{Ba}_{5} \mathrm{In}_{4} \mathrm{Bi}_{5}$ \\
\hline \multicolumn{3}{|r|}{ Space Group 107: $I 4 m m$} \\
\hline \multirow[t]{4}{*}{ NSOC } & $\Gamma_{5}(2), \mathrm{M}_{5}(2)$ & 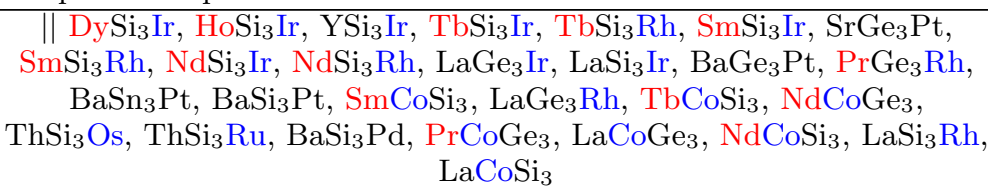 \\
\hline & $\mathrm{P}_{3} \mathrm{P}_{4}(2)$ & $\| \mathrm{HfGa}_{2} \mathrm{Ni}$ \\
\hline & $\mathrm{M}_{5}(2)$ & $\| \mathrm{HfGa}_{2} \mathrm{Co}$ \\
\hline & $\Gamma_{5}(2)$ & $\mathrm{CaSi}_{3} \mathrm{Pt}, \mathrm{SrGe}_{3} \mathrm{Pd}, \mathrm{SrSn}_{3} \mathrm{Pd}, \mathrm{SrNiSn}_{3}, \mathrm{SrSi}_{3} \mathrm{Ni}, \mathrm{CeSi}_{3} \mathrm{Os}, \mathrm{CeSi}_{3} \mathrm{Ru}$ \\
\hline \multicolumn{3}{|r|}{ Space Group 108: $I 4 \mathrm{~cm}$} \\
\hline \multirow{2}{*}{ NSOC } & $\mathrm{N}_{1} \mathrm{~N}_{2}(2), \mathrm{P}_{4} \mathrm{P}_{4}(2)$ & $\mathrm{KSnSe}_{2}$ \\
\hline & $\mathrm{N}_{1} \mathrm{~N}_{2}(2), \mathrm{P}_{1} \mathrm{P}_{2}(2)$ & $\| \mathrm{Cu}\left(\mathrm{BiO}_{2}\right)_{2}, \mathrm{Tl}(\mathrm{ZnSb})_{2}$ \\
\hline $\mathrm{SOC}$ & $\overline{\mathrm{P}}_{5} \overline{\mathrm{P}}_{5}(4)$ & $\| \mathrm{KSnSe}_{2}, \mathrm{Cu}\left(\mathrm{BiO}_{2}\right)_{2}, \mathrm{Tl}(\mathrm{ZnSb})_{2}$ \\
\hline \multicolumn{3}{|r|}{ Space Group 109: $I 4_{1} m d$} \\
\hline \multirow{3}{*}{ NSOC } & $\Gamma_{5}(2), \mathrm{M}_{5}(2), \mathrm{P}_{1}(2), \mathrm{X}_{1}(2)$ & \| NdPIr, PrPIr, LaAsRh, LaPRh, LaAsIr, LaPIr, LaGePt, LaSiNi \\
\hline & $\mathrm{M}_{5}(2), \mathrm{P}_{1}(2), \mathrm{X}_{1}(2)$ & $\|$ PrSiPt, SmSiPt, NdSiPt, CaAsPt \\
\hline & $\Gamma_{5}(2)$ & $\|$ ThSiNi \\
\hline \multirow{2}{*}{ SOC } & $\overline{\mathrm{M}}_{6} \overline{\mathrm{M}}_{7}(4), \overline{\mathrm{P}}_{2} \overline{\mathrm{P}}_{4}(2)$ & LaAsRh, LaPRh, PrPIr, NdPIr, LaAsIr, LaPIr, CaAsPt \\
\hline & $\overline{\mathrm{M}}_{6} \overline{\mathrm{M}}_{7}(4)$ & $\|$ LaGePt, PrSiPt, NdSiPt, SmSiPt, LaSiNi \\
\hline \multicolumn{3}{|r|}{ Space Group 111: $P \overline{4} 2 m$} \\
\hline NSOC & $\mathrm{M}_{5}(2)$ & $\mathrm{FeCuSe}_{2}$ \\
\hline \multicolumn{3}{|r|}{ Space Group 113: $P \overline{4} 2_{1} m$} \\
\hline \multirow{4}{*}{ NSOC } & $\begin{array}{c}\mathrm{A}_{5}(2), \Gamma_{5}(2), \mathrm{M}_{5}(2), \mathrm{R}_{1}(2) \\
\mathrm{X}_{1}(2), \mathrm{Z}_{5}(2)\end{array}$ & $\| \mathrm{Cu}_{3} \mathrm{Se}_{2}$ \\
\hline & $\begin{array}{c}\mathrm{A}_{5}(2), \mathrm{M}_{5}(2), \mathrm{R}_{1}(2), \mathrm{X}_{1}(2) \\
\mathrm{Z}_{5}(2)\end{array}$ & $\| \mathrm{Sr}_{2} \mathrm{P}_{6} \mathrm{Rh}_{7}$ \\
\hline & $\begin{array}{c}\mathrm{A}_{2} \mathrm{~A}_{4}(2), \Gamma_{5}(2), \mathrm{M}_{5}(2) \\
\mathrm{R}_{1}(2), \mathrm{X}_{1}(2)\end{array}$ & $\| \mathrm{NaSn}_{5}$ \\
\hline & $\mathrm{A}_{5}(2), \mathrm{M}_{5}(2), \mathrm{R}_{1}(2), \mathrm{X}_{1}(2)$ & $\| \mathrm{Ta}$ \\
\hline $\mathrm{SOC}$ & $\overline{\mathrm{A}}_{6} \overline{\mathrm{A}}_{7}(4), \overline{\mathrm{M}}_{6} \overline{\mathrm{M}}_{7}(4)$ & $\| \mathrm{NaSn}_{5}, \mathrm{Sr}_{2} \mathrm{P}_{6} \mathrm{Rh}_{7}, \mathrm{Cu}_{3} \mathrm{Se}_{2}, \mathrm{Ta}$ \\
\hline \multicolumn{3}{|r|}{ Space Group 114: $P \overline{4} 2_{1} c$} \\
\hline \multirow[t]{2}{*}{ NSOC } & $\begin{array}{c}\mathrm{A}_{2} \mathrm{~A}_{3}(2), \mathrm{M}_{2} \mathrm{M}_{4}(2), \mathrm{R}_{1}(2) \\
\mathrm{X}_{1}(2), \mathrm{Z}_{3} \mathrm{Z}_{4}(2)\end{array}$ & $\| \mathrm{Sm}_{11}(\mathrm{CoB})_{40}$ \\
\hline & $\Gamma_{5}(2)$ & $\mathrm{As}_{4} \mathrm{~S}_{11}\left(\mathrm{~N}_{6} \mathrm{Cl}_{7}\right)_{2}, \| \mathrm{Pd}_{4} \mathrm{Se}$ \\
\hline \multicolumn{3}{|r|}{ Space Group 115: $P \overline{4} m 2$} \\
\hline \multirow{4}{*}{ NSOC } & $\mathrm{A}_{5}(2), \Gamma_{5}(2)$ & $\| \mathrm{Zr}_{2} \mathrm{CuSb}_{3}, \mathrm{Hf}_{2} \mathrm{CuSb}_{3}, \mathrm{Ti}_{2} \mathrm{CuSb}_{3}$ \\
\hline & $\mathrm{Z}_{5}(2)$ & $\| \mathrm{Hf}_{2} \mathrm{GaSb}_{3}$ \\
\hline & $\Gamma_{5}(2)$ & $\mathrm{Ga}_{2} \mathrm{BiAs} \|$ \\
\hline & $\mathrm{A}_{5}(2)$ & $\| \mathrm{Li}_{2} \mathrm{~B}_{2} \mathrm{C}$ \\
\hline & & Space Group 116: $P \overline{4} c 2$ \\
\hline NSOC & $\mathrm{M}_{5}(2)$ & $\mathrm{Ga}_{17} \mathrm{Rh}_{10}$ \\
\hline & & Space Group 118: $P \overline{4} n 2$ \\
\hline NSOC & $\mathrm{A}_{5}(2), \Gamma_{5}(2)$ & $\| \mathrm{ZnSb}_{2}$ \\
\hline & & 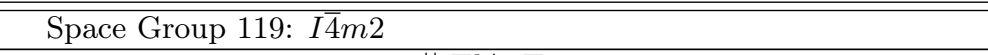 \\
\hline NSOC & $\Gamma_{5}(2), \mathrm{M}_{5}(2)$ & $\| \mathrm{TlAgTe}_{2}$ \\
\hline
\end{tabular}


Table II - continued

\begin{tabular}{|c|c|c|}
\hline $\begin{array}{l}\text { SOC or } \\
\text { NSOC }\end{array}$ & $\begin{array}{l}\text { Degenerate Irreducible } \\
\text { Representations }\end{array}$ & High Symmetry Point Semimetals \\
\hline & $\Gamma_{5}(2)$ & $\mathrm{AlPO}_{4}$ \\
\hline & & Space Group 120: $I \overline{4} c 2$ \\
\hline NSOC & $\mathrm{M}_{5}(2), \mathrm{N}_{1} \mathrm{~N}_{2}(2), \mathrm{P}_{1} \mathrm{P}_{2}(2)$ & $\mathrm{K}\left(\mathrm{SnAu}_{2}\right)_{2}$ \\
\hline & & Space Group 121: $I \overline{4} 2 m$ \\
\hline & $\Gamma_{5}(2), \mathrm{P}_{5}(2)$ & $\| \mathrm{Mo}_{3} \mathrm{P}$ \\
\hline NSOC & $\mathrm{M}_{5}(2)$ & || $\mathrm{FeAg}_{2} \mathrm{SnS}_{4}, \mathrm{~Pb}_{3} \mathrm{Au}$ \\
\hline & $\Gamma_{5}(2)$ & $\begin{array}{c}\mathrm{ZnCu}_{2} \mathrm{SiTe}_{4}, \mathrm{ZnCu}_{2} \mathrm{SnTe}_{4}, \mathrm{Cu}_{2} \mathrm{SnHgTe}_{4}, \mathrm{Cu}_{3} \mathrm{SbS}_{4}\left[62, \mathrm{ZnCu}_{2} \mathrm{GeSe}_{4},\right. \\
\mathrm{CdCu}_{2} \mathrm{SnTe}_{4}, \mathrm{CdCu}_{2} \mathrm{GeSe}_{4}, \mathrm{ZnAg}_{2} \mathrm{SnS}_{4}, \mathrm{CdCu}_{2} \mathrm{SnSe}_{4}, \\
\mathrm{Cu}_{2} \mathrm{SnHgS}_{4}\left[62, \mathrm{CdCu}_{2} \mathrm{SiTe}_{4}, \mathrm{ZnCu}_{2} \mathrm{GeTe}_{4}, \mathrm{Cu}_{2} \mathrm{SiHgTe}_{4}, \|\right. \\
\mathrm{CdCu}_{2} \mathrm{GeTe}_{4}, \mathrm{Cu}_{3} \mathrm{AsSe}_{4}, \mathrm{ZnCu}_{2} \mathrm{SnSe}_{4}\end{array}$ \\
\hline & & 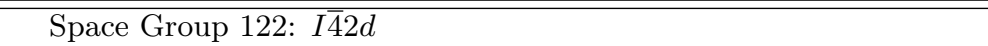 \\
\hline & $\mathrm{M}_{1} \mathrm{M}_{2}(2), \mathrm{P}_{2}(2), \mathrm{X}_{1}(2)$ & $\mathrm{FeCuS}_{2}$ \\
\hline NSOC & $\mathrm{M}_{1} \mathrm{M}_{2}(2), \mathrm{P}_{1}(2), \mathrm{X}_{1}(2)$ & $\| \mathrm{FeAgS}_{2}$ \\
\hline & $\Gamma_{5}(2)$ & $\mathrm{Ba}_{19} \mathrm{Li}_{44}, \mathrm{TlCuS}_{2}, \| \mathrm{UFeC}_{2}, \mathrm{Zr}_{11} \mathrm{Sb}_{18}$ \\
\hline $\mathrm{SOC}$ & $\overline{\mathrm{M}}_{6} \overline{\mathrm{M}}_{7}(4)$ & $\| \mathrm{FeCuS}_{2}, \mathrm{FeAgS}_{2}$ \\
\hline & & Space Group 123: $P 4 / \mathrm{mmm}$ \\
\hline & $\Gamma_{5}^{-}(2), \mathrm{M}_{5}^{+}(2), \mathrm{Z}_{5}^{-}(2)$ & $\| \mathrm{SiSnPd}_{5}$ \\
\hline & $\mathrm{A}_{5}^{-}(2), \Gamma_{5}^{-}(2), \mathrm{Z}_{5}^{+}(2)$ & $\mathrm{Ba}_{2} \mathrm{CaCu}_{2} \mathrm{HgO}_{6}$ \\
\hline & $\mathrm{A}_{5}^{-}(2), \Gamma_{5}^{-}(2), \mathrm{M}_{5}^{+}(2)$ & $\| \mathrm{Hg}_{2} \mathrm{Pt}$ \\
\hline & $\mathrm{A}_{5}^{+}(2), \mathrm{M}_{5}^{+}(2), \mathrm{Z}_{5}^{-}(2)$ & $\mathrm{KNb}_{4} \mathrm{O}_{5} \mathrm{~F}, \mathrm{CeIn}_{5} \mathrm{Ir}$ \\
\hline & $\mathrm{M}_{5}^{+}(2), \mathrm{Z}_{5}^{-}(2)$ & $\| \mathrm{CeIn}_{5} \mathrm{Co}$ \\
\hline & $\Gamma_{5}^{-}(2), \mathrm{Z}_{5}^{+}(2)$ & $\mathrm{BaMg}_{4} \mathrm{Si}_{3}, \mathrm{NaAs}, \mathrm{Ba}_{2} \mathrm{~Tb}_{2} \mathrm{Ti}_{2} \mathrm{Cu}_{2} \mathrm{O}_{11}, \mathrm{Sr}_{2} \mathrm{TlCuO}_{5}$ \\
\hline & $\Gamma_{5}^{-}(2), \mathrm{M}_{5}^{-}(2)$ & $\| \mathrm{Cu}_{3} \mathrm{~N}$ \\
\hline & $\Gamma_{5}^{-}(2), \mathrm{M}_{5}^{+}(2)$ & $\| \mathrm{GaPPd}_{5}, \mathrm{GaAsPd}_{5}$ \\
\hline & $\Gamma_{5}^{+}(2), Z_{5}^{-}(2)$ & $\| \mathrm{Sr}_{2} \mathrm{HoCu}_{3}\left(\mathrm{PbO}_{4}\right)_{2}, \mathrm{YbIn}_{5} \mathrm{Rh}, \mathrm{YbIn}{ }_{5} \mathrm{Ir}, \mathrm{MnAu}$ \\
\hline NSOC & $\Gamma_{5}^{+}(2), Z_{5}^{+}(2)$ & $\begin{array}{c}\| \mathrm{Ba}_{2} \mathrm{Sm}\left(\mathrm{CuO}_{2}\right)_{3}, \mathrm{Ba}_{2} \mathrm{Ho}\left(\mathrm{CuO}_{2}\right)_{3}, \mathrm{Ba}_{2} \mathrm{Y}\left(\mathrm{CuO}_{2}\right)_{3}, \mathrm{Ba}_{2} \mathrm{Lu}\left(\mathrm{CuO}_{2}\right)_{3}, \\
\mathrm{Ba}_{2} \mathrm{Nd}\left(\mathrm{CuO}_{2}\right)_{3}\end{array}$ \\
\hline & $\mathrm{A}_{5}^{-}(2), \mathrm{M}_{5}^{-}(2)$ & $\| \mathrm{HoS}, \mathrm{BaTi}_{2} \mathrm{Sb}_{2} \mathrm{O}, \mathrm{BaTi}_{2} \mathrm{As}_{2} \mathrm{O}, \mathrm{BaTi}_{2} \mathrm{Bi}_{2} \mathrm{O}$ \\
\hline & $\mathrm{A}_{5}^{-}(2), \mathrm{M}_{5}^{+}(2)$ & $\mathrm{Ti}_{3} \mathrm{Pd}_{5}(\mathrm{ICSD}: 105726), \mathrm{Ti}_{3} \mathrm{Pd}_{5}(\mathrm{ICSD}: 167651)$ \\
\hline & $\mathrm{A}_{5}^{+}(2), \mathrm{M}_{5}^{-}(2)$ & $\| \mathrm{AgPPd}_{5}, \mathrm{Ti}_{2} \mathrm{HPd}$ \\
\hline & $\mathrm{A}_{5}^{+}(2), \Gamma_{5}^{-}(2)$ & $\| \operatorname{InPPd}_{5}, \mathrm{HgPd}_{5} \mathrm{Se}$ \\
\hline & $\mathrm{Z}_{5}^{-}(2)$ & $\mathrm{CsZn}_{4} \mathrm{As}_{3}, \| \mathrm{LiBi}, \mathrm{NaBi}, \mathrm{U}_{2} \mathrm{Ga}_{8} \mathrm{Ru}$ \\
\hline & $\mathrm{Z}_{5}^{+}(2)$ & $\| \mathrm{Er}_{2} \mathrm{Ga}_{8} \mathrm{Fe}, \mathrm{Tm}_{2} \mathrm{Ga}_{8} \mathrm{Fe}$ \\
\hline & $\mathrm{M}_{5}^{-}(2)$ & ZnPd, CdPd, LiHPd, HgPd, $\| \mathrm{CuAu}, \mathrm{MgNi}$, NbIr, NbRh, RbN 3 , VIr \\
\hline & $\mathrm{M}_{5}^{+}(2)$ & $\begin{aligned} \| \mathrm{GaPPt}_{5}, \mathrm{InAsPt}_{5}, \mathrm{AlPPt}_{5}, \mathrm{SiPt}_{5} \mathrm{~Pb}, \mathrm{TlAsPt}_{5}, \mathrm{InPPt}_{5}, \mathrm{TlPPt}_{5} \\
\mathrm{BaNb}_{4} \mathrm{O}_{6}, \mathrm{TiCdHg}_{2}, \mathrm{FePt}, \mathrm{AgPPt}_{5}, \mathrm{Fe}_{3} \mathrm{Pt}\end{aligned}$ \\
\hline & $\Gamma_{5}^{-}(2)$ & $\begin{array}{c}\mathrm{SrCdO}_{2}, \mathrm{RbZn}_{4} \mathrm{As}_{3}, \| \mathrm{NdAs}_{\mathrm{A}}, \mathrm{SmAs}_{\mathrm{A}} \mathrm{PrAs}, \mathrm{PrBi}, \mathrm{PrSb}, \mathrm{NdSb}, \mathrm{SmBi} \\
\mathrm{LaAs}_{\mathrm{LaSb}}, \mathrm{TlPPd}_{5}, \mathrm{InAsPd}_{5}, \mathrm{TlAsPd}_{5}, \mathrm{BaMg}_{4} \mathrm{Ge}_{3}\end{array}$ \\
\hline & $\Gamma_{5}^{+}(2)$ & $\| \mathrm{MgTiH}_{4}, \mathrm{YbGa}_{5} \mathrm{Co}, \mathrm{YbIn}_{5} \mathrm{Co}, \mathrm{Nd}_{2} \mathrm{In}_{8} \mathrm{Pd}, \mathrm{Pr}_{2} \mathrm{In}_{8} \mathrm{Pd}, \mathrm{NiPt}, \mathrm{SrNb}_{4} \mathrm{O}_{6}$ \\
\hline & $\mathrm{A}_{5}^{+}(2)$ & $\begin{array}{c}\mathrm{Si}_{2} \mathrm{Ru}, \| \mathrm{Na}_{3} \mathrm{Cl}, \mathrm{ErTl}, \mathrm{SmTl}, \mathrm{Ba}(\mathrm{PPd})_{2}, \mathrm{Ba}(\mathrm{AsPd})_{2}, \mathrm{ZnPd}_{5} \mathrm{Se} \\
\mathrm{MgSc}_{2} \mathrm{Ru}, \mathrm{LaAl}_{2} \mathrm{BRu}_{2}, \mathrm{CdPd}_{5} \mathrm{Se}\end{array}$ \\
\hline & & Space Group 124: $P 4 / m c c$ \\
\hline & $\begin{array}{c}\mathrm{A}_{3} \mathrm{~A}_{4}(4), \Gamma_{5}^{+}(2), \mathrm{M}_{5}^{-}(2) \\
\mathrm{R}_{1}(2), \mathrm{Z}_{3} \mathrm{Z}_{4}(4)\end{array}$ & $\| \mathrm{Si}_{2} \mathrm{Bi}_{14} \mathrm{RhI}_{12}$ \\
\hline NSOC & $\begin{array}{l}\mathrm{A}_{3} \mathrm{~A}_{4}(4), \Gamma_{5}^{+}(2), \mathrm{M}_{5}^{+}(2) \\
\mathrm{R}_{1}(2), \mathrm{Z}_{3} \mathrm{Z}_{4}(4)\end{array}$ & $\| \mathrm{Si}_{2} \mathrm{Bi}_{14} \mathrm{IrI}_{12}$ \\
\hline & $\begin{array}{c}\mathrm{A}_{3} \mathrm{~A}_{4}(4), \mathrm{M}_{5}^{-}(2), \mathrm{R}_{2}(2) \\
\mathrm{Z}_{1}(2)\end{array}$ & $\| \mathrm{Nb}_{10} \mathrm{Se}_{40} \mathrm{I}_{3}$ \\
\hline & $\mathrm{A}_{2}(2), \mathrm{R}_{1}(2), \mathrm{Z}_{1}(2)$ & $\prod \mathrm{Nb}_{4} \mathrm{CoSi}, \mathrm{Ta}_{4} \mathrm{FeP}$ \\
\hline & $\mathrm{A}_{1}(2), \mathrm{R}_{1}(2), \mathrm{Z}_{2}(2)$ & $\mathrm{NbSe}_{4}, \mathrm{TaTe}_{4}, \mathrm{NbTe}_{4}$ \\
\hline & $\Gamma_{5}^{-}(2), \mathrm{M}_{5}^{-}(2)$ & $\| \mathrm{Nb}_{4} \mathrm{FeSi}$ \\
\hline & $\Gamma_{5}^{-}(2)$ & $\| \mathrm{Nb}_{4} \mathrm{SiNi}$ \\
\hline $\mathrm{SOC}$ & $\overline{\mathrm{A}}_{6} \overline{\mathrm{A}}_{8}(4), \overline{\mathrm{R}}_{3} \overline{\mathrm{R}}_{4}(4), \overline{\mathrm{Z}}_{5} \overline{\mathrm{Z}}_{7}(4)$ & $\mathrm{Nb}_{4} \mathrm{CoSi}, \mathrm{Ta}_{4} \mathrm{FeP}, \mathrm{Si}_{2} \mathrm{Bi}_{14} \mathrm{IrI}_{12}, \mathrm{Si}_{2} \mathrm{Bi}_{14} \mathrm{RhI}_{12}$ \\
\hline & $\overline{\mathrm{A}}_{5} \overline{\mathrm{A}}_{7}(4), \overline{\mathrm{R}}_{3} \overline{\mathrm{R}}_{4}(4), \overline{\mathrm{Z}}_{6} \overline{\mathrm{Z}}_{8}(4)$ & $\| \mathrm{NbSe}_{4}, \mathrm{TaTe}_{4}, \mathrm{NbTe}_{4}$ \\
\hline & & Space Group 125: P4/nbm \\
\hline & $\mathrm{A}_{3}(2), \mathrm{M}_{1}(2), \mathrm{R}_{1}(2), \mathrm{X}_{1}(2)$ & $\mathrm{Ce}_{2} \mathrm{Ga}_{12} \mathrm{Cu}$ \\
\hline
\end{tabular}


Table II - continued

\begin{tabular}{|c|c|c|}
\hline $\begin{array}{l}\text { SOC or } \\
\text { NSOC }\end{array}$ & $\begin{array}{c}\text { Degenerate Irreducible } \\
\text { Representations }\end{array}$ & High Symmetry Point Semimetals \\
\hline & $\mathrm{A}_{1}(2), \mathrm{M}_{1}(2), \mathrm{R}_{1}(2), \mathrm{X}_{2}(2)$ & $\| \mathrm{YGa}_{6}, \mathrm{LaGa}_{6}$ \\
\hline & $\Gamma_{5}^{-}(2)$ & $\mathrm{RbAg}_{5} \mathrm{Se}_{3}, \| \mathrm{PtPb}_{4}$ \\
\hline & $\Gamma_{5}^{+}(2)$ & $\| \mathrm{La}_{2} \mathrm{Ga}_{12} \mathrm{Pd}, \mathrm{La}_{2} \mathrm{Ga}_{12} \mathrm{Ni}$ \\
\hline $\mathrm{SOC}$ & $\begin{array}{c}\overline{\mathrm{A}}_{5}(4), \overline{\mathrm{M}}_{5}(4), \overline{\mathrm{R}}_{3} \overline{\mathrm{R}}_{4}(4) \\
\overline{\mathrm{X}}_{3} \overline{\mathrm{X}}_{4}(4)\end{array}$ & $\| \mathrm{YGa}_{6}, \mathrm{LaGa}_{6}, \mathrm{Ce}_{2} \mathrm{Ga}_{12} \mathrm{Cu}$ \\
\hline \multirow{27}{*}{ NSOC } & \multicolumn{2}{|r|}{ Space Group 127: $P 4 / m b m$} \\
\hline & $\begin{array}{c}\mathrm{A}_{5}^{-}(2), \Gamma_{5}^{-}(2), \mathrm{M}_{1}^{-} \mathrm{M}_{4}^{-}(2) \\
\mathrm{R}_{1}(2), \mathrm{X}_{1}(2), \mathrm{Z}_{5}^{+}(2)\end{array}$ & $\| \mathrm{Li}(\mathrm{SiRh})_{2}$ \\
\hline & $\begin{array}{c}\mathrm{A}_{5}^{+}(2), \Gamma_{5}^{+}(2), \mathrm{M}_{5}^{+}(2) \\
\mathrm{R}_{2}(2), \mathrm{X}_{1}(2), \mathrm{Z}_{5}^{+}(2)\end{array}$ & $\| \mathrm{Nb}_{3} \mathrm{Si}_{2}$ \\
\hline & $\begin{array}{c}\mathrm{A}_{2}^{-} \mathrm{A}_{3}^{-}(2), \Gamma_{5}^{-}(2) \\
\mathrm{M}_{1}^{+} \mathrm{M}_{4}^{+}(2), \mathrm{R}_{2}(2), \mathrm{X}_{2}(2) \\
\mathrm{Z}_{5}^{-}(2)\end{array}$ & $\| \mathrm{Sm}_{26} \mathrm{Ga}_{6} \mathrm{Co}_{11}$ \\
\hline & $\begin{array}{c}\mathrm{A}_{5}^{-}(2), \mathrm{M}_{5}^{+}(2), \mathrm{R}_{2}(2) \\
\mathrm{X}_{2}(2), \mathrm{Z}_{5}^{+}(2)\end{array}$ & $\| \mathrm{Y}_{2} \mathrm{InCu}_{2}$ \\
\hline & $\begin{array}{c}\mathrm{A}_{5}^{-}(2), \mathrm{M}_{5}^{+}(2), \mathrm{R}_{2}(2) \\
\mathrm{X}_{1}(2), \mathrm{Z}_{5}^{+}(2)\end{array}$ & $\| \mathrm{Be}_{2} \mathrm{Nb}_{3}$ \\
\hline & $\begin{array}{c}\mathrm{A}_{5}^{-}(2), \mathrm{M}_{5}^{+}(2), \mathrm{R}_{1}(2) \\
\mathrm{X}_{2}(2), \mathrm{Z}_{5}^{+}(2)\end{array}$ & $\| \mathrm{Lu}_{2} \mathrm{InAu}_{2}, \mathrm{Tm}_{2} \mathrm{InAu}_{2}, \mathrm{~V}_{2} \mathrm{Ga}_{5}$ \\
\hline & $\begin{array}{c}\mathrm{A}_{5}^{-}(2), \mathrm{M}_{2}^{-} \mathrm{M}_{3}^{-}(2), \mathrm{R}_{1}(2), \\
\mathrm{X}_{2}(2), \mathrm{Z}_{5}^{-}(2)\end{array}$ & $\| \mathrm{Sc}_{5}\left(\mathrm{Co}_{2} \mathrm{Si}_{5}\right)_{2}$ \\
\hline & $\begin{array}{c}\mathrm{A}_{5}^{-}(2), \mathrm{M}_{1}^{-} \mathrm{M}_{4}^{-}(2), \mathrm{R}_{2}(2) \\
\mathrm{X}_{2}(2), \mathrm{Z}_{5}^{+}(2)\end{array}$ & $\| \mathrm{Zr}_{2} \mathrm{InCo}_{2}$ \\
\hline & $\begin{array}{c}\mathrm{A}_{5}^{-}(2), \Gamma_{5}^{+}(2), \mathrm{M}_{5}^{-}(2) \\
\mathrm{R}_{1}(2), \mathrm{X}_{2}(2)\end{array}$ & $\| \mathrm{Nb}_{3} \mathrm{Ga}_{2}$ \\
\hline & $\begin{array}{c}\mathrm{A}_{5}^{-}(2), \Gamma_{5}^{+}(2), \mathrm{M}_{5}^{-}(2) \\
\mathrm{R}_{1}(2), \mathrm{X}_{1}(2)\end{array}$ & $\| \mathrm{Ba}_{3} \mathrm{Nb}_{5} \mathrm{O}_{15}, \mathrm{Ba}_{3} \mathrm{Ta}_{5} \mathrm{O}_{15}$ \\
\hline & $\begin{array}{c}\mathrm{A}_{5}^{+}(2), \mathrm{M}_{5}^{-}(2), \mathrm{R}_{2}(2) \\
\mathrm{X}_{1}(2), \mathrm{Z}_{5}^{-}(2)\end{array}$ & $\| \mathrm{Sc}_{2} \mathrm{AlSi}_{2}$ \\
\hline & $\begin{array}{c}\mathrm{A}_{5}^{+}(2), \mathrm{M}_{5}^{+}(2), \mathrm{R}_{2}(2) \\
\mathrm{X}_{2}(2), \mathrm{Z}_{5}^{-}(2)\end{array}$ & $\| \mathrm{Lu}_{2} \mathrm{InCu}_{2}$ \\
\hline & $\begin{array}{c}\mathrm{A}_{5}^{+}(2), \mathrm{M}_{5}^{+}(2), \mathrm{R}_{2}(2) \\
\mathrm{X}_{2}(2), \mathrm{Z}_{5}^{+}(2)\end{array}$ & $\| \mathrm{Er}_{2} \mathrm{InCu}_{2}$ \\
\hline & $\begin{array}{c}\mathrm{A}_{5}^{+}(2), \mathrm{M}_{1}^{+} \mathrm{M}_{4}^{+}(2), \mathrm{R}_{1}(2) \\
\mathrm{X}_{1}(2), \mathrm{Z}_{5}^{-}(2)\end{array}$ & $\| \mathrm{Li}\left(\mathrm{Sn}_{3} \mathrm{Pd}\right)_{2}$ \\
\hline & $\begin{array}{c}\mathrm{A}_{5}^{+}(2), \Gamma_{5}^{-}(2), \mathrm{M}_{5}^{+}(2) \\
\mathrm{R}_{2}(2), \mathrm{X}_{2}(2)\end{array}$ & $\| \mathrm{Hf}_{2} \operatorname{In}_{5}$ \\
\hline & $\begin{array}{c}\mathrm{A}_{5}^{+}(2), \Gamma_{5}^{-}(2), \mathrm{M}_{2}^{-} \mathrm{M}_{3}^{-}(2) \\
\mathrm{R}_{2}(2), \mathrm{X}_{2}(2)\end{array}$ & $\| \mathrm{La}_{2} \mathrm{InGe}_{2}, \mathrm{Dy}_{2} \mathrm{AlGe}_{2}, \mathrm{Er}_{3} \mathrm{Pd}_{2}, \mathrm{Ho}_{3} \mathrm{Pd}_{2}, \mathrm{Dy}_{3} \mathrm{Pd}_{2}$ \\
\hline & $\begin{array}{c}\mathrm{A}_{5}^{+}(2), \Gamma_{5}^{-}(2), \mathrm{M}_{2}^{-} \mathrm{M}_{3}^{-}(2) \\
\mathrm{R}_{1}(2), \mathrm{X}_{1}(2)\end{array}$ & $\| \mathrm{Pu}_{2} \operatorname{InPd}_{2}$ \\
\hline & $\begin{array}{c}\mathrm{A}_{2}^{-} \mathrm{A}_{3}^{-}(2), \mathrm{M}_{5}^{+}(2), \mathrm{R}_{2}(2) \\
\mathrm{X}_{2}(2), \mathrm{Z}_{5}^{-}(2)\end{array}$ & $\| \mathrm{Tm}_{2} \mathrm{InCu}_{2}$ \\
\hline & $\begin{array}{c}\mathrm{A}_{2}^{+} \mathrm{A}_{3}^{+}(2), \Gamma_{5}^{-}(2) \\
\mathrm{M}_{2}^{-} \mathrm{M}_{3}^{-}(2), \mathrm{R}_{1}(2), \mathrm{X}_{2}(2)\end{array}$ & $\| \mathrm{Ti}_{3} \mathrm{Co}_{5} \mathrm{~B}_{2}$ \\
\hline & $\begin{array}{c}\mathrm{A}_{1}^{-} \mathrm{A}_{4}^{-}(2), \mathrm{M}_{5}^{+}(2), \mathrm{R}_{2}(2), \\
\mathrm{X}_{1}(2), \mathrm{Z}_{5}^{+}(2)\end{array}$ & $\| \mathrm{Ta}_{3} \mathrm{Be}_{2}$ \\
\hline & $\begin{array}{c}\mathrm{A}_{1}^{-} \mathrm{A}_{4}^{-}(2), \mathrm{M}_{2}^{-} \mathrm{M}_{3}^{-}(2) \\
\mathrm{R}_{1}(2), \mathrm{X}_{2}(2), \mathrm{Z}_{5}^{-}(2)\end{array}$ & $\| \mathrm{Yb}_{2} \mathrm{InCu}_{2}$ \\
\hline & $\begin{array}{c}\mathrm{A}_{1}^{-} \mathrm{A}_{4}^{-}(2), \Gamma_{5}^{-}(2) \\
\mathrm{M}_{1}^{-} \mathrm{M}_{4}^{-}(2), \mathrm{R}_{2}(2), \mathrm{X}_{2}(2)\end{array}$ & $\| \mathrm{La}_{2} \mathrm{InRh}_{2}$ \\
\hline & $\begin{array}{c}\mathrm{A}_{1}^{+} \mathrm{A}_{4}^{+}(2), \mathrm{M}_{5}^{-}(2), \mathrm{R}_{1}(2) \\
\mathrm{X}_{1}(2), \mathrm{Z}_{5}^{+}(2)\end{array}$ & $\| \mathrm{Ta}(\mathrm{BMo})_{2}, \mathrm{Nb}(\mathrm{BMo})_{2}$ \\
\hline & $\begin{array}{c}\mathrm{A}_{1}^{+} \mathrm{A}_{4}^{+}(2), \mathrm{M}_{5}^{+}(2), \mathrm{R}_{1}(2), \\
\mathrm{X}_{2}(2), \mathrm{Z}_{5}^{-}(2)\end{array}$ & $\| \mathrm{Sc}_{2} \mathrm{InCu}_{2}$ \\
\hline & $\begin{array}{c}\mathrm{A}_{1}^{+} \mathrm{A}_{4}^{+}(2), \Gamma_{5}^{-}(2) \\
\mathrm{M}_{1}^{-} \mathrm{M}_{4}^{-}(2), \mathrm{R}_{2}(2), \mathrm{X}_{2}(2)\end{array}$ & $\| \mathrm{Nd}_{2} \mathrm{InRh}_{2}, \mathrm{Yb}_{2} \operatorname{InPd}_{2}$ \\
\hline & $\mathrm{A}_{5}^{-}(2), \mathrm{M}_{5}^{-}(2), \mathrm{R}_{1}(2), \mathrm{X}_{2}(2)$ & $\| \mathrm{ReN}_{2}, \mathrm{Ti}_{2} \mathrm{ReB}_{2}, \mathrm{Yb}_{2} \mathrm{InGe}_{2}, \mathrm{Yb}_{2} \mathrm{AlSi}_{2}$ \\
\hline
\end{tabular}


Table II - continued

\begin{tabular}{|c|c|c|}
\hline $\begin{array}{l}\text { SOC or } \\
\text { NSOC }\end{array}$ & $\begin{array}{l}\text { Degenerate Irreducible } \\
\text { Representations }\end{array}$ & High Symmetry Point Semimetals \\
\hline & $\mathrm{A}_{5}^{-}(2), \mathrm{M}_{5}^{-}(2), \mathrm{R}_{1}(2), \mathrm{X}_{1}(2)$ & $\mathrm{Mg}_{2} \mathrm{GeB}_{2} \mathrm{Rh}_{5}$ \\
\hline & $\mathrm{A}_{5}^{-}(2), \mathrm{M}_{5}^{+}(2), \mathrm{R}_{2}(2), \mathrm{X}_{2}(2)$ & $\| \mathrm{Zr}_{2} \mathrm{InNi}_{2}$ \\
\hline & $\mathrm{A}_{5}^{-}(2), \mathrm{M}_{5}^{+}(2), \mathrm{R}_{1}(2), \mathrm{X}_{2}(2)$ & $\mathrm{Nb}_{3} \mathrm{~B}_{2}, \mathrm{Lu}_{5}\left(\mathrm{Si}_{5} \mathrm{Ir}_{2}\right)_{2}, \mathrm{Sc}_{2} \mathrm{CrB}_{2} \mathrm{Rh}_{5}$ \\
\hline & $\mathrm{A}_{5}^{-}(2), \mathrm{M}_{5}^{+}(2), \mathrm{R}_{1}(2), \mathrm{X}_{1}(2)$ & $\| \mathrm{Li}(\mathrm{YSi})_{2}, \mathrm{Tm}_{5}\left(\mathrm{Ge}_{5} \mathrm{Rh}_{2}\right)_{2}$ \\
\hline & $\begin{array}{c}\mathrm{A}_{5}^{-}(2), \mathrm{M}_{2}^{-} \mathrm{M}_{3}^{-}(2), \mathrm{R}_{1}(2) \\
\mathrm{X}_{2}(2)\end{array}$ & $\| \mathrm{Li}(\mathrm{NdSi})_{2}$ \\
\hline & $\begin{array}{c}\mathrm{A}_{5}^{-}(2), \mathrm{M}_{2}^{+} \mathrm{M}_{3}^{+}(2), \mathrm{R}_{1}(2) \\
\mathrm{X}_{1}(2)\end{array}$ & $\| \mathrm{ReO}_{3}, \mathrm{Mg}_{2} \mathrm{ZnB}_{2} \mathrm{Ir}_{5}$ \\
\hline & $\begin{array}{c}\mathrm{A}_{5}^{-}(2), \mathrm{M}_{1}^{-} \mathrm{M}_{4}^{-}(2), \mathrm{R}_{1}(2) \\
\mathrm{X}_{2}(2)\end{array}$ & $\| \mathrm{Ho}_{5}\left(\mathrm{Ge}_{5} \mathrm{Rh}_{2}\right)_{2}$ \\
\hline & $\mathrm{A}_{5}^{+}(2), \mathrm{M}_{5}^{+}(2), \mathrm{R}_{2}(2), \mathrm{X}_{2}(2)$ & 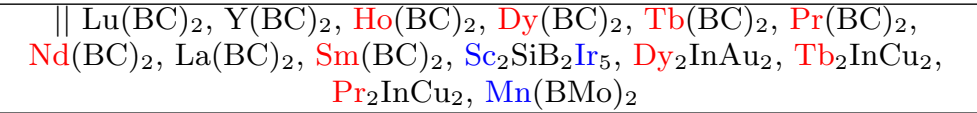 \\
\hline & $\mathrm{A}_{5}^{+}(2), \mathrm{M}_{5}^{+}(2), \mathrm{R}_{2}(2), \mathrm{X}_{1}(2)$ & $\| \mathrm{Mn}(\mathrm{BW})_{2}$ \\
\hline & $\mathrm{A}_{5}^{+}(2), \mathrm{M}_{5}^{+}(2), \mathrm{R}_{1}(2), \mathrm{X}_{2}(2)$ & $\| \mathrm{Hf}_{2} \operatorname{InPd}_{2}$ \\
\hline & $\begin{array}{c}\mathrm{A}_{5}^{+}(2), \mathrm{M}_{2}^{-} \mathrm{M}_{3}^{-}(2), \mathrm{R}_{2}(2) \\
\mathrm{X}_{2}(2)\end{array}$ & $\begin{array}{c}\| \mathrm{Dy}_{2} \mathrm{InGe}_{2}, \mathrm{~Tb}_{2} \mathrm{InGe}_{2}, \mathrm{Sm}_{2} \mathrm{InGe}_{2}, \mathrm{Nd}_{2} \mathrm{InGe}_{2}, \mathrm{Pr}_{2} \mathrm{InGe}_{2}, \mathrm{Ho}_{2} \mathrm{InGe}_{2}, \\
\mathrm{Zr}_{2} \mathrm{InAu}_{2}\end{array}$ \\
\hline & $\begin{array}{c}\mathrm{A}_{5}^{+}(2), \mathrm{M}_{2}^{-} \mathrm{M}_{3}^{-}(2), \mathrm{R}_{1}(2) \\
\mathrm{X}_{2}(2)\end{array}$ & $\| \mathrm{La}_{2} \mathrm{InNi}_{2}$ \\
\hline & $\begin{array}{c}\mathrm{A}_{2}^{-} \mathrm{A}_{3}^{-}(2), \mathrm{M}_{2}^{-} \mathrm{M}_{3}^{-}(2) \\
\mathrm{R}_{2}(2), \mathrm{X}_{2}(2)\end{array}$ & $\| \mathrm{KCuF}_{3}$ \\
\hline & $\begin{array}{c}\mathrm{A}_{2}^{+} \mathrm{A}_{3}^{+}(2), \mathrm{M}_{5}^{-}(2), \mathrm{R}_{1}(2) \\
\mathrm{X}_{2}(2)\end{array}$ & $\| \mathrm{Ta}_{3} \mathrm{Ga}_{2}$ \\
\hline & $\begin{array}{c}\mathrm{A}_{2}^{+} \mathrm{A}_{3}^{+}(2), \mathrm{M}_{5}^{-}(2), \mathrm{R}_{1}(2) \\
\mathrm{X}_{1}(2) \\
\end{array}$ & $\| \mathrm{Mg}_{2} \mathrm{SiB}_{2} \operatorname{Ir}_{5}$ \\
\hline & $\begin{array}{c}\mathrm{A}_{2}^{+} \mathrm{A}_{3}^{+}(2), \mathrm{M}_{5}^{+}(2), \mathrm{R}_{1}(2) \\
\mathrm{X}_{2}(2)\end{array}$ & $\| \mathrm{Ta}_{3} \mathrm{~B}_{2}, \mathrm{~V}_{3} \mathrm{~B}_{2}, \mathrm{Ho}_{5}\left(\mathrm{Ge}_{5} \mathrm{Ir}_{2}\right)_{2}$ \\
\hline & $\begin{array}{c}\mathrm{A}_{2}^{+} \mathrm{A}_{3}^{+}(2), \mathrm{M}_{2}^{+} \mathrm{M}_{3}^{+}(2) \\
\mathrm{R}_{1}(2), \mathrm{X}_{1}(2)\end{array}$ & $\| \mathrm{Al}(\mathrm{CuRe})_{2}$ \\
\hline & $\begin{array}{c}\mathrm{A}_{1}^{-} \mathrm{A}_{4}^{-}(2), \mathrm{M}_{5}^{+}(2), \mathrm{R}_{2}(2) \\
\mathrm{X}_{2}(2)\end{array}$ & $\| \mathrm{Tb}_{2} \mathrm{InAu}_{2}, \mathrm{Y}_{2} \operatorname{InAu_{2}}, \mathrm{Ho}_{2} \mathrm{InAu}_{2}, \mathrm{Er}_{2} \mathrm{InAu}_{2}$ \\
\hline & $\begin{array}{c}\mathrm{A}_{1}^{-} \mathrm{A}_{4}^{-}(2), \mathrm{M}_{2}^{-} \mathrm{M}_{3}^{-}(2) \\
\mathrm{R}_{1}(2), \mathrm{X}_{2}(2)\end{array}$ & $\begin{array}{c}\| \mathrm{Sc}_{2} \mathrm{InNi}_{2}, \mathrm{Lu}_{2} \mathrm{InPd}_{2}, \mathrm{Tm}_{2} \mathrm{InPd}_{2}, \mathrm{Er}_{2} \mathrm{InPd}_{2}, \mathrm{Ho}_{2} \mathrm{InPd}_{2}, \mathrm{Dy}_{2} \mathrm{InPd}_{2}, \\
\mathrm{~Tb}_{2} \mathrm{InPd}_{2}, \mathrm{Sm}_{2} \mathrm{InPd}_{2}, \mathrm{Dy}_{2} \mathrm{InNi}_{2}, \mathrm{Sm}_{2} \mathrm{InNi}_{2}, \mathrm{Ho}_{2} \mathrm{InNi}_{2}, \mathrm{Er}_{2} \mathrm{InNi}_{2}, \\
\mathrm{Y}_{2} \mathrm{InPd}_{2}, \mathrm{Lu}_{2} \mathrm{InNi}_{2}, \mathrm{Y}_{2} \mathrm{InNi}_{2}\end{array}$ \\
\hline & $\begin{array}{c}\mathrm{A}_{1}^{-} \mathrm{A}_{4}^{-}(2), \mathrm{M}_{1}^{-} \mathrm{M}_{4}^{-}(2), \\
\mathrm{R}_{2}(2), \mathrm{X}_{2}(2)\end{array}$ & 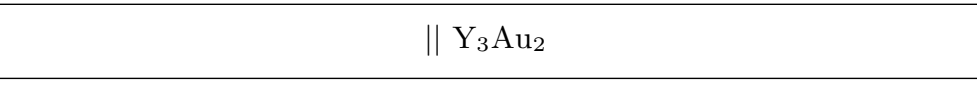 \\
\hline & $\begin{array}{c}\mathrm{A}_{1}^{-} \mathrm{A}_{4}^{-}(2), \mathrm{M}_{1}^{-} \mathrm{M}_{4}^{-}(2) \\
\mathrm{R}_{1}(2), \mathrm{X}_{2}(2)\end{array}$ & $\| \mathrm{Nd}_{2} \mathrm{InNi}_{2}, \mathrm{~Tb}_{2} \mathrm{InNi}_{2}$ \\
\hline & $\begin{array}{c}\mathrm{A}_{1}^{+} \mathrm{A}_{4}^{+}(2), \mathrm{M}_{5}^{+}(2), \mathrm{R}_{2}(2) \\
\mathrm{X}_{2}(2)\end{array}$ & $\| \mathrm{La}_{2} \mathrm{InCu}_{2}, \mathrm{Ce}_{2} \mathrm{InNi}_{2}, \mathrm{Ce}_{2} \mathrm{InPt}_{2}, \mathrm{Ce}_{2} \mathrm{InPd}_{2}$ \\
\hline & $\begin{array}{l}\mathrm{A}_{1}^{+} \mathrm{A}_{4}^{+}(2), \mathrm{M}_{2}^{-} \mathrm{M}_{3}^{-}(2) \\
\mathrm{R}_{1}(2), \mathrm{X}_{2}(2)\end{array}$ & $\| \mathrm{Nd}_{2} \operatorname{InPd}_{2}, \mathrm{Pr}_{2} \operatorname{InPd}_{2}, \mathrm{La}_{2} \operatorname{InPd}_{2}$ \\
\hline & $\begin{array}{c}\mathrm{A}_{1}^{+} \mathrm{A}_{4}^{+}(2), \mathrm{M}_{2}^{+} \mathrm{M}_{3}^{+}(2) \\
\mathrm{R}_{2}(2), \mathrm{X}_{1}(2)\end{array}$ & $\| \mathrm{AlPt}_{3}(\mathrm{ICSD}: 609153), \mathrm{GaPt}_{3}$ \\
\hline & $\begin{array}{l}\mathrm{A}_{1}^{+} \mathrm{A}_{4}^{+}(2), \mathrm{M}_{1}^{-} \mathrm{M}_{4}^{-}(2) \\
\mathrm{R}_{1}(2), \mathrm{X}_{2}(2)\end{array}$ & $\| \mathrm{Pr}_{2} \mathrm{InNi}_{2}, \mathrm{La}_{3} \mathrm{Si}_{2}$ \\
\hline & $\begin{array}{c}\mathrm{A}_{1}^{+} \mathrm{A}_{4}^{+}(2), \mathrm{M}_{1}^{+} \mathrm{M}_{4}^{+}(2), \\
\mathrm{R}_{1}(2), \mathrm{X}_{2}(2) \\
\end{array}$ & $\| \mathrm{Hf}_{3} \mathrm{~B}_{2} \operatorname{Ir}_{5}$ \\
\hline & $\begin{array}{l}\mathrm{A}_{1}^{+} \mathrm{A}_{4}^{+}(2), \mathrm{M}_{1}^{+} \mathrm{M}_{4}^{+}(2) \\
\mathrm{R}_{1}(2), \mathrm{X}_{1}(2) \\
\end{array}$ & $\| \mathrm{Np}_{2} \mathrm{InRh}_{2}$ \\
\hline & $\Gamma_{5}^{-}(2), \mathrm{Z}_{5}^{+}(2)$ & $\mathrm{La}_{2} \mathrm{Ni}_{5} \mathrm{C}_{3}, \mathrm{Hf}_{2} \mathrm{Ni}_{2} \mathrm{Sn}, \mathrm{Hg}_{5} \mathrm{Pd}_{2}, \mathrm{~Tb}_{2} \mathrm{MgPd}_{2}$ \\
\hline & $\Gamma_{5}^{+}(2), \mathrm{Z}_{5}^{-}(2)$ & $\| \mathrm{SiPt}_{3}$ \\
\hline & $\Gamma_{5}^{+}(2), \mathrm{Z}_{5}^{+}(2)$ & $\| \mathrm{Zr}_{9}\left(\mathrm{NiP}_{2}\right)_{2}$ \\
\hline & $\mathrm{Z}_{5}^{-}(2)$ & $\begin{array}{c}\| \mathrm{TmB}_{4}, \mathrm{TbB}_{4}, \mathrm{SmB}_{4}, \mathrm{LaB}_{4}, \mathrm{LuB}_{4}, \mathrm{HoB}_{4}, \mathrm{DyB}_{4}, \mathrm{NdB}_{4}, \mathrm{ErB}_{4}, \mathrm{YB} \mathrm{B}_{4}, \\
\mathrm{PrB}_{4}, \mathrm{U}_{2} \mathrm{Fe}_{2} \mathrm{Sn}, \mathrm{Sc}_{2} \mathrm{SnAu}_{2}, \mathrm{Ho}_{2} \mathrm{Pd}_{2} \mathrm{~Pb}, \mathrm{Dy}_{2} \mathrm{Pd}_{2} \mathrm{~Pb}, \mathrm{~Tb}_{2} \mathrm{Pd}_{2} \mathrm{~Pb} \\
\mathrm{La}_{2} \mathrm{Pd}_{2} \mathrm{~Pb}, \mathrm{Sm}_{2} \mathrm{Pd}_{2} \mathrm{~Pb}, \mathrm{Pr}_{2} \mathrm{Pd}_{2} \mathrm{~Pb}_{2} \mathrm{Hf}_{3} \mathrm{Si}_{2}, \mathrm{~Tb}_{2} \mathrm{Ni}_{2} \mathrm{Sn}, \mathrm{Tm}_{2} \mathrm{Ni}_{2} \mathrm{Sn} \\
\mathrm{Dy}_{2} \mathrm{Ni}_{2} \mathrm{Sn}, \mathrm{Ho}_{2} \mathrm{Ni}_{2} \mathrm{Sn}_{2} \mathrm{Er}_{2} \mathrm{Ni}_{2} \mathrm{Sn}, \mathrm{UB}_{4}, \mathrm{PuB}_{4}\end{array}$ \\
\hline & $\mathrm{Z}_{5}^{+}(2)$ & $\begin{array}{c}\| \mathrm{Yb}_{2} \mathrm{Ga}_{10} \mathrm{Ru}_{3}, \mathrm{Er}_{2} \mathrm{Ga}_{10} \mathrm{Os}_{3}, \mathrm{Zr}_{2} \mathrm{Ni}_{2} \mathrm{Sn}, \mathrm{Tb}_{2} \mathrm{SnAu}_{2}, \mathrm{ThB}_{4} \\
\mathrm{Tm}_{2} \mathrm{Ga}_{10} \mathrm{Os}_{3}, \mathrm{Fe}(\mathrm{BMo})_{2}, \mathrm{Th}_{2} \mathrm{Ru}_{6} \mathrm{C}_{5}, \mathrm{Sc}_{2} \mathrm{VB}_{2} \mathrm{Ir}_{5}, \mathrm{Ce}_{2} \mathrm{Pd}_{2} \mathrm{~Pb}\end{array}$ \\
\hline
\end{tabular}


Table II - continued

\begin{tabular}{|c|c|c|}
\hline $\begin{array}{l}\text { SOC or } \\
\text { NSOC }\end{array}$ & $\begin{array}{l}\text { Degenerate Irreducible } \\
\text { Representations }\end{array}$ & High Symmetry Point Semimetals \\
\hline & $\Gamma_{5}^{-}(2)$ & $\begin{array}{c}\mathrm{Y}_{2} \mathrm{MgGe}_{2}, \mathrm{~Tb}_{2} \mathrm{MgGe}_{2}, \mathrm{Lu}_{2} \mathrm{MgGe}_{2}, \mathrm{Er}_{2} \mathrm{MgGe}_{2}, \mathrm{Tm}_{2} \mathrm{MgGe}_{2}, \\
\mathrm{Ho}_{2} \mathrm{MgGe}_{2}, \mathrm{Dy}_{2} \mathrm{MgGe}_{2}, \mathrm{Sm}_{2} \mathrm{MgGe}_{2}, \mathrm{Tm}_{2} \mathrm{CdGe}_{2}, \mathrm{Er}_{2} \mathrm{CdGe}_{2}, \\
\mathrm{Dy}_{2} \mathrm{MgSi}_{2}, \mathrm{Y}_{2} \mathrm{CdGe}_{2}, \mathrm{Sm}_{2} \mathrm{CdGe}_{2}, \mathrm{Nd}_{2} \mathrm{MgGe}_{2}, \mathrm{Nd}_{2} \mathrm{CdGe}_{2}, \\
\mathrm{Pr}_{2} \mathrm{MgGe}_{2}, \mathrm{Yb}_{3} \mathrm{Ag}_{2}, \mathrm{Pr}_{2} \mathrm{MgNi}_{2}, \mathrm{Nd}_{2} \mathrm{MgNi}_{2}, \mathrm{La}_{2} \mathrm{MgNi}_{2}, \mathrm{Si}_{2} \mathrm{~W}_{3}, \\
\mathrm{Th}_{3} \mathrm{Si}_{2}\left(\mathrm{ICSD}: \text { 26571), } \mathrm{Ti}_{3} \mathrm{In}_{4}, \mathrm{CeB}_{4}, \mathrm{U}_{2} \mathrm{Co}_{2} \mathrm{Sn}, \mathrm{U}_{2} \mathrm{Ni}_{2} \mathrm{Sn}, \mathrm{Pu}_{2} \mathrm{SnPt}_{2},\right. \\
\mathrm{Pu}_{2} \mathrm{SnPd}_{2}\end{array}$ \\
\hline & $\Gamma_{5}^{+}(2)$ & $\| \mathrm{OsN}_{2}, \mathrm{Ta}_{2} \mathrm{CrB}_{2}, \mathrm{Mg}_{2} \mathrm{AlB}_{2} \mathrm{Ir}_{5}, \mathrm{Yb}_{2} \mathrm{MgSi}_{2}, \mathrm{Fe}(\mathrm{BW})_{2}$ \\
\hline \multirow[t]{4}{*}{$\mathrm{SOC}$} & $\begin{array}{c}\overline{\mathrm{A}}_{8} \overline{\mathrm{A}}_{9}(4), \overline{\mathrm{M}}_{8} \overline{\mathrm{M}}_{9}(4) \\
\overline{\mathrm{R}}_{3} \overline{\mathrm{R}}_{4}(4), \overline{\mathrm{X}}_{3} \overline{\mathrm{X}}_{4}(4)\end{array}$ & $\begin{array}{c}\| \mathrm{ReN}_{2}, \mathrm{Li}(\mathrm{SiRh})_{2}, \mathrm{Ti}_{2} \mathrm{ReB}_{2}, \mathrm{KCuF}_{3}, \mathrm{Mg}_{2} \mathrm{GeB}_{2} \mathrm{Rh}_{5}, \mathrm{Nb}_{3} \mathrm{Ga}_{2}, \\
\mathrm{Yb}_{2} \mathrm{InGe}_{2}, \mathrm{Lu}_{2} \mathrm{InPd}_{2}, \mathrm{Sc}_{2} \mathrm{InNi}_{2}, \mathrm{Yb}_{2} \mathrm{AlSi}_{2}, \mathrm{Ba}_{3} \mathrm{Nb}_{5} \mathrm{O}_{15}, \mathrm{Tm}_{2} \mathrm{InPd}_{2}, \\
\mathrm{Er}_{2} \mathrm{InPd}_{2}, \mathrm{La}_{2} \mathrm{InRh}_{2}, \mathrm{Ho}_{2} \mathrm{InPd}_{2}, \mathrm{Ba}_{3} \mathrm{Ta}_{5} \mathrm{O}_{15}, \mathrm{Dy}_{2} \mathrm{InPd}_{2}, \mathrm{Sm}_{2} \mathrm{InPd}_{2}, \\
\mathrm{~Tb}_{2} \mathrm{InPd}_{2}, \mathrm{Yb}_{2} \mathrm{InCu}_{2}, \mathrm{Dy}_{2} \mathrm{InNi}_{2}, \mathrm{Sm}_{2} \mathrm{InNi}_{2}, \mathrm{Li}\left(\mathrm{NdSi}_{2}, \mathrm{Ho}_{2} \mathrm{InNi}_{2},\right. \\
\mathrm{Er}_{2} \mathrm{InNi}_{2}, \mathrm{Nd}_{2} \mathrm{InNi}_{2}, \mathrm{Y}_{2} \mathrm{InPd}_{2}, \mathrm{Lu}_{2} \mathrm{InNi}_{2}, \mathrm{Y}_{2} \mathrm{InNi}_{2}, \mathrm{Y}_{3} \mathrm{Au}_{2}, \mathrm{Zr}_{2} \mathrm{InCo}_{2}, \\
\mathrm{~Tb}_{2} \mathrm{InNi}_{2}, \mathrm{Ho}_{5}\left(\mathrm{Ge}_{5} \mathrm{Rh}_{2}\right)_{2}, \mathrm{Sc}_{5}\left(\mathrm{Co}_{2} \mathrm{Si}_{5}\right)_{2}, \mathrm{Pu}_{2} \mathrm{InPd}_{2}\end{array}$ \\
\hline & $\begin{array}{c}\overline{\mathrm{A}}_{8} \overline{\mathrm{A}}_{9}(4), \overline{\mathrm{M}}_{6} \overline{\mathrm{M}}_{7}(4) \\
\overline{\mathrm{R}}_{3} \overline{\mathrm{R}}_{4}(4), \overline{\mathrm{X}}_{3} \overline{\mathrm{X}}_{4}(4)\end{array}$ & $\begin{array}{c}\| \mathrm{Nb}_{3} \mathrm{~B}_{2}, \mathrm{Sc}_{2} \mathrm{SiB}_{2} \mathrm{Ir}_{5}, \mathrm{Lu}_{2} \mathrm{InAu}_{2}, \mathrm{Tm}_{2} \mathrm{InAu}_{2}, \mathrm{Zr}_{2} \mathrm{InNi}_{2}, \mathrm{Be}_{2} \mathrm{Nb}_{3} \\
\mathrm{Tm}_{2} \mathrm{InCu}_{2}, \mathrm{Y}_{2} \mathrm{InCu}_{2}, \mathrm{~V}_{2} \mathrm{Ga}_{5}, \mathrm{Tm}_{5}\left(\mathrm{Ge}_{5} \mathrm{Rh}_{2}\right)_{2}, \mathrm{Lu}_{5}\left(\mathrm{Si}_{5} \mathrm{Ir}_{2}\right)_{2} \\
\mathrm{Sc}_{2} \mathrm{CrB}_{2} \mathrm{Rh}_{5}\end{array}$ \\
\hline & $\begin{array}{c}\overline{\mathrm{A}}_{6} \overline{\mathrm{A}}_{7}(4), \overline{\mathrm{M}}_{8} \overline{\mathrm{M}}_{9}(4) \\
\overline{\mathrm{R}}_{3} \overline{\mathrm{R}}_{4}(4), \overline{\mathrm{X}}_{3} \overline{\mathrm{X}}_{4}(4)\end{array}$ & $\begin{array}{c}\| \mathrm{Mg}_{2} \mathrm{SiB}_{2} \mathrm{Ir}_{5}, \mathrm{Ta}_{3} \mathrm{Ga}_{2}, \mathrm{Ta}(\mathrm{BMo})_{2}, \mathrm{Nd}_{2} \mathrm{InRh}_{2}, \mathrm{Yb}_{2} \mathrm{InPd}_{2}, \mathrm{~Tb}_{2} \mathrm{InGe}_{2}, \\
\mathrm{Dy}_{2} \mathrm{InGe}_{2}, \mathrm{Nb}\left(\mathrm{BMo}_{2}, \mathrm{Sm}_{2} \mathrm{InGe}_{2}, \mathrm{Nd}_{2} \mathrm{InGe}_{2}, \mathrm{La}_{2} \mathrm{InGe}_{2}, \mathrm{Pr}_{2} \mathrm{InGe}_{2},\right. \\
\mathrm{Dy}_{2} \mathrm{AlGe}_{2}, \mathrm{Zr}_{2} \mathrm{InAu}_{2}, \mathrm{Ho}_{2} \mathrm{InGe}_{2}, \mathrm{Nd}_{2} \mathrm{InPd}_{2}, \mathrm{Sc}_{2} \mathrm{AlSi}_{2}, \mathrm{Er}_{3} \mathrm{Pd}_{2}, \\
\mathrm{Pr}_{2} \mathrm{InNi}_{2}, \mathrm{Pr}_{2} \mathrm{InPd}_{2}, \mathrm{Dy}_{3} \mathrm{Pd}_{2}, \mathrm{Ho}_{3} \mathrm{Pd}_{2}, \mathrm{La}_{2} \mathrm{InNi}_{2}, \mathrm{La}_{2} \mathrm{InPd}_{2}, \\
\mathrm{Ti}_{3} \mathrm{Co}_{5} \mathrm{~B}_{2}\end{array}$ \\
\hline & $\begin{array}{c}\overline{\mathrm{A}}_{6} \overline{\mathrm{A}}_{7}(4), \overline{\mathrm{M}}_{6} \overline{\mathrm{M}}_{7}(4) \\
\overline{\mathrm{R}}_{3} \overline{\mathrm{R}}_{4}(4), \overline{\mathrm{X}}_{3} \overline{\mathrm{X}}_{4}(4)\end{array}$ & $\begin{array}{c}\mathrm{Tb}(\mathrm{BC})_{2}, \mathrm{La}(\mathrm{BC})_{2}, \mathrm{Ta}_{3} \mathrm{~B}_{2}, \mathrm{~V}_{3} \mathrm{~B}_{2}, \mathrm{GaPt}_{3}, \mathrm{AlPt}_{3}(\mathrm{ICSD}: 609153), \\
\mathrm{Hf}_{2} \mathrm{InPd}_{2}, \mathrm{Dy}_{2} \mathrm{InAu}_{2}, \mathrm{~Tb}_{2} \mathrm{InAu}_{2}, \mathrm{Li}\left(\mathrm{Sn}_{3} \mathrm{Pd}\right)_{2}, \mathrm{Ho}_{2} \mathrm{InAu}_{2}, \mathrm{Ta}_{3} \mathrm{Be}_{2}, \\
\mathrm{Hf}_{2} \mathrm{In}_{5}, \mathrm{Er}_{2} \mathrm{InAu}_{2}, \mathrm{Y}_{2} \mathrm{InAu}_{2}, \mathrm{La}_{2} \mathrm{InCu}_{2}, \mathrm{~Tb}_{2} \mathrm{InCu}_{2}, \mathrm{Al}\left(\mathrm{CuRe}_{2},\right. \\
\mathrm{Pr}_{2} \mathrm{InCu}_{2}, \mathrm{Mg}_{2} \mathrm{ZnB}_{2} \mathrm{Ir}_{5}, \mathrm{Er}_{2} \mathrm{InCu}_{2}, \mathrm{Hf}_{3} \mathrm{~B}_{2} \mathrm{Ir}_{5}, \mathrm{Sc}_{2} \mathrm{InCu}_{2}, \mathrm{Lu}_{2} \mathrm{InCu}_{2} \\
\mathrm{Mn}(\mathrm{BW})_{2}, \mathrm{Nb}_{3} \mathrm{Si}_{2}, \mathrm{Ho}_{5}\left(\mathrm{Ge}_{5} \mathrm{Ir}_{2}\right)_{2}, \mathrm{Ce}_{2} \mathrm{InNi}_{2}, \mathrm{Ce}_{2} \mathrm{InPt}_{2}, \mathrm{Ce}_{2} \mathrm{InPd}_{2} \\
\mathrm{~Np}_{2} \mathrm{InRh}_{2}, \mathrm{La}_{3} \mathrm{Si}_{2}\end{array}$ \\
\hline \multicolumn{3}{|r|}{ Space Group 128: P4/mnc } \\
\hline \multirow{7}{*}{ NSOC } & $\begin{array}{c}\mathrm{A}_{3} \mathrm{~A}_{4}(4), \Gamma_{5}^{-}(2), \mathrm{M}_{2}^{+} \mathrm{M}_{3}^{+}(2) \\
\mathrm{R}_{1}^{-}(2), \mathrm{X}_{1}(2), \mathrm{Z}_{3} \mathrm{Z}_{4}(4)\end{array}$ & $\| \mathrm{Sc}_{3} \mathrm{C}_{4}$ \\
\hline & $\begin{array}{c}\mathrm{A}_{3} \mathrm{~A}_{4}(4), \Gamma_{5}^{+}(2), \mathrm{M}_{5}^{+}(2) \\
\mathrm{R}_{1}^{+}(2), \mathrm{X}_{1}(2), \mathrm{Z}_{3} \mathrm{Z}_{4}(4)\end{array}$ & $\| \mathrm{Er}_{4} \mathrm{NiB}_{13}$ \\
\hline & $\mathrm{A}_{3} \mathrm{~A}_{4}(4), \Gamma_{5}^{-}(2), \mathrm{Z}_{3} \mathrm{Z}_{4}(4)$ & $\| \mathrm{Al}_{7} \mathrm{CoCu}_{2}$ \\
\hline & $\mathrm{A}_{3} \mathrm{~A}_{4}(4), \mathrm{Z}_{3} \mathrm{Z}_{4}(4)$ & $\| \mathrm{Lu}_{4} \mathrm{CoB}_{13}, \mathrm{Y}_{4} \mathrm{CoB}_{13}, \mathrm{~Tb}_{4} \mathrm{CoB}_{13}$ \\
\hline & $\mathrm{Z}_{3} \mathrm{Z}_{4}(4)$ & || $\mathrm{Nb}_{3} \mathrm{InSe}_{12}$ \\
\hline & $\Gamma_{5}^{+}(2)$ & $\| \mathrm{Tm}_{2} \mathrm{Mn}_{3} \mathrm{Si}_{5}$ \\
\hline & $\mathrm{A}_{3} \mathrm{~A}_{4}(4)$ & $\begin{array}{c}\| \mathrm{Tb}_{2} \mathrm{Si}_{5} \mathrm{Os}_{3}, \mathrm{Lu}_{2} \mathrm{Si}_{5} \mathrm{Ru}_{3}, \mathrm{~Tb}_{2} \mathrm{Fe}_{3} \mathrm{Si}_{5}, \mathrm{Dy}_{2} \mathrm{Fe}_{3} \mathrm{Si}_{5}, \mathrm{Er}_{2} \mathrm{Fe}_{3} \mathrm{Si}_{5} \\
\mathrm{Sc}_{2} \mathrm{Fe}_{3} \mathrm{Si}_{5}, \mathrm{Lu}_{2} \mathrm{Fe}_{3} \mathrm{Si}_{5}\end{array}$ \\
\hline \multirow[t]{2}{*}{$\mathrm{SOC}$} & $\begin{array}{l}\overline{\mathrm{A}}_{6} \overline{\mathrm{A}}_{8}(4), \overline{\mathrm{M}}_{6} \overline{\mathrm{M}}_{7}(4) \\
\overline{\mathrm{X}}_{3} \overline{\mathrm{X}}_{4}(4), \overline{\mathrm{Z}}_{6} \overline{\mathrm{Z}}_{8}(4)\end{array}$ & $\| \mathrm{Sc}_{3} \mathrm{C}_{4}$ \\
\hline & $\begin{array}{c}\overline{\mathrm{A}}_{5} \overline{\mathrm{A}}_{7}(4), \overline{\mathrm{M}}_{6} \overline{\mathrm{M}}_{7}(4) \\
\overline{\mathrm{X}}_{3} \overline{\mathrm{X}}_{4}(4), \overline{\mathrm{Z}}_{5} \overline{\mathrm{Z}}_{7}(4)\end{array}$ & $\| \mathrm{Er}_{4} \mathrm{NiB}_{13}$ \\
\hline \multicolumn{3}{|r|}{ Space Group 129: $P 4 / \mathrm{nmm}$} \\
\hline & \begin{tabular}{|c|}
$\mathrm{A}_{4}(2), \Gamma_{5}^{-}(2), \mathrm{M}_{4}(2), \mathrm{R}_{2}(2)$ \\
$\mathrm{X}_{2}(2), \mathrm{Z}_{5}^{-}(2)$
\end{tabular} & $\| \mathrm{YbBrO}$ \\
\hline & $\begin{array}{c}\mathrm{A}_{4}(2), \Gamma_{5}^{-}(2), \mathrm{M}_{4}(2), \mathrm{R}_{1}(2), \\
\mathrm{X}_{1}(2), \mathrm{Z}_{5}^{-}(2)\end{array}$ & $\| \mathrm{TmS}_{2}$ \\
\hline & $\begin{array}{c}\mathrm{A}_{4}(2), \Gamma_{5}^{-}(2), \mathrm{M}_{4}(2), \mathrm{R}_{1}(2) \\
\mathrm{X}_{1}(2), \mathrm{Z}_{5}^{+}(2)\end{array}$ & $\| \mathrm{CaSmCuClO}_{3}$ \\
\hline & $\begin{array}{c}\mathrm{A}_{3}(2), \Gamma_{5}^{-}(2), \mathrm{M}_{1}(2), \mathrm{R}_{1}(2) \\
\mathrm{X}_{2}(2), \mathrm{Z}_{5}^{-}(2)\end{array}$ & $\| \mathrm{MgCuGe}$ \\
\hline & $\begin{array}{c}\mathrm{A}_{2}(2), \Gamma_{5}^{-}(2), \mathrm{M}_{2}(2), \mathrm{R}_{2}(2) \\
\mathrm{X}_{2}(2), \mathrm{Z}_{5}^{-}(2)\end{array}$ & $\| \mathrm{CuSe}$ \\
\hline & $\begin{array}{c}\mathrm{A}_{2}(2), \Gamma_{5}^{-}(2), \mathrm{M}_{2}(2), \mathrm{R}_{1}(2) \\
\mathrm{X}_{2}(2), \mathrm{Z}_{5}^{-}(2)\end{array}$ & $\| \mathrm{Cu}_{2} \mathrm{Sb}$ \\
\hline & $\begin{array}{c}\mathrm{A}_{2}(2), \Gamma_{5}^{-}(2), \mathrm{M}_{2}(2), \mathrm{R}_{1}(2) \\
\mathrm{X}_{1}(2), \mathrm{Z}_{5}^{-}(2)\end{array}$ & || SiNiSb \\
\hline
\end{tabular}


Table II - continued

\begin{tabular}{|c|c|c|}
\hline $\begin{array}{l}\text { SOC or } \\
\text { NSOC }\end{array}$ & $\begin{array}{c}\text { Degenerate Irreducible } \\
\text { Representations }\end{array}$ & High Symmetry Point Semimetals \\
\hline & $\begin{array}{c}\mathrm{A}_{2}(2), \Gamma_{5}^{+}(2), \mathrm{M}_{3}(2), \mathrm{R}_{2}(2), \\
\mathrm{X}_{2}(2), \mathrm{Z}_{5}^{+}(2)\end{array}$ & \|ITiGe, TmTiGe \\
\hline & $\begin{array}{c}\mathrm{A}_{2}(2), \Gamma_{5}^{+}(2), \mathrm{M}_{3}(2), \mathrm{R}_{2}(2), \\
\mathrm{X}_{1}(2), \mathrm{Z}_{5}^{+}(2)\end{array}$ & || LuTiSi, ErTiSi, DyTiGe, YTiSi, HoTiSi, DyTiSi \\
\hline & $\begin{array}{c}\mathrm{A}_{2}(2), \Gamma_{5}^{+}(2), \mathrm{M}_{2}(2), \mathrm{R}_{2}(2), \\
\mathrm{X}_{2}(2), \mathrm{Z}_{5}^{+}(2)\end{array}$ & $\| \mathrm{SrSnP}$ \\
\hline & $\begin{array}{c}\mathrm{A}_{2}(2), \Gamma_{5}^{+}(2), \mathrm{M}_{2}(2), \mathrm{R}_{2}(2), \\
\mathrm{X}_{1}(2), \mathrm{Z}_{5}^{+}(2)\end{array}$ & || ErTiGe, HoTiGe \\
\hline & $\begin{array}{c}\mathrm{A}_{2}(2), \Gamma_{5}^{+}(2), \mathrm{M}_{2}(2), \mathrm{R}_{1}(2), \\
\mathrm{X}_{1}(2), \mathrm{Z}_{5}^{+}(2)\end{array}$ & |l HfAu, TiAu \\
\hline & $\begin{array}{c}\mathrm{A}_{1}(2), \Gamma_{5}^{-}(2), \mathrm{M}_{2}(2), \mathrm{R}_{2}(2), \\
\mathrm{X}_{2}(2), \mathrm{Z}_{5}^{+}(2)\end{array}$ & $\| \mathrm{YbCuAs}_{2}$ \\
\hline & $\begin{array}{c}\mathrm{A}_{1}(2), \Gamma_{5}^{-}(2), \mathrm{M}_{1}(2), \mathrm{R}_{2}(2), \\
\mathrm{X}_{2}(2), \mathrm{Z}_{5}^{+}(2)\end{array}$ & $\| \mathrm{YbCuSb}_{2}$ \\
\hline & $\begin{array}{c}\mathrm{A}_{1}(2), \Gamma_{5}^{+}(2), \mathrm{M}_{2}(2), \mathrm{R}_{1}(2), \\
\mathrm{X}_{2}(2), \mathrm{Z}_{5}^{+}(2)\end{array}$ & $\| \mathrm{TaPd}$ \\
\hline & $\begin{array}{c}\mathrm{A}_{1}(2), \Gamma_{5}^{+}(2), \mathrm{M}_{1}(2), \mathrm{R}_{1}(2), \\
\mathrm{X}_{1}(2), \mathrm{Z}_{5}^{+}(2)\end{array}$ & || LiMnP \\
\hline & $\begin{array}{c}\mathrm{A}_{4}(2), \mathrm{M}_{4}(2), \mathrm{R}_{1}(2), \mathrm{X}_{1}(2) \\
\mathrm{Z}_{5}^{-}(2)\end{array}$ & $\| \mathrm{LaS}_{2}, \mathrm{NdS}_{2}$ \\
\hline & $\begin{array}{c}\mathrm{A}_{4}(2), \mathrm{M}_{3}(2), \mathrm{R}_{1}(2), \mathrm{X}_{1}(2), \\
\mathrm{Z}_{5}^{+}(2)\end{array}$ & $\| \mathrm{Cr}_{2} \mathrm{As}$ \\
\hline & $\begin{array}{c}\mathrm{A}_{4}(2), \Gamma_{5}^{-}(2), \mathrm{M}_{4}(2), \mathrm{R}_{1}(2), \\
\mathrm{X}_{2}(2)\end{array}$ & $\| \mathrm{Fe}_{2} \mathrm{As}$ \\
\hline & $\begin{array}{c}\mathrm{A}_{3}(2), \mathrm{M}_{3}(2), \mathrm{R}_{2}(2), \mathrm{X}_{1}(2), \\
\mathrm{Z}_{5}^{-}(2)\end{array}$ & $\| \mathrm{Be}_{4} \mathrm{~B}$ \\
\hline & $\begin{array}{c}\mathrm{A}_{3}(2), \mathrm{M}_{1}(2), \mathrm{R}_{2}(2), \mathrm{X}_{1}(2), \\
\mathrm{Z}_{5}^{-}(2)\end{array}$ & $\| \mathrm{SN}$ \\
\hline & $\begin{array}{c}\mathrm{A}_{2}(2), \mathrm{M}_{4}(2), \mathrm{R}_{2}(2), \mathrm{X}_{1}(2), \\
\mathrm{Z}_{5}^{-}(2)\end{array}$ & $\| \mathrm{Tm}_{10} \operatorname{In}_{20} \mathrm{Rh}_{9}$ \\
\hline & $\begin{array}{c}\mathrm{A}_{2}(2), \mathrm{M}_{2}(2), \mathrm{R}_{2}(2), \mathrm{X}_{2}(2) \\
\mathrm{Z}_{5}^{-}(2)\end{array}$ & $\| \mathrm{Cu}_{2} \mathrm{As}$ \\
\hline & $\begin{array}{c}\mathrm{A}_{2}(2), \Gamma_{5}^{+}(2), \mathrm{M}_{3}(2), \mathrm{R}_{2}(2), \\
\mathrm{X}_{1}(2)\end{array}$ & \| LuTiGe \\
\hline & $\begin{array}{c}\mathrm{A}_{2}(2), \Gamma_{5}^{+}(2), \mathrm{M}_{2}(2), \mathrm{R}_{2}(2), \\
\mathrm{X}_{2}(2)\end{array}$ & \| SmTiGe \\
\hline & $\begin{array}{c}\mathrm{A}_{2}(2), \Gamma_{5}^{+}(2), \mathrm{M}_{2}(2), \mathrm{R}_{2}(2), \\
\mathrm{X}_{1}(2)\end{array}$ & \| TbTiSi \\
\hline & $\begin{array}{c}\mathrm{A}_{2}(2), \Gamma_{5}^{+}(2), \mathrm{M}_{2}(2), \mathrm{R}_{1}(2), \\
\mathrm{X}_{1}(2)\end{array}$ & $\| \mathrm{HfAg}, \mathrm{ZrAg}$ \\
\hline & $\begin{array}{c}\mathrm{A}_{1}(2), \mathrm{M}_{4}(2), \mathrm{R}_{2}(2), \mathrm{X}_{2}(2), \\
\mathrm{Z}_{5}^{-}(2)\end{array}$ & $\| \operatorname{Dy}\left(\mathrm{SiNi}_{5}\right)_{2}, \mathrm{Ho}\left(\mathrm{SiNi}_{5}\right)_{2}, \mathrm{~Tb}\left(\mathrm{SiNi}_{5}\right)_{2}$ \\
\hline & $\begin{array}{c}\mathrm{A}_{1}(2), \Gamma_{5}^{-}(2), \mathrm{M}_{2}(2), \mathrm{R}_{2}(2), \\
\mathrm{X}_{2}(2)\end{array}$ & $\| \mathrm{La}(\mathrm{NiAs})_{2}$ \\
\hline & $\begin{array}{c}\mathrm{A}_{1}(2), \Gamma_{5}^{-}(2), \mathrm{M}_{1}(2), \mathrm{R}_{2}(2), \\
\mathrm{X}_{2}(2)\end{array}$ & \| TiGeSb \\
\hline & $\begin{array}{c}\mathrm{A}_{1}(2), \Gamma_{5}^{+}(2), \mathrm{M}_{2}(2), \mathrm{R}_{1}(2), \\
\mathrm{X}_{1}(2)\end{array}$ & $\| \mathrm{TiCu}$ \\
\hline & $\begin{array}{c}\mathrm{A}_{1}(2), \Gamma_{5}^{+}(2), \mathrm{M}_{1}(2), \mathrm{R}_{1}(2), \\
\mathrm{X}_{1}(2)\end{array}$ & || KMnAs, CaMnGe \\
\hline & $\mathrm{A}_{4}(2), \mathrm{M}_{4}(2), \mathrm{R}_{2}(2), \mathrm{X}_{2}(2)$ & $\mathrm{Sm}(\mathrm{GeIr})_{2}, \mathrm{~Tb}(\mathrm{GeIr})_{2}$ \\
\hline & $\mathrm{A}_{4}(2), \mathrm{M}_{4}(2), \mathrm{R}_{1}(2), \mathrm{X}_{2}(2)$ & $\|$ MnVAs \\
\hline & $\mathrm{A}_{4}(2), \mathrm{M}_{4}(2), \mathrm{R}_{1}(2), \mathrm{X}_{1}(2)$ & $\begin{array}{c}\| \mathrm{LuS}_{2}, \mathrm{ErS}_{2}, \mathrm{HoS}_{2}, \mathrm{DyS}_{2}, \mathrm{SmSe}_{2}, \mathrm{NdSe}_{2}, \mathrm{LaSe}_{2}, \mathrm{TbS}_{2}, \mathrm{ThAsS} \\
\mathrm{PrSe}_{2}, \mathrm{ThBiTe}, \mathrm{MgCoGe}, \mathrm{NbCrN}_{2}, \mathrm{LiCoAs}, \mathrm{PrFeSi}, \mathrm{NdFeSi}, \mathrm{SmFeSi} \text {, } \\
\text { HoFeSi, SmFeSb }, \mathrm{LaFeSb}_{2}, \text { DyFeSi, TbFeSi }\end{array}$ \\
\hline & $\mathrm{A}_{4}(2), \mathrm{M}_{1}(2), \mathrm{R}_{1}(2), \mathrm{X}_{1}(2)$ & $\| \operatorname{Pr}(\mathrm{AsRh})_{2}, \mathrm{LaFeSi}$ \\
\hline & $\mathrm{A}_{3}(2), \mathrm{M}_{4}(2), \mathrm{R}_{2}(2), \mathrm{X}_{2}(2)$ & $\| \mathrm{Nd}(\mathrm{GeIr})_{2}$ \\
\hline & $\mathrm{A}_{3}(2), \mathrm{M}_{4}(2), \mathrm{R}_{1}(2), \mathrm{X}_{1}(2)$ & $\| \mathrm{ErSe}_{2}, \mathrm{HoSe}_{2}, \mathrm{DySe}_{2}, \mathrm{NdTe}_{2}, \mathrm{PrTe}_{2}$ \\
\hline
\end{tabular}


Table II - continued

\begin{tabular}{|c|c|c|}
\hline $\begin{array}{l}\text { SOC or } \\
\text { NSOC }\end{array}$ & $\begin{array}{l}\text { Degenerate Irreducible } \\
\text { Representations }\end{array}$ & High Symmetry Point Semimetals \\
\hline & $\mathrm{A}_{3}(2), \mathrm{M}_{3}(2), \mathrm{R}_{2}(2), \mathrm{X}_{2}(2)$ & $\begin{array}{c}\left.\left.\| \text { VSe, Ho(SiIr })_{2}, \text { Dy (SiIr }\right)_{2}, \text { Y }(\text { SiIr })_{2}, \text { Tb(SiIr }\right)_{2}, \operatorname{Pr}(\text { GeIr })_{2}, \\
\left.\text { La(GeIr })_{2}, \text { La(SiIr }\right)_{2}, \text { PuIO }\end{array}$ \\
\hline & $\mathrm{A}_{3}(2), \mathrm{M}_{3}(2), \mathrm{R}_{1}(2), \mathrm{X}_{1}(2)$ & $\| \mathrm{TmTe}_{2}, \mathrm{LuTe}_{2}, \mathrm{Li}_{2} \mathrm{NdSb}_{2}, \mathrm{Li}_{2} \mathrm{PrSb}_{2}, \mathrm{Sm}(\mathrm{PIr})_{2}, \mathrm{SmCuSeF}$ \\
\hline & $\mathrm{A}_{3}(2), \mathrm{M}_{2}(2), \mathrm{R}_{1}(2), \mathrm{X}_{1}(2)$ & 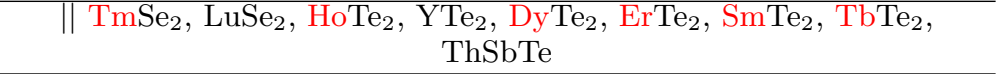 \\
\hline & $\mathrm{A}_{3}(2), \mathrm{M}_{1}(2), \mathrm{R}_{1}(2), \mathrm{X}_{1}(2)$ & || $\mathrm{Nd}(\mathrm{PRh})_{2}, \operatorname{Pr}(\mathrm{PRh})_{2}$ \\
\hline & $\mathrm{A}_{2}(2), \mathrm{M}_{3}(2), \mathrm{R}_{1}(2), \mathrm{X}_{1}(2)$ & || SmClF \\
\hline & $\mathrm{A}_{2}(2), \mathrm{M}_{2}(2), \mathrm{R}_{2}(2), \mathrm{X}_{2}(2)$ & $\| \mathrm{LaNiBN} \mathrm{HfCuSi}_{2}, \mathrm{ZrCuSi}_{2}, \mathrm{NdSb}_{2} \mathrm{Pd}, \mathrm{La}(\mathrm{GaPd})_{2}$ \\
\hline & $\mathrm{A}_{2}(2), \mathrm{M}_{2}(2), \mathrm{R}_{2}(2), \mathrm{X}_{1}(2)$ & $\begin{array}{c}\| \mathrm{Sm}(\mathrm{NiBi})_{2}, \mathrm{Nd}(\mathrm{NiBi})_{2}, \operatorname{Pr}(\mathrm{NiSb})_{2}, \operatorname{PrTiGe}, \mathrm{La}(\mathrm{AlPd})_{2}, \mathrm{Dy}(\mathrm{NiBi})_{2}, \\
\operatorname{Pr}(\mathrm{NiBi})_{2}, \mathrm{Ho}(\mathrm{NiSb})_{2}, \operatorname{Er}(\mathrm{NiSb})_{2}, \operatorname{Dy}(\mathrm{NiSb})_{2}, \mathrm{NdTiGe}, \mathrm{LaTiGe}\end{array}$ \\
\hline & $\mathrm{A}_{2}(2), \mathrm{M}_{2}(2), \mathrm{R}_{1}(2), \mathrm{X}_{2}(2)$ & ||$N d(\mathrm{NiSb})_{2}$ \\
\hline & $\mathrm{A}_{2}(2), \mathrm{M}_{2}(2), \mathrm{R}_{1}(2), \mathrm{X}_{1}(2)$ & $\begin{array}{c}\| \mathrm{ScNiC}_{2}, \mathrm{Nd}(\mathrm{NiAs})_{2}, \mathrm{Sm}(\mathrm{NiAs})_{2}, \operatorname{Pr}\left(\mathrm{NiAs}_{2}, \mathrm{La}(\mathrm{SbPd})_{2}, \mathrm{~Tb}(\mathrm{NiSb})_{2},\right. \\
\mathrm{Sm}(\mathrm{NiSb})_{2}, \mathrm{Ti}_{8} \mathrm{Bi}_{9}\end{array}$ \\
\hline & $\mathrm{A}_{2}(2), \mathrm{M}_{1}(2), \mathrm{R}_{2}(2), \mathrm{X}_{2}(2)$ & $\| \mathrm{TbZnSn}_{2}, \mathrm{PrSb}_{2} \mathrm{Pd}$ \\
\hline & $\mathrm{A}_{2}(2), \mathrm{M}_{1}(2), \mathrm{R}_{1}(2), \mathrm{X}_{2}(2)$ & $\mathrm{CaNiGeH}, \mathrm{Tb}(\mathrm{AlAu})_{2}, \mathrm{Sm}(\mathrm{AlAu})_{2}, \mathrm{Nd}_{17} \mathrm{Au}_{36}$ \\
\hline & $\mathrm{A}_{2}(2), \mathrm{M}_{1}(2), \mathrm{R}_{1}(2), \mathrm{X}_{1}(2)$ & $\| \mathrm{La}(\mathrm{AsIr})_{2}, \mathrm{Nd}(\mathrm{AlAu})_{2}, \operatorname{Pr}(\mathrm{AlAu})_{2}$ \\
\hline & $\mathrm{A}_{1}(2), \mathrm{M}_{4}(2), \mathrm{R}_{2}(2), \mathrm{X}_{2}(2)$ & $\| \mathrm{Tm}\left(\mathrm{SiNi}_{5}\right)_{2}$ \\
\hline & $\mathrm{A}_{1}(2), \mathrm{M}_{4}(2), \mathrm{R}_{1}(2), \mathrm{X}_{1}(2)$ & $\| \mathrm{Zr}_{2}\left(\mathrm{~V}_{2} \mathrm{Sb}_{3}\right)_{3}$ \\
\hline & $\mathrm{A}_{1}(2), \mathrm{M}_{3}(2), \mathrm{R}_{2}(2), \mathrm{X}_{2}(2)$ & $\| \mathrm{Sm}(\mathrm{SiIr})_{2}, \mathrm{Nd}(\mathrm{SiIr})_{2}$ \\
\hline & $\mathrm{A}_{1}(2), \mathrm{M}_{3}(2), \mathrm{R}_{2}(2), \mathrm{X}_{1}(2)$ & $\| \mathrm{Sc}_{2} \mathrm{Sb}$ \\
\hline & $\mathrm{A}_{1}(2), \mathrm{M}_{2}(2), \mathrm{R}_{2}(2), \mathrm{X}_{2}(2)$ & $\| \mathrm{HfCuGe}_{2}, \mathrm{ZrCuGe}_{2}, \mathrm{LaSb}_{2} \mathrm{Pd}, \mathrm{PrNiSb}_{2}$ \\
\hline & $\mathrm{A}_{1}(2), \mathrm{M}_{2}(2), \mathrm{R}_{2}(2), \mathrm{X}_{1}(2)$ & $\begin{array}{r}\| \mathrm{SmSb}_{2} \mathrm{Pd}, \mathrm{TbSb}_{2} \mathrm{Pd}, \mathrm{HoNiSb}_{2}, \mathrm{TbNiSb}_{2}, \mathrm{DyNiSb}_{2}, \mathrm{LaNiSb}_{2}, \\
\mathrm{SmNiSb}_{2}, \mathrm{NdNiSb}_{2}\end{array}$ \\
\hline & $\mathrm{A}_{1}(2), \mathrm{M}_{2}(2), \mathrm{R}_{1}(2), \mathrm{X}_{2}(2)$ & \begin{tabular}{l||l} 
& HfVSi, HfVGe, ZrVSi \\
\end{tabular} \\
\hline & $\mathrm{A}_{1}(2), \mathrm{M}_{2}(2), \mathrm{R}_{1}(2), \mathrm{X}_{1}(2)$ & $\begin{array}{l}\| \mathrm{LaTe}_{2}, \text { ThAsSe, ThPS, ThPSe, ThTeAs, ThSbSe, } \mathrm{PaP}_{2}, \mathrm{PaAs}_{2}, \\
\qquad \mathrm{PaSb}_{2}, \mathrm{LaS}\end{array}$ \\
\hline & $\mathrm{A}_{1}(2), \mathrm{M}_{1}(2), \mathrm{R}_{2}(2), \mathrm{X}_{2}(2)$ & $\|$ ZrGeSb, HoZnSn $2, \mathrm{LuZnSn}_{2}, \mathrm{TmZnSn}_{2}, \mathrm{YZnSn}_{2}$ \\
\hline & $\mathrm{A}_{1}(2), \mathrm{M}_{1}(2), \mathrm{R}_{2}(2), \mathrm{X}_{1}(2)$ & || NdSiRu, PrSiRu, SmSiRu, SmGeRu \\
\hline & $\mathrm{A}_{1}(2), \mathrm{M}_{1}(2), \mathrm{R}_{1}(2), \mathrm{X}_{2}(2)$ & || $\mathrm{Lu}(\mathrm{CuSn})_{2}, \mathrm{La}(\mathrm{CuSn})_{2}, \mathrm{Y}(\mathrm{CuSn})_{2}$ \\
\hline & $\mathrm{A}_{1}(2), \mathrm{M}_{1}(2), \mathrm{R}_{1}(2), \mathrm{X}_{1}(2)$ & 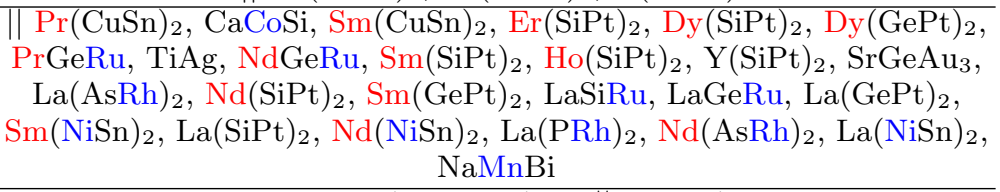 \\
\hline & $\Gamma_{5}^{-}(2), Z_{5}^{-}(2)$ & KCdSb, NaZnSb, NaAlGe, NaAlSi, \| LaSb $2 \mathrm{Au}, \mathrm{NdCuSb}_{2}$ \\
\hline & $\Gamma_{5}^{-}(2), \mathrm{Z}_{5}^{+}(2)$ & $\begin{array}{c}\| \mathrm{Nd}_{2} \mathrm{BiN}_{1} \mathrm{Pr}_{2} \mathrm{BiN}, \mathrm{PrSb}_{2} \mathrm{Au}, \mathrm{NdSb}_{2} \mathrm{Au}, \mathrm{SmSb}_{2} \mathrm{Au}, \mathrm{LuCuAs}_{2}, \\
\mathrm{PrAgSb}_{2}, \mathrm{ErCuAs}_{2}, \mathrm{HoCuAs}_{2}, \mathrm{DyCuAs}_{2}, \mathrm{TbCuAs}_{2}, \mathrm{YCuAs}_{2}, \\
\mathrm{HoCuSb}_{2}, \mathrm{LaCuSb}_{2}, \mathrm{SmCuSb}_{2}, \mathrm{BaNiS}_{2}, \mathrm{DyCuSb}_{2}, \mathrm{YCuSb}_{2}, \\
\mathrm{TbCuSb}_{2}, \mathrm{Ca}(\mathrm{BeGe})_{2}, \mathrm{HfSb}_{2}, \mathrm{ErCuSb} \mathrm{Cu}_{2}, \mathrm{TmCuSb}_{2}, \mathrm{LuCuSb}_{2}, \\
\mathrm{NdAsRuO}, \mathrm{SmAsRuO}\end{array}$ \\
\hline & $\Gamma_{5}^{+}(2), Z_{5}^{+}(2)$ & $\|$ NiGePd, NiPRh, CoPPd, Sc \\
\hline & $\mathrm{Z}_{5}^{-}(2)$ & $\begin{array}{c}\text { ThCuPO, } \| \text { DyAgSb } 2, \mathrm{NdAgAs}_{2}, \text { TaMoN, InBi(ICSD : 1251), } \\
\text { Th(NiP) })_{2}, \text { CeSiOs, } \mathrm{CeS}_{2}\end{array}$ \\
\hline & $\mathrm{Z}_{5}^{+}(2)$ & $\begin{array}{l}\text { NbSiAs, } \| \text { TaSiAs, } \mathrm{HoCuP}_{2}, \mathrm{TmCuAs}_{2}, \mathrm{YCuP}_{2}, \mathrm{ThBi}_{2}, \mathrm{NaFeAs}, \\
\mathrm{CeSb}_{2} \mathrm{Pd}, \mathrm{YMnSi}, \mathrm{Gd}(\mathrm{SiPt})_{2}\end{array}$ \\
\hline & $\Gamma_{5}^{-}(2)$ & $\begin{array}{l}\text { ThTeO, NaCuSe, SrZnSbF, LaZnSbO, PrN, } \| \mathrm{BaMgGe} \mathrm{BaMgSi}, \\
\mathrm{MgAgSb}, \mathrm{MgCuAs}, \mathrm{ZrNiP}_{2}, \mathrm{Th}(\mathrm{CuSn})_{2}, \mathrm{PrBi}_{2} \mathrm{Au}, \mathrm{SmBi}_{2} \mathrm{Au}, \\
\mathrm{Sr}(\mathrm{CuSb})_{2}, \mathrm{Ba}(\mathrm{MgPb})_{2}, \mathrm{Th}(\mathrm{SiPt})_{2}, \mathrm{Ba}(\mathrm{CuSb})_{2}, \mathrm{Sr}(\mathrm{SbPd})_{2}, \\
\mathrm{Sr}(\mathrm{AsPt})_{2}, \mathrm{Ce}(\mathrm{AsRh})_{2}, \mathrm{CeFeSb}{ }_{2}, \mathrm{Ce}(\mathrm{SiIr})_{2}, \mathrm{Ce}(\mathrm{GeIr})_{2}\end{array}$ \\
\hline & $\Gamma_{5}^{+}(2)$ & $\| \mathrm{TiH}_{2}, \mathrm{BaMgSn}, \mathrm{SrCuSeF}, \mathrm{Ca}\left(\mathrm{Al}_{5} \mathrm{Cr}\right)_{2}, \mathrm{Pu}(\mathrm{SiPt})_{2}$ \\
\hline
\end{tabular}


Table II - continued

\begin{tabular}{|c|c|c|}
\hline $\begin{array}{l}\text { SOC or } \\
\text { NSOC }\end{array}$ & $\begin{array}{c}\text { Degenerate Irreducible } \\
\text { Representations }\end{array}$ & High Symmetry Point Semimetals \\
\hline $\mathrm{SOC}$ & $\begin{aligned} & \overline{\mathrm{A}}_{5}(4), \overline{\mathrm{M}}_{5}(4), \overline{\mathrm{R}}_{3} \overline{\mathrm{R}}_{4}(4) \\
& \overline{\mathrm{X}}_{3} \overline{\mathrm{X}}_{4}(4)\end{aligned}$ & 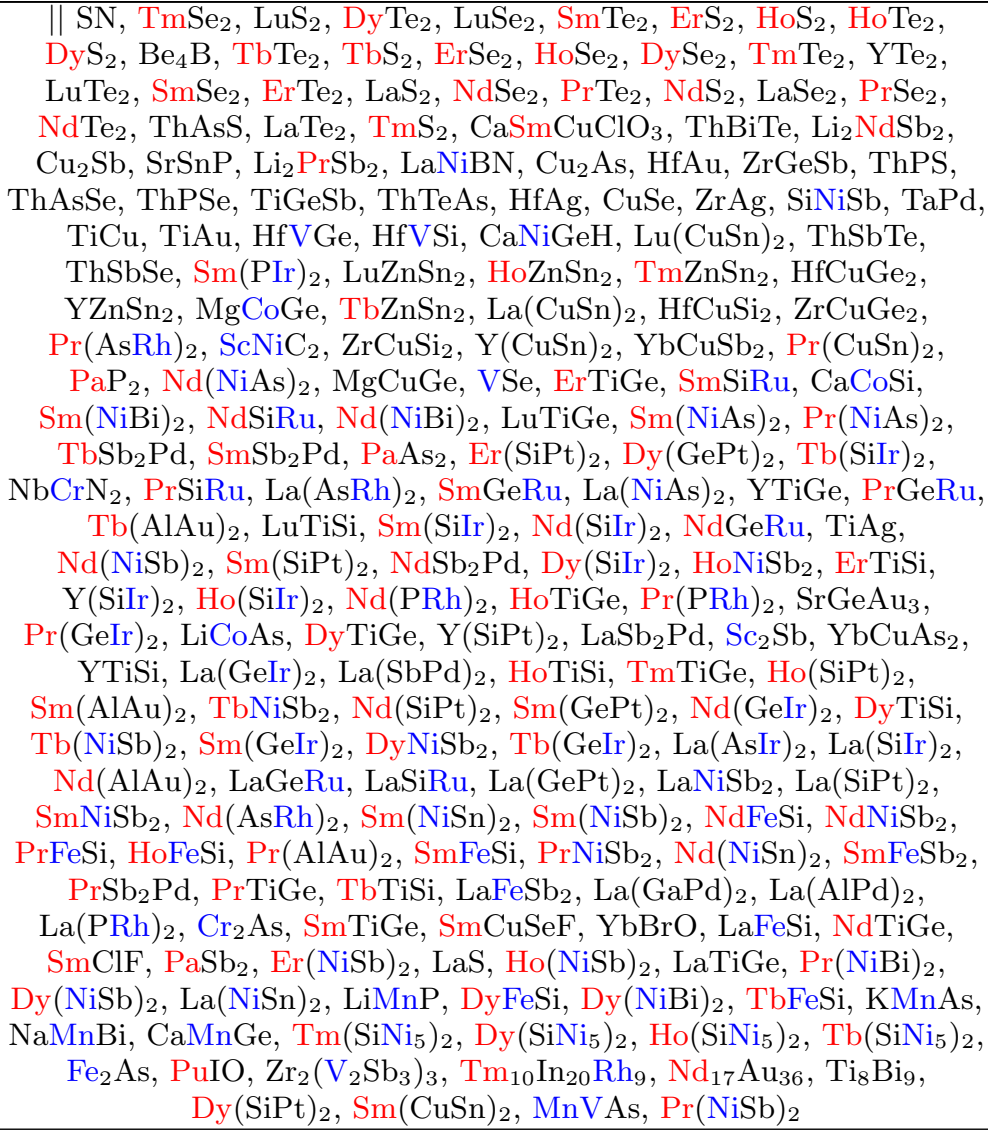 \\
\hline \multicolumn{3}{|r|}{ Space Group 130: P4/ncc } \\
\hline \multirow{4}{*}{ NSOC } & $\mathrm{A}_{1} \mathrm{~A}_{2}(4), \Gamma_{5}^{+}(2), \mathrm{R}_{1} \mathrm{R}_{2}(4)$ & $\| \mathrm{Pu}_{5} \mathrm{Rh}_{3}$ \\
\hline & $\mathrm{A}_{3} \mathrm{~A}_{4}(4), \mathrm{R}_{1} \mathrm{R}_{2}(4)$ & $\| \mathrm{Tl}_{5} \mathrm{Se}_{3}, \mathrm{TaTe}_{4}(\mathrm{ICSD}: 74414), \mathrm{Cu}\left(\mathrm{BiO}_{2}\right)_{2}, \mathrm{Bi}_{2} \mathrm{AuO}_{5}$ \\
\hline & $\mathrm{A}_{1} \mathrm{~A}_{2}(4), \mathrm{R}_{1} \mathrm{R}_{2}(4)$ & $\| \mathrm{Zr}_{2} \mathrm{FeH}_{5}, \mathrm{Pr}_{5} \mathrm{~B}_{2} \mathrm{C}_{5}, \mathrm{Dy}_{5} \mathrm{~B}_{2} \mathrm{C}_{5}$ \\
\hline & $\Gamma_{5}^{-}(2)$ & $\| \mathrm{Y}_{5} \mathrm{Ga}_{3}$ \\
\hline $\mathrm{SOC}$ & $\overline{\mathrm{A}}_{5} \overline{\mathrm{A}}_{5}(8)$ & $\begin{array}{c}\| \mathrm{Tl}_{5} \mathrm{Se}_{3}, \mathrm{Zr}_{2} \mathrm{FeH}_{5}, \mathrm{Pr}_{5} \mathrm{~B}_{2} \mathrm{C}_{5}, \mathrm{Dy}_{5} \mathrm{~B}_{2} \mathrm{C}_{5}, \mathrm{Cu}\left(\mathrm{BiO}_{2}\right)_{2}, \mathrm{Bi}_{2} \mathrm{AuO} \mathrm{O}_{5} \\
\mathrm{Pu}_{5} \mathrm{Rh}_{3}\end{array}$ \\
\hline \multicolumn{3}{|r|}{ Space Group 131: $P 4_{2} / m m c$} \\
\hline \multirow{12}{*}{ NSOC } & $\mathrm{A}_{4}(2), \Gamma_{5}^{+}(2), \mathrm{M}_{5}^{-}(2), \mathrm{Z}_{2}(2)$ & $\|$ OsN \\
\hline & $\mathrm{A}_{4}(2), \mathrm{M}_{5}^{-}(2), \mathrm{Z}_{1}(2)$ & $\| \operatorname{Tm}(\mathrm{BC})_{2}, \mathrm{Ho}(\mathrm{BC})_{2}, \operatorname{Er}(\mathrm{BC})_{2}$ \\
\hline & $\mathrm{A}_{3}(2), \mathrm{M}_{5}^{-}(2), \mathrm{Z}_{1}(2)$ & $\| \mathrm{Y}(\mathrm{BC})_{2}$ \\
\hline & $\mathrm{A}_{3}(2), \mathrm{M}_{5}^{+}(2), \mathrm{Z}_{3}(2)$ & CaNiN \\
\hline & $\Gamma_{5}^{-}(2), \mathrm{M}_{5}^{+}(2)$ & $\| \mathrm{TePdI}$ \\
\hline & $\mathrm{A}_{4}(2), \mathrm{Z}_{4}(2)$ & || $\mathrm{PdN}$ \\
\hline & $\mathrm{A}_{4}(2), \mathrm{Z}_{1}(2)$ & $\| \mathrm{La}(\mathrm{BC})_{2}, \mathrm{Nd}(\mathrm{BC})_{2}, \mathrm{Sm}(\mathrm{BC})_{2}, \operatorname{Pr}(\mathrm{BC})_{2}, \mathrm{~Tb}(\mathrm{BC})_{2}$ \\
\hline & $\mathrm{A}_{3}(2), \mathrm{Z}_{1}(2)$ & $\| \mathrm{Dy}(\mathrm{BC})_{2}$ \\
\hline & $\mathrm{A}_{2}(2), \mathrm{Z}_{1}(2)$ & $\| \mathrm{TiH}, \mathrm{ZrH}$ \\
\hline & $\mathrm{M}_{5}^{-}(2)$ & $\mathrm{PtO}, \mathrm{PdO}, \| \mathrm{Ca}\left(\mathrm{PtO}_{2}\right)_{2}$ \\
\hline & $\Gamma_{5}^{-}(2)$ & $\| \mathrm{SrPbF}_{6}$ \\
\hline & $\Gamma_{5}^{+}(2)$ & $\|$ TmCoC, ErCoC, HoCoC, DyCoC, TbCoC, YCoC \\
\hline $\mathrm{SOC}$ & $\overline{\mathrm{A}}_{5}(4), \overline{\mathrm{Z}}_{5}(4)$ & $\begin{array}{c}\| \mathrm{TiH}, \mathrm{ZrH}, \mathrm{Lu}(\mathrm{BC})_{2}, \mathrm{Tm}(\mathrm{BC})_{2}, \mathrm{La}(\mathrm{BC})_{2}, \mathrm{Ho}(\mathrm{BC})_{2}, \mathrm{Dy}(\mathrm{BC})_{2}, \\
\mathrm{Nd}(\mathrm{BC})_{2}, \mathrm{Y}(\mathrm{BC})_{2}, \mathrm{Sm}(\mathrm{BC})_{2}, \operatorname{Er}(\mathrm{BC})_{2}, \mathrm{~Tb}(\mathrm{BC})_{2}, \operatorname{Pr}(\mathrm{BC})_{2}, \mathrm{OsN}, \\
\mathrm{PdN}, \mathrm{CaNiN}\end{array}$ \\
\hline \multicolumn{3}{|r|}{ Space Group 132: $P 4_{2} / m c m$} \\
\hline NSOC & $\mathrm{A}_{2}(2), \Gamma_{5}^{+}(2), \mathrm{R}_{1}(2), \mathrm{Z}_{1}(2)$ & $\mathrm{Ti}_{2} \mathrm{H}_{3}$ \\
\hline SOC & $\overline{\mathrm{A}}_{5}(4), \overline{\mathrm{R}}_{3} \overline{\mathrm{R}}_{4}(4), \overline{\mathrm{Z}}_{5}(4)$ & $\mathrm{Ti}_{2} \mathrm{H}_{3}$ \\
\hline \multicolumn{3}{|r|}{ Space Group 133: $P 4_{2} / n b c$} \\
\hline
\end{tabular}


Table II - continued

\begin{tabular}{|c|c|c|}
\hline $\begin{array}{l}\text { SOC or } \\
\text { NSOC }\end{array}$ & $\begin{array}{l}\text { Degenerate Irreducible } \\
\text { Representations }\end{array}$ & High Symmetry Point Semimetals \\
\hline \multirow{2}{*}{ NSOC } & $\Gamma_{5}^{-}(2)$ & $\mathrm{V}_{3} \mathrm{~S}$ \\
\hline & $\Gamma_{5}^{+}(2)$ & $\mathrm{Ta}_{3} \mathrm{P}$ \\
\hline \multicolumn{3}{|r|}{ Space Group 134: $P 4_{2} / n n m$} \\
\hline \multirow{4}{*}{ NSOC } & $\begin{array}{l}\mathrm{A}_{5}^{+}(2), \mathrm{M}_{4}(2), \mathrm{R}_{2}(2) \\
\quad \mathrm{X}_{1}(2), \mathrm{Z}_{1}(2)\end{array}$ & $\| \mathrm{Li}_{3} \mathrm{Si}_{3} \mathrm{Ag}_{2}$ \\
\hline & $\begin{array}{l}\mathrm{A}_{5}^{+}(2), \mathrm{M}_{1}(2), \mathrm{R}_{2}(2) \\
\mathrm{X}_{1}(2), \mathrm{Z}_{4}(2)\end{array}$ & \| B(ICSD : 26636) \\
\hline & $\mathrm{M}_{3}(2), \mathrm{R}_{2}(2), \mathrm{X}_{2}(2), \mathrm{Z}_{3}(2)$ & \| B(ICSD : 22300) \\
\hline & $\Gamma_{5}^{+}(2)$ & B(ICSD : 240995) \\
\hline $\mathrm{SOC}$ & $\begin{array}{c}\overline{\mathrm{M}}_{5}(4), \overline{\mathrm{R}}_{3} \overline{\mathrm{R}}_{4}(4), \overline{\mathrm{X}}_{3} \overline{\mathrm{X}}_{4}(4), \\
\overline{\mathrm{Z}}_{5}(4)\end{array}$ & $\| \mathrm{Li}_{3} \mathrm{Si}_{3} \mathrm{Ag}_{2}$ \\
\hline \multicolumn{3}{|r|}{ Space Group 135: $P 4_{2} / m b c$} \\
\hline \multirow{2}{*}{ NSOC } & $\Gamma_{5}^{-}(2)$ & $\mathrm{Ca}_{2} \mathrm{GeN}_{2}, \| \mathrm{LuB}_{2} \mathrm{C}, \mathrm{TmB}_{2} \mathrm{C}, \mathrm{HoB}_{2} \mathrm{C}, \mathrm{DyB}_{2} \mathrm{C}, \mathrm{ScB}_{2} \mathrm{C}$ \\
\hline & $\Gamma_{5}^{+}(2)$ & $\| \mathrm{YbB}_{2} \mathrm{C}$ \\
\hline \multicolumn{3}{|r|}{ Space Group 136: $P 4_{2} / m n m$} \\
\hline \multirow{17}{*}{ NSOC } & $\begin{array}{l}\mathrm{A}_{3}(2), \Gamma_{5}^{-}(2), \mathrm{M}_{1}^{+} \mathrm{M}_{4}^{+}(2) \\
\mathrm{R}_{1}^{+}(2), \mathrm{X}_{2}(2), \mathrm{Z}_{3}(2)\end{array}$ & $\| \mathrm{Te}_{2} \mathrm{Ir}$ \\
\hline & $\begin{array}{c}\mathrm{A}_{2}(2), \Gamma_{5}^{-}(2), \mathrm{M}_{2}^{+} \mathrm{M}_{3}^{+}(2) \\
\mathrm{R}_{1}^{-}(2), \mathrm{X}_{2}(2), \mathrm{Z}_{1}(2)\end{array}$ & $\| \mathrm{Tb}\left(\mathrm{Ni}_{2} \mathrm{P}\right)_{2}$ \\
\hline & $\begin{array}{c}\mathrm{A}_{1}(2), \Gamma_{5}^{+}(2), \mathrm{M}_{1}^{+} \mathrm{M}_{4}^{+}(2) \\
\mathrm{R}_{1}^{+}(2), \mathrm{X}_{1}(2), \mathrm{Z}_{2}(2)\end{array}$ & || Ta(ICSD : 54203) \\
\hline & $\begin{array}{c}\mathrm{A}_{3}(2), \mathrm{M}_{5}^{-}(2), \mathrm{R}_{1}^{+}(2) \\
\mathrm{X}_{2}(2), \mathrm{Z}_{4}(2)\end{array}$ & $\| \mathrm{Y}\left(\mathrm{Re}_{2} \mathrm{Si}\right)_{2}$ \\
\hline & $\begin{array}{c}\mathrm{A}_{3}(2), \mathrm{M}_{5}^{+}(2), \mathrm{R}_{1}^{-}(2), \\
\mathrm{X}_{2}(2), \mathrm{Z}_{1}(2)\end{array}$ & \| Ta(ICSD : 54205) \\
\hline & $\begin{array}{c}\mathrm{A}_{3}(2), \mathrm{M}_{5}^{+}(2), \mathrm{R}_{1}^{-}(2) \\
\mathrm{X}_{1}(2), \mathrm{Z}_{3}(2) \\
\end{array}$ & $\| \mathrm{Nb}_{2} \mathrm{Al}$ \\
\hline & $\begin{array}{c}\mathrm{A}_{3}(2), \mathrm{M}_{2}^{+} \mathrm{M}_{3}^{+}(2), \mathrm{R}_{1}^{-}(2) \\
\mathrm{X}_{2}(2), \mathrm{Z}_{1}(2)\end{array}$ & || Dy $\left(\mathrm{Ni}_{2} \mathrm{As}\right)_{2}$ \\
\hline & $\begin{array}{c}\mathrm{A}_{3}(2), \mathrm{M}_{2}^{+} \mathrm{M}_{3}^{+}(2), \mathrm{R}_{1}^{+}(2) \\
\mathrm{X}_{2}(2), \mathrm{Z}_{3}(2)\end{array}$ & $\| \mathrm{IrO}_{2}, \mathrm{RhO}_{2}$ \\
\hline & $\begin{array}{c}\mathrm{A}_{3}(2), \mathrm{M}_{2}^{+} \mathrm{M}_{3}^{+}(2), \mathrm{R}_{1}^{+}(2) \\
\mathrm{X}_{2}(2), \mathrm{Z}_{1}(2) \\
\end{array}$ & $\| \mathrm{Dy}\left(\mathrm{Ni}_{2} \mathrm{P}\right)_{2}$ \\
\hline & $\begin{array}{c}\mathrm{A}_{2}(2), \mathrm{M}_{2}^{+} \mathrm{M}_{3}^{+}(2), \mathrm{R}_{1}^{-}(2), \\
\mathrm{X}_{2}(2), \mathrm{Z}_{1}(2)\end{array}$ & $\| \operatorname{Er}\left(\mathrm{Ni}_{2} \mathrm{As}\right)_{2}$ \\
\hline & $\begin{array}{c}\mathrm{A}_{2}(2), \mathrm{M}_{2}^{+} \mathrm{M}_{3}^{+}(2), \mathrm{R}_{1}^{+}(2), \\
\mathrm{X}_{1}(2), \mathrm{Z}_{1}(2)\end{array}$ & $\| \mathrm{Ti}_{2} \mathrm{~N}$ \\
\hline & $\begin{array}{c}\mathrm{A}_{2}(2), \mathrm{M}_{1}^{+} \mathrm{M}_{4}^{+}(2), \mathrm{R}_{1}^{-}(2), \\
\mathrm{X}_{2}(2), \mathrm{Z}_{2}(2) \\
\end{array}$ & $\| \mathrm{VO}_{2}(\mathrm{ICSD}: 647613)$ \\
\hline & $\begin{array}{c}\mathrm{A}_{2}(2), \mathrm{M}_{1}^{+} \mathrm{M}_{4}^{+}(2), \mathrm{R}_{1}^{+}(2), \\
\mathrm{X}_{2}(2), \mathrm{Z}_{2}(2)\end{array}$ & $\| \mathrm{TaO}_{2}, \mathrm{NbO}_{2}, \mathrm{VO}_{2}(\mathrm{ICSD}: 27482)$ \\
\hline & $\begin{array}{c}\mathrm{A}_{1}(2), \mathrm{M}_{5}^{+}(2), \mathrm{R}_{1}^{-}(2) \\
\mathrm{X}_{1}(2), \mathrm{Z}_{4}(2)\end{array}$ & $\| \mathrm{Ta}_{2} \mathrm{Al}$ \\
\hline & $\begin{array}{c}\mathrm{A}_{1}(2), \mathrm{M}_{5}^{+}(2), \mathrm{R}_{1}^{+}(2), \\
\mathrm{X}_{1}(2), \mathrm{Z}_{2}(2) \\
\end{array}$ & $\| \mathrm{MnO}_{2}$ \\
\hline & $\Gamma_{5}^{-}(2)$ & $\| \mathrm{In}_{3} \mathrm{Rh}, \mathrm{Y}_{3} \mathrm{Al}_{2}, \mathrm{Dy}_{3} \mathrm{Al}_{2}, \mathrm{Zr}_{3} \mathrm{Al}_{2}$ \\
\hline & $\Gamma_{5}^{+}(2)$ & 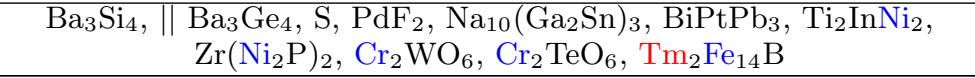 \\
\hline \multirow[t]{2}{*}{$\mathrm{SOC}$} & $\begin{array}{c}\overline{\mathrm{A}}_{5}(4), \overline{\mathrm{M}}_{8} \overline{\mathrm{M}}_{9}(4), \overline{\mathrm{X}}_{3} \overline{\mathrm{X}}_{4}(4), \\
\overline{\mathrm{Z}}_{5}(4)\end{array}$ & $\| \mathrm{Y}\left(\mathrm{Re}_{2} \mathrm{Si}\right)_{2}, \mathrm{Ta}(\mathrm{ICSD}: 54205)$ \\
\hline & $\overline{\mathrm{A}}_{5}(4), \overline{\mathrm{M}}_{6} \overline{\mathrm{Z}}_{7}(4), \overline{\mathrm{X}}_{3} \overline{\mathrm{X}}_{4}(4)$ & $\begin{array}{c}\| \mathrm{Ti}_{2} \mathrm{~N}, \mathrm{IrO}_{2}, \mathrm{NbO}_{2}, \mathrm{TaO}_{2}, \mathrm{RhO}_{2}, \mathrm{VO}_{2} \text { (ICSD : 647613), } \mathrm{Dy}\left(\mathrm{Ni}_{2} \mathrm{P}\right)_{2}, \\
\left.\mathrm{Er}\left(\mathrm{Ni}_{2} \mathrm{As}\right)_{2}, \mathrm{Dy}\left(\mathrm{Ni}_{2} \mathrm{As}\right)_{2}, \mathrm{VO}_{2} \text { (ICSD : } 27482\right), \mathrm{Tb}\left(\mathrm{Ni}_{2} \mathrm{P}\right)_{2}, \mathrm{Te}_{2} \mathrm{Ir}, \\
\mathrm{MnO}_{2}, \mathrm{Ta}_{2} \mathrm{Al}, \mathrm{Nb}_{2} \mathrm{Al}, \mathrm{Ta}(\mathrm{ICSD}: 54203), \mathrm{ReO}_{2}\end{array}$ \\
\hline \multicolumn{3}{|r|}{ Space Group 137: $P 4_{2} / n m c$} \\
\hline & $\begin{array}{l}\mathrm{A}_{3} \mathrm{~A}_{4}(4), \Gamma_{5}^{-}(2), \mathrm{M}_{4}(2) \\
\mathrm{R}_{1}(2), \mathrm{X}_{2}(2), \mathrm{Z}_{4}(2)\end{array}$ & $\| \operatorname{Sm}(\mathrm{BRh})_{4}, \mathrm{Nd}(\mathrm{BRh})_{4}$ \\
\hline
\end{tabular}


Table II - continued

\begin{tabular}{|c|c|c|}
\hline $\begin{array}{l}\text { SOC or } \\
\text { NSOC }\end{array}$ & $\begin{array}{l}\text { Degenerate Irreducible } \\
\text { Representations }\end{array}$ & High Symmetry Point Semimetals \\
\hline & $\begin{array}{l}\mathrm{A}_{3} \mathrm{~A}_{4}(4), \Gamma_{5}^{-}(2), \mathrm{M}_{4}(2) \\
\mathrm{R}_{1}(2), \mathrm{X}_{2}(2), \mathrm{Z}_{3}(2)\end{array}$ & $\| \mathrm{Y}(\mathrm{BRh})_{4}$ \\
\hline & $\begin{array}{c}\mathrm{A}_{3} \mathrm{~A}_{4}(4), \mathrm{M}_{4}(2), \mathrm{R}_{1}(2) \\
\mathrm{X}_{2}(2), \mathrm{Z}_{4}(2)\end{array}$ & $\| \operatorname{Er}(\mathrm{BRh})_{4}$ \\
\hline & $\Gamma_{5}^{-}(2)$ & $\mathrm{Cd}_{3} \mathrm{As}_{2}[63], \| \mathrm{U}(\mathrm{CoB})_{4}$ \\
\hline & $\mathrm{A}_{3} \mathrm{~A}_{4}(4)$ & $\| \mathrm{Nb}_{4} \mathrm{O}_{5}, \mathrm{Ce}(\mathrm{CoB})_{4}$ \\
\hline \multirow[t]{2}{*}{$\mathrm{SOC}$} & $\begin{array}{c}\overline{\mathrm{A}}_{6} \overline{\mathrm{A}}_{8}(4), \overline{\mathrm{M}}_{5}(4), \overline{\mathrm{R}}_{3} \overline{\mathrm{R}}_{4}(4) \\
\overline{\mathrm{X}}_{3} \overline{\mathrm{X}}_{4}(4), \overline{\mathrm{Z}}_{5}(4)\end{array}$ & $\| \operatorname{Er}(\mathrm{BRh})_{4}, \mathrm{Sm}(\mathrm{BRh})_{4}, \mathrm{Nd}(\mathrm{BRh})_{4}$ \\
\hline & $\begin{array}{c}\overline{\mathrm{A}}_{5} \overline{\mathrm{A}}_{7}(4), \overline{\mathrm{M}}_{5}(4), \overline{\mathrm{R}}_{3} \overline{\mathrm{R}}_{4}(4) \\
\overline{\mathrm{X}}_{3} \overline{\mathrm{X}}_{4}(4), \overline{\mathrm{Z}}_{5}(4)\end{array}$ & $\| \mathrm{Y}(\mathrm{BRh})_{4}$ \\
\hline \multicolumn{3}{|r|}{ Space Group 139: $I 4 / \mathrm{mmm}$} \\
\hline \multirow{14}{*}{ NSOC } & $\Gamma_{5}^{-}(2), \mathrm{M}_{5}^{-}(2), \mathrm{P}_{5}(2)$ & $\| \mathrm{Bi}_{2} \mathrm{Pd}, \mathrm{Tb}_{2} \mathrm{Al}_{4} \mathrm{NiGe}_{2}, \mathrm{U}(\mathrm{SiRh})_{2}$ \\
\hline & $\Gamma_{5}^{-}(2), \mathrm{M}_{5}^{+}(2), \mathrm{P}_{5}(2)$ & $\| \mathrm{Zr}_{3}\left(\mathrm{Cu}_{2} \mathrm{Si}_{3}\right)_{2}$ \\
\hline & $\Gamma_{5}^{+}(2), \mathrm{M}_{5}^{-}(2), \mathrm{P}_{5}(2)$ & $\| \mathrm{HfCuGe}, \mathrm{Ti}_{3} \mathrm{Cu}_{4}$ \\
\hline & $\mathrm{M}_{5}^{-}(2), \mathrm{P}_{5}(2)$ & $\begin{array}{l}\| \mathrm{CdHg}_{2}, \mathrm{Cd}_{2} \mathrm{Hg}, \mathrm{MgHg}_{2}, \mathrm{ThB}_{2} \mathrm{Pt}_{2} \mathrm{C}, \mathrm{Ce}(\mathrm{GePd})_{2}, \mathrm{Ce}(\mathrm{NiGe})_{2}, \\
\mathrm{CeNi}_{2} \mathrm{~B}_{2} \mathrm{C}, \mathrm{Th}(\mathrm{CrSi})_{2}, \mathrm{Th}\left(\mathrm{CrGe}_{2}, \mathrm{NdScGe}, \mathrm{SmScSi}, \mathrm{TbScGe},\right. \\
\mathrm{Yb}_{11} \mathrm{Sb}_{10}, \mathrm{Ca}_{11} \mathrm{Sb}_{10}, \mathrm{Ti}_{4} \mathrm{Si}_{7} \mathrm{Ni}_{4}, \mathrm{Pu}(\mathrm{FeSi})_{2}\end{array}$ \\
\hline & $\mathrm{M}_{5}^{+}(2), \mathrm{P}_{5}(2)$ & $\| \mathrm{Zr}\left(\mathrm{VGa}_{2}\right)_{2}, \mathrm{Hf}\left(\mathrm{VGa}_{2}\right)_{2}, \mathrm{NdMgSn}, \operatorname{PrMgSn}, \mathrm{ThMn}_{12}$ \\
\hline & $\Gamma_{5}^{-}(2), \mathrm{P}_{5}(2)$ & $\begin{array}{l}\| \mathrm{Sr}(\mathrm{SiAg})_{2}, \mathrm{Sr}(\mathrm{AgGe})_{2}, \mathrm{Ca}(\mathrm{AgGe})_{2}, \mathrm{Sr}(\mathrm{AgSn})_{2}, \mathrm{Ca}(\mathrm{AsPd})_{2}, \\
\mathrm{Sr}(\mathrm{AsPd})_{2}, \mathrm{Sr}(\mathrm{PPd})_{2}, \mathrm{Ca}(\mathrm{PPd})_{2}, \mathrm{Th}(\mathrm{SiNi})_{2}, \mathrm{Na}_{2} \mathrm{Ti}_{2} \mathrm{Sb}_{2} \mathrm{O}, \mathrm{U}(\mathrm{SiIr})_{2}, \\
\mathrm{ErNiB}_{4}, \mathrm{TbNiB}_{4}, \mathrm{SmNiB}_{4}, \mathrm{PrNiB}_{4}, \mathrm{U}(\mathrm{GeIr})_{2}, \mathrm{U}(\mathrm{CoGe})_{2}, \mathrm{PuGa}\end{array}$ \\
\hline & $\Gamma_{5}^{-}(2), \mathrm{M}_{5}^{-}(2)$ & $\begin{array}{c}\| \mathrm{Ba}_{2} \mathrm{CaTl}_{2}\left(\mathrm{CuO}_{4}\right)_{2}(\mathrm{ICSD}: 68198) \\
\mathrm{Ba}_{2} \mathrm{CaTl}_{2}\left(\mathrm{CuO}_{4}\right)_{2}(\mathrm{ICSD}: 78592), \mathrm{Ce}(\mathrm{MnSi})_{2}, \mathrm{SmTiGe}, \mathrm{TbTiGe} \\
\mathrm{U}(\mathrm{CrSi})_{2}, \mathrm{U}(\mathrm{GePd})_{2}, \mathrm{Sc}_{11}\left(\mathrm{AlGe}_{4}\right)_{2}, \mathrm{Sr}_{2} \mathrm{CaCu}_{2}\left(\mathrm{BiO}_{4}\right)_{2}\end{array}$ \\
\hline & $\Gamma_{5}^{-}(2), \mathrm{M}_{5}^{+}(2)$ & $\begin{array}{c}\| \mathrm{HfAl}_{3}(\mathrm{ICSD}: 608082), \mathrm{ZrAl}_{3}(\mathrm{ICSD}: 107130), \mathrm{Sr}_{2} \mathrm{Cu}_{2} \mathrm{Ni}(\mathrm{SO})_{2}, \\
\mathrm{La}_{2} \mathrm{PdO}_{4}\end{array}$ \\
\hline & $\Gamma_{5}^{+}(2), \mathrm{P}_{5}(2)$ & $\| \mathrm{CaN}_{2}, \mathrm{SrN}_{2}, \mathrm{CeC}_{2}, \mathrm{La}_{3} \mathrm{Ni}_{2} \mathrm{~B}_{2} \mathrm{~N}_{3}$ \\
\hline & $\Gamma_{5}^{+}(2), \mathrm{M}_{5}^{-}(2)$ & $\| \mathrm{Ba}(\mathrm{BIr})_{2}, \mathrm{Yb}(\mathrm{FeSi})_{2}$ \\
\hline & $\Gamma_{5}^{+}(2), M_{5}^{+}(2)$ & $\| \mathrm{Ba}_{4} \mathrm{Tl}_{2} \mathrm{Cu}_{2} \mathrm{HgO}_{10}, \mathrm{Sr}_{2} \mathrm{RuO}_{4}, \mathrm{~V}_{4} \mathrm{Zn}_{5}$ \\
\hline & $\mathrm{P}_{5}(2)$ & 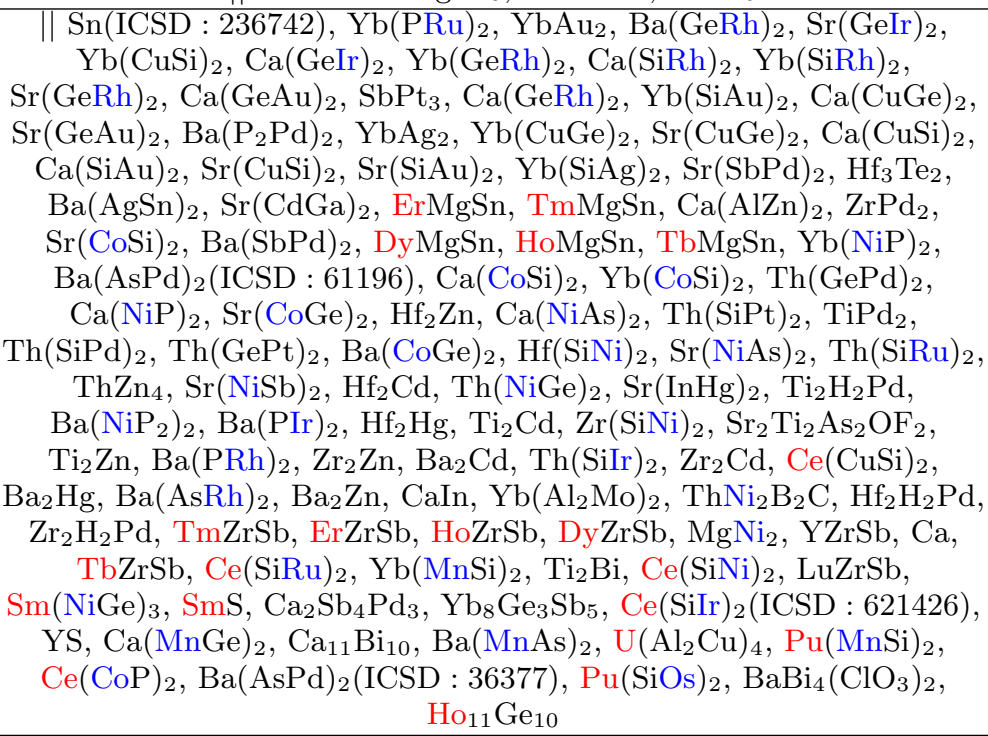 \\
\hline & $\mathrm{M}_{5}^{-}(2)$ & $\begin{array}{c}\mathrm{Si}_{2} \mathrm{Mo}, \mathrm{Si}(\mathrm{ICSD}: 163246), \| \mathrm{Mg}, \mathrm{Ba}(\mathrm{BRh})_{2}, \mathrm{BePd}_{2}, \mathrm{Ge}_{2} \mathrm{~W} \\
\mathrm{Ca}_{3} \mathrm{Cu}_{2}\left(\mathrm{ClO}_{2}\right)_{2}, \mathrm{CeB} \mathrm{Ir}_{2} \mathrm{C}, \mathrm{Ba}(\mathrm{POs})_{2}, \mathrm{Sr}(\mathrm{POs})_{2}, \mathrm{Ba}(\mathrm{PRu})_{2} \\
\mathrm{Ba}(\mathrm{AsRu})_{2}, \mathrm{U}(\mathrm{SiRu})_{2}, \mathrm{Ba}(\mathrm{FeAs})_{2}, \mathrm{PrScGe}_{1} \mathrm{YTiGe}_{\mathrm{N}} \mathrm{U}(\mathrm{NiP})_{2} \\
\mathrm{Sr}_{11} \mathrm{Bi}_{10}, \mathrm{Yb}_{11} \mathrm{Ge}_{10}, \mathrm{Ba}_{11} \mathrm{Bi}_{10}, \mathrm{Sr}_{11} \mathrm{Sb}_{10}\end{array}$ \\
\hline & $\mathrm{M}_{5}^{+}(2)$ & $\begin{array}{c}\mathrm{TlPd}_{3}(\mathrm{ICSD}: 247272), \mathrm{Si}_{2} \mathrm{~W}, \mathrm{CrSi}_{2}, \mathrm{NbAl}_{3}, \| \mathrm{NbGa}_{3}, \mathrm{TaAl}_{3}, \mathrm{Ge}_{2} \mathrm{Mo}, \\
\mathrm{TaGa}_{3}, \mathrm{BaCdBi}_{2}, \mathrm{Al}_{3} \mathrm{~V}, \mathrm{BaZnBi}_{2}, \mathrm{Cu}_{4} \mathrm{~W}(\mathrm{SCl})_{4}, \mathrm{Hf}_{4} \mathrm{Co}_{4} \mathrm{Ge}_{7}, \\
\mathrm{Zr}_{4} \mathrm{Co}_{4} \mathrm{Ge}_{7}, \mathrm{Hf}_{4} \mathrm{Co}_{4} \mathrm{Si}_{7}, \mathrm{Ce}_{2} \mathrm{Ga}_{10} \mathrm{Ni}\end{array}$ \\
\hline
\end{tabular}


Table II - continued

\begin{tabular}{|c|c|c|}
\hline $\begin{array}{l}\text { SOC or } \\
\text { NSOC }\end{array}$ & $\begin{array}{l}\text { Degenerate Irreducible } \\
\text { Representations }\end{array}$ & High Symmetry Point Semimetals \\
\hline & $\Gamma_{5}^{-}(2)$ & $\begin{array}{c}\mathrm{Sn}(\mathrm{ICSD}: 236710), \| \mathrm{TiS}_{2}\left[57, \mathrm{Zr}(\mathrm{NiP})_{2}, \mathrm{Ba}(\mathrm{AlSi})_{2}, \mathrm{Ba}(\mathrm{AlGe})_{2}\right. \\
\mathrm{Th}(\mathrm{CuSi})_{2}, \mathrm{Ca}(\mathrm{AlGa})_{2}, \mathrm{SmCuP}_{2}, \mathrm{Th}(\mathrm{CuGe})_{2}, \mathrm{CaO}, \mathrm{Ba}(\mathrm{CdGe})_{2} \\
\mathrm{U}(\mathrm{FeGe})_{2}, \mathrm{U}(\mathrm{FeSi})_{2}, \mathrm{U}(\mathrm{SiOs})_{2}, \mathrm{Ce}(\mathrm{NiP})_{2}, \mathrm{Ce}(\mathrm{NiAs})_{2}, \mathrm{Ce}(\mathrm{SiAu})_{2} \\
\mathrm{Ce}(\mathrm{AlGa})_{2}, \mathrm{RbAuO}, \mathrm{U}(\mathrm{NiGe})_{2}, \mathrm{CeAl}_{4}\end{array}$ \\
\hline & $\Gamma_{5}^{+}(2)$ & $\begin{array}{c}\mathrm{HfH}_{2}, \| \mathrm{ZrH}_{2}, \mathrm{TiAu}_{2}, \mathrm{ThH}_{2}, \mathrm{YbNi}_{2} \mathrm{~B}_{2} \mathrm{C}, \mathrm{Sr}(\mathrm{GeRu})_{2}, \mathrm{Yb}(\mathrm{SiRu})_{2}, \\
\mathrm{Ca}(\mathrm{GeRu})_{2}, \mathrm{Yb}(\mathrm{GeRu})_{2}, \mathrm{Th}(\mathrm{SiTc})_{2}, \mathrm{Sr}(\mathrm{CrAs})_{2}, \mathrm{U}_{2} \mathrm{Mo}, \mathrm{Th}(\mathrm{MnSi})_{2}, \\
\mathrm{Th}(\mathrm{MnGe})_{2}, \mathrm{BaBi}_{4}\left(\mathrm{IO}_{2}\right)_{2}, \mathrm{Ni}_{2} \mathrm{PdSe}_{2}\end{array}$ \\
\hline \multicolumn{3}{|r|}{ Space Group 140: $I 4 / \mathrm{mcm}$} \\
\hline \multirow{23}{*}{ NSOC } & $\Gamma_{5}^{-}(2), \mathrm{M}_{5}^{+}(2), \mathrm{N}_{1}(2), \mathrm{P}_{5}(2)$ & $\| \mathrm{Yb}_{5} \mathrm{Au}_{3}, \mathrm{Ca}_{5} \mathrm{Au}_{3}$ \\
\hline & $\Gamma_{5}^{+}(2), \mathrm{M}_{5}^{-}(2), \mathrm{N}_{1}(2), \mathrm{P}_{5}(2)$ & $\| \mathrm{Th}_{2} \mathrm{In}, \mathrm{Th}_{2} \mathrm{Ga}, \mathrm{Th}_{2} \mathrm{Al}$ \\
\hline & $\begin{array}{c}\Gamma_{5}^{+}(2), \mathrm{M}_{5}^{-}(2), \mathrm{N}_{1}(2) \\
\mathrm{P}_{2} \mathrm{P}_{4}(2)\end{array}$ & $\| \mathrm{Cr}_{5} \mathrm{~B}_{3}$ \\
\hline & $\Gamma_{5}^{+}(2), \mathrm{M}_{5}^{+}(2), \mathrm{N}_{1}(2), \mathrm{P}_{5}(2)$ & $\| \mathrm{Ti}_{2} \mathrm{~B}, \mathrm{Mn}_{3} \mathrm{GeC}$ \\
\hline & $\begin{array}{c}\Gamma_{5}^{+}(2), \mathrm{M}_{5}^{+}(2), \mathrm{N}_{1}(2) \\
\mathrm{P}_{1} \mathrm{P}_{3}(2)\end{array}$ & $\| \mathrm{Zr}_{2} \mathrm{Rh}$ \\
\hline & $\mathrm{M}_{5}^{-}(2), \mathrm{N}_{1}(2), \mathrm{P}_{5}(2)$ & $\begin{array}{l}\| \mathrm{Nb}_{5} \mathrm{Si}_{3}(\mathrm{ICSD}: 423763), \mathrm{Ta}_{5} \mathrm{Ge}_{3}(\mathrm{ICSD}: 195542) \\
\mathrm{Nb}_{5} \mathrm{Ge}_{3}(\mathrm{ICSD}: 2003), \mathrm{Sr}_{5} \mathrm{In}_{3}\end{array}$ \\
\hline & $\mathrm{M}_{5}^{-}(2), \mathrm{N}_{1}(2), \mathrm{P}_{2} \mathrm{P}_{4}(2)$ & $\| \mathrm{Ta}_{2} \mathrm{~B}, \mathrm{In}_{2} \mathrm{Cu}, \mathrm{Tl}_{4} \mathrm{CuTe}_{3}[5]$ \\
\hline & $\mathrm{M}_{5}^{-}(2), \mathrm{N}_{1}(2), \mathrm{P}_{1} \mathrm{P}_{3}(2)$ & $\| \mathrm{Pb}_{2} \mathrm{Au}, \mathrm{Mn}_{2} \mathrm{~B}$ \\
\hline & $\mathrm{M}_{5}^{+}(2), \mathrm{N}_{1}(2), \mathrm{P}_{5}(2)$ & $\mathrm{K}\left(\mathrm{InAu}_{2}\right)_{2}, \mathrm{Th}_{2} \mathrm{AlH}_{4}, \mathrm{Rb}\left(\mathrm{InAu}_{2}\right)_{2}, \mathrm{Yb}_{5} \mathrm{Ag}_{3}, \mathrm{La}_{10} \mathrm{Ga}_{5} \mathrm{Br}_{4}$ \\
\hline & $\mathrm{M}_{5}^{+}(2), \mathrm{N}_{1}(2), \mathrm{P}_{1} \mathrm{P}_{3}(2)$ & || $\mid \mathrm{Ta}_{5} \mathrm{Si}_{3}(\mathrm{ICSD}: 652304)$ \\
\hline & $\Gamma_{5}^{-}(2), \mathrm{N}_{1}(2), \mathrm{P}_{5}(2)$ & $\mathrm{Ni}_{2} \mathrm{~B}, \mathrm{Th}_{2} \mathrm{Cu}, \mathrm{Th}_{2} \mathrm{Au}, \mathrm{La}_{10} \mathrm{Al}_{5} \mathrm{Br}_{4}$ \\
\hline & $\Gamma_{5}^{-}(2), \mathrm{N}_{1}(2), \mathrm{P}_{2} \mathrm{P}_{4}(2)$ & $\| \mathrm{Na}_{2} \mathrm{Au}, \mathrm{Fe}_{2} \mathrm{~B}, \mathrm{U}_{6} \mathrm{Co}$ \\
\hline & $\Gamma_{5}^{+}(2), \mathrm{N}_{1}(2), \mathrm{P}_{5}(2)$ & $\mathrm{Sn}_{2} \mathrm{Rh}, \mathrm{In}_{2} \mathrm{Ag}, \mathrm{CoSn}_{2}, \mathrm{RhPb}_{2}, \mathrm{Ta}_{2} \mathrm{Co}, \mathrm{Hf}_{2} \mathrm{Ga}, \mathrm{Ti}_{5} \mathrm{CuSb}_{2}$ \\
\hline & $\Gamma_{5}^{+}(2), \mathrm{N}_{1}(2), \mathrm{P}_{2} \mathrm{P}_{4}(2)$ & $\| \mathrm{Tl}_{2} \mathrm{Au}, \mathrm{Hf}_{2} \mathrm{Al}, \mathrm{V}_{5} \mathrm{SiB}_{2}, \mathrm{Th}_{2} \mathrm{Tl}$ \\
\hline & $\mathrm{N}_{1}(2), \mathrm{P}_{5}(2)$ & $\begin{array}{c}\| \mathrm{SnI}_{3}, \mathrm{IrC}_{2}, \mathrm{Cd}_{5} \mathrm{Au}_{3}, \mathrm{BW}_{2}, \mathrm{Tl}_{5} \mathrm{Te}_{3}, \mathrm{BMo}_{2}, \mathrm{LiSn}_{4} \mathrm{Ru}, \mathrm{Zr}_{2} \mathrm{Ga}, \mathrm{Cr}_{2} \mathrm{~B} \\
\mathrm{BaCu}_{9} \mathrm{Ge}_{4}, \mathrm{VSb}_{2}, \mathrm{SrCu}_{9} \mathrm{Si}_{4}, \mathrm{BaCu}_{9} \mathrm{Si}_{4}, \mathrm{SrCu}_{9} \mathrm{Ge}_{4}, \mathrm{CaCu}_{9} \mathrm{Sn}_{4}, \\
\mathrm{YbCu}_{9} \mathrm{Sn}_{4}, \mathrm{Th}_{2} \mathrm{Ag}, \mathrm{Ca}_{5} \mathrm{Ag}_{3}, \mathrm{Co}_{2} \mathrm{~B}, \mathrm{Ti}_{5} \mathrm{Sb}_{2} \mathrm{Rh}, \mathrm{BaNd}_{2} \mathrm{MnS}_{5} \\
\mathrm{Ti}_{4} \mathrm{MnBi}_{2}, \mathrm{Pr}_{12} \mathrm{InPt}_{7}, \mathrm{Fe}_{5} \mathrm{~B}_{2} \mathrm{P}, \mathrm{Ho}_{12} \mathrm{InPt}_{7}, \mathrm{U}_{6} \mathrm{Mn}, \mathrm{BaCe}_{2} \mathrm{CoS}_{5} \\
\mathrm{BaNd}_{2} \mathrm{CoS}_{5}\end{array}$ \\
\hline & $\mathrm{N}_{1}(2), \mathrm{P}_{2} \mathrm{P}_{4}(2)$ & $\begin{array}{c}\| \mathrm{KCuF}_{3}(\mathrm{ICSD}: 9856), \mathrm{RbCuF}_{3}, \mathrm{RbAgF}_{3}, \mathrm{CsAgF}_{3}, \mathrm{Ga}_{5} \mathrm{Pd}, \mathrm{Ga} \mathrm{Ni}_{5} \\
\mathrm{Sc}_{2} \mathrm{Co}, \mathrm{Ti}_{4} \mathrm{CoBi}_{2}, \mathrm{Zr}_{2} \mathrm{Al}, \mathrm{MnSn}_{2}, \mathrm{Nb}_{5} \mathrm{SiSn}_{2}, \mathrm{La}_{5}(\mathrm{AlBr})_{4}, \mathrm{La}_{5} \mathrm{Si}_{3} \\
\mathrm{Nd}_{5} \mathrm{Si}_{3}, \mathrm{La}_{5} \mathrm{Sn}_{3}, \mathrm{LaSi}_{4} \mathrm{Ni}_{9}, \mathrm{Pr}_{5} \mathrm{Sn}_{3}, \mathrm{~Np}_{5} \mathrm{Ge}_{3}\end{array}$ \\
\hline & $\mathrm{N}_{1}(2), \mathrm{P}_{1} \mathrm{P}_{3}(2)$ & $\begin{array}{c}\| \mathrm{SiIr}_{3}, \mathrm{Al}_{2} \mathrm{Cu}, \mathrm{AlPt}_{3}, \mathrm{GaPt}_{3}, \mathrm{Ta}_{5} \mathrm{Ge}_{3}(\mathrm{ICSD}: 44743), \\
\mathrm{Nb}_{5} \mathrm{Si}_{3}(\mathrm{ICSD}: \\
\mathrm{Zr}_{5} \mathrm{Al}_{3}, \mathrm{Ca}_{5} \mathrm{Ge}_{3} \mathrm{H}, \mathrm{Nb}_{9} \mathrm{Co}_{4} \mathrm{Ge}, \mathrm{Ce}_{5} \mathrm{Ga}_{3}\end{array}$ \\
\hline & $\Gamma_{5}^{-}(2), \mathrm{M}_{5}^{-}(2)$ & $\| \mathrm{SrCu}_{2}\left(\mathrm{BO}_{3}\right)_{2}$ \\
\hline & $\Gamma_{5}^{+}(2), \mathrm{M}_{5}^{+}(2)$ & $\| \mathrm{Ti}_{4} \mathrm{FeBi}_{2}$ \\
\hline & $\mathrm{M}_{5}^{-}(2)$ & $\| \mathrm{Sr}, \mathrm{La}_{3} \mathrm{Ga}_{4} \mathrm{Cl}, \mathrm{Ba}, \mathrm{V}_{4} \mathrm{SiSb}_{2}, \mathrm{BaCe}_{2} \mathrm{FeS}_{5}, \mathrm{PrCo}_{9} \mathrm{Si}_{4}$ \\
\hline & $\mathrm{M}_{5}^{+}(2)$ & 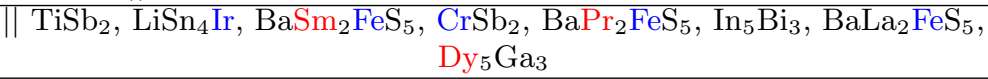 \\
\hline & $\Gamma_{5}^{-}(2)$ & $\begin{array}{c}\| \mathrm{Th}_{2} \mathrm{Pd}, \mathrm{Sc}, \mathrm{Nb}_{5} \mathrm{GaSn}_{2}, \mathrm{Er}_{5} \mathrm{BiPt}_{2}, \mathrm{Zr}_{2} \mathrm{Fe}, \mathrm{Si}_{3} \mathrm{Mo}_{5}, \mathrm{Dy}_{5} \mathrm{Ni}_{2} \mathrm{Sb} \\
\mathrm{Ho}_{5} \mathrm{Ni}_{2} \mathrm{Sb}, \mathrm{Tb}_{6} \mathrm{Al}_{3} \mathrm{Si}_{1} \mathrm{Ba}_{11} \mathrm{In}_{6} \mathrm{O}_{3}\end{array}$ \\
\hline & $\Gamma_{5}^{+}(2)$ & $\begin{array}{c}\text { \| } \mathrm{Tl}_{2} \mathrm{Pd}, \mathrm{Sb}, \mathrm{Sr}_{5} \mathrm{Si}_{3}(\mathrm{ICSD}: 93701), \mathrm{Ca}\left(\mathrm{Ni}_{2} \mathrm{Sn}\right)_{2}, \mathrm{SiB}_{2} \mathrm{Mo}_{5}, \mathrm{Sr}_{5} \mathrm{Sn}_{3}, \\
\qquad \mathrm{Sr}\left(\mathrm{Ni}_{2} \mathrm{Sn}\right)_{2}, \mathrm{~Tb}_{5} \mathrm{SbAu}_{2}, \mathrm{~Tb}_{5} \mathrm{BiAu}_{2}, \mathrm{Tl}_{3} \mathrm{FeCl}_{5}\end{array}$ \\
\hline
\end{tabular}


Table II - continued

\begin{tabular}{|c|c|c|}
\hline $\begin{array}{l}\text { SOC or } \\
\text { NSOC }\end{array}$ & $\begin{array}{l}\text { Degenerate Irreducible } \\
\text { Representations }\end{array}$ & High Symmetry Point Semimetals \\
\hline $\mathrm{SOC}$ & $\overline{\mathrm{N}}_{2} \overline{\mathrm{N}}_{2}(4), \overline{\mathrm{P}}_{6} \overline{\mathrm{P}}_{7}(4)$ & 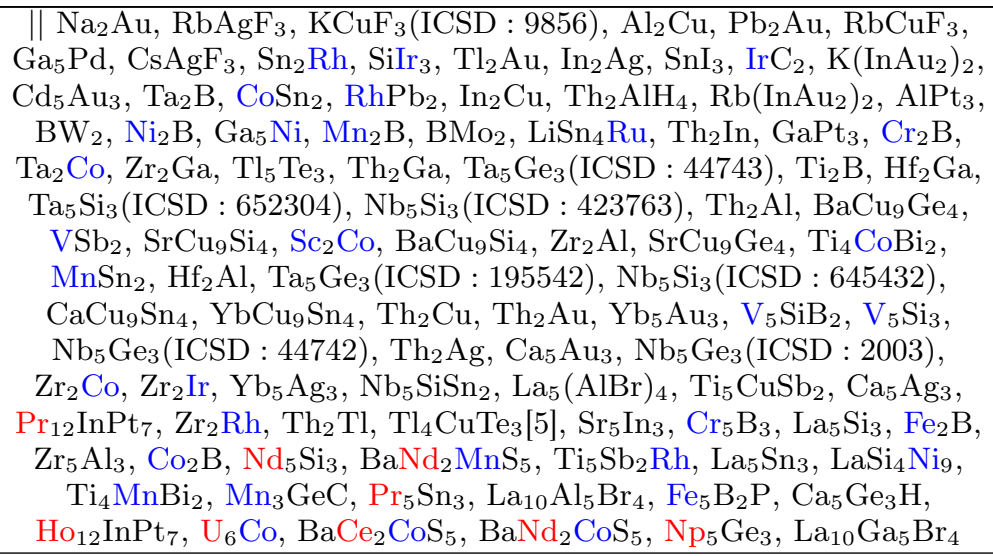 \\
\hline \multicolumn{3}{|r|}{ Space Group 141: $I 4_{1} / a m d$} \\
\hline \multirow{15}{*}{ NSOC } & $\Gamma_{5}^{-}(2), \mathrm{M}_{3}(2), \mathrm{P}_{1}(2), \mathrm{X}_{2}(2)$ & \multirow{3}{*}{$\begin{array}{l}\mathrm{YbAgS}_{2} \\
\mathrm{Mo}_{2} \mathrm{~N} \\
\mathrm{LiGe}\end{array}$} \\
\hline & $\Gamma_{5}^{+}(2), \mathrm{M}_{3}(2), \mathrm{P}_{1}(2), \mathrm{X}_{1}(2)$ & \\
\hline & $\Gamma_{5}^{+}(2), \mathrm{M}_{1}(2), \mathrm{P}_{2}(2), \mathrm{X}_{2}(2)$ & \\
\hline & $\Gamma_{5}^{+}(2), \mathrm{M}_{1}(2), \mathrm{P}_{1}(2), \mathrm{X}_{2}(2)$ & \multirow{2}{*}{$\begin{array}{c}\| \mathrm{LiSn}, \mathrm{NdZn}_{11}, \mathrm{LaZn}_{11}, \mathrm{PrZn}_{11} \\
\| \mathrm{Li}_{2} \mathrm{CuSn}_{2}, \mathrm{Li}_{2} \mathrm{Sn}_{2} \mathrm{Au}\end{array}$} \\
\hline & $\mathrm{M}_{4}(2), \mathrm{P}_{2}(2), \mathrm{X}_{2}(2)$ & \\
\hline & $\mathrm{M}_{4}(2), \mathrm{P}_{1}(2), \mathrm{X}_{2}(2)$ & $\begin{array}{c}\| \mathrm{Li}_{2} \mathrm{AgSn}_{2}, \mathrm{SmSi}_{2}, \mathrm{NdSi}_{2}, \mathrm{PrSi}_{2}, \mathrm{LaGe}_{2}, \mathrm{DySi}_{2}, \mathrm{YSi}_{2}, \mathrm{LaSi}_{2}, \\
\mathrm{Y}_{2} \mathrm{~B}_{5} \mathrm{Pd}_{14}\end{array}$ \\
\hline & $\mathrm{M}_{3}(2), \mathrm{P}_{2}(2), \mathrm{X}_{2}(2)$ & || $\mathrm{LiTiO}_{2}$ \\
\hline & $\mathrm{M}_{2}(2), \mathrm{P}_{2}(2), \mathrm{X}_{1}(2)$ & $\| \mathrm{Ti}_{2} \mathrm{~N}$ \\
\hline & $\mathrm{M}_{2}(2), \mathrm{P}_{1}(2), \mathrm{X}_{2}(2)$ & $\| \mathrm{ThCog}_{9} \mathrm{Si}_{2}$ \\
\hline & $\mathrm{M}_{1}(2), \mathrm{P}_{2}(2), \mathrm{X}_{2}(2)$ & $\| \mathrm{ErSi}_{2}, \mathrm{TbGe}_{2}$ \\
\hline & $\mathrm{M}_{1}(2), \mathrm{P}_{2}(2), \mathrm{X}_{1}(2)$ & $\| \mathrm{Rb}, \mathrm{Cs}, \mathrm{Sm}_{2}\left(\mathrm{PPt}_{2}\right)_{3}, \mathrm{YCrO}_{4}$ \\
\hline & $\mathrm{M}_{1}(2), \mathrm{P}_{1}(2), \mathrm{X}_{2}(2)$ & $\| \mathrm{TbSi}_{2}, \mathrm{HoSi}_{2}, \mathrm{PrGe}_{2}, \mathrm{NdGe}_{2}, \mathrm{SmGe}_{2}, \mathrm{YGe}_{2}, \mathrm{Ca}_{4} \mathrm{In}_{2} \mathrm{~N}, \mathrm{Sr}_{4} \mathrm{In}_{2} \mathrm{~N}$ \\
\hline & $\mathrm{M}_{1}(2), \mathrm{P}_{1}(2), \mathrm{X}_{1}(2)$ & $\| \mathrm{PrCrO}_{4}$ \\
\hline & $\Gamma_{5}^{-}(2)$ & $\| \mathrm{LaMgSi}_{2}, \mathrm{ReB}, \mathrm{PuSi}_{2}, \mathrm{EuVO}_{4}$ \\
\hline & $\Gamma_{5}^{+}(2)$ & \\
\hline $\mathrm{SOC}$ & $\overline{\mathrm{M}}_{5}(4), \overline{\mathrm{X}}_{3} \overline{\mathrm{X}}_{4}(4)$ & $\begin{array}{c}\| \mathrm{Rb}, \mathrm{Cs}, \mathrm{TbSi}_{2}, \mathrm{Li}_{2} \mathrm{AgSn}_{2}, \mathrm{NdGe}_{2}, \mathrm{PrGe}_{2}, \mathrm{SmGe}_{2}, \mathrm{YGe}_{2}, \mathrm{TbGe}_{2}, \\
\mathrm{Li}_{2} \mathrm{Sn}_{2} \mathrm{Au}, \mathrm{Ti}_{2} \mathrm{~N}, \mathrm{LiGe}_{\mathrm{Si}} \mathrm{SmSi}_{2}, \mathrm{NdSi}_{2}, \mathrm{LiTiO}_{2}, \mathrm{PrSi}_{2}, \mathrm{DySi}_{2}, \mathrm{LaGe}_{2}, \\
\mathrm{YSi}_{2}, \mathrm{LiSn}_{\mathrm{LaSi}}, \mathrm{YbAgS}_{2}, \mathrm{Mo}_{2} \mathrm{~N}, \mathrm{Ca}_{4} \mathrm{In}_{2} \mathrm{~N}, \mathrm{NdZn}_{11}, \mathrm{LaZn}_{11} \\
\mathrm{PrZn}_{11}, \mathrm{Sr}_{4} \mathrm{In}_{2} \mathrm{~N}, \mathrm{Sm}_{2}\left(\mathrm{PPt}_{2}\right)_{3}, \mathrm{PrCrO}_{4}, \mathrm{YCrO}_{4}, \mathrm{Y}_{2} \mathrm{~B}_{5} \mathrm{Pd}_{14}, \mathrm{Li}_{2} \mathrm{CuSn}_{2}\end{array}$ \\
\hline \multicolumn{3}{|r|}{ Space Group 142: I4 $/$ acd } \\
\hline \multirow{3}{*}{ NSOC } & $\mathrm{P}_{1} \mathrm{P}_{2}(4)$ & $\mathrm{Sn}_{4} \mathrm{Ir}, \mathrm{Sr}_{2} \mathrm{IrO}_{4}, \mathrm{Ga}_{8} \mathrm{BIr}_{4}, \operatorname{Er}(\mathrm{BRh})_{4}, \mathrm{Lu}(\mathrm{BRh})_{4}, \mathrm{Lu}(\mathrm{BRu})_{4}, \mathrm{Y}(\mathrm{BRu})_{4}$ \\
\hline & $\Gamma_{5}^{-}(2)$ & $\mathrm{Cd}_{3} \mathrm{As}_{2}, \| \mathrm{CoSn}_{3}$ \\
\hline & $\Gamma_{5}^{+}(2)$ & $\| \mathrm{ZnAu}_{3}$ \\
\hline $\mathrm{SOC}$ & $\overline{\mathrm{P}}_{7} \overline{\mathrm{P}}_{7}(4)$ & (Er(BRh $)_{4}, \mathrm{Lu}(\mathrm{BRh})_{4}, \mathrm{Y}(\mathrm{BRu})_{4}, \mathrm{Lu}(\mathrm{BRu})_{4}, \mathrm{Sr}_{2} \mathrm{IrO}_{4}$ \\
\hline \multicolumn{3}{|r|}{ Space Group 143: P3 } \\
\hline NSOC & $\mathrm{A}_{2} \mathrm{~A}_{3}(2)$ & $\| \mathrm{Hg}_{12} \mathrm{SbBr}\left(\mathrm{ClO}_{3}\right)_{2}$ \\
\hline \multicolumn{3}{|r|}{ Space Group 146: $R 3$} \\
\hline \multirow{3}{*}{ NSOC } & $\Gamma_{2} \Gamma_{3}(2), T_{2} T_{3}(2)$ & $\mathrm{Ba}_{3} \mathrm{Yb}_{4} \mathrm{O}_{9}$ \\
\hline & $\mathrm{T}_{2} \mathrm{~T}_{3}(2)$ & $\| \mathrm{La}_{4} \mathrm{Ni}_{3} \mathrm{~Pb}_{4}$ \\
\hline & $\Gamma_{2} \Gamma_{3}(2)$ & $\| \mathrm{P}_{3} \mathrm{Pd}_{7}$ \\
\hline & & Space Group 147: $P \overline{3}$ \\
\hline NSOC & $\Gamma_{2}^{-} \Gamma_{3}^{-}(2)$ & $\mathrm{In}_{3} \mathrm{Au}_{7}, \mathrm{TiNi}$ \\
\hline IVNOC & $\mathrm{A}_{2}^{+} \mathrm{A}_{3}^{+}(2)$ & $\mathrm{Mn}_{14} \mathrm{Al}_{56} \mathrm{Ge}_{3}$ \\
\hline & & Space Group 148: $R \overline{3}$ \\
\hline & $\Gamma_{2}^{-} \Gamma_{3}^{-}(2), \mathrm{T}_{2}^{-} \mathrm{T}_{3}^{-}(2)$ & $\begin{array}{c}\| \mathrm{Mo}_{6} \mathrm{PbSe}_{8}, \mathrm{Mo}_{3} \mathrm{~S}_{3} \mathrm{Br}, \mathrm{Hg}\left(\mathrm{Mo}_{3} \mathrm{~S}_{4}\right)_{2}, \mathrm{Yb}\left(\mathrm{Mo}_{3} \mathrm{~S}_{4}\right)_{2}, \mathrm{Ba}\left(\mathrm{Mo}_{3} \mathrm{~S}_{4}\right)_{2}, \\
\mathrm{Ca}\left(\mathrm{Mo}_{3} \mathrm{~S}_{4}\right)_{2}, \mathrm{Sr}\left(\mathrm{Mo}_{3} \mathrm{~S}_{4}\right)_{2}, \mathrm{Mo}_{6} \mathrm{PbS}_{8}, \mathrm{Sn}\left(\mathrm{Mo}_{3} \mathrm{~S}_{4}\right)_{2}, \mathrm{Rb}_{5} \mathrm{Mo}_{18} \mathrm{~S}_{19}, \mathrm{FeCl}_{3}\end{array}$ \\
\hline & $\Gamma_{2}^{-} \Gamma_{3}^{-}(2), \mathrm{T}_{2}^{+} \mathrm{T}_{3}^{+}(2)$ & $\| \mathrm{Cs}_{2} \mathrm{Te}_{10} \mathrm{Mo}_{9}$ \\
\hline & $\Gamma_{2}^{+} \Gamma_{3}^{+}(2), \mathrm{T}_{2}^{-} \mathrm{T}_{3}^{-}(2)$ & $\| \mathrm{CuSiO}_{3}$ \\
\hline NSOC & $\Gamma_{2}^{+} \Gamma_{3}^{+}(2), \mathrm{T}_{2}^{+} \mathrm{T}_{3}^{+}(2)$ & $\mathrm{Ca}_{3} \mathrm{Pt}_{2}, \mathrm{Sr}_{7} \mathrm{Nb}_{6} \mathrm{O}_{21}, \mathrm{Rb}_{4}\left(\mathrm{Mo}_{7} \mathrm{Se}_{8}\right)_{3}, \mathrm{TiCl}_{3}$ \\
\hline & $\mathrm{T}_{2}^{-} \mathrm{T}_{3}^{-}(2)$ & $\| \mathrm{Ti}_{3} \mathrm{Ni}_{4}, \mathrm{RbMo}_{6} \mathrm{Se}_{7}$ \\
\hline
\end{tabular}


Table II - continued

\begin{tabular}{|c|c|c|}
\hline $\begin{array}{l}\text { SOC or } \\
\text { NSOC }\end{array}$ & $\begin{array}{l}\text { Degenerate Irreducible } \\
\text { Representations }\end{array}$ & High Symmetry Point Semimetals \\
\hline & $\mathrm{T}_{2}^{+} \mathrm{T}_{3}^{+}(2)$ & $\| \mathrm{P}_{2} \mathrm{Pd}_{15}, \mathrm{Ba}_{3} \mathrm{Ag}_{2}, \mathrm{Zr}_{3} \mathrm{Pd}_{4}, \mathrm{Nd}_{3} \mathrm{Au}_{4}, \mathrm{CsTe}_{7} \mathrm{Mo}_{6}, \mathrm{Re}_{6} \mathrm{P}_{13}$ \\
\hline & $\Gamma_{2}^{-} \Gamma_{3}^{-}(2)$ & $\| \mathrm{AgBiO}_{3}, \mathrm{Pr}_{3} \mathrm{Pt}_{4}, \mathrm{Te}_{7} \mathrm{Pd}_{20}, \mathrm{Sr}_{3} \mathrm{Ag}_{2}, \mathrm{Rb}_{2} \mathrm{Mog}_{9} \mathrm{Se}_{10}$ \\
\hline & $\Gamma_{2}^{+} \Gamma_{3}^{+}(2)$ & $\| \mathrm{Yb}_{3} \mathrm{Pt}_{4}, \mathrm{Sc}_{7} \mathrm{BCl}_{12}$ \\
\hline & & Space Group 149: P312 \\
\hline NSOC & $\mathrm{A}_{3}(2), \Gamma_{3}(2)$ & $\mathrm{Ti}_{3} \mathrm{O}$ \\
\hline & & Space Group 150: P321 \\
\hline & $\mathrm{H}_{3}(2), \mathrm{K}_{3}(2)$ & $\mathrm{Al}_{5} \mathrm{Mo}$ \\
\hline NSOC & $\Gamma_{3}(2)$ & $\mathrm{Si}_{12} \mathrm{Ni}_{31}$ \\
\hline & & Space Group 152: $P 3_{1} 21$ \\
\hline NSOC & $\Gamma_{3}(2)$ & $\mathrm{T}_{\mathrm{C}}$ \\
\hline SOC & $\overline{\mathrm{H}}_{6}(2)$ & $\mathrm{Te} \|$ \\
\hline & & Space Group 154: $P 3_{2} 21$ \\
\hline $\mathrm{SOC}$ & $\overline{\mathrm{H}}_{6}(2)$ & Te 64 \\
\hline & & Space Group 155: R32 \\
\hline NSOC & $\mathrm{T}_{3}(2)$ & $\| \operatorname{Er}\left(\mathrm{Al}_{3} \mathrm{Ni}\right)_{3}$ \\
\hline NSUC & $\Gamma_{3}(2)$ & $\mathrm{Ag}_{3} \mathrm{BO}_{3}, \| \mathrm{Ni}_{3} \mathrm{~S}_{2}, \mathrm{Ni}_{3} \mathrm{Se}_{2}, \mathrm{Zr}_{3} \mathrm{O}$ \\
\hline & & Space Group 156: P3m1 \\
\hline & $\mathrm{A}_{3}(2), \Gamma_{3}(2)$ & $\mathrm{Zn}\left(\operatorname{InS}_{2}\right)_{2} \|$ \\
\hline NSOC & $\Gamma_{3}(2)$ & $\|$ ZnGaNO \\
\hline & $\mathrm{A}_{3}(2)$ & BiTeI, $\| \mathrm{Cu}_{7} \mathrm{Te}_{4}$ \\
\hline & & Space Group 157: P31m \\
\hline & $\mathrm{A}_{3}(2), \Gamma_{3}(2)$ & $\mathrm{Cu}_{2} \mathrm{SiS}_{3}$ \\
\hline NSOC & $\Gamma_{3}(2)$ & \begin{tabular}{l||l}
$\mathrm{TiNi}$ \\
\end{tabular} \\
\hline & $\mathrm{A}_{3}(2)$ & || $\mathrm{Li}_{17}\left(\mathrm{AgSn}_{2}\right)_{3}, \mathrm{Ba}_{21} \mathrm{Al}_{40}$ \\
\hline & & Space Group 159: P31c \\
\hline NSOC & $\Gamma_{3}(2)$ & $\| \mathrm{Si}_{3} \mathrm{~N}_{4}(\mathrm{ICSD}: 16752)$ \\
\hline & & Space Group 160: $R 3 m$ \\
\hline & $\Gamma_{3}(2), \mathrm{T}_{3}(2)$ & $\mathrm{NaCu}_{3} \mathrm{Te}_{2}, \| \mathrm{In}_{2} \mathrm{Se}_{3}, \mathrm{TaSe}_{2}$ \\
\hline NSOC & $\mathrm{T}_{3}(2)$ & $\mathrm{Ge}_{3}\left(\mathrm{BiTe}_{3}\right)_{2}, \| \mathrm{Zn}_{2} \mathrm{In}_{2} \mathrm{~S}_{5}, \mathrm{Al}_{8} \mathrm{Cr}_{5}$ \\
\hline & $\Gamma_{3}(2)$ & $\begin{array}{c}\mathrm{Zn}\left(\mathrm{InS}_{2}\right)_{2}(\mathrm{ICSD}: 65725), \mathrm{Zn}_{\left(\mathrm{InS}_{2}\right)_{2}(\mathrm{ICSD}: 42667),} \| \mathrm{Al}_{2} \mathrm{ZnS}_{4}, \\
\mathrm{NaLi}_{5} \mathrm{Sn}_{4}, \mathrm{NiS}, \mathrm{NiSe}\end{array}$ \\
\hline & & Space Group 161: $R 3 c$ \\
\hline & $\mathrm{L}_{1} \mathrm{~L}_{2}(2), \mathrm{T}_{3} \mathrm{~T}_{3}(4)$ & $\| \mathrm{LiOsO}_{3}$ \\
\hline $\mathrm{NSOC}$ & $\mathrm{L}_{1} \mathrm{~L}_{2}(2), \mathrm{T}_{1} \mathrm{~T}_{2}(2)$ & $\mathrm{Sr}_{9} \mathrm{YbV}_{7} \mathrm{O}_{28}$ \\
\hline NSUC & $\Gamma_{3}(2), \mathrm{T}_{3} \mathrm{~T}_{3}(4)$ & $\| \mathrm{Li}_{8} \mathrm{Zn}_{2} \mathrm{Ge}_{3}$ \\
\hline & $\Gamma_{3}(2)$ & $\mathrm{ZnPbO}_{3}[65$ \\
\hline & & Space Group 162: $P \overline{3} 1 m$ \\
\hline & $\mathrm{A}_{3}^{+}(2), \Gamma_{3}^{+}(2), \mathrm{H}_{3}(2), \mathrm{K}_{3}(2)$ & $\mathrm{As}_{2} \mathrm{PdO}_{6}$ \\
\hline NSOC & $\mathrm{K}_{3}(2)$ & $\mathrm{Cu}_{10} \mathrm{Sn}_{3}$ \\
\hline & $\Gamma_{3}^{+}(2)$ & $\| \mathrm{Ti}_{6} \mathrm{O}$ \\
\hline & & Space Group 163: $P \overline{3} 1 c$ \\
\hline & $\mathrm{A}_{3}(2), \mathrm{H}_{3}(2), \mathrm{K}_{3}(2), \mathrm{L}_{1}(2)$ & $\| \mathrm{K}\left(\mathrm{Mo}_{6} \mathrm{~S}_{7}\right)_{2}$ \\
\hline & $\mathrm{A}_{3}(2), \mathrm{L}_{1}(2)$ & $\| \mathrm{CsTa}_{6} \mathrm{PbCl}_{18}$ \\
\hline NSOC & $\mathrm{A}_{1} \mathrm{~A}_{2}(4), \Gamma_{3}^{-}(2)$ & $\| \mathrm{Ti}_{6} \mathrm{O}$ \\
\hline & $\mathrm{K}_{3}(2)$ & $\mathrm{SiO}_{2}(\mathrm{ICSD}: 170495)$ \\
\hline & $\mathrm{A}_{1} \mathrm{~A}_{2}(4)$ & $\| \mathrm{Ti}_{3} \mathrm{O}$ \\
\hline $\mathrm{SOC}$ & $\overline{\mathrm{A}}_{5} \overline{\mathrm{A}}_{6}(4), \overline{\mathrm{L}}_{2} \overline{\mathrm{L}}_{2}(4)$ & $\mathrm{K}\left(\mathrm{Mo}_{6} \mathrm{~S}_{7}\right)_{2}$ \\
\hline & & Space Group 164: $P \overline{3} m 1$ \\
\hline & $\mathrm{A}_{3}^{-}(2), \Gamma_{3}^{-}(2), \mathrm{H}_{3}(2), \mathrm{K}_{3}(2)$ & $\| \mathrm{Er}_{5} \mathrm{Si}_{3} \mathrm{C}$ \\
\hline & $\mathrm{A}_{3}^{-}(2), \mathrm{H}_{3}(2), \mathrm{K}_{3}(2)$ & $\| \mathrm{PrAlSi}_{2}, \mathrm{Cr}_{3} \mathrm{Te}_{4}$ \\
\hline & $\mathrm{A}_{3}^{+}(2), \Gamma_{3}^{+}(2), \mathrm{H}_{3}(2)$ & $\| \mathrm{LiVS}_{2}$ \\
\hline & $\mathrm{H}_{3}(2), \mathrm{K}_{3}(2)$ & $\| \mathrm{LaAlSi}_{2}, \mathrm{LaGeI}, \mathrm{CoO}_{2}(\mathrm{ICSD}: 53994), \mathrm{CoO}_{2}(\mathrm{ICSD}: 26763)$ \\
\hline & $\Gamma_{3}^{+}(2), \mathrm{H}_{3}(2)$ & $\| \mathrm{Al}_{3} \mathrm{Pt}_{2}(\mathrm{ICSD}: 58134)$ \\
\hline & $\mathrm{A}_{3}^{-}(2), \mathrm{H}_{3}(2)$ & $\| \mathrm{Ta}_{2} \mathrm{CS}_{2}, \mathrm{ZrN}_{2}, \mathrm{Nb}_{2} \mathrm{CS}_{2}$ \\
\hline end & $\mathrm{A}_{3}^{-}(2), \Gamma_{3}^{-}(2)$ & $\| \mathrm{Li}_{2} \mathrm{ZnSi}\left(\mathrm{ICSD}:\right.$ 642380), $\mathrm{Ca}_{3} \mathrm{SiBr}_{2}, \mathrm{Ag}_{2} \mathrm{O}, \mathrm{Yb}_{2} \mathrm{SeO}_{2}$ \\
\hline
\end{tabular}


Table II - continued

\begin{tabular}{|c|c|c|}
\hline $\begin{array}{l}\text { SOC or } \\
\text { NSOC }\end{array}$ & $\begin{array}{l}\text { Degenerate Irreducible } \\
\text { Representations }\end{array}$ & High Symmetry Point Semimetals \\
\hline & $\mathrm{A}_{3}^{-}(2), \Gamma_{3}^{+}(2)$ & $\mathrm{Ge}_{5}\left(\mathrm{Te}_{4} \mathrm{As}\right)_{2}, \mathrm{BiSe} \|$ \\
\hline & $\mathrm{A}_{3}^{+}(2), \Gamma_{3}^{+}(2)$ & $\mathrm{Ta}_{2} \mathrm{C}, \| \mathrm{V}_{2} \mathrm{C}, \mathrm{Nb}_{2} \mathrm{C}$ \\
\hline & $\mathrm{K}_{3}(2)$ & $\mathrm{W}_{2} \mathrm{C}, \mathrm{Rb}_{2} \mathrm{Hg}_{7}, \mathrm{~K}_{2} \mathrm{Hg}_{7}$ \\
\hline & $\mathrm{H}_{3}(2)$ & $\| \mathrm{Sc}_{2} \mathrm{C}, \mathrm{U}(\mathrm{CuP})_{2}$ \\
\hline & $\Gamma_{3}^{-}(2)$ & 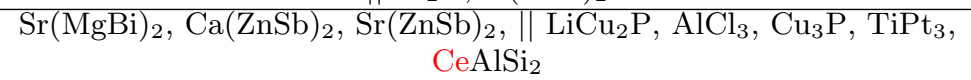 \\
\hline & $\Gamma_{3}^{+}(2)$ & $\begin{array}{c}\mathrm{CdInGaS}_{4}, \mathrm{Bi}_{2} \mathrm{Te}_{5} \mathrm{~Pb}_{2}, \mathrm{Hf}(\mathrm{CuP})_{2}, \mathrm{Zr}(\mathrm{CuP})_{2}, \| \mathrm{Ge}_{2} \mathrm{Sb}_{2} \mathrm{Te}_{5}, \mathrm{Te}_{2} \mathrm{Pt} \\
\mathrm{Te}_{2} \mathrm{Pd}, \mathrm{YbHg}_{2}, \mathrm{NiTe}_{2}, \mathrm{HfTe}_{2}\end{array}$ \\
\hline & $\mathrm{A}_{3}^{-}(2)$ & $\mathrm{BiTe}[5], \| \mathrm{Yb}_{2} \mathrm{SO}_{2}, \mathrm{Ba}_{3} \mathrm{CaIr}_{2} \mathrm{O}_{9}$ \\
\hline & $\mathrm{A}_{3}^{+}(2)$ & $\mathrm{CaSi}_{2}, \| \mathrm{Ti}_{2} \mathrm{O}, \mathrm{CaHg}_{2}, \mathrm{AgBiTe}{ }_{2}$ \\
\hline \multicolumn{3}{|r|}{ Space Group 165: $P \overline{3} c 1$} \\
\hline \multirow{6}{*}{ NSOC } & $\begin{array}{l}\mathrm{A}_{3}(2), \mathrm{H}_{3} \mathrm{H}_{3}(4), \mathrm{K}_{3}(2) \\
\mathrm{L}_{1}(2)\end{array}$ & $\| \mathrm{YbZr}_{6}\left(\mathrm{PO}_{4}\right)_{9}$ \\
\hline & $\mathrm{A}_{3}(2), \mathrm{H}_{3} \mathrm{H}_{3}(4), \mathrm{L}_{1}(2)$ & $\| \mathrm{Mg}_{3} \mathrm{Au}$ \\
\hline & $\mathrm{A}_{1} \mathrm{~A}_{2}(4), \Gamma_{3}^{-}(2)$ & $\mathrm{K}_{2} \mathrm{Sn}\left(\mathrm{GeO}_{3}\right)_{3}$ \\
\hline & $\mathrm{A}_{1} \mathrm{~A}_{2}(4), \Gamma_{3}^{+}(2)$ & $\mathrm{Cu}_{3} \mathrm{As}(\mathrm{ICSD}: 26776)$ \\
\hline & $\Gamma_{3}^{-}(2)$ & $\| \mathrm{Rb}_{2} \mathrm{Sn}\left(\mathrm{GeO}_{3}\right)_{3}$ \\
\hline & $\Gamma_{3}^{+}(2)$ & $\| \mathrm{Cu}_{3} \mathrm{P}(\mathrm{ICSD}: 26775), \mathrm{Cu}_{3} \mathrm{P}(\mathrm{ICSD}: 16841)$ \\
\hline \multirow{2}{*}{$\mathrm{SOC}$} & $\overline{\mathrm{A}}_{5} \overline{\mathrm{A}}_{6}(4), \overline{\mathrm{H}}_{6} \overline{\mathrm{H}}_{6}(4), \overline{\mathrm{L}}_{2} \overline{\mathrm{L}}_{2}(4)$ & $\| \mathrm{YbZr}_{6}\left(\mathrm{PO}_{4}\right)_{9}$ \\
\hline & $\overline{\mathrm{A}}_{5} \overline{\mathrm{A}}_{6}(4), \overline{\mathrm{L}}_{2} \overline{\mathrm{L}}_{2}(4)$ & $\| \mathrm{Mg}_{3} \mathrm{Au}$ \\
\hline \multirow{9}{*}{ NSOC } & \multicolumn{2}{|r|}{ Space Group 166: $R \overline{3} m$} \\
\hline & $\Gamma_{3}^{-}(2), \mathrm{T}_{3}^{-}(2)$ & 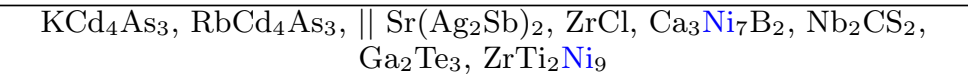 \\
\hline & $\Gamma_{3}^{-}(2), \mathrm{T}_{3}^{+}(2)$ & $\begin{array}{c}\mathrm{Ca}_{3} \mathrm{SiBr}_{2}, \| \mathrm{LuHCl}, \mathrm{ZrBr}, \mathrm{ErHCl}, \mathrm{ScHCl}, \mathrm{Tb}_{2} \mathrm{Al}_{6} \mathrm{Si}_{4} \mathrm{Pt} \\
\mathrm{TaCuN}_{2}(\mathrm{ICSD}: 190938)\end{array}$ \\
\hline & $\Gamma_{3}^{+}(2), \mathrm{T}_{3}^{-}(2)$ & $\| \mathrm{Re}_{2} \mathrm{PbO}_{6}$ \\
\hline & $\Gamma_{3}^{+}(2), \mathrm{T}_{3}^{+}(2)$ & $\begin{array}{c}\text { I| } \mathrm{NaVSe}_{2}, \mathrm{NaVS}_{2}, \mathrm{NiI}_{2}, \mathrm{NiBr}_{2}, \mathrm{LaRh}_{3}, \mathrm{Nd}_{2} \mathrm{Fe}, \mathrm{PrCo}_{3}, \mathrm{Sm}_{2} \mathrm{Cr}_{2} \mathrm{Fe}_{15}, \\
\mathrm{SiMo}_{12} \mathrm{H}_{24} \mathrm{C}_{6} \mathrm{~N}_{3} \mathrm{O}_{40}\end{array}$ \\
\hline & $\mathrm{T}_{3}^{-}(2)$ & $\begin{array}{c}\| \mathrm{MoS}_{2}, \mathrm{Ta}_{2} \mathrm{CS}_{2}, \mathrm{InRh}_{3} \mathrm{PbS}_{2}, \mathrm{Ni}_{3}(\mathrm{BiS})_{2}, \mathrm{Ni}_{3}(\mathrm{PbS})_{2}, \mathrm{Bi}_{2} \mathrm{Pd}_{3} \mathrm{~S}_{2}, \\
\mathrm{TaS}_{2}(\mathrm{ICSD}: 24757), \mathrm{HfNi}_{3}, \mathrm{Be}_{17} \mathrm{Nb}_{2}, \mathrm{Pr}_{2} \mathrm{Zn}_{17}, \mathrm{Nd}_{2} \mathrm{Zn}_{17}, \mathrm{La}_{2} \mathrm{Zn}_{17}, \\
\mathrm{Sm}_{2} \mathrm{Zn}_{17}, \mathrm{~Tb}_{2} \mathrm{Zn}_{17}, \mathrm{Y}_{2} \mathrm{Zn}_{17}, \mathrm{Dy}_{2} \mathrm{Zn}_{17}, \mathrm{Tm}_{2} \mathrm{Zn}_{17}, \mathrm{Lu}_{2} \mathrm{Zn}_{17}, \mathrm{Er}_{2} \mathrm{Zn}_{17} \\
\mathrm{Ho}_{2} \mathrm{Zn}_{17}, \mathrm{HoAl}_{3}, \mathrm{Th}_{2} \mathrm{Zn}_{17}, \mathrm{~K}_{2} \mathrm{~Pb}\left(\mathrm{SO}_{4}\right)_{2}(\mathrm{ICSD}: 29335), \mathrm{U}_{2} \mathrm{Zn}_{17}\end{array}$ \\
\hline & $\mathrm{T}_{3}^{+}(2)$ & $\begin{array}{c}\mathrm{Bi}_{2} \mathrm{TeI} 66, \| \mathrm{B}_{3} \mathrm{Mo}, \mathrm{Ca}_{2} \mathrm{GePd}_{3}, \mathrm{Dy}_{2} \mathrm{Al}_{6} \mathrm{Si}_{4} \mathrm{Pt}, \mathrm{Ni}_{3}(\mathrm{SnS})_{2}, \mathrm{TbAl}_{3} \\
\mathrm{YAl}_{3}, \mathrm{Ni}_{3}(\mathrm{PbSe})_{2}, \mathrm{YEr}_{4} \mathrm{Al}_{15}, \mathrm{ErAl}_{3}, \mathrm{HoGa}_{3}, \mathrm{Ca}\left(\mathrm{Ni}_{2} \mathrm{~B}\right)_{6}, \mathrm{ThFe}_{3} \\
\text { B(ICSD : 43431), } \mathrm{Nd}_{2} \mathrm{Fe}_{17}, \mathrm{Pr}_{2} \mathrm{Fe}_{17}, \mathrm{PuB}_{2} \mathrm{C}\end{array}$ \\
\hline & $\Gamma_{3}^{-}(2)$ & $\begin{array}{c}\mathrm{Al}_{8} \mathrm{C}_{3} \mathrm{~N}_{4}, \mathrm{CuI} 67, \mathrm{NaCd}_{4} \mathrm{As}_{3}, \| \mathrm{Ba}\left(\mathrm{Cu}_{2} \mathrm{As}_{2}, \mathrm{Sr}\left(\mathrm{Cu}_{2} \mathrm{As}\right)_{2}\right. \\
\mathrm{Ca}\left(\mathrm{Cu}_{2} \mathrm{As}\right)_{2}, \mathrm{~Pb}, \mathrm{Zr}_{2} \mathrm{Be}_{17}, \mathrm{Ti}_{2} \mathrm{Be}_{17}, \mathrm{Na}_{2} \mathrm{Li}_{5} \mathrm{Sn}_{4}, \mathrm{BaPb}_{3}, \mathrm{CsGa}_{7} \\
\mathrm{RbGa}, \mathrm{Nd}_{2} \mathrm{Zn}_{15} \mathrm{Ge}_{2}, \mathrm{Ba}_{2} \mathrm{Mg}_{17}, \mathrm{SrSn}_{3}\end{array}$ \\
\hline & $\Gamma_{3}^{+}(2)$ & $\begin{array}{c}\| \mathrm{Li}_{2} \mathrm{Si}, \mathrm{ErAl}_{4} \mathrm{Ge}_{2} \mathrm{Au}, \mathrm{GeP}_{3}, \mathrm{NdAl}_{4} \mathrm{Ge}_{2} \mathrm{Au}, \mathrm{B}_{2} \mathrm{Mo}, \mathrm{InAgSe}, \mathrm{SnP}_{3}, \\
\mathrm{WN}_{2}, \mathrm{Li}_{8} \mathrm{~Pb}_{3}, \mathrm{Tl}_{2} \mathrm{Pd}_{3} \mathrm{Se}_{2}, \mathrm{Li}_{4} \mathrm{ZrGe}_{2}, \mathrm{Ni}_{17} \mathrm{~S}_{18}, \mathrm{YCo}_{3}, \mathrm{HoCo}_{3}, \mathrm{ErCo}_{3}, \\
\mathrm{Ti}_{2} \mathrm{CuS}_{4}, \mathrm{Cs}_{2} \mathrm{NaVF}_{6}\end{array}$ \\
\hline & & Space Group 167: $R \overline{3} c$ \\
\hline & $\Gamma_{3}^{-}(2), \mathrm{L}_{1}(2), \mathrm{T}_{3}(2)$ & $\| \mathrm{BaPrO}_{3}$ \\
\hline & $\Gamma_{3}^{-}(2), \mathrm{L}_{1}(2), \mathrm{T}_{1} \mathrm{~T}_{2}(4)$ & $\mathrm{Zn}_{6} \mathrm{Sb}_{5}, \mathrm{Cd}_{6} \mathrm{Sb}_{5}$ \\
\hline & $\Gamma_{3}^{+}(2), \mathrm{L}_{1}(2), \mathrm{T}_{3}(2)$ & $\| \mathrm{Nb}_{2}\left(\mathrm{PO}_{4}\right)_{3}$ \\
\hline NSOC & $\mathrm{L}_{1}(2), \mathrm{T}_{3}(2)$ & $\begin{array}{c}\| \mathrm{HoAl}_{7} \mathrm{Au}_{3}, \mathrm{ErAl}_{7} \mathrm{Au}_{3}, \mathrm{DyAl}_{7} \mathrm{Au}_{3}, \mathrm{TbAl}_{7} \mathrm{Au}_{3}, \mathrm{TmAl}_{7} \mathrm{Au}_{3}, \\
\mathrm{SmAl}_{7} \mathrm{Au}_{3}, \mathrm{LuAl}_{7} \mathrm{Au}_{3}, \mathrm{NdAl}_{7} \mathrm{Au}_{3}, \mathrm{PrAl}_{7} \mathrm{Au}_{3}, \mathrm{Rb}_{8} \mathrm{Tl}_{11}, \mathrm{MgSi}_{7} \mathrm{Ir}_{3}, \\
\mathrm{~K}_{8} \mathrm{Tl}_{11}\end{array}$ \\
\hline & $\mathrm{L}_{1}(2), \mathrm{T}_{1} \mathrm{~T}_{2}(4)$ & $\begin{array}{c}\| \mathrm{Pd}_{3} \mathrm{~N}, \mathrm{Al}_{5} \mathrm{Mo}, \mathrm{LiOsO}_{3}, \mathrm{Sr}_{3} \mathrm{CdIrO}_{6}, \mathrm{Mg}_{13} \mathrm{Ir}_{3}, \mathrm{Ca}_{4} \mathrm{IrO}_{6}, \mathrm{Sr}_{4} \mathrm{IrO}_{6}, \\
\mathrm{Sr}_{3} \mathrm{ZnIrO}_{6}, \mathrm{Sr}_{3} \mathrm{MgIrO}_{6}, \mathrm{Sr}_{3} \mathrm{MgRhO}_{6}, \mathrm{Ba}_{14} \mathrm{Na}_{7} \mathrm{CaN}_{6}, \mathrm{Zr}_{21} \mathrm{Re}_{25}\end{array}$ \\
\hline & $\Gamma_{3}^{+}(2), \mathrm{T}_{1} \mathrm{~T}_{2}(4)$ & $\| \mathrm{NaSr}_{3} \mathrm{IrO}_{6}, \mathrm{Sr}_{3} \mathrm{LiIrO}_{6}, \mathrm{Ba}_{3} \mathrm{NaIrO}_{6}$ \\
\hline & $\mathrm{T}_{1} \mathrm{~T}_{2}(4)$ & $\begin{array}{c}\| \mathrm{LaCuO}_{3}, \mathrm{Ni}_{3} \mathrm{C}, \mathrm{K}_{2} \mathrm{Mog}_{9} \mathrm{~S}_{11}, \mathrm{Rb}_{2} \mathrm{Mo}_{9} \mathrm{~S}_{11}, \mathrm{Yb}_{8} \mathrm{In}_{3}, \mathrm{Na}_{8} \mathrm{Hg}_{3}, \\
\mathrm{Rb}_{2} \mathrm{Mo}_{15} \mathrm{~S}_{19}, \mathrm{Rb}_{4} \mathrm{Mo}_{21} \mathrm{~S}_{25}, \mathrm{~K}_{2} \mathrm{Mo}_{15} \mathrm{~S}_{19}, \mathrm{Cs}_{2} \mathrm{Mo}_{15} \mathrm{~S}_{19}\end{array}$ \\
\hline & $\Gamma_{3}^{-}(2)$ & $\| \mathrm{SiO}_{2}(\mathrm{ICSD}: 170515), \mathrm{YbAl}_{7} \mathrm{Au}_{3}$ \\
\hline & $\Gamma_{3}^{+}(2)$ & $\mathrm{SnHgO}_{3}, \mathrm{Ag}_{5} \mathrm{IO}_{6}, \| \mathrm{Ti}_{2} \mathrm{O}_{3}, \mathrm{Hf}\left(\mathrm{TeCl}_{6}\right)_{2}, \mathrm{Al}_{3} \mathrm{Au}_{8}, \mathrm{~V}_{2} \mathrm{O}_{3}, \mathrm{Cr}_{2} \mathrm{O}_{3}$ \\
\hline
\end{tabular}


Table II - continued

\begin{tabular}{|c|c|c|}
\hline $\begin{array}{l}\text { SOC or } \\
\text { NSOC }\end{array}$ & $\begin{array}{l}\text { Degenerate Irreducible } \\
\text { Representations }\end{array}$ & High Symmetry Point Semimetals \\
\hline \multirow[t]{2}{*}{$\mathrm{SOC}$} & $\overline{\mathrm{L}}_{2} \overline{\mathrm{L}}_{2}(4), \overline{\mathrm{T}}_{5} \overline{\mathrm{T}}_{6}(4)$ & $\begin{array}{c}\| \mathrm{Pd}_{3} \mathrm{~N}, \mathrm{HoAl}_{7} \mathrm{Au}_{3}, \mathrm{TmAl}_{7} \mathrm{Au}_{3}, \mathrm{LuAl}_{7} \mathrm{Au}_{3}, \mathrm{ErAl}_{7} \mathrm{Au}_{3}, \mathrm{DyAl}_{7} \mathrm{Au}_{3}, \\
\mathrm{TbAl}_{7} \mathrm{Au}_{3}, \mathrm{SmAl}_{7} \mathrm{Au}_{3}, \mathrm{NdAl}_{7} \mathrm{Au}_{3}, \mathrm{PrAl}_{7} \mathrm{Au}_{3}, \mathrm{BaPrO}_{3}, \mathrm{MgSi}_{7} \mathrm{Ir}_{3}, \\
\mathrm{Ca}_{4} \mathrm{IrO}_{6}, \mathrm{Sr}_{3} \mathrm{MgIrO}_{6}, \mathrm{~K}_{8} \mathrm{Tl}_{11}, \mathrm{Mg}_{13} \mathrm{Ir}_{3}, \mathrm{Sr}_{3} \mathrm{MgRhO}_{6}, \mathrm{Zr}_{21} \mathrm{Re}_{25}, \\
\mathrm{Sr}_{3} \mathrm{CdIrO}_{6}, \mathrm{Sr}_{3} \mathrm{ZnIrO}_{6}, \mathrm{Sr}_{4} \mathrm{IrO}_{6}, \mathrm{Rb}_{8} \mathrm{Tl}_{11}, \mathrm{Nb}_{2}\left(\mathrm{PO}_{4}\right)_{3}\end{array}$ \\
\hline & $\overline{\mathrm{L}}_{2} \overline{\mathrm{L}}_{2}(4), \overline{\mathrm{T}}_{4} \overline{\mathrm{T}}_{4}(4)$ & $\| \mathrm{Al}_{5} \mathrm{Mo}, \mathrm{Zn}_{6} \mathrm{Sb}_{5}, \mathrm{Cd}_{6} \mathrm{Sb}_{5}, \mathrm{Ba}_{14} \mathrm{Na}_{7} \mathrm{CaN}_{6}, \mathrm{LiOsO}_{3}$ \\
\hline \multicolumn{3}{|r|}{ Space Group 174: $P \overline{6}$} \\
\hline NSOC & $\Gamma_{3} \Gamma_{5}(2)$ & $\| \mathrm{La}_{2} \mathrm{~B}_{3} \mathrm{Cl}$ \\
\hline \multicolumn{3}{|r|}{ Space Group 175: $P 6 / m$} \\
\hline NSOC & $\Gamma_{3}^{+} \Gamma_{5}^{+}(2)$ & $\mathrm{ErNi}_{3} \mathrm{Ge}_{2}$ \\
\hline \multicolumn{3}{|r|}{ Space Group 176: $P 6_{3} / m$} \\
\hline \multirow{24}{*}{ NSOC } & $\begin{array}{c}\mathrm{A}_{2} \mathrm{~A}_{3}(4), \Gamma_{4}^{+} \Gamma_{6}^{+}(2) \\
\mathrm{H}_{4} \mathrm{H}_{5}(2), \mathrm{K}_{3} \mathrm{~K}_{5}(2), \mathrm{L}_{1}(2)\end{array}$ & $\| \mathrm{Rb}_{5} \mathrm{Mo}_{27} \mathrm{Se}_{31}$ \\
\hline & $\begin{array}{c}\mathrm{A}_{2} \mathrm{~A}_{3}(4), \Gamma_{3}^{-} \Gamma_{5}^{-}(2) \\
\mathrm{H}_{4} \mathrm{H}_{5}(2), \mathrm{K}_{3} \mathrm{~K}_{5}(2), \mathrm{L}_{1}(2) \\
\end{array}$ & $\| \mathrm{Cs}_{3} \mathrm{Te}_{17} \mathrm{Mo}_{15}, \mathrm{Cs}_{3} \mathrm{Mo}_{15} \mathrm{Se}_{17}$ \\
\hline & $\begin{array}{c}\mathrm{A}_{2} \mathrm{~A}_{3}(4), \Gamma_{3}^{-} \Gamma_{5}^{-}(2) \\
\mathrm{H}_{3} \mathrm{H}_{6}(2), \mathrm{K}_{3} \mathrm{~K}_{5}(2), \mathrm{L}_{1}(2)\end{array}$ & $\| \mathrm{Rb}_{3} \mathrm{Mo}_{15} \mathrm{Se}_{17}$ \\
\hline & $\begin{array}{c}\mathrm{A}_{1}(2), \Gamma_{4}^{+} \Gamma_{6}^{+}(2), \mathrm{H}_{1} \mathrm{H}_{2}(2) \\
\mathrm{K}_{4} \mathrm{~K}_{6}(2), \mathrm{L}_{1}(2)\end{array}$ & $\| \mathrm{V}_{3} \mathrm{~S}_{4}$ \\
\hline & $\begin{array}{c}\mathrm{A}_{2} \mathrm{~A}_{3}(4), \mathrm{H}_{4} \mathrm{H}_{5}(2) \\
\mathrm{K}_{4} \mathrm{~K}_{6}(2), \mathrm{L}_{1}(2)\end{array}$ & $\| \mathrm{Ba}_{3} \mathrm{FeN}_{3}$ \\
\hline & $\begin{array}{c}\mathrm{A}_{2} \mathrm{~A}_{3}(4), \mathrm{H}_{4} \mathrm{H}_{5}(2), \\
\mathrm{K}_{3} \mathrm{~K}_{5}(2), \mathrm{L}_{1}(2)\end{array}$ & $\| \mathrm{Sm}_{5} \mathrm{Si}_{3} \mathrm{O}_{13}$ \\
\hline & $\begin{array}{c}\mathrm{A}_{2} \mathrm{~A}_{3}(4), \mathrm{H}_{1} \mathrm{H}_{2}(2), \\
\mathrm{K}_{4} \mathrm{~K}_{6}(2), \mathrm{L}_{1}(2)\end{array}$ & $\| \mathrm{Nb}_{3} \mathrm{Se}_{4}, \mathrm{Nb}_{3} \mathrm{Te}_{4}$ \\
\hline & $\begin{array}{c}\mathrm{A}_{2} \mathrm{~A}_{3}(4), \Gamma_{4}^{-} \Gamma_{6}^{-}(2), \\
\mathrm{H}_{3} \mathrm{H}_{6}(2), \mathrm{L}_{1}(2)\end{array}$ & $\| \operatorname{Pr}_{15} \mathrm{Si}_{10} \mathrm{Ni}_{7}$ \\
\hline & $\begin{array}{c}\mathrm{A}_{2} \mathrm{~A}_{3}(4), \Gamma_{4}^{+} \Gamma_{6}^{+}(2) \\
\mathrm{H}_{1} \mathrm{H}_{2}(2), \mathrm{L}_{1}(2) \\
\end{array}$ & $\| \mathrm{Nb}_{3} \mathrm{~S}_{4}$ \\
\hline & $\begin{array}{l}\mathrm{A}_{1}(2), \mathrm{H}_{1} \mathrm{H}_{2}(2), \mathrm{K}_{3} \mathrm{~K}_{5}(2) \\
\mathrm{L}_{1}(2)\end{array}$ & $\| \mathrm{HfB}_{4} \mathrm{Ir}_{3}, \mathrm{In}_{3} \mathrm{Au}_{10}$ \\
\hline & $\mathrm{A}_{2} \mathrm{~A}_{3}(4), \mathrm{H}_{4} \mathrm{H}_{5}(2), \mathrm{L}_{1}(2)$ & $\| \mathrm{Tb}_{3}\left(\mathrm{MnC}_{3}\right)_{2}$ \\
\hline & $\mathrm{A}_{2} \mathrm{~A}_{3}(4), \mathrm{H}_{3} \mathrm{H}_{6}(2), \mathrm{L}_{1}(2)$ & $\| \mathrm{SmSi}_{3} \mathrm{Pt}_{5}$ \\
\hline & $\mathrm{A}_{2} \mathrm{~A}_{3}(4), \mathrm{H}_{1} \mathrm{H}_{2}(2), \mathrm{L}_{1}(2)$ & $\| \mathrm{Sr}_{3} \mathrm{CrN}_{3}, \mathrm{Er}_{3} \mathrm{Ru}_{2}$ \\
\hline & $\mathrm{A}_{1}(2), \mathrm{H}_{3} \mathrm{H}_{6}(2), \mathrm{L}_{1}(2)$ & $\| \mathrm{Cu}_{10} \mathrm{Sb}_{3}$ \\
\hline & $\mathrm{A}_{1}(2), \mathrm{H}_{1} \mathrm{H}_{2}(2), \mathrm{L}_{1}(2)$ & $\begin{array}{l}\mathrm{K}(\mathrm{MoS})_{3}\left[68, \mathrm{Rb}(\mathrm{MoS})_{3}\left[68, \mathrm{Cs}(\mathrm{MoS})_{3}, \mathrm{Tl}(\mathrm{MoSe})_{3}\left[68, \mathrm{Zr}_{3} \mathrm{Cd}_{3} \mathrm{AgF}_{20},\right.\right.\right. \\
\quad \operatorname{In}(\mathrm{MoSe})_{3}\left[68, \operatorname{In}(\mathrm{TeMo})_{3}[68], \mathrm{Tl}(\mathrm{TeMo})_{3}\left[\underline{68}, \mathrm{Ba}_{3} \mathrm{CrN}_{3} \|\right.\right.\end{array}$ \\
\hline & $\Gamma_{4}^{+} \Gamma_{6}^{+}(2), \mathrm{K}_{4} \mathrm{~K}_{6}(2)$ & $\| \mathrm{Nb}_{3} \mathrm{Te}_{3} \mathrm{As}$ \\
\hline & $\Gamma_{3}^{+} \Gamma_{5}^{+}(2), \mathrm{K}_{3} \mathrm{~K}_{5}(2)$ & $\| \mathrm{Sn}_{4} \mathrm{~B}_{3} \mathrm{Ir}_{7}$ \\
\hline & $\mathrm{A}_{2} \mathrm{~A}_{3}(4), \mathrm{K}_{3} \mathrm{~K}_{5}(2)$ & $\pi \mathrm{Mg}_{3} \mathrm{Ge}, \mathrm{Mg}_{3} \mathrm{Sn}$ \\
\hline & $\mathrm{A}_{2} \mathrm{~A}_{3}(4), \Gamma_{4}^{+} \Gamma_{6}^{+}(2)$ & $\| \mathrm{Ba}_{3} \mathrm{MnN}_{3}$ \\
\hline & $\mathrm{A}_{2} \mathrm{~A}_{3}(4), \Gamma_{3}^{-} \Gamma_{5}^{-}(2)$ & $\| \mathrm{Sr}_{3} \mathrm{MnN}_{3}$ \\
\hline & $\mathrm{K}_{3} \mathrm{~K}_{5}(2)$ & $\| \mathrm{Ti}_{3} \mathrm{Se}_{4}, \mathrm{U}_{5} \mathrm{Re}_{5} \mathrm{Si}_{9}$ \\
\hline & $\Gamma_{4}^{+} \Gamma_{6}^{+}(2)$ & $\| \mathrm{LiCaAl}_{3}$ \\
\hline & $\Gamma_{3}^{-} \Gamma_{5}^{-}(2)$ & $\mathrm{Na}_{2} \mathrm{Zn}_{2}\left(\mathrm{TeO}_{3}\right)_{3}$ \\
\hline & $\mathrm{A}_{2} \mathrm{~A}_{3}(4)$ & $\| \mathrm{SmSi}_{3} \mathrm{Rh}_{5}, \mathrm{HoSi}_{3} \mathrm{Rh}_{5}, \mathrm{TmSi}_{3} \mathrm{Rh}_{5}$ \\
\hline \multirow[t]{3}{*}{$\mathrm{SOC}$} & $\overline{\mathrm{A}}_{5} \overline{\mathrm{A}}_{6}(4), \overline{\mathrm{L}}_{2} \overline{\mathrm{L}}_{2}(4)$ & $\begin{array}{c}\mathrm{K}(\mathrm{MoS})_{3}[6], \mathrm{Rb}(\mathrm{MoS})_{3}\left[68, \mathrm{Cs}(\mathrm{MoS})_{3}, \mathrm{Zr}_{3} \mathrm{Cd}_{3} \mathrm{AgF}_{20}, \mathrm{Tl}(\mathrm{MoSe})_{3}[68,\right. \\
\mathrm{In}(\mathrm{MoSe})_{3}\left[68, \mathrm{In}(\mathrm{TeMo})_{3}\left[68, \mathrm{Tl}(\mathrm{TeM})_{3}\left[68, \mathrm{Ba}_{3} \mathrm{CrN}_{3}, \| \mathrm{HfB}_{4} \mathrm{Ir}_{3},\right.\right.\right. \\
\mathrm{Cu}_{10} \mathrm{Sb}_{3}, \mathrm{~V}_{3} \mathrm{~S}_{4}, \mathrm{Er}_{3} \mathrm{Ru}_{2}, \mathrm{In}_{3} \mathrm{Au}_{10}, \mathrm{Nb}_{3} \mathrm{Se}_{4}, \mathrm{Nb}_{3} \mathrm{Te}_{4}, \mathrm{Rb}_{5} \mathrm{Mo}_{27} \mathrm{Se}_{31}, \\
\mathrm{Cs}_{3} \mathrm{Te}_{17} \mathrm{Mo}_{15}, \mathrm{Rb}_{3} \mathrm{Mo}_{15} \mathrm{Se}_{17}, \mathrm{Cs}_{3} \mathrm{Mo}_{15} \mathrm{Se}_{17}\end{array}$ \\
\hline & $\overline{\mathrm{A}}_{4} \overline{\mathrm{A}}_{4}(4), \overline{\mathrm{L}}_{2} \overline{\mathrm{L}}_{2}(4)$ & $\| \mathrm{Sm}_{5} \mathrm{Si}_{3} \mathrm{O}_{13}, \mathrm{Nb}_{3} \mathrm{~S}_{4}, \mathrm{Ba}_{3} \mathrm{FeN}_{3}, \mathrm{SmSi}_{3} \mathrm{Pt}_{5}, \mathrm{Pr}_{15} \mathrm{Si}_{10} \mathrm{Ni}_{7}$ \\
\hline & $\overline{\mathrm{H}}_{9} \overline{\mathrm{H}}_{11}(2)$ & $\| \mathrm{Tb}_{15}\left(\mathrm{Ni}_{4} \mathrm{P}_{3}\right)_{7}$ \\
\hline \multicolumn{3}{|r|}{ Space Group 178: $P 6_{1} 22$} \\
\hline \multirow[t]{2}{*}{ NSOC } & $\begin{array}{c}\mathrm{A}_{2}(2), \Gamma_{6}(2), \mathrm{H}_{1} \mathrm{H}_{2}(2), \\
\mathrm{L}_{1}(2)\end{array}$ & $\| \mathrm{Sc}$ \\
\hline & $\mathrm{A}_{1}(2), \mathrm{H}_{1} \mathrm{H}_{2}(2), \mathrm{L}_{1}(2)$ & $\| \mathrm{Zr}_{5} \operatorname{Ir}_{3}$ \\
\hline \multicolumn{3}{|r|}{ Space Group 180: $P 6_{2} 22$} \\
\hline & $\mathrm{A}_{5}(2), \Gamma_{6}(2)$ & $\| \mathrm{NdBPt}_{2}$ \\
\hline
\end{tabular}


Table II - continued

\begin{tabular}{|c|c|c|}
\hline $\begin{array}{l}\text { SOC or } \\
\text { NSOC }\end{array}$ & $\begin{array}{c}\text { Degenerate Irreducible } \\
\text { Representations }\end{array}$ & High Symmetry Point Semimetals \\
\hline \multirow[b]{4}{*}{$\mathrm{SOC}$} & $\Gamma_{6}(2)$ & $\mathrm{LuBPt}_{2}, \mathrm{YBPt}_{2}, \mathrm{HfSn}_{2}$ \\
\hline & $\Gamma_{5}(2)$ & $\| \mathrm{LaBPt}_{2}$ \\
\hline & $\mathrm{A}_{5}(2)$ & || MgBIr \\
\hline & $\overline{\mathrm{K}}_{6}(2)$ & $\mathrm{Sn}_{2} \mathrm{Mo}$ \\
\hline \multicolumn{3}{|r|}{ Space Group 181: $P 6_{4} 22$} \\
\hline \multirow{2}{*}{ NSOC } & $\Gamma_{5}(2)$ & $\mathrm{MgNi}_{3} \mathrm{~B}_{2}$ \\
\hline & $\mathrm{A}_{6}(2)$ & $\| \mathrm{Al}_{2} \mathrm{~W}$ \\
\hline \multicolumn{3}{|r|}{ Space Group 182: $P 6_{3} 22$} \\
\hline \multirow{9}{*}{ NSOC } & $\begin{array}{c}\mathrm{A}_{3}(2), \Gamma_{5}(2), \mathrm{H}_{3}(2), \mathrm{K}_{3}(2), \\
\mathrm{L}_{1}(2)\end{array}$ & $\| \mathrm{Nb}_{3} \mathrm{FeS}_{6}$ \\
\hline & $\begin{array}{c}\mathrm{A}_{1}(2), \Gamma_{6}(2), \mathrm{H}_{3}(2), \mathrm{K}_{3}(2), \\
\mathrm{L}_{1}(2)\end{array}$ & $\| \mathrm{Al}_{5} \mathrm{~W}, \mathrm{Al}_{5} \mathrm{Mo}$ \\
\hline & $\begin{array}{c}\mathrm{A}_{2}(2), \mathrm{H}_{1} \mathrm{H}_{2}(2), \mathrm{K}_{3}(2) \\
\mathrm{L}_{1}(2)\end{array}$ & $\| \mathrm{ReO}_{3}$ \\
\hline & $\mathrm{A}_{1}(2), \mathrm{H}_{3}(2), \mathrm{K}_{3}(2), \mathrm{L}_{1}(2)$ & $\pi \mathrm{Nb}_{3} \mathrm{SnS}_{6}$ \\
\hline & $\mathrm{A}_{1}(2), \mathrm{H}_{3}(2), \mathrm{L}_{1}(2)$ & $\| \mathrm{Pd}_{3} \mathrm{~N}, \mathrm{Ni}_{3} \mathrm{~N}$ \\
\hline & $\mathrm{A}_{1}(2), \mathrm{H}_{1} \mathrm{H}_{2}(2), \mathrm{L}_{1}(2)$ & $\| \mathrm{LiSmAlF}_{6}$ \\
\hline & $\Gamma_{5}(2), \mathrm{K}_{3}(2)$ & $\| \mathrm{Nb}_{3} \mathrm{VS}_{6}$ \\
\hline & $\mathrm{K}_{3}(2)$ & $\| \mathrm{RbPbIO}_{6}$ \\
\hline & $\Gamma_{6}(2)$ & $\mathrm{NaCu}_{5} \mathrm{~S}_{3}, \mathrm{Zr}_{3} \mathrm{O}$ \\
\hline $\mathrm{SOC}$ & $\overline{\mathrm{K}_{6}(2)}$ & $\| \mathrm{Nb}_{3} \mathrm{SnS}_{6}$ \\
\hline \multicolumn{3}{|r|}{ Space Group 183: $P 6 \mathrm{~mm}$} \\
\hline NSOC & $\mathrm{A}_{5}(2)$ & $\| \mathrm{Ta}_{21} \mathrm{Te}_{13}$ \\
\hline \multicolumn{3}{|r|}{ Space Group 185: $P 6_{3} \mathrm{~cm}$} \\
\hline \multirow{6}{*}{ NSOC } & $\begin{array}{c}\mathrm{A}_{2} \mathrm{~A}_{4}(2), \mathrm{H}_{3} \mathrm{H}_{3}(4), \mathrm{K}_{3}(2) \\
\mathrm{L}_{1} \mathrm{~L}_{3}(2)\end{array}$ & $\| \mathrm{Ba}_{3} \mathrm{Yb}\left(\mathrm{BO}_{3}\right)_{3}$ \\
\hline & $\mathrm{A}_{2} \mathrm{~A}_{4}(2), \mathrm{H}_{3} \mathrm{H}_{3}(4), \mathrm{L}_{1} \mathrm{~L}_{3}(2)$ & $\| \mathrm{Mg}_{3} \mathrm{Ir}$ \\
\hline & $\mathrm{A}_{1} \mathrm{~A}_{3}(2), \mathrm{H}_{3} \mathrm{H}_{3}(4), \mathrm{L}_{2} \mathrm{~L}_{4}(2)$ & $\| \mathrm{Cd}_{3} \mathrm{Au}, \mathrm{Mg}_{3} \mathrm{Au}, \mathrm{Mg}_{3} \mathrm{Rh}$ \\
\hline & $\mathrm{A}_{5} \mathrm{~A}_{6}(4), \Gamma_{6}(2)$ & $\| \mathrm{Mg}_{3} \mathrm{Pt}, \mathrm{Mg}_{3} \mathrm{Pd}$ \\
\hline & $\Gamma_{6}(2)$ & $\| \mathrm{RbTiI}_{3}$ \\
\hline & $\Gamma_{5}(2)$ & $\mathrm{Cu}_{3} \mathrm{P}, \mathrm{Yb}_{2} \mathrm{~S}_{3}$ \\
\hline \multirow{3}{*}{$\mathrm{SOC}$} & $\overline{\mathrm{A}}_{8} \overline{\mathrm{A}}_{9}(4), \overline{\mathrm{K}}_{6}(2), \overline{\mathrm{L}}_{5} \overline{\mathrm{L}}_{5}(4)$ & $\mathrm{Ba}_{3} \mathrm{Yb}\left(\mathrm{BO}_{3}\right)_{3}$ \\
\hline & $\overline{\mathrm{A}}_{8} \overline{\mathrm{A}}_{9}(4), \overline{\mathrm{H}}_{6} \overline{\mathrm{H}}_{6}(4), \overline{\mathrm{L}}_{5} \overline{\mathrm{L}}_{5}(4)$ & $\| \mathrm{Cd}_{3} \mathrm{Au}$ \\
\hline & $\overline{\mathrm{A}}_{8} \overline{\mathrm{A}}_{9}(4), \overline{\mathrm{L}}_{5} \overline{\mathrm{L}}_{5}(4)$ & $\| \mathrm{Mg}_{3} \mathrm{Au}, \mathrm{Mg}_{3} \mathrm{Ir}, \mathrm{Mg}_{3} \mathrm{Rh}$ \\
\hline \multicolumn{3}{|r|}{ Space Group 186: $P 6_{3} m c$} \\
\hline \multirow{12}{*}{ NSOC } & $\begin{array}{c}\mathrm{A}_{5} \mathrm{~A}_{6}(4), \Gamma_{6}(2), \mathrm{H}_{3}(2), \\
\mathrm{K}_{3}(2), \mathrm{L}_{2} \mathrm{~L}_{3}(2)\end{array}$ & $\| \mathrm{Y}_{3}\left(\mathrm{Co}_{2} \mathrm{Sn}\right)_{4}$ \\
\hline & $\begin{array}{c}\mathrm{A}_{5} \mathrm{~A}_{6}(4), \Gamma_{6}(2), \mathrm{H}_{3}(2), \\
\mathrm{K}_{3}(2), \mathrm{L}_{1} \mathrm{~L}_{4}(2)\end{array}$ & || YbCuGe \\
\hline & $\begin{array}{c}\mathrm{A}_{5} \mathrm{~A}_{6}(4), \mathrm{H}_{3}(2), \mathrm{K}_{3}(2) \\
\mathrm{L}_{1} \mathrm{~L}_{4}(2)\end{array}$ & |l MoN(ICSD : 168369) \\
\hline & $\begin{array}{c}\mathrm{A}_{5} \mathrm{~A}_{6}(4), \mathrm{H}_{1} \mathrm{H}_{2}(2), \mathrm{K}_{3}(2) \\
\mathrm{L}_{1} \mathrm{~L}_{4}(2)\end{array}$ & $\| \mathrm{B}_{3} \mathrm{Ru}_{7}$ \\
\hline & $\begin{array}{c}\mathrm{A}_{5} \mathrm{~A}_{6}(4), \Gamma_{6}(2), \mathrm{H}_{3}(2) \\
\mathrm{L}_{1} \mathrm{~L}_{4}(2)\end{array}$ & || TiCuSn \\
\hline & $\begin{array}{c}\mathrm{A}_{5} \mathrm{~A}_{6}(4), \Gamma_{5}(2), \mathrm{H}_{3}(2) \\
\mathrm{L}_{1} \mathrm{~L}_{4}(2)\end{array}$ & $\| \mathrm{MnWN}_{2}$ \\
\hline & $\begin{array}{c}\mathrm{A}_{5} \mathrm{~A}_{6}(4), \Gamma_{5}(2), \mathrm{H}_{1} \mathrm{H}_{2}(2) \\
\mathrm{L}_{2} \mathrm{~L}_{3}(2)\end{array}$ & $\| \mathrm{CrH}$ \\
\hline & $\begin{array}{c}\mathrm{A}_{5} \mathrm{~A}_{6}(4), \Gamma_{5}(2), \mathrm{H}_{1} \mathrm{H}_{2}(2), \\
\mathrm{L}_{1} \mathrm{~L}_{4}(2)\end{array}$ & $\| \mathrm{CoO}$ \\
\hline & $\begin{array}{c}\mathrm{A}_{2} \mathrm{~A}_{3}(2), \mathrm{H}_{1} \mathrm{H}_{2}(2), \mathrm{K}_{3}(2) \\
\mathrm{L}_{2} \mathrm{~L}_{3}(2)\end{array}$ & $\| \mathrm{La}_{7} \mathrm{Ni}_{3}$ \\
\hline & $\begin{array}{c}\mathrm{A}_{1} \mathrm{~A}_{4}(2), \mathrm{H}_{3}(2), \mathrm{K}_{3}(2) \\
\mathrm{L}_{1} \mathrm{~L}_{4}(2)\end{array}$ & $\| \mathrm{Si}_{3} \mathrm{Ir}, \mathrm{CeCuSn}$ \\
\hline & $\mathrm{A}_{5} \mathrm{~A}_{6}(4), \mathrm{H}_{3}(2), \mathrm{L}_{2} \mathrm{~L}_{3}(2)$ & $\| \mathrm{Th}_{7} \mathrm{Rh}_{3}, \mathrm{Th}_{7} \mathrm{Ir}_{3}, \mathrm{Th}_{7} \mathrm{Co}_{3}$ \\
\hline & $\mathrm{A}_{5} \mathrm{~A}_{6}(4), \mathrm{H}_{3}(2), \mathrm{L}_{1} \mathrm{~L}_{4}(2)$ & $\mathrm{HfCuSn}, \mathrm{Yb}_{7} \mathrm{Au}_{3}, \mathrm{ZnBi}, \mathrm{Sr}_{7} \mathrm{Au}_{3}$ \\
\hline
\end{tabular}


Table II - continued

\begin{tabular}{|c|c|c|}
\hline $\begin{array}{l}\text { SOC or } \\
\text { NSOC }\end{array}$ & $\begin{array}{c}\text { Degenerate Irreducible } \\
\text { Representations }\end{array}$ & High Symmetry Point Semimetals \\
\hline & $\mathrm{A}_{5} \mathrm{~A}_{6}(4), \mathrm{H}_{1} \mathrm{H}_{2}(2), \mathrm{L}_{2} \mathrm{~L}_{3}(2)$ & $\| \mathrm{PdN}$ \\
\hline & $\mathrm{A}_{5} \mathrm{~A}_{6}(4), \mathrm{H}_{1} \mathrm{H}_{2}(2), \mathrm{L}_{1} \mathrm{~L}_{4}(2)$ & TbSe, SmSe \\
\hline & $\mathrm{A}_{2} \mathrm{~A}_{3}(2), \mathrm{H}_{3}(2), \mathrm{L}_{1} \mathrm{~L}_{4}(2)$ & $\| \mathrm{La}_{23} \mathrm{Cd}_{4} \mathrm{Pt}_{7}$ \\
\hline & $\mathrm{A}_{2} \mathrm{~A}_{3}(2), \mathrm{H}_{1} \mathrm{H}_{2}(2), \mathrm{L}_{2} \mathrm{~L}_{3}(2)$ & $\| \mathrm{SrYbSi}_{4} \mathrm{~N}_{7}, \mathrm{BaYbSi}_{4} \mathrm{~N}_{7}$ \\
\hline & $\mathrm{A}_{1} \mathrm{~A}_{4}(2), \mathrm{H}_{3}(2), \mathrm{L}_{2} \mathrm{~L}_{3}(2)$ & MnS(ICSD : 44765), MnS(ICSD : 643455) \\
\hline & $\mathrm{A}_{1} \mathrm{~A}_{4}(2), \mathrm{H}_{1} \mathrm{H}_{2}(2), \mathrm{L}_{1} \mathrm{~L}_{4}(2)$ & || $\mathrm{ZrN}$ \\
\hline & $\Gamma_{5}(2), K_{3}(2)$ & || ErGeAu \\
\hline & $\mathrm{A}_{5} \mathrm{~A}_{6}(4), \mathrm{K}_{3}(2)$ & $\mathrm{LiSn}_{4} \mathrm{Au}_{3}, \mathrm{Ca}_{3}\left(\mathrm{InNi}_{2}\right)_{4}$ \\
\hline & $\mathrm{A}_{5} \mathrm{~A}_{6}(4), \Gamma_{6}(2)$ & $\| \mathrm{Tc}_{7} \mathrm{~B}_{3}$ \\
\hline & $\mathrm{K}_{3}(2)$ & Ge, $\|$ MoN(ICSD : 99453), $\mathrm{Ni}_{5} \mathrm{P}_{4}, \mathrm{Th}_{7} \mathrm{Fe}_{3}$ \\
\hline & $\Gamma_{6}(2)$ & $\begin{array}{c}\text { InSb, InN, CdO, TlN, YbAgBi, CaAgBi } 69], \mathrm{CaBiAu}, \mathrm{SrSnHg}, \| \\
\text { CaZnSn, YbZnSn, YbSnHg, LaSnAu, SmAsPd, } \mathrm{NdAsPd}, \mathrm{Al}_{7}(\mathrm{CN})_{3}, \\
\mathrm{Pr}_{8} \mathrm{Ga}_{3} \mathrm{Co}, \mathrm{Ca}_{5} \mathrm{~Pb}_{3}, \mathrm{Th}_{7} \mathrm{Pt}_{3}\end{array}$ \\
\hline & $\Gamma_{5}(2)$ & $\begin{array}{c}\text { \| ErSnAu, TmSnAu, TbSnAu, DySnAu, NdSnAu, PrSnAu, Er } \mathrm{Er}_{7} \mathrm{Rh}_{3}, \\
\mathrm{Th}_{7} \mathrm{Ru}_{3}, \mathrm{Th}_{7} \mathrm{Os}_{3}\end{array}$ \\
\hline & $\mathrm{A}_{5} \mathrm{~A}_{6}(4)$ & $\mathrm{Zn}\left(\mathrm{InS}_{2}\right)_{2}, \| \mathrm{NbN}, \mathrm{Zn}_{2} \mathrm{In}_{2} \mathrm{~S}_{5}, \mathrm{Sm}_{4} \mathrm{I}_{6} \mathrm{O}, \mathrm{Sm}_{4} \mathrm{Br}_{6} \mathrm{O}, \mathrm{Nd}_{7} \mathrm{Co}_{3}, \mathrm{LuMn}_{5}$ \\
\hline \multirow{5}{*}{$\mathrm{SOC}$} & $\overline{\mathrm{A}}_{8} \overline{\mathrm{A}}_{9}(4), \overline{\mathrm{K}}_{6}(2), \overline{\mathrm{L}}_{5} \overline{\mathrm{L}}_{5}(4)$ & $\| \mathrm{Si}_{3} \mathrm{Ir}, \mathrm{La}_{7} \mathrm{Ni}_{3}, \mathrm{CeCuSn}$ \\
\hline & $\overline{\mathrm{A}}_{7} \overline{\mathrm{A}}_{7}(4), \overline{\mathrm{K}}_{6}(2), \overline{\mathrm{L}}_{5} \overline{\mathrm{L}}_{5}(4)$ & \begin{tabular}{l||l} 
& MoN(ICSD : 168369) \\
\end{tabular} \\
\hline & $\overline{\mathrm{A}}_{8} \overline{\mathrm{A}}_{9}(4), \overline{\mathrm{L}}_{5} \overline{\mathrm{L}}_{5}(4)$ & $\begin{array}{l}\| \mathrm{HfCuSn}, \mathrm{ZrN}, \mathrm{CrH}, \mathrm{TbSe}, \mathrm{SmSe}, \mathrm{SrYbSi}_{4} \mathrm{~N}_{7}, \mathrm{BaYbSi}_{4} \mathrm{~N}_{7}, \mathrm{Yb}_{7} \mathrm{Au}_{3}, \\
\mathrm{MnWN}_{2}, \mathrm{MnS}\left(\mathrm{ICSD}: \text { 643455), MnS(ICSD : 44765), } \mathrm{Th}_{7} \mathrm{Co}_{3}\right.\end{array}$ \\
\hline & $\overline{\mathrm{A}}_{7} \overline{\mathrm{A}}_{7}(4), \overline{\mathrm{L}}_{5} \overline{\mathrm{L}}_{5}(4)$ & $\begin{array}{c}\| \mathrm{PdN}, \mathrm{TiCuSn}, \mathrm{ZnBi}, \mathrm{CoO}, \mathrm{YbCuGe} \mathrm{Sr}_{7} \mathrm{Au}_{3}, \mathrm{Th}_{7} \mathrm{Rh}_{3}, \mathrm{Th}_{7} \mathrm{Ir}_{3} \\
\mathrm{~B}_{3} \mathrm{Ru}_{7}\end{array}$ \\
\hline & $\overline{\mathrm{K}_{6}(2)}$ & 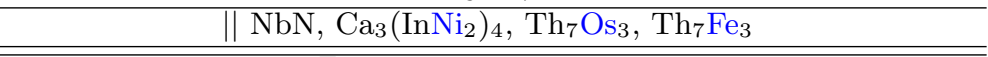 \\
\hline \multicolumn{3}{|r|}{ Space Group 187: $P \overline{6} m 2$} \\
\hline \multirow{7}{*}{ NSOC } & $\mathrm{A}_{6}(2), \Gamma_{6}(2)$ & $\mathrm{TcB}$ \\
\hline & $\mathrm{A}_{6}(2), \Gamma_{5}(2)$ & $\| \mathrm{AgN}$ \\
\hline & $\mathrm{A}_{5}(2), \Gamma_{5}(2)$ & $\prod$ LiNiN, $\mathrm{La}_{2} \mathrm{~B}_{3} \mathrm{Br}$ \\
\hline & $\Gamma_{6}(2)$ & $\|$ OsC, TcN, ZrTaNO \\
\hline & $\Gamma_{5}(2)$ & $\|$ LuPPt, TmPPt, YPPt, DyPPt, TbPPt, SmPPt \\
\hline & $\mathrm{A}_{6}(2)$ & $\begin{array}{c}\text { TaN [0], } \mathrm{NbN}, \| \mathrm{Re}_{3} \mathrm{~N}, \mathrm{Mg}\left(\mathrm{As}_{2} \mathrm{Rh}_{3}\right)_{2}, \mathrm{Mg}\left(\mathrm{P}_{2} \mathrm{Rh}_{3}\right)_{2}, \mathrm{Yb}\left(\mathrm{As}_{2} \mathrm{Rh}_{3}\right)_{2}, \\
\mathrm{Ca}\left(\mathrm{As}_{2} \mathrm{Rh}_{3}\right)_{2}, \operatorname{Sr}\left(\mathrm{As}_{2} \mathrm{Rh}_{3}\right)_{2}, \mathrm{Mg}\left(\mathrm{Co}_{3} \mathrm{P}_{2}\right)_{2}\end{array}$ \\
\hline & $\mathrm{A}_{5}(2)$ & ZrTe[71], VN, TiS[72], WC, MoC[73], ||$C$ CrC \\
\hline \multicolumn{3}{|r|}{ Space Group 188: $P \overline{6} c 2$} \\
\hline NSOC & $\mathrm{A}_{1}(2), \mathrm{H}_{1} \mathrm{H}_{2}(2), \mathrm{L}_{1}(2)$ & $\mathrm{LiScI}_{3}$ \\
\hline $\mathrm{SOC}$ & $\overline{\mathrm{A}}_{8} \overline{\mathrm{A}}_{9}(4)$ & $\mathrm{LiScI}_{3}$ \\
\hline \multicolumn{3}{|r|}{ Space Group 189: $P \overline{6} 2 m$} \\
\hline \multirow{20}{*}{ NSOC } & $\mathrm{A}_{6}(2), \Gamma_{6}(2), \mathrm{H}_{5}(2), \mathrm{K}_{6}(2)$ & $\| \mathrm{Hf}_{2} \mathrm{Co}_{4} \mathrm{P}_{3}$ \\
\hline & $\mathrm{A}_{6}(2), \Gamma_{5}(2), \mathrm{H}_{5}(2), \mathrm{K}_{5}(2)$ & $\mathrm{Yb}_{3} \mathrm{Si}_{5}, \mathrm{~Tb}_{3} \mathrm{Mn}_{3} \mathrm{Ga}_{2} \mathrm{Si}$ \\
\hline & $\Gamma_{5}(2), \mathrm{H}_{6}(2), \mathrm{K}_{6}(2)$ & $\| \mathrm{YbMgPb}$ \\
\hline & $\Gamma_{5}(2), \mathrm{H}_{5}(2), \mathrm{K}_{5}(2)$ & $\| \mathrm{Dy}_{3} \mathrm{Mn}_{3} \mathrm{Ga}_{2} \mathrm{Si}$ \\
\hline & $\mathrm{A}_{6}(2), \mathrm{H}_{6}(2), \mathrm{K}_{5}(2)$ & $\| \mathrm{Y}_{3} \mathrm{Al}_{3} \mathrm{NiGe}_{2}$ \\
\hline & $\mathrm{A}_{6}(2), \mathrm{H}_{5}(2), \mathrm{K}_{5}(2)$ & $\mathrm{Yb}_{3} \mathrm{Ge}_{5}, \mathrm{Er}_{3} \mathrm{Al}_{3} \mathrm{NiGe}_{2}, \mathrm{Ti}_{4} \mathrm{Ga}_{3} \mathrm{Ni}_{2}$ \\
\hline & $\mathrm{H}_{5}(2), \mathrm{K}_{5}(2)$ & || $\mathrm{Hf}_{6} \mathrm{NiSb}_{2}$ \\
\hline & $\Gamma_{6}(2), \mathrm{K}_{6}(2)$ & $\| \mathrm{K}_{2} \mathrm{UF}_{6}$ \\
\hline & $\Gamma_{6}(2), \mathrm{K}_{5}(2)$ & $\| \mathrm{Mg}_{3} \mathrm{Al}_{8} \mathrm{FeSi}_{6}$ \\
\hline & $\Gamma_{5}(2), \mathrm{K}_{6}(2)$ & $\| \mathrm{HfSiOs}, \mathrm{Zr}_{6} \mathrm{Ga}_{2} \mathrm{Ni}$ \\
\hline & $\Gamma_{5}(2), \mathrm{K}_{5}(2)$ & $\begin{array}{l}\text { IIfGeRu, HfGeOs, ZrGeOs, ZrSiRu, Lu } \mathrm{Hu}_{8} \mathrm{Te}, \mathrm{Lu}_{6} \mathrm{FeSb}_{2}, \mathrm{Tm}_{6} \mathrm{FeSb}_{2} \text {, } \\
\mathrm{Ho}_{6} \mathrm{FeBi}_{2}, \mathrm{Dy}_{6} \mathrm{FeSb}_{2}, \mathrm{Y}_{6} \mathrm{FeSb}_{2}, \mathrm{Ho}_{6} \mathrm{FeSb}_{2}, \mathrm{~Tb}_{6} \mathrm{FeSb}_{2}, \mathrm{~Tb}_{6} \mathrm{FeBi}_{2}\end{array}$ \\
\hline & $\Gamma_{5}(2), \mathrm{H}_{6}(2)$ & || DyCdCu, YMgAg, SmCdCu, ErMgAg, $\mathrm{La}_{5} \mathrm{Cu}_{19} \mathrm{P}_{12}$ \\
\hline & $\Gamma_{5}(2), \mathrm{H}_{5}(2)$ & $\| \mathrm{SmInPt}, \mathrm{YMgCu}, \mathrm{Zr}_{6} \mathrm{Sb}_{2} \mathrm{Pt}$ \\
\hline & $\mathrm{A}_{6}(2), \Gamma_{5}(2)$ & || TaN, PrTlPd \\
\hline & $\mathrm{A}_{5}(2), \mathrm{H}_{6}(2)$ & $\| \mathrm{Zr}_{6} \mathrm{Al}_{2} \mathrm{Fe}$ \\
\hline & $\mathrm{A}_{5}(2), \mathrm{H}_{5}(2)$ & $\mathrm{K}_{5}\left(\mathrm{Dy}_{4}\right)_{3}$ \\
\hline & $\mathrm{A}_{5}(2), \Gamma_{5}(2)$ & $\| \mathrm{YbPdPb}$ \\
\hline & $\mathrm{K}_{6}(2)$ & $\| \mathrm{CaCdPb}$, TaReSi \\
\hline & $\mathrm{K}_{5}(2)$ & $\begin{array}{c}\text { \| TiGePd, ScPRu, HfSiRu, ScGeRh, HfFeGe, TbAlNi, PrAlPd, } \\
\text { TbAlPd, } \mathrm{Zr}_{6} \mathrm{FeTe}_{2}, \text { NdAlNi, PrAlNi }\end{array}$ \\
\hline & $\mathrm{H}_{6}(2)$ & $\mathrm{ZrGaRh}, \mathrm{Hf}_{6} \mathrm{Al}_{2} \mathrm{Pt}, \mathrm{ZrGaCo}, \mathrm{PrCdCu}, \mathrm{DyMgAg}, \mathrm{LaMgAg}, \mathrm{CeMgPd}$ \\
\hline
\end{tabular}


Table II - continued

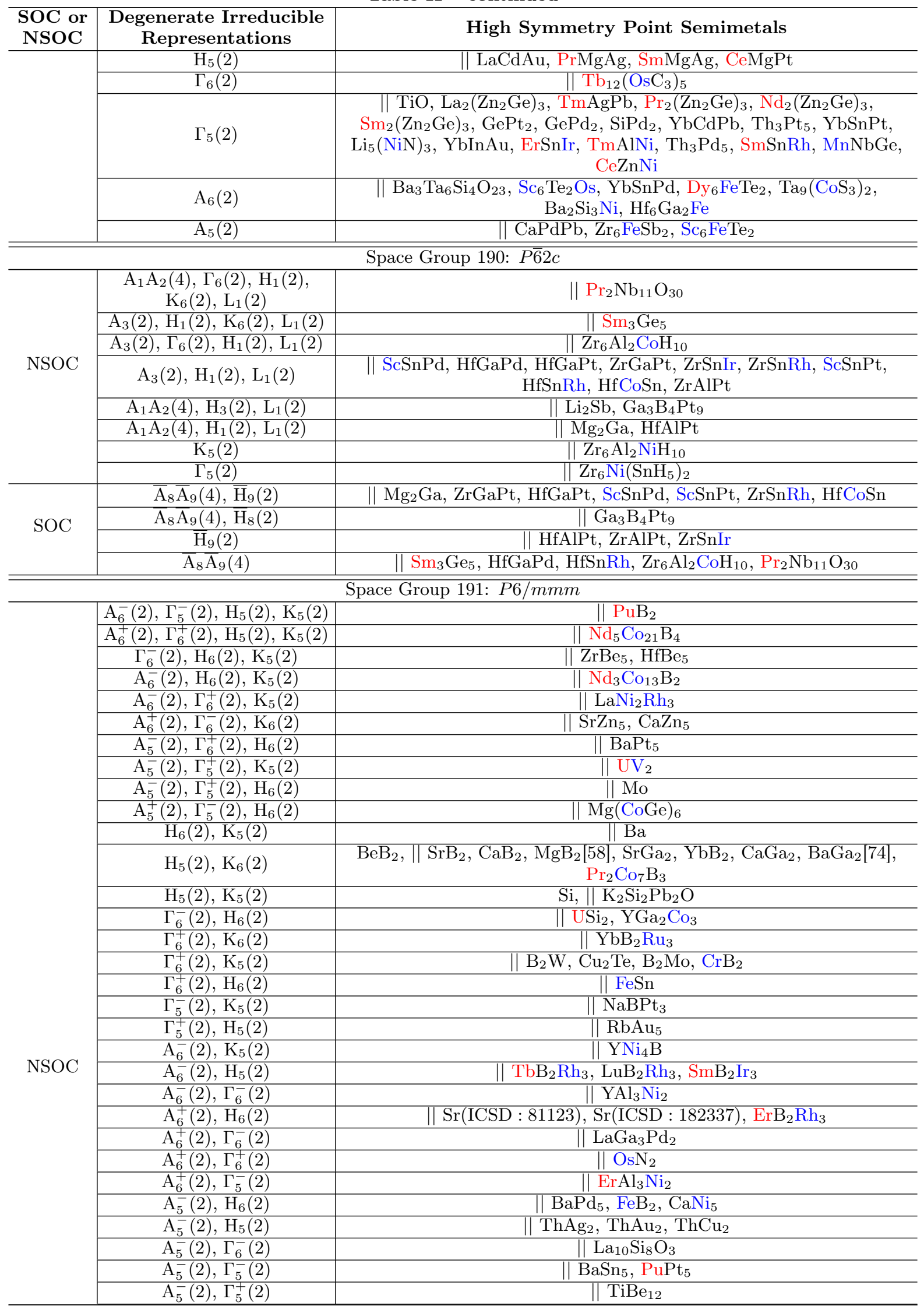


Table II - continued

\begin{tabular}{|c|c|c|}
\hline $\begin{array}{l}\text { SOC or } \\
\text { NSOC }\end{array}$ & $\begin{array}{c}\text { Degenerate Irreducible } \\
\text { Representations }\end{array}$ & High Symmetry Point Semimetals \\
\hline & $\mathrm{A}_{5}^{+}(2), \mathrm{H}_{5}(2)$ & $\| \mathrm{TmB}_{2} \mathrm{Rh}_{3}$ \\
\hline & $\mathrm{A}_{5}^{+}(2), \Gamma_{5}^{+}(2)$ & \| TaN(ICSD : 186417), $\mathrm{ThFe}_{5}$ \\
\hline & $\mathrm{K}_{6}(2)$ & $\| \mathrm{ZrBe}_{2}, \mathrm{ThZn}_{2}, \mathrm{Sc}(\mathrm{NiGe})_{6}, \mathrm{CeCo}_{4} \mathrm{~B}$ \\
\hline & $\mathrm{K}_{5}(2)$ & $\begin{array}{l}\text { IfhSi }, \mathrm{LaGe}_{2} \mathrm{Ir}_{3}, \mathrm{Ta}_{2} \mathrm{BN}_{3}, \mathrm{MoN}(\mathrm{ICSD}: 60168), \mathrm{Zr}_{2} \mathrm{TaN}_{3}, \mathrm{TaTi}_{2} \mathrm{~N}_{3} \text {, } \\
\mathrm{Hf}_{2} \mathrm{TaN}_{3}, \mathrm{TbNi}_{4} \mathrm{~B}, \mathrm{HoNi}_{4} \mathrm{~B}, \mathrm{TmNi}_{4} \mathrm{~B}, \mathrm{NdNi}_{4} \mathrm{~B}, \mathrm{ErNi}_{4} \mathrm{~B}, \mathrm{LaNi}_{4} \mathrm{~B}, \\
\mathrm{UGa}_{2}, \mathrm{Dy}_{3} \mathrm{Co}_{11} \mathrm{~B}_{4}, \mathrm{Ni}_{3} \mathrm{Co}_{11} \mathrm{~B}_{4}, \mathrm{Pr}_{3} \mathrm{Co}_{11} \mathrm{~B}_{4}, \mathrm{Hf}(\mathrm{MnGe})_{6}, \mathrm{Ti}_{13} \mathrm{Si}_{31} \mathrm{Ni}_{40}\end{array}$ \\
\hline & $\mathrm{H}_{6}(2)$ & $\begin{aligned} \| \mathrm{LaB}_{2} \mathrm{Ir}_{3}, \mathrm{PrB}_{2} \mathrm{Ir}_{3}, \mathrm{SmB}_{2} \mathrm{Rh}_{3}, \mathrm{CaPt}_{5}, \mathrm{SrPt}_{5}, \mathrm{NdB}_{2} \mathrm{Rh}_{3}, \mathrm{LaB}_{2} \mathrm{Rh}_{3}, \\
\mathrm{PrB}_{2} \mathrm{Rh}_{3}, \mathrm{Dy}_{4} \mathrm{In}_{3} \mathrm{Cu}_{2}, \mathrm{CaPd}_{5}, \mathrm{UFe}_{3} \mathrm{~B}_{2}, \mathrm{SrPd}_{5}\end{aligned}$ \\
\hline & $\mathrm{H}_{5}(2)$ & $\begin{array}{c}\| \mathrm{KAu}_{5}, \mathrm{TbB}_{2} \mathrm{Ir}_{3}, \mathrm{NdB}_{2} \mathrm{Ir}_{3}, \mathrm{DyB}_{2} \mathrm{Rh}_{3}, \mathrm{HoB}_{2} \mathrm{Rh}_{3}, \mathrm{YB}_{2} \mathrm{Rh}_{3}, \mathrm{U}_{2} \mathrm{Ti} \\
\mathrm{YCo}_{4} \mathrm{~B}, \mathrm{NdCo}_{4} \mathrm{~B}, \mathrm{PrCo}_{4} \mathrm{~B}, \mathrm{Sm}_{2} \mathrm{Co}_{7} \mathrm{~B}_{3}, \mathrm{Mg}(\mathrm{FeGe})_{6}\end{array}$ \\
\hline & $\Gamma_{6}^{-}(2)$ & $\begin{array}{c}\mathrm{NaLi}_{2} \mathrm{~N}(\mathrm{ICSD}: 92308), \mathrm{Na}_{2} \mathrm{LiN}, \mathrm{Na}_{3} \mathrm{~N}, \| \mathrm{Hf}, \mathrm{NaLi}_{2} \mathrm{~N}(\mathrm{ICSD}: 92307), \\
\mathrm{LaGa}_{2} \mathrm{Rh}_{3}, \mathrm{UB}_{2} \mathrm{Ru}_{3}\end{array}$ \\
\hline & $\Gamma_{6}^{+}(2)$ & $\| \mathrm{UB}_{2} \mathrm{Os}_{3}, \mathrm{TbRh}_{5}, \mathrm{DyRh}_{5}, \mathrm{ErRh}_{5}, \mathrm{YRh}_{5}, \mathrm{PrCo}_{5}, \mathrm{YCo}_{5}$ \\
\hline & $\Gamma_{5}^{+}(2)$ & $\| \mathrm{BaAu}_{2}, \mathrm{ThNi}_{2}, \mathrm{CaZn}_{3} \mathrm{Ni}_{2}, \mathrm{CeFe}_{5}$ \\
\hline & $\mathrm{A}_{6}^{-}(2)$ & $\| \mathrm{ScB}_{2} \mathrm{Ir}_{3}, \mathrm{TmB}_{2} \mathrm{Ir}_{3}, \mathrm{HoB}_{2} \mathrm{Ir}_{3}, \mathrm{PrBPt}_{4}, \mathrm{Hf}(\mathrm{FeGe})_{6}$ \\
\hline & $\mathrm{A}_{6}^{+}(2)$ & $\begin{array}{c}\mathrm{ZrB}_{2}\left[75, \mathrm{TiB}_{2}\left[75, \mathrm{HfB}_{2}, \mathrm{ThGa}_{2}, \| \mathrm{YbSi}_{2}, \mathrm{CaHg}_{2}, \mathrm{NdAl}_{3} \mathrm{Pd}_{2},\right.\right. \\
\mathrm{LaAl}_{3} \mathrm{Pd}_{2}, \mathrm{DyGa}_{2} \mathrm{Cu}_{3}, \mathrm{PrAl}_{3} \mathrm{Ni}_{2}, \mathrm{NdAl}_{3} \mathrm{Ni}_{2}, \mathrm{ScCo}_{3} \mathrm{~B}_{2}, \mathrm{LuCo}_{3} \mathrm{~B}_{2} \\
\mathrm{TaN}\left(\mathrm{ICSD}: \text { : 76457), } \mathrm{LaAl}_{2} \mathrm{Ag}_{3}, \mathrm{CeAl}_{2} \mathrm{Pt}_{3}, \mathrm{DyGa}_{3} \mathrm{Ni}_{2}, \mathrm{CeZn}_{3} \mathrm{Cu}_{2}\right. \\
\mathrm{CeSi}_{2} \mathrm{Ru}_{3}\end{array}$ \\
\hline & $\mathrm{A}_{5}^{-}(2)$ & $\begin{array}{l}\mathrm{ThAl}_{2}, \| \mathrm{Hg}, \mathrm{TmCo}_{3} \mathrm{~B}_{2}, \mathrm{ErCo}_{3} \mathrm{~B}_{2}, \mathrm{DyCo}_{3} \mathrm{~B}_{2}, \mathrm{HoCo}_{3} \mathrm{~B}_{2}, \mathrm{TbCo}_{3} \mathrm{~B}_{2}, \\
\mathrm{YCo}_{3} \mathrm{~B}_{2}, \mathrm{ThSi}_{2} \mathrm{Ru}_{3}, \mathrm{KIn}_{9} \mathrm{Ni}_{2}, \mathrm{CeB}_{2} \mathrm{Ru}_{3}, \mathrm{ThB}_{2} \mathrm{Ru}_{3}, \mathrm{CeNi}_{5}, \mathrm{ThNi}_{5}\end{array}$ \\
\hline & $\mathrm{A}_{5}^{+}(2)$ & $\| \mathrm{SmAl}_{3} \mathrm{Pd}_{2}, \mathrm{TaNO}$ \\
\hline \multirow{19}{*}{ NSOC } & & Space Group 193: $P 6_{3} / \mathrm{mcm}$ \\
\hline & $\begin{array}{c}\mathrm{A}_{3}(4), \Gamma_{6}^{+}(2), \mathrm{H}_{2} \mathrm{H}_{4}(2), \\
\mathrm{K}_{5}(2), \mathrm{L}_{2}(2)\end{array}$ & $\| \mathrm{Sm}_{5} \mathrm{Sb}_{3} \mathrm{H}$ \\
\hline & $\begin{array}{c}\mathrm{A}_{3}(4), \Gamma_{5}^{+}(2), \mathrm{H}_{5} \mathrm{H}_{6}(4) \\
\mathrm{K}_{5}(2), \mathrm{L}_{2}(2)\end{array}$ & $\| \mathrm{Sc}_{5} \mathrm{~Pb}_{3}$ \\
\hline & $\begin{array}{c}\mathrm{A}_{3}(4), \Gamma_{5}^{+}(2), \mathrm{H}_{5} \mathrm{H}_{6}(4), \\
\mathrm{K}_{5}(2), \mathrm{L}_{1}(2)\end{array}$ & $\| \mathrm{Ta}_{3}\left(\mathrm{BiS}_{3}\right)_{2}, \mathrm{Nb}_{3}\left(\mathrm{BiS}_{3}\right)_{2}$ \\
\hline & $\begin{array}{c}\mathrm{A}_{2}(2), \Gamma_{6}^{+}(2), \mathrm{H}_{2} \mathrm{H}_{4}(2), \\
\mathrm{K}_{5}(2), \mathrm{L}_{2}(2)\end{array}$ & $\| \mathrm{La}_{5} \mathrm{Sb}_{3} \mathrm{Br}$ \\
\hline & $\begin{array}{c}\mathrm{A}_{3}(4), \mathrm{H}_{5} \mathrm{H}_{6}(4), \mathrm{K}_{6}(2) \\
\mathrm{L}_{2}(2)\end{array}$ & $\| \mathrm{Ta}_{5} \mathrm{~N}_{6}, \mathrm{Nb}_{5} \mathrm{~N}_{6}, \mathrm{Hf}_{5} \mathrm{CuSn}_{3}, \mathrm{Hf}_{5} \mathrm{CuPb}_{3}$ \\
\hline & $\begin{array}{c}\mathrm{A}_{3}(4), \mathrm{H}_{5} \mathrm{H}_{6}(4), \mathrm{K}_{5}(2) \\
\mathrm{L}_{2}(2)\end{array}$ & $\begin{array}{c}\text { \| } \mathrm{Dy}_{5} \mathrm{Sn}_{3}, \mathrm{Lu}_{5} \mathrm{NiPb}_{3}, \mathrm{Tm}_{5} \mathrm{NiPb}_{3}, \mathrm{~Tb}_{5} \mathrm{Si}_{3}, \mathrm{H}_{5} \mathrm{NiPb}_{3}, \mathrm{Dy}_{5} \mathrm{NiPb}_{3}, \\
\mathrm{~Tb}_{5} \mathrm{NiPb}_{3}, \mathrm{Er}_{5} \mathrm{NiPb}_{3}\end{array}$ \\
\hline & $\begin{array}{c}\mathrm{A}_{3}(4), \mathrm{H}_{5} \mathrm{H}_{6}(4), \mathrm{K}_{5}(2), \\
\mathrm{L}_{1}(2)\end{array}$ & $\| \mathrm{Zr}_{3} \mathrm{Ti}_{2} \mathrm{Ga}_{3}$ \\
\hline & $\begin{array}{c}\mathrm{A}_{3}(4), \mathrm{H}_{2} \mathrm{H}_{4}(2), \mathrm{K}_{5}(2) \\
\mathrm{L}_{2}(2)\end{array}$ & $\| \mathrm{V}_{5} \mathrm{P}_{3} \mathrm{~N}$ \\
\hline & $\begin{array}{c}\mathrm{A}_{3}(4), \Gamma_{6}^{-}(2), \mathrm{H}_{1} \mathrm{H}_{3}(2) \\
\mathrm{L}_{1}(2)\end{array}$ & $\| \mathrm{Zr}_{5} \mathrm{SiSb}_{3}$ \\
\hline & $\begin{array}{l}\mathrm{A}_{3}(4), \Gamma_{6}^{+}(2), \mathrm{H}_{5} \mathrm{H}_{6}(4) \\
\mathrm{L}_{2}(2)\end{array}$ & $\| \mathrm{Nb}_{5} \mathrm{Pt}_{3} \mathrm{O}$ \\
\hline & $\begin{array}{c}\mathrm{A}_{3}(4), \Gamma_{6}^{+}(2), \mathrm{H}_{5} \mathrm{H}_{6}(4) \\
\mathrm{L}_{1}(2)\end{array}$ & || $\mathrm{Dy}_{6} \mathrm{Ti}_{4} \mathrm{Al}_{43}$ \\
\hline & $\begin{array}{c}\mathrm{A}_{3}(4), \Gamma_{6}^{+}(2), \mathrm{H}_{2} \mathrm{H}_{4}(2) \\
\mathrm{L}_{1}(2)\end{array}$ & $\| \mathrm{Zr}_{5} \mathrm{Al}_{3} \mathrm{C}$ \\
\hline & $\begin{array}{l}\mathrm{A}_{3}(4), \Gamma_{5}^{+}(2), \mathrm{H}_{5} \mathrm{H}_{6}(4) \\
\mathrm{L}_{2}(2)\end{array}$ & $\| \mathrm{Tb}_{5} \mathrm{Ge}_{3}, \mathrm{Y}_{5} \mathrm{Sn}_{3}, \mathrm{Sc}_{5} \mathrm{Sn}_{3}, \mathrm{Sc}_{5} \mathrm{Si}_{3}, \mathrm{Nd}_{5} \mathrm{Ge}_{3}(\mathrm{ICSD}: 637262)$ \\
\hline & $\begin{array}{l}\mathrm{A}_{2}(2), \mathrm{H}_{5} \mathrm{H}_{6}(4), \mathrm{K}_{5}(2) \\
\mathrm{L}_{1}(2)\end{array}$ & $\| \mathrm{Zr}_{5} \mathrm{Sb}_{3}$ \\
\hline & $\begin{array}{c}\mathrm{A}_{2}(2), \mathrm{H}_{2} \mathrm{H}_{4}(2), \mathrm{K}_{5}(2) \\
\mathrm{L}_{2}(2)\end{array}$ & $\| \mathrm{La}_{5} \mathrm{Bi}_{3} \mathrm{Br}$ \\
\hline & $\begin{array}{c}\mathrm{A}_{2}(2), \mathrm{H}_{1} \mathrm{H}_{3}(2), \mathrm{K}_{6}(2), \\
\mathrm{L}_{2}(2)\end{array}$ & $\| \mathrm{K}_{3} \mathrm{Nb}_{8} \mathrm{O}_{21}$ \\
\hline & $\begin{array}{c}\mathrm{A}_{2}(2), \Gamma_{6}^{-}(2), \mathrm{H}_{1} \mathrm{H}_{3}(2) \\
\mathrm{L}_{1}(2)\end{array}$ & $\| \mathrm{Hf}_{5} \mathrm{ZnSb}_{3}$ \\
\hline & $\begin{array}{c}\mathrm{A}_{1}(2), \mathrm{H}_{5} \mathrm{H}_{6}(4), \mathrm{K}_{5}(2), \\
\mathrm{L}_{2}(2)\end{array}$ & $\| \mathrm{Nb}_{3} \mathrm{PbS}_{6}, \mathrm{Cs}_{3} \mathrm{O}$ \\
\hline
\end{tabular}


Table II - continued

\begin{tabular}{|c|c|c|}
\hline $\begin{array}{l}\text { SOC or } \\
\text { NSOC }\end{array}$ & $\begin{array}{c}\text { Degenerate Irreducible } \\
\text { Representations }\end{array}$ & High Symmetry Point Semimetals \\
\hline & $\begin{array}{c}\mathrm{A}_{1}(2), \mathrm{H}_{5} \mathrm{H}_{6}(4), \mathrm{K}_{5}(2) \\
\mathrm{L}_{1}(2)\end{array}$ & $\| \mathrm{Nd}_{3} \mathrm{MnBi}_{5}, \mathrm{La}_{3} \mathrm{MnBi}_{5}, \mathrm{Ba}_{10} \mathrm{Al}_{3} \mathrm{Ge}_{7}$ \\
\hline & $\begin{array}{l}\mathrm{A}_{1}(2), \mathrm{H}_{1} \mathrm{H}_{3}(2), \mathrm{K}_{6}(2) \\
\mathrm{L}_{2}(2)\end{array}$ & $\| \mathrm{La}_{5} \mathrm{In}_{3} \mathrm{Br}$ \\
\hline & $\begin{array}{c}\mathrm{A}_{1}(2), \mathrm{H}_{1} \mathrm{H}_{3}(2), \mathrm{K}_{5}(2), \\
\mathrm{L}_{2}(2)\end{array}$ & $\| \mathrm{Ta}_{3} \mathrm{SnS}_{6}$ \\
\hline & $\begin{array}{c}\mathrm{A}_{1}(2), \Gamma_{6}^{-}(2), \mathrm{H}_{1} \mathrm{H}_{3}(2), \\
\mathrm{L}_{1}(2)\end{array}$ & $\| \mathrm{Zr}_{5} \mathrm{ZnSb}_{3}$ \\
\hline & $\Gamma_{6}^{-}(2), \mathrm{H}_{5} \mathrm{H}_{6}(4), \mathrm{K}_{6}(2)$ & $\| \mathrm{Th}_{5} \mathrm{Sn}_{4}$ \\
\hline & $\mathrm{A}_{3}(4), \mathrm{H}_{5} \mathrm{H}_{6}(4), \mathrm{L}_{2}(2)$ & $\begin{array}{c}\| \mathrm{Ho}_{5} \mathrm{Si}_{3}, \mathrm{Dy}_{5} \mathrm{Si}_{3}, \mathrm{Er}_{5} \mathrm{Ge}_{3}, \mathrm{Er}_{5} \mathrm{Sn}_{3}, \mathrm{Er}_{5} \mathrm{Si}_{3}, \mathrm{RuCl}_{3}, \mathrm{Y}_{5} \mathrm{Si}_{3}, \mathrm{Ho}_{5} \mathrm{Sn}_{3}, \\
\mathrm{Pr}_{5} \mathrm{Ge}_{3}, \mathrm{Ho}_{5} \mathrm{~Pb}_{3}, \mathrm{Y}_{5} \mathrm{Ge}_{3}, \mathrm{Dy}_{5} \mathrm{~Pb}_{3}, \mathrm{Nd}_{5} \mathrm{Ge}_{3}(\mathrm{ICSD}: 637271), \mathrm{Sc}_{5} \mathrm{Ge}_{3}, \\
\mathrm{Lu}_{5} \mathrm{Si}_{3}, \mathrm{Nd}_{5} \mathrm{Sn}_{3}, \mathrm{Mg}_{13} \mathrm{Au}_{41}, \mathrm{La}_{5} \mathrm{NiPb}_{3}, \mathrm{Pr}_{5} \mathrm{NiPb}_{3} \\
\end{array}$ \\
\hline & $\mathrm{A}_{3}(4), \mathrm{H}_{5} \mathrm{H}_{6}(4), \mathrm{L}_{1}(2)$ & $\mathrm{TiCl}_{3}\left[76, \| \mathrm{La}_{3} \mathrm{ScBi}_{5}, \mathrm{TiI}_{3}, \mathrm{Ca}_{5} \mathrm{CuPb}_{3}, \mathrm{Hf}_{5} \mathrm{Ga}_{3}, \mathrm{~Np}_{5} \mathrm{Ge}_{4}\right.$ \\
\hline & $\mathrm{A}_{3}(4), \mathrm{H}_{1} \mathrm{H}_{3}(2), \mathrm{L}_{2}(2)$ & $\| \mathrm{RuBr}_{3}$ \\
\hline & $\mathrm{A}_{3}(4), \Gamma_{6}^{-}(2), \mathrm{H}_{5} \mathrm{H}_{6}(4)$ & $\| \mathrm{La}_{3} \mathrm{HfSb}_{5}$ \\
\hline & $\mathrm{A}_{3}(4), \Gamma_{6}^{+}(2), \mathrm{H}_{5} \mathrm{H}_{6}(4)$ & $\| \mathrm{Hf}_{5} \mathrm{Al}_{3} \mathrm{~N}$ \\
\hline & $\mathrm{A}_{3}(4), \Gamma_{5}^{+}(2), \mathrm{K}_{6}(2)$ & $\| \mathrm{Dy}_{5} \mathrm{Si}_{3} \mathrm{~B}, \mathrm{Ho}_{5} \mathrm{Si}_{3} \mathrm{~B}$ \\
\hline & $\mathrm{A}_{3}(4), \Gamma_{5}^{+}(2), \mathrm{H}_{5} \mathrm{H}_{6}(4)$ & $\mathrm{La}_{3} \mathrm{ZrSb}_{5}, \mathrm{La}_{3} \mathrm{TiSb}_{5}$ \\
\hline & $\mathrm{A}_{2}(2), \mathrm{H}_{5} \mathrm{H}_{6}(4), \mathrm{L}_{1}(2)$ & $\| \mathrm{Ba}_{3} \mathrm{~N}$ \\
\hline & $\mathrm{A}_{2}(2), \mathrm{H}_{2} \mathrm{H}_{4}(2), \mathrm{L}_{2}(2)$ & $\mathrm{K}_{3} \mathrm{YbSi}_{2} \mathrm{O}_{7}$ \\
\hline & $\mathrm{A}_{2}(2), \mathrm{H}_{2} \mathrm{H}_{4}(2), \mathrm{L}_{1}(2)$ & $\mathrm{Hf}_{3} \mathrm{Nb}_{2} \mathrm{Ga}_{3}$ \\
\hline & $\mathrm{A}_{1}(2), \mathrm{H}_{5} \mathrm{H}_{6}(4), \mathrm{L}_{2}(2)$ & $\| \mathrm{Zr}_{5} \mathrm{AlSn}_{3}$ \\
\hline & $\mathrm{A}_{1}(2), \mathrm{H}_{5} \mathrm{H}_{6}(4), \mathrm{L}_{1}(2)$ & $\| \mathrm{ZrI}_{3}, \mathrm{HfI}_{3}, \mathrm{Zr}_{5} \mathrm{GaSn}_{3}, \mathrm{Zr}_{5} \mathrm{Al}_{3}, \mathrm{Hf}_{5} \mathrm{Al}_{3}, \mathrm{Pr}_{3} \mathrm{MnBi}_{5}$ \\
\hline & $\mathrm{A}_{1}(2), \mathrm{H}_{1} \mathrm{H}_{3}(2), \mathrm{L}_{2}(2)$ & $\| \mathrm{Ta}_{3} \mathrm{PbS}_{6}, \mathrm{Nb}_{3} \mathrm{SnS}_{6}, \mathrm{Nb}_{3} \mathrm{GeS}_{6}$ \\
\hline & $\mathrm{A}_{1}(2), \mathrm{H}_{1} \mathrm{H}_{3}(2), \mathrm{L}_{1}(2)$ & $\begin{array}{c}\mathrm{ZrCl}_{3}, \| \mathrm{ZrBr}_{3}, \mathrm{Nb}_{5} \mathrm{Ga}_{4}, \mathrm{Ca}_{5} \mathrm{As}_{3}, \mathrm{Sr}_{5} \mathrm{As}_{3}, \mathrm{Ca}_{5} \mathrm{Sb}_{3}, \mathrm{Yb}_{5} \mathrm{Sb}_{3}, \mathrm{Ta}_{5} \mathrm{Si}_{3}, \\
\mathrm{Sr}_{5} \mathrm{Sb}_{3}, \mathrm{Nb}_{5} \mathrm{Si}_{3}, \mathrm{Sr}_{5} \mathrm{Bi}_{3}, \mathrm{Nb}_{5} \mathrm{Ge}_{3}, \mathrm{Ba}_{5} \mathrm{As}_{3}, \mathrm{Ba}_{5} \mathrm{Sb}_{3}, \mathrm{Ta}_{5} \mathrm{Ge}_{3}, \mathrm{Ba}_{5} \mathrm{Bi}_{3}, \\
\mathrm{~V}_{5} \mathrm{Ge}_{3}\end{array}$ \\
\hline & $\mathrm{H}_{5} \mathrm{H}_{6}(4), \mathrm{K}_{6}(2)$ & $\| \mathrm{Fe}_{5} \mathrm{Si}_{3}$ \\
\hline & $\mathrm{H}_{5} \mathrm{H}_{6}(4), \mathrm{K}_{5}(2)$ & $\mathrm{Mg}_{5} \mathrm{Hg}_{3}$ \\
\hline & $\Gamma_{6}^{-}(2), \mathrm{K}_{6}(2)$ & $\| \mathrm{Zr}_{5} \mathrm{Sn}_{4}$ \\
\hline & $\Gamma_{6}^{-}(2), \mathrm{K}_{5}(2)$ & $\mathrm{Er}_{5} \mathrm{Rh}_{3}, \mathrm{Ho}_{5} \mathrm{Rh}_{3}$ \\
\hline & $\Gamma_{6}^{+}(2), \mathrm{H}_{5} \mathrm{H}_{6}(4)$ & $\| \mathrm{Zr}_{5} \mathrm{ZnPb}_{3}$ \\
\hline & $\Gamma_{5}^{-}(2), \mathrm{K}_{5}(2)$ & $\| \mathrm{Ge}_{3} \mathrm{Mo}_{5} \mathrm{C}$ \\
\hline & $\Gamma_{5}^{+}(2), \mathrm{K}_{6}(2)$ & $\| \mathrm{Tb}_{5} \mathrm{Tl}_{3}$ \\
\hline & $\mathrm{A}_{3}(4), \mathrm{K}_{6}(2)$ & $\| \mathrm{Lu}_{5} \mathrm{Si}_{3} \mathrm{~B}$ \\
\hline & $\mathrm{A}_{3}(4), \mathrm{K}_{5}(2)$ & $\| \mathrm{Zr}_{5} \mathrm{Sb}_{4}$ \\
\hline & $\mathrm{A}_{3}(4), \mathrm{H}_{5} \mathrm{H}_{6}(4)$ & $\mathrm{Y}_{5} \mathrm{CuPb}_{3}, \mathrm{La}_{5} \mathrm{~Pb}_{3} \mathrm{I}$ \\
\hline & $\mathrm{A}_{3}(4), \Gamma_{6}^{-}(2)$ & $\| \mathrm{TlAgSe}_{2}$ \\
\hline & $\mathrm{A}_{3}(4), \Gamma_{5}^{-}(2)$ & $\| \mathrm{Zr}_{5} \mathrm{Al}_{4}$ \\
\hline & $\mathrm{K}_{6}(2)$ & $\| \mathrm{Hf}_{5} \mathrm{Sn}_{4}$ \\
\hline & $\mathrm{K}_{5}(2)$ & $\| \mathrm{Ho}_{5} \mathrm{Sb}_{3}$ \\
\hline & $\mathrm{H}_{5} \mathrm{H}_{6}(4)$ & $\| \mathrm{Ta}_{5} \mathrm{Ga}_{3}$ \\
\hline & $\Gamma_{6}^{-}(2)$ & $\mathrm{Lu}_{5} \mathrm{Rh}_{3}, \mathrm{Sc}_{5} \mathrm{Ga}_{3}$ \\
\hline & $\Gamma_{6}^{+}(2)$ & $\| \mathrm{Ti}_{3} \mathrm{O}, \mathrm{Hg}_{5} \mathrm{Au}_{6}$ \\
\hline & $\mathrm{A}_{3}(4)$ & $\begin{array}{c}\| \mathrm{Hf}_{5} \mathrm{Si}_{3}, \mathrm{Zr}_{5} \mathrm{Si}_{3}, \mathrm{Yb}_{5} \mathrm{Sn}_{3}, \mathrm{Th}_{5} \mathrm{Sn}_{3}, \mathrm{Yb}_{5} \mathrm{~Pb}_{3}, \mathrm{Ti}_{5} \mathrm{Ga}_{4}, \mathrm{LaSiNO}_{2} \\
\mathrm{Yb}_{5} \mathrm{Ge}_{3}, \mathrm{Ho}_{5} \mathrm{Ga}_{3}, \mathrm{Er}_{5} \mathrm{Ga}_{3}, \mathrm{Ce}_{5} \mathrm{Ge}_{3}\end{array}$ \\
\hline \multirow[t]{2}{*}{$\mathrm{SOC}$} & $\overline{\mathrm{A}}_{6}(4), \overline{\mathrm{H}}_{8} \overline{\mathrm{H}}_{9}(4), \overline{\mathrm{L}}_{3} \overline{\mathrm{L}}_{4}(4)$ & $\begin{array}{c}\mathrm{ZrCl}_{3}, \| \mathrm{Ta}_{3} \mathrm{SnS}_{6}, \mathrm{Cs}_{3} \mathrm{O}, \mathrm{Nb}_{5} \mathrm{Ga}_{4}, \mathrm{Ca}_{5} \mathrm{As}_{3}, \mathrm{Ta}_{3} \mathrm{PbS}_{6}, \mathrm{Hf}_{5} \mathrm{ZnSb}_{3}, \\
\mathrm{Nb}_{3} \mathrm{SnS}_{6}, \mathrm{Nb}_{3} \mathrm{GeS}_{6}, \mathrm{Zr}_{5} \mathrm{ZnSb}_{3}, \mathrm{Sr}_{5} \mathrm{As}_{3}, \mathrm{RuCl}_{3}, \mathrm{Ca}_{5} \mathrm{Sb}_{3}, \mathrm{Yb}_{5} \mathrm{Sb}_{3}, \\
\mathrm{RuBr}_{3}, \mathrm{Sr}_{5} \mathrm{Sb}_{3}, \mathrm{Nb}_{5} \mathrm{Ge}_{3}, \mathrm{Zr}_{5} \mathrm{SiSb}_{3}, \mathrm{Ba}_{5} \mathrm{As}_{3}, \mathrm{~K}_{3} \mathrm{Nb}_{8} \mathrm{O}_{21}, \mathrm{La}_{5} \mathrm{Sb}_{3} \mathrm{Br} \\
\mathrm{Sc}_{5} \mathrm{Ge}_{3}, \mathrm{Nb}_{5} \mathrm{Si}_{3}, \mathrm{Ba}_{5} \mathrm{Sb}_{3}, \mathrm{Y}_{5} \mathrm{Sn}_{3}, \mathrm{Ba}_{5} \mathrm{Bi}_{3}, \mathrm{Ta}_{5} \mathrm{Si}_{3}, \mathrm{Hf}_{3} \mathrm{Nb}_{2} \mathrm{Ga}_{3}, \\
\mathrm{Ta}_{5} \mathrm{Ge}_{3}, \mathrm{Sr}_{5} \mathrm{Bi}_{3}, \mathrm{Zr}_{5} \mathrm{Sb}_{3}, \mathrm{Zr}_{5} \mathrm{Al}_{3}, \mathrm{Hf}_{5} \mathrm{Al}_{3}, \mathrm{La}_{3} \mathrm{MnBi}_{5}, \mathrm{Nd}_{3} \mathrm{MnBi}_{5} \\
\mathrm{Pr}_{3} \mathrm{MnBi}_{5}, \mathrm{La}_{5} \mathrm{In}_{3} \mathrm{Br}, \mathrm{Np}_{5} \mathrm{Ge}_{4}, \mathrm{~V}_{5} \mathrm{Ge}_{3}, \mathrm{~K}_{3} \mathrm{YbSi}_{2} \mathrm{O}_{7}, \mathrm{La}_{5} \mathrm{Bi}_{3} \mathrm{Br}\end{array}$ \\
\hline & $\overline{\mathrm{A}}_{6}(4), \overline{\mathrm{H}}_{7} \overline{\mathrm{H}}_{7}(4), \overline{\mathrm{L}}_{3} \overline{\mathrm{L}}_{4}(4)$ & $\begin{array}{l}\| \mathrm{Nb}_{3} \mathrm{PbS}_{6}, \mathrm{ZrBr}_{3}, \mathrm{Ba}_{3} \mathrm{~N}, \mathrm{HfI}_{3}, \mathrm{La}_{3} \mathrm{ScBi}_{5}, \mathrm{Ho}_{5} \mathrm{Si}_{3}, \mathrm{Dy}_{5} \mathrm{Si}_{3}, \mathrm{Er}_{5} \mathrm{Ge}_{3}, \\
\mathrm{Er}_{5} \mathrm{Sn}_{3}, \mathrm{Y}_{5} \mathrm{Si}_{3}, \mathrm{Er}_{5} \mathrm{Si}_{3}, \mathrm{Ho}_{5} \mathrm{Sn}_{3}, \mathrm{Pr}_{5} \mathrm{Ge}_{3}, \mathrm{Dy}_{5} \mathrm{Sn}_{3}, \mathrm{Y}_{5} \mathrm{Ge}_{3}, \mathrm{TiI}_{3}, \\
\mathrm{~Tb}_{5} \mathrm{Ge}_{3}, \mathrm{Nd}_{5} \mathrm{Ge}_{3}(\mathrm{ICSD}: 637271), \mathrm{Ir}_{5} \mathrm{GaSn}_{3}, \mathrm{Lu}_{5} \mathrm{NiPb}_{3}, \mathrm{Hf}_{5} \mathrm{CuSn}_{3}, \\
\mathrm{Tm}_{5} \mathrm{NiPb}_{3}, \mathrm{Lu}_{5} \mathrm{Si}_{3}, \mathrm{Nd}_{5} \mathrm{Sn}_{3}, \mathrm{Er}_{5} \mathrm{NiPb}_{3}, \mathrm{Ho}_{5} \mathrm{NiPb}_{3}, \mathrm{~Tb}_{5} \mathrm{Si}_{3}, \mathrm{Sc}_{5} \mathrm{Si}_{3}, \\
\mathrm{Dy}_{5} \mathrm{NiPb}_{3}, \mathrm{Nd}_{5} \mathrm{Ge}_{3}(\mathrm{ICSD}: 637262), \mathrm{Hf}_{5} \mathrm{CuPb}_{3}, \mathrm{Zr}_{5} \mathrm{AlSl}_{3}, \mathrm{~Tb}_{5} \mathrm{NiPb}_{3}, \mathrm{Ni}_{3}, \mathrm{Pr}_{5} \mathrm{NiPb}_{3}, \mathrm{Ba}_{10} \mathrm{Al}_{3} \mathrm{Ge}_{7}\end{array}$ \\
\hline
\end{tabular}


Table II - continued

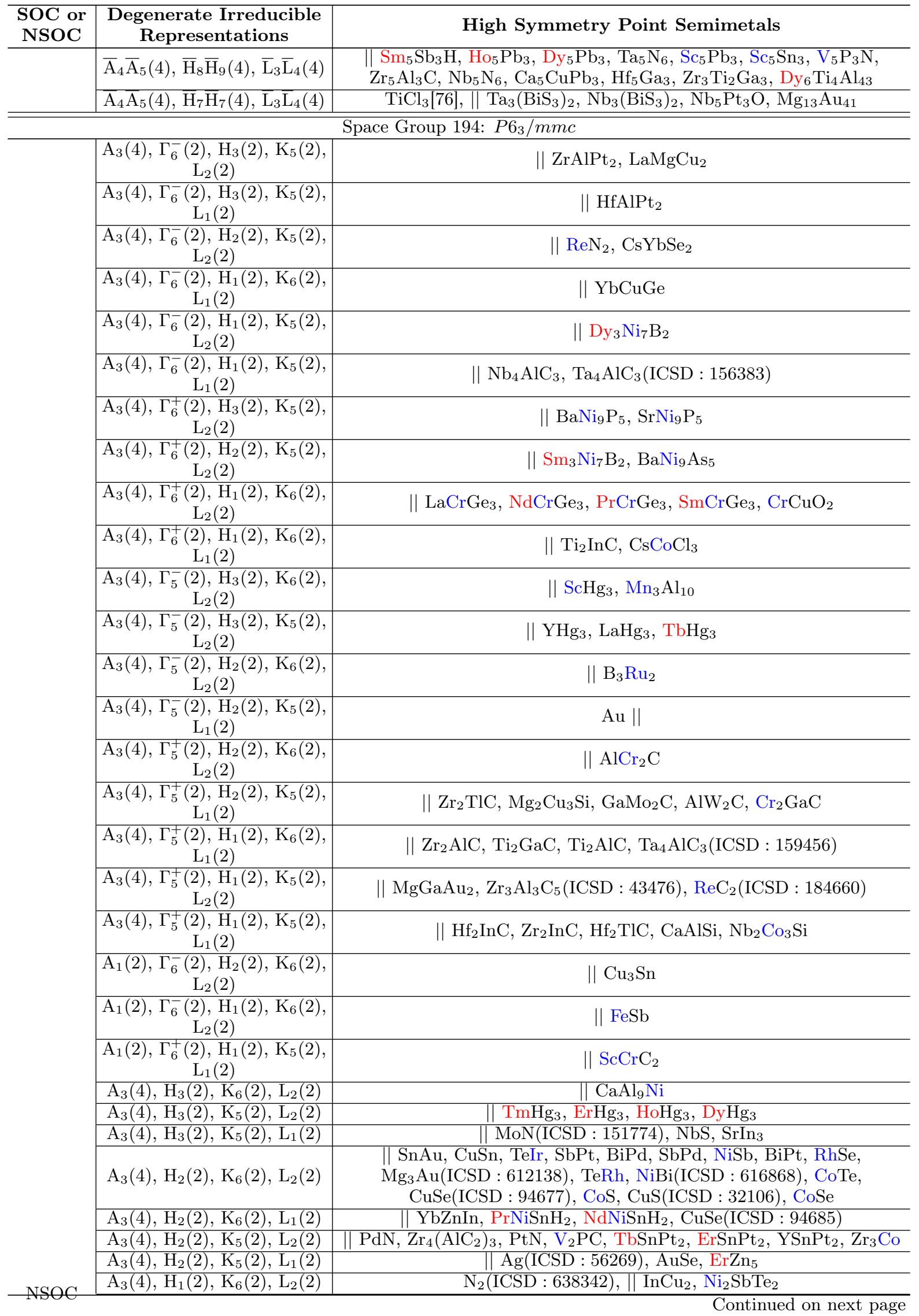


Table II - continued

\begin{tabular}{|c|c|c|}
\hline $\begin{array}{l}\text { SOC or } \\
\text { NSOC }\end{array}$ & $\begin{array}{l}\text { Degenerate Irreducible } \\
\text { Representations }\end{array}$ & High Symmetry Point Semimetals \\
\hline & $\mathrm{A}_{3}(4), \mathrm{H}_{1}(2), \mathrm{K}_{6}(2), \mathrm{L}_{1}(2)$ & $\mathrm{YbCuPb}, \mathrm{LaNiSnH}_{2}, \mathrm{SrAsPt}, \mathrm{La}_{2} \mathrm{Al}_{2} \mathrm{I}, \mathrm{SrAsPd}$ \\
\hline & $\mathrm{A}_{3}(4), \mathrm{H}_{1}(2), \mathrm{K}_{5}(2), \mathrm{L}_{2}(2)$ & $\| \mathrm{Tc}_{2} \mathrm{~N}$ \\
\hline & $\mathrm{A}_{3}(4), \mathrm{H}_{1}(2), \mathrm{K}_{5}(2), \mathrm{L}_{1}(2)$ & $\| \mathrm{TaPd}_{3}$ \\
\hline & $\mathrm{A}_{3}(4), \Gamma_{6}^{-}(2), \mathrm{H}_{3}(2), \mathrm{L}_{2}(2)$ & $\mathrm{Ba}_{3} \mathrm{LuIr}_{2} \mathrm{O}_{9}$ \\
\hline & $\mathrm{A}_{3}(4), \Gamma_{6}^{-}(2), \mathrm{H}_{3}(2), \mathrm{L}_{1}(2)$ & $\| \mathrm{AlV}_{4} \mathrm{C}_{3}$ \\
\hline & $\mathrm{A}_{3}(4), \Gamma_{6}^{-}(2), \mathrm{H}_{2}(2), \mathrm{L}_{2}(2)$ & $\| \mathrm{Tm}_{3} \mathrm{Ni}_{7} \mathrm{~B}_{2}$ \\
\hline & $\mathrm{A}_{3}(4), \Gamma_{6}^{-}(2), \mathrm{H}_{1}(2), \mathrm{L}_{2}(2)$ & $\| \mathrm{La}_{2} \mathrm{In}$ \\
\hline & $\mathrm{A}_{3}(4), \Gamma_{6}^{-}(2), \mathrm{H}_{1}(2), \mathrm{L}_{1}(2)$ & $\| \mathrm{Pr}_{2} \mathrm{In}, \mathrm{Nd}_{2} \mathrm{In}, \mathrm{Pr}_{2} \mathrm{Tl}$ \\
\hline & $\mathrm{A}_{3}(4), \Gamma_{6}^{+}(2), \mathrm{H}_{3}(2), \mathrm{L}_{2}(2)$ & $\| \mathrm{Nb}_{2} \mathrm{GaC}, \mathrm{V}_{2} \mathrm{GaC}, \mathrm{ScCd}_{3}, \mathrm{VTe}$ \\
\hline & $\mathrm{A}_{3}(4), \Gamma_{6}^{+}(2), \mathrm{H}_{3}(2), \mathrm{L}_{1}(2)$ & $\begin{array}{l}\text { II } \mathrm{Nb}_{2} \mathrm{AlC}, \mathrm{Ta}_{2} \mathrm{InC}, \mathrm{Ta}_{2} \mathrm{GaC}_{,} \mathrm{Nb}_{2} \mathrm{InC}, \mathrm{Ta}_{2} \mathrm{AlC}(\mathrm{ICSD}: 187514), \\
\mathrm{AlV}_{2} \mathrm{C}, \mathrm{Lu}_{2} \mathrm{In}, \mathrm{Tm}_{2} \mathrm{In}, \mathrm{Er}_{2} \mathrm{In}, \mathrm{Sc}_{2} \mathrm{Al}, \mathrm{Ho}_{2} \mathrm{In}, \mathrm{Y}_{2} \mathrm{In}, \mathrm{Sm}_{2} \mathrm{In}, \mathrm{Dy}_{2} \mathrm{In}\end{array}$ \\
\hline & $\mathrm{A}_{3}(4), \Gamma_{6}^{+}(2), \mathrm{H}_{2}(2), \mathrm{L}_{2}(2)$ & $\|$ CrCoGe \\
\hline & $\mathrm{A}_{3}(4), \Gamma_{6}^{+}(2), \mathrm{H}_{2}(2), \mathrm{L}_{1}(2)$ & $\|$ VS(ICSD : 52211), $\mathrm{Ta}_{2} \mathrm{AlC}(\mathrm{ICSD}: 181247)$ \\
\hline & $\mathrm{A}_{3}(4), \Gamma_{6}^{+}(2), \mathrm{H}_{1}(2), \mathrm{L}_{1}(2)$ & TiAs(ICSD : 44074), TiSb, $\mathrm{Tb}_{2} \mathrm{Tl}, \mathrm{Dy}_{2} \mathrm{Tl}, \mathrm{Nd}_{2} \mathrm{Tl}$ \\
\hline & $\mathrm{A}_{3}(4), \Gamma_{5}^{-}(2), \mathrm{H}_{3}(2), \mathrm{L}_{2}(2)$ & $\| \mathrm{TcB}_{2}, \mathrm{ReB}_{2}, \mathrm{TbCd}_{3}, \mathrm{~B}_{4} \mathrm{Rh}_{5}, \mathrm{CsYb}_{3} \mathrm{Se}_{4}, \mathrm{InMoS}_{2}$ \\
\hline & $\mathrm{A}_{3}(4), \Gamma_{5}^{-}(2), \mathrm{H}_{3}(2), \mathrm{L}_{1}(2)$ & $\| \mathrm{VS}_{2}$ \\
\hline & $\mathrm{A}_{3}(4), \Gamma_{5}^{-}(2), \mathrm{H}_{2}(2), \mathrm{L}_{1}(2)$ & $\begin{array}{c}\| \mathrm{Hf}_{9} \mathrm{BMo}_{4}, \mathrm{Ba}_{3} \mathrm{ErRu}_{2} \mathrm{O}_{9}, \mathrm{Ba}_{3} \mathrm{YRu}_{2} \mathrm{O}_{9}, \mathrm{Ba}_{3} \mathrm{DyRu}_{2} \mathrm{O}_{9}, \mathrm{Ba}_{3} \mathrm{TbRu}_{2} \mathrm{O}_{9}, \\
\mathrm{Ba}_{3} \mathrm{NdRu}_{2} \mathrm{O}_{9}, \mathrm{Ba}_{3} \mathrm{SmRu}_{2} \mathrm{O}_{9}, \mathrm{Ba}_{3} \mathrm{PrRu}_{2} \mathrm{O}_{9}\end{array}$ \\
\hline & $\mathrm{A}_{3}(4), \Gamma_{5}^{-}(2), \mathrm{H}_{1}(2), \mathrm{L}_{2}(2)$ & $\| \mathrm{B}_{3} \mathrm{Os}_{2}$ \\
\hline & $\mathrm{A}_{3}(4), \Gamma_{5}^{-}(2), \mathrm{H}_{1}(2), \mathrm{L}_{1}(2)$ & $\mathrm{BPt}_{2}, \mathrm{Sn}_{4} \mathrm{BRh}_{6}, \mathrm{CeMg}_{2} \mathrm{Cu}_{9}$ \\
\hline & $\mathrm{A}_{3}(4), \Gamma_{5}^{+}(2), \mathrm{H}_{3}(2), \mathrm{L}_{2}(2)$ & $\| \mathrm{HW}$ \\
\hline & $\mathrm{A}_{3}(4), \Gamma_{5}^{+}(2), \mathrm{H}_{3}(2), \mathrm{L}_{1}(2)$ & $\mathrm{Hf}_{2} \mathrm{Al}_{3} \mathrm{C}_{4}, \mathrm{Mg}_{3} \mathrm{Ir}, \mathrm{In}_{2} \mathrm{Cu}, \mathrm{TiH}$ \\
\hline & $\mathrm{A}_{3}(4), \Gamma_{5}^{+}(2), \mathrm{H}_{2}(2), \mathrm{L}_{2}(2)$ & $\| \mathrm{YbMg}_{2} \mathrm{Cu}_{9}, \mathrm{Sm}_{3} \mathrm{Mg}_{13} \mathrm{Zn}_{30}$ \\
\hline & $\mathrm{A}_{3}(4), \Gamma_{5}^{+}(2), \mathrm{H}_{2}(2), \mathrm{L}_{1}(2)$ & $\mathrm{MnMoN}_{2}, \mathrm{Sc}_{2} \mathrm{Al}_{3} \mathrm{Ru}, \mathrm{Hf}_{9} \mathrm{CoMo}_{4}$ \\
\hline & $\mathrm{A}_{3}(4), \Gamma_{5}^{+}(2), \mathrm{H}_{1}(2), \mathrm{L}_{2}(2)$ & $\| \mathrm{Zr}_{2} \mathrm{Al}, \mathrm{Ti}_{2} \mathrm{Ga}$ \\
\hline & $\mathrm{A}_{3}(4), \Gamma_{5}^{+}(2), \mathrm{H}_{1}(2), \mathrm{L}_{1}(2)$ & $\mathrm{MoH}, \mathrm{Ti}_{2} \mathrm{TlC}$ \\
\hline & $\mathrm{A}_{2}(2), \mathrm{H}_{1}(2), \mathrm{K}_{5}(2), \mathrm{L}_{1}(2)$ & $\| \mathrm{PaPt}_{3}$ \\
\hline & $\mathrm{A}_{1}(2), \mathrm{H}_{3}(2), \mathrm{K}_{6}(2), \mathrm{L}_{1}(2)$ & $\mathrm{Hf}_{9} \mathrm{AsW}_{4}$ \\
\hline & $\mathrm{A}_{1}(2), \mathrm{H}_{2}(2), \mathrm{K}_{6}(2), \mathrm{L}_{2}(2)$ & $\| \mathrm{Zr}_{2} \mathrm{Al}_{3} \mathrm{C}_{5}$ \\
\hline & $\mathrm{A}_{1}(2), \mathrm{H}_{2}(2), \mathrm{K}_{6}(2), \mathrm{L}_{1}(2)$ & $\mathrm{TaPbS}_{2}, \mathrm{TaSnS}_{2}, \mathrm{NbPbS}_{2}$ \\
\hline & $\mathrm{A}_{1}(2), \mathrm{H}_{2}(2), \mathrm{K}_{5}(2), \mathrm{L}_{2}(2)$ & $\| \operatorname{Ir}_{3} \mathrm{~W}$ \\
\hline & $\mathrm{A}_{1}(2), \mathrm{H}_{2}(2), \mathrm{K}_{5}(2), \mathrm{L}_{1}(2)$ & $\mathrm{In}_{2} \mathrm{Bi}, \mathrm{LaTiGe}_{3}, \mathrm{TaSe}_{2}(\mathrm{ICSD}: 651950), \mathrm{NbS}_{2}, \mathrm{NbSe}_{2}, \mathrm{CeCuSi}$ \\
\hline & $\mathrm{A}_{1}(2), \mathrm{H}_{1}(2), \mathrm{K}_{6}(2), \mathrm{L}_{2}(2)$ & $\| \mathrm{Zr}_{3} \mathrm{Al}_{3} \mathrm{C}_{5}(\mathrm{ICSD}: 159412)$ \\
\hline & $\mathrm{A}_{1}(2), \mathrm{H}_{1}(2), \mathrm{K}_{5}(2), \mathrm{L}_{2}(2)$ & $\mathrm{Hf}_{3} \mathrm{Al}_{3} \mathrm{C}_{5}, \mathrm{ReC}_{2}(\mathrm{ICSD}: 184663)$ \\
\hline & $\mathrm{A}_{1}(2), \mathrm{H}_{1}(2), \mathrm{K}_{5}(2), \mathrm{L}_{1}(2)$ & $\| \mathrm{Sc}_{2} \mathrm{InC}, \mathrm{Si}_{3} \mathrm{Ir}$ \\
\hline & $\mathrm{A}_{1}(2), \Gamma_{6}^{-}(2), \mathrm{H}_{3}(2), \mathrm{L}_{1}(2)$ & Sm(ICSD : 52483) \\
\hline & $\mathrm{A}_{1}(2), \Gamma_{6}^{-}(2), \mathrm{H}_{1}(2), \mathrm{L}_{2}(2)$ & $\| \mathrm{Er}_{3} \mathrm{Ni}_{7} \mathrm{~B}_{2}$ \\
\hline & $\mathrm{A}_{1}(2), \Gamma_{6}^{-}(2), \mathrm{H}_{1}(2), \mathrm{L}_{1}(2)$ & $\mathrm{BPt}, \mathrm{USnAu}$ \\
\hline & $\mathrm{A}_{1}(2), \Gamma_{6}^{+}(2), \mathrm{H}_{2}(2), \mathrm{L}_{1}(2)$ & $\| \mathrm{Ce}_{2} \mathrm{Co}_{17}$ \\
\hline & $\mathrm{A}_{1}(2), \Gamma_{5}^{+}(2), \mathrm{H}_{3}(2), \mathrm{L}_{2}(2)$ & $\| \mathrm{Ta}_{4} \mathrm{MnS}_{8}$ \\
\hline & $\mathrm{A}_{1}(2), \Gamma_{5}^{+}(2), \mathrm{H}_{2}(2), \mathrm{L}_{1}(2)$ & $\|$ TiGaPt, TiAlPt, BaVSe $3, \mathrm{Mn}\left(\mathrm{NbS}_{2}\right)_{4}(\mathrm{ICSD}: 643021)$ \\
\hline & $\mathrm{A}_{3}(4), \mathrm{H}_{3}(2), \mathrm{L}_{2}(2)$ & $\begin{array}{l}\| \mathrm{Sb}, \mathrm{Zr}_{2} \mathrm{Al}_{3} \mathrm{C}_{4}, \mathrm{In}(\mathrm{GaAu})_{2}, \mathrm{VS}(\mathrm{ICSD}: 33613), \mathrm{CuS}(\mathrm{ICSD}: 24586), \\
\mathrm{Na}_{9} \mathrm{Sn}_{4}, \mathrm{Pr}_{13} \mathrm{Zn}_{58}, \mathrm{Pr}_{13} \mathrm{Cd}_{58}, \mathrm{Al}_{23} \mathrm{~V}_{4}, \mathrm{Sm}_{13} \mathrm{Cd}_{58}, \mathrm{La}_{13} \mathrm{Cd}_{58}, \mathrm{Nd}_{13} \mathrm{Cd}_{58}\end{array}$ \\
\hline & $\mathrm{A}_{3}(4), \mathrm{H}_{3}(2), \mathrm{L}_{1}(2)$ & $\begin{array}{c}\text { \|Re, } \mathrm{Tc}, \mathrm{ErH}_{2}, \mathrm{~W}_{2} \mathrm{~N}_{3}, \mathrm{Ti}_{3} \mathrm{AlC}_{2}, \mathrm{Mn}\left(\mathrm{NbS}_{2}\right)_{4}(\mathrm{ICSD}: 643016), \mathrm{Cs}_{6} \mathrm{~K}_{7}, \\
\mathrm{~V}_{3} \mathrm{Sn}\end{array}$ \\
\hline & $\mathrm{A}_{3}(4), \mathrm{H}_{2}(2), \mathrm{L}_{2}(2)$ & $\begin{array}{c}\| \mathrm{LiCu}_{2} \mathrm{Sn}, \mathrm{Nb}_{2} \mathrm{PC}, \mathrm{Mg}_{3} \mathrm{Au}(\mathrm{ICSD}: 58541), \mathrm{Nb}_{2} \mathrm{AsC}, \mathrm{V}_{2} \mathrm{AsC}, \\
\mathrm{Lu}_{3} \mathrm{Ni}_{7} \mathrm{~B}_{2}, \mathrm{Er}_{2} \mathrm{Co}_{17}\end{array}$ \\
\hline & $\mathrm{A}_{3}(4), \mathrm{H}_{2}(2), \mathrm{L}_{1}(2)$ & $\begin{array}{c}\| \mathrm{CuSe}(\mathrm{ICSD}: 94686), \mathrm{BaVS}_{3}, \mathrm{BaNbS}_{3}, \mathrm{Zr}_{9} \mathrm{CoMo}_{4}, \mathrm{RbVCl}_{3} \\
\mathrm{Y}_{13} \mathrm{Cd}_{58}\end{array}$ \\
\hline & $\mathrm{A}_{3}(4), \mathrm{H}_{1}(2), \mathrm{L}_{2}(2)$ & $\begin{array}{c}\| \mathrm{Re}_{2} \mathrm{~N}, \mathrm{NiBi}(\mathrm{ICSD}: 58820), \mathrm{MoIr}_{3}, \mathrm{MoRh}_{3}, \mathrm{Rh}_{3} \mathrm{~W}, \mathrm{Co}_{3} \mathrm{~W}, \mathrm{~V}_{6} \mathrm{Ga}_{5} \\
\mathrm{Co}_{3} \mathrm{Mo}, \mathrm{InNi}_{3}, \mathrm{Ta}_{2} \mathrm{~V}_{3} \mathrm{Si}_{,} \mathrm{Sc}_{3} \mathrm{Si}_{4} \mathrm{Ni}_{11}\end{array}$ \\
\hline & $\mathrm{A}_{3}(4), \mathrm{H}_{1}(2), \mathrm{L}_{1}(2)$ & $\| \mathrm{Y}_{3} \mathrm{H}_{2}, \mathrm{Y}_{2} \mathrm{Co}_{17}, \mathrm{Ho}_{2} \mathrm{Co}_{17}, \mathrm{Dy}_{2} \mathrm{Co}_{17}$ \\
\hline & $\mathrm{A}_{3}(4), \Gamma_{6}^{-}(2), \mathrm{K}_{6}(2)$ & $\| \mathrm{KTl}, \mathrm{SrNi}_{3} \mathrm{Ge}_{2}$ \\
\hline & $\mathrm{A}_{3}(4), \Gamma_{6}^{-}(2), \mathrm{K}_{5}(2)$ & $\|$ HfP \\
\hline & $\mathrm{A}_{3}(4), \Gamma_{6}^{+}(2), \mathrm{K}_{6}(2)$ & $\| \mathrm{Mn}_{2} \mathrm{Nb}, \mathrm{TaMn}_{2}$ \\
\hline & $\mathrm{A}_{3}(4), \Gamma_{6}^{+}(2), \mathrm{K}_{5}(2)$ & $\begin{array}{l}\| \mathrm{Hf}_{2} \mathrm{SbP}, \mathrm{HfAs}, \mathrm{Zr}_{2} \mathrm{SbP}, \mathrm{ZrAs}, \mathrm{NaAl}_{2} \mathrm{Sb}_{2} \mathrm{Pr}_{2} \mathrm{Zn}_{17}, \mathrm{La}_{2} \mathrm{Cd}_{17}, \mathrm{ThRe}_{2}, \\
\mathrm{LaRh}_{3}\end{array}$ \\
\hline & $\mathrm{A}_{3}(4), \Gamma_{5}^{-}(2), \mathrm{K}_{6}(2)$ & $\| \mathrm{YbOs}_{2}$ \\
\hline
\end{tabular}


Table II - continued

\begin{tabular}{|c|c|c|}
\hline $\begin{array}{l}\text { SOC or } \\
\text { NSOC }\end{array}$ & $\begin{array}{c}\text { Degenerate Irreducible } \\
\text { Representations }\end{array}$ & High Symmetry Point Semimetals \\
\hline & $\mathrm{A}_{3}(4), \Gamma_{5}^{-}(2), \mathrm{K}_{5}(2)$ & $\mathrm{SrZn}_{2}, \mathrm{BaMg}_{2}, \mathrm{BaRuO}_{3}(\mathrm{ICSD}: 172176)$ \\
\hline & $\mathrm{A}_{3}(4), \Gamma_{5}^{+}(2), \mathrm{K}_{6}(2)$ & $\|$ TiSe \\
\hline & $\mathrm{A}_{3}(4), \Gamma_{5}^{+}(2), \mathrm{K}_{5}(2)$ & 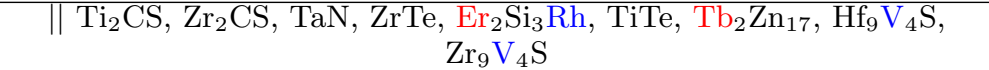 \\
\hline & $\mathrm{A}_{2}(2), \mathrm{H}_{3}(2), \mathrm{L}_{1}(2)$ & $\| \mathrm{Yb}_{23} \mathrm{Mg}_{4} \mathrm{Cu}_{7}$ \\
\hline & $\mathrm{A}_{1}(2), \mathrm{H}_{3}(2), \mathrm{L}_{2}(2)$ & $\| \mathrm{ThAl}_{3}, \mathrm{Pr}_{2} \mathrm{Co}_{2} \mathrm{I}$ \\
\hline & $\mathrm{A}_{1}(2), \mathrm{H}_{3}(2), \mathrm{L}_{1}(2)$ & $\begin{array}{l}\| \mathrm{Mg}_{3} \mathrm{In}, \mathrm{Dy}, \mathrm{Pr}_{2} \mathrm{CBr}, \mathrm{Tb}(\mathrm{ICSD}: 52495), \mathrm{Lu}, \mathrm{V}_{2} \mathrm{InC}, \mathrm{Ho}, \mathrm{Tm}, \mathrm{Er} \\
\quad \mathrm{ZrNi}_{2} \mathrm{Sb}, \mathrm{HfNi}_{2} \mathrm{Sb}_{2} \mathrm{Pr}_{2} \mathrm{Ni}_{2} \mathrm{I}, \mathrm{Ba}_{3} \mathrm{Al}_{5}, \mathrm{Ba}_{3} \mathrm{Al}_{3} \mathrm{Ga}_{2}, \mathrm{Hf}_{9} \mathrm{Mo}_{4} \mathrm{As}\end{array}$ \\
\hline & $\mathrm{A}_{1}(2), \mathrm{H}_{2}(2), \mathrm{L}_{1}(2)$ & $\begin{array}{c}\| \mathrm{Tl}, \mathrm{Li}, \mathrm{Na}(\mathrm{ICSD}: 44758), \mathrm{LiHg}_{3}, \mathrm{BaTaS}_{3}, \mathrm{BaTaSe}_{3}, \mathrm{~B}_{5} \mathrm{~W}_{2}, \mathrm{FeN} \\
\mathrm{Ti}_{3} \mathrm{In}, \mathrm{Sc}_{2} \mathrm{Co}_{3} \mathrm{Si}_{1}, \mathrm{Ba}_{3} \mathrm{SmIr}_{2} \mathrm{O}_{9}, \mathrm{Ba}_{4} \mathrm{Al}_{5}\end{array}$ \\
\hline & $\mathrm{A}_{1}(2), \mathrm{H}_{1}(2), \mathrm{L}_{2}(2)$ & \begin{tabular}{l|ll}
$\mathrm{CoMoP}_{2}, \mathrm{YSiRh}_{2}$ \\
\end{tabular} \\
\hline & $\mathrm{A}_{1}(2), \mathrm{H}_{1}(2), \mathrm{L}_{1}(2)$ & $\begin{array}{c}1 \| \mathrm{Cs}(\mathrm{ICSD}: 57180), \mathrm{ZrN} \mathrm{Ti}_{3} \mathrm{Al}, \mathrm{Sc}_{2} \mathrm{TlC}, \mathrm{SnIr}, \mathrm{IrPb}, \mathrm{TlNi}, \mathrm{GaNi}_{2}, \\
\mathrm{TmTe}, \mathrm{Sc}_{2} \mathrm{AlC}, \mathrm{Ti}_{3} \mathrm{Ga}, \mathrm{InNi}_{2}, \mathrm{ScTe}(\mathrm{ICSD}: 43593), \mathrm{Sc}_{2} \mathrm{GaC}, \mathrm{CoNiSn}, \\
\mathrm{ZrNiP}, \mathrm{Ce}_{3} \mathrm{Al}\end{array}$ \\
\hline & $\Gamma_{6}^{-}(2), \mathrm{K}_{6}(2)$ & $\|$ CeAlIr \\
\hline & $\Gamma_{6}^{-}(2), \mathrm{K}_{5}(2)$ & $\mathrm{BaGe}_{3}, \mathrm{BaSn}_{3}, \mathrm{NdOs}_{2}, \mathrm{PrOs}_{2}, \mathrm{ScRu}_{2}, \mathrm{SmOs}_{2}$ \\
\hline & $\Gamma_{6}^{+}(2), \mathrm{K}_{6}(2)$ & $\mathrm{CrS}(\mathrm{ICSD}: 16723), \mathrm{CrS}(\mathrm{ICSD}: 626613), \mathrm{URe}_{2}$ \\
\hline & $\Gamma_{6}^{+}(2), \mathrm{K}_{5}(2)$ & $\|$ Os, $\mathrm{Ag}(\mathrm{ICSD}: 64707), \mathrm{Ru}, \mathrm{Fe}, \mathrm{OsC}, \mathrm{ZrP}$ \\
\hline & $\Gamma_{5}^{-}(2), \mathrm{K}_{5}(2)$ & $\| \mathrm{Li}_{2} \mathrm{ZnSi}, \mathrm{YRu}_{2}, \mathrm{ErRu}_{2}, \mathrm{LuRu}_{2}$ \\
\hline & $\Gamma_{5}^{+}(2), \mathrm{K}_{6}(2)$ & $\| \mathrm{Al}_{5} \mathrm{Co}_{2}, \mathrm{BaNa}_{2}, \mathrm{TaFe}_{2}$ \\
\hline & $\Gamma_{5}^{+}(2), \mathrm{K}_{5}(2)$ & $\mathrm{Ge}(\mathrm{ICSD}: 189805), \mathrm{Pb}, \mathrm{Hf}_{2} \mathrm{CS}, \mathrm{ScTe}(\mathrm{ICSD}: 262042), \mathrm{Ti}_{6} \mathrm{Sn}_{5}$ \\
\hline & $\mathrm{A}_{3}(4), \mathrm{K}_{6}(2)$ & $\| \mathrm{O}_{2}, \mathrm{Yb}_{3} \mathrm{Ni}_{7} \mathrm{~B}_{2}, \mathrm{VH}, \mathrm{KPb}_{2}, \mathrm{ThMn}_{2}$ \\
\hline & $\mathrm{A}_{3}(4), \mathrm{K}_{5}(2)$ & $\begin{array}{c}\| \mathrm{Ti}_{2} \mathrm{SbP}, \mathrm{MgZn}_{2}, \mathrm{TiAs}(\mathrm{ICSD}: 16773), \mathrm{CaCd}_{2}, \mathrm{CaZn}_{2}, \mathrm{CaMg}_{2}, \\
\mathrm{MoC}(\mathrm{ICSD}: 618295), \mathrm{Dy}_{2} \mathrm{Zn}_{17}, \mathrm{CsNa}_{2}, \mathrm{CsK} 2, \mathrm{VSb}, \mathrm{La}_{2} \mathrm{Mg}_{17}, \\
\mathrm{Ba}_{3} \mathrm{CeRu}_{2} \mathrm{O}_{9}, \mathrm{BaLi}_{4}, \mathrm{Ho}_{2} \mathrm{Si}_{3} \mathrm{Rh}, \mathrm{MnCoGe}, \mathrm{Ce}_{2} \mathrm{Zn}_{17}\end{array}$ \\
\hline & $\mathrm{A}_{3}(4), \Gamma_{6}^{-}(2)$ & $\begin{array}{c}\mathrm{HfBeSi}, \mathrm{ZrBeSi}, \| \mathrm{CaSnHg}, \mathrm{InCu}, \mathrm{Mg}_{2} \mathrm{SiPt}, \mathrm{CaZnPb}, \mathrm{CaHgPb} \\
\text { YbHgPb, NaTl, Mo, CeAlAu }\end{array}$ \\
\hline & $\mathrm{A}_{3}(4), \Gamma_{6}^{+}(2)$ & $\| \mathrm{NiS}_{2}, \mathrm{Ti}_{2} \mathrm{GeC}, \mathrm{Hf}_{2} \mathrm{~S}, \mathrm{Ti}_{3} \mathrm{GeC}_{2}, \mathrm{MnCoSi}, \mathrm{ScMn}_{2}, \mathrm{NdRh}_{3}, \mathrm{NdMn}_{2}$, \\
\hline & $\mathrm{A}_{3}(4), \Gamma_{5}^{-}(2)$ & $\| \mathrm{U}_{2} \mathrm{Fe}_{3} \mathrm{Ge}$ \\
\hline & $\mathrm{A}_{3}(4), \Gamma_{5}^{+}(2)$ & $\begin{array}{c}\mathrm{Na}_{2} \mathrm{Sn}_{3} \mathrm{Au}, \| \mathrm{CuBi}, \mathrm{CuAs}, \mathrm{VP}, \mathrm{ReN}, \mathrm{TcN}, \mathrm{NiS}(\mathrm{ICSD}: 646352) \\
\mathrm{YAlPd}_{2}, \mathrm{LaInPd}_{2}, \mathrm{Cr}, \mathrm{TaSe}_{2}(\mathrm{ICSD}: 651948), \mathrm{TaCr}_{2}, \mathrm{HfNi}_{3} \\
\operatorname{Th}\left(\mathrm{TiH}_{3}\right)_{2}\end{array}$ \\
\hline & $\mathrm{K}_{6}(2)$ & $\begin{array}{c}\mathrm{C}(\mathrm{ICSD}: 193439)\left[58, \| \mathrm{LiAl}, \mathrm{MgCd}_{3}, \mathrm{Co}_{2} \mathrm{Sn}, \mathrm{MnAs},\right. \\
\mathrm{HfCr}_{2}(\mathrm{ICSD}: 626144), \mathrm{Mg}_{5} \mathrm{Rh}_{2}, \mathrm{TmMg}_{2}, \mathrm{HoMg}_{2}, \mathrm{DyMg}_{2}, \mathrm{CeNi}_{5} \mathrm{Sn}, \\
\mathrm{Ca}_{5} \mathrm{Ni}_{15} \mathrm{~B}_{4}, \mathrm{ZrCr}_{2}(\mathrm{ICSD}: 626949), \mathrm{U}_{2} \mathrm{Cr}_{3} \mathrm{Si}_{2} \mathrm{Zr}_{2} \mathrm{Fe}_{3} \mathrm{Si}, \mathrm{Tm}_{2} \mathrm{Ni}_{17} \\
\mathrm{Er}_{2} \mathrm{Ni}_{17}\end{array}$ \\
\hline & $\mathrm{K}_{5}(2)$ & $\begin{array}{l}\mathrm{SrMg}_{4}, \mathrm{Be}[7], \| \mathrm{BaPt}[74], \mathrm{Mg}, \mathrm{SnH}_{4}, \mathrm{Zr}_{2} \mathrm{NiAs}_{2}, \mathrm{Ba}, \mathrm{KAu}_{2}, \mathrm{~B} \mathrm{~B}_{2} \mathrm{~W}, \\
\mathrm{Sn}_{3} \mathrm{Pt}_{2}, \mathrm{KAg}_{2}, \mathrm{Ni}_{3} \mathrm{Sn}, \mathrm{Ta}_{4} \mathrm{FeS}_{8}, \mathrm{KNa}_{2}, \mathrm{ZrCr}_{2}(\mathrm{ICSD}: 106984), \mathrm{HfRe}_{2}, \\
\mathrm{Ce}(\mathrm{ICSD}: 52845), \mathrm{ScOs}_{2}, \mathrm{LuOs}_{2}, \mathrm{Nb}_{2} \mathrm{CrSe}_{4}, \mathrm{YOs}_{2}, \mathrm{TbOs}_{2}, \mathrm{La}_{3} \mathrm{Al}, \\
\quad \mathrm{ZrMn}_{2}(\mathrm{ICSD}: 643951), \mathrm{Ce}(\mathrm{ICSD}: 43381), \mathrm{HfMn}_{2}, \mathrm{Th}_{2} \mathrm{Fe}_{7}\end{array}$ \\
\hline & $\Gamma_{6}^{-}(2)$ & 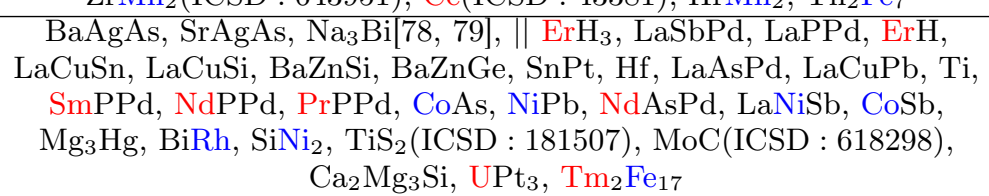 \\
\hline & $\Gamma_{6}^{+}(2)$ & $\begin{array}{c}\text { Ge(ICSD : 636533), Ge(ICSD : 167204), } \mathrm{YbGa}_{2}, \mathrm{YbIn}_{2}, \mathrm{IrN}, \\
\mathrm{ThHg}_{2}, \mathrm{YZnGe}, \mathrm{RhN}, \mathrm{VN}, \mathrm{SmAl}_{3}, \mathrm{YAl}_{3}, \mathrm{Nb}_{2} \mathrm{CS}, \mathrm{TbGa}_{3}, \mathrm{LaAl}_{3}, \mathrm{TiS}, \\
\mathrm{PrAl}_{3}, \mathrm{NdAl}_{3}, \mathrm{NbN}(\mathrm{ICSD}: 644558), \mathrm{Mg}_{5} \mathrm{Pd}_{2}, \mathrm{FeS}(\mathrm{ICSD}: 35008), \\
\mathrm{FeS}(\mathrm{ICSD}: 35008), \mathrm{TiCr}_{2}(\mathrm{ICSD}: 626919), \mathrm{FeSe}, \mathrm{Be}_{2} \mathrm{Fe}, \mathrm{CsFeBr}_{3}, \\
\mathrm{Th}_{2} \mathrm{Ni}_{17}, \mathrm{ReC}\end{array}$ \\
\hline & $\Gamma_{5}^{-}(2)$ & $\begin{array}{c}\mathrm{NdSbAu}, \| \mathrm{CaIn}_{2}(\mathrm{ICSD}: 58686), \mathrm{SmZnSn}, \mathrm{PrZnSn}, \mathrm{NdZnSn}, \mathrm{DyAl}_{3}, \\
\mathrm{DyMg}_{2} \mathrm{Cu}_{9}, \mathrm{PrMg}_{2} \mathrm{Cu}_{9}, \mathrm{NdMg}_{2} \mathrm{Cu}_{9}, \mathrm{TbMg}_{2} \mathrm{Cu}_{9}, \mathrm{SmMg}_{2} \mathrm{Cu}_{9}, \\
\mathrm{YMg}_{2} \mathrm{Cu}_{9}, \mathrm{LaMg}_{2} \mathrm{Cu}_{9}, \mathrm{Ce}_{2} \mathrm{Co}_{5} \mathrm{~B}_{2}\end{array}$ \\
\hline
\end{tabular}


Table II - continued

\begin{tabular}{|c|c|c|}
\hline $\begin{array}{l}\text { SOC or } \\
\text { NSOC }\end{array}$ & $\begin{array}{l}\text { Degenerate Irreducible } \\
\text { Representations }\end{array}$ & High Symmetry Point Semimetals \\
\hline & $\Gamma_{5}^{+}(2)$ & 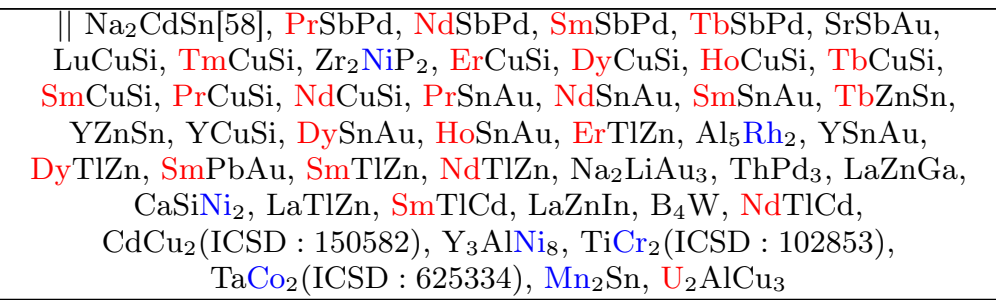 \\
\hline & $\mathrm{A}_{3}(4)$ & 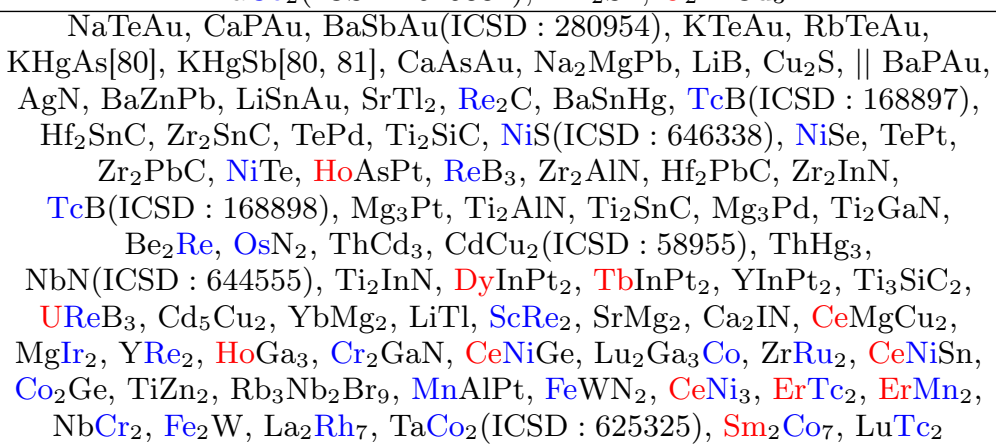 \\
\hline \multirow[b]{3}{*}{$\mathrm{SOC}$} & $\overline{\mathrm{A}}_{6}(4), \overline{\mathrm{H}}_{9}(2), \overline{\mathrm{L}}_{3} \overline{\mathrm{L}}_{4}(4)$ & $\mathrm{N}_{2}(\mathrm{ICSD}: 638342) \|$ \\
\hline & $\overline{\mathrm{A}}_{6}(4), \overline{\mathrm{H}}_{8}(2), \overline{\mathrm{L}}_{3} \overline{\mathrm{L}}_{4}(4)$ & $\| \mathrm{Li}$ \\
\hline & $\overline{\mathrm{A}}_{6}(4), \overline{\mathrm{L}}_{3} \overline{\mathrm{L}}_{4}(4)$ & 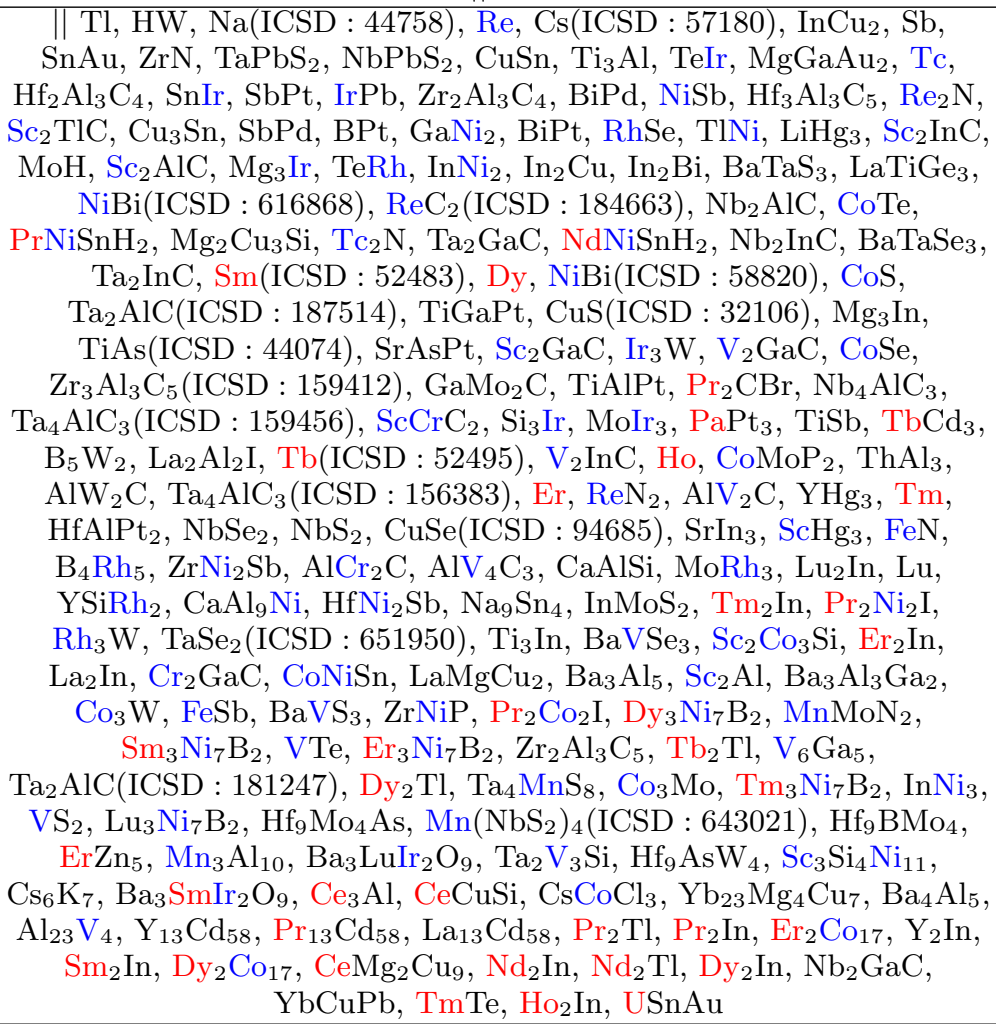 \\
\hline
\end{tabular}


Table II - continued

\begin{tabular}{|c|c|c|}
\hline $\begin{array}{l}\text { SOC or } \\
\text { NSOC }\end{array}$ & $\begin{array}{l}\text { Degenerate Irreducible } \\
\text { Representations }\end{array}$ & High Symmetry Point Semimetals \\
\hline & $\overline{\mathrm{A}}_{4} \overline{\mathrm{A}}_{5}(4), \overline{\mathrm{L}}_{3} \overline{\mathrm{L}}_{4}(4)$ & 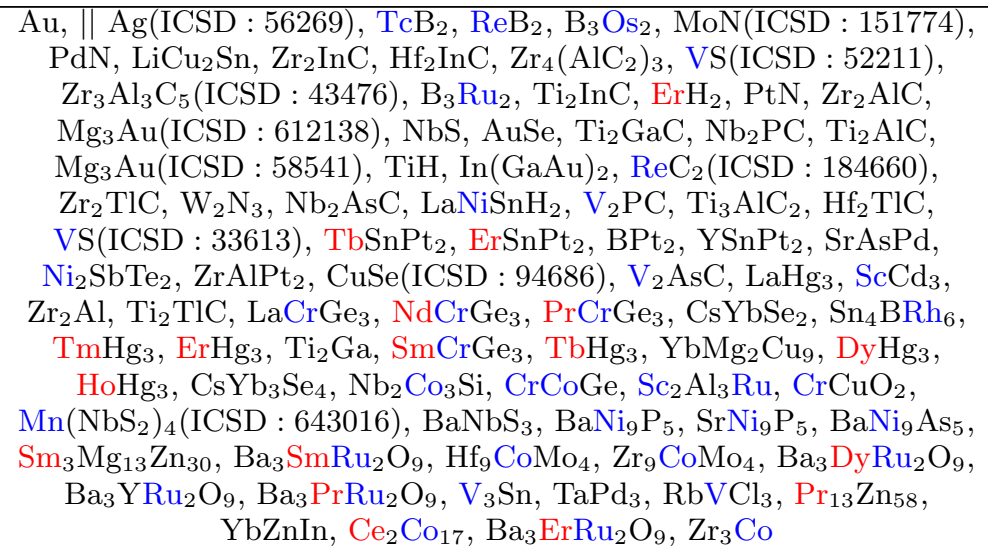 \\
\hline & $\overline{\mathrm{L}}_{3} \overline{\mathrm{L}}_{4}(4)$ & $\| \mathrm{Sm}_{13} \mathrm{Cd}_{58}, \mathrm{Nd}_{13} \mathrm{Cd}_{58}$ \\
\hline & & Space Group 197: I23 \\
\hline & $\Gamma_{4}(3), \mathrm{H}_{4}(3), \mathrm{P}_{4}(3)$ & $\mathrm{Zn}\left(\mathrm{Bi}_{3} \mathrm{O}_{5}\right)_{4}$ \\
\hline $\mathrm{NSOC}$ & $\mathrm{H}_{4}(3), \mathrm{P}_{4}(3)$ & $\mathrm{Ba}_{2}\left(\mathrm{IrO}_{3}\right)_{3}$ \\
\hline NSUC & $\Gamma_{4}(3), \mathrm{P}_{4}(3)$ & $\| \mathrm{La}_{4} \mathrm{Re}_{6} \mathrm{O}_{19}$ \\
\hline & $\Gamma_{2} \Gamma_{3}(2)$ & $\mathrm{Nd}_{4} \mathrm{O}_{6} \mathrm{O}_{19}, \mathrm{La}_{4} \mathrm{Ru}_{6} \mathrm{O}_{19}$ \\
\hline $\mathrm{SOC}$ & $\overline{\mathrm{H}}_{6} \overline{\mathrm{H}}_{7}(4)$ & $\| \mathrm{Ba}_{2}\left(\mathrm{IrO}_{3}\right)_{3}$ \\
\hline & $\bar{\Gamma}_{6} \bar{\Gamma}_{7}(4)$ & $\| \mathrm{La}_{4} \mathrm{Re}_{6} \mathrm{O}_{19}, \mathrm{La}_{4} \mathrm{Ru}_{6} \mathrm{O}_{19}, \mathrm{Zn}\left(\mathrm{Bi}_{3} \mathrm{O}_{5}\right)_{4}$ \\
\hline & & Space Group 198: $P 2_{1} 3$ \\
\hline & $\Gamma_{4}(3), \mathrm{R}_{2} \mathrm{R}_{2}(4)$ & $\|$ BaAsPt, HfSb, ZrSb \\
\hline NSOC & $\Gamma_{4}(3), \mathrm{R}_{1} \mathrm{R}_{3}(4)$ & $\begin{array}{c}\text { GeRh }[82, \| \text { SiRh }[82] \text {, AlPt, SnRh, GaPt, CoGe, CoSi, AlPd, BaPPt, } \\
\text { BeAu, GaPd, AlAu }, \text { SbPdSe, NiSbS, BiTePt, SbTePd, BiPdSe, } \\
\text { BiTePd, AsPdS, NiSbSe, NiAsSe, NiAsS, AsPdSe, NiPS }\end{array}$ \\
\hline & $\mathrm{R}_{1} \mathrm{R}_{3}(4)$ & $\|$ ReSi, SiTc, MnSi \\
\hline & $\Gamma_{4}(3)$ & $\mathrm{Ga}\left(\mathrm{Ag}_{3} \mathrm{Se}_{2}\right)_{3}, \mathrm{NaClO}_{3}(\mathrm{ICSD}: 31056), \| \mathrm{SiNi}, \mathrm{CrSi}, \mathrm{Mn}_{3} \mathrm{SiIr}, \mathrm{Mn}_{3} \mathrm{GeIr}$ \\
\hline & $\Gamma_{2} \Gamma_{3}(2)$ & $\begin{array}{l}\text { "I CaSiPt, SrSiPt, SrGePt, SrSiPd, SrPIr, BaGePt, BaPIr, LaSiIr, } \\
\text { SrAl }\end{array}$ \\
\hline $\mathrm{C} \cap \mathrm{C}$ & $\bar{\Gamma}_{6} \bar{\Gamma}_{7}(4), \overline{\mathrm{R}}_{7} \overline{\mathrm{R}}_{7}(6)$ & $\begin{array}{c}\text { GeRh[82], } \| \text { SiRh }[82 \text {, SnRh, AlPt, CoSi, GaPt, BaAsPt, AlPd, BeAu, } \\
\text { GaPd, BaPPt, HfSb, ZrSb, SbTePd, BiTePd, BiPdSe }\end{array}$ \\
\hline SUC & $\overline{\mathrm{R}}_{7} \overline{\mathrm{R}}_{7}(6)$ & $\begin{array}{c}\| \text { SbPdSe, NiSbS, SiTc, ReSi, } \mathrm{AlAu}_{4}, \mathrm{BiTePt}, \mathrm{AsPdS}, \mathrm{NiSbSe} \\
\text { NiAsSe, AsPdSe, MnSi, NiAsS, NiPS }\end{array}$ \\
\hline & $\bar{\Gamma}_{6} \bar{\Gamma}_{7}(4)$ & $\begin{array}{c}\mathrm{Ga}\left(\mathrm{Ag}_{3} \mathrm{Se}_{2}\right)_{3}, \mathrm{NaClO}_{3}(\mathrm{ICSD}: 31056), \| \mathrm{SiNi}, \mathrm{CaSiPt}, \mathrm{SrSiPt} \\
\text { SrGePt, SrSiPd, BaGePt, SrPIr, LaSiIr, BaPIr, HfSn, Mn }{ }_{3} \mathrm{GeIr}, \mathrm{SrAl}\end{array}$ \\
\hline & & Space Group 199: $I 2_{1} 3$ \\
\hline $\mathrm{NSOC}$ & $\Gamma_{4}(3), \mathrm{P}_{1}(2)$ & $\| \operatorname{Pr}_{5} \mathrm{Al}_{2} \mathrm{Ru}_{3}$ \\
\hline & $\Gamma_{4}(3), \mathrm{H}_{4}(3)$ & $\| \mathrm{Bi}_{2} \mathrm{Pd}_{3} \mathrm{~S}_{2}, \mathrm{Ni}_{3}(\mathrm{BiS})_{2}$ \\
\hline $\mathrm{SOC}$ & $\bar{\Gamma}_{6} \bar{\Gamma}_{7}(4), \overline{\mathrm{P}}_{7}(3)$ & $\| \operatorname{Pr}_{5} \mathrm{Al}_{2} \mathrm{Ru}_{3}$ \\
\hline & $\bar{\Gamma}_{6} \bar{\Gamma}_{7}(4), \overline{\mathrm{H}}_{6} \overline{\mathrm{H}}_{7}(4)$ & $\| \mathrm{Bi}_{2} \mathrm{Pd}_{3} \mathrm{~S}_{2}, \mathrm{Ni}_{3}(\mathrm{BiS})_{2}$ \\
\hline & & Space Group 200: $P m \overline{3}$ \\
\hline & $\Gamma_{4}^{-}(3), \mathrm{R}_{4}^{-}(3)$ & $\mathrm{Mn}_{4} \mathrm{Al}_{19}$ \\
\hline NSOC & $\mathrm{R}_{4}^{-}(3)$ & $\| \mathrm{Mg}_{2} \mathrm{Zn}_{11}$ \\
\hline & $\Gamma_{4}^{-}(3)$ & $\mathrm{NaLu}\left(\mathrm{Pd}_{3} \mathrm{O}_{4}\right)_{2}$ \\
\hline & & Space Group 204: $\operatorname{Im} \overline{3}$ \\
\hline & $\Gamma_{4}^{-}(3), \mathrm{H}_{4}^{+}(3), \mathrm{P}_{4}(3)$ & $\| \mathrm{Sc}_{3} \mathrm{Zn}_{17}$ \\
\hline & $\Gamma_{4}^{+}(3), \mathrm{H}_{4}^{-}(3), \mathrm{P}_{4}(3)$ & $\mathrm{Co}_{4} \mathrm{SnSb}_{12}, \mathrm{NiP}_{3}, \mathrm{Be}_{17} \mathrm{Ru}_{3}, \mathrm{Nd}_{12} \mathrm{Ni}_{6} \mathrm{Sn}$ \\
\hline & $\Gamma_{4}^{+}(3), \mathrm{H}_{2}^{-} \mathrm{H}_{3}^{-}(2), \mathrm{P}_{4}(3)$ & $\| \mathrm{ReO}_{3}$ \\
\hline & $\Gamma_{2}^{+} \Gamma_{3}^{+}(2), \mathrm{H}_{4}^{-}(3), \mathrm{P}_{4}(3)$ & $\mathrm{U}\left(\mathrm{FeP}_{3}\right)_{4}$ \\
\hline & $\mathrm{H}_{4}^{-}(3), \mathrm{P}_{4}(3)$ & $\| \mathrm{P}_{3} \mathrm{Pd}$ \\
\hline & $\Gamma_{4}^{+}(3), \mathrm{P}_{4}(3)$ & $\| \mathrm{Dy}_{4} \mathrm{Ni}_{12} \mathrm{Sn}_{25}$ \\
\hline NSOC & $\mathrm{P}_{4}(3)$ & $\| \mathrm{Ba}\left(\mathrm{Ge}_{3} \mathrm{Pt}\right)_{4}, \mathrm{Li}_{13}\left(\mathrm{Ga}_{7} \mathrm{Cu}_{2}\right)_{3}$ \\
\hline & $\mathrm{H}_{4}^{-}(3)$ & $\| \mathrm{Sr}\left(\mathrm{Sb}_{3} \mathrm{Ru}\right)_{4}, \mathrm{Ba}\left(\mathrm{Sb}_{3} \mathrm{Ru}\right)_{4}, \mathrm{Yb}\left(\mathrm{FeP}_{3}\right)_{4}$ \\
\hline & $\mathrm{H}_{4}^{+}(3)$ & $\| \mathrm{Al}_{12} \mathrm{~W}, \mathrm{Al}_{12} \mathrm{Mo}, \mathrm{Nd}_{12} \mathrm{Co}_{6} \mathrm{Sn}$ \\
\hline
\end{tabular}


Table II - continued

\begin{tabular}{|c|c|c|}
\hline $\begin{array}{l}\text { SOC or } \\
\text { NSOC }\end{array}$ & $\begin{array}{l}\text { Degenerate Irreducible } \\
\text { Representations }\end{array}$ & High Symmetry Point Semimetals \\
\hline & $\mathrm{H}_{2}^{+} \mathrm{H}_{3}^{+}(2)$ & $\| \mathrm{Y}_{12} \mathrm{Ni}_{6} \mathrm{~Pb}$ \\
\hline & $\Gamma_{4}^{-}(3)$ & $\| \mathrm{SmC}_{10}$ \\
\hline & $\Gamma_{4}^{+}(3)$ & $\mathrm{Sb}_{3} \mathrm{Ir}, \mathrm{CoAs}_{3}, \mathrm{As}_{3} \mathrm{Rh}[83], \mathrm{Sb}_{3} \mathrm{Rh}, \| \mathrm{NaCu}_{3}\left(\mathrm{RuO}_{3}\right)_{4}, \mathrm{Cs}_{2} \mathrm{Hg}_{27}$ \\
\hline & $\Gamma_{2}^{+} \Gamma_{3}^{+}(2)$ & $\mathrm{CoP}_{3}, \mathrm{RhN}_{3}, \mathrm{P}_{3} \mathrm{Rh}, \| \mathrm{CoN}_{3}$ \\
\hline \multirow{7}{*}{$\mathrm{SOC}$} & $\bar{\Gamma}_{9} \bar{\Gamma}_{10}(4), \overline{\mathrm{H}}_{6} \overline{\mathrm{H}}_{7}(4), \overline{\mathrm{P}}_{6} \overline{\mathrm{P}}_{7}(4)$ & \\
\hline & $\bar{\Gamma}_{6} \bar{\Gamma}_{7}(4), \overline{\mathrm{H}}_{9} \overline{\mathrm{H}}_{10}(4), \overline{\mathrm{P}}_{6} \overline{\mathrm{P}}_{7}(4)$ & $\mathrm{Nd}_{12} \mathrm{Ni}_{6} \mathrm{Sn}$ \\
\hline & $\bar{\Gamma}_{6} \bar{\Gamma}_{7}(4), \overline{\mathrm{H}}_{9} \overline{\mathrm{H}}_{10}(4)$ & $\| \mathrm{U}\left(\mathrm{FeP}_{3}\right)_{4}$ \\
\hline & $\overline{\mathrm{P}}_{6} \overline{\mathrm{P}}_{7}(4)$ & $\mathrm{Li}_{13}\left(\mathrm{Ga}_{7} \mathrm{Cu}_{2}\right)_{3}, \mathrm{P}_{3} \mathrm{Pd}$ \\
\hline & $\overline{\mathrm{H}}_{9} \overline{\mathrm{H}}_{10}(4)$ & $\| \mathrm{ReO}_{3}, \mathrm{Be}_{17} \mathrm{Ru}_{3}$ \\
\hline & $\overline{\mathrm{H}}_{6} \overline{\mathrm{H}}_{7}(4)$ & $\| \mathrm{Nd}_{12} \mathrm{Co}_{6} \mathrm{Sn}, \mathrm{Y}_{12} \mathrm{Ni}_{6} \mathrm{~Pb}$ \\
\hline & $\bar{\Gamma}_{6} \bar{\Gamma}_{7}(4)$ & 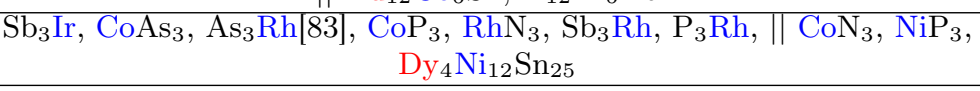 \\
\hline \multicolumn{3}{|r|}{ Space Group 205: $P a \overline{3}$} \\
\hline \multirow{6}{*}{ NSOC } & $\Gamma_{4}^{-}(3), \mathrm{M}_{1} \mathrm{M}_{2}(4), \mathrm{R}_{1}^{-} \mathrm{R}_{3}^{-}(4)$ & $\| \mathrm{Sb}_{2} \mathrm{Au}$ \\
\hline & $\Gamma_{4}^{+}(3), \mathrm{M}_{1} \mathrm{M}_{2}(4), \mathrm{R}_{1}^{+} \mathrm{R}_{3}^{+}(4)$ & \multirow{2}{*}{$\begin{array}{c}\mathrm{Te}_{2} \mathrm{Rh}, \mathrm{Te}_{2} \mathrm{Ir}, \mathrm{RhSe}_{2}, \mathrm{RhS}_{2}, \mathrm{CoS}_{2}, \mathrm{CoS}_{2}, \mathrm{MnS}_{2} \\
\| \mathrm{CuS}_{2}, \mathrm{CuSe}_{2}\end{array}$} \\
\hline & $\mathrm{M}_{1} \mathrm{M}_{2}(4), \mathrm{R}_{2}^{+} \mathrm{R}_{2}^{+}(4)$ & \\
\hline & $\Gamma_{4}^{-}(3)$ & AlSb, GaAs, InAs $\|$ \\
\hline & $\Gamma_{4}^{+}(3)$ & $\mathrm{Cu}_{3} \mathrm{WO}_{6}, \mathrm{OsS}_{2}, \mathrm{In}_{5} \mathrm{~S}_{4}$ \\
\hline & $\Gamma_{2}^{+} \Gamma_{3}^{+}(2)$ & $\| \mathrm{PdF}_{2}, \mathrm{ThC}_{2}$ \\
\hline \multirow{5}{*}{$\mathrm{SOC}$} & $\bar{\Gamma}_{9} \bar{\Gamma}_{10}(4), \overline{\mathrm{R}}_{11} \overline{\mathrm{R}}_{11}(6)$ & $\| \mathrm{Sb}_{2} \mathrm{Au}$ \\
\hline & $\bar{\Gamma}_{6} \bar{\Gamma}_{7}(4), \overline{\mathrm{R}}_{10} \overline{\mathrm{R}}_{10}(6)$ & $\mathrm{Te}_{2} \mathrm{Rh}, \mathrm{Te}_{2} \mathrm{Ir}, \mathrm{RhSe}_{2}, \mathrm{RhS}_{2}$ \\
\hline & $\overline{\mathrm{R}}_{10} \overline{\mathrm{R}}_{10}(6)$ & $\mathrm{CuS}_{2}, \mathrm{CuSe}_{2}, \mathrm{CoS}_{2}, \mathrm{CoS}_{2}$ \\
\hline & $\bar{\Gamma}_{9} \bar{\Gamma}_{10}(4)$ & AlSb, InAs, GaAs $\|$ \\
\hline & $\bar{\Gamma}_{6} \bar{\Gamma}_{7}(4)$ & $\| \mathrm{PdF}_{2}, \mathrm{ThC}_{2}$ \\
\hline & & Space Group 206: $I a \overline{3}$ \\
\hline & $\mathrm{P}_{2} \mathrm{P}_{2}(4)$ & $\mathrm{Cu}_{3} \mathrm{TeO}_{6}[5]$ \\
\hline & $\mathrm{H}_{4}^{-}(3)$ & $\mathrm{Pt}\left(\mathrm{OF}_{3}\right)_{2}, \mathrm{Ge}$ \\
\hline NSOC & $\mathrm{H}_{4}^{+}(3)$ & $\mathrm{Si} \|$ \\
\hline & $\Gamma_{4}^{-}(3)$ & $\mathrm{Zn}_{3} \mathrm{~N}_{2}, \mathrm{Cd}_{3} \mathrm{~N}_{2} \|$ \\
\hline & $\Gamma_{4}^{+}(3)$ & $\mathrm{Tl}_{2} \mathrm{O}_{3}, \| \mathrm{V}_{2} \mathrm{O}_{3}$ \\
\hline & $\overline{\mathrm{P}}_{7} \overline{\mathrm{P}}_{7}(6)$ & $\| \mathrm{Cu}_{3} \mathrm{TeO}_{6}[5]$ \\
\hline $\mathrm{SOC}$ & $\overline{\mathrm{H}}_{9} \overline{\mathrm{H}}_{10}(4)$ & $\mathrm{Pt}\left(\mathrm{OF}_{3}\right)_{2}, \mathrm{Ge}$ \\
\hline & $\overline{\mathrm{H}}_{6} \overline{\mathrm{H}}_{7}(4)$ & $\mathrm{Si} \|$ \\
\hline & & Space Group 212: $P 4_{3} 32$ \\
\hline & $\Gamma_{4}(3), \mathrm{R}_{3}(4)$ & $\mathrm{Li}_{2} \mathrm{BPt}_{3}$ \\
\hline NSOC & $\mathrm{R}_{3}(4)$ & $\mathrm{Li}_{2} \mathrm{BPd}_{3}$ \\
\hline & $\Gamma_{5}(3)$ & $\| \mathrm{K}_{6} \mathrm{Sn}_{25}$ \\
\hline $\mathrm{SOC}$ & $\overline{\mathrm{R}}_{7} \overline{\mathrm{R}}_{8}(6)$ & $\mathrm{Li}_{2} \mathrm{BPt}_{3}, \mathrm{Li}_{2} \mathrm{BPd}_{3}$ \\
\hline & $\bar{\Gamma}_{8}(4)$ & $\| \mathrm{K}_{6} \mathrm{Sn}_{25}$ \\
\hline & & Space Group 213: $P 4_{1} 32$ \\
\hline & $\Gamma_{5}(3), \mathrm{R}_{3}(4)$ & $\| \mathrm{Co}_{2} \mathrm{Mo}_{3} \mathrm{~N}, \mathrm{Mn}$ \\
\hline & $\Gamma_{4}(3), \mathrm{R}_{3}(4)$ & Li $\|$ \\
\hline NSOC & $\mathrm{R}_{3}(4)$ & $\mathrm{Mo}_{3} \mathrm{Pt}_{2} \mathrm{~N}, \mathrm{Ni}_{2} \mathrm{~W}_{3} \mathrm{~N}, \mathrm{Ni}_{2} \mathrm{Mo}_{3} \mathrm{~N}, \mathrm{Fe}_{2} \mathrm{Re}_{3}$ \\
\hline & $\mathrm{R}_{1} \mathrm{R}_{2}(4)$ & $\| \mathrm{Mo}_{3} \mathrm{Pd}_{2} \mathrm{~N}$ \\
\hline & $\Gamma_{5}(3)$ & $\mathrm{Mg}_{3} \mathrm{Ru}_{2}, \mathrm{Re}_{2} \mathrm{~W}_{3} \mathrm{C}$ \\
\hline & $\bar{\Gamma}_{8}(4), \overline{\mathrm{R}}_{7} \overline{\mathrm{R}}_{8}(6)$ & $\mathrm{Li}, \| \mathrm{Fe}_{2} \mathrm{Re}_{3}, \mathrm{Mn}$ \\
\hline $\mathrm{SOC}$ & $\overline{\mathrm{R}}_{7} \overline{\mathrm{R}}_{8}(6)$ & $\mathrm{Mo}_{3} \mathrm{Pt}_{2} \mathrm{~N}, \mathrm{Mo}_{3} \mathrm{Pd}_{2} \mathrm{~N}, \mathrm{Ni}_{2} \mathrm{~W}_{3} \mathrm{~N}, \mathrm{Ni}_{2} \mathrm{Mo}_{3} \mathrm{~N}, \mathrm{Co}_{2} \mathrm{Mo}_{3} \mathrm{~N}$ \\
\hline & $\bar{\Gamma}_{8}(4)$ & $\| \mathrm{Mg}_{3} \mathrm{Ru}_{2}$ \\
\hline & & Space Group 214: $I 4_{1} 32$ \\
\hline & $\Gamma_{5}(3), \mathrm{H}_{4}(3)$ & $\| \mathrm{La}_{3} \mathrm{PbI}_{3}, \mathrm{~Tb}_{3} \mathrm{SiBr}_{3}, \mathrm{La}_{3} \mathrm{SiBr}_{3}$ \\
\hline $\mathrm{NSOC}$ & $\Gamma_{4}(3), \mathrm{H}_{5}(3)$ & \begin{tabular}{l||l}
$\mathrm{C}$ \\
\end{tabular} \\
\hline NSOC & $\mathrm{H}_{5}(3)$ & $\mathrm{La}_{3} \mathrm{Sn}_{13} \mathrm{Rh}_{4}$ \\
\hline & $\mathrm{H}_{4}(3)$ & $\| \mathrm{Pr}_{3} \mathrm{PtI}_{3}$ \\
\hline & $\bar{\Gamma}_{8}(4), \overline{\mathrm{P}}_{7}(3)$ & $\| \mathrm{Tb}_{3} \mathrm{SiBr}_{3}$ \\
\hline $\mathrm{SOC}$ & $\overline{\mathrm{H}}_{8}(4)$ & $\mathrm{Pr}_{3} \mathrm{PtI}_{3}, \mathrm{La}_{3} \mathrm{Sn}_{13} \mathrm{Rh}_{4}$ \\
\hline & $\bar{\Gamma}_{8}(4)$ & $\mathrm{C}, \mathrm{La}_{3} \mathrm{PbI}_{3}, \mathrm{La}_{3} \mathrm{SiBr}_{3}$ \\
\hline
\end{tabular}


Table II - continued

\begin{tabular}{|c|c|c|}
\hline $\begin{array}{l}\text { SOC or } \\
\text { NSOC }\end{array}$ & $\begin{array}{l}\text { Degenerate Irreducible } \\
\text { Representations }\end{array}$ & High Symmetry Point Semimetals \\
\hline & & Space Group 215: $P \overline{4} 3 m$ \\
\hline \multirow{8}{*}{ NSOC } & $\Gamma_{5}(3), \mathrm{R}_{5}(3), \mathrm{X}_{5}(2)$ & $\| \mathrm{Al}_{4} \mathrm{Cu}_{9}, \mathrm{In}_{9}\left(\mathrm{CuSe}_{4}\right)_{4}$ \\
\hline & $\mathrm{R}_{5}(3), \mathrm{X}_{5}(2)$ & $\| \operatorname{In}_{4} \mathrm{Au}_{9}$ \\
\hline & $\Gamma_{5}(3), \mathrm{R}_{4}(3)$ & $\mathrm{In}_{4} \mathrm{Cu}_{9}, \mathrm{In}_{4} \mathrm{Ag}_{9}, \mathrm{Fe}_{4} \mathrm{C}$ \\
\hline & $\Gamma_{4}(3), \mathrm{R}_{5}(3)$ & $\mathrm{Cu}_{3} \mathrm{AsS}_{4}, \mathrm{Ga}_{4} \mathrm{BiAs}_{3}$ \\
\hline & $\Gamma_{3}(2), \mathrm{R}_{4}(3)$ & $\| \mathrm{Li}_{10} \mathrm{~Pb}_{3}$ \\
\hline & $\mathrm{R}_{3}(2)$ & LiMgIr $\|$ \\
\hline & $\Gamma_{5}(3)$ & $\| \mathrm{Ca}_{4} \mathrm{Hg}_{9}$ \\
\hline & $\Gamma_{4}(3)$ & $\mathrm{As}_{4} \mathrm{C}_{3} \|$ \\
\hline \multirow{2}{*}{ SOC } & $\bar{\Gamma}_{8}(4), \overline{\mathrm{R}}_{8}(4)$ & $\mathrm{In}_{4} \mathrm{Ag}_{9}, \mathrm{Li}_{10} \mathrm{~Pb}_{3}, \mathrm{Ca}_{4} \mathrm{Hg}_{9}, \mathrm{Ga}_{4} \mathrm{BiAs}_{3}, \mathrm{Fe}_{4} \mathrm{C}$ \\
\hline & $\bar{\Gamma}_{8}(4)$ & $\| \operatorname{In}_{4} \mathrm{Cu}_{9}$ \\
\hline \multicolumn{3}{|r|}{ Space Group 216: $F \overline{4} 3 m$} \\
\hline \multirow{14}{*}{ NSOC } & $\Gamma_{5}(3), \mathrm{L}_{3}(2), \mathrm{X}_{5}(2)$ & $\| \mathrm{Dy}_{4} \mathrm{CdCo}, \mathrm{Tb}_{4} \mathrm{CdCo}, \mathrm{Ho}_{4} \mathrm{CdCo}$ \\
\hline & $\Gamma_{4}(3), \mathrm{L}_{3}(2), \mathrm{X}_{5}(2)$ & $\begin{array}{c}\text { \| } \mathrm{Li}_{2} \mathrm{GaAu}, \mathrm{Li}_{2} \mathrm{InAu}, \mathrm{Li}_{2} \mathrm{TlAu}, \mathrm{Li}_{2} \mathrm{InAg}, \mathrm{Li}_{2} \mathrm{AlAg}, \mathrm{CdC}, \mathrm{AgN} \\
\text { TiGaRh, Tb }{ }_{4} \mathrm{CdRh}, \mathrm{Dy}_{4} \mathrm{CdRh}, \mathrm{Ho}_{4} \mathrm{CdRh}, \mathrm{Tb}_{4} \mathrm{MgIr}, \mathrm{Dy}{ }_{4} \mathrm{MgRh}, \\
\mathrm{Mn}_{5}\left(\mathrm{NiBi}_{2}\right)_{2}\end{array}$ \\
\hline & $\mathrm{L}_{3}(2), \mathrm{X}_{5}(2)$ & $\begin{array}{c}\text { T/ } \mathrm{TmCu}_{5}, \mathrm{ErCu}_{5}, \mathrm{DyCu}_{5}, \mathrm{HoCu}_{5}, \mathrm{SmCu}_{4} \mathrm{Ag}, \mathrm{LuCu}_{5}, \mathrm{TbCu}_{4} \mathrm{Au}, \\
\mathrm{HoCu}_{4} \mathrm{Ag}, \mathrm{HoCu}_{4} \mathrm{Au}, \mathrm{TmCu}_{4} \mathrm{Ag}, \mathrm{TbCu}_{5}, \mathrm{YbCdCu}, \mathrm{TbCu}_{4} \mathrm{Ag} \\
\mathrm{ErCu}_{4} \mathrm{Ag}, \mathrm{DyCu} \mathrm{Cu}_{4} \mathrm{Ag}, \mathrm{MnSbPt}_{4} \mathrm{ErCu}_{4} \mathrm{Au}\end{array}$ \\
\hline & $\Gamma_{5}(3), \mathrm{X}_{5}(2)$ & $\mathrm{Ba}_{19} \mathrm{Na}_{29} \mathrm{Li}_{13} \|$ \\
\hline & $\Gamma_{5}(3), \mathrm{L}_{3}(2)$ & $\| \mathrm{ZrZnNi}_{4}, \mathrm{LaInPt}_{4}, \mathrm{UPt}_{5}, \mathrm{Mg}_{3} \mathrm{Si}_{8} \mathrm{Ir}_{3}, \mathrm{Mg}_{29} \mathrm{Ir}_{4}$ \\
\hline & $\Gamma_{4}(3), \mathrm{X}_{5}(2)$ & $\begin{array}{c}\| \mathrm{YbBiAu}, \mathrm{Ag}_{7} \mathrm{NO}_{6}, \mathrm{Ta}_{5} \mathrm{AuS}, \mathrm{La}_{4} \mathrm{CdCo}, \mathrm{La}_{4} \mathrm{MgIr}, \mathrm{La}_{4} \mathrm{MgCo} \\
\mathrm{Ba}_{14} \mathrm{Na}_{11} \mathrm{Li}_{5} \mathrm{~N}_{6}, \mathrm{Pr}_{4} \mathrm{MgCo}, \mathrm{Nd}_{4} \mathrm{MgIr}, \mathrm{Sm}_{4} \mathrm{MgRh}\end{array}$ \\
\hline & $\Gamma_{4}(3), \mathrm{L}_{3}(2)$ & $\mathrm{Na}_{2} \mathrm{CdPb}, \mathrm{TiFeSn}, \mathrm{Mn}_{2} \mathrm{GaCo}, \mathrm{Mn}_{2} \mathrm{InCo}, \mathrm{Yb}_{4} \mathrm{MgPd}, \mathrm{Mg}_{44} \mathrm{Rh}_{7}$ \\
\hline & $\Gamma_{3}(2), \mathrm{X}_{5}(2)$ & $\| \mathrm{Nd}_{4} \mathrm{MgRu}$ \\
\hline & $\Gamma_{3}(2), \mathrm{L}_{3}(2)$ & 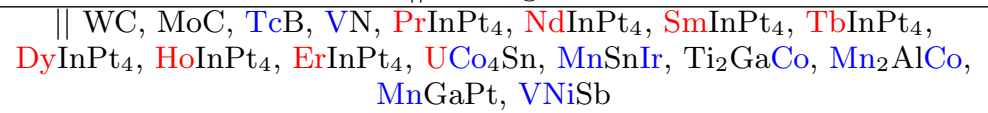 \\
\hline & $\mathrm{X}_{5}(2)$ & $\begin{array}{c}\text { LiNdSn, LiPrSn, } \| \text { ThNiSn(ICSD : 646771), ZrNiSn(ICSD : 646828), } \\
\mathrm{Li}_{21} \mathrm{Si}_{5}, \mathrm{HfSnPt}, \mathrm{HfNiSn}(\mathrm{ICSD}: 104249), \mathrm{TiNiSn}(\mathrm{ICSD}: 185078) \\
\mathrm{NdCu}_{4} \mathrm{Ag}, \mathrm{UNi}_{4} \mathrm{Sn}\end{array}$ \\
\hline & $\mathrm{L}_{3}(2)$ & $\|$ ReN \\
\hline & $\Gamma_{5}(3)$ & $\mathrm{UNi}_{5}, \| \mathrm{TmInNi}_{4}, \mathrm{HfNi}_{5}, \mathrm{La}_{4} \mathrm{MgRu}, \mathrm{La}_{4} \mathrm{CdRu}$ \\
\hline & $\Gamma_{4}(3)$ & 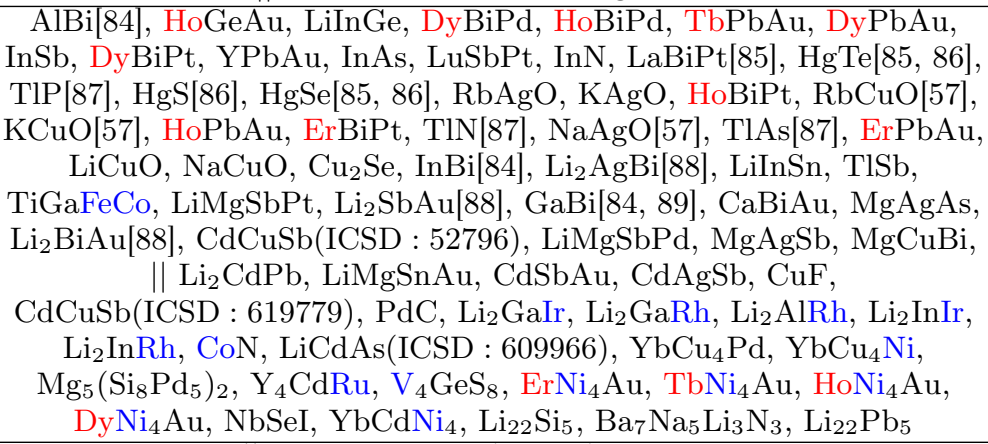 \\
\hline & $\Gamma_{3}(2)$ & $\| \mathrm{Pr}_{4} \mathrm{MgRu}, \mathrm{Mg}_{5}\left(\mathrm{Si}_{8} \mathrm{Pt}_{5}\right)_{2}, \mathrm{Mg}_{44} \mathrm{Ir}_{7}$ \\
\hline
\end{tabular}


Table II - continued

\begin{tabular}{|c|c|c|}
\hline $\begin{array}{l}\text { SOC or } \\
\text { NSOC }\end{array}$ & $\begin{array}{c}\text { Degenerate Irreducible } \\
\text { Representations }\end{array}$ & High Symmetry Point Semimetals \\
\hline $\mathrm{SOC}$ & $\bar{\Gamma}_{8}(4)$ & 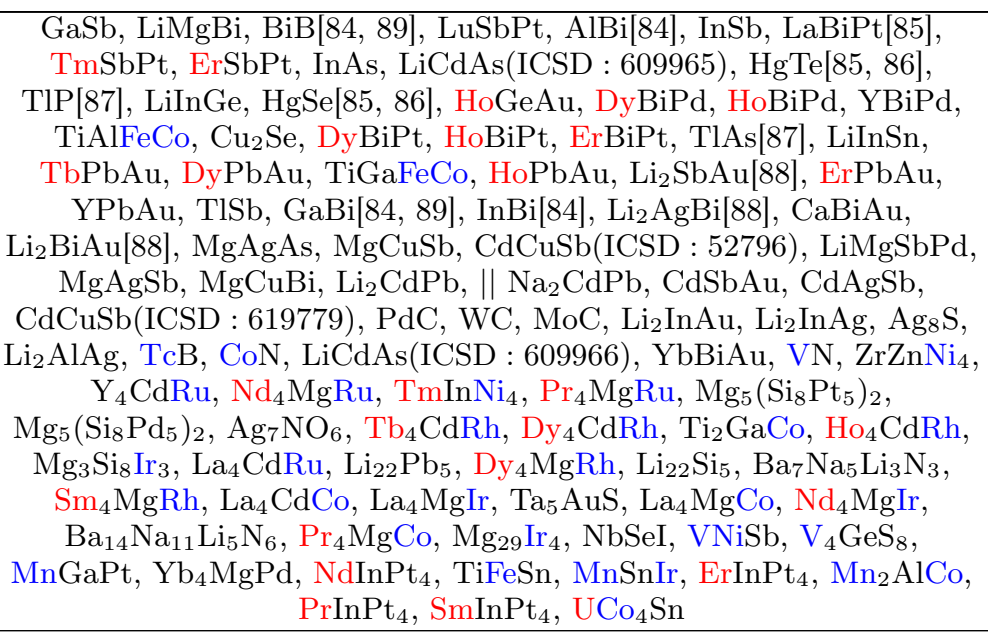 \\
\hline \multicolumn{3}{|r|}{ Space Group 217: $I \overline{4} 3 m$} \\
\hline \multirow{13}{*}{ NSOC } & $\Gamma_{5}(3), \mathrm{H}_{5}(3), \mathrm{P}_{4}(3)$ & $\| \mathrm{Ta}_{2} \mathrm{O}$ \\
\hline & $\Gamma_{5}(3), \mathrm{H}_{5}(3), \mathrm{P}_{3}(2)$ & $\mathrm{Re}_{24} \mathrm{Mo}_{5}$ \\
\hline & $\Gamma_{5}(3), \mathrm{H}_{4}(3), \mathrm{P}_{5}(3)$ & $\| \mathrm{Pd}_{16} \mathrm{~S}_{7}$ \\
\hline & $\Gamma_{5}(3), \mathrm{H}_{4}(3), \mathrm{P}_{4}(3)$ & $\| \mathrm{Ca}_{3}(\mathrm{GaNi})_{4}$ \\
\hline & $\Gamma_{4}(3), \mathrm{H}_{4}(3), \mathrm{P}_{5}(3)$ & $\| \mathrm{Zn}_{8} \mathrm{Ag}_{5}, \mathrm{Zn}_{8} \mathrm{Cu}_{5}, \mathrm{Cd}_{8} \mathrm{Ag}_{5}$ \\
\hline & $\Gamma_{3}(2), \mathrm{H}_{4}(3), \mathrm{P}_{4}(3)$ & $\mathrm{Ca}_{3}(\mathrm{GeIr})_{4}, \mathrm{Sr}_{3}(\mathrm{SnIr})_{4}, \mathrm{Ca}_{3}(\mathrm{SiIr})_{4}$ \\
\hline & $\mathrm{H}_{5}(3), \mathrm{P}_{5}(3)$ & $\| \mathrm{Cu}_{12} \mathrm{Sb}_{4} \mathrm{~S}_{13}$ \\
\hline & $\mathrm{H}_{4}(3), \mathrm{P}_{5}(3)$ & $\mathrm{Zn}_{11} \mathrm{Ir}_{2}, \mathrm{Cu}_{12} \mathrm{As}_{4} \mathrm{~S}_{13}$ \\
\hline & $\Gamma_{4}(3), \mathrm{P}_{5}(3)$ & $\| \mathrm{Mg}_{17} \mathrm{Al}_{12}$ \\
\hline & $\Gamma_{4}(3), \mathrm{H}_{5}(3)$ & $\| \mathrm{Th}_{11}\left(\mathrm{Ru}_{2} \mathrm{C}_{3}\right)_{6}$ \\
\hline & $\mathrm{P}_{5}(3)$ & $\| \mathrm{Cu}_{3} \mathrm{SbS}_{3}, \mathrm{Cu}_{3} \mathrm{AsS}_{3}$ \\
\hline & $\mathrm{P}_{4}(3)$ & $\mathrm{Zn}_{11} \mathrm{Rh}_{2}, \mathrm{Ti}_{5} \mathrm{Re}_{24}, \mathrm{Zr}_{5} \mathrm{Re}_{24}, \mathrm{Hf}_{5} \mathrm{Re}_{24}$ \\
\hline & $\Gamma_{4}(3)$ & $\| \mathrm{Sr}_{3}(\mathrm{GaIr})_{4}$ \\
\hline \multirow{6}{*}{$\mathrm{SOC}$} & $\bar{\Gamma}_{8}(4), \overline{\mathrm{H}}_{8}(4), \overline{\mathrm{P}}_{8}(4)$ & $\mathrm{Sr}_{3}(\mathrm{SnIr})_{4}, \mathrm{Pd}_{16} \mathrm{~S}_{7}, \mathrm{Ca}_{3}(\mathrm{GeIr})_{4}$ \\
\hline & $\overline{\mathrm{H}}_{8}(4), \overline{\mathrm{P}}_{8}(4)$ & $\| \mathrm{Re}_{24} \mathrm{Mo}_{5}$ \\
\hline & $\bar{\Gamma}_{8}(4), \overline{\mathrm{P}}_{8}(4)$ & $\| \mathrm{Ca}_{3}(\mathrm{SiIr})_{4}$ \\
\hline & $\overline{\mathrm{P}}_{8}(4)$ & $\| \mathrm{Ca}_{3}(\mathrm{GaNi})_{4}, \mathrm{Hf}_{5} \mathrm{Re}_{24}, \mathrm{Zr}_{5} \mathrm{Re}_{24}$ \\
\hline & $\overline{\mathrm{H}}_{8}(4)$ & $\mathrm{Zn}_{8} \mathrm{Cu}_{5}, \mathrm{Cd}_{8} \mathrm{Ag}_{5}, \mathrm{Zn}_{11} \mathrm{Ir}_{2}, \mathrm{Th}_{11}\left(\mathrm{Ru}_{2} \mathrm{C}_{3}\right)_{6}, \mathrm{Cu}_{12} \mathrm{As}_{4} \mathrm{~S}_{13}$ \\
\hline & $\bar{\Gamma}_{8}(4)$ & $\| \mathrm{Zn}_{8} \mathrm{Ag}_{5}, \mathrm{Ta}_{2} \mathrm{O}, \mathrm{Mg}_{17} \mathrm{Al}_{12}, \mathrm{Sr}_{3}(\mathrm{GaIr})_{4}$ \\
\hline \multirow{13}{*}{ NSOC } & \multicolumn{2}{|r|}{ Space Group 220: $I \overline{4} 3 d$} \\
\hline & $\begin{array}{c}\Gamma_{4}(3), \mathrm{H}_{4} \mathrm{H}_{5}(6), \mathrm{N}_{1}(2) \\
\mathrm{P}_{3}(4)\end{array}$ & $\begin{array}{c}\| \mathrm{Pr}_{4} \mathrm{As}_{3}, \mathrm{Sm}_{4} \mathrm{Sb}_{3}, \mathrm{Er}_{4} \mathrm{Sb}_{3}, \mathrm{Ho}_{4} \mathrm{Sb}_{3}, \mathrm{Dy}_{4} \mathrm{Sb}_{3}, \mathrm{Nd}_{4} \mathrm{Sb}_{3}, \mathrm{~Tb}_{4} \mathrm{Sb}_{3} \\
\mathrm{Pr}_{4} \mathrm{Sb}_{3}, \mathrm{Y}_{4} \mathrm{Sb}_{3}, \mathrm{La}_{4} \mathrm{Sb}_{3}, \mathrm{La}_{4} \mathrm{Bi}_{3}, \mathrm{La}_{4} \mathrm{As}_{3}, \mathrm{~Tb}_{4} \mathrm{Bi}_{3}, \mathrm{Pr}_{4} \mathrm{Bi}_{3}, \mathrm{~Pa}_{3} \mathrm{P}_{4} \\
\mathrm{Sm}_{4} \mathrm{Bi}_{3}, \mathrm{~Pa}_{3} \mathrm{As}_{4}\end{array}$ \\
\hline & $\begin{array}{c}\Gamma_{4}(3), \mathrm{H}_{4} \mathrm{H}_{5}(6), \mathrm{N}_{1}(2) \\
\mathrm{P}_{2}(2)\end{array}$ & $\| \mathrm{Yb}_{4} \mathrm{Sb}_{3}$ \\
\hline & $\begin{array}{c}\Gamma_{4}(3), \mathrm{H}_{4} \mathrm{H}_{5}(6), \mathrm{N}_{1}(2) \\
\mathrm{P}_{1}(2)\end{array}$ & $\| \mathrm{Yb}_{4} \mathrm{Bi}_{3}, \mathrm{Yb}_{4} \mathrm{As}_{3}, \mathrm{Sr}_{4} \mathrm{Bi}_{3}, \mathrm{Ba}_{4} \mathrm{Bi}_{3}$ \\
\hline & $\begin{array}{c}\Gamma_{4}(3), \mathrm{H}_{3} \mathrm{H}_{3}(4), \mathrm{N}_{1}(2) \\
\mathrm{P}_{3}(4)\end{array}$ & $\| \mathrm{Li}_{15} \mathrm{Ge}_{4}[5], \mathrm{Li}_{15} \mathrm{Si}_{4}, \mathrm{Na}_{15} \mathrm{~Pb}_{4}, \mathrm{Na}_{15} \mathrm{Sn}_{4}$ \\
\hline & $\begin{array}{c}\Gamma_{3}(2), \mathrm{H}_{4} \mathrm{H}_{5}(6), \mathrm{N}_{1}(2) \\
\mathrm{P}_{3}(4)\end{array}$ & $\| \mathrm{Sb}_{3} \mathrm{H}_{36}\left(\mathrm{C}_{3} \mathrm{I}_{2}\right)_{4}, \mathrm{La}_{3} \mathrm{Te}_{4}, \mathrm{La}_{3} \mathrm{Se}_{4}$ \\
\hline & $\begin{array}{c}\Gamma_{3}(2), \mathrm{H}_{4} \mathrm{H}_{5}(6), \mathrm{N}_{1}(2) \\
\mathrm{P}_{2}(2)\end{array}$ & $\begin{array}{c}\| \mathrm{Sm}_{3} \mathrm{~S}_{4}, \mathrm{~Tb}_{3} \mathrm{Se}_{4}, \mathrm{Dy}_{3} \mathrm{~S}_{4}, \mathrm{Nd}_{3} \mathrm{Se}_{4}, \mathrm{Pr}_{3} \mathrm{Te}_{4}, \mathrm{Pr}_{3} \mathrm{Se}_{4}, \mathrm{Sm}_{3} \mathrm{Se}_{4}, \mathrm{Nd}_{3} \mathrm{~S}_{4} \\
\mathrm{Dy}_{3} \mathrm{Se}_{4}, \mathrm{Sm}_{3} \mathrm{Te}_{4}, \mathrm{Li}_{12} \mathrm{Al}_{3} \mathrm{Si}_{4}, \mathrm{Th}_{4} \mathrm{H}_{15}\end{array}$ \\
\hline & $\begin{array}{c}\Gamma_{3}(2), \mathrm{H}_{4} \mathrm{H}_{5}(6), \mathrm{N}_{1}(2), \\
\mathrm{P}_{1}(2)\end{array}$ & $\| \mathrm{Ti}_{4} \mathrm{P}_{3}, \mathrm{Ti}_{4} \mathrm{As}_{3}, \mathrm{Nd}_{3} \mathrm{Te}_{4}, \operatorname{Pr}_{3} \mathrm{~S}_{4}$ \\
\hline & $\mathrm{H}_{4} \mathrm{H}_{5}(6), \mathrm{N}_{1}(2), \mathrm{P}_{3}(4)$ & $\mathrm{Ga}, \mathrm{Ca}_{6} \mathrm{Al}_{7} \mathrm{O}_{16}, \mathrm{Li}_{15} \mathrm{Au}_{4}, \mathrm{La}_{4} \mathrm{Rh}_{3}$ \\
\hline & $\mathrm{H}_{3} \mathrm{H}_{3}(4), \mathrm{N}_{1}(2), \mathrm{P}_{3}(4)$ & $\| \mathrm{Cu}_{15} \mathrm{Si}_{4}$ \\
\hline & $\Gamma_{4}(3), \mathrm{H}_{4} \mathrm{H}_{5}(6), \mathrm{P}_{3}(4)$ & $\| \mathrm{Yb}_{2} \mathrm{C}_{3}$ \\
\hline & $\Gamma_{3}(2), \mathrm{H}_{4} \mathrm{H}_{5}(6), \mathrm{P}_{3}(4)$ & $\mathrm{V}_{2} \mathrm{Ga}_{8} \mathrm{Cu}_{3}$ \\
\hline & $\mathrm{H}_{3} \mathrm{H}_{3}(4), \mathrm{P}_{3}(4)$ & $\| \mathrm{Ce}_{2} \mathrm{C}_{3}$ \\
\hline
\end{tabular}


Table II - continued

\begin{tabular}{|c|c|c|}
\hline $\begin{array}{l}\text { SOC or } \\
\text { NSOC }\end{array}$ & $\begin{array}{l}\text { Degenerate Irreducible } \\
\text { Representations }\end{array}$ & High Symmetry Point Semimetals \\
\hline & $\Gamma_{5}(3), \mathrm{H}_{4} \mathrm{H}_{5}(6)$ & $\mathrm{Li}, \mathrm{Li}\left(\mathrm{H}_{3} \mathrm{~N}\right)_{4}, \mathrm{U}_{3} \mathrm{Se}_{4}$ \\
\hline & $\mathrm{H}_{4} \mathrm{H}_{5}(6)$ & $\| \mathrm{Na}, \mathrm{Li}_{12} \mathrm{Mg}_{3} \mathrm{Si}_{4}$ \\
\hline & $\Gamma_{5}(3)$ & $\| \mathrm{Tb}_{2} \mathrm{C}_{3}, \mathrm{Dy}_{2} \mathrm{C}_{3}$ \\
\hline & $\Gamma_{4}(3)$ & $\mathrm{Pr}_{4} \mathrm{Ge}_{3}, \mathrm{Sm}_{4} \mathrm{Sn}_{3}, \mathrm{La}_{4} \mathrm{~Pb}_{3} \|$ \\
\hline \multirow{9}{*}{$\mathrm{SOC}$} & $\bar{\Gamma}_{8}(4), \overline{\mathrm{H}}_{8} \overline{\mathrm{H}}_{8}(8), \overline{\mathrm{P}}_{8}(3)$ & $\begin{array}{l}\| \mathrm{Yb}_{4} \mathrm{Sb}_{3}, \mathrm{Sm}_{3} \mathrm{~S}_{4}, \mathrm{Dy}_{3} \mathrm{~S}_{4}, \mathrm{Pr}_{3} \mathrm{Te}_{4}, \mathrm{~Tb}_{3} \mathrm{Se}_{4}, \mathrm{Dy}_{3} \mathrm{Se}_{4}, \mathrm{Nd}_{3} \mathrm{Se}_{4}, \mathrm{Pr}_{3} \mathrm{Se}_{4}, \\
\quad \mathrm{Nd}_{3} \mathrm{~S}_{4}, \mathrm{Sm}_{3} \mathrm{Se}_{4}, \mathrm{Sm}_{3} \mathrm{Te}_{4}, \mathrm{Li}_{12} \mathrm{Al}_{3} \mathrm{Si}_{4}, \mathrm{Li}_{15} \mathrm{Si}_{4}, \mathrm{Th}_{4} \mathrm{H}_{15}\end{array}$ \\
\hline & $\bar{\Gamma}_{8}(4), \overline{\mathrm{H}}_{8} \overline{\mathrm{H}}_{8}(8), \overline{\mathrm{P}}_{7}(3)$ & $\begin{array}{c}\| \mathrm{Sr}_{4} \mathrm{Bi}_{3}, \mathrm{Ba}_{4} \mathrm{Bi}_{3}, \mathrm{Ti}_{4} \mathrm{P}_{3}, \mathrm{Nd}_{3} \mathrm{Te}_{4}, \mathrm{Ti}_{4} \mathrm{As}_{3}, \mathrm{Pr}_{3} \mathrm{~S}_{4}, \mathrm{Yb}_{4} \mathrm{As}_{3}, \mathrm{Li}_{15} \mathrm{Ge}_{4}[\underline{5}], \\
\mathrm{Pa}_{3} \mathrm{P}_{4}\end{array}$ \\
\hline & $\bar{\Gamma}_{8}(4), \overline{\mathrm{H}}_{6} \overline{\mathrm{H}}_{7}(4), \overline{\mathrm{P}}_{8}(3)$ & $\| \mathrm{La}_{3} \mathrm{Te}_{4}$ \\
\hline & $\bar{\Gamma}_{8}(4), \overline{\mathrm{H}}_{6} \overline{\mathrm{H}}_{7}(4), \overline{\mathrm{P}}_{7}(3)$ & $\| \mathrm{La}_{3} \mathrm{Se}_{4}$ \\
\hline & $\overline{\mathrm{H}}_{8} \overline{\mathrm{H}}_{8}(8), \overline{\mathrm{P}}_{8}(3)$ & $\begin{array}{c}\| \mathrm{Na}_{15} \mathrm{~Pb}_{4}, \mathrm{Li}_{15} \mathrm{Au}_{4}, \mathrm{Pr}_{4} \mathrm{Sb}_{3}, \mathrm{Sm}_{4} \mathrm{Sb}_{3}, \mathrm{Er}_{4} \mathrm{Sb}_{3}, \mathrm{~Tb}_{4} \mathrm{Sb}_{3}, \mathrm{Y}_{4} \mathrm{Sb}_{3} \\
\mathrm{Na}_{15} \mathrm{Sn}_{4}, \mathrm{Dy}_{4} \mathrm{Sb}_{3}, \mathrm{Ho}_{4} \mathrm{Sb}_{3}\end{array}$ \\
\hline & $\overline{\mathrm{H}}_{8} \overline{\mathrm{H}}_{8}(8), \overline{\mathrm{P}}_{7}(3)$ & $\| \mathrm{Ga}, \mathrm{Yb}_{4} \mathrm{Bi}_{3}, \mathrm{Pr}_{4} \mathrm{As}_{3}, \mathrm{Nd}_{4} \mathrm{Sb}_{3}, \mathrm{La}_{4} \mathrm{Rh}_{3}, \mathrm{La}_{4} \mathrm{As}_{3}, \mathrm{~Tb}_{4} \mathrm{Bi}_{3}$ \\
\hline & $\bar{\Gamma}_{8}(4), \overline{\mathrm{P}}_{6}(2)$ & $\| \mathrm{Yb}_{2} \mathrm{C}_{3}, \mathrm{~V}_{2} \mathrm{Ga}_{8} \mathrm{Cu}_{3}$ \\
\hline & $\bar{\Gamma}_{8}(4), \overline{\mathrm{H}}_{8} \overline{\mathrm{H}}_{8}(8)$ & $\| \mathrm{Sb}_{3} \mathrm{H}_{36}\left(\mathrm{C}_{3} \mathrm{I}_{2}\right)_{4}$ \\
\hline & $\overline{\mathrm{H}}_{8} \overline{\mathrm{H}}_{8}(8)$ & $\| \mathrm{Cu}_{15} \mathrm{Si}_{4}$ \\
\hline \multicolumn{3}{|r|}{ Space Group 221: $P m \overline{3} m$} \\
\hline & $\begin{array}{c}\Gamma_{4}^{-}(3), \mathrm{M}_{5}^{-}(2), \mathrm{R}_{5}^{+}(3) \\
\mathrm{X}_{5}^{+}(2)\end{array}$ & $\| \mathrm{YBPd}_{3}, \mathrm{DyBPd}_{3}, \mathrm{TbBPd}_{3}, \mathrm{SmBPd}_{3}, \mathrm{NdBPd}_{3}, \mathrm{PrBPd}_{3}, \mathrm{LaBPd}_{3}$ \\
\hline & $\begin{array}{l}\Gamma_{4}^{-}(3), \mathrm{M}_{5}^{-}(2), \mathrm{R}_{4}^{-}(3) \\
\mathrm{X}_{5}^{+}(2)\end{array}$ & $\| \mathrm{HoBPd}_{3}$ \\
\hline & $\begin{array}{c}\Gamma_{4}^{+}(3), \mathrm{M}_{5}^{-}(2), \mathrm{R}_{5}^{+}(3) \\
\mathrm{X}_{5}^{+}(2)\end{array}$ & $\| \mathrm{LaBPt}_{3}$ \\
\hline & $\begin{array}{l}\Gamma_{4}^{+}(3), \mathrm{M}_{5}^{-}(2), \mathrm{R}_{4}^{-}(3) \\
\mathrm{X}_{5}^{-}(2)\end{array}$ & $\| \mathrm{ZrOs}_{3}, \mathrm{HfOs}_{3}$ \\
\hline & $\begin{array}{l}\Gamma_{4}^{+}(3), \mathrm{M}_{5}^{-}(2), \mathrm{R}_{4}^{-}(3) \\
\mathrm{X}_{5}^{+}(2)\end{array}$ & $\| \mathrm{LuBPd}_{3}, \mathrm{ScBPt}_{3}, \mathrm{TmBPd}_{3}, \mathrm{YBPt}_{3}, \mathrm{ErBPd}_{3}, \mathrm{CaPd}_{3} \mathrm{C}, \mathrm{ScBPd}_{3}$ \\
\hline & $\mathrm{M}_{5}^{-}(2), \mathrm{R}_{5}^{+}(3), \mathrm{X}_{5}^{+}(2)$ & $\mathrm{Rh}_{17} \mathrm{~S}_{15}$ \\
\hline & $\mathrm{M}_{5}^{-}(2), \mathrm{R}_{4}^{-}(3), \mathrm{X}_{5}^{+}(2)$ & $\| \mathrm{TmAg}_{3}$ \\
\hline & $\mathrm{M}_{5}^{-}(2), \mathrm{R}_{4}^{+}(3), \mathrm{X}_{5}^{+}(2)$ & $\mathrm{ZnNi}_{3} \mathrm{C}, \mathrm{MgNi}_{3} \mathrm{C}$ \\
\hline & $\Gamma_{5}^{-}(3), \mathrm{R}_{5}^{-}(3), \mathrm{X}_{5}^{-}(2)$ & $\| \mathrm{ThPb}_{3}$ \\
\hline & $\Gamma_{5}^{-}(3), \mathrm{M}_{5}^{-}(2), \mathrm{R}_{5}^{-}(3)$ & $\mathrm{CePb}_{3}, \mathrm{NpGa}_{3}$ \\
\hline & $\Gamma_{5}^{+}(3), \mathrm{R}_{4}^{-}(3), \mathrm{X}_{5}^{+}(2)$ & $\| \mathrm{Cr}_{3} \mathrm{Fe}$ \\
\hline & $\Gamma_{5}^{+}(3), \mathrm{R}_{3}^{-}(2), \mathrm{X}_{5}^{-}(2)$ & $\| \mathrm{MnAu}$ \\
\hline & $\Gamma_{5}^{+}(3), \mathrm{M}_{5}^{-}(2), \mathrm{R}_{5}^{+}(3)$ & $\| \mathrm{Nd}_{3} \mathrm{SnN}, \mathrm{Nd}_{3} \mathrm{PbN}$ \\
\hline & $\Gamma_{5}^{+}(3), \mathrm{M}_{5}^{-}(2), \mathrm{R}_{4}^{-}(3)$ & $\mathrm{Sm}_{3} \mathrm{Al}, \mathrm{Pr}_{3} \mathrm{Al}, \mathrm{Tb}_{3} \mathrm{Al}, \mathrm{Sm}_{3} \mathrm{In}, \mathrm{Nd}_{3} \mathrm{In}, \mathrm{Nd}_{3} \mathrm{Ga}, \mathrm{Nd}_{3} \mathrm{Tl}, \mathrm{Pr}_{3} \mathrm{Ga}, \mathrm{La}_{3} \mathrm{Ga}$ \\
\hline & $\Gamma_{5}^{+}(3), \mathrm{M}_{5}^{+}(2), \mathrm{R}_{5}^{+}(3)$ & $\|$ AlRe, $\mathrm{NbF}_{3}$ \\
\hline & $\Gamma_{5}^{+}(3), \mathrm{M}_{5}^{+}(2), \mathrm{R}_{4}^{-}(3)$ & $\| \mathrm{Cr}_{3} \mathrm{Ni}$ \\
\hline & $\Gamma_{5}^{+}(3), \mathrm{M}_{5}^{+}(2), \mathrm{R}_{3}^{+}(2)$ & $\| \mathrm{TcB}$ \\
\hline & $\Gamma_{4}^{-}(3), \mathrm{R}_{5}^{-}(3), \mathrm{X}_{5}^{+}(2)$ & $\| \mathrm{NdGaO}_{3}$ \\
\hline & $\Gamma_{4}^{-}(3), \mathrm{R}_{5}^{+}(3), \mathrm{X}_{5}^{+}(2)$ & $\| \mathrm{Te}, \mathrm{SrSi}, \mathrm{CuN}$ \\
\hline & $\Gamma_{4}^{-}(3), \mathrm{R}_{4}^{-}(3), \mathrm{X}_{5}^{-}(2)$ & $\| \mathrm{Se} \mathrm{TiOs}_{3}$ \\
\hline & $\Gamma_{4}^{-}(3), \mathrm{R}_{4}^{+}(3), \mathrm{X}_{5}^{+}(2)$ & $\| \mathrm{Ti}_{3} \mathrm{Sn}$ \\
\hline & $\Gamma_{4}^{-}(3), \mathrm{M}_{5}^{-}(2), \mathrm{X}_{5}^{-}(2)$ & $\mathrm{InAg}_{3}, \mathrm{AlCu}_{3}$ \\
\hline & $\Gamma_{4}^{-}(3), \mathrm{M}_{5}^{-}(2), \mathrm{R}_{5}^{+}(3)$ & $\|$ LiTl \\
\hline & $\Gamma_{4}^{-}(3), \mathrm{M}_{5}^{-}(2), \mathrm{R}_{4}^{-}(3)$ & $\| \mathrm{MgHg}, \mathrm{LiAu}_{3}$ \\
\hline & $\Gamma_{4}^{-}(3), \mathrm{M}_{5}^{+}(2), \mathrm{R}_{4}^{-}(3)$ & $\| \mathrm{TiZn}_{3}, \mathrm{ZrCd}_{3}, \mathrm{ZrHg}_{3}$ \\
\hline & $\Gamma_{4}^{+}(3), \mathrm{R}_{5}^{+}(3), \mathrm{X}_{5}^{-}(2)$ & $\| \mathrm{Pd}_{17} \mathrm{Se}_{15}$ \\
\hline & $\Gamma_{4}^{+}(3), \mathrm{M}_{5}^{-}(2), \mathrm{R}_{4}^{-}(3)$ & $\| \mathrm{HfRu}_{3}$ \\
\hline & $\Gamma_{4}^{+}(3), \mathrm{M}_{5}^{-}(2), \mathrm{R}_{4}^{+}(3)$ & $\mathrm{ZrRu}_{3}$ \\
\hline & $\Gamma_{4}^{+}(3), \mathrm{M}_{5}^{+}(2), \mathrm{R}_{3}^{+}(2)$ & $\mathrm{Fe}_{3} \mathrm{Pt}$ \\
\hline & $\Gamma_{3}^{+}(2), \mathrm{R}_{4}^{-}(3), \mathrm{X}_{5}^{-}(2)$ & $\mathrm{LiAl}_{3}$ \\
\hline & $\Gamma_{3}^{+}(2), \mathrm{R}_{4}^{+}(3), \mathrm{X}_{5}^{+}(2)$ & $\| \mathrm{TiPt}_{3}$ \\
\hline & $\Gamma_{3}^{+}(2), \mathrm{M}_{5}^{-}(2), \mathrm{R}_{5}^{+}(3)$ & $\begin{array}{c}\| \mathrm{YbZn}, \mathrm{DyCu}, \mathrm{YCu}, \mathrm{TmAg}, \mathrm{DyAg}, \mathrm{CaCd}, \mathrm{CaHg}, \mathrm{BaHg}, \mathrm{BaZn}, \\
\text { BaCd, HfPd, ZrPd }\end{array}$ \\
\hline
\end{tabular}


Table II - continued

\begin{tabular}{|c|c|c|}
\hline $\begin{array}{l}\text { SOC or } \\
\text { NSOC }\end{array}$ & $\begin{array}{l}\text { Degenerate Irreducible } \\
\text { Representations }\end{array}$ & High Symmetry Point Semimetals \\
\hline & $\Gamma_{3}^{+}(2), \mathrm{M}_{5}^{-}(2), \mathrm{R}_{4}^{-}(3)$ & $\begin{array}{c}\text { If } \mathrm{BrN}, \mathrm{TmCu}, \mathrm{ErAu}, \mathrm{ErCu}, \mathrm{IN}, \mathrm{HoCu}, \mathrm{TmAu}, \mathrm{LuAu}, \mathrm{HoAu}, \mathrm{YbHg}, \\
\mathrm{DyAu}, \mathrm{YAu}, \mathrm{ErAg}, \mathrm{TbAu}, \mathrm{SrHg}, \mathrm{YbCd}, \mathrm{TbAg}, \mathrm{ScCu}, \mathrm{HoAg}, \mathrm{ScAu} \\
\mathrm{SrCd}, \mathrm{YAg}, \mathrm{SrMg}, \mathrm{ScAg}, \mathrm{SmAu}, \mathrm{NdAu}, \operatorname{PrAu}, \mathrm{Mg}_{3} \mathrm{ZnC}, \mathrm{Pr}_{3} \mathrm{InO} \\
\mathrm{Ca}_{3} \mathrm{AuN}, \mathrm{TiPd}\end{array}$ \\
\hline & $\Gamma_{3}^{+}(2), \mathrm{M}_{5}^{-}(2), \mathrm{R}_{3}^{-}(2)$ & $\| \mathrm{HfPt}, \mathrm{TiPt}$ \\
\hline & $\Gamma_{3}^{+}(2), \mathrm{M}_{5}^{-}(2), \mathrm{R}_{3}^{+}(2)$ & ZrPt, TiNi \\
\hline & $\Gamma_{3}^{+}(2), \mathrm{M}_{5}^{+}(2), \mathrm{X}_{5}^{-}(2)$ & $\| \mathrm{Cr}_{3} \mathrm{RhN}$ \\
\hline & $\mathrm{R}_{4}^{-}(3), \mathrm{X}_{5}^{-}(2)$ & $\mathrm{Ca}_{3} \mathrm{TlN}$ \\
\hline & $\mathrm{R}_{4}^{+}(3), \mathrm{X}_{5}^{-}(2)$ & $\mathrm{Cr}_{3} \mathrm{GaN}$ \\
\hline & $\mathrm{R}_{4}^{+}(3), \mathrm{X}_{5}^{+}(2)$ & $\| \mathrm{CuNi}_{3} \mathrm{~N}$ \\
\hline & $\mathrm{M}_{5}^{-}(2), \mathrm{R}_{5}^{-}(3)$ & $\| \mathrm{USi}_{3}, \mathrm{UGe}_{3}, \mathrm{USn}_{3}$ \\
\hline & $\mathrm{M}_{5}^{-}(2), \mathrm{R}_{5}^{+}(3)$ & $\| \mathrm{MgPd}, \mathrm{MgNi}, \mathrm{NpAs}$ \\
\hline & $\mathrm{M}_{5}^{-}(2), \mathrm{R}_{4}^{-}(3)$ & $\mathrm{LiAg}, \mathrm{URu}_{3}, \mathrm{Mn}_{3} \mathrm{Rh}$ \\
\hline & $\mathrm{M}_{5}^{-}(2), \mathrm{R}_{3}^{-}(2)$ & $\| \mathrm{Ba}_{6} \mathrm{Ni}_{25} \mathrm{~S}_{27}$ \\
\hline & $\mathrm{M}_{5}^{+}(2), \mathrm{R}_{4}^{-}(3)$ & $\| \mathrm{MoPt}_{3}$ \\
\hline & $\Gamma_{5}^{-}(3), \mathrm{R}_{5}^{-}(3)$ & $\| \mathrm{NaN}_{3}, \mathrm{CeSn}_{3}$ \\
\hline & $\Gamma_{5}^{-}(3), \mathrm{R}_{4}^{-}(3)$ & $\mathrm{CePd}_{3}, \| \mathrm{Ce}_{2} \mathrm{Si}_{7} \mathrm{Rh}_{15}$ \\
\hline & $\Gamma_{5}^{-}(3), \mathrm{R}_{3}^{-}(2)$ & $\| \mathrm{PuRu}$ \\
\hline & $\Gamma_{5}^{-}(3), \mathrm{M}_{5}^{-}(2)$ & $\mathrm{ThB}_{6}, \mathrm{CeB}_{6}$ \\
\hline & $\Gamma_{5}^{+}(3), \mathrm{R}_{5}^{-}(3)$ & $\| \mathrm{TbGa}_{3} \mathrm{Os}$ \\
\hline & $\Gamma_{5}^{+}(3), \mathrm{R}_{5}^{+}(3)$ & $\| \operatorname{IrN}($ ICSD $: 183155)$ \\
\hline & $\Gamma_{5}^{+}(3), \mathrm{R}_{4}^{-}(3)$ & $\mathrm{Pr}_{3} \mathrm{In}_{2} \mathrm{Pr}_{3} \mathrm{Tl}, \mathrm{Y}_{3} \mathrm{Al}, \mathrm{Mn}_{3} \mathrm{PdN}, \mathrm{La}_{3} \mathrm{In}, \mathrm{La}_{3} \mathrm{Al}, \mathrm{Sm}_{3} \mathrm{Ga}$ \\
\hline & $\Gamma_{5}^{+}(3), \mathrm{R}_{4}^{+}(3)$ & TcN(ICSD : 187707), ReN(ICSD : 187711), TmGas Ru \\
\hline & $\Gamma_{5}^{+}(3), \mathrm{R}_{3}^{+}(2)$ & $\| \mathrm{Ni}_{3} \mathrm{Pt}$ \\
\hline & $\Gamma_{5}^{+}(3), \mathrm{M}_{5}^{+}(2)$ & $\| \mathrm{FePt}_{3}, \mathrm{FeNi}_{3}$ \\
\hline & $\Gamma_{4}^{-}(3), \mathrm{R}_{5}^{+}(3)$ & TaN, $\|$ AlAu, InAg, ThTe, PtC, TlBi, TlSb, LuAs \\
\hline & $\Gamma_{4}^{-}(3), \mathrm{R}_{4}^{-}(3)$ & $\mathrm{HfPt}_{3}, \mathrm{TlHPd}_{3}, \| \mathrm{ZrPt}_{3}, \mathrm{GaAg}, \mathrm{Cu}_{3} \mathrm{Au}, \mathrm{CuAu}_{3}$ \\
\hline & $\Gamma_{4}^{-}(3), \mathrm{R}_{4}^{+}(3)$ & $\mathrm{Pd}_{3} \mathrm{~Pb}, \mathrm{NpRh}_{3}, \| \mathrm{BaPbO}_{3}(\mathrm{ICSD}: 51659)$ \\
\hline & $\Gamma_{4}^{-}(3), \mathrm{R}_{3}^{+}(2)$ & $\mathrm{NbN}, \| \mathrm{VN}$ \\
\hline & $\Gamma_{4}^{-}(3), \mathrm{M}_{5}^{+}(2)$ & $\| \mathrm{WC}$ \\
\hline & $\Gamma_{4}^{+}(3), \mathrm{X}_{5}^{-}(2)$ & $\| \mathrm{Cu}_{3} \mathrm{~N}(\mathrm{ICSD}: 55222)$ \\
\hline & $\Gamma_{4}^{+}(3), \mathrm{X}_{5}^{+}(2)$ & $\| \mathrm{VIr}_{3}$ \\
\hline & $\Gamma_{4}^{+}(3), \mathrm{R}_{5}^{+}(3)$ & $\| \mathrm{SmRh}_{3} \mathrm{C}, \mathrm{NdRh}_{3} \mathrm{C}, \mathrm{PrRh}_{3} \mathrm{C}, \mathrm{Mn}_{3} \mathrm{PtN}$ \\
\hline & $\Gamma_{4}^{+}(3), \mathrm{R}_{4}^{-}(3)$ & $\begin{array}{c}\mathrm{HfBRh}_{3}, \mathrm{ScRh}_{3} \mathrm{C}, \mathrm{ZrBRh}_{3}, \mathrm{ScCo}_{3} \mathrm{C}, \mathrm{LuRh}_{3} \mathrm{C}, \mathrm{TmRh}_{3} \mathrm{C}, \mathrm{HoRh}_{3} \mathrm{C} \\
\mathrm{TbRh}_{3} \mathrm{C}, \mathrm{ErRh}_{3} \mathrm{C}, \mathrm{YRh}_{3} \mathrm{C}, \| \mathrm{DyRh}_{3} \mathrm{C}, \mathrm{ScIr}_{3}\end{array}$ \\
\hline & $\Gamma_{4}^{+}(3), \mathrm{R}_{4}^{+}(3)$ & $\begin{array}{c}\| \mathrm{SnPd}_{3}, \mathrm{Pt}_{3} \mathrm{~Pb}, \mathrm{SnPt}_{3}, \mathrm{Ni}_{3} \mathrm{Sn}, \mathrm{AlCo}_{3} \mathrm{C}, \mathrm{SiNi}_{3}, \mathrm{Ni}_{3} \mathrm{Ge}, \mathrm{NdIr}_{3} \mathrm{C} \\
\text { Sch }\end{array}$ \\
\hline & $\Gamma_{3}^{+}(2), \mathrm{X}_{5}^{-}(2)$ & $\| \mathrm{Cr}_{3} \operatorname{IrN}$ \\
\hline & $\Gamma_{3}^{+}(2), \mathrm{R}_{5}^{+}(3)$ & $\| \mathrm{PBr}, \mathrm{ThSe}$ \\
\hline & $\Gamma_{3}^{+}(2), \mathrm{R}_{4}^{-}(3)$ & $\begin{array}{c}\| \mathrm{HfPd}_{3}, \mathrm{TbCu}, \mathrm{ZrPd}_{3}, \mathrm{TiPd}_{3}, \mathrm{SmAg}, \mathrm{SmCu}, \mathrm{LaAg}, \mathrm{NdAg}, \mathrm{PrAg}, \\
\mathrm{La}_{3} \mathrm{Tl}\end{array}$ \\
\hline & $\Gamma_{3}^{+}(2), \mathrm{R}_{3}^{+}(2)$ & $\| \mathrm{KPdF}_{3}, \mathrm{RbPdF}_{3}$ \\
\hline & $\mathrm{X}_{5}^{-}(2)$ & $\| \mathrm{CaSn}_{3}, \mathrm{YbSn}_{3}, \mathrm{Nd}_{3} \mathrm{GaC}, \mathrm{SmCoO}_{3}, \mathrm{Nb}_{4} \mathrm{C}_{3}, \mathrm{LaCoO}_{3}$ \\
\hline & $\mathrm{X}_{5}^{+}(2)$ & $\begin{array}{l}\| \mathrm{Pr}_{3} \mathrm{InC}_{2} \mathrm{Tm}_{3} \mathrm{AlC}, \mathrm{Er}_{3} \mathrm{AlC}\left(\mathrm{ICSD}: \text { : 606192), } \mathrm{Tb}_{3} \mathrm{AlC}(\mathrm{ICSD}: \text { : 606265), }\right. \\
\quad \mathrm{Sm}_{3} \mathrm{InC}_{\mathrm{InC}} \mathrm{Pr}_{3} \mathrm{GaC}, \mathrm{GeTe}, \mathrm{Ca} \mathrm{Hg}_{3}, \mathrm{MnRh}, \mathrm{CaHg}_{11}, \mathrm{BaHg}_{11}\end{array}$ \\
\hline & $\mathrm{R}_{5}^{-}(3)$ & $\mathrm{KIO}_{3}, \mathrm{TIIO}_{3} \|$ \\
\hline & $\mathrm{R}_{5}^{+}(3)$ & $\begin{array}{l}\text { Ca, } \mathrm{Ca}_{3} \mathrm{~Pb}, \| \text { AlCo, BeNi, DyIn, HoIn, CaNi, HoTl, SmTl, TbTl, } \\
\text { ErTl, NdTl, PrTl, DyTl, LaTl, Ti }{ }_{3} \text { AlN, } \mathrm{Ti}_{3} \mathrm{TlN}, \mathrm{Ti}_{3} \mathrm{InN}, \mathrm{CaPd}, \mathrm{CeHg}\end{array}$ \\
\hline & $\mathrm{R}_{4}^{-}(3)$ & 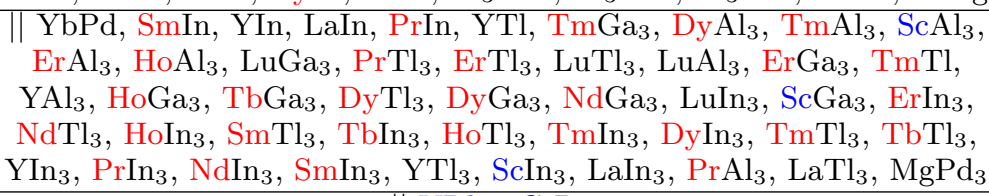 \\
\hline & $\mathrm{R}_{4}^{+}(3)$ & $\| \mathrm{VRh}_{3}, \mathrm{CrPt}_{3}$ \\
\hline & $\mathrm{R}_{3}^{+}(2)$ & $\| \mathrm{Mn}_{3} \mathrm{SnN}$ \\
\hline & $\mathrm{M}_{5}^{-}(2)$ & $\begin{array}{c}\mathrm{ZrZn}, \mathrm{BaLiH}_{3}(\mathrm{ICSD}: 23977), \text { TaTc, } \| \mathrm{VTc}, \mathrm{CeMg}, \mathrm{Ti}_{3} \mathrm{Hg}, \\
\text { IrN(ICSD : 187719) }\end{array}$ \\
\hline & $\mathrm{M}_{5}^{+}(2)$ & || ReN(ICSD : 187714), TcN(ICSD : 187710) \\
\hline
\end{tabular}


Table II - continued

\begin{tabular}{|c|c|c|}
\hline $\begin{array}{l}\text { SOC or } \\
\text { NSOC }\end{array}$ & $\begin{array}{c}\text { Degenerate Irreducible } \\
\text { Representations }\end{array}$ & High Symmetry Point Semimetals \\
\hline \multirow{13}{*}{$\mathrm{SOC}$} & $\Gamma_{5}^{-}(3)$ & $\| \mathrm{SrPb}_{3}, \mathrm{ThSn}_{3}$ \\
\hline & $\Gamma_{5}^{+}(3)$ & $\begin{array}{c}\mathrm{Ba}_{3} \mathrm{SnO}, \mathrm{Ba}_{3} \mathrm{PbO}[90], \mathrm{Sc}_{3} \mathrm{BPb}, \mathrm{Sc}_{3} \mathrm{SnB}_{2} \mathrm{Sc}_{3} \mathrm{TlC}, \mathrm{Y}_{3} \mathrm{TlC}, \mathrm{Sc}_{3} \mathrm{GaC} \\
\mathrm{Sc}_{3} \mathrm{InC}, \mathrm{Dy}_{3} \mathrm{InC}, \mathrm{Y}_{3} \mathrm{InC}, \mathrm{Y}_{3} \mathrm{GaC}, \mathrm{Lu}_{3} \mathrm{TlC}, \mathrm{Y}_{3} \mathrm{AlC}, \mathrm{Sc} \mathrm{c}_{3} \mathrm{AlC}, \| \\
\mathrm{Er}_{3} \mathrm{AlC}(\mathrm{ICSD}: 43849), \mathrm{Ho}_{3} \mathrm{AlC}\end{array}$ \\
\hline & $\Gamma_{4}^{-}(3)$ & 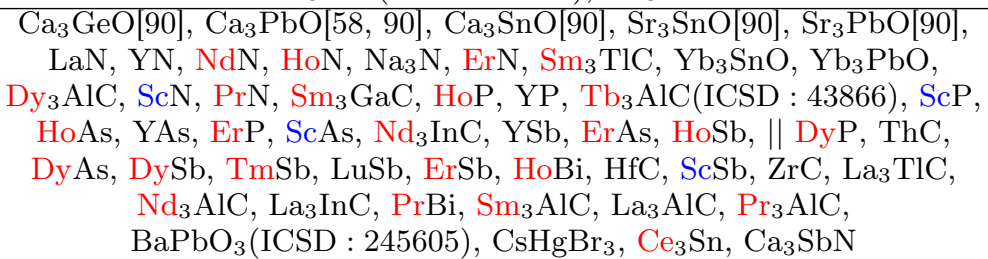 \\
\hline & $\Gamma_{4}^{+}(3)$ & $\| \mathrm{Fe}_{3} \mathrm{SnC}, \mathrm{YbBRh}_{3}, \mathrm{ScRh}_{3}, \mathrm{LaRh}_{3}$ \\
\hline & $\Gamma_{3}^{+}(2)$ & $\| \mathrm{Mn}_{3} \mathrm{ZnN}, \mathrm{NCl}$ \\
\hline & $\bar{\Gamma}_{11}(4), \overline{\mathrm{R}}_{11}(4)$ & \multirow{2}{*}{$\begin{array}{c}\mathrm{HfPt}_{3}, \mathrm{ZrPt}_{3}, \| \mathrm{Se}, \mathrm{LiAu}_{3}, \mathrm{PuRu}, \mathrm{CePd}_{3}, \mathrm{TiO}_{3}, \mathrm{NdGaO}_{3} \\
\text { TaN, NbN, \| Te, VN, ThSe, ThTe, TiPt }, \mathrm{BaPbO}_{3}(\mathrm{ICSD}: 51659) \\
\text { TlSb, TlBi, LuAs }\end{array}$} \\
\hline & $\bar{\Gamma}_{11}(4), \overline{\mathrm{R}}_{10}(4)$ & \\
\hline & $\bar{\Gamma}_{10}(4), \overline{\mathrm{R}}_{11}(4)$ & $\begin{array}{l}\mathrm{ScRh}_{3} \mathrm{C}, \mathrm{ScCo}_{3} \mathrm{C}, \mathrm{ErAu}, \mathrm{TmAu}, \mathrm{HoAu}, \mathrm{YAu}, \mathrm{DyAu}, \mathrm{LuAu}, \mathrm{ErAg}, \\
\mathrm{TbAu}, \mathrm{YbCd}, \mathrm{ScCu}, \mathrm{HoAg}, \mathrm{TbAg}, \mathrm{YAg}, \mathrm{ScAg}, \mathrm{SmAu}, \mathrm{LiAl}_{3}, \mathrm{HfPt}, \\
\mathrm{NdAu}, \mathrm{ScAu}, \mathrm{PrAu}, \mathrm{SmAg}, \mathrm{LaAg}, \mathrm{NdAg}, \mathrm{PrAg}, \mathrm{LuBPd}_{3}, \mathrm{TmBPd}_{3}, \\
\text { TiPt}, \mathrm{TiPd}, \mathrm{La}_{3} \mathrm{Tl}, \mathrm{La}_{3} \mathrm{In}, \mathrm{Mn}_{3} \mathrm{PdN}, \mathrm{BrN}, \mathrm{MnAu}, \mathrm{IN}\end{array}$ \\
\hline & $\bar{\Gamma}_{10}(4), \overline{\mathrm{R}}_{10}(4)$ & $\begin{array}{c}\| \mathrm{YbZn}, \mathrm{TmAg}, \mathrm{YCu}, \mathrm{DyAg}, \mathrm{CaCd}, \mathrm{IrN}(\mathrm{ICSD}: 183155), \mathrm{KPdF}_{3} \\
\mathrm{RbPdF}_{3}, \mathrm{LaBPt}_{3}, \mathrm{HfPd}, \mathrm{ZrPt}_{\mathrm{Pr}} \mathrm{Pr}_{3} \mathrm{InO}, \mathrm{ZrPd}, \mathrm{NdIr}_{3}, \mathrm{DyIr}_{3}, \mathrm{PBr} \\
\mathrm{Fe}_{3} \mathrm{Pt}, \mathrm{NbF}_{3}, \mathrm{TiNi}\end{array}$ \\
\hline & $\overline{\mathrm{R}}_{11}(4)$ & $\begin{array}{c}\mathrm{C}[91], \| \mathrm{YRh}_{3} \mathrm{C}, \mathrm{SmIn}, \mathrm{YIn}, \mathrm{LaIn}, \mathrm{PrIn}, \mathrm{TmIn}, \mathrm{YTl}, \mathrm{DyAl} \mathrm{Al}_{3}, \mathrm{ScAl}_{3}, \\
\mathrm{HoAl}_{3}, \mathrm{TmAl}_{3}, \mathrm{ErAl}_{3}, \mathrm{LuGa}_{3}, \mathrm{TmGa}_{3}, \mathrm{ErGa}_{3}, \mathrm{HoGa}_{3}, \mathrm{Dy}_{3} \mathrm{Ga}_{3}, \\
\mathrm{TbGa}_{3}, \mathrm{TmTl}_{3}, \mathrm{YAl}_{3}, \mathrm{LuAl}_{3}, \mathrm{TiZn}_{3}, \mathrm{TmIn}_{3}, \mathrm{ErIn}_{3}, \mathrm{LuIn}_{3}, \mathrm{HoIn}_{3}, \\
\mathrm{DyIn}_{3}, \mathrm{TbIn}_{3}, \mathrm{SmIn}_{3}, \mathrm{PrIn}_{3}, \mathrm{NdIn}_{3}, \mathrm{NdGa}_{3}, \mathrm{ErBPd}_{3}, \mathrm{LaIn}_{3}, \mathrm{PrAl}_{3}, \\
\mathrm{ZrHg}_{3}, \mathrm{ZrCd}_{3}, \mathrm{Ca}_{3} \mathrm{TIN}, \mathrm{MgPd}_{3}, \mathrm{USi}_{3}, \mathrm{Cr}_{3} \mathrm{Ni}, \mathrm{ZrOs}_{3}, \\
\mathrm{IrN}(\mathrm{ICSD}: 187719), \mathrm{HfOs}_{3}, \mathrm{UGe}_{3}, \mathrm{CeSn}_{3}, \mathrm{Pr}_{3} \mathrm{Tl}, \mathrm{Nd}_{3} \mathrm{Tl}, \mathrm{Mn}_{3} \mathrm{Rh}, \\
\mathrm{ZrBRh}_{3}\end{array}$ \\
\hline & $\overline{\mathrm{R}}_{10}(4)$ & 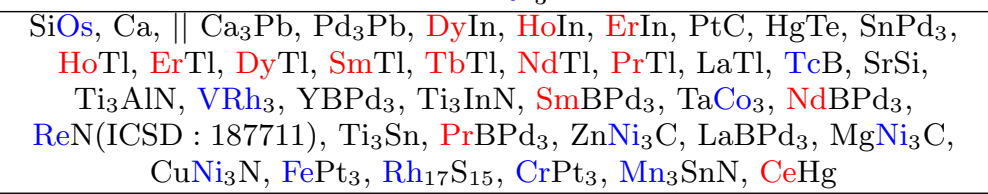 \\
\hline & $\bar{\Gamma}_{11}(4)$ & $\begin{array}{c}\mathrm{Na}_{3} \mathrm{~N}, \mathrm{~Tb}_{3} \mathrm{AlC}(\mathrm{ICSD}: 43866), \| \mathrm{Tm}_{3} \mathrm{AlC}, \mathrm{WC}, \mathrm{CuAu} \mathrm{A}_{3}, \mathrm{InAg}_{3}, \\
\mathrm{SrPb}_{3}, \mathrm{CsHgBr}_{3}, \mathrm{BaPbO}_{3}(\mathrm{ICSD}: 245605), \mathrm{Sm} \mathrm{AlC}_{3} \mathrm{AlC}, \mathrm{Sm}_{3} \mathrm{GaC} \\
\mathrm{Nd}_{3} \mathrm{InC}, \mathrm{Nd}_{3} \mathrm{AlC}, \mathrm{Pr}_{3} \mathrm{AlC}_{2} \mathrm{La}_{3} \mathrm{AlC}, \mathrm{La}_{3} \mathrm{InC}, \mathrm{La}_{3} \mathrm{TlC}, \mathrm{Sm}_{3} \mathrm{TlC} \\
\mathrm{NpRh}_{3}, \mathrm{Ce}_{2} \mathrm{Si}_{7} \mathrm{Rh}_{15}, \mathrm{Ca}_{3} \mathrm{SbN}, \mathrm{CuN}, \mathrm{NpGa}_{3}\end{array}$ \\
\hline & $\bar{\Gamma}_{10}(4)$ & 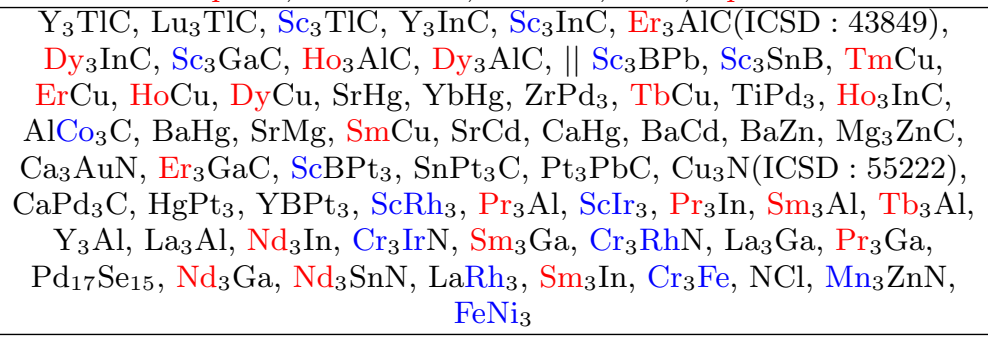 \\
\hline \multicolumn{3}{|r|}{ Space Group 223: $P m \overline{3} n$} \\
\hline & $\begin{array}{c}\Gamma_{4}^{+}(3), \mathrm{M}_{5}^{-}(2), \mathrm{R}_{2} \mathrm{R}_{3}(4) \\
\mathrm{X}_{4}(2)\end{array}$ & $\| \mathrm{Ba}_{4} \mathrm{Ge}_{20} \mathrm{Au}_{3}$ \\
\hline & $\begin{array}{c}\Gamma_{4}^{+}(3), \mathrm{M}_{5}^{-}(2), \mathrm{R}_{2} \mathrm{R}_{3}(4) \\
\mathrm{X}_{3}(2)\end{array}$ & $\| \mathrm{Ba}_{4} \mathrm{Cu}_{3} \mathrm{Ge}_{20}, \mathrm{Ba}_{4} \mathrm{Ag}_{3} \mathrm{Ge}_{20}$ \\
\hline & $\mathrm{M}_{5}^{-}(2), \mathrm{R}_{4}(6), \mathrm{X}_{2}(2)$ & $\| \mathrm{V}_{3} \mathrm{Ni}$ \\
\hline & $\Gamma_{5}^{-}(3), \mathrm{R}_{4}(6), \mathrm{X}_{3}(2)$ & $\mathrm{Nb}_{3} \mathrm{Os}, \mathrm{NaPd}_{3} \mathrm{O}_{4}, \mathrm{Tl}_{3} \mathrm{Pt}, \mathrm{Ti}_{3} \mathrm{Ir}$ \\
\hline & $\Gamma_{5}^{-}(3), \mathrm{R}_{4}(6), \mathrm{X}_{1}(2)$ & $\| \mathrm{La}_{3} \mathrm{Co}_{4} \mathrm{Sn}_{13}, \mathrm{Zr}_{3} \mathrm{Au}$ \\
\hline & $\Gamma_{5}^{-}(3), \mathrm{R}_{1}(2), \mathrm{X}_{4}(2)$ & $\| \mathrm{NaPt}_{3} \mathrm{O}_{4}$ \\
\hline & $\Gamma_{5}^{-}(3), \mathrm{M}_{5}^{-}(2), \mathrm{R}_{4}(6)$ & $\| \mathrm{Tl}_{3} \mathrm{Au}$ \\
\hline & $\Gamma_{5}^{+}(3), \mathrm{R}_{4}(6), \mathrm{X}_{2}(2)$ & $\mathrm{Ti}_{3} \mathrm{Sb}, \mathrm{Ba}_{4} \mathrm{Yb}\left(\mathrm{CuO}_{3}\right)_{3}$ \\
\hline
\end{tabular}


Table II - continued

\begin{tabular}{|c|c|c|}
\hline $\begin{array}{l}\text { SOC or } \\
\text { NSOC }\end{array}$ & $\begin{array}{l}\text { Degenerate Irreducible } \\
\text { Representations }\end{array}$ & High Symmetry Point Semimetals \\
\hline & $\Gamma_{5}^{+}(3), \mathrm{R}_{2} \mathrm{R}_{3}(4), \mathrm{X}_{2}(2)$ & $\mathrm{Ti}_{3} \mathrm{As}$ \\
\hline & $\Gamma_{5}^{+}(3), \mathrm{M}_{5}^{+}(2), \mathrm{R}_{4}(6)$ & $\mathrm{Mo}_{3} \mathrm{Pt}$ \\
\hline & $\Gamma_{5}^{+}(3), \mathrm{M}_{5}^{+}(2), \mathrm{R}_{2} \mathrm{R}_{3}(4)$ & $\mathrm{Zr}_{3} \mathrm{Sn}$ \\
\hline & $\Gamma_{4}^{-}(3), \mathrm{R}_{4}(6), \mathrm{X}_{4}(2)$ & $\| \mathrm{Pd}_{3} \mathrm{~N}$ \\
\hline & $\Gamma_{4}^{-}(3), \mathrm{R}_{4}(6), \mathrm{X}_{1}(2)$ & $\mathrm{AlMo}_{3}, \mathrm{GaMo}_{3}, \mathrm{~K}_{4} \mathrm{In}_{3} \mathrm{Ge}_{20}$ \\
\hline & $\Gamma_{4}^{-}(3), \mathrm{R}_{2} \mathrm{R}_{3}(4), \mathrm{X}_{3}(2)$ & $\| \mathrm{Ba}_{4} \mathrm{Si}_{20} \mathrm{Au}_{3}$ \\
\hline & $\Gamma_{4}^{-}(3), \mathrm{R}_{2} \mathrm{R}_{3}(4), \mathrm{X}_{1}(2)$ & $\| \mathrm{Cr}_{3} \mathrm{Ga}$ \\
\hline & $\Gamma_{4}^{+}(3), \mathrm{R}_{4}(6), \mathrm{X}_{2}(2)$ & $\mathrm{Cs}_{3} \mathrm{Hg}_{20}, \mathrm{~K}_{3}\left(\mathrm{Na}_{13} \mathrm{In}_{24}\right)_{2}$ \\
\hline & $\Gamma_{4}^{+}(3), \mathrm{R}_{4}(6), \mathrm{X}_{1}(2)$ & $\| \mathrm{Rb}_{3} \mathrm{Hg}_{20}$ \\
\hline & $\Gamma_{3}^{+}(2), \mathrm{R}_{4}(6), \mathrm{X}_{1}(2)$ & $\| \mathrm{Ti}_{3} \mathrm{Au}, \mathrm{V}_{3} \mathrm{~Pb}, \mathrm{Nb}_{3} \mathrm{Si}, \mathrm{Nb}_{3} \mathrm{Ge}, \mathrm{V}_{3} \mathrm{Sn}, \mathrm{V}_{3} \mathrm{Si}, \mathrm{Nb}_{3} \mathrm{~Pb}, \mathrm{~V}_{3} \mathrm{Ge}, \mathrm{Nb}_{3} \mathrm{Sn}$ \\
\hline & $\Gamma_{3}^{+}(2), \mathrm{M}_{5}^{+}(2), \mathrm{R}_{4}(6)$ & $\| \mathrm{K}_{4} \mathrm{Si}_{23}$ \\
\hline & $\mathrm{R}_{4}(6), \mathrm{X}_{4}(2)$ & $\mathrm{Mo}_{3} \mathrm{Ir}, \mathrm{Cr}_{3} \mathrm{Rh}, \mathrm{Cr}_{3} \mathrm{Ir}$ \\
\hline & $\mathrm{R}_{4}(6), \mathrm{X}_{3}(2)$ & $\| \mathrm{V}_{3} \mathrm{Fe}, \mathrm{Y}_{3} \mathrm{Ge}_{13} \mathrm{Ru}_{4}$ \\
\hline & $\mathrm{R}_{4}(6), \mathrm{X}_{2}(2)$ & $\mathrm{LaPd}_{3} \mathrm{~S}_{4}, \mathrm{NdPd}_{3} \mathrm{~S}_{4}, \mathrm{~V}_{3} \mathrm{Pt}, \mathrm{Nb}_{3} \mathrm{Pt}, \mathrm{V}_{3} \mathrm{Pd}$ \\
\hline & $\mathrm{R}_{4}(6), \mathrm{X}_{1}(2)$ & $\| \mathrm{Nb}_{3} \mathrm{Te}, \mathrm{La}_{3} \mathrm{Sn}_{13} \mathrm{Rh}_{4}, \mathrm{Ba}_{3} \mathrm{NaSi}_{23}$ \\
\hline & $\mathrm{R}_{1}(2), \mathrm{X}_{1}(2)$ & $\mathrm{Lu}_{3} \mathrm{Ge}_{13} \mathrm{Rh}_{4}, \mathrm{Pr}_{3} \mathrm{Sn}_{13} \mathrm{Rh}_{4}, \mathrm{Nd}_{3} \mathrm{Sn}_{13} \mathrm{Rh}_{4}, \mathrm{Sm}_{3} \mathrm{Sn}_{13} \mathrm{Rh}_{4}$ \\
\hline & $\mathrm{M}_{5}^{-}(2), \mathrm{R}_{4}(6)$ & $\| \mathrm{V}_{3} \mathrm{Ga}$ \\
\hline & $\mathrm{M}_{5}^{+}(2), \mathrm{R}_{4}(6)$ & $\mathrm{BeMo}_{3}, \mathrm{BaSi}_{23}, \mathrm{Ti}_{3} \mathrm{Pt}, \mathrm{LaSn}_{3} \mathrm{Ru}$ \\
\hline & $\Gamma_{5}^{-}(3), \mathrm{M}_{5}^{-}(2)$ & $\| \mathrm{Nb}_{3} \mathrm{Rh}, \mathrm{Nb}_{3} \mathrm{Ir}$ \\
\hline & $\Gamma_{5}^{+}(3), \mathrm{R}_{4}(6)$ & $\mathrm{Mo}_{3} \mathrm{Os}, \mathrm{Cr}_{3} \mathrm{Ru}, \mathrm{Cr}_{3} \mathrm{Os}, \mathrm{Cr}_{3} \mathrm{Pt}$ \\
\hline & $\Gamma_{5}^{+}(3), \mathrm{M}_{5}^{+}(2)$ & $\| \mathrm{Ba}_{3} \mathrm{Si}_{23}$ \\
\hline & $\Gamma_{4}^{-}(3), \mathrm{R}_{4}(6)$ & $\mathrm{SiMo}_{3}, \mathrm{GeMo}_{3}, \mathrm{SnMo}_{3}, \mathrm{Cr}_{3} \mathrm{Si}_{2} \mathrm{Zn}_{3} \mathrm{Au}, \mathrm{Ti}_{3} \mathrm{Pd}$ \\
\hline & $\Gamma_{4}^{-}(3), \mathrm{R}_{2} \mathrm{R}_{3}(4)$ & $\| \mathrm{NaCl}_{3}, \mathrm{Cr}_{3} \mathrm{Ge}$ \\
\hline & $\Gamma_{4}^{+}(3), \mathrm{R}_{4}(6)$ & $\| \mathrm{Ba}_{4} \mathrm{Si}_{23}$ \\
\hline & $\Gamma_{4}^{+}(3), \mathrm{M}_{5}^{+}(2)$ & $\| \mathrm{Cr}$ \\
\hline & $\Gamma_{3}^{+}(2), \mathrm{R}_{4}(6)$ & $\| \mathrm{Nb}_{3} \mathrm{Au}$ \\
\hline & $\Gamma_{3}^{+}(2), \mathrm{M}_{5}^{-}(2)$ & $\mathrm{V}_{3} \mathrm{Rh}, \mathrm{V}_{3} \mathrm{Co}$ \\
\hline & $\Gamma_{3}^{+}(2), \mathrm{M}_{5}^{+}(2)$ & $\| \mathrm{V}_{3} \operatorname{Ir}$ \\
\hline & $\mathrm{R}_{4}(6)$ & $\begin{array}{c}\| \mathrm{K}_{4} \mathrm{Sn}_{23}, \mathrm{Nb}_{3} \mathrm{Sb}, \mathrm{Ta}_{3} \mathrm{Sb}, \mathrm{ZrMo}_{3}, \mathrm{Pt}_{3} \mathrm{O}_{4}, \mathrm{Ag}_{3} \mathrm{PO}_{4}, \mathrm{Th}_{3} \mathrm{Sn}_{13} \mathrm{Rh}_{4}, \\
\mathrm{Nb}_{3} \mathrm{Tl}, \mathrm{Sr}_{3} \mathrm{Rh}_{4} \mathrm{~Pb}_{13}, \mathrm{Ca}_{3} \mathrm{Sn}_{13} \mathrm{Rh}_{4}, \mathrm{Yb}_{3} \mathrm{Sn}_{13} \mathrm{Rh}_{4}, \mathrm{~K}_{4} \mathrm{Ge}_{23}, \mathrm{Sr}_{3} \mathrm{Sn}_{13} \mathrm{Rh}_{4}, \\
\mathrm{Na}_{4} \mathrm{Si}_{23}, \mathrm{~K}_{29} \mathrm{NaHg}_{48}, \mathrm{Cs}_{4} \mathrm{Sn}_{23}\end{array}$ \\
\hline & $\mathrm{M}_{5}^{+}(2)$ & $\| \mathrm{V}_{3} \mathrm{As}, \mathrm{V}_{3} \mathrm{Sb}$ \\
\hline & $\Gamma_{5}^{-}(3)$ & $\mathrm{SrPd}_{3} \mathrm{O}_{4}, \mathrm{CaPd}_{3} \mathrm{O}_{4}, \| \mathrm{CdPd}_{3} \mathrm{O}_{4}$ \\
\hline & $\Gamma_{4}^{-}(3)$ & $\mathrm{W}_{3} \mathrm{O}, \mathrm{SiO}_{2}(\mathrm{ICSD}: 170488), \mathrm{Ba}_{4} \mathrm{Ni}_{3} \mathrm{Ge}_{20}, \mathrm{Ba}_{4} \mathrm{Ge}_{20} \mathrm{Pd}_{3}$ \\
\hline \multirow{11}{*}{$\mathrm{SOC}$} & $\bar{\Gamma}_{11}(4), \overline{\mathrm{R}}_{6} \overline{\mathrm{R}}_{7}(8), \overline{\mathrm{X}}_{5}(4)$ & $\| \mathrm{NaPd}_{3} \mathrm{O}_{4}, \mathrm{Ti}_{3} \mathrm{Ir}, \mathrm{La}_{3} \mathrm{Co}_{4} \mathrm{Sn}_{13}$ \\
\hline & $\bar{\Gamma}_{11}(4), \overline{\mathrm{R}}_{5}(4), \overline{\mathrm{X}}_{5}(4)$ & $\| \mathrm{NaPt}_{3} \mathrm{O}_{4}$ \\
\hline & $\bar{\Gamma}_{10}(4), \overline{\mathrm{R}}_{6} \overline{\mathrm{R}}_{7}(8), \overline{\mathrm{X}}_{5}(4)$ & $\begin{array}{c}\| \mathrm{Ti}_{3} \mathrm{Au}, \mathrm{V}_{3} \mathrm{~Pb}, \mathrm{Ti}_{3} \mathrm{As}, \mathrm{Ba}_{4} \mathrm{Cu}_{3} \mathrm{Ge}_{20}, \mathrm{Ba}_{4} \mathrm{Ag}_{3} \mathrm{Ge}_{20}, \mathrm{~V}_{3} \mathrm{Sn}, \mathrm{Nb}_{3} \mathrm{~Pb} \text {, } \\
\mathrm{V}_{3} \mathrm{Ge}\end{array}$ \\
\hline & $\bar{\Gamma}_{10}(4), \overline{\mathrm{R}}_{5}(4), \overline{\mathrm{X}}_{5}(4)$ & $\| \mathrm{Nb}_{3} \mathrm{Sn}, \mathrm{V}_{3} \mathrm{Si}, \mathrm{Nb}_{3} \mathrm{Ge}, \mathrm{Nb}_{3} \mathrm{Si}$ \\
\hline & $\overline{\mathrm{R}}_{6} \overline{\mathrm{R}}_{7}(8), \overline{\mathrm{X}}_{5}(4)$ & 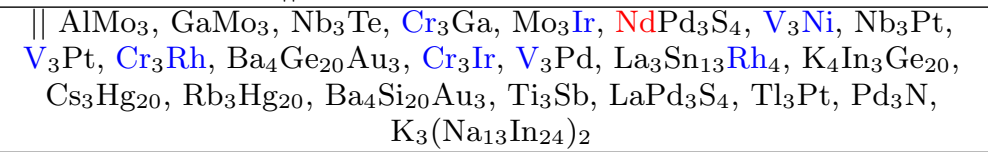 \\
\hline & $\overline{\mathrm{R}}_{5}(4), \overline{\mathrm{X}}_{5}(4)$ & $\begin{array}{l}\| \mathrm{Nb}_{3} \mathrm{Os}, \mathrm{Lu}_{3} \mathrm{Ge}_{13} \mathrm{Rh}_{4}, \mathrm{Ba}_{4} \mathrm{Yb}\left(\mathrm{CuO}_{3}\right)_{3}, \mathrm{Zr}_{3} \mathrm{Au}, \mathrm{Sm}_{3} \mathrm{Sn}_{13} \mathrm{Rh}_{4} \\
\mathrm{Nd}_{3} \mathrm{Sn}_{13} \mathrm{Rh}_{4}, \mathrm{Pr}_{3} \mathrm{Sn}_{13} \mathrm{Rh}_{4}, \mathrm{Y}_{3} \mathrm{Ge}_{13} \mathrm{Ru}_{4}, \mathrm{Ba}_{3} \mathrm{NaSi}_{23}, \mathrm{~V}_{3} \mathrm{Fe}\end{array}$ \\
\hline & $\bar{\Gamma}_{11}(4), \overline{\mathrm{R}}_{6} \overline{\mathrm{R}}_{7}(8)$ & $\mathrm{SiMo}_{3}, \mathrm{GeMo}_{3}, \mathrm{SnMo}_{3}, \mathrm{Cr}_{3} \mathrm{Si}, \mathrm{Cr}_{3} \mathrm{Ge}, \mathrm{Sr}_{3} \mathrm{Rh}_{4} \mathrm{~Pb}_{13}, \mathrm{Sr}_{3} \mathrm{Sn}_{13} \mathrm{Rh}_{4}$ \\
\hline & $\bar{\Gamma}_{10}(4), \overline{\mathrm{R}}_{6} \overline{\mathrm{R}}_{7}(8)$ & $\| \mathrm{Mo}_{3} \mathrm{Os}, \mathrm{Cr}_{3} \mathrm{Os}, \mathrm{Cr}_{3} \mathrm{Ru}, \mathrm{Nb}_{3} \mathrm{Au}, \mathrm{K}_{4} \mathrm{Si}_{23}, \mathrm{~K}_{29} \mathrm{NaHg}_{48}$ \\
\hline & $\overline{\mathrm{R}}_{6} \overline{\mathrm{R}}_{7}(8)$ & $\begin{array}{c}\| \mathrm{ZrMo}_{3}, \mathrm{BaSi}_{23}, \mathrm{Zr}_{3} \mathrm{Sn}, \mathrm{Ca}_{3} \mathrm{Sn}_{13} \mathrm{Rh}_{4}, \mathrm{Na}_{4} \mathrm{Si}_{23}, \mathrm{Nb}_{3} \mathrm{Tl}, \mathrm{Tl}_{3} \mathrm{Au}, \mathrm{NaCl}_{3}, \\
\mathrm{Ti}_{3} \mathrm{Pd}, \mathrm{V}_{3} \mathrm{Ga}\end{array}$ \\
\hline & $\bar{\Gamma}_{11}(4)$ & $\begin{array}{c}\mathrm{CdPd}_{3} \mathrm{O}_{4}, \| \mathrm{Nb}_{3} \mathrm{Ir}, \mathrm{SiO}_{2}(\mathrm{ICSD}: 170488), \mathrm{Ba}_{3} \mathrm{Si}_{23}, \mathrm{Ba}_{4} \mathrm{Ni}_{3} \mathrm{Ge}_{20} \\
\mathrm{Ba}_{4} \mathrm{Ge}_{20} \mathrm{Pd}_{3}\end{array}$ \\
\hline & $\bar{\Gamma}_{10}(4)$ & $\| \mathrm{V}_{3} \mathrm{Ir}, \mathrm{V}_{3} \mathrm{Rh}, \mathrm{V}_{3} \mathrm{Co}, \mathrm{Mo}_{3} \mathrm{Pt}, \mathrm{Cr}_{3} \mathrm{Pt}$ \\
\hline \multirow{6}{*}{ NSOC } & \multicolumn{2}{|r|}{$\overline{\text { Space Group 224: } P n \overline{3} m}$} \\
\hline & $\Gamma_{5}^{+}(3), \mathrm{M}_{1}(2), \mathrm{R}_{3}^{-}(2), \mathrm{X}_{2}(2)$ & $\| \mathrm{Ti}_{2} \mathrm{H}$ \\
\hline & $\Gamma_{4}^{+}(3), \mathrm{R}_{5}^{-}(3)$ & $\mathrm{Ag}_{2} \mathrm{O}_{3}$ \\
\hline & $\Gamma_{3}^{-}(2), \mathrm{R}_{4}^{-}(3)$ & $\mathrm{Pd}_{2} \mathrm{O}$ \\
\hline & $\Gamma_{3}^{-}(2), \mathrm{R}_{3}^{+}(2)$ & $\| \mathrm{PtO}_{2}$ \\
\hline & $\mathrm{R}_{4}^{-}(3)$ & $\mathrm{Pb}_{2} \mathrm{O}[5]$ \\
\hline
\end{tabular}


Table II - continued

\begin{tabular}{|c|c|c|}
\hline $\begin{array}{l}\text { SOC or } \\
\text { NSOC }\end{array}$ & $\begin{array}{l}\text { Degenerate Irreducible } \\
\text { Representations }\end{array}$ & High Symmetry Point Semimetals \\
\hline & $\Gamma_{4}^{-}(3)$ & $\mathrm{Zn}_{3} \mathrm{P}_{2}, \mathrm{CdB}_{2}$ \\
\hline & $\Gamma_{4}^{+}(3)$ & $\| \mathrm{Bi}_{2} \mathrm{O}_{3}$ \\
\hline \multirow{4}{*}{$\mathrm{SOC}$} & $\bar{\Gamma}_{10}(4), \overline{\mathrm{M}}_{5}(4), \overline{\mathrm{R}}_{11}(4)$ & $\| \mathrm{Ti}_{2} \mathrm{H}$ \\
\hline & $\bar{\Gamma}_{11}(4), \overline{\mathrm{R}}_{10}(4)$ & $\mathrm{PtO}_{2}$ \\
\hline & $\overline{\Gamma_{10}(4), \overline{\mathrm{R}}_{11}(4)}$ & $\| \mathrm{Pd}_{2} \mathrm{O}$ \\
\hline & $\overline{\mathrm{R}}_{11}(4)$ & $\| \mathrm{Pb}_{2} \mathrm{O}[5], \mathrm{Ag}_{2} \mathrm{O}_{3}$ \\
\hline \multicolumn{3}{|r|}{ Space Group 225: $F m \overline{3} m$} \\
\hline \multirow{41}{*}{ NSOC } & $\begin{array}{c}\Gamma_{5}^{+}(3), \mathrm{L}_{3}^{-}(2), \mathrm{W}_{5}(2) \\
\mathrm{X}_{5}^{-}(2)\end{array}$ & $\begin{array}{c}\| \mathrm{MgCdAg}_{2}, \operatorname{LuInPd}_{2}, \mathrm{YbSnPd}_{2}, \mathrm{TmInPd}_{2}, \mathrm{ErInPd}_{2}, \mathrm{ScAlPd}_{2}, \\
\operatorname{DyInPd}_{2}, \mathrm{ScInPd}_{2}, \mathrm{YbNi}_{2} \mathrm{Sn}\end{array}$ \\
\hline & $\begin{array}{c}\Gamma_{5}^{+}(3), \mathrm{L}_{3}^{+}(2), \mathrm{W}_{5}(2) \\
\mathrm{X}_{5}^{-}(2)\end{array}$ & $\| \mathrm{MgZnAg}_{2}, \mathrm{YInPd}_{2}$ \\
\hline & $\begin{array}{c}\Gamma_{4}^{-}(3), \mathrm{L}_{3}^{-}(2), \mathrm{W}_{5}(2) \\
\mathrm{X}_{5}^{-}(2)\end{array}$ & $\| \mathrm{Zr}_{6} \mathrm{Al}_{16} \mathrm{Ru}_{7}$ \\
\hline & $\begin{array}{c}\Gamma_{4}^{-}(3), \mathrm{L}_{3}^{+}(2), \mathrm{W}_{5}(2) \\
\mathrm{X}_{5}^{-}(2)\end{array}$ & $\| \mathrm{Sr}_{21} \mathrm{In}_{8} \mathrm{~Pb}_{7}$ \\
\hline & $\mathrm{L}_{3}^{+}(2), \mathrm{W}_{5}(2), \mathrm{X}_{5}^{+}(2)$ & $\mathrm{Zr}_{6} \mathrm{Al}_{7} \mathrm{Cu}_{16} \mathrm{H}_{9}$ \\
\hline & $\Gamma_{5}^{-}(3), \mathrm{L}_{3}^{-}(2), \mathrm{W}_{5}(2)$ & $\| \mathrm{UB}_{12}$ \\
\hline & $\Gamma_{5}^{-}(3), \mathrm{L}_{3}^{+}(2), \mathrm{W}_{5}(2)$ & $\| \mathrm{Fe}_{23} \mathrm{~B}_{6}$ \\
\hline & $\Gamma_{5}^{+}(3), \mathrm{L}_{3}^{-}(2), \mathrm{X}_{5}^{-}(2)$ & $\| \mathrm{Ga}_{2} \mathrm{Pt}, \mathrm{LiInAg}_{2}, \mathrm{In}_{2} \mathrm{Pt}$ \\
\hline & $\Gamma_{5}^{+}(3), \mathrm{L}_{3}^{-}(2), \mathrm{W}_{5}(2)$ & $\begin{array}{l}\text { \|TaN, NbN, ScInPt }{ }_{2}, \mathrm{MgNi}_{2} \mathrm{Sn}, \mathrm{ScGaPd}_{2}, \mathrm{ScAlNi}_{2}, \mathrm{ScGaNi}_{2}, \\
\mathrm{HfCO}_{2} \mathrm{Sn}, \mathrm{TaGaCo}_{2}, \mathrm{InCu}_{6} \mathrm{ClO}_{8}, \mathrm{VGaCo}_{2}, \mathrm{NbGaCo}_{2}\end{array}$ \\
\hline & $\Gamma_{5}^{+}(3), \mathrm{L}_{3}^{+}(2), \mathrm{X}_{5}^{-}(2)$ & $\| \mathrm{LiAlAg}_{2}$ \\
\hline & $\Gamma_{5}^{+}(3), \mathrm{L}_{3}^{+}(2), \mathrm{X}_{5}^{+}(2)$ & $\| \mathrm{Ba}_{2} \mathrm{TbIrO}_{6}, \mathrm{~K}_{2} \mathrm{OsBr}_{6}$ \\
\hline & $\Gamma_{5}^{+}(3), \mathrm{L}_{3}^{+}(2), \mathrm{W}_{5}(2)$ & $\| \mathrm{AlCu}_{3}, \mathrm{ZrS}, \mathrm{Zr}, \mathrm{VN}, \mathrm{ScInNi}_{2}, \mathrm{TiCo}_{2} \mathrm{Sn}$ \\
\hline & $\Gamma_{4}^{-}(3), \mathrm{L}_{3}^{-}(2), \mathrm{X}_{5}^{-}(2)$ & $\| \mathrm{Zn}_{2} \mathrm{CuAu}, \mathrm{Al}_{2} \mathrm{Pd}, \mathrm{Al}_{2} \mathrm{Pt}, \mathrm{Li}_{3} \mathrm{Tl}, \mathrm{S}$ \\
\hline & $\Gamma_{4}^{-}(3), \mathrm{L}_{3}^{+}(2), \mathrm{X}_{5}^{-}(2)$ & $\| \mathrm{Zn}_{2} \mathrm{AgAu}, \mathrm{CdC}$ \\
\hline & $\Gamma_{4}^{-}(3), \mathrm{L}_{3}^{+}(2), \mathrm{W}_{5}(2)$ & $\| \mathrm{CuNi}_{2} \mathrm{Sb}, \mathrm{ZnNi}_{2} \mathrm{Ge}$ \\
\hline & $\Gamma_{4}^{+}(3), \mathrm{W}_{5}(2), \mathrm{X}_{5}^{+}(2)$ & $\mathrm{Sr}_{11} \mathrm{Al}_{4} \mathrm{In}_{3}, \mathrm{Sr}_{11} \mathrm{In}_{3} \mathrm{Ga}_{4}, \mathrm{Sr}_{11} \mathrm{In}_{7}, \mathrm{Ca}_{11} \mathrm{Ga}_{7}$ \\
\hline & $\Gamma_{4}^{+}(3), \mathrm{L}_{3}^{+}(2), \mathrm{W}_{5}(2)$ & $\| \mathrm{Zr}_{6} \mathrm{Co}_{16} \mathrm{Ge}_{7}$ \\
\hline & $\Gamma_{3}^{-}(2), \mathrm{L}_{3}^{-}(2), \mathrm{W}_{5}(2)$ & $\| \mathrm{TiCO}_{2} \mathrm{Si}, \mathrm{AlVCO}_{2}$ \\
\hline & $\Gamma_{3}^{-}(2), \mathrm{L}_{3}^{+}(2), \mathrm{W}_{5}(2)$ & $\| \mathrm{TaAlCO}_{2}, \mathrm{TiCo}_{2} \mathrm{Ge}, \mathrm{NbAlCo}_{2}$ \\
\hline & $\Gamma_{3}^{+}(2), \mathrm{L}_{3}^{-}(2), \mathrm{W}_{5}(2)$ & $\| \mathrm{MoC}, \mathrm{MnGa}_{2} \mathrm{Co}$ \\
\hline & $\Gamma_{3}^{+}(2), \mathrm{L}_{3}^{+}(2), \mathrm{X}_{5}^{-}(2)$ & $\| \mathrm{TcB}$ \\
\hline & $\Gamma_{3}^{+}(2), \mathrm{L}_{3}^{+}(2), \mathrm{X}_{5}^{+}(2)$ & $\| \mathrm{PdO}$ \\
\hline & $\Gamma_{3}^{+}(2), \mathrm{L}_{3}^{+}(2), \mathrm{W}_{5}(2)$ & $\mathrm{MnGaNi}_{2}, \mathrm{MnCo}_{2} \mathrm{Sb}$ \\
\hline & $\mathrm{W}_{5}(2), \mathrm{X}_{5}^{+}(2)$ & $\mathrm{Ni}_{3} \mathrm{Sn}, \mathrm{Cu}_{6} \mathrm{PbO}_{8}(\mathrm{ICSD}: 280596), \mathrm{Cr}_{21}\left(\mathrm{WC}_{3}\right)_{2}$ \\
\hline & $\mathrm{L}_{3}^{-}(2), \mathrm{X}_{5}^{-}(2)$ & $\| \mathrm{Pt}\left(\mathrm{Cl}_{3} \mathrm{O}\right)_{2}$ \\
\hline & $\mathrm{L}_{3}^{+}(2), \mathrm{X}_{5}^{-}(2)$ & $\mathrm{Se}\left(\mathrm{Br}_{3} \mathrm{O}\right)_{2}$ \\
\hline & $\mathrm{L}_{3}^{+}(2), \mathrm{X}_{5}^{+}(2)$ & $\| \mathrm{PtO}$ \\
\hline & $\mathrm{L}_{3}^{+}(2), \mathrm{W}_{5}(2)$ & $\mathrm{U}_{3} \mathrm{Si}_{11} \mathrm{Pt}_{23}$ \\
\hline & $\Gamma_{5}^{-}(3), \mathrm{W}_{5}(2)$ & $\| \mathrm{PuTe}$ \\
\hline & $\Gamma_{5}^{-}(3), \mathrm{L}_{3}^{-}(2)$ & $\| \mathrm{HfB}_{12}, \mathrm{ZrB}_{12}, \mathrm{ThB}_{12}, \mathrm{U}_{6} \mathrm{Fe}_{16} \mathrm{Si}_{7} \mathrm{C}$ \\
\hline & $\Gamma_{5}^{-}(3), \mathrm{L}_{3}^{+}(2)$ & $\| \mathrm{NpN}, \mathrm{Ti}_{6} \mathrm{Si}_{7} \mathrm{Ni}_{16}, \mathrm{Zr}_{6} \mathrm{Co}_{16} \mathrm{Si}_{7}$ \\
\hline & $\Gamma_{5}^{+}(3), \mathrm{X}_{5}^{-}(2)$ & $\| \mathrm{Ta}_{6} \mathrm{Co}_{16} \mathrm{Si}_{7}, \mathrm{Nb}_{6} \mathrm{Co}_{16} \mathrm{Si}_{7}$ \\
\hline & $\Gamma_{5}^{+}(3), \mathrm{W}_{5}(2)$ & $\begin{array}{l}\| \mathrm{ZrO}, \mathrm{TiO}, \mathrm{ThS}, \mathrm{ThSe}, \mathrm{ErH}, \mathrm{Hf}, \mathrm{Ti}, \mathrm{Ba}_{2} \mathrm{HoReO}_{6}, \mathrm{Ba}_{2} \mathrm{YbRuO}_{6}, \\
\qquad \mathrm{Ba}_{6} \mathrm{Mg}_{23}, \mathrm{Sc}_{6} \mathrm{Ni}_{16} \mathrm{Ge}_{7}, \mathrm{Sr}_{6} \mathrm{Mg}_{23}\end{array}$ \\
\hline & $\Gamma_{5}^{+}(3), \mathrm{L}_{3}^{-}(2)$ & $\| \mathrm{Ti}_{6} \mathrm{Al}_{16} \mathrm{Pt}_{7}$ \\
\hline & $\Gamma_{5}^{+}(3), \mathrm{L}_{3}^{+}(2)$ & $\| \mathrm{VH}, \mathrm{ZrH}_{2}, \mathrm{ThH}_{2}, \mathrm{TiH}_{2}, \mathrm{US}$ \\
\hline & $\Gamma_{4}^{-}(3), \mathrm{X}_{5}^{-}(2)$ & $\begin{array}{c}\| \mathrm{Li}_{2} \mathrm{ZnGe}, \mathrm{Li}_{2} \mathrm{CdPb}, \mathrm{NiC}, \mathrm{Li}_{2} \mathrm{CdSn}, \mathrm{Li}_{2} \mathrm{ZnSn}, \mathrm{PdC}, \mathrm{Li}_{2} \mathrm{HgGe}, \\
\mathrm{Li}_{2} \mathrm{CdGe}, \mathrm{HgSe}, \mathrm{Pu}, \mathrm{Th}_{6} \mathrm{Mg}_{23}, \mathrm{Ba}_{14} \mathrm{Na}_{14} \mathrm{CaN}_{6}\end{array}$ \\
\hline & $\Gamma_{4}^{-}(3), \mathrm{X}_{5}^{+}(2)$ & $\| \mathrm{PtC}, \mathrm{UC}_{2}, \mathrm{FeCu}_{2} \mathrm{Sn}, \mathrm{Mg}_{6} \mathrm{Si}_{7} \mathrm{Ni}_{16}, \mathrm{Mg}_{6} \mathrm{Ni}_{16} \mathrm{Ge}_{7}$ \\
\hline & $\Gamma_{4}^{-}(3), \mathrm{W}_{5}(2)$ & $\| \mathrm{Pb}, \mathrm{Ge}, \mathrm{NaB}, \mathrm{Li}_{2} \mathrm{GaIr}, \mathrm{Li}_{3} \mathrm{Au}, \mathrm{Li}_{2} \operatorname{InIr}, \mathrm{KB}, \mathrm{Hf}_{6} \mathrm{Al}_{16} \mathrm{Os}_{7}$ \\
\hline & $\Gamma_{4}^{-}(3), \mathrm{L}_{3}^{-}(2)$ & $\begin{array}{c}\| \mathrm{H}_{2} \mathrm{~S}, \mathrm{Cu}_{3} \mathrm{Sb}, \mathrm{H}_{2} \mathrm{Se}, \mathrm{Li}_{2} \mathrm{GePd}, \mathrm{LiIn}_{2} \mathrm{Ir}, \mathrm{LiGa}_{2} \mathrm{Ir}, \mathrm{LiAl}_{2} \mathrm{Ir}, \mathrm{LiGa}_{2} \mathrm{Rh}, \\
\mathrm{Si}_{2} \mathrm{Ru}\end{array}$ \\
\hline & $\Gamma_{4}^{-}(3), \mathrm{L}_{3}^{+}(2)$ & $\| \mathrm{IN}, \mathrm{AgN}, \mathrm{AuN}, \mathrm{CuN}, \mathrm{OsN}_{2}, \mathrm{LiAl}_{2} \mathrm{Rh}, \mathrm{LiIn}_{2} \mathrm{Rh}, \mathrm{USnAu}_{2}$ \\
\hline & $\Gamma_{4}^{+}(3), \mathrm{X}_{5}^{+}(2)$ & $\| \mathrm{Ni}_{21}\left(\mathrm{SnB}_{3}\right)_{2}$ \\
\hline
\end{tabular}


Table II - continued

\begin{tabular}{|c|c|c|}
\hline $\begin{array}{l}\text { SOC or } \\
\text { NSOC }\end{array}$ & $\begin{array}{l}\text { Degenerate Irreducible } \\
\text { Representations }\end{array}$ & High Symmetry Point Semimetals \\
\hline & $\Gamma_{4}^{+}(3), \mathrm{W}_{5}(2)$ & $\mathrm{Zn}_{7} \mathrm{Sb}_{8} \mathrm{Ru}_{9}, \mathrm{NaPb}_{2} \mathrm{IO}_{6}, \mathrm{Cu}_{3} \mathrm{SbS}_{4}$ \\
\hline & $\Gamma_{4}^{+}(3), \mathrm{L}_{3}^{-}(2)$ & $\| \mathrm{Ni}_{21}\left(\mathrm{~B}_{3} \mathrm{Sb}\right)_{2}, \mathrm{Ho}_{6} \mathrm{Fe}_{23}$ \\
\hline & $\Gamma_{3}^{+}(2), \mathrm{L}_{3}^{-}(2)$ & $\| \mathrm{HRh}$ \\
\hline & $\Gamma_{3}^{+}(2), \mathrm{L}_{3}^{+}(2)$ & $\| \mathrm{NiO}, \mathrm{Pt}, \mathrm{Cs}_{2} \mathrm{AgAuCl}_{6}, \mathrm{Ni}$ \\
\hline & $\mathrm{X}_{5}^{-}(2)$ & 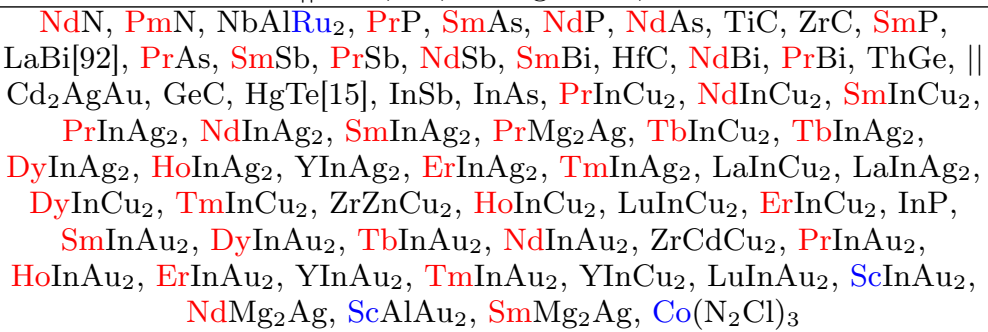 \\
\hline & $\mathrm{X}_{5}^{+}(2)$ & $\begin{array}{c}\| \mathrm{Ru}, \mathrm{Sn}_{2} \mathrm{MoOsO}_{6}, \mathrm{Ce}_{3}\left(\mathrm{Si}_{3} \mathrm{Pd}_{10}\right)_{2}, \mathrm{~K}_{3} \mathrm{Rh}\left(\mathrm{NO}_{2}\right)_{6}, \mathrm{Rb}_{3} \operatorname{Ir}\left(\mathrm{NO}_{2}\right)_{6} \\
\mathrm{~K}_{3} \operatorname{Ir}\left(\mathrm{NO}_{2}\right)_{6}\end{array}$ \\
\hline & $\mathrm{W}_{5}(2)$ & $\begin{array}{c}\mathrm{Mg}, \| \mathrm{CeMg}_{3}, \mathrm{Th}, \mathrm{PdN}_{2}, \mathrm{PaN}, \mathrm{UC}, \mathrm{Ni}_{20}\left(\mathrm{SnB}_{2}\right)_{3}, \mathrm{CeO}, \mathrm{CeS}, \mathrm{Ce}, \\
\mathrm{PaAs}, \mathrm{UNi}_{2} \mathrm{Sn}, \mathrm{Ba}_{2} \mathrm{YSbO}_{6}(\mathrm{ICSD}: 33624), \mathrm{CeCd}_{3}, \mathrm{CeSe}, \mathrm{Ca}_{7} \mathrm{Ge}, \\
\mathrm{Ba}_{2} \mathrm{ScSbO}_{6}, \mathrm{Zn}_{3}\left(\mathrm{Ni}_{10} \mathrm{~B}_{3}\right)_{2}, \mathrm{Ta}_{6} \mathrm{Si}_{7} \mathrm{Ni}_{16}, \mathrm{Cr}_{21}\left(\mathrm{MoC}_{3}\right)_{2}, \mathrm{U}_{2}\left(\mathrm{Ni}_{7} \mathrm{~B}_{2}\right)_{3}, \\
\mathrm{Nb}_{6} \mathrm{Ni}_{16} \mathrm{Ge}_{7}, \mathrm{Nb}_{6} \mathrm{Si}_{7} \mathrm{Ni}_{16}, \mathrm{Ta}_{6} \mathrm{Ni}_{16} \mathrm{Ge}_{7}\end{array}$ \\
\hline & $\mathrm{L}_{3}^{-}(2)$ & $\mathrm{BiI}_{3}, \| \mathrm{Hf}_{6} \mathrm{Si}_{7} \mathrm{Ni}_{16}, \mathrm{Cu}_{6} \mathrm{PbO}_{8}(\mathrm{ICSD}: 27585), \mathrm{Zr}_{6} \mathrm{Ni}_{16} \mathrm{Ge}_{7}$ \\
\hline & $\mathrm{L}_{3}^{+}(2)$ & 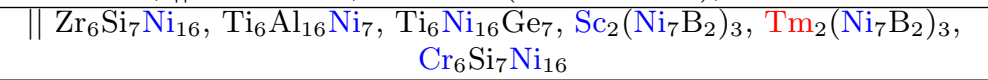 \\
\hline & $\overline{\Gamma_{5}^{-}(3)}$ & $\| \mathrm{Mg}_{6} \mathrm{Cu}_{16} \mathrm{Si}_{7}, \mathrm{Sc}_{11} \mathrm{Ir}_{4}, \mathrm{Sc}_{6} \mathrm{Ga}_{16} \mathrm{Ru}_{7}, \mathrm{Ti}_{6} \mathrm{Co}_{16} \mathrm{Si}_{7}$ \\
\hline & $\Gamma_{5}^{+}(3)$ & $\begin{array}{c}\mathrm{LaH}_{3}, \mathrm{TiFe}_{2} \mathrm{Sn}, \mathrm{TbH}_{3}, \mathrm{ErH}_{3}, \mathrm{NCl}, \| \mathrm{Si}_{2} \mathrm{Ni}, \mathrm{Sn}_{2} \mathrm{Pt}, \mathrm{YBiPd}_{2}, \mathrm{Mo}, \mathrm{W}, \\
\mathrm{TcN}, \mathrm{ReN}, \mathrm{Al}_{2} \mathrm{O}, \mathrm{Cr}, \mathrm{SiHgP}_{2}, \mathrm{Sr}_{11} \mathrm{Al}_{4} \mathrm{Sn}_{3}, \mathrm{Er}_{6} \mathrm{Zn}_{23}, \mathrm{Sr}_{11} \mathrm{Ga}_{4} \mathrm{Sn}_{3}, \\
\mathrm{Sc}_{6} \mathrm{Si}_{7} \mathrm{Ni}_{16}, \mathrm{Nb}_{6} \mathrm{Co}_{16} \mathrm{Ge}_{7}, \mathrm{Ta}_{6} \mathrm{Co}_{16} \mathrm{Ge}_{7}\end{array}$ \\
\hline & $\Gamma_{4}^{-}(3)$ & $\begin{array}{c}\mathrm{Cu}_{2} \mathrm{Se} 93, \mathrm{Cu}_{2} \mathrm{~S}[3], \mathrm{HCl}, \mid \mathrm{PbO}_{2}, \mathrm{BrN}, \mathrm{Li}_{2} \mathrm{MgPb}, \mathrm{TlN}, \mathrm{WC}, \mathrm{HBr}, \\
\mathrm{BeSiP}_{2}, \mathrm{MgSiP}_{2}, \mathrm{Cu}_{2} \mathrm{NiSn}, \mathrm{CdSiP}_{2}, \mathrm{RbB}, \mathrm{MnN}, \mathrm{Ni}_{21}\left(\mathrm{SnP}_{3}\right)_{2}, \mathrm{CsB}, \\
\mathrm{Ce}_{2}\left(\mathrm{Ni}_{7} \mathrm{~B}_{2}\right)_{3}\end{array}$ \\
\hline & $\Gamma_{4}^{+}(3)$ & $\mathrm{Ba}_{2} \mathrm{NaIO}_{6}, \| \mathrm{Ba}_{2} \mathrm{ZnTeO}_{6}, \mathrm{Cr}_{23} \mathrm{C}_{6}, \mathrm{Zr}_{2}\left(\mathrm{Ni}_{7} \mathrm{~B}_{2}\right)_{3}, \mathrm{Y}_{6} \mathrm{Fe}_{23}$ \\
\hline & $\Gamma_{3}^{-}(2)$ & $\| \mathrm{Sc}_{11} \mathrm{Os}_{4}$ \\
\hline & $\Gamma_{3}^{+}(2)$ & $\| \mathrm{Pd}, \mathrm{MnSbPd}_{2}$ \\
\hline \multirow[t]{2}{*}{$\mathrm{SOC}$} & $\bar{\Gamma}_{11}(4)$ & 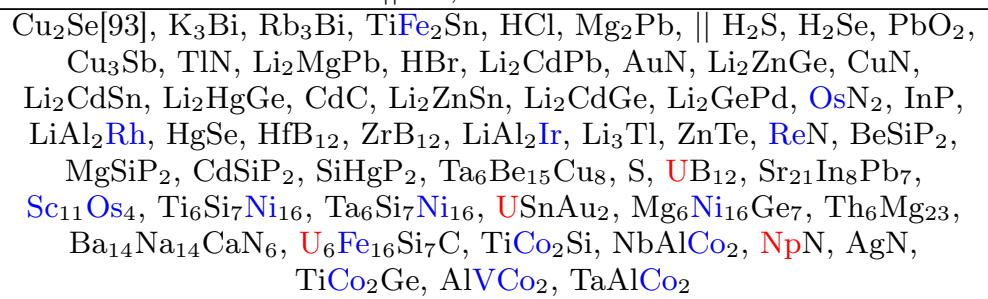 \\
\hline & $\bar{\Gamma}_{10}(4)$ & $\begin{array}{l}\mathrm{TbH}_{3}, \mathrm{ErH}_{3}, \| \mathrm{ThH}_{2}, \mathrm{TaN}, \mathrm{NbN}, \mathrm{ZrO}, \mathrm{Sn}_{2} \mathrm{Pt}, \mathrm{TiO}, \mathrm{MgCdAg}_{2}, \\
\mathrm{AlCu}_{3}, \mathrm{LiAlAg}_{2}, \mathrm{ThS}, \mathrm{ZrS}, \mathrm{MoC}, \mathrm{YBiPd} \\
2\end{array}$ \\
\hline \multicolumn{3}{|r|}{ Space Group 226: $F m \overline{3} c$} \\
\hline \multirow{8}{*}{ NSOC } & $\mathrm{L}_{1} \mathrm{~L}_{2}(4), \mathrm{W}_{5}(2), \mathrm{X}_{5}^{-}(2)$ & $\| \mathrm{LuBe}_{13}, \mathrm{DyBe}_{13}$ \\
\hline & $\mathrm{L}_{1} \mathrm{~L}_{2}(4), \mathrm{W}_{1} \mathrm{~W}_{2}(2), \mathrm{X}_{5}^{-}(2)$ & $\| \mathrm{TbBe}_{13}, \mathrm{NdBe}_{13}, \mathrm{ScBe}_{13}$ \\
\hline & $\Gamma_{5}^{-}(3), \mathrm{L}_{3}(2), \mathrm{W}_{5}(2)$ & $\| \mathrm{NaZn}_{13}$ \\
\hline & $\Gamma_{5}^{-}(3), \mathrm{L}_{3}(2), \mathrm{W}_{3} \mathrm{~W}_{4}(2)$ & $\mathrm{KZn}_{13}, \mathrm{KCd}_{13}, \mathrm{RbCd}_{13}, \mathrm{RbZn}_{13}, \mathrm{CsCd}_{13}$ \\
\hline & $\Gamma_{4}^{-}(3), \mathrm{L}_{3}(2), \mathrm{W}_{1} \mathrm{~W}_{2}(2)$ & $\| \mathrm{Ag}_{13} \mathrm{OsO}_{6}$ \\
\hline & $\mathrm{L}_{1} \mathrm{~L}_{2}(4), \mathrm{W}_{3} \mathrm{~W}_{4}(2)$ & $\| \mathrm{BaCu}_{13}$ \\
\hline & $\mathrm{L}_{1} \mathrm{~L}_{2}(4), \mathrm{W}_{1} \mathrm{~W}_{2}(2)$ & $\mathrm{Be}_{13} \mathrm{Sb}$ \\
\hline & $\Gamma_{5}^{-}(3), \mathrm{X}_{5}^{-}(2)$ & $\mathrm{BaBe}_{13}$ \\
\hline
\end{tabular}


Table II - continued

\begin{tabular}{|c|c|c|}
\hline $\begin{array}{l}\text { SOC or } \\
\text { NSOC }\end{array}$ & $\begin{array}{l}\text { Degenerate Irreducible } \\
\text { Representations }\end{array}$ & High Symmetry Point Semimetals \\
\hline & $\Gamma_{5}^{+}(3), \mathrm{L}_{1} \mathrm{~L}_{2}(4)$ & $\mathrm{ThBe}_{13}$ \\
\hline & $\Gamma_{4}^{+}(3), \mathrm{L}_{1} \mathrm{~L}_{2}(4)$ & $\| \mathrm{CeBe}_{13}$ \\
\hline & $\Gamma_{3}^{-}(2), \mathrm{L}_{1} \mathrm{~L}_{2}(4)$ & $\mathrm{ZrBe}_{13}, \mathrm{HfBe}_{13}$ \\
\hline & $\mathrm{L}_{1} \mathrm{~L}_{2}(4)$ & $\| \mathrm{LaCo}_{13}$ \\
\hline & $\Gamma_{5}^{-}(3)$ & $\mathrm{SrBe}_{13}, \mathrm{YbBe}_{13}, \mathrm{CaBe}_{13}, \mathrm{MgBe}_{13}, \mathrm{CaZn}_{13}, \mathrm{BaZn}_{13}, \mathrm{SrZn}_{13}$ \\
\hline & $\Gamma_{3}^{+}(2)$ & $\| \mathrm{LaCoCu}_{12}$ \\
\hline \multirow{5}{*}{ SOC } & $\bar{\Gamma}_{11}(4), \overline{\mathrm{L}}_{5} \overline{\mathrm{L}}_{6}(4), \overline{\mathrm{W}}_{6} \overline{\mathrm{W}}_{7}(4)$ & $\| \mathrm{Ag}_{13} \mathrm{OsO}_{6}$ \\
\hline & $\overline{\mathrm{L}}_{5} \overline{\mathrm{L}}_{6}(4), \overline{\mathrm{W}}_{6} \overline{\mathrm{W}}_{7}(4)$ & $\begin{array}{c}\text { I| } \mathrm{Be}_{13} \mathrm{Sb}, \mathrm{NaZn}_{13}, \mathrm{KZn}_{13}, \mathrm{RbZn}_{13}, \mathrm{RbCd}_{13}, \mathrm{CsCd}_{13}, \mathrm{DyBe}_{13}, \\
\mathrm{BaCu}_{13}, \mathrm{TbBe}_{13}, \mathrm{NdBe}_{13}\end{array}$ \\
\hline & $\overline{\mathrm{L}}_{4} \overline{\mathrm{L}}_{4}(4), \overline{\mathrm{W}}_{6} \overline{\mathrm{W}}_{7}(4)$ & $\| \mathrm{KCd}_{13}, \mathrm{LuBe}_{13}, \mathrm{ScBe}_{13}$ \\
\hline & $\bar{\Gamma}_{11}(4)$ & $\mathrm{MgBe}_{13}, \mathrm{ZrBe}_{13}, \mathrm{CaZn}_{13}, \mathrm{BaZn}_{13}, \mathrm{HfBe}_{13}, \mathrm{SrZn}_{13}, \mathrm{CeBe}_{13}$ \\
\hline & $\bar{\Gamma}_{10}(4)$ & $\| \mathrm{ThBe}_{13}, \mathrm{LaCoCu}_{12}, \mathrm{PuBe}_{13}$ \\
\hline \multicolumn{3}{|r|}{ Space Group 227: $F d \overline{3} m$} \\
\hline \multirow{39}{*}{ NSOC } & $\Gamma_{5}^{-}(3), \mathrm{L}_{3}^{-}(2), \mathrm{W}_{2}(2), \mathrm{X}_{3}(2)$ & $\mathrm{Mg}_{8} \mathrm{BPt}_{4}$ \\
\hline & $\Gamma_{5}^{-}(3), \mathrm{L}_{3}^{-}(2), \mathrm{W}_{2}(2), \mathrm{X}_{1}(2)$ & $\| \mathrm{NpFe}_{2}$ \\
\hline & $\Gamma_{5}^{+}(3), \mathrm{L}_{3}^{-}(2), \mathrm{W}_{2}(2), \mathrm{X}_{3}(2)$ & || LiZn \\
\hline & $\Gamma_{5}^{+}(3), \mathrm{L}_{3}^{+}(2), \mathrm{W}_{1}(2), \mathrm{X}_{4}(2)$ & $\mathrm{TaCr}_{2}, \mathrm{NbCr}_{2}$ \\
\hline & $\Gamma_{5}^{+}(3), \mathrm{L}_{3}^{+}(2), \mathrm{W}_{1}(2), \mathrm{X}_{3}(2)$ & $\| \mathrm{LiCd}$ \\
\hline & $\Gamma_{5}^{+}(3), \mathrm{L}_{3}^{+}(2), \mathrm{W}_{1}(2), \mathrm{X}_{2}(2)$ & $\| \mathrm{Be}_{2} \mathrm{Nb}, \mathrm{TaBe}_{2}$ \\
\hline & $\Gamma_{4}^{-}(3), \mathrm{L}_{3}^{+}(2), \mathrm{W}_{2}(2), \mathrm{X}_{4}(2)$ & $\mathrm{Cu}\left(\mathrm{IrS}_{2}\right)_{2}, \mathrm{Co}\left(\mathrm{NiS}_{2}\right)_{2}, \mathrm{Cu}\left(\mathrm{RhSe}_{2}\right)_{2}, \mathrm{Cu}\left(\mathrm{RhS}_{2}\right)_{2}, \mathrm{Co}_{2} \mathrm{CuS}_{4}$ \\
\hline & $\Gamma_{4}^{-}(3), \mathrm{L}_{3}^{+}(2), \mathrm{W}_{1}(2), \mathrm{X}_{3}(2)$ & $\| \mathrm{LaAl}_{2}, \mathrm{PrAl}_{2}, \mathrm{SmAl}_{2}$ \\
\hline & $\Gamma_{4}^{+}(3), \mathrm{L}_{3}^{-}(2), \mathrm{W}_{1}(2), \mathrm{X}_{3}(2)$ & $\| \mathrm{NpMn}_{2}$ \\
\hline & $\Gamma_{3}^{-}(2), \mathrm{L}_{3}^{+}(2), \mathrm{W}_{1}(2), \mathrm{X}_{3}(2)$ & $\| \mathrm{TmAl}_{2}, \mathrm{ScAl}_{2}, \mathrm{YAl}_{2}, \mathrm{ErAl}_{2}, \mathrm{HoAl}_{2}, \mathrm{DyAl}_{2}, \mathrm{TbAl}_{2}$ \\
\hline & $\Gamma_{3}^{-}(2), \mathrm{L}_{3}^{+}(2), \mathrm{W}_{1}(2), \mathrm{X}_{1}(2)$ & $\| \mathrm{LuAl}_{2}$ \\
\hline & $\Gamma_{3}^{+}(2), \mathrm{L}_{3}^{-}(2), \mathrm{W}_{2}(2), \mathrm{X}_{1}(2)$ & $\mathrm{LiTi}_{2} \mathrm{O}_{4}$ \\
\hline & $\mathrm{L}_{3}^{-}(2), \mathrm{W}_{2}(2), \mathrm{X}_{4}(2)$ & $\| \mathrm{KBi}_{2}$ \\
\hline & $\mathrm{L}_{3}^{-}(2), \mathrm{W}_{2}(2), \mathrm{X}_{2}(2)$ & $\mathrm{RbBi}_{2}$ \\
\hline & $\mathrm{L}_{3}^{-}(2), \mathrm{W}_{2}(2), \mathrm{X}_{1}(2)$ & $\| \mathrm{Nd}_{2} \mathrm{Al}$ \\
\hline & $\mathrm{L}_{3}^{-}(2), \mathrm{W}_{1}(2), \mathrm{X}_{4}(2)$ & $\mathrm{HoMn}_{2}, \mathrm{YMn}_{2}$ \\
\hline & $\mathrm{L}_{3}^{-}(2), \mathrm{W}_{1}(2), \mathrm{X}_{1}(2)$ & $\| \mathrm{Zr}_{2} \mathrm{CuS}_{4}$ \\
\hline & $\mathrm{L}_{3}^{+}(2), \mathrm{W}_{2}(2), \mathrm{X}_{2}(2)$ & $\| \mathrm{Ca}_{2} \mathrm{Ta}_{2} \mathrm{O}_{6} \mathrm{~F}$ \\
\hline & $\mathrm{L}_{3}^{+}(2), \mathrm{W}_{2}(2), \mathrm{X}_{1}(2)$ & $\| \mathrm{DyNi}_{2}, \mathrm{TbNi}_{2}, \mathrm{YNi}_{2}, \mathrm{SmNi}_{2}, \underset{\mathrm{ScNi}_{2}}{\mathrm{TbPt}_{2}}, \mathrm{NdNi}_{2}, \mathrm{PrNi}_{2}, \mathrm{YPt}_{2}, \mathrm{LaNi}_{2}$ \\
\hline & $\mathrm{L}_{3}^{+}(2), \mathrm{W}_{1}(2), \mathrm{X}_{2}(2)$ & $\| \mathrm{CsBi}_{2}$ \\
\hline & $\mathrm{L}_{3}^{+}(2), \mathrm{W}_{1}(2), \mathrm{X}_{1}(2)$ & $\| \mathrm{Ti}_{2} \mathrm{Co}$ \\
\hline & $\Gamma_{5}^{-}(3), \mathrm{W}_{2}(2), \mathrm{X}_{1}(2)$ & $\| \mathrm{NpRu}_{2}, \mathrm{NpOs}_{2}, \mathrm{NpNi}_{2}$ \\
\hline & $\Gamma_{5}^{+}(3), \mathrm{W}_{2}(2), \mathrm{X}_{3}(2)$ & $\mathrm{NdS}_{2}, \mathrm{LuS}_{2}, \mathrm{TmS}_{2}, \mathrm{ErS}_{2}, \mathrm{PrS}_{2}$ \\
\hline & $\Gamma_{5}^{+}(3), \mathrm{W}_{2}(2), \mathrm{X}_{2}(2)$ & $\| \mathrm{Sc}_{2} \mathrm{MnSe}_{4}$ \\
\hline & $\Gamma_{5}^{+}(3), \mathrm{W}_{2}(2), \mathrm{X}_{1}(2)$ & $\mathrm{ThZr}_{2} \mathrm{H}_{7}$ \\
\hline & $\Gamma_{5}^{+}(3), \mathrm{W}_{1}(2), \mathrm{X}_{1}(2)$ & $\| \mathrm{BiAu}_{2}$ \\
\hline & $\Gamma_{4}^{-}(3), \mathrm{W}_{2}(2), \mathrm{X}_{3}(2)$ & $\| \mathrm{NaAu}_{2}[5], \mathrm{NaAg}_{2}, \mathrm{YCu}_{2}$ \\
\hline & $\Gamma_{4}^{-}(3), \mathrm{W}_{2}(2), \mathrm{X}_{1}(2)$ & $\mathrm{Dy}\left(\mathrm{Zn}_{10} \mathrm{Ru}\right)_{2}, \mathrm{Y}\left(\mathrm{Zn}_{10} \mathrm{Ru}\right)_{2}, \mathrm{~Tb}\left(\mathrm{Zn}_{10} \mathrm{Fe}\right)_{2}$ \\
\hline & $\Gamma_{4}^{-}(3), \mathrm{W}_{1}(2), \mathrm{X}_{4}(2)$ & $\| \mathrm{RbNa}_{2} \mathrm{Ge}_{17}, \mathrm{CsNa}_{2} \mathrm{Ge}_{17}, \mathrm{Er}\left(\mathrm{Al}_{10} \mathrm{Cr}\right)_{2}$ \\
\hline & $\Gamma_{4}^{-}(3), \mathrm{W}_{1}(2), \mathrm{X}_{3}(2)$ & $\| \operatorname{PrRe}_{2}$ \\
\hline & $\Gamma_{4}^{-}(3), \mathrm{W}_{1}(2), \mathrm{X}_{2}(2)$ & $\begin{array}{c}\| \mathrm{PrIr}_{2}, \mathrm{NdIr}_{2}, \mathrm{LaIr}_{2}, \mathrm{TbIr}_{2}, \mathrm{DyIr}_{2}, \mathrm{HoIr}_{2}, \mathrm{YIr}_{2}, \mathrm{ErIr}_{2}, \mathrm{TmIr}_{2}, \\
\mathrm{NdRh}_{2}, \mathrm{PrRh}_{2}, \mathrm{LuIr}_{2}, \mathrm{SmRh}_{2}, \mathrm{TbRh}_{2}, \mathrm{HoRh}_{2}, \mathrm{DyRh}_{2}, \mathrm{LaRh}_{2}, \\
\mathrm{YRh}_{2}, \mathrm{ErRh}_{2}, \mathrm{TmRh}_{2}, \mathrm{LuRh}_{2}, \mathrm{ScIr}_{2}, \mathrm{NdCo}_{2}, \mathrm{PrCo}_{2}, \mathrm{SmCo}_{2}, \mathrm{TbCo}_{2}, \\
\mathrm{DyCo}_{2}, \mathrm{HoCo}_{2}, \mathrm{ErCo}_{2}, \mathrm{TmCo}_{2}, \mathrm{YCo}_{2}, \mathrm{LuCo}_{2}\end{array}$ \\
\hline & $\Gamma_{4}^{+}(3), \mathrm{W}_{2}(2), \mathrm{X}_{3}(2)$ & $\| \mathrm{SmRu}_{2}, \mathrm{NdRu}_{2}, \mathrm{PrOs}_{2}, \underset{\mathrm{PrRu}_{2}, \mathrm{ScFe}_{2}, \mathrm{ErFe}_{2}, \mathrm{HoFe}_{2}, \mathrm{YFe}_{2}, \mathrm{DyFe}_{2},}{ }$ \\
\hline & $\Gamma_{4}^{+}(3), \mathrm{W}_{2}(2), \mathrm{X}_{2}(2)$ & $\| \mathrm{Be}_{22} \mathrm{Re}$ \\
\hline & $\Gamma_{4}^{+}(3), \mathrm{W}_{1}(2), \mathrm{X}_{2}(2)$ & $\| \mathrm{NaPt}_{2}$ \\
\hline & $\Gamma_{3}^{-}(2), \mathrm{W}_{2}(2), \mathrm{X}_{2}(2)$ & $\mathrm{Yb}_{2} \mathrm{MnS}_{4}$ \\
\hline & $\Gamma_{3}^{-}(2), \mathrm{W}_{2}(2), \mathrm{X}_{1}(2)$ & $\mathrm{Yb}_{2} \mathrm{MnSe}_{4}$ \\
\hline & $\Gamma_{3}^{-}(2), \mathrm{W}_{1}(2), \mathrm{X}_{3}(2)$ & $\| \mathrm{ScB}_{2}$ \\
\hline & $\Gamma_{3}^{+}(2), \mathrm{W}_{2}(2), \mathrm{X}_{3}(2)$ & $\mathrm{LaOs}_{2}, \mathrm{LaRu}_{2}$ \\
\hline & $\mathrm{W}_{2}(2), \mathrm{X}_{3}(2)$ & $\| \mathrm{RbNa}_{2} \mathrm{Si}_{17}$ \\
\hline
\end{tabular}


Table II - continued

\begin{tabular}{|c|c|c|}
\hline $\begin{array}{l}\text { SOC or } \\
\text { NSOC }\end{array}$ & $\begin{array}{c}\text { Degenerate Irreducible } \\
\text { Representations }\end{array}$ & High Symmetry Point Semimetals \\
\hline & $\mathrm{W}_{2}(2), \mathrm{X}_{2}(2)$ & $\| \mathrm{Mg}_{8} \mathrm{BRh}_{4}$ \\
\hline & $\mathrm{W}_{2}(2), \mathrm{X}_{1}(2)$ & $\begin{array}{l}\mathrm{Rb}\left(\mathrm{TeO}_{3}\right)_{2}, \| \mathrm{NdPt}_{2}, \mathrm{PrPt}_{2}, \mathrm{SmPt}_{2}, \mathrm{ErNi}_{2}, \mathrm{HoNi}_{2}, \mathrm{LaPt}_{2}, \mathrm{LuNi}_{2}, \\
\quad \mathrm{ErPt}_{2}, \mathrm{DyPt}_{2}, \mathrm{TmPt}_{2}, \mathrm{HoPt}_{2}, \mathrm{In}_{3} \mathrm{~S}_{4}, \mathrm{NbCo}_{2}, \mathrm{TaV}_{2}, \mathrm{TaCo}_{2}\end{array}$ \\
\hline & $\mathrm{W}_{1}(2), \mathrm{X}_{4}(2)$ & $\| \mathrm{NdSi}_{2}, \mathrm{Dy}\left(\mathrm{Zn}_{10} \mathrm{Rh}\right)_{2}$ \\
\hline & $\mathrm{W}_{1}(2), \mathrm{X}_{3}(2)$ & $\mathrm{YZn}_{2}, \mathrm{NdMg}_{2}, \mathrm{PrMg}_{2}, \mathrm{SmMg}_{2}, \mathrm{CsNa}_{2} \mathrm{Si}_{17}$ \\
\hline & $\mathrm{W}_{1}(2), \mathrm{X}_{2}(2)$ & $\| \mathrm{LiPt}_{2}, \mathrm{LaMg}_{2}, \mathrm{~V}_{2} \mathrm{CuS}_{4}$ \\
\hline & $\mathrm{W}_{1}(2), \mathrm{X}_{1}(2)$ & $\begin{array}{c}\mathrm{Hg}_{2} \mathrm{~N}, \| \mathrm{Be}_{2} \mathrm{Ag}, \mathrm{Be}_{2} \mathrm{Cu}, \mathrm{CsU}_{2} \mathrm{O}_{6}, \mathrm{Co}_{3} \mathrm{~S}_{4}, \mathrm{Co}_{3} \mathrm{Se}_{4}, \mathrm{Cs}\left(\mathrm{OsO}_{3}\right)_{2}, \\
\left.\mathrm{Rb}_{(\mathrm{OsO}}\right)_{2}, \mathrm{~K}\left(\mathrm{OsO}_{3}\right)_{2}, \mathrm{Na}\left(\mathrm{OsO}_{3}\right)_{2}, \mathrm{NpIr}_{2}\end{array}$ \\
\hline & $\Gamma_{5}^{-}(3), \mathrm{L}_{3}^{-}(2)$ & $\| \mathrm{Mg}_{2} \mathrm{Pd}$ \\
\hline & $\Gamma_{5}^{-}(3), \mathrm{L}_{3}^{+}(2)$ & $\mathrm{CeRh}_{2}, \mathrm{Na}_{3} \mathrm{In}_{2} \mathrm{Ag}, \mathrm{Na}_{3} \mathrm{In}_{2} \mathrm{Au}, \mathrm{Nb}_{3} \mathrm{SiNi}_{2}, \mathrm{Ta}_{4} \mathrm{Ni}_{2} \mathrm{C}, \mathrm{Ta}_{3} \mathrm{SiNi}_{2}$ \\
\hline & $\Gamma_{5}^{+}(3), \mathrm{L}_{3}^{-}(2)$ & $\begin{array}{c}\| \mathrm{NaTl}, \mathrm{Pr}_{2} \mathrm{Ir}_{2} \mathrm{O}_{7}, \mathrm{Bi}_{2} \mathrm{Ir}_{2} \mathrm{O}_{7}, \mathrm{Ti}_{3} \mathrm{Al}_{2} \mathrm{NiO}, \mathrm{Bi}_{2} \mathrm{Rh}_{2} \mathrm{O}_{7}, \mathrm{Mn}_{3} \mathrm{~W}_{3} \mathrm{C} \\
\mathrm{Mn}_{3} \mathrm{Mo}_{3} \mathrm{C}\end{array}$ \\
\hline & $\Gamma_{5}^{+}(3), \mathrm{L}_{3}^{+}(2)$ & $\| \mathrm{ZrZn}_{2}, \mathrm{Ta}_{3} \mathrm{Al}_{2} \mathrm{CoC}, \mathrm{Cd}_{2} \mathrm{Re}_{2} \mathrm{O}_{7}, \mathrm{Al}_{2} \mathrm{Re}_{3} \mathrm{~B}, \mathrm{Ni}_{3} \mathrm{Mo}_{3} \mathrm{C}, \mathrm{Ce}\left(\mathrm{Al}_{10} \mathrm{Cr}\right)_{2}$ \\
\hline & $\Gamma_{4}^{-}(3), \mathrm{L}_{3}^{-}(2)$ & $\| \mathrm{Se}, \mathrm{Ti}_{3} \mathrm{Al}_{2} \mathrm{NiN}, \mathrm{Zr}_{2} \mathrm{Fe}, \mathrm{Ti}_{3} \mathrm{Cu}_{3} \mathrm{O}$ \\
\hline & $\Gamma_{4}^{-}(3), \mathrm{L}_{3}^{+}(2)$ & $\begin{array}{c}\| \mathrm{O}_{2}, \mathrm{MgCu}_{2}, \mathrm{BaAl}_{2}, \mathrm{ZrIr}_{2}, \mathrm{ThIr}_{2}, \mathrm{Nb}_{3} \mathrm{Cr}_{3} \mathrm{C}, \mathrm{CaIr}_{2}, \mathrm{Hf}_{3} \mathrm{Zn}_{3} \mathrm{C}, \\
\mathrm{NaSbO}_{3}, \mathrm{Ti}_{3} \mathrm{Zn}_{3} \mathrm{C}, \mathrm{Zr}_{3} \mathrm{Zn}_{3} \mathrm{C}\end{array}$ \\
\hline & $\Gamma_{4}^{+}(3), \mathrm{L}_{3}^{-}(2)$ & $\| \mathrm{Cr}_{2} \mathrm{Ni}, \mathrm{Al}_{10} \mathrm{~V}, \mathrm{Fe}_{3} \mathrm{~W}_{3} \mathrm{C}$ \\
\hline & $\Gamma_{4}^{+}(3), \mathrm{L}_{3}^{+}(2)$ & 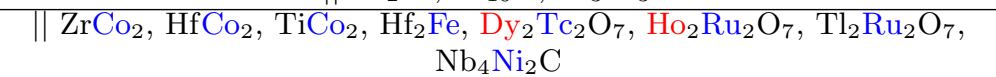 \\
\hline & $\Gamma_{3}^{-}(2), \mathrm{L}_{3}^{-}(2)$ & $\| \mathrm{Fe}\left(\mathrm{RhS}_{2}\right)_{2}$ \\
\hline & $\Gamma_{3}^{+}(2), \mathrm{L}_{3}^{-}(2)$ & $\| \mathrm{PuFe}_{2}$ \\
\hline & $\mathrm{L}_{3}^{-}(2)$ & $\begin{array}{l}\mathrm{K}_{17} \mathrm{In}_{41}, \| \mathrm{YbIr}_{2}, \mathrm{SrIr}_{2}, \mathrm{ThRu}_{2}, \mathrm{YbRh}_{2}, \mathrm{ZrFe}_{2}, \mathrm{HfFe}_{2}, \mathrm{SrRh}_{2} \\
\mathrm{Nb}_{8} \mathrm{Zn}_{4} \mathrm{C}_{3}, \mathrm{Na}_{17} \mathrm{In}_{12} \mathrm{Ga}_{29}, \mathrm{YbCo}_{2}, \mathrm{Nb}_{5} \mathrm{Ni}, \mathrm{Zr}_{4} \mathrm{Pd}_{2} \mathrm{O}, \mathrm{Co}_{3} \mathrm{Mo}_{3} \mathrm{~N}\end{array}$ \\
\hline & $\mathrm{L}_{3}^{+}(2)$ & $\begin{array}{l}\| \mathrm{CeOs}_{2}, \mathrm{SrAl}_{2}, \mathrm{ThOs}_{2}, \mathrm{CeRu}_{2}, \mathrm{CeFe}_{2}, \mathrm{CaRh}_{2}, \mathrm{CeCo}_{2}, \mathrm{U}\left(\mathrm{Al}_{10} \mathrm{~V}\right)_{2}, \\
\quad \mathrm{BaRh}_{2}, \mathrm{Ce}\left(\mathrm{Al}_{10} \mathrm{Co}\right)_{2}, \mathrm{Co}_{2} \mathrm{~W}_{4} \mathrm{C}, \mathrm{Ni}_{2} \mathrm{Mo}_{4} \mathrm{~N}, \mathrm{Fe}_{2} \mathrm{~W}_{2} \mathrm{C}\end{array}$ \\
\hline & $\Gamma_{5}^{-}(3)$ & $\begin{array}{l}\| \mathrm{La}_{2} \mathrm{Zr}_{2} \mathrm{O}_{7}(\mathrm{ICSD}: 184089), \mathrm{SbO}_{2}(\mathrm{ICSD}: 31103), \mathrm{UCo}_{2}, \mathrm{UOs}_{2}, \\
\quad \mathrm{SbAsPd}_{5}, \mathrm{UMn}_{2}, \mathrm{Co}_{2} \mathrm{Mo}_{4} \mathrm{~N}, \mathrm{Ni}_{2} \mathrm{Mo}_{4} \mathrm{C}, \mathrm{Zr}_{2} \mathrm{Cu}, \mathrm{V}_{3} \mathrm{SiNi}_{2}\end{array}$ \\
\hline & $\Gamma_{5}^{+}(3)$ & $\begin{array}{l}\mathrm{Sn}, \mathrm{Nb}_{2} \mathrm{Tl}_{2} \mathrm{O}_{7}, \| \mathrm{LiIn}, \mathrm{Sb}_{2} \mathrm{O}_{5}, \mathrm{NaIn}, \mathrm{ZrW}_{2}, \mathrm{HfW}_{2}, \mathrm{HfMo}_{2}, \mathrm{TiCr}_{2}, \\
\mathrm{ZrMo}_{2}, \mathrm{HfCr}_{2}, \mathrm{ZrCr}_{2}, \mathrm{Sb}_{2} \mathrm{~Pb}_{2} \mathrm{O}_{7}, \mathrm{AgSbO}_{3}, \mathrm{Zn}_{2} \mathrm{PdO}_{4}, \mathrm{HfZn}_{2}, \mathrm{ZrZn}_{22}, \\
\mathrm{Lu}_{2} \mathrm{FeS}_{4}, \mathrm{Sc}_{2} \mathrm{FeS}_{4}, \mathrm{Nd}_{2} \mathrm{Zr}_{2} \mathrm{O}_{7}(\mathrm{ICSD}: 261854)\end{array}$ \\
\hline & $\Gamma_{4}^{-}(3)$ & $\begin{array}{c}\mathrm{Yb}_{2} \mathrm{~V}_{2} \mathrm{O}_{7}, \| \mathrm{YbPt}_{2}, \mathrm{Nb}_{2} \mathrm{Cd}_{2} \mathrm{O}_{7}(\mathrm{ICSD}: 33672), \mathrm{ThMg}_{2}, \mathrm{Fe}\left(\mathrm{NiS}_{2}\right)_{2} \\
\mathrm{Co}_{2} \mathrm{NiS}_{4}, \mathrm{Yb}_{2} \mathrm{CdSe}_{4}, \mathrm{Zn}_{3} \mathrm{Ni}_{2} \mathrm{Ge}_{2} \mathrm{Zn}_{3} \mathrm{SiNi}_{2}, \mathrm{ZrV}_{2}, \mathrm{HfV}_{2}, \mathrm{Yb}_{2} \mathrm{MgSe}_{4} \\
\mathrm{Yb}_{2} \mathrm{CdS}_{4}, \mathrm{Yb}_{2} \mathrm{MgS}_{4}, \mathrm{PuCo}_{2}, \mathrm{Nd}_{2} \mathrm{Zr}_{2} \mathrm{O}_{7}(\mathrm{ICSD}: 160164), \mathrm{PuRh}_{4} \\
\mathrm{PuIr}_{2}, \mathrm{Ti}_{4} \mathrm{Cu}_{2} \mathrm{O}, \mathrm{Yb}_{2} \mathrm{Ti}_{2} \mathrm{O}_{7}, \mathrm{Ti}_{3} \mathrm{Al}_{2} \mathrm{NiC}_{3} \mathrm{Cr}_{3} \mathrm{SiNi}_{2}, \mathrm{Ba}_{10} \mathrm{Ga}_{2} \\
\mathrm{Ba}_{21} \mathrm{Si}_{2} \mathrm{O}_{5}\end{array}$ \\
\hline & $\Gamma_{4}^{+}(3)$ & $\begin{array}{c}\| \mathrm{Sc}_{2} \mathrm{Ni}, \mathrm{BaPd}_{2}, \mathrm{BaPt}_{2}, \mathrm{HfNi}_{2}, \mathrm{SrPt}_{2}, \mathrm{ZrNi}_{2}, \mathrm{OsPbO}_{3}, \mathrm{Y}_{2} \mathrm{Ru}_{2} \mathrm{O}_{7} \\
\mathrm{MnO}_{2}, \mathrm{Sb}_{2} \mathrm{Pt}_{2} \mathrm{O}_{7}, \mathrm{CdNi}_{2} \mathrm{Nb}_{3} \mathrm{Co}_{2} \mathrm{Si}_{1} \mathrm{Yb}_{2} \mathrm{Sn}_{2} \mathrm{O}_{7}\end{array}$ \\
\hline & $\Gamma_{3}^{-}(2)$ & $\| \mathrm{SrPd}_{2}, \mathrm{CaPt}_{2}, \mathrm{CaPd}_{2}, \mathrm{CaNi}_{2}, \mathrm{YbNi}_{2}, \mathrm{Cr}\left(\mathrm{GaS}_{2}\right)_{2}, \mathrm{Te}_{3} \mathrm{Pd}_{10}$ \\
\hline & $\Gamma_{3}^{+}(2)$ & $\| \mathrm{Nb}_{3} \mathrm{Co}_{3} \mathrm{C}, \mathrm{Ta}_{3} \mathrm{Co}_{3} \mathrm{C}, \mathrm{PuOs}_{2}, \mathrm{Mg}_{3}\left(\mathrm{TiAl}_{9}\right)_{2}, \mathrm{Zr}_{4} \mathrm{AlNi}_{2}, \mathrm{Nb}_{4} \mathrm{Zn}_{2} \mathrm{C}$ \\
\hline \multirow{3}{*}{$\mathrm{SOC}$} & $\bar{\Gamma}_{11}(4), \bar{X}_{5}(4)$ & $\begin{array}{c}\| \mathrm{NaAu}_{2}\left[5, \mathrm{YNi}_{2}, \mathrm{NaAg}_{2}, \mathrm{ScB}_{2}, \mathrm{YAl}_{2}, \mathrm{LuAl}_{2}, \mathrm{TmAl}_{2}, \mathrm{ScAl}_{2}, \mathrm{LuNi}_{2},\right. \\
\mathrm{ErAl}_{2}, \mathrm{HoAl} \mathrm{H}_{2}, \mathrm{DyAl}_{2}, \mathrm{TbAl}_{2}, \mathrm{LaAl}_{2}, \mathrm{Cu}\left(\mathrm{RhSe}_{2}\right)_{2}, \mathrm{PrAl}_{2}, \mathrm{Co}\left(\mathrm{NiS}_{2}\right)_{2}, \\
\mathrm{SmAl}_{2}, \mathrm{Cu}\left(\mathrm{IrS}_{2}\right)_{2}, \mathrm{Cu}\left(\mathrm{RhS}_{2}\right)_{2}, \mathrm{RbNa}_{2} \mathrm{Ge}_{17}, \mathrm{Co}_{2} \mathrm{CuS}_{4}, \mathrm{CsNa}_{2} \mathrm{Ge}_{17}, \\
\mathrm{NpNi}_{2}, \mathrm{YZn}_{2}, \mathrm{Yb}_{2} \mathrm{MnSe}_{4}, \mathrm{Yb}_{2} \mathrm{MnS}_{4}, \mathrm{YCu}_{2}\end{array}$ \\
\hline & $\bar{\Gamma}_{10}(4), \overline{\mathrm{X}}_{5}(4)$ & \begin{tabular}{l|l} 
& $\mathrm{LiZn}, \mathrm{TbNi}_{2}, \mathrm{DyNi}_{2}, \mathrm{SmNi}_{2}, \mathrm{HoNi}_{2}, \mathrm{ErNi}_{2}, \mathrm{NaPt}_{2}, \mathrm{Be}_{2} \mathrm{Nb}, \mathrm{LiPt}_{2}$, \\
& $\mathrm{TaBe}_{2}, \mathrm{LiTi}_{2} \mathrm{O}_{4}, \mathrm{PrRe}_{2}, \mathrm{SmMg}_{2}, \operatorname{Er}\left(\mathrm{Al}_{10} \mathrm{Cr}\right)_{2}, \mathrm{Sc}_{2} \mathrm{MnSe}_{4}$
\end{tabular} \\
\hline & $\overline{\mathrm{X}}_{5}(4)$ & 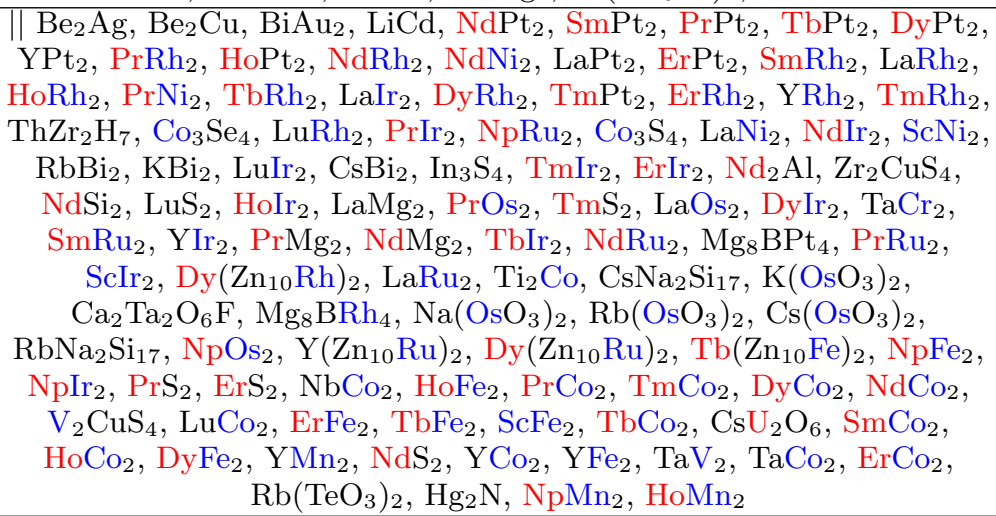 \\
\hline
\end{tabular}


Table II - continued

\begin{tabular}{|c|c|c|}
\hline $\begin{array}{l}\text { SOC or } \\
\text { NSOC }\end{array}$ & $\begin{array}{c}\text { Degenerate Irreducible } \\
\text { Representations }\end{array}$ & High Symmetry Point Semimetals \\
\hline & $\bar{\Gamma}_{11}(4)$ & $\begin{array}{c}\mathrm{Yb}_{2} \mathrm{~V}_{2} \mathrm{O}_{7}, \| \mathrm{KBiF}_{4}, \mathrm{SrPd}_{2}, \mathrm{MgCu}_{2}, \mathrm{CaPd}_{2}, \\
\mathrm{Nb}_{2} \mathrm{Cd}_{2} \mathrm{O}_{7}\left(\mathrm{ICSD}_{2}: 33672\right), \mathrm{SbO}_{2}(\mathrm{ICSD}: 31103), \mathrm{Na}_{3} \mathrm{In}_{2} \mathrm{Ag}, \mathrm{CaNi}_{2}, \\
\mathrm{YbNi}_{2}, \mathrm{Zn}_{3} \mathrm{SiNi}_{2}, \mathrm{Zn}_{3} \mathrm{Ni}_{2} \mathrm{Ge}, \mathrm{HfV}_{2}, \mathrm{ZrV}_{2}, \mathrm{Nd}_{2} \mathrm{Zr}_{2} \mathrm{O}_{7}(\mathrm{ICSD}: 160164), \\
\mathrm{SbAs}_{5} \mathrm{Id}_{5}, \mathrm{Hf}_{3} \mathrm{Zn}_{3} \mathrm{C}, \mathrm{Cr}\left(\mathrm{GaS}_{2}\right)_{2}, \mathrm{Te}_{3} \mathrm{Pd}_{10}, \mathrm{Ti}_{3} \mathrm{Zn}_{3} \mathrm{C}, \mathrm{Nb}_{3} \mathrm{SiNi}_{2}, \\
\mathrm{Ni}_{2} \mathrm{Mo}_{4} \mathrm{C}, \mathrm{Ti}_{3} \mathrm{Al}_{2} \mathrm{NiC}_{\mathrm{CiC}} \mathrm{CriNi}_{2}, \mathrm{Ta}_{3} \mathrm{SiNi}_{2}, \mathrm{RbBiF}_{4}, \mathrm{Ti}_{3} \mathrm{Cu}_{3} \mathrm{O}, \\
\mathrm{Zr}_{3} \mathrm{Zn}_{3} \mathrm{C}, \mathrm{V}_{3} \mathrm{SiNi}_{2}, \mathrm{Fe}\left(\mathrm{RhS}_{2}\right)_{2}, \mathrm{UMn}_{2}, \mathrm{UIr}_{2}, \mathrm{HfCo}_{2}, \mathrm{NaSbO}_{3}, \mathrm{O}_{2}\end{array}$ \\
\hline & $\bar{\Gamma}_{10}(4)$ & $\begin{array}{c}\mathrm{Ge}, \mathrm{Sn}, \| \mathrm{LiIn}, \mathrm{NaIn}, \mathrm{ThOs} \mathrm{S}_{2}, \mathrm{BaPd}_{2}, \mathrm{HfW}_{2}, \mathrm{ZrW}_{2}, \mathrm{HfMo}_{2}, \mathrm{TiCr}_{2}, \\
\mathrm{ZrMo}_{2}, \mathrm{HfCr}_{2}, \mathrm{ZrCr}_{2}, \mathrm{AgSbO}_{3}, \mathrm{Sb}_{2} \mathrm{~Pb}_{2} \mathrm{O}_{7}, \mathrm{Pr}_{2} \mathrm{Ir}_{2} \mathrm{O}_{7}, \mathrm{Ta}_{3} \mathrm{Al}_{2} \mathrm{CoC}, \\
\mathrm{Sb}_{2} \mathrm{Pt}_{2} \mathrm{O}_{7}, \mathrm{Ti}_{3} \mathrm{Al}_{2} \mathrm{NiO}_{2} \mathrm{Bi}_{2} \mathrm{Rh}_{2} \mathrm{O}_{7}, \mathrm{Bi}_{2} \mathrm{Ir}_{2} \mathrm{O}_{7}, \mathrm{OsPbO}_{3}, \mathrm{Hf}_{2} \mathrm{Fe}, \\
\mathrm{Mg}_{3}\left(\mathrm{TiAl}_{9}\right)_{2}, \mathrm{Nd}_{2} \mathrm{Zr}_{2} \mathrm{O}_{7}(\mathrm{ICSD}: 261854), \mathrm{Zr}_{4} \mathrm{AlNi}_{2}, \mathrm{Ni}_{3} \mathrm{Mo}_{3} \mathrm{C} \\
\mathrm{Dy}_{2} \mathrm{Tc}_{2} \mathrm{O}_{7}, \mathrm{Sc}_{2} \mathrm{Ni}_{2}, \mathrm{Nb}_{2} \mathrm{Tl}_{2} \mathrm{O}_{7}, \mathrm{Ce}\left(\mathrm{Al}_{10} \mathrm{Cr}_{2}, \mathrm{Ta}_{3} \mathrm{Co}_{3} \mathrm{C}, \mathrm{Nb}_{3} \mathrm{Co}_{3} \mathrm{C}\right. \\
\mathrm{Yb}_{2} \mathrm{Sn}_{2} \mathrm{O}_{7}, \mathrm{Fe}_{3} \mathrm{Mo}_{3} \mathrm{~N}, \mathrm{MnO}_{2}\end{array}$ \\
\hline & & Space Group 229: $\operatorname{Im} \overline{3} m$ \\
\hline \multirow{26}{*}{ NSOC } & $\Gamma_{5}^{-}(3), \mathrm{H}_{4}^{-}(3), \mathrm{P}_{4}(3)$ & $\mathrm{Pb}, \| \mathrm{Sn}$ \\
\hline & $\Gamma_{5}^{+}(3), \mathrm{H}_{4}^{-}(3), \mathrm{P}_{5}(3)$ & $\mathrm{Ga}_{7} \mathrm{Ni}_{3}, \mathrm{In}_{7} \mathrm{Pd}_{3}, \mathrm{In}_{7} \mathrm{Pt}_{3}$ \\
\hline & $\Gamma_{5}^{+}(3), \mathrm{H}_{4}^{+}(3), \mathrm{P}_{4}(3)$ & $\| \mathrm{Tb}_{4} \mathrm{Ga}_{6} \mathrm{FeGe}_{6}$ \\
\hline & $\Gamma_{4}^{-}(3), \mathrm{H}_{4}^{-}(3), \mathrm{P}_{4}(3)$ & $\mathrm{Se}, \mathrm{Ge}, \| \mathrm{Sb}_{3} \mathrm{Au}$ \\
\hline & $\Gamma_{4}^{+}(3), \mathrm{H}_{4}^{-}(3), \mathrm{P}_{4}(3)$ & $\| \mathrm{Sb}_{7} \mathrm{Mo}_{3}$ \\
\hline & $\Gamma_{4}^{+}(3), \mathrm{H}_{3}^{+}(2), \mathrm{P}_{4}(3)$ & $\mathrm{Mg}_{13} \mathrm{Al}_{14}$ \\
\hline & $\mathrm{H}_{5}^{-}(3), \mathrm{P}_{5}(3)$ & $\mathrm{Yb}_{11}\left(\mathrm{Ni}_{10} \mathrm{C}\right)_{6}$ \\
\hline & $\mathrm{H}_{5}^{+}(3), \mathrm{P}_{5}(3)$ & $\| \mathrm{Lu}_{4} \mathrm{Ge}_{6} \mathrm{Ru}_{7}$ \\
\hline & $\mathrm{H}_{5}^{+}(3), \mathrm{P}_{4}(3)$ & $\mathrm{Pr}_{6} \mathrm{Cd}_{4} \mathrm{Pd}_{13}$ \\
\hline & $\mathrm{H}_{4}^{-}(3), \mathrm{P}_{4}(3)$ & $\mathrm{Be} \|$ \\
\hline & $\mathrm{H}_{4}^{+}(3), \mathrm{P}_{3}(2)$ & $\| \mathrm{Pt}_{3} \mathrm{O}_{4}$ \\
\hline & $\mathrm{H}_{3}^{+}(2), \mathrm{P}_{4}(3)$ & $\mathrm{Mg}, \mathrm{Yb}, \| \mathrm{Sr}, \mathrm{Ba}, \mathrm{Sm}_{3}\left(\mathrm{GaNi}_{3}\right)_{2}, \mathrm{Sm}_{3}\left(\mathrm{AlNi}_{3}\right)_{2}$ \\
\hline & $\mathrm{H}_{3}^{+}(2), \mathrm{P}_{3}(2)$ & $\| \mathrm{Er}_{3}\left(\mathrm{AlNi}_{3}\right)_{2}, \mathrm{Y}_{3}\left(\mathrm{AlNi}_{3}\right)_{2}, \mathrm{Dy}_{3}\left(\mathrm{AlNi}_{3}\right)_{2}, \mathrm{Ho}_{3}\left(\mathrm{AlNi}_{3}\right)_{2}$ \\
\hline & $\Gamma_{5}^{-}(3), \mathrm{P}_{5}(3)$ & $\| \mathrm{Sr}_{17}\left(\mathrm{Li}_{2} \mathrm{Hg}_{3}\right)_{3}$ \\
\hline & $\Gamma_{5}^{-}(3), \mathrm{P}_{4}(3)$ & $\| \mathrm{Th}, \mathrm{Ce}, \mathrm{U}$ \\
\hline & $\Gamma_{5}^{-}(3), \mathrm{H}_{5}^{+}(3)$ & $\mathrm{Ce}_{3}\left(\mathrm{SiNi}_{3}\right)_{2}$ \\
\hline & $\Gamma_{5}^{+}(3), \mathrm{P}_{4}(3)$ & $\| \mathrm{Zr}, \mathrm{Hf}, \mathrm{Ti}$ \\
\hline & $\Gamma_{5}^{+}(3), \mathrm{H}_{5}^{+}(3)$ & $\| \mathrm{Mo}, \mathrm{Cr}$ \\
\hline & $\Gamma_{3}^{+}(2), \mathrm{H}_{3}^{+}(2)$ & $\| \mathrm{SiW}_{3}$ \\
\hline & $\mathrm{P}_{4}(3)$ & $\| \mathrm{Ca}_{3} \mathrm{Ag}_{8}$ \\
\hline & $\mathrm{H}_{4}^{-}(3)$ & $\| \mathrm{Pu}, \mathrm{Sn}_{7} \mathrm{Os}_{3}$ \\
\hline & $\mathrm{H}_{3}^{+}(2)$ & $\| \mathrm{Tb}_{4} \mathrm{Ga}_{12} \mathrm{Pd}, \mathrm{Y}_{4} \mathrm{Ga}_{12} \mathrm{Pd}, \mathrm{Tb}_{4} \mathrm{Ga}_{12} \mathrm{Pt}, \mathrm{Y}_{4} \mathrm{Ga}_{12} \mathrm{Pt}$ \\
\hline & $\Gamma_{5}^{-}(3)$ & $\mathrm{CrGa}_{4} \|$ \\
\hline & $\Gamma_{5}^{+}(3)$ & $\mathrm{W} \|$ \\
\hline & $\Gamma_{4}^{+}(3)$ & $\mathrm{Ba}_{4} \mathrm{Na}\left(\mathrm{BiO}_{4}\right)_{3}, \| \mathrm{Sn}_{7} \mathrm{Ir}_{3}$ \\
\hline & $\Gamma_{3}^{+}(2)$ & $\| \operatorname{Re}_{3} \mathrm{As}_{7}$ \\
\hline \multirow{14}{*}{$\mathrm{SOC}$} & $\bar{\Gamma}_{11}(4), \overline{\mathrm{H}}_{11}(4), \overline{\mathrm{P}}_{8}(4)$ & Se \| \\
\hline & $\bar{\Gamma}_{10}(4), \overline{\mathrm{H}}_{11}(4), \overline{\mathrm{P}}_{8}(4)$ & $\| \mathrm{Ga}_{7} \mathrm{Ni}_{3}$ \\
\hline & $\bar{\Gamma}_{10}(4), \overline{\mathrm{H}}_{10}(4), \overline{\mathrm{P}}_{8}(4)$ & $\| \mathrm{Mg}_{13} \mathrm{Al}_{14}$ \\
\hline & $\mathrm{H}_{11}(4), \mathrm{P}_{8}(4)$ & $\| \mathrm{Yb}_{11}\left(\mathrm{Ni}_{10} \mathrm{C}\right)_{6}$ \\
\hline & $\overline{\mathrm{H}}_{10}(4), \overline{\mathrm{P}}_{8}(4)$ & $\| \mathrm{Y}_{3}\left(\mathrm{AlNi}_{3}\right)_{2}$ \\
\hline & $\bar{\Gamma}_{11}(4), \overline{\mathrm{P}}_{8}(4)$ & $\| \mathrm{Sb}_{3} \mathrm{Au}, \mathrm{U}$ \\
\hline & $\bar{\Gamma}_{10}(4), \overline{\mathrm{P}}_{8}(4)$ & $\mathrm{Zr}, \mathrm{Hf}, \mathrm{Ti}, \mathrm{In}_{7} \mathrm{Pd}_{3}$ \\
\hline & $\bar{\Gamma}_{10}(4), \overline{\mathrm{H}}_{11}(4)$ & $\| \mathrm{In}_{7} \mathrm{Pt}_{3}, \mathrm{Sb}_{7} \mathrm{Mo}_{3}$ \\
\hline & $\bar{\Gamma}_{10}(4), \overline{\mathrm{H}}_{10}(4)$ & $\| \mathrm{SiW}_{3}, \mathrm{Pt}_{3} \mathrm{O}_{4}$ \\
\hline & $\overline{\mathrm{P}}_{8}(4)$ & $\| \mathrm{Th}, \mathrm{Ce}, \mathrm{Ca}_{3} \mathrm{Ag}_{8}$ \\
\hline & $\mathrm{H}_{11}(4)$ & $\mathrm{Sn}, \| \mathrm{Pu}, \mathrm{Sn}_{7} \mathrm{Ru}_{3}, \mathrm{Sr}_{17}\left(\mathrm{Li}_{2} \mathrm{Hg}_{3}\right)_{3}$ \\
\hline & $\overline{\mathrm{H}}_{10}(4)$ & $\begin{array}{c}\mathrm{Mg}, \mathrm{Yb}, \| \mathrm{Sr}, \mathrm{Mo}, \mathrm{Cr}, \mathrm{Ba}, \mathrm{Y}_{4} \mathrm{Ga}_{12} \mathrm{Pd}, \mathrm{Tb}_{4} \mathrm{Ga}_{12} \mathrm{Pd}, \mathrm{Tb}_{4} \mathrm{Ga}_{12} \mathrm{Pt}, \\
\mathrm{Y}_{4} \mathrm{Ga}_{12} \mathrm{Pt}, \mathrm{Pr}_{6} \mathrm{Cd}_{4} \mathrm{Pd}_{13}, \mathrm{Er}_{3}\left(\mathrm{AlNi}_{3}\right)_{2}, \mathrm{Ho}_{3}\left(\mathrm{AlNi}_{3}\right)_{2}, \mathrm{Dy}_{3}\left(\mathrm{AlNi}_{3}\right)_{2}, \\
\mathrm{Sm}_{3}\left(\mathrm{GaNi}_{3}\right)_{2}, \mathrm{Sm}_{3}\left(\mathrm{AlNi}_{3}\right)_{2}, \mathrm{Sn}_{7} \mathrm{Os}_{3}\end{array}$ \\
\hline & $\bar{\Gamma}_{11}(4)$ & $\| \mathrm{CrGa}_{4}$ \\
\hline & $\bar{\Gamma}_{10}(4)$ & $\mathrm{Ba}_{4} \mathrm{Na}\left(\mathrm{BiO}_{4}\right)_{3}, \| \mathrm{Tb}_{4} \mathrm{Ga}_{6} \mathrm{FeGe}_{6}, \mathrm{Ge}_{7} \mathrm{Ir}_{3}, \mathrm{Sn}_{7} \mathrm{Ir}_{3}, \mathrm{Re}_{3} \mathrm{As}_{7}, \mathrm{Ce}_{3}\left(\mathrm{SiNi}_{3}\right)_{2}$ \\
\hline \multicolumn{3}{|r|}{ Space Group 230: $I a \overline{3} d$} \\
\hline & $\Gamma_{5}^{-}(3), \mathrm{H}_{4}(6), \mathrm{P}_{1} \mathrm{P}_{2}(4)$ & $\| \mathrm{Bi}_{4} \mathrm{Rh}$ \\
\hline
\end{tabular}


Table II - continued

\begin{tabular}{c|c|c}
\hline $\begin{array}{c}\text { SOC or } \\
\text { NSOC }\end{array}$ & $\begin{array}{c}\text { Degenerate Irreducible } \\
\text { Representations }\end{array}$ & \multicolumn{2}{c}{ High Symmetry Point Semimetals } \\
\hline \multirow{5}{*}{} & $\mathrm{H}_{4}(6), \mathrm{P}_{1} \mathrm{P}_{2}(4)$ & $\| \mathrm{Yb}_{3} \mathrm{Al}_{5} \mathrm{O}_{12}$ \\
\cline { 2 - 3 } & $\Gamma_{4}^{-}(3), \mathrm{H}_{4}(6)$ & $\mathrm{Ca}_{3} \mathrm{Y}_{2}\left(\mathrm{GeO}_{4}\right)_{3}$ \\
\cline { 2 - 3 } & $\mathrm{H}_{4}(6)$ & $\mathrm{Ta}_{2} \mathrm{Br}_{5}, \mathrm{Ta}_{2} \mathrm{Cl}_{5} \|$ \\
\cline { 2 - 3 } & $\Gamma_{5}^{-}(3)$ & $\| \mathrm{Al}_{4} \mathrm{Ni}_{3}$ \\
\cline { 2 - 3 } & $\Gamma_{4}^{+}(3)$ & $\| \mathrm{Zn}\left(\mathrm{GaNi}_{3}\right.$ \\
\hline \multirow{5}{*}{$\mathrm{SOC}$} & $\bar{\Gamma}_{11}(4), \overline{\mathrm{H}}_{6} \overline{\mathrm{H}}_{7}(8), \overline{\mathrm{P}}_{7} \overline{\mathrm{P}}_{8}(6)$ & $\| \mathrm{Bi}_{4} \mathrm{Rh}$ \\
\cline { 2 - 3 } & $\bar{\Gamma}_{11}(4), \overline{\mathrm{H}}_{6} \overline{\mathrm{H}}_{7}(8)$ & $\mathrm{Ca}_{3} \mathrm{Y}_{2}\left(\mathrm{GeO}_{4}\right)_{3}$ \\
\cline { 2 - 3 } & $\overline{\mathrm{P}}_{7} \overline{\mathrm{P}}_{8}(6)$ & $\| \mathrm{Yb}_{3} \mathrm{Al}_{5} \mathrm{O}_{12}$ \\
\cline { 2 - 3 } & $\overline{\mathrm{H}}_{6} \overline{\mathrm{H}}_{7}(8)$ & $\mathrm{Ta}_{2} \mathrm{Br}_{5}, \mathrm{Ta}_{2} \mathrm{Cl}_{5} \|$ \\
\cline { 2 - 3 } & $\bar{\Gamma}_{10}(4)$ & $\| \mathrm{Zn}\left(\mathrm{GaNi}_{3}\right.$ \\
\hline \hline
\end{tabular}

Table III: List of High Symmetry Line Semimetal

\begin{tabular}{|c|c|c|}
\hline \multicolumn{3}{|c|}{ List of High Symmetry Line Semimetal } \\
\hline $\begin{array}{l}\text { SOC or } \\
\text { NSOC }\end{array}$ & $\begin{array}{l}\text { HSL with Band } \\
\text { Crossing }\end{array}$ & High Symmetry Line Semimetals \\
\hline \multicolumn{3}{|r|}{ Space Group 4: $P 2_{1}$} \\
\hline \multirow{3}{*}{ NSOC } & E-A, D-B & $\| \mathrm{KNa}_{2} \mathrm{AlH}_{6}$ \\
\hline & E-A & $\mathrm{KCuPO}_{4}$ \\
\hline & $\mathrm{Z}-\Gamma$ & $\mathrm{Ag}_{2} \mathrm{~S}(\mathrm{ICSD}: 98454)$ \\
\hline \multicolumn{3}{|r|}{ Space Group 5: $C 2$} \\
\hline \multirow{2}{*}{ NSOC } & $\mathrm{M}-\mathrm{A}, \mathrm{Y}-\Gamma$ & $\mathrm{KSe}_{3}, \mathrm{Sr}_{10} \mathrm{Cu}_{5} \mathrm{Bi}_{10} \mathrm{O}_{29}$ \\
\hline & $\mathrm{Y}-\Gamma$ & $\mathrm{Na}_{5} \mathrm{LiN}_{2}, \| \mathrm{TlV}_{5} \mathrm{~S}_{8}, \mathrm{RbCuCl}_{3}, \mathrm{Sc}_{3} \mathrm{Re}_{2} \mathrm{Si}_{3}$ \\
\hline \multicolumn{3}{|r|}{ Space Group 6: Pm } \\
\hline NSOC & $\begin{array}{c}\mathrm{Y}-\Gamma, \Gamma-\mathrm{B}, \Gamma-\mathrm{A}, \mathrm{Z}-\mathrm{E}, \mathrm{Z}-\mathrm{D}, \\
\mathrm{Z}-\mathrm{C}\end{array}$ & $\| \mathrm{La}_{2} \mathrm{AlNi}_{9} \mathrm{H}$ \\
\hline \multicolumn{3}{|r|}{ Space Group 7: $P c$} \\
\hline NSOC & $\mathrm{Y}-\Gamma, \Gamma-\mathrm{B}, \Gamma-\mathrm{A}$ & $\| \mathrm{VO}_{2}$ \\
\hline \multirow[t]{2}{*}{$\mathrm{SOC}$} & $\begin{array}{l}\text { Y-B, Y-A, Г-B, Г-A, Z-E, } \\
\text { Z-D, E-D, E-C, D-C }\end{array}$ & $\| \mathrm{Ga}_{9} \mathrm{Rh}_{2}, \mathrm{Ga}_{9} \mathrm{Ir}_{2}$ \\
\hline & $\begin{array}{c}\mathrm{Y}-\mathrm{B}, \mathrm{Y}-\mathrm{A}, \Gamma-\mathrm{B}, \Gamma-\mathrm{A}, \mathrm{Z}-\mathrm{E} \\
\mathrm{Z}-\mathrm{D}, \mathrm{E}-\mathrm{C}, \mathrm{D}-\mathrm{C}\end{array}$ & \| $\mathrm{ReN}_{2}$ \\
\hline \multicolumn{3}{|r|}{ Space Group 8: $\mathrm{Cm}$} \\
\hline \multirow{4}{*}{ NSOC } & $\mathrm{Y}-\Gamma, \mathrm{Y}-\mathrm{A}, \mathrm{M}-\Gamma, \mathrm{M}-\mathrm{A}$ & $\mathrm{Ca}_{3} \mathrm{SiBr}_{2}(\mathrm{ICSD}: 89545), \mathrm{SiGeN}_{2} \mathrm{O}, \mathrm{VHO}_{3}, \mathrm{ZrCu}, \mathrm{Er}_{10} \mathrm{Ru}_{10} \mathrm{C}_{19}$ \\
\hline & $\mathrm{Y}-\mathrm{A}, \mathrm{M}-\mathrm{A}, \Gamma-\mathrm{A}$ & $\mathrm{KNO}_{2}(\mathrm{ICSD}: 36202) \|$ \\
\hline & $\mathrm{Y}-\Gamma, \mathrm{M}-\Gamma, \Gamma-\mathrm{A}$ & $\| \mathrm{Nd}_{2} \mathrm{Cu}\left(\mathrm{GeO}_{4}\right)_{2}, \mathrm{Ca}_{3} \mathrm{SiBr}_{2}(\mathrm{ICSD}: 89544), \mathrm{Re}_{4} \mathrm{Si}_{7}, \mathrm{Al}_{4} \mathrm{Mo}$ \\
\hline & $\mathrm{Y}-\mathrm{M}, \mathrm{M}-\Gamma, \mathrm{M}-\mathrm{A}$ & $\| \mathrm{BiPd}_{2} \mathrm{~Pb}$ \\
\hline \multicolumn{3}{|r|}{ Space Group 10: $P 2 / m$} \\
\hline \multirow{8}{*}{ NSOC } & 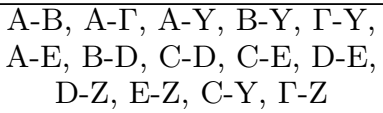 & \| NbRh \\
\hline & $\begin{array}{l}\text { A-B, A- } \Gamma, A-Y, B-Y, \Gamma-Y, \\
\text { C-D, C-E, C-Z, D-E, E-Z }\end{array}$ & $\| \mathrm{Bi}$ \\
\hline & $\begin{array}{c}\mathrm{A}-\Gamma, \mathrm{B}-\Gamma, \Gamma-\mathrm{Y}, \mathrm{B}-\mathrm{D}, \mathrm{C}-\mathrm{Y}, \\
\Gamma-\mathrm{Z}\end{array}$ & $\| \mathrm{Th}_{3} \mathrm{~B}_{2} \mathrm{C}_{3}$ \\
\hline & A-E, B-D, C-Y, $\Gamma-Z$ & $\mathrm{O}_{2} \|$ \\
\hline & C-E, D-E, E-Z, C-Y & $\mathrm{Tb}_{4} \mathrm{Mg}_{3} \mathrm{Co}_{2}$ \\
\hline & A-E, B-D, C-Y & $\mathrm{Na}_{7}\left(\mathrm{Cu}_{6} \mathrm{~S}_{5}\right)_{2}$ \\
\hline & $\mathrm{B}-\mathrm{D}, \Gamma-\mathrm{Z}$ & $\| \mathrm{TiBrO}$ \\
\hline & $\mathrm{C}-\mathrm{Y}, \Gamma-\mathrm{Z}$ & $\mathrm{ZrTiSe}_{4} \|$ \\
\hline \multicolumn{3}{|r|}{ Space Group 11: $P 2_{1} / m$} \\
\hline & $\begin{array}{c}\text { A-B, A- } \Gamma, A-Y, B-\Gamma, B-Y, \\
\text { C-Y, }-\mathrm{Z} \text { - }\end{array}$ & $\| \mathrm{Si}_{5} \mathrm{Pt}_{6}$ \\
\hline & $\begin{array}{c}\mathrm{A}-\mathrm{B}, \mathrm{A}-\Gamma, \mathrm{B}-\Gamma, \mathrm{B}-\mathrm{Y}, \Gamma-\mathrm{Y}, \\
\mathrm{A}-\mathrm{E}, \Gamma-\mathrm{Z}\end{array}$ & $\| \mathrm{Si}_{5} \mathrm{Rh}_{4}$ \\
\hline & $\begin{array}{c}\mathrm{A}-\mathrm{B}, \mathrm{A}-\Gamma, \mathrm{B}-\mathrm{Y}, \Gamma-\mathrm{Y}, \mathrm{A}-\mathrm{E}, \\
\mathrm{B}-\mathrm{D}, \Gamma-\mathrm{Z}\end{array}$ & $\| \mathrm{CeSi}_{2} \mathrm{Ru}$ \\
\hline
\end{tabular}


Table III - continued

\begin{tabular}{|c|c|c|}
\hline $\begin{array}{l}\text { SOC or } \\
\text { NSOC }\end{array}$ & $\begin{array}{l}\text { HSL with Band } \\
\text { Crossing }\end{array}$ & High Symmetry Line Semimetals \\
\hline & $\begin{array}{l}\text { A- } \Gamma, A-Y, B-\Gamma, B-Y, A-E, \\
\text { B-D, } \Gamma-Z\end{array}$ & $\| \mathrm{NbCuTe}_{2}$ \\
\hline & $\begin{array}{c}\mathrm{A}-\mathrm{B}, \mathrm{A}-\Gamma, \underset{\Gamma-\mathrm{A}}{\mathrm{A}} \mathrm{Y}, \mathrm{B}-\mathrm{Y}, \Gamma-\mathrm{Y} \\
\end{array}$ & $\| \mathrm{TaPt}_{3}$ \\
\hline & 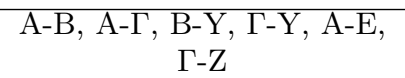 & $\| \mathrm{TiPd}$ \\
\hline & $\mathrm{A}-\mathrm{B}, \mathrm{A}-\Gamma, \mathrm{A}-\mathrm{Y}, \mathrm{A}-\mathrm{E}, \Gamma-\mathrm{Z}$ & $\pi \mathrm{Yb}_{2} \mathrm{~S}_{3}$ \\
\hline & $\mathrm{A}-\mathrm{B}, \mathrm{A}-\mathrm{\Gamma}, \mathrm{A}-\mathrm{Y}, \mathrm{B}-\mathrm{D}, \mathrm{C}-\mathrm{Y}$ & HfGePd \\
\hline & $\mathrm{A}-\mathrm{B}, \mathrm{A}-\mathrm{Y}, \mathrm{B}-\Gamma, \Gamma-\mathrm{Y}, \mathrm{A}-\mathrm{E}$ & $\mathrm{Ho}_{2} \mathrm{Sb}_{5}, \mathrm{Yb}_{2} \mathrm{PrDyS}_{6}, \mathrm{TbYb}_{2} \mathrm{PrS}_{6}, \mathrm{Yb}_{3} \mathrm{CeS}_{6}$ \\
\hline & $\mathrm{A}-\mathrm{B}, \mathrm{A}-\mathrm{Y}, \mathrm{B}-\Gamma, \Gamma-\mathrm{Y}, \mathrm{B}-\mathrm{D}$ & $\| \mathrm{Nb}_{2} \mathrm{Se}_{3}$ \\
\hline & $\mathrm{A}-\Gamma, \mathrm{A}-\mathrm{Y}, \mathrm{B}-\Gamma, \mathrm{B}-\mathrm{Y}, \Gamma-\mathrm{Z}$ & "IbNi \\
\hline & A-Y, B-Y, $\Gamma-Y, A-E, B-D$ & $\| \mathrm{Ni}_{3} \mathrm{Te}_{2}$ \\
\hline & $\mathrm{A}-\mathrm{Y}, \mathrm{B}-\mathrm{Y}, \Gamma-\mathrm{Y}, \mathrm{B}-\mathrm{D}, \Gamma-\mathrm{Z}$ & $\mathrm{NbPt}_{3}, \mathrm{TaNi}_{3}$ \\
\hline & $\mathrm{A}-\mathrm{Y}, \mathrm{B}-\mathrm{Y}, \Gamma-\mathrm{Y}, \mathrm{C}-\mathrm{Y}, \Gamma-\mathrm{Z}$ & $\| \mathrm{Sr}(\mathrm{ZnSn})_{2}$ \\
\hline & $\mathrm{A}-\mathrm{B}, \mathrm{A}-\Gamma, \mathrm{A}-\mathrm{Y}, \mathrm{A}-\mathrm{E}$ & $\| \mathrm{TiO}_{2}$ \\
\hline & $\mathrm{A}-\mathrm{B}, \mathrm{A}-\Gamma, \mathrm{A}-\mathrm{Y}, \mathrm{B}-\mathrm{D}$ & $\| \mathrm{ZrTe}_{3}$ \\
\hline & $\mathrm{A}-\mathrm{B}, \mathrm{A}-\Gamma, \mathrm{A}-\mathrm{Y}, \mathrm{C}-\mathrm{Y}$ & $\mathrm{Cs}_{2} \mathrm{Bi}_{8} \mathrm{Se}_{13}$ \\
\hline & $\mathrm{A}-\mathrm{B}, \mathrm{A}-\mathrm{Y}, \mathrm{B}-\Gamma, \Gamma-\mathrm{Y}$ & $\mathrm{Ba}(\mathrm{SbAu})_{2}, \mathrm{Dy}_{2} \mathrm{Sb}_{5}, \mathrm{~Tb}_{2} \mathrm{Sb}_{5}$ \\
\hline & $\mathrm{A}-\mathrm{B}, \mathrm{B}-\Gamma, \mathrm{B}-\mathrm{Y}, \Gamma-\mathrm{Z}$ & $\| \mathrm{Cu}_{2} \mathrm{H}_{3} \mathrm{ClO}_{3}$ \\
\hline & $\mathrm{A}-\Gamma, \mathrm{A}-\mathrm{Y}, \mathrm{B}-\Gamma, \mathrm{B}-\mathrm{Y}$ & $\| \mathrm{SiS}_{4}$ \\
\hline & $\mathrm{A}-\Gamma, \mathrm{B}-\Gamma, \Gamma-\mathrm{Y}, \mathrm{A}-\mathrm{E}$ & $\mathrm{TaSe}_{3} \|$ \\
\hline & $\mathrm{A}-\Gamma, \mathrm{B}-\Gamma, \Gamma-\mathrm{Y}, \Gamma-\mathrm{Z}$ & $\| \mathrm{Hf}_{2} \mathrm{CoP}$ \\
\hline & $\mathrm{A}-\mathrm{Y}, \mathrm{B}-\mathrm{Y}, \Gamma-\mathrm{Y}, \mathrm{A}-\mathrm{E}$ & $\| \mathrm{Ce}(\mathrm{InPt})_{2}$ \\
\hline & A-E, B-D, C-Y & $\| \mathrm{LiBeB}$ \\
\hline & $\mathrm{A}-\mathrm{E}, \mathrm{C}-\mathrm{Y}, \Gamma-\mathrm{Z}$ & $\| \mathrm{Mo}_{2} \mathrm{~S}_{3}$ \\
\hline & $\mathrm{A}-\Gamma, \mathrm{B}-\Gamma, \Gamma-\mathrm{Y}$ & $\| \mathrm{ErB}_{3} \mathrm{Mo}, \mathrm{TiNi}_{\mathrm{C}} \mathrm{CdNCl}_{3}$ \\
\hline & $\mathrm{A}-\mathrm{Y}, \mathrm{B}-\mathrm{Y}, \Gamma-\mathrm{Y}$ & $\| \mathrm{Ta}_{2} \mathrm{Se}_{3}, \mathrm{C}_{3} \mathrm{IN}$ \\
\hline & $\mathrm{B}-\mathrm{D}, \Gamma-\mathrm{Z}$ & $\| \mathrm{La}_{2} \mathrm{CuSnO}_{6}$ \\
\hline & $\mathrm{C}-\mathrm{Y}, \Gamma-\mathrm{Z}$ & $\mathrm{SrSb}_{2}, \mathrm{BaSb}_{2}(\mathrm{ICSD}: 409517), \| \mathrm{BaSb}_{2}(\mathrm{ICSD}: 413519), \mathrm{Ba}_{3}(\mathrm{GeN})_{2}$ \\
\hline & A-E & $\| \mathrm{CaSb}_{2}, \mathrm{Bi}(\mathrm{ICSD}: 42679)$ \\
\hline & B-D & $\| \mathrm{Ag}_{2} \mathrm{~S}$ \\
\hline & $\mathrm{C}-\mathrm{Y}$ & $\| \operatorname{In}_{3} \mathrm{Bi}_{7}\left(\mathrm{~Pb}_{2} \mathrm{~S}_{9}\right)_{2}$ \\
\hline & $\Gamma-\mathrm{Z}$ & $\mathrm{BaNd}_{2} \mathrm{Ti}_{3} \mathrm{O}_{10}, \| \mathrm{AlAu}, \mathrm{Te}_{2} \mathrm{Mo}, \mathrm{Zr}_{2} \mathrm{CoP}$ \\
\hline \multicolumn{3}{|r|}{ Space Group 12: $C 2 / m$} \\
\hline \multirow{15}{*}{ NSOC } & $\begin{array}{c}\mathrm{A}-\Gamma, \mathrm{A}-\mathrm{Y}, \Gamma-\mathrm{M}, \mathrm{M}-\mathrm{Y}, \mathrm{A}-\mathrm{M}, \\
\Gamma-\mathrm{Y}\end{array}$ & $\| \mathrm{Zr}_{2} \mathrm{HBr}_{2}, \mathrm{Pr}_{2} \mathrm{Al}_{19} \mathrm{Co}_{6}$ \\
\hline & $\mathrm{A}-\Gamma, \mathrm{A}-\mathrm{M}, \mathrm{A}-\mathrm{Y}, \Gamma-\mathrm{M}, \Gamma-\mathrm{Y}$ & $\| \mathrm{Mg}_{4} \mathrm{Zn}_{7}$ \\
\hline & $\mathrm{A}-\Gamma, \mathrm{A}-\mathrm{M}, \Gamma-\mathrm{M}, \Gamma-\mathrm{Y}, \mathrm{M}-\mathrm{Y}$ & $\| \mathrm{Ba}_{2} \mathrm{Mg}_{3} \mathrm{Si}_{4}$ \\
\hline & $\mathrm{A}-\Gamma, \mathrm{A}-\mathrm{Y}, \Gamma-\mathrm{M}, \Gamma-\mathrm{Y}, \mathrm{M}-\mathrm{Y}$ & $\| \mathrm{AlCu}$ \\
\hline & $\mathrm{A}-\Gamma, \mathrm{A}-\mathrm{Y}, \Gamma-\mathrm{M}, \mathrm{M}-\mathrm{Y}, \mathrm{A}-\mathrm{M}$ & $\| \mathrm{La}_{3} \mathrm{Ge}_{3} \mathrm{Br}_{2}, \mathrm{La}_{3} \mathrm{Ge}_{3} \mathrm{Cl}_{2}, \mathrm{La}_{3} \mathrm{Si}_{3} \mathrm{Cl}_{2}$ \\
\hline & $\mathrm{A}-\Gamma, \mathrm{A}-\mathrm{Y}, \Gamma-\mathrm{M}, \mathrm{M}-\mathrm{Y}, \Gamma-\mathrm{Y}$ & $\| \mathrm{Y}_{3} \mathrm{Si}_{3} \mathrm{Rh}, \mathrm{Cr}_{3} \mathrm{Ga}_{4}$ \\
\hline & $\mathrm{A}-\mathrm{M}, \mathrm{A}-\mathrm{Y}, \Gamma-\mathrm{M}, \Gamma-\mathrm{Y}, \mathrm{M}-\mathrm{Y}$ & $\| \mathrm{LiEu}_{2} \mathrm{Si}_{3}$ \\
\hline & $\mathrm{A}-\Gamma, \mathrm{A}-\mathrm{M}, \mathrm{A}-\mathrm{Y}, \Gamma-\mathrm{Y}$ & $\| \mathrm{Ta}_{2} \mathrm{NiSe}_{7}, \mathrm{Ta}_{2} \mathrm{Te}_{3}, \mathrm{Ce}_{2} \mathrm{Mo}_{2} \mathrm{C}_{3}, \mathrm{Nb}_{2} \mathrm{Se}, \mathrm{Ti}_{4} \mathrm{FeS}_{8}, \mathrm{Nb}_{2} \mathrm{Te}_{3}, \mathrm{Sc}_{8} \mathrm{Te}_{3}$ \\
\hline & A- $\Gamma$, A-M, $\Gamma-Y, M-Y$ & $\begin{array}{l}\| \mathrm{K}_{4} \mathrm{Cu}_{8} \mathrm{Te}_{11}, \mathrm{Ho}_{2} \mathrm{Mo}_{2} \mathrm{C}_{3}, \mathrm{Bi}_{2} \mathrm{Pd}, \mathrm{Ni}_{3}(\mathrm{BiSe})_{2}, \mathrm{Yb}_{2} \mathrm{Ge}_{2} \mathrm{Ir}, \mathrm{Sc}_{2} \mathrm{Si} \mathrm{Si}_{2} \mathrm{Ru}, \\
\mathrm{Tb}_{2} \mathrm{Ge}_{2} \mathrm{Ru}, \mathrm{Sc}_{2} \mathrm{FeSi}_{2}, \mathrm{Cr}_{2} \mathrm{NiS}_{4}, \mathrm{Co}_{2} \mathrm{NiSe}_{4}, \mathrm{~Tb}_{4} \mathrm{In}_{20} \mathrm{Ni}_{11}, \mathrm{LaIO}_{3}, \mathrm{WBr}\end{array}$ \\
\hline & A-Г, A-Y, Г-M, M-Y & $\begin{array}{c}\mathrm{Ba}_{3}\left(\mathrm{ZnAs}_{2}\right)_{2}, \mathrm{Ba}_{3}\left(\mathrm{CdAs}_{2}\right)_{2}, \| \mathrm{Ba}_{3}\left(\mathrm{CdSb}_{2}\right)_{2}, \mathrm{Ba}_{2} \mathrm{LaBiO}_{6}, \mathrm{AsPd}_{5} \\
\mathrm{Na}_{3} \mathrm{Cd}_{2} \mathrm{IrO}_{6}, \mathrm{Ni}_{3}(\mathrm{BiS})_{2}\end{array}$ \\
\hline & $\mathrm{A}-\Gamma, \Gamma-\mathrm{M}, \Gamma-\mathrm{Y}, \mathrm{A}-\mathrm{M}$ & $\begin{array}{c}\| \mathrm{Bi}, \mathrm{Er}_{2} \mathrm{Mo}_{2} \mathrm{C}_{3}(\mathrm{ICSD}: 88515), \mathrm{Yb}_{3}\left(\mathrm{Ga}_{2} \mathrm{Ge}_{3}\right)_{2}, \mathrm{Zn}_{13} \mathrm{Fe}, \mathrm{Ba}_{5} \mathrm{P}_{5} \mathrm{I}_{3}, \\
\mathrm{La}_{5} \mathrm{Si}_{5} \mathrm{I}_{3}, \mathrm{NaN}_{3}(\mathrm{ICSD}: 29375), \mathrm{Ba}(\mathrm{NiAs})_{2}\end{array}$ \\
\hline & A-M, A-Y, Г-M, Г-Y & $\begin{array}{c}\mathrm{SrAs}_{3}, \mathrm{BaAs}_{3}[94], \| \mathrm{YCI}, \mathrm{BaSb}_{3}, \mathrm{Nb}_{2} \mathrm{PdS}_{6}, \mathrm{IrC}_{2}, \mathrm{YCBr}, \mathrm{MgNb}_{2} \\
\mathrm{Yb}_{3} \mathrm{H}_{8}, \mathrm{Sm}_{2} \mathrm{AlGe}_{6}, \mathrm{Bi}_{2} \mathrm{Rh}_{3} \mathrm{~S}_{2}, \mathrm{Hf}_{2} \mathrm{Te}, \mathrm{LaSe}_{2} \mathrm{Zr}_{2} \mathrm{Ni}_{7}, \mathrm{Ba}_{7} \mathrm{Ir}_{6} \mathrm{O}_{19} \\
\mathrm{KNp}\left(\mathrm{CO}_{3}\right)_{2}, \mathrm{BaIrO}_{3}, \mathrm{AsN}\left(\mathrm{OF}_{3}\right)_{2}, \mathrm{U}_{8} \mathrm{CrS}_{17}\end{array}$ \\
\hline & A-M, Г-M, M-Y, Г-Y & $\| \mathrm{Yb}_{2}(\mathrm{ZnGe})_{3}, \mathrm{As}_{3} \mathrm{~W}_{2}, \mathrm{Si}_{2} \mathrm{NiSe}_{4}, \mathrm{Bi}_{2} \mathrm{Pd}_{5}, \mathrm{Zr}_{2} \mathrm{CoSi}_{2}, \mathrm{MoCl}_{5}$ \\
\hline & $\mathrm{A}-\mathrm{Y}, \mathrm{\Gamma}-\mathrm{Y}, \mathrm{M}-\mathrm{Y}, \mathrm{A}-\mathrm{M}$ & $\mathrm{Mg}_{5} \mathrm{Si}_{6}(\mathrm{ICSD}: 54780), \mathrm{MgZn}_{5}, \mathrm{Ba}\left(\mathrm{Cu}_{5} \mathrm{P}_{2}\right)_{2}, \mathrm{Zr}_{7} \mathrm{P}_{4}, \mathrm{Pr}_{2} \mathrm{Os}_{3}, \mathrm{Ga}_{5} \mathrm{Fe}_{6}$ \\
\hline & $\mathrm{A}-\Gamma, \mathrm{A}-\mathrm{M}, \mathrm{A}-\mathrm{Y}$ & $\begin{array}{c}\mathrm{SrP}_{3}(\mathrm{ICSD}: \text { : 96543) } 94], \| \mathrm{Li}_{2} \mathrm{Ta}_{3} \mathrm{~N}_{5}, \mathrm{Ni}\left(\mathrm{RhSe}_{2}\right)_{2}, \mathrm{PtN}_{4}(\mathrm{ClO})_{2} \\
\mathrm{Mg}\left(\mathrm{BrO}_{3}\right)_{2}, \mathrm{Mg}\left(\mathrm{ClO}_{3}\right)_{2}\end{array}$ \\
\hline
\end{tabular}


Table III - continued

\begin{tabular}{|c|c|c|}
\hline $\begin{array}{l}\text { SOC or } \\
\text { NSOC }\end{array}$ & $\begin{array}{l}\text { HSL with Band } \\
\text { Crossing }\end{array}$ & High Symmetry Line Semimetals \\
\hline & $\mathrm{A}-\Gamma, \Gamma-\mathrm{M}, \Gamma-\mathrm{Y}$ & $\begin{array}{c}\mathrm{Na}_{2} \mathrm{Zr}\left(\mathrm{CuS}_{2}\right)_{2}, \mathrm{Sr}_{2} \mathrm{MgTeO}_{6}, \mathrm{Na}_{6} \mathrm{ZnSn}_{2}, \| \mathrm{YbGa}_{4}, \mathrm{CaGa}_{4}, \\
\mathrm{Ba}_{3}\left(\mathrm{LiSn}_{2}\right)_{4}, \mathrm{Ni}_{3} \mathrm{Se}_{4}, \mathrm{TlFeS}_{2}, \mathrm{Ti}_{3} \mathrm{Te}_{4}, \mathrm{Nd}_{2} \mathrm{Ni}_{5} \mathrm{~B}_{4}, \mathrm{La}_{2} \mathrm{Ni}_{5} \mathrm{~B}_{4}, \mathrm{Y}_{2} \mathrm{Si}_{2} \mathrm{Rh}, \\
\mathrm{Sc}_{2} \mathrm{CoSi}_{2}, \mathrm{Er}_{2} \mathrm{CoSi}_{2}, \mathrm{Lu}_{2} \mathrm{CoGe}_{2}, \mathrm{Tm}_{2} \mathrm{CoGe}_{2}, \mathrm{Dy}_{2} \mathrm{CoSi}_{2}, \mathrm{LiO}_{2}, \mathrm{Hf}_{7} \mathrm{P}_{4}, \\
\mathrm{Sb}_{2} \mathrm{Te}_{3}(\mathrm{ICSD}: 187496)\end{array}$ \\
\hline & A-M, Г-M, M-Y & $\begin{array}{c}\| \mathrm{CaC}_{2}(\mathrm{ICSD}: 252716), \mathrm{SrIn}_{4}, \mathrm{Mg}_{5} \mathrm{Si}_{6}(\mathrm{ICSD}: 85529), \mathrm{Nb}_{6} \mathrm{C}_{5}, \mathrm{Hf}_{2} \mathrm{Bi}, \\
\mathrm{Pr}_{2} \mathrm{CdCu}_{2}, \mathrm{Ti}_{3} \mathrm{O}_{5}, \mathrm{Nd}_{6} \mathrm{In}_{22} \mathrm{Pt}_{13}, \mathrm{Al}_{45} \mathrm{~V}_{7}, \mathrm{Ce}_{4} \mathrm{Ru}_{3}, \mathrm{Cr}_{4} \mathrm{As}_{3}\end{array}$ \\
\hline & A-Y, Г-Y, M-Y & $\begin{array}{c}\| \mathrm{Sr}(\mathrm{SnHg})_{2}, \mathrm{NaN}_{3}(\mathrm{ICSD}: 29375), \mathrm{Al}_{3} \mathrm{Mo}, \mathrm{Sm}_{2} \mathrm{Ge}_{2} \mathrm{Ru}, \mathrm{SnPS}_{3}, \mathrm{CoGe} \\
\mathrm{Ho}_{2} \mathrm{Ge}_{2} \mathrm{Os}, \mathrm{Er}_{2} \mathrm{FeSi}_{2}, \mathrm{~K}_{4} \mathrm{GaAu}_{8}, \mathrm{~Tb}_{2} \mathrm{FeSi}_{2}, \mathrm{Zr}_{2} \mathrm{Cu}, \mathrm{Nb}_{7} \mathrm{P}_{4}, \mathrm{TlCr}_{3} \mathrm{~S}_{5}, \\
\mathrm{U}_{4} \mathrm{Ga}_{20} \mathrm{Ni}_{11}\end{array}$ \\
\hline & A-M, $\Gamma-Y$ & 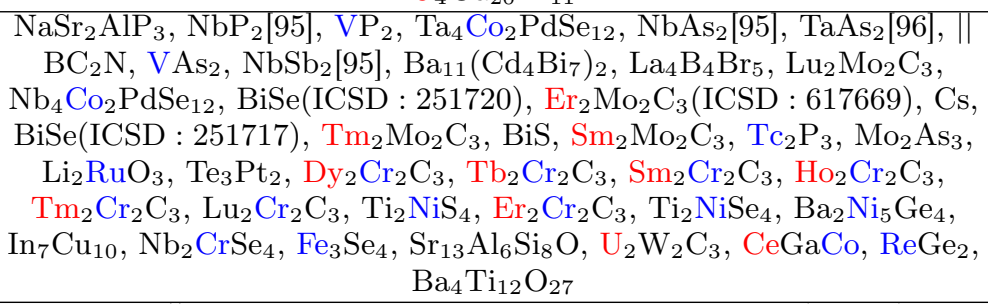 \\
\hline & A-M & $\begin{array}{c}\mathrm{P}_{3} \mathrm{Au}_{2}, \| \mathrm{Te}, \mathrm{Ni}_{3} \mathrm{Sn}_{4}, \mathrm{Te}_{4} \mathrm{Pt}_{3}, \mathrm{NiP}_{3} \mathrm{~W}_{2}, \mathrm{Nd}_{2} \mathrm{BC}, \mathrm{Sr}_{11}\left(\mathrm{MgSi}_{5}\right)_{2} \\
\mathrm{Er}_{3} \mathrm{Si}_{3} \mathrm{Ni}, \mathrm{K}_{2} \mathrm{Ca}\left(\mathrm{NO}_{2}\right)_{4}, \mathrm{Cu}_{4} \mathrm{Bi}_{5} \mathrm{PbS}_{11}, \mathrm{Ga}_{4} \mathrm{Fe}_{3}\end{array}$ \\
\hline & $\Gamma-Y$ & $\begin{array}{c}\mathrm{Sr}_{3}\left(\mathrm{AlGe}_{2}\right)_{2}, \mathrm{Cs}_{2} \mathrm{Pd}\left(\mathrm{IBr}_{2}\right)_{2}, \| \mathrm{TaSb}_{2}, \mathrm{Hg}, \mathrm{Ta}_{2} \mathrm{PdSe}, \mathrm{Ca}(\mathrm{CuSn})_{2}, \\
\mathrm{BaThTe}_{4}, \mathrm{Sr}(\mathrm{CuSn})_{2}, \mathrm{Ta}_{2} \mathrm{PtSe}_{7}, \mathrm{Ho}_{2} \mathrm{Fe}_{2} \mathrm{Si}_{2} \mathrm{C}, \mathrm{Dy}_{2} \mathrm{Fe}_{2} \mathrm{Si}_{2} \mathrm{C}, \\
\mathrm{Er}_{2} \mathrm{Fe}_{2} \mathrm{Si}_{2} \mathrm{C}, \mathrm{Tb}_{2} \mathrm{Fe}_{2} \mathrm{Si}_{2} \mathrm{C}, \mathrm{Tm}_{2} \mathrm{Fe}_{2} \mathrm{Si}_{2} \mathrm{C}, \mathrm{Y}_{2} \mathrm{Fe}_{2} \mathrm{Si}_{2} \mathrm{C}, \mathrm{Pr}_{2} \mathrm{Fe}_{2} \mathrm{Si}_{2} \mathrm{C}, \mathrm{Pr}, \\
\mathrm{Nd}_{2} \mathrm{Fe}_{2} \mathrm{Si}_{2} \mathrm{C}, \mathrm{Ba}_{2} \mathrm{Hg}_{3}\left(\mathrm{PdO}_{2}\right)_{7}, \mathrm{TiO}, \mathrm{Ca}_{6} \mathrm{Cu}_{2} \mathrm{Sn}_{7}, \mathrm{Rb}_{2} \mathrm{TeO}_{3}, \mathrm{~V}_{2} \mathrm{NiS}_{4}, \\
\mathrm{La}_{4} \mathrm{C}_{5} \mathrm{Cl}_{2}, \mathrm{~V}_{2} \mathrm{NiSe}_{4}, \mathrm{Tm}_{3} \mathrm{FeSi}_{3}, \mathrm{Er}_{3} \mathrm{FeSi}_{3}, \mathrm{Ta}_{4} \mathrm{Te}_{16} \mathrm{Pd}_{3}, \mathrm{Ba}_{5} \mathrm{Al}_{2} \mathrm{Ge}_{7}, \\
\mathrm{Ce}_{2} \mathrm{Ni}_{5} \mathrm{~B}_{4}, \mathrm{ZrSc} \mathrm{Zr}_{2}, \mathrm{CeSnRu}, \mathrm{FeGe}, \mathrm{Ba}_{2} \mathrm{UCu}_{2} \mathrm{~S}_{5}, \mathrm{Sm}_{10} \mathrm{Pd}_{21}, \mathrm{Sn}_{4} \mathrm{Sb}_{6} \mathrm{~S}_{13}, \\
\mathrm{~K}_{3} \mathrm{Rh}, \mathrm{Mn}_{3} \mathrm{As}_{2}, \mathrm{Ti}_{3} \mathrm{H}_{2} \mathrm{O}_{7}, \mathrm{~K}_{2} \mathrm{PtS}_{6}\end{array}$ \\
\hline \multicolumn{3}{|r|}{ Space Group 13: $P 2 / c$} \\
\hline \multirow[t]{2}{*}{ NSOC } & $\begin{array}{l}\mathrm{A}-\Gamma, \mathrm{A}-\mathrm{Y}, \mathrm{B}-\Gamma, \mathrm{B}-\mathrm{Y}, \mathrm{C}-\mathrm{D} \\
\mathrm{C}-\mathrm{E}, \mathrm{D}-\mathrm{Z}, \mathrm{E}-\mathrm{Z}\end{array}$ & $\| \mathrm{VAg}\left(\mathrm{PS}_{3}\right)_{2}$ \\
\hline & $\Gamma-\mathrm{Z}$ & $\mathrm{Nd}_{7}\left(\mathrm{~S}_{2} \mathrm{Cl}_{3}\right)_{3}$ \\
\hline \multirow{16}{*}{ NSOC } & \multicolumn{2}{|r|}{ Space Group 14: $P 2_{1} / c$} \\
\hline & $\begin{array}{l}\text { A- } \Gamma, \mathrm{A}-\mathrm{Y}, \mathrm{B}-\Gamma, \mathrm{B}-\mathrm{Y}, \Gamma-\mathrm{Y} \\
\mathrm{C}-\mathrm{Y}, \Gamma-\mathrm{Z}\end{array}$ & $\| \mathrm{Cu}_{2} \mathrm{H}_{2} \mathrm{CO}_{5}$ \\
\hline & $\begin{array}{c}\mathrm{A}-\Gamma, \mathrm{A}-\mathrm{Y}, \mathrm{B}-\Gamma, \mathrm{B}-\mathrm{Y}, \mathrm{C}-\mathrm{Y}, \\
\Gamma-\mathrm{Z}\end{array}$ & $\| \mathrm{LiSm}_{2} \mathrm{IrO}_{6}$ \\
\hline & $\begin{array}{c}\mathrm{A}-\Gamma, \mathrm{A}-\mathrm{Y}, \mathrm{B}-\Gamma, \mathrm{B}-\mathrm{Y}, \Gamma-\mathrm{Y} \\
\mathrm{C}-\mathrm{Y}\end{array}$ & $\| \mathrm{Te}_{4} \mathrm{Pd}_{9}$ \\
\hline & $\mathrm{A}-\Gamma, \mathrm{A}-\mathrm{Y}, \mathrm{B}-\Gamma, \mathrm{B}-\mathrm{Y}, \mathrm{C}-\mathrm{Y}$ & $\|$ S(ICSD : 50338) \\
\hline & $\mathrm{A}-\Gamma, \mathrm{A}-\mathrm{Y}, \mathrm{B}-\Gamma, \mathrm{B}-\mathrm{Y}, \Gamma-\mathrm{Z}$ & $\mathrm{Cu}_{5} \mathrm{Sn}_{4}, \mathrm{NbCoTe}_{2}, \mathrm{Hg}_{2} \mathrm{~Pb}(\mathrm{SBr})_{2}, \mathrm{Er}_{5} \mathrm{Si}_{4}, \mathrm{CuH}_{5} \mathrm{~Pb}_{4} \mathrm{SO}_{11}$ \\
\hline & $\mathrm{A}-\Gamma, \mathrm{B}-\Gamma, \Gamma-\mathrm{Y}, \mathrm{C}-\mathrm{Y}, \Gamma-\mathrm{Z}$ & $\| \mathrm{SnHg}_{2}(\mathrm{SBr})_{2}(\mathrm{ICSD}: 85584)$ \\
\hline & $\mathrm{A}-\mathrm{Y}, \mathrm{B}-\mathrm{Y}, \Gamma-\mathrm{Y}, \mathrm{C}-\mathrm{Y}, \Gamma-\mathrm{Z}$ & $\| \mathrm{OsF}_{5}$ \\
\hline & $\mathrm{A}-\Gamma, \mathrm{A}-\mathrm{Y}, \mathrm{B}-\Gamma, \mathrm{B}-\mathrm{Y}$ & $\begin{array}{c}\mathrm{CsNb}_{3} \mathrm{SBr}_{7}, \| \mathrm{Sm}_{2} \mathrm{~B}_{5}, \mathrm{CuHClO}, \mathrm{CuHOF}^{\mathrm{CuHa}} \mathrm{LuO}_{5}, \mathrm{CoH}_{2}(\mathrm{SN})_{4}, \\
\mathrm{CuH}_{7} \mathrm{C}_{3} \mathrm{SN}_{3} \mathrm{O}_{4}\end{array}$ \\
\hline & $\mathrm{A}-\Gamma, \mathrm{B}-\Gamma, \Gamma-\mathrm{Y}, \mathrm{C}-\mathrm{Y}$ & $\| \mathrm{La}(\mathrm{GePt})_{2}, \mathrm{Pr}_{2} \mathrm{Mo}_{2} \mathrm{C}_{3}, \mathrm{Li}_{6} \mathrm{Yb}\left(\mathrm{BO}_{3}\right)_{3}$ \\
\hline & $\mathrm{A}-\Gamma, \mathrm{B}-\Gamma, \Gamma-\mathrm{Y}, \Gamma-\mathrm{Z}$ & $\begin{array}{l}\| \mathrm{Nd}_{2} \mathrm{P}_{3} \mathrm{Au}, \mathrm{OsN}_{2}, \mathrm{TaCoTe}_{2}, \mathrm{ICl}(\mathrm{ICSD}: 26032), \mathrm{Al}_{3} \mathrm{Fe}_{2} \mathrm{Si}_{3}, \mathrm{Re}_{2} \mathrm{Si} \\
\mathrm{Sm}\left(\mathrm{IO}_{3}\right)_{3}\end{array}$ \\
\hline & A-Y, B-Y, Г-Y, C-Y & $\begin{array}{c}\| \mathrm{Sr}_{2} \mathrm{TbIrO}_{6}, \mathrm{Sr}_{2} \mathrm{YIrO}_{6}, \mathrm{LiNd}_{2} \mathrm{IrO}_{6}, \mathrm{LiPr}_{2} \mathrm{IrO}_{6}, \mathrm{NaSm}_{2} \mathrm{IrO}_{6} \\
\mathrm{NaPr}_{2} \operatorname{IrO}_{6}\end{array}$ \\
\hline & A-Y, B-Y, Г-Y, Г-Z & 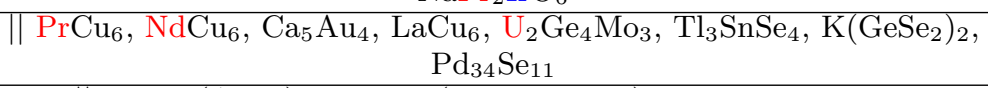 \\
\hline & $\mathrm{A}-\Gamma, \mathrm{B}-\Gamma, \Gamma-\mathrm{Y}$ & $\begin{array}{c}\| \mathrm{BaCu}_{2}\left(\mathrm{AsO}_{4}\right)_{2}, \mathrm{CaSiO}_{3}(\mathrm{ICSD}: 36311), \mathrm{CuTeO}_{3}, \mathrm{CaSiPd} \\
\mathrm{Sr}(\mathrm{AsPt})_{2}, \mathrm{PrSiPd}, \mathrm{H}_{8} \mathrm{~N}_{2} \mathrm{O}, \mathrm{Ba}_{2} \mathrm{P}_{3} \mathrm{Pd}, \mathrm{Yb}_{2} \mathrm{~S}_{2} \mathrm{O}, \mathrm{KSm}_{2} \mathrm{Cl}_{5}, \mathrm{Cs}_{11} \mathrm{O}_{3} \\
\mathrm{CuH}_{8} \mathrm{CN}_{5} \mathrm{Cl}_{3}, \mathrm{RhF}_{5}, \mathrm{MoP}_{2} \mathrm{NCl}_{12}\end{array}$ \\
\hline & A-Y, B-Y, Г-Y & $\begin{array}{c}\| \mathrm{Y}_{4} \mathrm{C}_{7}, \mathrm{Ho}_{4} \mathrm{C}_{7}(\mathrm{ICSD}: 83381), \mathrm{Bi}_{2} \mathrm{Rh}, \mathrm{MoO}_{2}, \mathrm{KCuCl}_{3}, \mathrm{CuTe}_{2} \mathrm{O}_{5}, \\
\mathrm{TcO}_{2}, \mathrm{Sn}_{3} \mathrm{PO}_{6}, \mathrm{Pd}_{7} \mathrm{Se}_{2}, \mathrm{CuSeO}_{3}, \mathrm{U}_{2} \mathrm{Si}_{4} \mathrm{~W}_{3}, \mathrm{La}_{6} \mathrm{Ge}_{11}, \mathrm{Ho}_{2}\left(\mathrm{Ni}_{5} \mathrm{~B}_{2}\right)_{3}, \\
\mathrm{Os}, \mathrm{K}_{3} \mathrm{Pu}\left(\mathrm{PS}_{4}\right)_{2}, \mathrm{ReO}_{2}, \mathrm{ReCl}_{5}\end{array}$ \\
\hline & C-Y, Г-Z & $\begin{array}{l}\mathrm{Sr}_{2} \mathrm{Cu}_{2} \mathrm{Te}\left(\mathrm{BrO}_{3}\right)_{2}, \| \mathrm{SN}(\mathrm{ICSD}: 41967), \mathrm{LaGaNi}, \mathrm{La}_{3} \mathrm{C}_{3} \mathrm{Cl}_{2} \\
\mathrm{La}_{11} \mathrm{C}_{11} \mathrm{Cl}_{7}, \mathrm{HgH}_{4} \mathrm{C}_{4}\left(\mathrm{Br}_{2} \mathrm{~N}\right)_{2}, \mathrm{HgH}_{4} \mathrm{C}_{4}\left(\mathrm{NCl}_{2}\right)_{2}, \mathrm{CaCuAs}_{2} \mathrm{O}_{7}\end{array}$ \\
\hline
\end{tabular}


Table III - continued

\begin{tabular}{|c|c|c|}
\hline $\begin{array}{l}\text { SOC or } \\
\text { NSOC }\end{array}$ & $\begin{array}{l}\text { HSL with Band } \\
\text { Crossing }\end{array}$ & High Symmetry Line Semimetals \\
\hline & $\mathrm{C}-\mathrm{Y}$ & $\begin{array}{c}\mathrm{As}_{5} \mathrm{~Pb}_{3} \mathrm{~S}_{10}, \mid \mathrm{ZnP}_{2}(\mathrm{ICSD}: 43334), \mathrm{Be}\left(\mathrm{B}_{3} \mathrm{H}_{8}\right)_{2}, \mathrm{Ba}(\mathrm{SiRh})_{2}, \mathrm{NaCuPO}_{4}, \\
\mathrm{Ba}(\mathrm{GaPt})_{2}, \mathrm{H}_{2} \mathrm{WO}_{5}, \mathrm{UMo}, \mathrm{LaCrO}_{4}, \mathrm{CuClF}_{10}(\mathrm{ICSD}: 35387)\end{array}$ \\
\hline & $\Gamma-\mathrm{Z}$ & $\begin{array}{c}\mathrm{Na}_{3} \mathrm{InBi}_{2}, \mathrm{Ag}_{2} \mathrm{Te}[97], \| \mathrm{CaSiPt}, \mathrm{Y}_{2} \mathrm{Si}_{4} \mathrm{Mo}_{3}, \mathrm{Bi}_{2} \mathrm{O}_{3}(\mathrm{ICSD}: 15752) \\
\mathrm{Pt}_{5} \mathrm{Se}_{4}\end{array}$ \\
\hline \multirow{5}{*}{$\mathrm{SOC}$} & C-D, C-E, D-E, D-Z, E-Z & $\| \mathrm{ReCl}_{5}$ \\
\hline & C-D, D-E, D-Z & $\|$ BiSe \\
\hline & C-E, D-E, E-Z & $\| \mathrm{Sr}(\mathrm{AsPt})_{2}$ \\
\hline & $\mathrm{A}-\mathrm{E}$ & $\| \mathrm{PPd}_{6}, \mathrm{Yb}_{2} \mathrm{~S}_{2} \mathrm{O}, \mathrm{Tl}_{3} \mathrm{SnSe}_{4}$ \\
\hline & B-D & $\| \mathrm{Pt}_{5} \mathrm{Se}_{4}, \mathrm{U}_{2} \mathrm{Si}_{4} \mathrm{~W}_{3}$ \\
\hline \multirow{6}{*}{ NSOC } & \multicolumn{2}{|r|}{ Space Group 15: $C 2 / c$} \\
\hline & $\mathrm{A}-\Gamma, \mathrm{A}-\mathrm{Y}, \Gamma-\mathrm{M}, \mathrm{M}-\mathrm{Y}, \Gamma-\mathrm{Y}$ & $\| \mathrm{Yb}_{2} \mathrm{Ge}_{4} \mathrm{Ru}_{3}$ \\
\hline & $\mathrm{A}-\Gamma, \mathrm{A}-\mathrm{Y}, \Gamma-\mathrm{M}, \mathrm{M}-\mathrm{Y}$ & || $\mathrm{Ca}_{4} \mathrm{Si}_{3} \mathrm{Pd}_{4}, \mathrm{Sm}_{4} \mathrm{Si}_{3} \mathrm{Pd}_{4}, \mathrm{RePO}_{5}$ \\
\hline & A-Г, Г-M, Г-Y & $\begin{array}{c}\| \mathrm{Ag}_{4} \mathrm{TeO}_{5}, \mathrm{GeH}_{4}, \mathrm{BaH}_{8} \mathrm{PtC}_{4}(\mathrm{NO})_{4}(\mathrm{ICSD}: 4412), \mathrm{V}_{2} \mathrm{Cu}_{2} \mathrm{O}_{7} \\
\mathrm{Ca}_{2} \mathrm{SiIr}_{2}, \mathrm{Pr}_{2} \mathrm{~B}_{5}, \mathrm{Nd}_{2} \mathrm{~B}_{5}, \mathrm{Zr}_{2} \mathrm{Si}_{4} \mathrm{Ru}_{3}, \mathrm{Cu}_{2} \mathrm{Te}_{3} \mathrm{O}_{8}, \mathrm{Hf}_{2} \mathrm{Si}_{4} \mathrm{Ru}_{3}, \\
\mathrm{Na}_{6} \mathrm{TlSb}_{4}, \mathrm{NiH}_{2} \mathrm{SO}_{5}, \mathrm{Sr}_{2} \mathrm{LiFe}_{2} \mathrm{~N}_{3}, \mathrm{Ba}_{2} \mathrm{LiFe}_{2} \mathrm{~N}_{3}, \mathrm{Ta}_{3} \mathrm{As}_{4} \mathrm{Ta}_{4} \mathrm{Te}_{9} \mathrm{I}_{4} \mathrm{O} \\
\mathrm{Nb}_{4} \mathrm{Te}_{9} \mathrm{I}_{4} \mathrm{O}, \mathrm{Yb}_{2} \mathrm{SiO}_{5}, \mathrm{YbP}_{5} \mathrm{O}_{14}\end{array}$ \\
\hline & A-Y, Г-Y, M-Y & $\begin{array}{c}\| \mathrm{Mo}_{9} \mathrm{O}_{26}, \mathrm{Ca}_{2} \mathrm{GePt}_{2}, \mathrm{Cu}_{6} \mathrm{Sn}_{5}, \mathrm{Hf}_{3} \mathrm{As}, \mathrm{LiTm}_{2} \mathrm{Cl}_{5}, \mathrm{~Tb}_{10} \mathrm{~B}_{7} \mathrm{C}_{10} \\
\mathrm{LaNdI}_{4}, \mathrm{~K}_{3} \mathrm{Na}\left(\mathrm{RuO}_{4}\right)_{2}, \mathrm{Na}_{2} \mathrm{TbO}_{3}, \mathrm{Sc}_{3} \mathrm{Re}_{2} \mathrm{Si}_{3}\end{array}$ \\
\hline & $\Gamma-Y$ & $\begin{array}{c}\mathrm{TlCuPO}_{4}, \mathrm{TlCuAsO}_{4}, \mathrm{Ca}_{3}\left(\mathrm{AlAs}_{2}\right)_{2}, \mathrm{BaCuN}, \| \mathrm{MoP}_{4}, \mathrm{CrP}_{4}, \mathrm{Sb}_{2} \mathrm{Te}_{3}, \\
\mathrm{~B}_{2} \mathrm{Pd}_{5}, \mathrm{Yb}_{2} \mathrm{Te}_{4} \mathrm{O}_{11}, \mathrm{Ti}_{3} \mathrm{O}_{5}, \mathrm{Nd}_{4} \mathrm{Ge}_{3} \mathrm{Rh}_{4}, \mathrm{Cu}_{2} \mathrm{As}_{2} \mathrm{O}_{7}, \mathrm{LiDy}_{2} \mathrm{Cl}_{5}, \mathrm{Ta}_{6} \mathrm{~S}, \\
\mathrm{Sc}_{3}\left(\mathrm{ReSi}_{2}\right)_{2}, \mathrm{RbCuCl}_{3}, \mathrm{Cu}_{2} \mathrm{P}_{2} \mathrm{O}_{7}, \mathrm{Na}_{2} \mathrm{PrO}_{3}, \mathrm{RbB}_{6} \mathrm{H}_{8} \mathrm{O}_{7}, \\
\mathrm{Cu}_{4} \mathrm{Ag}\left(\mathrm{AsO}_{4}\right)_{3}\end{array}$ \\
\hline & & Space Group 17: P2221 \\
\hline NSOC & $\Gamma-\mathrm{X}, \mathrm{S}-\mathrm{Y}$ & $\| \mathrm{Sr}_{2} \mathrm{YCu}_{3}\left(\mathrm{PbO}_{4}\right)_{2}$ \\
\hline & $\Gamma-\mathrm{Z}, \mathrm{T}-\mathrm{Y}$ & $\| \mathrm{Ag}_{2} \mathrm{Se}$ \\
\hline & & Space Group 18: $P 2_{1} 2_{1} 2$ \\
\hline NSOC & $\Gamma-\mathrm{X}, \mathrm{T}-\mathrm{Z}$ & $\mathrm{Pd}_{7} \mathrm{Se}_{4}$ \\
\hline NSUC & $\Gamma-\mathrm{X}, \mathrm{U}-\mathrm{Z}$ & $\mathrm{CaCuO}_{2}$ \\
\hline & & Space Group 19: $P 2_{1} 2_{1} 2_{1}$ \\
\hline & $\Gamma-Y, \Gamma-\mathrm{X}$ & $\mathrm{Ag}_{2} \mathrm{~S} \|$ \\
\hline NSOC & $\Gamma-\mathrm{Y}, \Gamma-\mathrm{Z}$ & $\mathrm{H}_{5} \mathrm{NO}, \| \mathrm{PrBC}, \mathrm{NdBC}, \mathrm{LaBC}$ \\
\hline & $\Gamma-\mathrm{Z}, \Gamma-\mathrm{X}$ & $\| \mathrm{Ag}_{2} \mathrm{SnO}_{3}$ \\
\hline $\mathrm{SOC}$ & $\mathrm{R}-\mathrm{S}$ & $\| \mathrm{ThP}_{7}$ \\
\hline SUC & $\mathrm{R}-\mathrm{T}$ & $\mathrm{CuP}(\mathrm{HO})_{5} \|$ \\
\hline & & Space Group 20: $C 222_{1}$ \\
\hline NSOC & $\Gamma-Y, \Gamma-Z$ & $\| \mathrm{Nd}_{4} \mathrm{Ge}_{7}, \mathrm{Pr}_{4} \mathrm{Ge}_{7}$ \\
\hline & & Space Group 21: $C 222$ \\
\hline NSOC & $\Gamma-\mathrm{Z}, \Gamma-\mathrm{Y}$ & $\mathrm{Ni}_{9} \mathrm{~S}_{8}$ \\
\hline & & Space Group 23: I222 \\
\hline NSOC & $\mathrm{W}-\mathrm{S}$ & $\mathrm{NaFeS}_{2}$ \\
\hline & & Space Group 25: Pmm2 \\
\hline & $\begin{array}{l}\mathrm{Z}-\Gamma, \mathrm{Y}-\Gamma, \mathrm{Y}-\mathrm{T}, \mathrm{T}-\mathrm{Z}, \mathrm{X}-\mathrm{Z} \\
\mathrm{X}-\Gamma, \mathrm{U}-\mathrm{Z}, \mathrm{U}-\Gamma, \mathrm{S}-\mathrm{R}, \mathrm{Y}-\mathrm{S} \\
\mathrm{T}-\mathrm{S}\end{array}$ & $\| \mathrm{CuAgTe}_{2}$ \\
\hline NSOC & $\begin{array}{l}\mathrm{X}-\mathrm{Z}, \mathrm{X}-\Gamma, \mathrm{U}-\mathrm{Z}, \mathrm{U}-\Gamma, \mathrm{S}-\mathrm{R} \\
\mathrm{T}-\mathrm{R}, \mathrm{X}-\mathrm{U}, \mathrm{X}-\mathrm{S}, \mathrm{U}-\mathrm{R}, \mathrm{Y}-\mathrm{R}\end{array}$ & $\| \mathrm{Ag}_{3} \mathrm{Sb}$ \\
\hline & $\begin{array}{l}\text { X-U, U-Z, U- } \Gamma, S-R, T-R, \\
\text { X-S, U-R, Y-R }\end{array}$ & $\|$ GaAs \\
\hline & $\begin{array}{l}\text { Y- } \Gamma, \text { Y-Z, T-Z, T- } \Gamma, S-R \\
\text { Y-S, T-S }\end{array}$ & \| $\mathrm{HgBrN}$ \\
\hline & S-R, T-R, X-S, U-S, Y-R & $\| \mathrm{InSb}$ \\
\hline & & Space Group 26: $P m c 2_{1}$ \\
\hline $\mathrm{NSOC}$ & $\begin{array}{l}\mathrm{Z}-\Gamma, \mathrm{Y}-\Gamma, \mathrm{T}-\Gamma, \mathrm{S}-\mathrm{R}, \mathrm{X}-\mathrm{S} \\
\mathrm{U}-\mathrm{S}, \mathrm{Y}-\mathrm{S}, \mathrm{T}-\mathrm{S}\end{array}$ & $\| \mathrm{Si}_{3} \mathrm{MoPt}_{2}$ \\
\hline NSUC & $\begin{array}{l}\mathrm{Z}-\Gamma, \mathrm{Y}-\Gamma, \mathrm{T}-\Gamma, \mathrm{X}-\mathrm{U}, \mathrm{X}-\mathrm{Z} \\
\mathrm{U}-\Gamma, \mathrm{X}-\mathrm{S}, \mathrm{X}-\mathrm{R}\end{array}$ & $\| \mathrm{TiCdO}_{3}$ \\
\hline & $\mathrm{X}-\mathrm{U}, \mathrm{X}-\mathrm{Z}, \mathrm{X}-\Gamma, \mathrm{X}-\mathrm{S}, \mathrm{X}-\mathrm{R}$ & $\| \mathrm{TiFeH}_{2}$ \\
\hline
\end{tabular}


Table III - continued

\begin{tabular}{|c|c|c|}
\hline $\begin{array}{l}\text { SOC or } \\
\text { NSOC }\end{array}$ & $\begin{array}{l}\text { HSL with Band } \\
\text { Crossing }\end{array}$ & High Symmetry Line Semimetals \\
\hline \multicolumn{3}{|r|}{ Space Group 28: Pma2 } \\
\hline NSOC & Y-T, T-Z, T-, T-R, T-S & $\mathrm{Te}_{2} \mathrm{Au}$ \\
\hline \multicolumn{3}{|r|}{ Space Group 29: $P c a 2_{1}$} \\
\hline \multirow{2}{*}{ NSOC } & $\begin{array}{l}\mathrm{Z}-\Gamma, \mathrm{Y}-\Gamma, \mathrm{T}-\Gamma, \mathrm{X}-\Gamma, \mathrm{U}-\Gamma \\
\mathrm{T}-\mathrm{R}, \mathrm{Y}-\mathrm{T}, \mathrm{T}-\mathrm{S}\end{array}$ & || CoAsS(ICSD : 41857) \\
\hline & $\mathrm{Z}-\Gamma, \mathrm{X}-\mathrm{Z}, \mathrm{X}-\Gamma, \mathrm{U}-\mathrm{Z}, \mathrm{U}-\Gamma$ & $\mathrm{Ni}_{6} \mathrm{Se}_{5}$ \\
\hline \multicolumn{3}{|r|}{ Space Group 30: Pnc2 } \\
\hline NSOC & $\mathrm{Z}-\Gamma, \mathrm{X}-\mathrm{U}, \mathrm{X}-\mathrm{Z}, \mathrm{U}-\Gamma$ & $\mathrm{Nb}_{3}\left(\mathrm{BiO}_{3}\right)_{5}$ \\
\hline SOC & T-R, Y-T, T-S & $\mathrm{Nb}_{3}\left(\mathrm{BiO}_{3}\right)_{5}$ \\
\hline \multicolumn{3}{|r|}{ Space Group 31: Pmn2 } \\
\hline \multirow{5}{*}{ NSOC } & $\begin{array}{l}\mathrm{Z}-\Gamma, \mathrm{Y}-\Gamma, \mathrm{Y}-\mathrm{Z}, \mathrm{T}-\mathrm{Z}, \mathrm{T}-\Gamma \\
\mathrm{X}-\Gamma, \mathrm{U}-\Gamma\end{array}$ & $\| \mathrm{Ca}_{2} \mathrm{Ni}_{7} \mathrm{P}_{4}$ \\
\hline & $\mathrm{Z}-\Gamma, \mathrm{Y}-\Gamma, \mathrm{T}-\Gamma, \mathrm{X}-\Gamma, \mathrm{U}-\Gamma$ & $\| \mathrm{Nd}_{2} \mathrm{Ni}_{7} \mathrm{P}_{4}, \mathrm{Pr}_{2} \mathrm{Ni}_{7} \mathrm{P}_{4}$ \\
\hline & Z-Г, Y-T, Y-Z, T-Г & $\| \mathrm{Ce}_{2} \mathrm{Ni}_{7} \mathrm{P}_{4}$ \\
\hline & $\mathrm{Z}-\Gamma, \mathrm{X}-\Gamma, \mathrm{U}-\Gamma$ & $\mathrm{Ag}_{8} \mathrm{GeSe}_{6}, \mathrm{Ag}_{8} \mathrm{SnSe}_{6}, \mathrm{Ag}_{3} \mathrm{PSe}_{4}, \mathrm{TaTe}_{4} \mathrm{Ir}, \| \mathrm{NbTe}_{4} \mathrm{Ir}$ \\
\hline & $\mathrm{Z}-\Gamma, \mathrm{Y}-\Gamma, \mathrm{T}-\Gamma$ & $\mathrm{Ag}_{2} \mathrm{SnHgSe}_{4} \|$ \\
\hline \multicolumn{3}{|r|}{ Space Group 33: Pna $2_{1}$} \\
\hline \multirow{5}{*}{ NSOC } & $\begin{array}{l}\mathrm{Z}-\Gamma, \mathrm{Y}-\Gamma, \mathrm{T}-\Gamma, \mathrm{X}-\mathrm{Z}, \mathrm{X}-\Gamma \\
\mathrm{U}-\mathrm{Z}, \mathrm{U}-\Gamma\end{array}$ & $\| \mathrm{PNF}_{2}$ \\
\hline & $\mathrm{Z}-\Gamma, \mathrm{Y}-\Gamma, \mathrm{T}-\Gamma, \mathrm{X}-\Gamma, \mathrm{U}-\Gamma$ & $\| \mathrm{H}_{4} \mathrm{~N}_{2} \mathrm{O}_{3}, \mathrm{TlSbAu}, \mathrm{H}_{3} \mathrm{NO}_{4}(\mathrm{ICSD}: 1901)$ \\
\hline & $\mathrm{Z}-\Gamma, \mathrm{X}-\Gamma, \mathrm{U}-\Gamma$ & || $\mathrm{Fe}_{2} \mathrm{O}_{3}$ \\
\hline & $\mathrm{Z}-\Gamma, \mathrm{Y}-\Gamma, \mathrm{T}-\Gamma$ & $\mathrm{LiAlSiH}_{2} \mathrm{O}_{5}(\mathrm{ICSD}: 161494), \| \mathrm{Sc}_{2} \mathrm{Cu}_{2} \mathrm{O}_{5}$ \\
\hline & $\mathrm{S}-\mathrm{R}$ & $\| \mathrm{Mo}_{4} \mathrm{O}_{11}$ \\
\hline $\mathrm{SOC}$ & $\mathrm{X}-\mathrm{U}, \mathrm{U}-\mathrm{S}, \mathrm{U}-\mathrm{R}$ & $\mathrm{LaYbO}_{3}$ \\
\hline \multicolumn{3}{|r|}{ Space Group 36: $C m c 2_{1}$} \\
\hline \multirow{8}{*}{ NSOC } & Y-Г, Y-T, Y-Z, T-Z, T-Г & $\| \mathrm{BiPd}$ \\
\hline & $\mathrm{Y}-\Gamma, \mathrm{Y}-\mathrm{Z}, \mathrm{T}-\mathrm{Z}, \mathrm{T}-\Gamma, \mathrm{S}-\mathrm{R}$ & ||$C \mathrm{Ce}\left(\mathrm{Ni}_{2} \mathrm{~B}\right)_{6}$ \\
\hline & $\mathrm{Z}-\Gamma, \mathrm{Y}-\Gamma, \mathrm{T}-\Gamma, \mathrm{Y}-\mathrm{T}, \mathrm{Y}-\mathrm{Z}$ & $\mathrm{Tm}_{4} \mathrm{Zn}_{5} \mathrm{Ge}_{6}, \mathrm{Lu}_{4} \mathrm{Zn}_{5} \mathrm{Ge}_{6}, \| \mathrm{Tb}_{4} \mathrm{Zn}_{5} \mathrm{Ge}_{6}$ \\
\hline & $\mathrm{Z}-\Gamma, \mathrm{Y}-\Gamma, \mathrm{Y}-\mathrm{T}, \mathrm{Y}-\mathrm{Z}, \mathrm{T}-\Gamma$ & $\| \mathrm{BiPd}_{2} \mathrm{~Pb}$ \\
\hline & $\mathrm{Z}-\Gamma, \mathrm{Y}-\mathrm{T}, \mathrm{Y}-\mathrm{Z}, \mathrm{T}-\Gamma, \mathrm{Y}-\Gamma$ & $\| \mathrm{La}_{2} \mathrm{Fe}_{2} \mathrm{~S}_{5}$ \\
\hline & $\mathrm{Z}-\Gamma, \mathrm{Y}-\mathrm{T}, \mathrm{Y}-\mathrm{Z}, \mathrm{T}-\Gamma$ & $\mathrm{SrSm}_{2} \mathrm{Nb}_{2} \mathrm{O}_{9}, \| \mathrm{YbGa}_{2} \mathrm{Ge}_{3}, \mathrm{Ca}_{3} \mathrm{Ru}_{2} \mathrm{O}_{7}$ \\
\hline & $\mathrm{Z}-\Gamma, \mathrm{Y}-\Gamma, \mathrm{T}-\Gamma$ & $\mathrm{Al}_{10} \mathrm{Ge}_{2} \mathrm{O}_{19} \|$ \\
\hline & $\mathrm{S}-\mathrm{R}$ & $\mathrm{K}_{2} \mathrm{Be}_{2}\left(\mathrm{Si}_{2} \mathrm{O}_{5}\right)_{3}$ \\
\hline \multirow{16}{*}{ NSOC } & \multicolumn{2}{|r|}{ Space Group 38: $A m m 2$} \\
\hline & $\begin{array}{c}\mathrm{Y}-\Gamma, \mathrm{Z}-\Gamma, \mathrm{Z}-\mathrm{T}, \mathrm{Z}-\mathrm{Y}, \mathrm{Y}-\mathrm{S}, \\
\mathrm{T}-\mathrm{R}\end{array}$ & || $\mathrm{BaTiO}_{3}(\mathrm{ICSD}: 154346)$ \\
\hline & 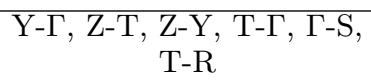 & || $\mathrm{BaTiO}_{3}(\mathrm{ICSD}: 161341)$ \\
\hline & $\begin{array}{c}\mathrm{Y}-\Gamma, \mathrm{Z}-\mathrm{T}, \mathrm{Z}-\mathrm{Y}, \mathrm{T}-\Gamma, \Gamma-\mathrm{S} \\
\text { Z-R }\end{array}$ & || MoSeS \\
\hline & $\begin{array}{c}\mathrm{Y}-\Gamma, \mathrm{Z}-\mathrm{T}, \mathrm{Z}-\mathrm{Y}, \mathrm{T}-\Gamma, \mathrm{Y}-\mathrm{S}, \\
\Gamma-\mathrm{S}\end{array}$ & $\| \mathrm{BiS}$ \\
\hline & $\begin{array}{c}\mathrm{Z}-\Gamma, \mathrm{Z}-\mathrm{T}, \mathrm{Z}-\mathrm{Y}, \mathrm{Y}-\mathrm{S}, \mathrm{\Gamma}-\mathrm{S} \\
\mathrm{T}-\mathrm{R}\end{array}$ & || TiSi, TiSi \\
\hline & $\begin{array}{c}\mathrm{Z}-\Gamma, \mathrm{Z}-\mathrm{Y}, \mathrm{T}-\mathrm{Y}, \mathrm{T}-\Gamma, \mathrm{Z}-\mathrm{T}, \\
\text { T-R }\end{array}$ & $\| \mathrm{Yb}_{2} \mathrm{Ge}_{6} \mathrm{Pd}, \mathrm{TmSn}_{3}, \mathrm{ErSn}_{3}, \mathrm{YSn}_{3}$ \\
\hline & $\begin{array}{c}\mathrm{Z}-\Gamma, \mathrm{Z}-\mathrm{Y}, \mathrm{T}-\mathrm{Y}, \mathrm{T}-\Gamma, \mathrm{Z}-\mathrm{T}, \\
\text { Z-R }\end{array}$ & $\| \mathrm{HoSn}_{3}, \mathrm{TbSn}_{3}, \mathrm{DySn}_{3}$ \\
\hline & Y- $\Gamma$, Y-S, $\Gamma-S, Z-R, T-R$ & $\mathrm{Sc}_{5}\left(\mathrm{Re}_{2} \mathrm{Si}_{3}\right)_{4}$ \\
\hline & Y-Г, Z-Г, Z-T, Z-Y, Г-S & $\| \mathrm{Cu}_{8} \mathrm{O}$ \\
\hline & Y- $\Gamma, Z-T, Z-Y, T-\Gamma, \Gamma-S$ & $\| \mathrm{CeNiC}_{2}$ \\
\hline & Y-Г, Z-Y, T-Y, Г-S & $\| \mathrm{BaTiO}_{3}(\mathrm{ICSD}: 161419)$ \\
\hline & $\mathrm{Z}-\Gamma, \mathrm{Z}-\mathrm{T}, \mathrm{Z}-\mathrm{Y}, \mathrm{Z}-\mathrm{R}$ & $\begin{array}{c}\| \mathrm{SmRhC}_{2}, \mathrm{NdRhC}_{2}, \mathrm{PrRhC}_{2}, \mathrm{ErCoC}_{2}, \mathrm{TbCoC}_{2}, \mathrm{DyCoC}_{2}, \mathrm{SmCoC}_{2}, \\
\mathrm{HoCoC}_{2}, \mathrm{TmCoC}_{2}, \mathrm{YCoC}_{2}, \mathrm{OsN}_{2}\end{array}$ \\
\hline & $\mathrm{Y}-\Gamma, \mathrm{Z}-\Gamma, \mathrm{T}-\Gamma$ & $\| \mathrm{Rb}_{4} \mathrm{CO}_{4}$ \\
\hline & Y-Г, Z-Y, T-Y & $\mathrm{Rb}_{5} \mathrm{Tl}_{11} \mathrm{Cd}_{2}, \| \mathrm{Cs}_{5} \mathrm{Tl}_{11} \mathrm{Cd}_{2}$ \\
\hline & $\mathrm{Y}-\Gamma, \mathrm{Y}-\mathrm{S}$ & $\| \mathrm{Cu}_{11} \mathrm{Sb}_{3}$ \\
\hline
\end{tabular}


Table III - continued

\begin{tabular}{|c|c|c|}
\hline $\begin{array}{l}\text { SOC or } \\
\text { NSOC }\end{array}$ & $\begin{array}{l}\text { HSL with Band } \\
\text { Crossing }\end{array}$ & High Symmetry Line Semimetals \\
\hline & Z-R, T-R & $\pi \mathrm{Ho}_{2} \mathrm{NiGe}_{6}, \mathrm{Ho}_{2} \mathrm{Ge}_{6} \mathrm{Pd}$ \\
\hline & & Space Group 39: Aem2 \\
\hline & $\begin{array}{c}\mathrm{Y}-\Gamma, \mathrm{Z}-\Gamma, \mathrm{Z}-\mathrm{T}, \mathrm{Z}-\mathrm{Y}, \mathrm{Y}-\mathrm{S}, \\
\Gamma-\mathrm{S}\end{array}$ & $\| \mathrm{Y}_{7}\left(\mathrm{O}_{2} \mathrm{~F}_{3}\right)_{3}$ \\
\hline $\mathrm{NSOC}$ & $\mathrm{Y}-\Gamma, \mathrm{Y}-\mathrm{S}, \Gamma-\mathrm{S}$ & $\| \mathrm{K}_{2} \mathrm{Mo}_{2} \mathrm{H}_{12} \mathrm{C}_{4} \mathrm{~N}\left(\mathrm{OF}_{3}\right)_{3}$ \\
\hline & $\mathrm{Y}-\Gamma, \Gamma-\mathrm{S}$ & $\mathrm{Ta}_{3} \mathrm{~S}_{2} \|$ \\
\hline & & Space Group 40: $A m a 2$ \\
\hline NSOC & $\mathrm{Y}-\Gamma, \Gamma-\mathrm{S}$ & $\| \mathrm{MgCl}_{2}$ \\
\hline SOC & Z-Г, Z-T, Z-Y, T-Y, T-Г & $\| \mathrm{PrTe}_{3}$ \\
\hline & & Space Group 43: $F d d 2$ \\
\hline $\mathrm{NSOC}$ & $\mathrm{Z}-\Gamma, \mathrm{Y}-\Gamma, \mathrm{T}-\Gamma$ & $\mathrm{RbP}\left(\mathrm{HO}_{2}\right)_{2}, \| \mathrm{Ta}_{2} \mathrm{H}_{4} \mathrm{O}_{7}, \mathrm{Ca}_{2} \mathrm{GePd}_{2}, \mathrm{BIrPd}$ \\
\hline & & Space Group 44: Imm2 \\
\hline & $\mathrm{X}-\Gamma, \mathrm{X}-\mathrm{S}, \Gamma-\mathrm{S}, \mathrm{X}-\mathrm{R}, \Gamma-\mathrm{R}$ & $\| \mathrm{LiRh}_{3}$ \\
\hline & $\mathrm{X}-\Gamma, \mathrm{X}-\mathrm{S}, \Gamma-\mathrm{S}, \mathrm{X}-\mathrm{R}$ & $\begin{array}{c}\mathrm{Cu}_{2} \mathrm{SnTe}_{3}, \| \mathrm{Cu}_{2} \mathrm{GeSe}_{3}, \mathrm{Cu}_{2} \mathrm{GeS}_{3}, \mathrm{Cu}_{2} \mathrm{GeTe}_{3}, \mathrm{Ho}_{10} \mathrm{Si}_{17}, \mathrm{Dy}_{10} \mathrm{Si}_{17}, \\
\mathrm{CaCuSn}\end{array}$ \\
\hline & $\mathrm{X}-\Gamma, \mathrm{X}-\mathrm{S}, \mathrm{X}-\mathrm{R}, \Gamma-\mathrm{R}$ & || $\operatorname{LiIr}_{3}$ \\
\hline & $\mathrm{X}-\Gamma, \Gamma-\mathrm{R}, \mathrm{W}-\mathrm{T}$ & $\| \mathrm{Cr}_{2} \mathrm{P}$ \\
\hline NSOC & $\mathrm{X}-\Gamma, \Gamma-\mathrm{S}, \mathrm{W}-\mathrm{T}$ & TmGePd \\
\hline & $\mathrm{X}-\Gamma, \Gamma-\mathrm{S}, \mathrm{X}-\mathrm{R}$ & YbGePd \\
\hline & $\mathrm{X}-\Gamma, \mathrm{X}-\mathrm{R}, \Gamma-\mathrm{R}$ & $\mathrm{NaGeAu}$ \\
\hline & $\mathrm{X}-\Gamma, \mathrm{X}-\mathrm{S}, \Gamma-\mathrm{S}$ & YbSnAu \\
\hline & $\mathrm{X}-\Gamma, \mathrm{X}-\mathrm{S}, \mathrm{X}-\mathrm{R}$ & $\|$ CaSnAu \\
\hline & $\mathrm{X}-\Gamma, \mathrm{X}-\mathrm{R}$ & $\| \mathrm{YbSiAu}, \mathrm{NaSi}_{2} \mathrm{Pd}_{3}, \mathrm{Yb}_{8} \mathrm{Si}_{11}$ \\
\hline & $\mathrm{X}-\mathrm{S}, \mathrm{\Gamma}-\mathrm{S}$ & $\| \mathrm{GaAs}, \mathrm{Bi}_{2} \mathrm{CO}_{5}$ \\
\hline & & Space Group 46: Ima2 \\
\hline & $\mathrm{X}-\Gamma, \mathrm{X}-\mathrm{S}, \Gamma-\mathrm{S}, \mathrm{X}-\mathrm{R}, \Gamma-\mathrm{R}$ & $\| \mathrm{Sr}_{2} \mathrm{YAlCu}_{2} \mathrm{O}_{7}$ \\
\hline NSOC & $\mathrm{X}-\Gamma, \mathrm{X}-\mathrm{R}, \Gamma-\mathrm{R}$ & $\begin{array}{l}\mathrm{Sr}_{2} \mathrm{HoGaCu}_{2} \mathrm{O}_{7}, \mathrm{Sr}_{2} \mathrm{ErGaCu}_{2} \mathrm{O}_{7}, \mathrm{Sr}_{2} \mathrm{DyGaCu}_{2} \mathrm{O}_{7}, \mathrm{Sr}_{2} \mathrm{SmGaCu}_{2} \mathrm{O}_{7}, \\
\mathrm{Sr}_{2} \mathrm{LaGaCu}_{2} \mathrm{O}_{7}, \mathrm{Sr}_{2} \mathrm{PrGaCu}_{2} \mathrm{O}_{7}, \mathrm{Sr}_{2} \mathrm{EuGaCu}_{2} \mathrm{O}_{7}, \mathrm{Sr}_{2} \mathrm{YGaCu}_{2} \mathrm{O}_{7}\end{array}$ \\
\hline & $\mathrm{X}-\Gamma, \Gamma-\mathrm{R}$ & $\| \mathrm{Sb}_{2} \mathrm{~Pb}_{2} \mathrm{O}_{7}$ \\
\hline & $\mathrm{X}-\Gamma$ & || TiSiRu, TiFeSi, ZrGeRu \\
\hline & & Space Group 47: Pmmm \\
\hline & $\begin{array}{l}\Gamma-Y, \Gamma-X, \Gamma-T, T-Z, Y-Z, \\
\text {-U, U-Z, X-Z, R-T, R-U, } \\
\text { R-X, S-U, S-X, R-Y, S-T, } \\
\text { S-Y, R-Z, T-U }\end{array}$ & $\| \mathrm{ZnB}_{3} \operatorname{Ir}_{4}$ \\
\hline NSOC & 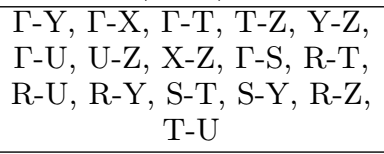 & $\| \mathrm{Ba}_{2} \mathrm{YCu}_{3} \mathrm{O}_{7}, \mathrm{Ba}_{2} \mathrm{LuCu}_{3} \mathrm{O}_{7}$ \\
\hline & $\begin{array}{c}\text { T-Y, }-\mathrm{X}, \mathrm{\Gamma}-\mathrm{T}, \mathrm{T}-\mathrm{Z}, \mathrm{Y}-\mathrm{Z} \\
\mathrm{\Gamma -U}, \mathrm{U}-\mathrm{Z}, \mathrm{X}-\mathrm{Z}, \Gamma-\mathrm{S}, \mathrm{R}-\mathrm{T} \\
\mathrm{R}-\mathrm{Y}, \mathrm{S}-\mathrm{T}, \mathrm{S}-\mathrm{Y}, \mathrm{R}-\mathrm{Z} \\
\end{array}$ & $\| \mathrm{Ba}_{2} \mathrm{SmCu}_{3} \mathrm{O}_{7}$ \\
\hline & $\begin{array}{c}-\mathrm{Y}, \Gamma-\mathrm{X}, \Gamma-\mathrm{T}, \mathrm{T}-\mathrm{Z}, \mathrm{Y}-\mathrm{Z} \\
\Gamma-\mathrm{U}, \mathrm{U}-\mathrm{Z}, \mathrm{X}-\mathrm{Z}, \Gamma-\mathrm{S}, \mathrm{R}-\mathrm{U} \\
\mathrm{R}-\mathrm{X}, \mathrm{S}-\mathrm{U}, \mathrm{S}-\mathrm{X}, \mathrm{R}-\mathrm{Z}\end{array}$ & $\| \mathrm{Ba}_{2} \mathrm{PrCu}_{3} \mathrm{O}_{7}$ \\
\hline & $\begin{array}{l}\Gamma-\mathrm{Y}, \Gamma-\mathrm{X}, \Gamma-\mathrm{T}, \mathrm{T}-\mathrm{Z}, \mathrm{Y}-\mathrm{Z} \\
\mathrm{\Gamma -U}, \mathrm{U}-\mathrm{Z}, \mathrm{X}-\mathrm{Z}, \Gamma-\mathrm{S}, \mathrm{R}-\mathrm{T} \\
\mathrm{S}-\mathrm{T}, \mathrm{T}-\mathrm{Y}, \mathrm{R}-\mathrm{Z}\end{array}$ & $\begin{array}{c}\| \mathrm{Ba}_{2} \mathrm{TmCu}_{3} \mathrm{O}_{7}, \mathrm{Ba}_{2} \mathrm{ErCu}_{3} \mathrm{O}_{7}, \mathrm{Ba}_{2} \mathrm{HoCu}_{3} \mathrm{O}_{7}, \mathrm{Ba}_{2} \mathrm{DyCu}_{3} \mathrm{O}_{7}, \\
\mathrm{Ba}_{2} \mathrm{LaCu}_{3} \mathrm{O}_{7}, \mathrm{Ba}_{2} \mathrm{NdCu}_{3} \mathrm{O}_{7}\end{array}$ \\
\hline & $\begin{array}{c}\text { Г-Y, Г-X, Г-T, T-Z, Y-Z, } \\
\Gamma-\mathrm{U}, \mathrm{U}-\mathrm{Z}, \mathrm{X}-\mathrm{Z}, \Gamma-\mathrm{S}, \mathrm{S}-\mathrm{X} \\
\text { S-Y }\end{array}$ & $\| \mathrm{Sr}_{2}(\mathrm{PPd})_{3}$ \\
\hline & & Space Group 51: Pmma \\
\hline & 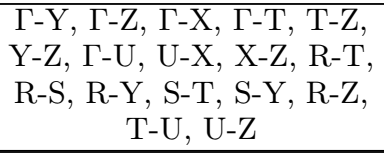 & $\| \mathrm{Fe}_{4} \mathrm{Cu}_{2} \mathrm{~S}_{7}$ \\
\hline
\end{tabular}


Table III - continued

\begin{tabular}{|c|c|c|}
\hline $\begin{array}{l}\text { SOC or } \\
\text { NSOC }\end{array}$ & $\begin{array}{c}\text { HSL with Band } \\
\text { Crossing }\end{array}$ & High Symmetry Line Semimetals \\
\hline & 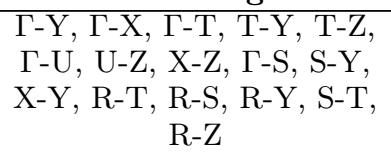 & $\| \mathrm{AgTe}_{2} \mathrm{Au}$ \\
\hline & $\begin{array}{c}\text { Г-Y, } \mathrm{\Gamma -X}, \Gamma-\mathrm{T}, \mathrm{T}-\mathrm{Z}, \mathrm{Y}-\mathrm{Z} \\
\Gamma-\mathrm{U}, \mathrm{U}-\mathrm{Z}, \mathrm{X}-\mathrm{Z}, \Gamma-\mathrm{S}, \mathrm{S}-\mathrm{Y}, \\
\mathrm{X}-\mathrm{Y}, \mathrm{R}-\mathrm{T}, \mathrm{R}-\mathrm{S}, \mathrm{S}-\mathrm{T}, \mathrm{T}-\mathrm{Y} \\
\text { T-U }\end{array}$ & $\| \mathrm{TbInCo} 2$ \\
\hline & 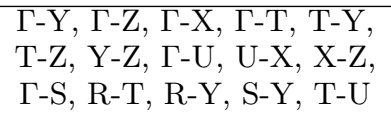 & $\|$ TaIr, NbIr \\
\hline & 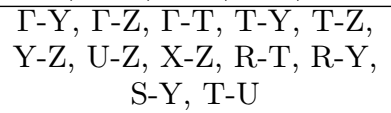 & $\| \mathrm{PrGaCo}_{2}$ \\
\hline & $\begin{array}{c}\Gamma-Y, \Gamma-Z, \Gamma-T, T-Z, Y-Z \\
\Gamma-U, U-X, U-Z, X-Z, S-Y \\
\text { X-Y, R-Z }\end{array}$ & $\| \mathrm{WO}_{3}$ \\
\hline & 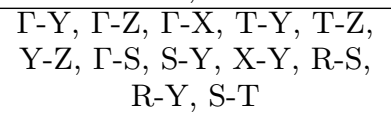 & $\| \mathrm{Y}_{5} \mathrm{Mg}_{8} \mathrm{Cu}_{5}$ \\
\hline & $\begin{array}{c}\text { T-Y, }-\mathrm{T}-\mathrm{T}, \mathrm{T}-\mathrm{Z}, \mathrm{Y}-\mathrm{Z}, \mathrm{S}-\mathrm{Y}, \\
\mathrm{X}-\mathrm{Y}, \mathrm{R}-\mathrm{T}, \mathrm{R}-\mathrm{Y}, \mathrm{S}-\mathrm{T}, \mathrm{R}-\mathrm{Z}, \\
\text { U-Z }\end{array}$ & $\| \mathrm{MoPt}$ \\
\hline & $\begin{array}{c}\text { T-Y, } \mathrm{\Gamma -X}, \Gamma-\mathrm{T}, \mathrm{T}-\mathrm{Z}, \mathrm{Y}-\mathrm{Z} \\
\mathrm{U}-\mathrm{X}, \mathrm{X}-\mathrm{Z}, \mathrm{S}-\mathrm{Y}, \mathrm{X}-\mathrm{Y}, \mathrm{R}-\mathrm{T} \\
\text { T-U }\end{array}$ & $\| \mathrm{Ca}_{5}(\mathrm{PPd})_{6}$ \\
\hline & $\begin{array}{l}\text { T-Y, } \Gamma-Z, \Gamma-X, \Gamma-T, \Gamma-U \\
\text { R-Y, S-Y, T-Y, R-Z, T-Z, } \\
\text { U-Z }\end{array}$ & $\| \mathrm{NdSbPd}$ \\
\hline & $\begin{array}{c}\Gamma-\mathrm{Y}, \Gamma-\mathrm{Z}, \Gamma-\mathrm{X}, \Gamma-\mathrm{T}, \mathrm{T}-\mathrm{Y} \\
\mathrm{T}-\mathrm{Z}, \mathrm{Y}-\mathrm{Z}, \Gamma-\mathrm{U}, \mathrm{U}-\mathrm{X}, \mathrm{U}-\mathrm{Z} \\
\Gamma-\mathrm{S}\end{array}$ & $\| \mathrm{GeH}_{4}$ \\
\hline & $\begin{array}{c}\text { T-Y, } \Gamma-Z, \Gamma-X, \Gamma-T, T-Y, \\
\text { Y-Z, } \Gamma-U, U-X, U-Z, X-Z, \\
\Gamma-S\end{array}$ & $\| \mathrm{Te}$ \\
\hline & 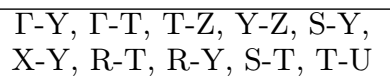 & $\| \mathrm{NdInNi}_{2}, \mathrm{LaInNi}_{2}, \mathrm{PrInNi}_{2}$ \\
\hline & $\begin{array}{l}\Gamma-Y, \Gamma-Z, \Gamma-U, U-X, U-Z, \\
\text { X-Z, S-Y, X-Y, R-Y, T-Y }\end{array}$ & $\| \mathrm{GaPt}_{2}$ \\
\hline & $\begin{array}{l}\Gamma-Y, \Gamma-Z, \Gamma-X, T-Y, T-Z \\
\Gamma-U, \Gamma-S, S-Y, X-Y, R-Y\end{array}$ & $\| \mathrm{Zn}_{5} \mathrm{~B}_{3} \operatorname{Ir}_{7}$ \\
\hline & 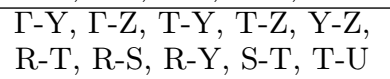 & $\| \mathrm{Zn}_{5} \mathrm{~B}_{3} \mathrm{Rh}_{7}$ \\
\hline & $\begin{array}{c}\text { Г-X, Г-U, U-Z, X-Z, Г-S } \\
\text { S-Y, X-Y, R-Y, T-Y }\end{array}$ & $\| \mathrm{AlPt}_{2}$ \\
\hline & 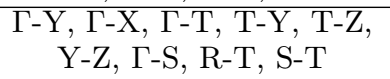 & $\| \mathrm{TiPd}$ \\
\hline & 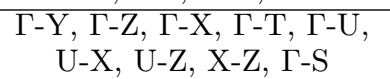 & $\| \mathrm{Li}_{2} \mathrm{Ca}_{3} \mathrm{~N}_{6}$ \\
\hline & $\begin{array}{c}\Gamma-\mathrm{Y}, \Gamma-\mathrm{Z}, \Gamma-\mathrm{T}, \mathrm{U}-\mathrm{Z}, \mathrm{X}-\mathrm{Z} \\
\mathrm{R}-\mathrm{S}, \mathrm{S}-\mathrm{T}, \mathrm{S}-\mathrm{Y}\end{array}$ & $\| \mathrm{BiPd}_{3}$ \\
\hline & $\begin{array}{l}\text { Г-Z, } \mathrm{\Gamma -X}, \mathrm{T}-\mathrm{Z}, \mathrm{Y}-\mathrm{Z}, \Gamma-\mathrm{U} \\
\mathrm{R}-\mathrm{T}, \mathrm{S}-\mathrm{T}, \mathrm{T}-\mathrm{Y}\end{array}$ & $\| \mathrm{NdAl}_{4} \mathrm{Co}, \mathrm{PrAl}_{4} \mathrm{Co}$ \\
\hline & $\begin{array}{c}\text { T-T, T-Y, T-Z, R-Y, S-Y, } \\
\text { R-Z, U-Z }\end{array}$ & $\mathrm{Rb}_{2} \mathrm{TlAu}_{3} \|$ \\
\hline & 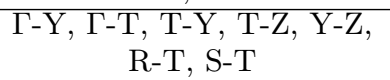 & $\| \mathrm{NbRh}$ \\
\hline & $\begin{array}{c}\Gamma-\mathrm{Y}, \Gamma-\mathrm{Z}, \Gamma-\mathrm{T}, \mathrm{T}-\mathrm{Z}, \mathrm{Y}-\mathrm{Z} \\
\text { S-Y, X-Y }\end{array}$ & $\| \mathrm{MgCd}$ \\
\hline
\end{tabular}


Table III - continued

\begin{tabular}{|c|c|c|}
\hline $\begin{array}{l}\text { SOC or } \\
\text { NSOC }\end{array}$ & $\begin{array}{c}\text { HSL with Band } \\
\text { Crossing }\end{array}$ & High Symmetry Line Semimetals \\
\hline & $\begin{array}{c}\text { T-Z, Г-T, T-Y, T-Z, Y-Z, } \\
\text { R-T, S-T }\end{array}$ & $\| \mathrm{TiPt}$ \\
\hline & $\begin{array}{c}\Gamma-\mathrm{Z}, \Gamma-\mathrm{X}, \mathrm{T}-\mathrm{Z}, \mathrm{Y}-\mathrm{Z}, \Gamma-\mathrm{U} \\
\mathrm{R}-\mathrm{T}, \mathrm{T}-\mathrm{U}\end{array}$ & $\| \mathrm{LaAl}_{4} \mathrm{Co}$ \\
\hline & $\Gamma-\mathrm{Y}, \Gamma-\mathrm{X}, \mathrm{T}-\mathrm{Y}, \mathrm{Y}-\mathrm{Z}, \Gamma-\mathrm{S}$ & $\| \mathrm{TiNi}$ \\
\hline & $\Gamma-\mathrm{Y}, \Gamma-\mathrm{Z}, \Gamma-\mathrm{X}, \Gamma-\mathrm{U}, \Gamma-\mathrm{S}$ & $\| \mathrm{MgCl}_{2}$ \\
\hline \multicolumn{3}{|r|}{ Space Group 52: Pnna } \\
\hline \multirow[t]{2}{*}{ NSOC } & $\begin{array}{c}\Gamma-\mathrm{Y}, \Gamma-\mathrm{Z}, \Gamma-\mathrm{X}, \Gamma-\mathrm{T}, \Gamma-\mathrm{S} \\
\mathrm{R}-\mathrm{U}\end{array}$ & $\| \mathrm{ErP}_{2} \mathrm{H}_{7} \mathrm{O}_{10}$ \\
\hline & $\Gamma-\mathrm{Z}, \Gamma-\mathrm{X}, \Gamma-\mathrm{U}$ & || $\mathrm{TaSbO}_{4}$ \\
\hline $\mathrm{SOC}$ & $\mathrm{S}-\mathrm{X}$ & $\mathrm{Ag}_{2} \mathrm{BiO}_{3}, \| \mathrm{Sr}_{2} \mathrm{Bi}_{3}$ \\
\hline \multicolumn{3}{|r|}{ Space Group 53: Pmna } \\
\hline \multirow{3}{*}{ NSOC } & 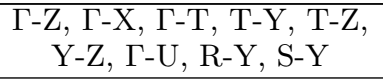 & $\| \mathrm{KTa}_{3} \mathrm{CuO}_{9}$ \\
\hline & $\begin{array}{c}-\mathrm{Y}, \Gamma-\mathrm{Z}, \Gamma-\mathrm{X}, \Gamma-\mathrm{T}, \mathrm{T}-\mathrm{Y} \\
\mathrm{Y}-\mathrm{Z}, \Gamma-\mathrm{S}\end{array}$ & \| $\mathrm{TaNiTe}_{2}$ \\
\hline & $\begin{array}{c}\Gamma-\mathrm{Z}, \Gamma-\mathrm{X}, \Gamma-\mathrm{T}, \mathrm{T}-\mathrm{Y}, \mathrm{Y}-\mathrm{Z} \\
\Gamma-\mathrm{U}\end{array}$ & $\| \mathrm{NbNiTe}_{2}$ \\
\hline \multirow{4}{*}{ NSOC } & \multicolumn{2}{|r|}{ Space Group 54: Pcca } \\
\hline & $\begin{array}{c}\Gamma-Y, \Gamma-Z, \Gamma-X, \Gamma-U, U-X, \\
\text { X-Z, Г-S, R-Y, S-Y, T-Y }\end{array}$ & \| C(ICSD : 88813) \\
\hline & $\begin{array}{c}\text { Г-Y, Г-Z, Г-X, Г-T, T-Y, } \\
\text { Y-Z, Г-S }\end{array}$ & $\| \mathrm{FeS}_{2}$ \\
\hline & $\Gamma-\mathrm{Y}, \mathrm{S}-\mathrm{Y}, \mathrm{X}-\mathrm{Y}$ & $\mathrm{La}_{2} \mathrm{Re}_{3} \mathrm{~B}_{7}$ \\
\hline \multirow{14}{*}{ NSOC } & & Space Group 55: Pbam \\
\hline & $\begin{array}{l}\text { T-Y, }-\mathrm{Z}, \Gamma-\mathrm{X}, \Gamma-\mathrm{T}, \Gamma-\mathrm{S} \\
\mathrm{S}-\mathrm{X}, \mathrm{X}-\mathrm{Y}, \mathrm{R}-\mathrm{U}, \mathrm{R}-\mathrm{S}, \mathrm{R}-\mathrm{Z} \\
\mathrm{T}-\mathrm{U}, \mathrm{T}-\mathrm{Z}, \mathrm{U}-\mathrm{Z}\end{array}$ & $\| \mathrm{B}_{8} \mathrm{Ru}_{11}$ \\
\hline & $\begin{array}{l}\Gamma-\mathrm{Y}, \Gamma-\mathrm{Z}, \Gamma-\mathrm{X}, \mathrm{T}-\mathrm{Z}, \mathrm{Y}-\mathrm{Z} \\
\Gamma-\mathrm{U}, \Gamma-\mathrm{S}, \mathrm{S}-\mathrm{Y}, \mathrm{X}-\mathrm{Y}, \mathrm{R}-\mathrm{T} \\
\mathrm{R}-\mathrm{Z}, \mathrm{T}-\mathrm{U}, \mathrm{U}-\mathrm{Z}\end{array}$ & $\| \mathrm{Ca}\left(\mathrm{Al}_{4} \mathrm{Co}\right)_{2}$ \\
\hline & 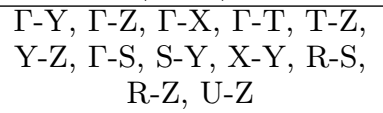 & $\| \mathrm{Fe}(\mathrm{BrN})_{2}$ \\
\hline & $\begin{array}{c}\Gamma-Y, \Gamma-Z, \Gamma-X, \Gamma-T, U-Z, \\
\text { X-Z, }-\mathrm{S}, \mathrm{S}-\mathrm{Y}, \mathrm{X}-\mathrm{Y}, \mathrm{R}-\mathrm{Z} \\
\text { T-Z }\end{array}$ & \| $\mathrm{SmFeB}_{4}$ \\
\hline & $\begin{array}{c}\text { Г-Y, Г-Z, }-\mathrm{X} \text { X, T-Z, Y-Z, } \\
\Gamma-\mathrm{U}, \mathrm{U}-\mathrm{Z}, \mathrm{X}-\mathrm{Z}, \Gamma-\mathrm{S}, \mathrm{S}-\mathrm{Y} \\
\mathrm{X}-\mathrm{Y}\end{array}$ & $\| \mathrm{Zn}_{12} \mathrm{Pt}_{7}$ \\
\hline & 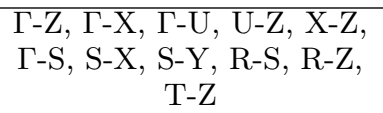 & || $\mathrm{TiMnSi}_{2}$ \\
\hline & $\begin{array}{c}\text { T-Z, } \mathrm{T}-\mathrm{X}, \mathrm{T}-\mathrm{Z}, \mathrm{Y}-\mathrm{Z}, \mathrm{\Gamma -U} \\
\mathrm{U}-\mathrm{Z}, \mathrm{X}-\mathrm{Z}, \mathrm{R}-\mathrm{U}, \mathrm{R}-\mathrm{S}, \mathrm{R}-\mathrm{Z} \\
\text { T-U }\end{array}$ & $\| \mathrm{ZrCrSi}_{2}$ \\
\hline & $\begin{array}{c}\text { Г-Y, Г-X, Г-T, T-Z, Y-Z, } \\
\Gamma-\mathrm{S}, \mathrm{S}-\mathrm{Y}, \mathrm{X}-\mathrm{Y}, \mathrm{R}-\mathrm{Z}, \mathrm{U}-\mathrm{Z}\end{array}$ & $\| \mathrm{Nd}_{2} \mathrm{NiB}_{10}$ \\
\hline & $\begin{array}{c}\Gamma-\mathrm{Y}, \Gamma-\mathrm{Z}, \Gamma-\mathrm{X}, \Gamma-\mathrm{T}, \Gamma-\mathrm{U} \\
\mathrm{U}-\mathrm{Z}, \mathrm{X}-\mathrm{Z}, \Gamma-\mathrm{S}, \mathrm{S}-\mathrm{Y}, \mathrm{X}-\mathrm{Y}\end{array}$ & $\| \mathrm{MgGa}_{2}$ \\
\hline & $\begin{array}{c}\Gamma-\mathrm{Y}, \Gamma-\mathrm{Z}, \Gamma-\mathrm{X}, \Gamma-\mathrm{T}, \mathrm{U}-\mathrm{Z} \\
\mathrm{X}-\mathrm{Z}, \Gamma-\mathrm{S}, \mathrm{S}-\mathrm{X}, \mathrm{S}-\mathrm{Y}, \mathrm{R}-\mathrm{S}\end{array}$ & $\| \mathrm{YbB}_{4} \mathrm{Rh}$ \\
\hline & $\begin{array}{c}-Y, \Gamma-Z, \Gamma-X, T-Z, Y-Z, \\
\Gamma-U, \Gamma-S, \text { R-S, R-Z, U-Z }\end{array}$ & $\| \mathrm{ScNiB}_{4}, \mathrm{CeReB}_{4}$ \\
\hline & 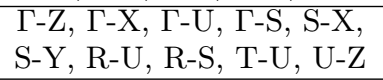 & || HfReSi 2 \\
\hline & 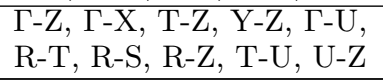 & $\| \mathrm{Zr}_{5}\left(\operatorname{In}_{2} \mathrm{Rh}\right)_{2}$ \\
\hline
\end{tabular}


Table III - continued

\begin{tabular}{|c|c|c|}
\hline $\begin{array}{l}\text { SOC or } \\
\text { NSOC }\end{array}$ & $\begin{array}{l}\text { HSL with Band } \\
\text { Crossing }\end{array}$ & High Symmetry Line Semimetals \\
\hline & $\begin{array}{l}\Gamma-Z, \Gamma-X, U-Z, X-Z, \Gamma-S, \\
\text { S-Y, X-Y, R-S, R-Z, T-Z }\end{array}$ & $\| \mathrm{LuNiB}_{4}$ \\
\hline & $\begin{array}{l}\Gamma-Y, \Gamma-Z, \Gamma-X, \Gamma-T, \Gamma-U, \\
\text { U-Z, X-Z, Г-S, R-S }\end{array}$ & $\| \mathrm{ZrGe}_{2} \mathrm{Rh}$ \\
\hline & 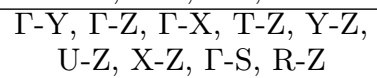 & $\| \mathrm{Ca}_{3}\left(\mathrm{Bi}_{2} \mathrm{Pd}\right)_{4}$ \\
\hline & $\begin{array}{l}\Gamma-Y, \Gamma-Z, \Gamma-T, R-T, R-Z, \\
\text { T-U, T-Z, U-Z }\end{array}$ & $\| \mathrm{Ho}_{4} \mathrm{C}_{5}$ \\
\hline & $\begin{array}{l}\Gamma-\mathrm{Y}, \Gamma-\mathrm{Z}, \Gamma-\mathrm{T}, \mathrm{T}-\mathrm{Z}, \mathrm{Y}-\mathrm{Z} \\
\Gamma-\mathrm{S}, \mathrm{S}-\mathrm{X}, \mathrm{X}-\mathrm{Y}\end{array}$ & \| $\mathrm{HoReB}_{4}, \mathrm{YReB}_{4}$ \\
\hline & $\begin{array}{l}\Gamma-\mathrm{Y}, \Gamma-\mathrm{Z}, \Gamma-\mathrm{X}, \Gamma-\mathrm{T}, \Gamma-\mathrm{S} \\
\mathrm{S}-\mathrm{Y}, \mathrm{X}-\mathrm{Y}, \mathrm{R}-\mathrm{S}\end{array}$ & || NiSn \\
\hline & $\begin{array}{l}\Gamma-\mathrm{Y}, \Gamma-\mathrm{Z}, \Gamma-\mathrm{X}, \Gamma-\mathrm{T}, \Gamma-\mathrm{U} \\
\text { U-Z, X-Z, R-S }\end{array}$ & $\| \operatorname{Sr}\left(\operatorname{In}_{4} \mathrm{Rh}\right)_{2}$ \\
\hline & $\begin{array}{l}\Gamma-\mathrm{Z}, \Gamma-\mathrm{X}, \Gamma-\mathrm{U}, \mathrm{R}-\mathrm{T}, \mathrm{R}-\mathrm{S}, \\
\text { R-Z, T-U, U-Z }\end{array}$ & $\| \mathrm{Sc}_{2} \mathrm{~B}_{6} \mathrm{Rh}$ \\
\hline & 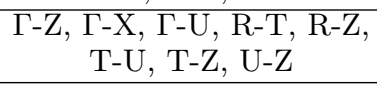 & $\| \mathrm{Tb}_{4} \mathrm{C}_{5}, \mathrm{Y}_{4} \mathrm{C}_{5}$ \\
\hline & $\begin{array}{l}\Gamma-\mathrm{Y}, \Gamma-\mathrm{Z}, \Gamma-\mathrm{X}, \Gamma-\mathrm{U}, \Gamma-\mathrm{S} \\
\text { S-X, S-Y }\end{array}$ & $\| \mathrm{Yb}\left(\mathrm{Ga}_{4} \mathrm{Co}\right)_{2}$ \\
\hline & $\begin{array}{c}\Gamma-\mathrm{Y}, \Gamma-\mathrm{Z}, \Gamma-\mathrm{X}, \Gamma-\mathrm{U}, \mathrm{U}-\mathrm{Z} \\
\mathrm{X}-\mathrm{Z}, \Gamma-\mathrm{S} \\
\end{array}$ & $\| \mathrm{LuAlB}_{4}$ \\
\hline & $\begin{array}{l}\Gamma-Y, S-Y, X-Y, R-S, R-Z, \\
\text { T-Z, U-Z }\end{array}$ & $\| \mathrm{Ti}_{9} \mathrm{Se}_{2}$ \\
\hline & $\begin{array}{l}\Gamma-\mathrm{Z}, \Gamma-\mathrm{X}, \Gamma-\mathrm{U}, \mathrm{U}-\mathrm{Z}, \mathrm{X}-\mathrm{Z} \\
\mathrm{R}-\mathrm{U}, \mathrm{T}-\mathrm{U}\end{array}$ & $\| \mathrm{TiFeSi}_{2}$ \\
\hline & $\begin{array}{l}\Gamma-\mathrm{Z}, \Gamma-\mathrm{X}, \mathrm{U}-\mathrm{Z}, \mathrm{X}-\mathrm{Z}, \Gamma-\mathrm{S} \\
\text { S-Y, X-Y }\end{array}$ & $\| \mathrm{SmMnB}_{4}$ \\
\hline & $\begin{array}{l}\text { T-Z, T-Z, Y-Z, R-U, R-Z, } \\
\text { T-U, U-Z }\end{array}$ & $\| \mathrm{Sb}_{4} \mathrm{~Pb}_{4} \mathrm{~S}_{11}$ \\
\hline & $\begin{array}{c}\Gamma-\mathrm{Y}, \Gamma-\mathrm{X}, \underset{\Gamma-\mathrm{T}}{\Gamma}, \mathrm{T}-\mathrm{Z}, \mathrm{Y}-\mathrm{Z}, \\
\end{array}$ & $\| \mathrm{YbCoB}_{4}$ \\
\hline & $\begin{array}{l}\Gamma-\mathrm{Y}, \Gamma-\mathrm{Z}, \Gamma-\mathrm{T}, \mathrm{U}-\mathrm{Z}, \mathrm{X}-\mathrm{Z}, \\
\mathrm{R}-\mathrm{S}\end{array}$ & $\| \mathrm{Hf}_{5}\left(\operatorname{In}_{2} \mathrm{Rh}\right)_{2}$ \\
\hline & $\begin{array}{c}\Gamma-\mathrm{Y}, \Gamma-\mathrm{Z}, \Gamma-\mathrm{X}, \Gamma-\mathrm{T}, \Gamma-\mathrm{S} \\
\text { R-S }\end{array}$ & 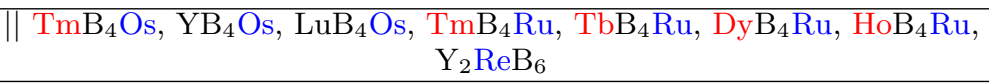 \\
\hline & $\begin{array}{c}\Gamma-\mathrm{Y}, \Gamma-\mathrm{Z}, \Gamma-\mathrm{X}, \Gamma-\mathrm{T}, \Gamma-\mathrm{U} \\
\Gamma-\mathrm{S}\end{array}$ & $\| \mathrm{Sc}(\mathrm{BC})_{2}, \mathrm{Lu}_{2} \mathrm{FeB}_{6}, \mathrm{Sr}_{2} \mathrm{Cu}_{2} \mathrm{O}_{5}, \mathrm{Ga}_{2} \mathrm{AsRh}_{5}$ \\
\hline & $\begin{array}{c}\Gamma-\mathrm{Z}, \Gamma-\mathrm{X}, \mathrm{\Gamma -U}, \Gamma-\mathrm{S}, \mathrm{S}-\mathrm{X} \\
\mathrm{S}-\mathrm{Y}\end{array}$ & $\| \mathrm{YbB}_{4} \mathrm{Ru}, \mathrm{YbB}_{4} \mathrm{Os}$ \\
\hline & $\begin{array}{l}\Gamma-\mathrm{Z}, \mathrm{T}-\mathrm{Z}, \mathrm{Y}-\mathrm{Z}, \mathrm{R}-\mathrm{S}, \mathrm{R}-\mathrm{Z} \\
\mathrm{U}-\mathrm{Z}\end{array}$ & $\| \mathrm{Tb}_{4} \mathrm{SiBr}_{6}$ \\
\hline & $\begin{array}{l}\Gamma-\mathrm{Z}, \mathrm{T}-\mathrm{Z}, \mathrm{Y}-\mathrm{Z}, \mathrm{U}-\mathrm{Z}, \mathrm{X}-\mathrm{Z} \\
\mathrm{R}-\mathrm{Z}\end{array}$ & $\| \mathrm{Th}_{2} \mathrm{NiB}_{10}$ \\
\hline & $\Gamma-\mathrm{Y}, \Gamma-\mathrm{X}, \Gamma-\mathrm{S}, \mathrm{S}-\mathrm{X}, \mathrm{X}-\mathrm{Y}$ & $\operatorname{Pr}\left(\mathrm{Al}_{4} \mathrm{Co}\right)_{2}$ \\
\hline & $\Gamma-\mathrm{Y}, \Gamma-\mathrm{X}, \Gamma-\mathrm{S}, \mathrm{S}-\mathrm{Y}, \mathrm{X}-\mathrm{Y}$ & $\mathrm{Sr}_{5}\left(\mathrm{InBi}_{3}\right)_{2}$ \\
\hline & $\Gamma-\mathrm{Y}, \Gamma-\mathrm{Z}, \Gamma-\mathrm{T}, \mathrm{T}-\mathrm{Z}, \mathrm{Y}-\mathrm{Z}$ & $\| \mathrm{ThCrB}_{4}$ \\
\hline & $\Gamma-\mathrm{Y}, \Gamma-\mathrm{Z}, \Gamma-\mathrm{X}, \Gamma-\mathrm{T}, \Gamma-\mathrm{U}$ & $\| \mathrm{Th}_{6} \mathrm{Cd}_{7}$ \\
\hline & $\Gamma-\mathrm{Y}, \Gamma-\mathrm{Z}, \Gamma-\mathrm{X}, \Gamma-\mathrm{U}, \Gamma-\mathrm{S}$ & $\| \mathrm{HfGe}_{2} \mathrm{Rh}$ \\
\hline & $\Gamma-\mathrm{Z}, \Gamma-\mathrm{X}, \Gamma-\mathrm{U}, \mathrm{S}-\mathrm{X}, \mathrm{X}-\mathrm{Y}$ & $\mathrm{Nd}\left(\mathrm{Al}_{4} \mathrm{Co}\right)_{2}$ \\
\hline & $\Gamma-\mathrm{Z}, \Gamma-\mathrm{X}, \mathrm{R}-\mathrm{T}, \mathrm{R}-\mathrm{U}, \mathrm{R}-\mathrm{Z}$ & $\| \mathrm{Yb}_{2} \mathrm{AlB}_{6}$ \\
\hline & Г-Z, T-Z, Y-Z, U-Z, X-Z & $\| \mathrm{Sc}_{2} \mathrm{CrC}_{3}$ \\
\hline & $\Gamma-\mathrm{X}, \Gamma-\mathrm{S}, \mathrm{S}-\mathrm{Y}, \mathrm{X}-\mathrm{Y}$ & $\mathrm{Yb}_{5}\left(\mathrm{AlSb}_{3}\right)_{2}, \mathrm{Yb}_{5}\left(\mathrm{InSb}_{3}\right)_{2}, \mathrm{Ca}_{5}\left(\mathrm{AlBi}_{3}\right)_{2}$ \\
\hline & $\Gamma-\mathrm{X}, \Gamma-\mathrm{U}, \mathrm{U}-\mathrm{Z}, \mathrm{X}-\mathrm{Z}$ & $\| \mathrm{Th}_{2} \mathrm{FeB}_{10}$ \\
\hline & $\Gamma-\mathrm{Y}, \Gamma-\mathrm{S}, \mathrm{S}-\mathrm{X}, \mathrm{X}-\mathrm{Y}$ & $\mathrm{Ca}_{5}\left(\mathrm{GaSb}_{3}\right)_{2} \|$ \\
\hline & $\Gamma-\mathrm{Y}, \Gamma-\mathrm{Z}, \Gamma-\mathrm{X}, \Gamma-\mathrm{S}$ & $\| \mathrm{Sr}_{13}\left(\mathrm{MgSi}_{10}\right)_{2}$ \\
\hline & $\Gamma-\mathrm{Z}, \Gamma-\mathrm{X}, \Gamma-\mathrm{U}, \mathrm{R}-\mathrm{S}$ & $\| \mathrm{ErVB}_{4}, \mathrm{HoVB}_{4}, \mathrm{TbVB}_{4}, \mathrm{Sc}_{2} \mathrm{CrB}_{6}$ \\
\hline & $\overline{\Gamma-Z, ~ U-Z, ~ X-Z, ~ R-S ~}$ & $\| \mathrm{Pb}_{3} \mathrm{O}_{4}(\mathrm{ICSD}: 97282)$ \\
\hline & R-T, R-Z, T-U, U-Z & $\| \mathrm{Ce}_{2} \mathrm{NiB}_{10}$ \\
\hline
\end{tabular}


Table III - continued

\begin{tabular}{|c|c|c|}
\hline \multirow{8}{*}{$\begin{array}{l}\text { SOC or } \\
\text { NSOC }\end{array}$} & & \multirow[b]{2}{*}{ High Symmetry Line Semimetals } \\
\hline & $\begin{array}{l}\text { HSL with Band } \\
\text { Crossing }\end{array}$ & \\
\hline & $\Gamma-\mathrm{X}, \mathrm{S}-\mathrm{X}, \mathrm{X}-\mathrm{Y}$ & $\| \mathrm{Li}_{7} \mathrm{Si}_{2}$ \\
\hline & $\Gamma-\mathrm{Y}, \Gamma-\mathrm{Z}, \Gamma-\mathrm{T}$ & $\| \mathrm{ErB}_{4} \mathrm{Rh}, \mathrm{YB}_{4} \mathrm{Rh}, \mathrm{ScCoGe}_{2}$ \\
\hline & $\Gamma-Y, S-Y, X-Y$ & $\mathrm{Ba}_{5}\left(\mathrm{InSb}_{3}\right)_{2} \|$ \\
\hline & $\Gamma-\mathrm{Z}, \Gamma-\mathrm{X}, \Gamma-\mathrm{U}$ & $\mathrm{TmB}_{4} \mathrm{Rh}, \mathrm{La}_{2} \mathrm{Cu}_{2} \mathrm{O}_{5}, \mathrm{Nd}_{2} \mathrm{Cu}_{2} \mathrm{O}_{5}$ \\
\hline & $\Gamma-\mathrm{Z}, \mathrm{U}-\mathrm{Z}, \mathrm{X}-\mathrm{Z}$ & $\| \mathrm{Yb}\left(\mathrm{Al}_{4} \mathrm{Co}\right)_{2}, \mathrm{Mg}_{2} \mathrm{~B}_{4} \mathrm{Ru}_{5}$ \\
\hline & $\Gamma-\mathrm{Z}, \mathrm{R}-\mathrm{S}$ & $\| \mathrm{Ce}\left(\mathrm{Al}_{4} \mathrm{Fe}\right)_{2}$ \\
\hline \multicolumn{3}{|r|}{ Space Group 56: Pccn } \\
\hline $\mathrm{NSOC}$ & $\Gamma-\mathrm{Z}, \Gamma-\mathrm{T}, \mathrm{T}-\mathrm{Y}, \mathrm{Y}-\mathrm{Z}$ & $\mathrm{ZnCuTeCl}_{2} \mathrm{O}_{3}$ \\
\hline \multicolumn{3}{|r|}{ Space Group 57: $\mathrm{Pbcm}$} \\
\hline \multirow{14}{*}{ NSOC } & $\begin{array}{l}\Gamma-Y, \Gamma-Z, \Gamma-T, T-Z, Y-Z, \\
\Gamma-U, U-X, X-Z, \Gamma-S, S-X, \\
\text { S-Y, X-Y, R-U, R-X, S-U }\end{array}$ & $\| \mathrm{LaCoSb}_{3}$ \\
\hline & $\begin{array}{l}\text { Г-Y, Г-Z, Г-T, T-Z, Y-Z, } \\
\Gamma-\mathrm{U}, \mathrm{U}-\mathrm{X}, \mathrm{X}-\mathrm{Z}, \Gamma-\mathrm{S}, \mathrm{S}-\mathrm{X} \\
\quad \mathrm{X}-\mathrm{Y}, \mathrm{R}-\mathrm{U}, \mathrm{R}-\mathrm{X}, \mathrm{S}-\mathrm{U}\end{array}$ & $\| \mathrm{LaNiSb}_{3}, \mathrm{SmCoSb}_{3}, \mathrm{PrCoSb}_{3}, \mathrm{NdCoSb}_{3}$ \\
\hline & $\begin{array}{l}\Gamma-\mathrm{Y}, \Gamma-\mathrm{Z}, \Gamma-\mathrm{X}, \Gamma-\mathrm{T}, \mathrm{T}-\mathrm{Z} \\
\mathrm{Y}-\mathrm{Z}, \Gamma-\mathrm{S}, \mathrm{S}-\mathrm{X}, \mathrm{X}-\mathrm{Y}, \mathrm{R}-\mathrm{U} \\
\mathrm{R}-\mathrm{X}, \mathrm{S}-\mathrm{U}, \mathrm{U}-\mathrm{X}\end{array}$ & $\| \mathrm{PrNiSb}_{3}, \mathrm{NdNiSb}_{3}, \mathrm{SmNiSb}_{3}$ \\
\hline & $\begin{array}{c}\Gamma-Y, \Gamma-Z, \Gamma-X, \Gamma-U, U-X \\
\text { X-Z, }-\mathrm{S}, \mathrm{S}-\mathrm{X}, \mathrm{S}-\mathrm{Y}, \mathrm{X}-\mathrm{Y} \\
\text { R-X }\end{array}$ & || NdAl, SmAl \\
\hline & $\begin{array}{l}\Gamma-Y, \Gamma-X, U-X, X-Z, \Gamma-S \\
\text { S-Y, X-Y, R-U, S-U, S-X }\end{array}$ & $\| \mathrm{W}$ \\
\hline & 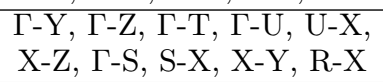 & $\| \mathrm{TmAl}$ \\
\hline & $\begin{array}{l}\Gamma-\mathrm{Y}, \Gamma-\mathrm{Z}, \Gamma-\mathrm{X}, \Gamma-\mathrm{U}, \mathrm{U}-\mathrm{X} \\
\mathrm{X}-\mathrm{Z}, \Gamma-\mathrm{S}, \mathrm{S}-\mathrm{X}, \mathrm{X}-\mathrm{Y}, \mathrm{R}-\mathrm{X}\end{array}$ & \| TbAl, DyAl \\
\hline & $\begin{array}{l}\text { Г-Y, } \Gamma-\mathrm{Z}, \Gamma-\mathrm{U}, \mathrm{U}-\mathrm{X}, \mathrm{X}-\mathrm{Z} \\
\text { Г-S, S-X, X-Y, R-X }\end{array}$ & $\| \mathrm{Hf}(\mathrm{FeSi})_{2}$ \\
\hline & $\begin{array}{l}\text { Г-Y, } \Gamma-\mathrm{Z}, \Gamma-\mathrm{X}, \Gamma-\mathrm{T}, \mathrm{T}-\mathrm{Z} \\
\mathrm{Y}-\mathrm{Z}, \Gamma-\mathrm{S}, \mathrm{S}-\mathrm{X}, \mathrm{X}-\mathrm{Y}\end{array}$ & $\| \mathrm{Ca}_{3}(\mathrm{GaPd})_{2}$ \\
\hline & $\begin{array}{l}\Gamma-\mathrm{Y}, \Gamma-\mathrm{X}, \Gamma-\mathrm{T}, \mathrm{T}-\mathrm{Z}, \mathrm{Y}-\mathrm{Z} \\
\mathrm{U}-\mathrm{X}, \mathrm{X}-\mathrm{Z}\end{array}$ & $\| \mathrm{Ta}_{2} \mathrm{~S}$ \\
\hline & 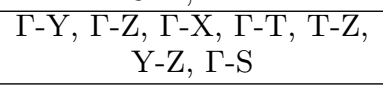 & $\| \mathrm{Ca}_{3}(\mathrm{GaPt})_{2}$ \\
\hline & $\begin{array}{c}\text { Г-Y, } \Gamma-\mathrm{Z}, \Gamma-\mathrm{X}, \Gamma-\mathrm{U}, \mathrm{U}-\mathrm{X} \\
\mathrm{X}-\mathrm{Z}, \Gamma-\mathrm{S}\end{array}$ & $\| \mathrm{LuAl}$ \\
\hline & $\Gamma-\mathrm{Y}, \Gamma-\mathrm{Z}, \Gamma-\mathrm{X}, \Gamma-\mathrm{T}, \Gamma-\mathrm{S}$ & $\mathrm{Pr}_{6} \mathrm{Si}_{4} \mathrm{Ni}_{7}$ \\
\hline & $\Gamma-Y, \Gamma-X, \Gamma-S$ & $\| \mathrm{LiSn}_{5} \mathrm{Rh}_{3}$ \\
\hline \multirow{2}{*}{$\mathrm{SOC}$} & R-T, R-S, R-Y, S-T, T-Y & $\| \mathrm{Cs}, \mathrm{HfGa}, \mathrm{Nd}_{3}(\mathrm{GeRu})_{2}, \mathrm{YbCrSb}_{3}$ \\
\hline & R-S, R-Y, S-T, T-Y & $\begin{array}{c}\| \mathrm{K}_{2} \mathrm{SnBi}, \mathrm{CaAlPd}, \mathrm{Ca}_{4} \mathrm{MgAl}_{3}, \mathrm{La}_{3}(\mathrm{GaNi})_{2}, \mathrm{ThTl}, \mathrm{Y}_{3}(\mathrm{SiRh})_{2}, \\
\qquad \mathrm{Na}_{8} \mathrm{In}_{6} \mathrm{Au}_{11}\end{array}$ \\
\hline \multicolumn{3}{|r|}{ Space Group 58: Pnnm } \\
\hline \multirow{9}{*}{ NSOC } & $\begin{array}{l}\Gamma-\mathrm{Y}, \Gamma-\mathrm{Z}, \Gamma-\mathrm{X}, \Gamma-\mathrm{T}, \Gamma-\mathrm{S} \\
\text { S-Y, X-Y, R-S }\end{array}$ & $\| \mathrm{Ti}_{2} \mathrm{~S}$ \\
\hline & $\begin{array}{l}\Gamma-\mathrm{Y}, \Gamma-\mathrm{Z}, \Gamma-\mathrm{X}, \Gamma-\mathrm{T}, \Gamma-\mathrm{U} \\
\Gamma-\mathrm{S}, \mathrm{S}-\mathrm{X}, \mathrm{S}-\mathrm{Y}\end{array}$ & $\| \mathrm{NiAs}_{2}$ \\
\hline & $\begin{array}{l}\Gamma-\mathrm{Y}, \Gamma-\mathrm{Z}, \Gamma-\mathrm{X}, \Gamma-\mathrm{T}, \Gamma-\mathrm{U} \\
\Gamma-\mathrm{S}, \mathrm{S}-\mathrm{Y}, \mathrm{X}-\mathrm{Y}\end{array}$ & $\| \mathrm{Ti}_{7} \mathrm{Cl}_{16}, \mathrm{Ti}_{7} \mathrm{Br}_{16}$ \\
\hline & $\begin{array}{l}\Gamma-\mathrm{Y}, \Gamma-\mathrm{Z}, \Gamma-\mathrm{X}, \Gamma-\mathrm{S}, \mathrm{S}-\mathrm{Y} \\
\text { X-Y, R-S }\end{array}$ & $\| \mathrm{B}_{2} \mathrm{Mo}_{2} \mathrm{Ir}$ \\
\hline & $\begin{array}{c}\Gamma-\mathrm{Y}, \Gamma-\mathrm{Z}, \Gamma-\mathrm{X}, \Gamma-\mathrm{T}, \Gamma-\mathrm{U} \\
\Gamma-\mathrm{S}, \mathrm{R}-\mathrm{S}\end{array}$ & $\| \mathrm{NiSb}_{2}, \mathrm{Th}\left(\mathrm{Fe}_{2} \mathrm{P}\right)_{2}$ \\
\hline & $\begin{array}{c}\Gamma-\mathrm{Y}, \Gamma-\mathrm{Z}, \Gamma-\mathrm{X}, \Gamma-\mathrm{T}, \Gamma-\mathrm{U} \\
\Gamma-\mathrm{S}\end{array}$ & $\| \mathrm{Th}\left(\mathrm{Re}_{2} \mathrm{Si}\right)_{2}, \mathrm{Ta}_{2} \mathrm{P}$ \\
\hline & $\begin{array}{c}\Gamma-\mathrm{Y}, \Gamma-\mathrm{Z}, \Gamma-\mathrm{X}, \Gamma-\mathrm{T}, \Gamma-\mathrm{U} \\
\mathrm{R}-\mathrm{S}\end{array}$ & $\| \mathrm{LiCa}_{2} \mathrm{Si}_{3}$ \\
\hline & $\Gamma-\mathrm{Y}, \Gamma-\mathrm{Z}, \Gamma-\mathrm{X}, \Gamma-\mathrm{S}, \mathrm{R}-\mathrm{S}$ & || $\mathrm{Hf}_{2} \mathrm{P}$ \\
\hline & $\Gamma-\mathrm{Y}, \Gamma-\mathrm{Z}, \Gamma-\mathrm{X}, \Gamma-\mathrm{U}, \Gamma-\mathrm{S}$ & $\mathrm{RuN}_{2}, \mathrm{FeB}_{4}, \mathrm{Ta}_{2} \mathrm{As}$ \\
\hline
\end{tabular}


Table III - continued

\begin{tabular}{|c|c|c|}
\hline $\begin{array}{l}\text { SOC or } \\
\text { NSOC }\end{array}$ & $\begin{array}{l}\text { HSL with Band } \\
\text { Crossing }\end{array}$ & High Symmetry Line Semimetals \\
\hline \multirow{13}{*}{$\mathrm{SOC}$} & $\Gamma-\mathrm{Z}, \Gamma-\mathrm{X}, \Gamma-\mathrm{U}, \mathrm{S}-\mathrm{X}, \mathrm{X}-\mathrm{Y}$ & $\| \mathrm{NiSe}_{2}, \mathrm{NiS}_{2}$ \\
\hline & $\Gamma-\mathrm{X}, \Gamma-\mathrm{S}, \mathrm{S}-\mathrm{Y}, \mathrm{X}-\mathrm{Y}$ & $\| \mathrm{C}$ \\
\hline & $\Gamma-\mathrm{Y}, \Gamma-\mathrm{Z}, \Gamma-\mathrm{T}, \mathrm{R}-\mathrm{S}$ & $\| \mathrm{In}_{2} \mathrm{Se}, \mathrm{Fe}_{2} \mathrm{C}, \mathrm{Ti}_{2} \mathrm{Se}$ \\
\hline & $\Gamma-\mathrm{Z}, \Gamma-\mathrm{X}, \Gamma-\mathrm{U}, \mathrm{R}-\mathrm{S}$ & $\| \mathrm{Th}_{2} \mathrm{Al}_{2} \mathrm{C}_{3}$ \\
\hline & $\Gamma-\mathrm{X}, \mathrm{S}-\mathrm{X}, \mathrm{X}-\mathrm{Y}$ & $\| \mathrm{Co}_{2} \mathrm{C}$ \\
\hline & $\Gamma-\mathrm{Y}, \Gamma-\mathrm{X}, \Gamma-\mathrm{S}$ & $\mathrm{CrB}_{4}, \mathrm{Zr}_{2} \mathrm{Se}$ \\
\hline & $\Gamma-\mathrm{Y}, \Gamma-\mathrm{Z}, \Gamma-\mathrm{T}$ & $\| \mathrm{LiCa}_{2} \mathrm{Ge}_{3}$ \\
\hline & $\Gamma-\mathrm{Z}, \Gamma-\mathrm{X}, \Gamma-\mathrm{U}$ & $\mathrm{TiS}_{2} \|$ \\
\hline & $\Gamma-Z$ & $\pi \mathrm{OsN}_{2}, \mathrm{RbBi}_{3} \mathrm{~S}_{5}, \mathrm{Zr}_{14} \mathrm{P}_{9}$ \\
\hline & R-S & $\| \mathrm{Ge}_{2} \mathrm{Pt}, \mathrm{Nd}\left(\mathrm{Re}_{2} \mathrm{Si}\right)_{2}$ \\
\hline & R-U, S-U, U-X, T-Z & $\| \mathrm{Nd}\left(\mathrm{Re}_{2} \mathrm{Si}\right)_{2}$ \\
\hline & R-T, S-T, T-Y & $\operatorname{Th}\left(\mathrm{Re}_{2} \mathrm{Si}\right)_{2}$ \\
\hline & $\mathrm{U}-\mathrm{Z}$ & $\| \mathrm{Ta}_{2} \mathrm{As}, \mathrm{Ta}_{2} \mathrm{P}, \mathrm{Hf}_{2} \mathrm{P}$ \\
\hline \multicolumn{3}{|r|}{ Space Group 59: Pmmn } \\
\hline \multirow{24}{*}{ NSOC } & $\begin{array}{c}\Gamma-\mathrm{Y}, \Gamma-\mathrm{Z}, \Gamma-\mathrm{X}, \Gamma-\mathrm{T}, \mathrm{T}-\mathrm{Y}, \\
\mathrm{T}-\mathrm{Z}, \mathrm{Y}-\mathrm{Z}, \Gamma-\mathrm{U}, \mathrm{U}-\mathrm{Z}, \mathrm{X}-\mathrm{Z} \\
\Gamma-\mathrm{S}, \mathrm{R}-\mathrm{Z}\end{array}$ & \| SmGePt, YSiPd, ErSiPd, PrGePt \\
\hline & $\begin{array}{c}\Gamma-\mathrm{Y}, \Gamma-\mathrm{X}, \Gamma-\mathrm{T}, \mathrm{T}-\mathrm{Z}, \mathrm{Y}-\mathrm{Z}, \\
\Gamma-\mathrm{U}, \mathrm{U}-\mathrm{X}, \mathrm{U}-\mathrm{Z}, \mathrm{X}-\mathrm{Z}, \Gamma-\mathrm{S}, \\
\mathrm{R}-\mathrm{Z}\end{array}$ & $\| \mathrm{Dy}_{2} \mathrm{Sn}_{5}$ \\
\hline & $\begin{array}{c}\Gamma-\mathrm{Y}, \Gamma-\mathrm{Z}, \Gamma-\mathrm{X}, \Gamma-\mathrm{T}, \mathrm{T}-\mathrm{Z}, \\
\mathrm{Y}-\mathrm{Z}, \Gamma-\mathrm{U}, \mathrm{U}-\mathrm{X}, \mathrm{U}-\mathrm{Z}, \mathrm{X}-\mathrm{Z} \\
\mathrm{R}-\mathrm{S}\end{array}$ & $\| \mathrm{Ni}_{3} \mathrm{Mo}$ \\
\hline & $\begin{array}{c}\Gamma-\mathrm{Y}, \Gamma-\mathrm{Z}, \Gamma-\mathrm{X}, \Gamma-\mathrm{T}, \mathrm{T}-\mathrm{Z} \\
\mathrm{Y}-\mathrm{Z}, \Gamma-\mathrm{U}, \mathrm{U}-\mathrm{X}, \mathrm{X}-\mathrm{Z}, \Gamma-\mathrm{S} \\
\mathrm{R}-\mathrm{S}\end{array}$ & $\| \mathrm{AlCu}_{3}$ \\
\hline & $\begin{array}{c}\Gamma-\mathrm{Y}, \Gamma-\mathrm{X}, \Gamma-\mathrm{T}, \mathrm{T}-\mathrm{Z}, \mathrm{Y}-\mathrm{Z}, \\
\Gamma-\mathrm{U}, \mathrm{U}-\mathrm{Z}, \mathrm{X}-\mathrm{Z}, \Gamma-\mathrm{S}, \mathrm{R}-\mathrm{S}\end{array}$ & $\| \mathrm{LuAu}_{3}$ \\
\hline & $\begin{array}{c}\Gamma-\mathrm{Y}, \Gamma-\mathrm{X}, \Gamma-\mathrm{T}, \mathrm{T}-\mathrm{Z}, \mathrm{Y}-\mathrm{Z} \\
\Gamma-\mathrm{U}, \mathrm{U}-\mathrm{Z}, \mathrm{X}-\mathrm{Z}, \mathrm{\Gamma}-\mathrm{S}, \mathrm{R}-\mathrm{Z}\end{array}$ & $\| \mathrm{TbAu}_{3}, \mathrm{Er}_{2} \mathrm{Ge}_{5}$ \\
\hline & 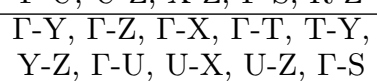 & $\| \mathrm{Zn}_{2} \mathrm{CuAu}$ \\
\hline & $\begin{array}{l}\Gamma-\mathrm{Y}, \Gamma-\mathrm{X}, \Gamma-\mathrm{U}, \mathrm{U}-\mathrm{X}, \mathrm{U}-\mathrm{Z} \\
\quad \mathrm{X}-\mathrm{Z}, \Gamma-\mathrm{S}, \mathrm{R}-\mathrm{Z}, \mathrm{T}-\mathrm{Z}\end{array}$ & $\| \mathrm{Te}_{2} \mathrm{Pd}_{3} \mathrm{~Pb}_{2}$ \\
\hline & $\begin{array}{c}\Gamma-\mathrm{Y}, \Gamma-\mathrm{Z}, \Gamma-\mathrm{X}, \Gamma-\mathrm{T}, \Gamma-\mathrm{U} \\
\mathrm{U}-\mathrm{X}, \mathrm{X}-\mathrm{Z}, \Gamma-\mathrm{S}\end{array}$ & $\| \mathrm{InAu}_{3}$ \\
\hline & $\begin{array}{c}\Gamma-Y, \Gamma-Z, \Gamma-X, \Gamma-\mathrm{T}, \mathrm{T}-\mathrm{Y}, \\
\mathrm{T}-\mathrm{Z}, \Gamma-\mathrm{U}, \Gamma-\mathrm{S}\end{array}$ & $\| \mathrm{SmAu}_{3}$ \\
\hline & $\begin{array}{c}\Gamma-\mathrm{Y}, \Gamma-\mathrm{Z}, \Gamma-\mathrm{X}, \Gamma-\mathrm{T}, \mathrm{T}-\mathrm{Z} \\
\mathrm{Y}-\mathrm{Z}, \Gamma-\mathrm{U}, \Gamma-\mathrm{S}\end{array}$ & $\| \mathrm{TmAu}_{3}, \mathrm{YAu}_{3}, \mathrm{DyAu}_{3}, \mathrm{HoAu}_{3}, \mathrm{ErAu}_{3}$ \\
\hline & $\begin{array}{c}\Gamma-\mathrm{Y}, \Gamma-\mathrm{Z}, \Gamma-\mathrm{X}, \Gamma-\mathrm{U}, \mathrm{U}-\mathrm{X}, \\
\mathrm{X}-\mathrm{Z}, \Gamma-\mathrm{S}, \mathrm{R}-\mathrm{S}\end{array}$ & $\| \operatorname{Sr}(\operatorname{InAu})_{3}$ \\
\hline & $\begin{array}{c}\Gamma-\mathrm{Y}, \Gamma-\mathrm{Z}, \Gamma-\mathrm{X}, \Gamma-\mathrm{T}, \mathrm{U}-\mathrm{Z} \\
\mathrm{X}-\mathrm{Z}, \Gamma-\mathrm{S}\end{array}$ & |l $\mathrm{Er}_{5} \mathrm{Al}_{3} \mathrm{Ni}_{3} \mathrm{Ge}_{4}, \mathrm{Nd}_{3} \mathrm{Sb}_{5} \mathrm{Pd}_{6}, \mathrm{Pr}_{3} \mathrm{Sb}_{5} \mathrm{Pd}_{6}$ \\
\hline & $\begin{array}{c}\Gamma-\mathrm{Z}, \Gamma-\mathrm{X}, \Gamma-\mathrm{U}, \mathrm{R}-\mathrm{S}, \mathrm{R}-\mathrm{Z} \\
\mathrm{T}-\mathrm{Z}, \mathrm{U}-\mathrm{Z}\end{array}$ & $\| \mathrm{Hf}_{8} \mathrm{FeTe}_{6}$ \\
\hline & 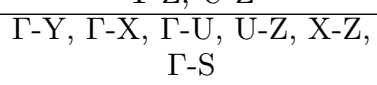 & $\| \mathrm{K}(\mathrm{SnAu})_{3}$ \\
\hline & $\begin{array}{c}\Gamma-\mathrm{Y}, \Gamma-\mathrm{Z}, \Gamma-\mathrm{X}, \Gamma-\mathrm{T}, \Gamma-\mathrm{U}, \\
\Gamma-\mathrm{S}\end{array}$ & $\| \mathrm{Cu}_{3} \mathrm{Sb}, \mathrm{Ag}_{3} \mathrm{Sb}$ \\
\hline & $\begin{array}{c}\Gamma-\mathrm{Z}, \Gamma-\mathrm{X}, \Gamma-\mathrm{U}, \mathrm{U}-\mathrm{X}, \mathrm{U}-\mathrm{Z}, \\
\mathrm{R}-\mathrm{S}\end{array}$ & $\| \mathrm{Rb}_{3} \mathrm{~Pb}_{4} \mathrm{Au}$ \\
\hline & $\Gamma-\mathrm{X}, \Gamma-\mathrm{U}, \mathrm{U}-\mathrm{Z}, \mathrm{X}-\mathrm{Z}, \mathrm{R}-\mathrm{S}$ & $\| \mathrm{Sr}_{6} \mathrm{Ge}_{5} \mathrm{~N}_{2}$ \\
\hline & $\Gamma-\mathrm{Y}, \Gamma-\mathrm{Z}, \Gamma-\mathrm{T}, \mathrm{U}-\mathrm{Z}, \mathrm{X}-\mathrm{Z}$ & $\| \mathrm{Ni}_{3} \mathrm{Sn}$ \\
\hline & $\Gamma-\mathrm{Y}, \Gamma-\mathrm{Z}, \Gamma-\mathrm{X}, \Gamma-\mathrm{T}, \Gamma-\mathrm{S}$ & $\| \mathrm{BaLi}_{2} \mathrm{Si}$ \\
\hline & $\Gamma-\mathrm{Y}, \Gamma-\mathrm{Z}, \Gamma-\mathrm{X}, \Gamma-\mathrm{U}, \Gamma-\mathrm{S}$ & $\| \mathrm{Yb}_{4} \mathrm{Cu}_{2} \mathrm{Sn}_{5}$ \\
\hline & $\Gamma-\mathrm{Z}, \Gamma-\mathrm{X}, \Gamma-\mathrm{U}, \mathrm{U}-\mathrm{Z}, \mathrm{X}-\mathrm{Z}$ & $\| \mathrm{SiNi}$ \\
\hline & $\Gamma-\mathrm{Z}, \Gamma-\mathrm{X}, \mathrm{U}-\mathrm{X}, \mathrm{U}-\mathrm{Z}, \mathrm{R}-\mathrm{S}$ & $\| \mathrm{MgCu}_{2} \mathrm{O}_{3}$ \\
\hline & $\Gamma-\mathrm{Z}, \mathrm{U}-\mathrm{Z}, \mathrm{X}-\mathrm{Z}, \mathrm{R}-\mathrm{Z}, \mathrm{T}-\mathrm{Z}$ & $\|$ InSb \\
\hline
\end{tabular}


Table III - continued

\begin{tabular}{|c|c|c|}
\hline $\begin{array}{l}\text { SOC or } \\
\text { NSOC }\end{array}$ & $\begin{array}{l}\text { HSL with Band } \\
\text { Crossing }\end{array}$ & High Symmetry Line Semimetals \\
\hline & $\Gamma-\mathrm{X}, \Gamma-\mathrm{U}, \mathrm{U}-\mathrm{Z}, \mathrm{X}-\mathrm{Z}$ & $\| \mathrm{B}_{2} \mathrm{Os}, \mathrm{B}_{2} \mathrm{Ru}, \mathrm{Ba}_{6} \mathrm{Ge}_{5} \mathrm{~N}_{2}$ \\
\hline & $\Gamma-\mathrm{Y}, \Gamma-\mathrm{T}, \mathrm{T}-\mathrm{Z}, \mathrm{Y}-\mathrm{Z}$ & $\| \mathrm{CaBiCO}_{4} \mathrm{~F}$ \\
\hline & $\Gamma-\mathrm{Z}, \Gamma-\mathrm{X}, \Gamma-\mathrm{U}, \mathrm{R}-\mathrm{S}$ & $\mathrm{K}_{3} \mathrm{Sn}_{4} \mathrm{Au}, \mathrm{Rb}_{3} \mathrm{Sn}_{4} \mathrm{Au}$ \\
\hline & $\Gamma-\mathrm{Z}, \Gamma-\mathrm{X}, \Gamma-\mathrm{U}$ & $\| \mathrm{CaCu}_{2} \mathrm{O}_{3}, \mathrm{SrCu}_{2} \mathrm{O}_{3}$ \\
\hline & & Space Group 60: Pbcn \\
\hline & $\begin{array}{c}\Gamma-\mathrm{Y}, \Gamma-\mathrm{Z}, \Gamma-\mathrm{X}, \Gamma-\mathrm{T}, \mathrm{T}-\mathrm{Z} \\
\mathrm{Y}-\mathrm{Z}, \Gamma-\mathrm{U}, \Gamma-\mathrm{S}\end{array}$ & $\| \mathrm{W}_{2} \mathrm{C}$ \\
\hline NSOC & $\begin{array}{c}\Gamma-\mathrm{Y}, \Gamma-\mathrm{X}, \Gamma-\mathrm{T}, \mathrm{T}, \mathrm{T}-\mathrm{Z}, \mathrm{Y}-\mathrm{Z}, \\
\Gamma-\mathrm{S}\end{array}$ & $\mathrm{NbBi}_{4} \mathrm{ClO}_{8} \|$ \\
\hline & $\Gamma-\mathrm{Y}, \Gamma-\mathrm{Z}, \Gamma-\mathrm{T}, \mathrm{T}-\mathrm{Z}, \mathrm{Y}-\mathrm{Z}$ & $\| \mathrm{Mo}_{2} \mathrm{C}$ \\
\hline & $\Gamma-\mathrm{Y}, \Gamma-\mathrm{Z}, \Gamma-\mathrm{X}, \Gamma-\mathrm{T}, \Gamma-\mathrm{S}$ & $\| \mathrm{V}_{2} \mathrm{C}$ \\
\hline & $\Gamma-\mathrm{Y}, \Gamma-\mathrm{X}, \Gamma-\mathrm{S}$ & $\mathrm{CuAsSe}, \mathrm{PbO}_{2}, \| \mathrm{GeTe}$ \\
\hline $\mathrm{SOC}$ & $\begin{array}{c}\text { R-U, R-S, R-X, T-U, U-Z, } \\
\text { T-Y }\end{array}$ & II $\mathrm{ReO}_{2}, \mathrm{Al}_{3} \mathrm{FeSi}_{2}, \mathrm{RbCuCl}_{3}, \mathrm{Hf}_{2} \mathrm{Co}_{3} \mathrm{Si}_{4}, \mathrm{Mn}_{2} \mathrm{~N}, \mathrm{Fe}_{2} \mathrm{~N}$ \\
\hline & & Space Group 61: Pbca \\
\hline & $\begin{array}{l}\Gamma-\mathrm{Y}, \Gamma-\mathrm{Z}, \Gamma-\mathrm{X}, \Gamma-\mathrm{T}, \Gamma-\mathrm{S} \\
\text { S-Y, X-Y }\end{array}$ & $\| \mathrm{Ni}_{5} \mathrm{Ge}_{2} \mathrm{P}_{3}$ \\
\hline & $\begin{array}{c}\Gamma-\mathrm{Y}, \Gamma-\mathrm{Z}, \Gamma-\mathrm{X}, \Gamma-\mathrm{T}, \mathrm{T}-\mathrm{Z} \\
\mathrm{Y}-\mathrm{Z}, \Gamma-\mathrm{U}\end{array}$ & $\| \mathrm{Ca}_{7} \mathrm{Au}_{3}$ \\
\hline & $\begin{array}{c}\Gamma-\mathrm{Y}, \Gamma-\mathrm{Z}, \Gamma-\mathrm{X}, \mathrm{T}-\mathrm{Z}, \mathrm{Y}-\mathrm{Z} \\
\Gamma-\mathrm{U}, \Gamma-\mathrm{S}\end{array}$ & $\| \mathrm{Zr}_{7} \mathrm{Ni}_{10}$ \\
\hline NSOC & $\begin{array}{c}\Gamma-\mathrm{Y}, \Gamma-\mathrm{Z}, \Gamma-\mathrm{X}, \Gamma-\mathrm{T}, \Gamma-\mathrm{U}, \\
\Gamma-\mathrm{S}\end{array}$ & $\| \mathrm{Sn}_{2} \mathrm{Au}$ \\
\hline & $\begin{array}{c}\Gamma-\mathrm{Y}, \Gamma-\mathrm{Z}, \Gamma-\mathrm{X}, \Gamma-\mathrm{T}, \mathrm{U}-\mathrm{X} \\
\mathrm{X}-\mathrm{Z}\end{array}$ & $\| \mathrm{TmCl}_{2}, \mathrm{DyBr}_{2}$ \\
\hline & $\begin{array}{c}\Gamma-\mathrm{Z}, \Gamma-\mathrm{X}, \Gamma-\mathrm{U}, \Gamma-\mathrm{S}, \mathrm{S}-\mathrm{Y} \\
\mathrm{X}-\mathrm{Y}\end{array}$ & $\| \mathrm{Si}_{2} \mathrm{Ni}_{5} \mathrm{P}_{3}$ \\
\hline & $\Gamma-\mathrm{Y}, \Gamma-\mathrm{Z}, \Gamma-\mathrm{T}, \mathrm{T}-\mathrm{Z}, \mathrm{Y}-\mathrm{Z}$ & $\|$ SnTePd \\
\hline & $\Gamma-\mathrm{Z}, \Gamma-\mathrm{X}, \Gamma-\mathrm{S}, \mathrm{S}-\mathrm{Y}, \mathrm{X}-\mathrm{Y}$ & $\| \mathrm{LaSiAs}_{3}$ \\
\hline & $\Gamma-\mathrm{Z}, \Gamma-\mathrm{X}, \mathrm{T}-\mathrm{Z}, \mathrm{Y}-\mathrm{Z}, \Gamma-\mathrm{U}$ & $\| \mathrm{Bi}_{2} \mathrm{Pt}$ \\
\hline & $\Gamma-\mathrm{X}, \Gamma-\mathrm{S}, \mathrm{S}-\mathrm{Y}, \mathrm{X}-\mathrm{Y}$ & $\| \mathrm{Ho}_{2}\left(\mathrm{Ni}_{2} \mathrm{~B}\right)_{5}$ \\
\hline & $\Gamma-\mathrm{Y}, \Gamma-\mathrm{Z}, \Gamma-\mathrm{T}$ & $\| \mathrm{SmI}_{2}$ \\
\hline & $\Gamma-\mathrm{Z}, \mathrm{T}-\mathrm{Z}, \mathrm{Y}-\mathrm{Z}$ & $\mathrm{NiAs}_{2}, \mathrm{SiNi}_{2} \mathrm{P}$ \\
\hline $\mathrm{SOC}$ & $\begin{array}{l}\text { R-T, R-U, R-X, S-U, S-X, } \\
\text { S-T, T-Y, T-U, U-Z }\end{array}$ & $\| \mathrm{AgF}_{2}$ \\
\hline & R-U, R-S, R-X & $\| \mathrm{Ca}_{7} \mathrm{Au}_{3}$ \\
\hline & & Space Group 62: Pnma \\
\hline & $\begin{array}{l}\Gamma-\mathrm{Y}, \Gamma-\mathrm{Z}, \Gamma-\mathrm{X}, \Gamma-\mathrm{T}, \Gamma-\mathrm{U} \\
\mathrm{U}-\mathrm{X}, \mathrm{U}-\mathrm{Z}, \Gamma-\mathrm{S}, \mathrm{S}-\mathrm{Y}, \mathrm{X}-\mathrm{Y}\end{array}$ & $\| \mathrm{Mg}_{2} \mathrm{Hg}$ \\
\hline & $\begin{array}{l}\Gamma-\mathrm{Y}, \Gamma-\mathrm{Z}, \Gamma-\mathrm{X}, \Gamma-\mathrm{U}, \mathrm{U}-\mathrm{X} \\
\mathrm{X}-\mathrm{Z}, \Gamma-\mathrm{S}, \mathrm{S}-\mathrm{Y}, \mathrm{X}-\mathrm{Y}, \mathrm{R}-\mathrm{U}\end{array}$ & || NbNiP \\
\hline & $\begin{array}{c}\Gamma-\mathrm{Y}, \Gamma-\mathrm{Z}, \Gamma-\mathrm{X}, \Gamma-\mathrm{T}, \Gamma-\mathrm{U} \\
\Gamma-\mathrm{S}, \mathrm{S}-\mathrm{Y}, \mathrm{X}-\mathrm{Y}, \mathrm{R}-\mathrm{U}\end{array}$ & $\| \mathrm{Tb}_{2} \mathrm{Pt}$ \\
\hline & $\begin{array}{l}\Gamma-\mathrm{Y}, \Gamma-\mathrm{Z}, \Gamma-\mathrm{X}, \Gamma-\mathrm{T}, \Gamma-\mathrm{U} \\
\quad \mathrm{U}-\mathrm{X}, \mathrm{U}-\mathrm{Z}, \Gamma-\mathrm{S}, \mathrm{R}-\mathrm{U}\end{array}$ & $\| \mathrm{Ca}(\mathrm{BH})_{2}$ \\
\hline & $\begin{array}{l}\Gamma-\mathrm{Y}, \Gamma-\mathrm{Z}, \Gamma-\mathrm{X}, \Gamma-\mathrm{T}, \Gamma-\mathrm{U} \\
\quad \mathrm{U}-\mathrm{Z}, \mathrm{X}-\mathrm{Z}, \Gamma-\mathrm{S}, \mathrm{R}-\mathrm{U}\end{array}$ & $\| \mathrm{Pr}_{2} \mathrm{P}_{3} \mathrm{Au}, \mathrm{FeS}, \mathrm{PrCu}$ \\
\hline & $\begin{array}{l}\Gamma-\mathrm{Y}, \Gamma-Z, \Gamma-\mathrm{X}, \Gamma-\mathrm{T}, \Gamma-\mathrm{U} \\
\mathrm{U}-\mathrm{Z}, \mathrm{X}-\mathrm{Z}, \mathrm{S}-\mathrm{Y}, \mathrm{X}-\mathrm{Y}\end{array}$ & $\| \mathrm{Si}_{4} \mathrm{MoPt}_{3}$ \\
\hline & $\begin{array}{l}\Gamma-\mathrm{Y}, \Gamma-\mathrm{Z}, \Gamma-\mathrm{X}, \Gamma-\mathrm{U}, \mathrm{U}-\mathrm{Z} \\
\quad \mathrm{X}-\mathrm{Z}, \Gamma-\mathrm{S}, \mathrm{S}-\mathrm{Y}, \mathrm{X}-\mathrm{Y}\end{array}$ & $\| \mathrm{Pr}_{3} \mathrm{Co}, \mathrm{La}_{3} \mathrm{Co}$ \\
\hline & $\begin{array}{l}\text { Г-Y, }-\mathrm{-Z}, \Gamma-\mathrm{X}, \Gamma-\mathrm{U}, \mathrm{U}-\mathrm{Z} \\
\mathrm{X}-\mathrm{Z}, \mathrm{S}-\mathrm{Y}, \mathrm{X}-\mathrm{Y}, \mathrm{R}-\mathrm{T}\end{array}$ & $\| \mathrm{Sm}_{3} \mathrm{Co}$ \\
\hline & $\begin{array}{l}\Gamma-\mathrm{Z}, \Gamma-\mathrm{X}, \Gamma-\mathrm{U}, \mathrm{U}-\mathrm{X}, \mathrm{X}-\mathrm{Z} \\
\mathrm{\Gamma}-\mathrm{S}, \mathrm{S}-\mathrm{Y}, \mathrm{X}-\mathrm{Y}, \mathrm{R}-\mathrm{T}\end{array}$ & $\| \mathrm{Sr}_{7} \mathrm{Pt}_{3}$ \\
\hline & $\begin{array}{c}\Gamma-\mathrm{Y}, \Gamma-\mathrm{X}, \Gamma-\mathrm{U}, \mathrm{U}-\mathrm{Z}, \mathrm{X}-\mathrm{Z} \\
\Gamma-\mathrm{S}, \mathrm{R}-\mathrm{T}, \mathrm{R}-\mathrm{U}\end{array}$ & $\| \mathrm{Si}_{3} \mathrm{Ru}_{4}$ \\
\hline & $\begin{array}{l}\Gamma-\mathrm{Y}, \Gamma-\mathrm{Z}, \Gamma-\mathrm{X}, \Gamma-\mathrm{T}, \Gamma-\mathrm{S} \\
\quad \mathrm{S}-\mathrm{Y}, \mathrm{X}-\mathrm{Y}, \mathrm{R}-\mathrm{U}\end{array}$ & || USi \\
\hline
\end{tabular}


Table III - continued

\begin{tabular}{|c|c|c|}
\hline $\begin{array}{l}\text { SOC or } \\
\text { NSOC }\end{array}$ & $\begin{array}{l}\text { HSL with Band } \\
\text { Crossing }\end{array}$ & High Symmetry Line Semimetals \\
\hline & $\begin{array}{c}\Gamma-\mathrm{Y}, \Gamma-\mathrm{Z}, \Gamma-\mathrm{X}, \Gamma-\mathrm{T}, \Gamma-\mathrm{U} \\
\mathrm{U}-\mathrm{X}, \mathrm{U}-\mathrm{Z}, \Gamma-\mathrm{S}\end{array}$ & || NiTePd, MnP \\
\hline & $\begin{array}{l}\Gamma-Y, \Gamma-Z, \Gamma-\mathrm{X}, \Gamma-\mathrm{T}, \Gamma-\mathrm{U} \\
\text { U-X, X-Z, } \Gamma-\mathrm{S}\end{array}$ & $\| \mathrm{CsAl}, \mathrm{Hf}_{5} \mathrm{Ge}_{4}, \mathrm{Ce}\left(\mathrm{In}_{2} \mathrm{Pd}\right)_{2}, \mathrm{Nb}_{5} \mathrm{P}_{3}, \mathrm{KTiF}_{4}$ \\
\hline & $\begin{array}{l}\Gamma-Y, \Gamma-Z, \Gamma-X, \Gamma-T, \Gamma-U \\
\text { U-X, X-Z, R-U }\end{array}$ & \| CoMoP \\
\hline & $\begin{array}{c}\Gamma-\mathrm{Y}, \Gamma-\mathrm{Z}, \Gamma-\mathrm{X}, \Gamma-\mathrm{T}, \Gamma-\mathrm{U} \\
\mathrm{U}-\mathrm{Z}, \mathrm{X}-\mathrm{Z}, \Gamma-\mathrm{S}\end{array}$ & 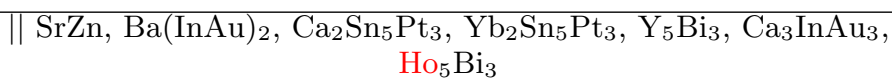 \\
\hline & $\begin{array}{c}\Gamma-\mathrm{Y}, \Gamma-\mathrm{Z}, \Gamma-\mathrm{X}, \Gamma-\mathrm{T}, \mathrm{U}-\mathrm{X} \\
\mathrm{U}-\mathrm{Z}, \mathrm{S}-\mathrm{Y}, \mathrm{X}-\mathrm{Y}\end{array}$ & ( \\
\hline & $\begin{array}{c}\Gamma-\mathrm{Y}, \Gamma-\mathrm{Z}, \Gamma-\mathrm{X}, \Gamma-\mathrm{U}, \mathrm{U}-\mathrm{X} \\
\mathrm{U}-\mathrm{Z}, \mathrm{S}-\mathrm{Y}, \mathrm{X}-\mathrm{Y}\end{array}$ & $\| \mathrm{Sm}_{3} \mathrm{Ir}$ \\
\hline & $\begin{array}{c}\text { T-Y, }, \text {-Z, }, \Gamma-\mathrm{X}, \Gamma-\mathrm{U}, \mathrm{U}-\mathrm{Z} \\
\mathrm{X}-\mathrm{Z}, \Gamma-\mathrm{S}, \mathrm{R}-\mathrm{U}\end{array}$ & $\| \mathrm{CaYb}_{2} \mathrm{O}_{4}$ \\
\hline & $\begin{array}{c}\Gamma-\mathrm{Y}, \Gamma-\mathrm{X}, \Gamma-\mathrm{U}, \mathrm{U}-\mathrm{Z}, \mathrm{X}-\mathrm{Z}, \\
\Gamma-\mathrm{S}, \mathrm{R}-\mathrm{T}\end{array}$ & || CeAlRh \\
\hline & $\begin{array}{c}\Gamma-\mathrm{Y}, \Gamma-\mathrm{Z}, \Gamma-\mathrm{T}, \Gamma-\mathrm{U}, \mathrm{U}-\mathrm{X}, \\
\text { X-Z, R-U }\end{array}$ & $\| \operatorname{PrAgAs}{ }_{2}$ \\
\hline & $\begin{array}{c}\Gamma-\mathrm{Y}, \Gamma-\mathrm{Z}, \Gamma-\mathrm{X}, \Gamma-\mathrm{T}, \Gamma-\mathrm{S} \\
\text { S-Y }, \mathrm{X}-\mathrm{Y}\end{array}$ & $\| \mathrm{SiRu}_{2}$ \\
\hline & $\begin{array}{c}\Gamma-\mathrm{Y}, \Gamma-\mathrm{Z}, \Gamma-\mathrm{X}, \Gamma-\mathrm{T}, \Gamma-\mathrm{U} \\
\Gamma-\mathrm{S}, \mathrm{R}-\mathrm{U}\end{array}$ & $\| \mathrm{Ba}_{2} \mathrm{Tl}_{7} \mathrm{Au}$ \\
\hline & $\begin{array}{c}\Gamma-\mathrm{Y}, \Gamma-\mathrm{Z}, \Gamma-\mathrm{X}, \Gamma-\mathrm{T}, \Gamma-\mathrm{U} \\
\mathrm{U}-\mathrm{X}, \mathrm{U}-\mathrm{Z}\end{array}$ & $\mathrm{Sm}_{2}(\mathrm{CuAs})_{3} \|$ \\
\hline & $\begin{array}{c}\Gamma-\mathrm{Y}, \Gamma-\mathrm{Z}, \Gamma-\mathrm{X}, \Gamma-\mathrm{T}, \Gamma-\mathrm{U} \\
\mathrm{U}-\mathrm{X}, \mathrm{X}-\mathrm{Z}\end{array}$ & $\| \mathrm{Ta}_{2} \mathrm{Te}_{5} \mathrm{Pd}_{3}, \mathrm{SiNi}_{2}, \mathrm{YbSiPt}_{2}, \mathrm{Ni}_{2} \mathrm{Ge}$ \\
\hline & $\begin{array}{c}\Gamma-\mathrm{Y}, \Gamma-\mathrm{Z}, \Gamma-\mathrm{X}, \Gamma-\mathrm{T}, \Gamma-\mathrm{U} \\
\mathrm{U}-\mathrm{Z}, \mathrm{X}-\mathrm{Z}\end{array}$ & $\| \mathrm{ZrAs}_{2}, \mathrm{TiP}_{2}, \mathrm{HfGeMo}, \mathrm{AsRh}, \mathrm{MnSiIr}, \mathrm{UCrC}_{2}$ \\
\hline & $\begin{array}{c}\Gamma-\mathrm{Y}, \Gamma-\mathrm{Z}, \Gamma-\mathrm{X}, \Gamma-\mathrm{T}, \mathrm{U}-\mathrm{X} \\
\mathrm{X}-\mathrm{Z}, \mathrm{R}-\mathrm{T}\end{array}$ & || DyZnRh, TbZnRh, SmZnRh \\
\hline & $\begin{array}{c}\Gamma-\mathrm{Y}, \Gamma-\mathrm{Z}, \Gamma-\mathrm{X}, \Gamma-\mathrm{T}, \mathrm{U}-\mathrm{Z} \\
\mathrm{X}-\mathrm{Z}, \Gamma-\mathrm{S}\end{array}$ & $\| \mathrm{Sr}(\mathrm{InAu})_{2}, \mathrm{LaNi}_{5} \mathrm{As}_{3}$ \\
\hline & $\begin{array}{c}\Gamma-\mathrm{Y}, \Gamma-\mathrm{Z}, \Gamma-\mathrm{X}, \Gamma-\mathrm{U}, \Gamma-\mathrm{S} \\
\mathrm{R}-\mathrm{T}, \mathrm{R}-\mathrm{U}\end{array}$ & $\| \mathrm{Hf}_{6} \mathrm{Cr}_{5} \mathrm{Si}_{7}$ \\
\hline & $\begin{array}{c}\Gamma-\mathrm{Y}, \Gamma-\mathrm{Z}, \Gamma-\mathrm{X}, \Gamma-\mathrm{U}, \Gamma-\mathrm{S} \\
\text { S-Y, X-Y }\end{array}$ & $\| \mathrm{Cr}_{7} \mathrm{C}_{3}$ \\
\hline & $\begin{array}{c}\Gamma-Y, \Gamma-Z, \Gamma-X, \Gamma-U, S-Y, \\
\text { X-Y, R-U }\end{array}$ & $\| \mathrm{CeGe}_{3} \mathrm{Pd}_{5}$ \\
\hline & $\begin{array}{c}\Gamma-\mathrm{Y}, \Gamma-\mathrm{Z}, \Gamma-\mathrm{X}, \Gamma-\mathrm{U}, \mathrm{U}-\mathrm{X}, \\
\mathrm{U}-\mathrm{Z}, \Gamma-\mathrm{S} \\
\end{array}$ & $\| \mathrm{TePb}(\mathrm{ICSD}: 190438), \mathrm{Co}_{3} \mathrm{~B}$ \\
\hline & $\begin{array}{c}-\mathrm{Y}, \Gamma-\mathrm{Z}, \Gamma-\mathrm{X}, \Gamma-\mathrm{U}, \mathrm{U}-\mathrm{X}, \\
\text { X-Z, } \Gamma-\mathrm{S}\end{array}$ & $\| \mathrm{In}_{3} \mathrm{Ir}, \mathrm{SiRh}_{2}, \mathrm{GeRh}_{2}, \mathrm{SnRh}_{2}, \mathrm{Sc}_{3} \mathrm{Co}$ \\
\hline & $\begin{array}{c}\Gamma-\mathrm{Y}, \Gamma-\mathrm{Z}, \Gamma-\mathrm{X}, \Gamma-\mathrm{U}, \mathrm{U}-\mathrm{Z} \\
\mathrm{X}-\mathrm{Z}, \Gamma-\mathrm{S}\end{array}$ & || HfSiMo, Np, USeS \\
\hline & $\begin{array}{c}\Gamma-\mathrm{Y}, \Gamma-\mathrm{Z}, \Gamma-\mathrm{X}, \mathrm{U}-\mathrm{Z}, \mathrm{X}-\mathrm{Z} \\
\Gamma-\mathrm{S}, \mathrm{R}-\mathrm{U}\end{array}$ & \| $\mathrm{Ta}_{8} \mathrm{NiSe}_{8}$ \\
\hline & $\begin{array}{c}\Gamma-\mathrm{Y}, \Gamma-\mathrm{U}, \mathrm{U}-\mathrm{X}, \mathrm{U}-\mathrm{Z}, \mathrm{S}-\mathrm{Y}, \\
\mathrm{X}-\mathrm{Y}\end{array}$ & $\| \mathrm{Yb}_{3} \mathrm{~S}_{4}$ \\
\hline & $\begin{array}{c}\Gamma-\mathrm{Y}, \Gamma-\mathrm{X}, \Gamma-\mathrm{U}, \mathrm{U}-\mathrm{X}, \mathrm{U}-\mathrm{Z}, \\
\Gamma-\mathrm{S}\end{array}$ & $\| \mathrm{TeS}_{7} \mathrm{Cl}_{2}$ \\
\hline & $\begin{array}{c}\Gamma-\mathrm{Y}, \Gamma-\mathrm{X}, \Gamma-\mathrm{U}, \mathrm{U}-\mathrm{Z}, \mathrm{X}-\mathrm{Z}, \\
\Gamma-\mathrm{S}\end{array}$ & $\| \mathrm{Ca}_{3} \mathrm{Si}_{2} \mathrm{Ni}_{3}$ \\
\hline & $\begin{array}{c}\Gamma-\mathrm{Y}, \Gamma-\mathrm{Z}, \Gamma-\mathrm{T}, \Gamma-\mathrm{U}, \mathrm{U}-\mathrm{X}, \\
\mathrm{U}-\mathrm{Z}\end{array}$ & $\| \mathrm{ZrP}_{2}, \mathrm{Ni}_{3} \mathrm{Sn}_{2}, \mathrm{ThNi}$ \\
\hline & $\begin{array}{c}\Gamma-\mathrm{Y}, \Gamma-\mathrm{Z}, \Gamma-\mathrm{T}, \Gamma-\mathrm{U}, \mathrm{U}-\mathrm{X}, \\
\mathrm{X}-\mathrm{Z}\end{array}$ & $\| \mathrm{SrZn}_{5}, \mathrm{Mo}_{4} \mathrm{P}_{3}$ \\
\hline
\end{tabular}


Table III - continued

\begin{tabular}{|c|c|c|}
\hline $\begin{array}{l}\text { SOC or } \\
\text { NSOC }\end{array}$ & $\begin{array}{l}\text { HSL with Band } \\
\text { Crossing }\end{array}$ & High Symmetry Line Semimetals \\
\hline & $\begin{array}{c}\Gamma-\mathrm{Y}, \Gamma-\mathrm{Z}, \Gamma-\mathrm{X}, \Gamma-\mathrm{T}, \Gamma-\mathrm{U}, \\
\Gamma-\mathrm{S}\end{array}$ & 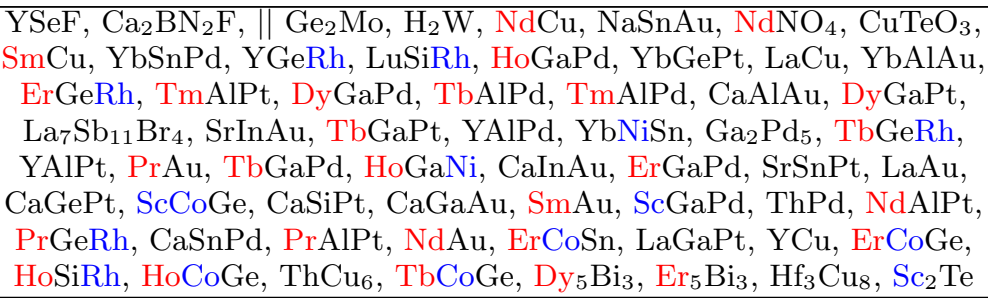 \\
\hline & $\begin{array}{c}\Gamma-\mathrm{Y}, \Gamma-\mathrm{Z}, \Gamma-\mathrm{X}, \Gamma-\mathrm{T}, \Gamma-\mathrm{U} \\
\mathrm{R}-\mathrm{U}\end{array}$ & e \\
\hline & $\begin{array}{c}\Gamma-\mathrm{Y}, \Gamma-\mathrm{Z}, \Gamma-\mathrm{X}, \Gamma-\mathrm{T}, \mathrm{U}-\mathrm{X} \\
\mathrm{X}-\mathrm{Z}\end{array}$ & |l HoZnRh, YbInRh, CaInRh \\
\hline & $\begin{array}{c}\Gamma-\mathrm{Y}, \Gamma-\mathrm{Z}, \Gamma-\mathrm{X}, \Gamma-\mathrm{U}, \Gamma-\mathrm{S} \\
\text { R-U }\end{array}$ & $\| \mathrm{Hf}_{5} \mathrm{CoAs}_{3}, \mathrm{SrYb}_{2} \mathrm{O}_{4}$ \\
\hline & $\begin{array}{c}\Gamma-\mathrm{Y}, \Gamma-\mathrm{Z}, \Gamma-\mathrm{X}, \Gamma-\mathrm{U}, \mathrm{S}-\mathrm{Y} \\
\mathrm{X}-\mathrm{Y}\end{array}$ & $\| \mathrm{Si}_{2} \mathrm{Pd}_{9}$ \\
\hline & $\begin{array}{c}\Gamma-\mathrm{Y}, \Gamma-\mathrm{Z}, \Gamma-\mathrm{X}, \Gamma-\mathrm{U}, \mathrm{U}-\mathrm{Z} \\
\mathrm{X}-\mathrm{Z}\end{array}$ & $\| \mathrm{VAs}$ \\
\hline & $\begin{array}{c}\Gamma-\mathrm{Y}, \Gamma-\mathrm{Z}, \Gamma-\mathrm{X}, \mathrm{U}-\mathrm{Z}, \mathrm{X}-\mathrm{Z}, \\
\Gamma-\mathrm{S}\end{array}$ & $\| \mathrm{SrMgSi}_{2}$ \\
\hline & $\begin{array}{c}\Gamma-\mathrm{Z}, \Gamma-\mathrm{X}, \Gamma-\mathrm{U}, \mathrm{U}-\mathrm{X}, \mathrm{X}-\mathrm{Z}, \\
\mathrm{R}-\mathrm{U}\end{array}$ & $\| \mathrm{SmAgAs}_{2}, \mathrm{NdAgAs}_{2}, \mathrm{SmAs}_{2} \mathrm{Au}$ \\
\hline & $\Gamma-Y, \Gamma-Z, \Gamma-T, S-Y, X-Y$ & || $\mathrm{Na}$ \\
\hline & $\Gamma-\mathrm{Y}, \Gamma-\mathrm{Z}, \Gamma-\mathrm{T}, \mathrm{U}-\mathrm{Z}, \mathrm{X}-\mathrm{Z}$ & $\|$ HfNbP, ZrNbP \\
\hline & $\Gamma-\mathrm{Y}, \Gamma-\mathrm{Z}, \Gamma-\mathrm{U}, \mathrm{U}-\mathrm{X}, \mathrm{X}-\mathrm{Z}$ & $\| \mathrm{ThGa}$ \\
\hline & $\Gamma-\mathrm{Y}, \Gamma-\mathrm{Z}, \Gamma-\mathrm{X}, \Gamma-\mathrm{T}, \Gamma-\mathrm{S}$ & $\mathrm{Nd}_{2} \mathrm{Te}_{3}, \| \mathrm{Tb}_{5} \mathrm{Bi}_{3}, \mathrm{Tm}_{5} \mathrm{Sb}_{3}, \mathrm{Er}_{5} \mathrm{Sb}_{3}$ \\
\hline & $\Gamma-Y, \Gamma-Z, \Gamma-X, \Gamma-\mathrm{T}, \Gamma-\mathrm{U}$ & $\begin{array}{l}\mathrm{LiYb}_{2} \mathrm{InGe}_{2}, \mathrm{LiCa}_{2} \mathrm{InGe}_{2}, \| \mathrm{Ca}_{3} \mathrm{Al}_{2} \mathrm{Ge}_{3}, \mathrm{SiPt}, \mathrm{GePt}, \mathrm{Hf}_{3}\left(\mathrm{NbGe}_{2}\right)_{2}, \\
\mathrm{SnPd}, \mathrm{GePd}, \mathrm{SiPd}, \mathrm{SiNi}, \mathrm{NiGe}, \mathrm{SbRh}, \mathrm{VCoSi}, \mathrm{CoP}, \mathrm{SnPd}_{2}, \mathrm{CoAs}\end{array}$ \\
\hline & $\Gamma-\mathrm{Y}, \Gamma-\mathrm{Z}, \Gamma-\mathrm{X}, \Gamma-\mathrm{U}, \Gamma-\mathrm{S}$ & $\begin{array}{c}\text { "I NbGeIr, TaSiIr, NbSiIr, ZrTe, TaFeP, TiSe, TaFeAs, } \mathrm{Ba}_{2} \mathrm{SnTe}_{5} \\
\text { HfVP, ZrVP, YAl } \mathrm{YA}_{3} \mathrm{Ni}, \mathrm{Bi}_{3} \mathrm{Rh}, \mathrm{CaIn}_{2} \mathrm{Ir}, \mathrm{Sc}_{2} \mathrm{Pt}, \mathrm{Sc}_{5} \mathrm{Bi}_{3}, \mathrm{Al}_{14} \mathrm{Co}_{5} \mathrm{Si}_{2} \\
\mathrm{Zr}_{3}\left(\mathrm{VGa}_{2}\right)_{2}\end{array}$ \\
\hline & $\Gamma-\mathrm{Z}, \Gamma-\mathrm{X}, \Gamma-\mathrm{U}, \mathrm{U}-\mathrm{X}, \mathrm{U}-\mathrm{Z}$ & HfSiPt, HfSiPd, || TiSiNi, $\mathrm{Ho}_{3} \mathrm{Co}, \mathrm{Er}_{3} \mathrm{Rh}, \mathrm{Dy}_{3} \mathrm{Co}$ \\
\hline & $\Gamma-\mathrm{Z}, \Gamma-\mathrm{X}, \Gamma-\mathrm{U}, \mathrm{U}-\mathrm{X}, \mathrm{X}-\mathrm{Z}$ & $\| \mathrm{PtF}_{6}, \mathrm{Nb}_{5} \mathrm{As}_{3}, \mathrm{~Tb}_{2} \mathrm{Ti}_{3} \mathrm{Ge}_{4}$ \\
\hline & $\Gamma-\mathrm{Z}, \Gamma-\mathrm{X}, \Gamma-\mathrm{U}, \mathrm{U}-\mathrm{Z}, \mathrm{X}-\mathrm{Z}$ & CoBW, PrNiSnH, LaNiSnH, $\mathrm{Sm}_{2} \mathrm{NiSn}_{4}, \mathrm{NdNiSnH}, \mathrm{Ta}_{5} \mathrm{P}_{3}$ \\
\hline & $\Gamma-\mathrm{Z}, \Gamma-\mathrm{X}, \mathrm{U}-\mathrm{X}, \mathrm{U}-\mathrm{Z}, \mathrm{R}-\mathrm{T}$ & $\| \mathrm{CaRuO}_{3}$ \\
\hline & $\Gamma-\mathrm{X}, \Gamma-\mathrm{S}, \mathrm{S}-\mathrm{Y}, \mathrm{X}-\mathrm{Y}$ & $\mathrm{Ca}_{7} \mathrm{Sn}_{6}, \mathrm{Ca}_{7} \mathrm{Ge}_{6}$ \\
\hline & $\Gamma-\mathrm{X}, \Gamma-\mathrm{U}, \mathrm{U}-\mathrm{Z}, \mathrm{X}-\mathrm{Z}$ & $\mathrm{NbCoB}_{2}, \mathrm{UFeSi}$ \\
\hline & $\Gamma-\mathrm{Y}, \Gamma-\mathrm{X}, \Gamma-\mathrm{S}, \mathrm{R}-\mathrm{T}$ & $\| \mathrm{KGa}$ \\
\hline & $\Gamma-\mathrm{Y}, \Gamma-\mathrm{Z}, \Gamma-\mathrm{T}, \mathrm{R}-\mathrm{T}$ & $\|$ PrMgRh \\
\hline & $\Gamma-\mathrm{Y}, \Gamma-\mathrm{Z}, \Gamma-\mathrm{T}, \mathrm{R}-\mathrm{U}$ & $\mathrm{SrZnSb}_{2}, \| \mathrm{Ho}_{5} \mathrm{CoSb}_{2}, \mathrm{~Tb}_{5} \mathrm{CoSb}_{2}, \mathrm{Dy}_{5} \mathrm{CoSb}_{2}, \mathrm{Er}_{5} \mathrm{CoSb}_{2}, \mathrm{Hf}_{5} \mathrm{FeTe}_{3}$ \\
\hline & $\Gamma-\mathrm{Y}, \Gamma-\mathrm{Z}, \Gamma-\mathrm{X}, \Gamma-\mathrm{T}$ & $\| \mathrm{Hf}_{3} \mathrm{P}_{2}, \mathrm{Zr}_{3} \mathrm{As}_{2}$ \\
\hline & $\Gamma-\mathrm{Z}, \Gamma-\mathrm{U}, \mathrm{U}-\mathrm{X}, \mathrm{X}-\mathrm{Z}$ & LuAsSe, $\mathrm{BaCu}_{2} \mathrm{Ge}_{2} \mathrm{O}_{7}$, YAsSe, $\| \mathrm{Ge}_{3} \mathrm{Pt}_{2}$ \\
\hline & $\Gamma-\mathrm{Z}, \Gamma-\mathrm{X}, \Gamma-\mathrm{U}, \mathrm{R}-\mathrm{U}$ & \begin{tabular}{l||l}
$\mathrm{ErCuP}_{2}$ \\
\end{tabular} \\
\hline & $\Gamma-\mathrm{U}, \mathrm{U}-\mathrm{X}, \mathrm{U}-\mathrm{Z}$ & $\mathrm{Cr}_{3} \mathrm{C}_{2}, \mathrm{Yb}_{5} \mathrm{Ge}_{4}, \mathrm{Sr}\left(\mathrm{Ni}_{5} \mathrm{P}_{3}\right)_{2}$ \\
\hline & $\Gamma-\mathrm{X}, \mathrm{U}-\mathrm{X}, \mathrm{X}-\mathrm{Z}$ & $\| \mathrm{MgPd}_{2}$ \\
\hline & $\Gamma-\mathrm{Y}, \Gamma-\mathrm{X}, \Gamma-\mathrm{S}$ & $\begin{array}{c}\| \mathrm{Lu}_{5} \mathrm{Sb}_{3}, \mathrm{GaAu}, \mathrm{ScSiIr}, \mathrm{ScSi}_{2} \mathrm{Rh}, \mathrm{LuSiIr}, \mathrm{Nb}_{2} \mathrm{C}, \mathrm{ErSiIr}, \mathrm{HoSiIr}, \\
\mathrm{H}_{4} \mathrm{~N}_{2} \mathrm{O}_{3}(\mathrm{ICSD}: 24012), \mathrm{DySiIr}, \mathrm{TiSiRu}, \mathrm{Er}_{2} \mathrm{Pt}, \mathrm{Sc}_{5} \mathrm{As}_{3}, \mathrm{BaFe}_{2} \mathrm{Se}_{3}, \\
\text { CeAlRu, } \mathrm{Zr}_{2} \mathrm{Te}\end{array}$ \\
\hline & $\Gamma-Y, \Gamma-Z, \Gamma-\mathrm{T}$ & $\begin{array}{c}\| \mathrm{HfAs}_{2}, \mathrm{AlHO}_{2}(\mathrm{ICSD}: 17014), \mathrm{KNOF}_{2}, \mathrm{SrSi}, \mathrm{SiPt}_{3}, \mathrm{YbZnPt}, \mathrm{K}, \\
\text { ErSiRh, YbMgPd, YbZnPd, } \mathrm{Ge}_{2} \mathrm{Pt}_{3}, \mathrm{ZrMnP}, \mathrm{SrRuO}_{3}, \mathrm{Ti}_{2} \mathrm{Se}, \\
\mathrm{HoMnSi}_{\mathrm{No} \mathrm{N}_{14} \mathrm{~S}_{5}}\end{array}$ \\
\hline & $\Gamma-\mathrm{Y}, \mathrm{S}-\mathrm{Y}, \mathrm{X}-\mathrm{Y}$ & $\|$ LaMgRh, NdZnRh, PrZnRh, Yb ${ }_{3} \mathrm{Se}_{4}$ \\
\hline & $\Gamma-\mathrm{Z}, \Gamma-\mathrm{X}, \Gamma-\mathrm{U}$ & 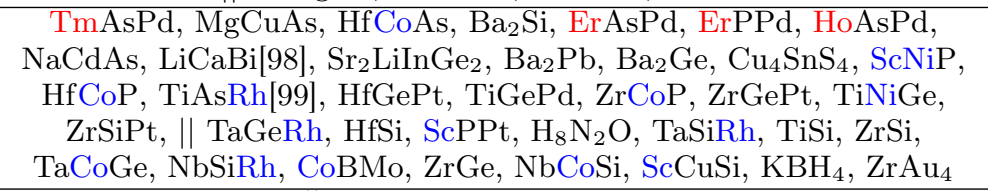 \\
\hline & $\Gamma-\mathrm{Z}, \mathrm{U}-\mathrm{Z}, \mathrm{X}-\mathrm{Z}$ & 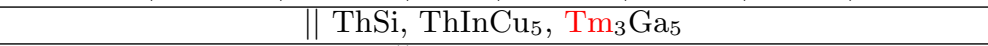 \\
\hline & $\Gamma-\mathrm{Y}, \Gamma-\mathrm{Z}$ & $\| \mathrm{CsK}_{5} \mathrm{Ru}_{2} \mathrm{O}_{9}$ \\
\hline
\end{tabular}


Table III - continued

\begin{tabular}{|c|c|c|}
\hline $\begin{array}{l}\text { SOC or } \\
\text { NSOC }\end{array}$ & $\begin{array}{l}\text { HSL with Band } \\
\text { Crossing }\end{array}$ & High Symmetry Line Semimetals \\
\hline & $\Gamma-Y, R-U$ & $2 \mathrm{LiB} \|$ \\
\hline & $\Gamma-\mathrm{Z}, \mathrm{R}-\mathrm{U}$ & $\mathrm{BaCu}_{2} \mathrm{Si}_{2} \mathrm{O}_{7} \|$ \\
\hline & $\mathrm{R}-\mathrm{T}$ & $\| \mathrm{Cu}_{2} \mathrm{BiPO}_{6}, \mathrm{KPd}_{2} \mathrm{~F}_{5}, \mathrm{Fe}_{7} \mathrm{C}_{3}$ \\
\hline & $\mathrm{R}-\mathrm{U}$ & SmAsS, ErAsS, $\| \mathrm{La}_{3} \mathrm{Si}_{2} \mathrm{Rh}_{3}, \mathrm{Th}(\mathrm{NiP})_{2}, \mathrm{HoB}_{7} \mathrm{Mo}_{3}$ \\
\hline $\mathrm{SOC}$ & R-S, S-U, S-X & 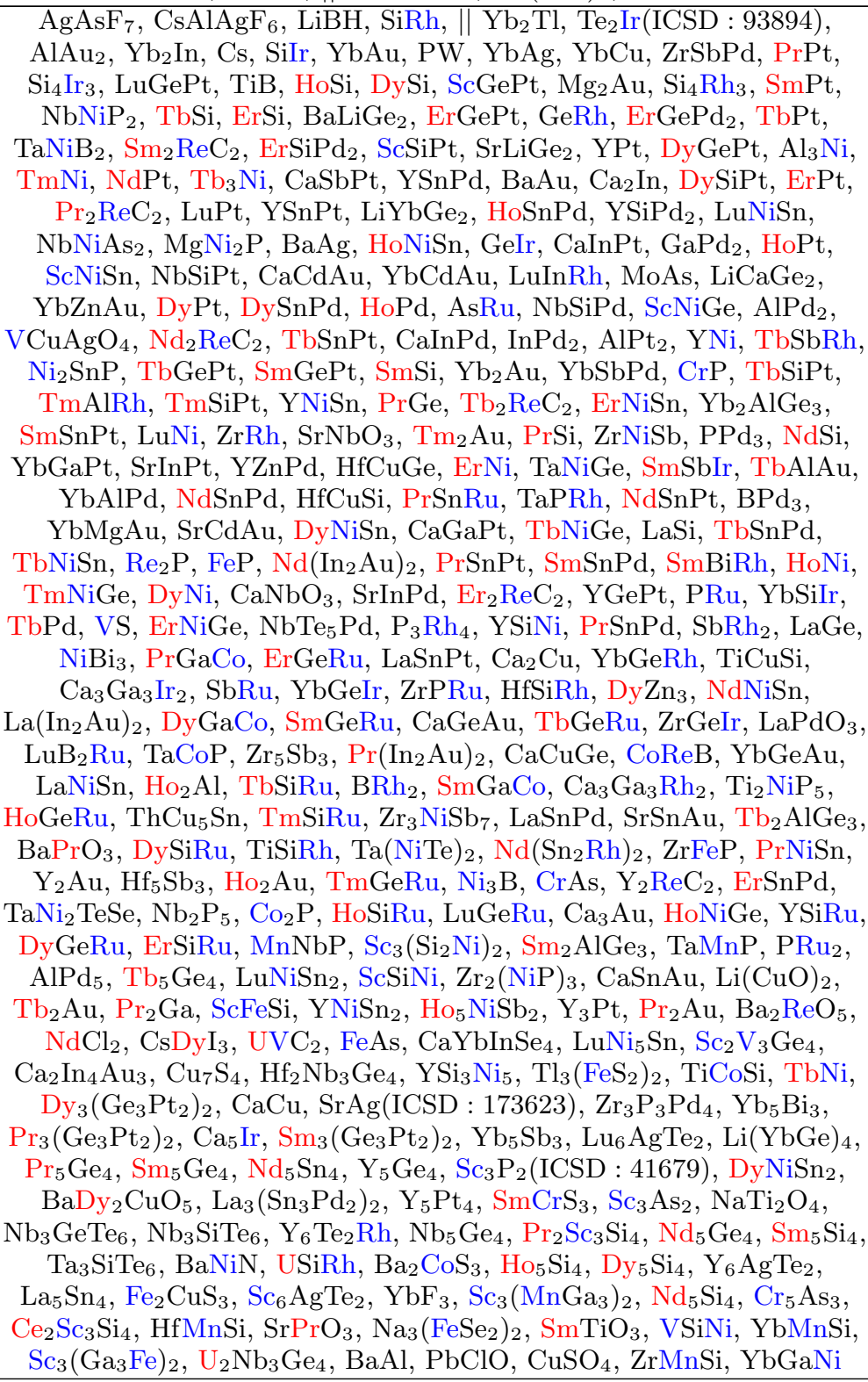 \\
\hline \multicolumn{3}{|r|}{ Space Group 63: $\mathrm{Cmcm}$} \\
\hline & $\begin{array}{l}\Gamma-\mathrm{Z}, \Gamma-\mathrm{Y}, \Gamma-\mathrm{T}, \mathrm{T}-\mathrm{Y}, \mathrm{T}-\mathrm{Z} \\
\quad \mathrm{Y}-\mathrm{Z}, \mathrm{S}-\Gamma, \mathrm{S}-\mathrm{Y}, \mathrm{R}-\mathrm{S}\end{array}$ & $\begin{array}{c}\| \mathrm{PrCuGe}_{2}, \mathrm{ThSn}_{2}, \mathrm{ZrGe}_{2}, \mathrm{LiNdSn}_{2}, \mathrm{LiTbSn}_{2}, \mathrm{LaCuGe}_{2}, \mathrm{LiHoSn}_{2}, \\
\mathrm{LiErSn}_{2}, \mathrm{LiTmSn}_{2}, \mathrm{SmIn}_{2} \mathrm{Rh}, \mathrm{YAl}_{2} \mathrm{Co}, \mathrm{LiLuSn}_{2}\end{array}$ \\
\hline & $\begin{array}{c}\Gamma-\mathrm{Y}, \Gamma-\mathrm{Z}, \Gamma-\mathrm{T}, \mathrm{T}-\mathrm{Y}, \mathrm{T}-\mathrm{Z} \\
\mathrm{Y}-\mathrm{Z}, \mathrm{S}-\Gamma, \mathrm{R}-\mathrm{S}\end{array}$ & e \\
\hline & $\begin{array}{c}\Gamma-Y, \Gamma-Z, \Gamma-T, T-Y, T-Z, \\
Y-Z, S-\Gamma, S-Y\end{array}$ & $\| \mathrm{Na}_{3} \mathrm{~B}_{5} \mathrm{Pt}_{9}, \mathrm{CeNiGe}_{2}, \mathrm{Hf}_{3} \mathrm{Si}_{3} \mathrm{Ni}_{2}, \mathrm{CeSi}_{2} \mathrm{Ni}$ \\
\hline & $\begin{array}{c}\Gamma-Y, \Gamma-Z, \Gamma-T, T-Y, T-Z, \\
\text { Y-Z, S-Y, R-S }\end{array}$ & $\| \mathrm{BaIn}_{2} \mathrm{Pt}$ \\
\hline & $\begin{array}{l}\Gamma-\mathrm{Y}, \Gamma-\mathrm{Z}, \mathrm{T}-\mathrm{Y}, \mathrm{T}-\mathrm{Z}, \mathrm{Y}-\mathrm{Z} \\
\Gamma-\mathrm{T}, \mathrm{S}-\Gamma, \mathrm{S}-\mathrm{Y}\end{array}$ & $\| \mathrm{Nb}_{3} \mathrm{Co}_{4} \mathrm{~B}_{7}$ \\
\hline
\end{tabular}


Table III - continued

\begin{tabular}{|c|c|c|}
\hline $\begin{array}{l}\text { SOC or } \\
\text { NSOC }\end{array}$ & $\begin{array}{l}\text { HSL with Band } \\
\text { Crossing }\end{array}$ & High Symmetry Line Semimetals \\
\hline & $\begin{array}{l}\Gamma-Z, \Gamma-Y, \Gamma-T, T-Y, T-Z, \\
\text { Y-Z, S- } \Gamma, S-Y\end{array}$ & $\| \mathrm{MgZn}_{2}, \mathrm{LiDySn}_{2}$ \\
\hline & $\begin{array}{c}\Gamma-\mathrm{Z}, \Gamma-\mathrm{Y}, \Gamma-\mathrm{T}, \mathrm{T}-\mathrm{Y}, \mathrm{Y}-\mathrm{Z} \\
\mathrm{S}-\Gamma, \mathrm{S}-\mathrm{Y}, \mathrm{R}-\mathrm{S}\end{array}$ & $\begin{array}{c}\| \mathrm{HfSi}_{2}, \mathrm{LiPrSn}_{2}, \mathrm{LiSmSn}_{2}, \mathrm{LiLaSn}_{2}, \mathrm{NdCuGe}_{2}, \mathrm{LaIn}_{2} \mathrm{Rh}, \mathrm{LaIn}_{2} \mathrm{Ir}, \\
\mathrm{NdIn}_{2} \mathrm{Ir}, \mathrm{SmCoSi}_{2}\end{array}$ \\
\hline & $\begin{array}{l}\Gamma-\mathrm{Z}, \Gamma-\mathrm{Y}, \mathrm{T}-\mathrm{Z}, \mathrm{Y}-\mathrm{Z}, \Gamma-\mathrm{T}, \\
\mathrm{T}-\mathrm{Y}, \mathrm{S}-\Gamma, \mathrm{S}-\mathrm{Y}\end{array}$ & ( \\
\hline & $\begin{array}{l}\Gamma-\mathrm{Y}, \Gamma-\mathrm{T}, \mathrm{T}-\mathrm{Y}, \mathrm{T}-\mathrm{Z}, \mathrm{Y}-\mathrm{Z} \\
\text { S- } \Gamma, \mathrm{S}-\mathrm{Y}\end{array}$ & $\| \mathrm{Co}(\mathrm{BW})_{3}$ \\
\hline & $\begin{array}{c}\Gamma-Y, \Gamma-Z, \Gamma-T, T-Y, T-Z \\
\text { Y-Z, S- } \Gamma\end{array}$ & $\| \mathrm{Pd}_{3} \mathrm{~S}, \mathrm{NaIn}_{2} \mathrm{Au}, \mathrm{YbAl}_{2} \mathrm{Pd}, \mathrm{SrIn}_{2} \mathrm{Pd}, \mathrm{SrIn}_{2} \mathrm{Pt}, \mathrm{CaGa}_{2} \mathrm{Ni}, \mathrm{YbGa}_{2} \mathrm{Ni}$ \\
\hline & $\begin{array}{c}\Gamma-\mathrm{Y}, \Gamma-\mathrm{Z}, \Gamma-\mathrm{T}, \mathrm{T}-\mathrm{Y}, \mathrm{T}-\mathrm{Z} \\
\mathrm{Y}-\mathrm{Z}, \mathrm{S}-\mathrm{Y}\end{array}$ & $\| \mathrm{Si}_{2} \mathrm{Ni}_{3}, \mathrm{SrIn}_{6} \mathrm{Cu}_{7}$ \\
\hline & $\begin{array}{l}\Gamma-\mathrm{Y}, \Gamma-\mathrm{Z}, \Gamma-\mathrm{T}, \mathrm{T}-\mathrm{Y}, \mathrm{Y}-\mathrm{Z} \\
\mathrm{S}-\Gamma, \mathrm{R}-\mathrm{S}\end{array}$ & $\| \mathrm{SiAg}_{2}, \mathrm{Er}_{2}\left(\mathrm{Ga}_{3} \mathrm{Co}\right)_{3}, \mathrm{Ho}_{2}\left(\mathrm{Ga}_{3} \mathrm{Co}\right)_{3}, \mathrm{Sm}_{2}\left(\mathrm{Ga}_{3} \mathrm{Co}\right)_{3}$ \\
\hline & $\begin{array}{c}\Gamma-Y, \Gamma-Z, \Gamma-T, T-Y, Y-Z, \\
\text { S- } \Gamma, S-Y\end{array}$ & $\begin{array}{c}\| \mathrm{Li}_{2} \mathrm{Nd}_{2} \mathrm{Si}_{3}, \mathrm{YbSb}_{2}, \mathrm{La}_{2}\left(\mathrm{ZnGe}_{2}\right)_{3}, \mathrm{CaIn}_{4} \mathrm{Ni}_{1} \mathrm{Cr}_{3} \mathrm{C}_{2}, \mathrm{Ba}_{3} \mathrm{~Pb}_{5}, \mathrm{Cr} \\
\mathrm{Y}_{2}\left(\mathrm{Ga}_{3} \mathrm{Co}\right)_{3}, \mathrm{Mn}_{3} \mathrm{As}\end{array}$ \\
\hline & 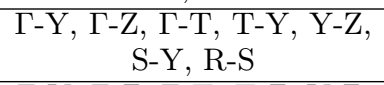 & $\| \mathrm{Zr}_{3} \mathrm{AlN}$ \\
\hline & $\begin{array}{l}\Gamma-\mathrm{Y}, \Gamma-\mathrm{Z}, \Gamma-\mathrm{T}, \mathrm{T}-\mathrm{Z}, \mathrm{Y}-\mathrm{Z} \\
\mathrm{S}-\Gamma, \mathrm{S}-\mathrm{Y}\end{array}$ & $\| \mathrm{YbIn}_{2} \mathrm{Pd}, \mathrm{CaIn}_{2} \mathrm{Pd}$ \\
\hline & $\begin{array}{l}\Gamma-\mathrm{Y}, \Gamma-\mathrm{Z}, \mathrm{T}-\mathrm{Y}, \mathrm{Y}-\mathrm{Z}, \Gamma-\mathrm{T} \\
\mathrm{S}-\Gamma, \mathrm{S}-\mathrm{Y}\end{array}$ & $\| \mathrm{SnH}_{4}$ \\
\hline & $\begin{array}{l}\Gamma-\mathrm{Z}, \Gamma-\mathrm{Y}, \Gamma-\mathrm{T}, \mathrm{T}-\mathrm{Y}, \mathrm{Y}-\mathrm{Z} \\
\text { S- } \Gamma, \mathrm{S}-\mathrm{Y}\end{array}$ & $\begin{array}{c}\| \mathrm{HfGe}_{2}, \mathrm{Ba}(\mathrm{AlSi})_{2}, \mathrm{Nd}_{2}\left(\mathrm{ZnGe}_{2}\right)_{3}, \mathrm{Pr}_{2}\left(\mathrm{ZnGe}_{2}\right)_{3}, \mathrm{Nd}_{2} \mathrm{Te}_{5}, \mathrm{Nb}_{3} \mathrm{~B}_{3} \mathrm{C}, \\
\mathrm{ZrSi}_{2}, \mathrm{BaZn}_{5}\end{array}$ \\
\hline & $\begin{array}{l}\Gamma-\mathrm{Z}, \Gamma-\mathrm{Y}, \mathrm{T}-\mathrm{Z}, \mathrm{Y}-\mathrm{Z}, \mathrm{T}-\mathrm{Y} \\
\text { S- } \Gamma, \mathrm{S}-\mathrm{Y}\end{array}$ & ॥ Ga(ICSD : 2795) \\
\hline & $\begin{array}{l}\Gamma-\mathrm{Y}, \Gamma-\mathrm{T}, \mathrm{T}-\mathrm{Y}, \mathrm{T}-\mathrm{Z}, \mathrm{Y}-\mathrm{Z} \\
\mathrm{S}-\mathrm{\Gamma}\end{array}$ & $\| \mathrm{CeSi}_{2} \mathrm{Pt}$ \\
\hline & $\begin{array}{c}\Gamma-\mathrm{Y}, \Gamma-\mathrm{T}, \mathrm{T}-\mathrm{Z}, \mathrm{Y}-\mathrm{Z}, \mathrm{T}-\mathrm{Y}, \\
\mathrm{S}-\Gamma\end{array}$ & $\| \mathrm{Al}_{6} \mathrm{Ru}, \mathrm{Al}_{6} \mathrm{Fe}$ \\
\hline & $\begin{array}{c}\Gamma-\mathrm{Y}, \Gamma-\mathrm{Z}, \Gamma-\mathrm{T}, \mathrm{S}-\Gamma, \mathrm{S}-\mathrm{Y} \\
\mathrm{R}-\mathrm{S}\end{array}$ & $\| \mathrm{Mn}_{5} \mathrm{SiC}$ \\
\hline & $\begin{array}{c}\Gamma-\mathrm{Y}, \Gamma-\mathrm{Z}, \Gamma-\mathrm{T}, \mathrm{T}-\mathrm{Y}, \mathrm{T}-\mathrm{Z} \\
\mathrm{Y}-\mathrm{Z} \\
\end{array}$ & $\| \mathrm{BaSb}_{2} \mathrm{Pd}, \mathrm{BaAs}_{2} \mathrm{Pd}$ \\
\hline & $\begin{array}{c}\Gamma-\mathrm{Y}, \Gamma-\mathrm{Z}, \Gamma-\mathrm{T}, \mathrm{T}-\mathrm{Y}, \mathrm{Y}-\mathrm{Z} \\
\mathrm{R}-\mathrm{S}\end{array}$ & $\| \mathrm{SmGa}_{4} \mathrm{Co}$ \\
\hline & $\begin{array}{c}\Gamma-\mathrm{Y}, \Gamma-\mathrm{Z}, \Gamma-\mathrm{T}, \mathrm{T}-\mathrm{Y}, \mathrm{Y}-\mathrm{Z} \\
\mathrm{S}-\Gamma\end{array}$ & $\begin{array}{c}\| \mathrm{BaAs}_{2} \mathrm{Pt}, \mathrm{SrTl}_{2} \mathrm{Pd}, \mathrm{NdMnSi}_{2}, \mathrm{Y}_{2}\left(\mathrm{Al}_{3} \mathrm{Co}\right)_{3}, \mathrm{Nd}_{2}\left(\mathrm{Al}_{3} \mathrm{Co}\right)_{3} \\
\mathrm{MgAu}_{3}(\mathrm{ICSD}: 58545)\end{array}$ \\
\hline & $\begin{array}{c}\Gamma-\mathrm{Y}, \Gamma-\mathrm{Z}, \Gamma-\mathrm{T}, \mathrm{T}-\mathrm{Y}, \mathrm{Y}-\mathrm{Z}, \\
\mathrm{S}-\mathrm{Y}\end{array}$ & $\| \mathrm{LaGa}, \mathrm{YbIn}_{2} \mathrm{Pt}, \mathrm{UPt}_{2}, \mathrm{MgAu}_{3}(\mathrm{ICSD}: 58546)$ \\
\hline & $\begin{array}{c}\Gamma-\mathrm{Y}, \Gamma-\mathrm{Z}, \Gamma-\mathrm{T}, \mathrm{T}-\mathrm{Z}, \mathrm{Y}-\mathrm{Z} \\
\mathrm{S}-\Gamma\end{array}$ & $\| \mathrm{BePd}_{3}$ \\
\hline & $\begin{array}{c}\Gamma-\mathrm{Y}, \Gamma-\mathrm{Z}, \Gamma-\mathrm{T}, \mathrm{T}-\mathrm{Z}, \mathrm{Y}-\mathrm{Z} \\
\mathrm{S}-\mathrm{Y}\end{array}$ & $\| \mathrm{YbIn}_{2} \mathrm{Ni}, \mathrm{CaIn}_{2} \mathrm{Ni}, \mathrm{Ti}_{2} \mathrm{Pd}_{3}$ \\
\hline & $\begin{array}{l}\text { T-Y, Г-Z, T-Y, T-Z, Y-Z, } \\
\text { S- } \Gamma\end{array}$ & $\| \operatorname{TiZn}_{16}$ \\
\hline & $\begin{array}{l}\Gamma-Y, \Gamma-Z, T-Y, T-Z, Y-Z, \\
\text { S-Y }\end{array}$ & $\| \mathrm{ErCd}_{2} \mathrm{Cu}$ \\
\hline & $\begin{array}{c}\Gamma-\mathrm{Y}, \Gamma-\mathrm{Z}, \mathrm{T}-\mathrm{Y}, \mathrm{Y}-\mathrm{Z}, \Gamma-\mathrm{T}, \\
\mathrm{S}-\Gamma\end{array}$ & $\| \mathrm{Na}_{2} \mathrm{HgPb}, \mathrm{LaAl}, \mathrm{PrAl}, \mathrm{NaTl}$ \\
\hline & $\begin{array}{c}\Gamma-Y, \Gamma-Z, T-Y, Y-Z, \Gamma-T, \\
\text { S-Y }\end{array}$ & $\mathrm{Ta}_{2} \mathrm{NiSe}_{5} \|$ \\
\hline & $\begin{array}{l}\Gamma-\mathrm{Z}, \Gamma-\mathrm{T}, \mathrm{T}-\mathrm{Y}, \mathrm{Y}-\mathrm{Z}, \mathrm{S}-\Gamma \\
\mathrm{S}-\mathrm{Y}\end{array}$ & $\| \mathrm{V}_{4} \mathrm{As}_{3}$ \\
\hline & $\begin{array}{c}\Gamma-Z, \Gamma-Y, \Gamma-T, T-Y, T-Z, \\
Y-Z\end{array}$ & II $\mathrm{ThGe}_{2}, \mathrm{CaIn}_{2} \mathrm{Pt}, \mathrm{YbAl}_{2} \mathrm{Ni}, \mathrm{Sr}_{3} \mathrm{In}_{4} \mathrm{~Pb}$ \\
\hline & $\begin{array}{c}\Gamma-\mathrm{Z}, \Gamma-\mathrm{Y}, \Gamma-\mathrm{T}, \mathrm{T}-\mathrm{Y}, \mathrm{Y}-\mathrm{Z} \\
\mathrm{R}-\mathrm{S}\end{array}$ & \| DyCoSi ${ }_{2}$ \\
\hline & $\begin{array}{c}\Gamma-Z, \Gamma-Y, T-Z, Y-Z, \Gamma-T, \\
\text { T-Y }\end{array}$ & $\| \mathrm{CaBi}_{2}$ \\
\hline
\end{tabular}


Table III - continued

\begin{tabular}{|c|c|c|}
\hline $\begin{array}{l}\text { SOC or } \\
\text { NSOC }\end{array}$ & $\begin{array}{l}\text { HSL with Band } \\
\text { Crossing }\end{array}$ & High Symmetry Line Semimetals \\
\hline & $\Gamma-\mathrm{Y}, \Gamma-\mathrm{T}, \mathrm{T}-\mathrm{Y}, \mathrm{T}-\mathrm{Z}, \mathrm{S}-\mathrm{Y}$ & \begin{tabular}{l||l} 
& $\mathrm{Th}\left(\mathrm{Al}_{5} \mathrm{Fe}\right)_{2}$ \\
\end{tabular} \\
\hline & $\Gamma-\mathrm{Y}, \Gamma-\mathrm{Z}, \Gamma-\mathrm{T}, \mathrm{S}-\Gamma, \mathrm{S}-\mathrm{Y}$ & $\operatorname{InSb}(\mathrm{ICSD}: 156995), \mathrm{YbGa}_{2} \mathrm{Pd}$ \\
\hline & $\Gamma-\mathrm{Y}, \Gamma-\mathrm{Z}, \Gamma-\mathrm{T}, \mathrm{S}-\mathrm{Y}, \mathrm{R}-\mathrm{S}$ & ||$l$ LuNiGe \\
\hline & $\Gamma-\mathrm{Y}, \Gamma-\mathrm{Z}, \Gamma-\mathrm{T}, \mathrm{T}-\mathrm{Y}, \mathrm{Y}-\mathrm{Z}$ & $\mathrm{YbIn}_{4} \mathrm{Pt}, \mathrm{SrIn}_{4} \mathrm{Pt}, \mathrm{YbIn}{ }_{4} \mathrm{Pd}, \mathrm{CaIn}_{4} \mathrm{Pd}, \mathrm{SrIn}_{4} \mathrm{Pd}$ \\
\hline & $\Gamma-Y, \Gamma-Z, T-Y, T-Z, Y-Z$ & $\mathrm{Ga}(\mathrm{ICSD}: 165979) \|$ \\
\hline & $\Gamma-\mathrm{Y}, \mathrm{T}-\mathrm{Y}, \mathrm{Y}-\mathrm{Z}, \mathrm{S}-\Gamma, \mathrm{R}-\mathrm{S}$ & $\| \mathrm{Ge}_{2} \mathrm{Pt}_{3}$ \\
\hline & $\Gamma-\mathrm{Y}, \mathrm{T}-\mathrm{Y}, \mathrm{Y}-\mathrm{Z}, \mathrm{S}-\Gamma, \mathrm{S}-\mathrm{Y}$ & $\mathrm{ZrNi}, \mathrm{ThPt}, \mathrm{ZrPd}, \mathrm{SrIn}_{4} \mathrm{Ni}, \mathrm{NbAgO}_{3}, \mathrm{Ti}_{3} \mathrm{O}_{5}$ \\
\hline & $\Gamma-Y, T-Y, Y-Z, S-Y, R-S$ & $\| \mathrm{Hf}_{3} \mathrm{AlN}, \mathrm{Y}_{5} \mathrm{Mg}_{16} \mathrm{Cu}_{5}$ \\
\hline & $\Gamma-\mathrm{Z}, \Gamma-\mathrm{T}, \mathrm{T}-\mathrm{Y}, \mathrm{T}-\mathrm{Z}, \mathrm{Y}-\mathrm{Z}$ & $\mathrm{SmPRu}_{2} \mathrm{C}, \mathrm{TbPRu}_{2} \mathrm{C}, \mathrm{ThFe}_{2} \mathrm{SiC}, \mathrm{Nd}_{3} \mathrm{IrO}_{7}, \mathrm{Pr}_{3} \mathrm{IrO}_{7}, \mathrm{Sm}_{3} \mathrm{IrO}_{7}$ \\
\hline & $\Gamma-\mathrm{Z}, \Gamma-\mathrm{T}, \mathrm{T}-\mathrm{Y}, \mathrm{Y}-\mathrm{Z}, \mathrm{R}-\mathrm{S}$ & $\| \mathrm{CaC}_{2}$ \\
\hline & $\Gamma-\mathrm{Z}, \Gamma-\mathrm{Y}, \Gamma-\mathrm{T}, \mathrm{S}-\Gamma, \mathrm{S}-\mathrm{Y}$ & $\| \mathrm{Y}_{5} \mathrm{Mg}_{13} \mathrm{Cu}_{5}$ \\
\hline & $\Gamma-\mathrm{Z}, \Gamma-\mathrm{Y}, \Gamma-\mathrm{T}, \mathrm{T}-\mathrm{Y}, \mathrm{Y}-\mathrm{Z}$ & 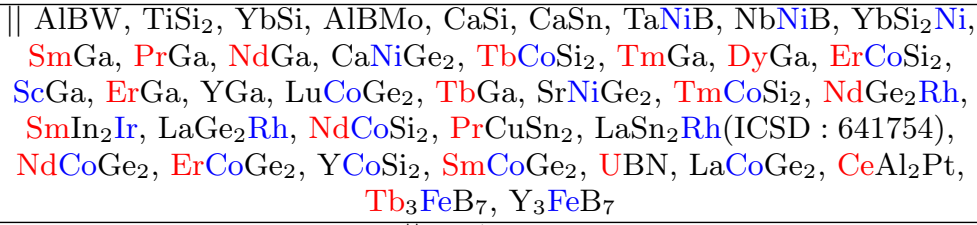 \\
\hline & $\Gamma-\mathrm{Y}, \Gamma-\mathrm{T}, \mathrm{T}-\mathrm{Z}, \mathrm{Y}-\mathrm{Z}$ & $\| \mathrm{Y}_{3} \mathrm{Al}_{19} \mathrm{Ni}_{5}$ \\
\hline & $\Gamma-\mathrm{Y}, \Gamma-\mathrm{Z}, \Gamma-\mathrm{T}, \mathrm{S}-\Gamma$ & $\mathrm{Ta}_{2} \mathrm{NiS}_{5}, \| \mathrm{Ba}(\mathrm{SiNi})_{2}, \mathrm{BaCd}_{2} \mathrm{Pt}, \mathrm{Ce}, \mathrm{La}_{5} \mathrm{Al}_{3} \mathrm{Ni}_{2}$ \\
\hline & $\Gamma-\mathrm{Y}, \Gamma-\mathrm{Z}, \Gamma-\mathrm{T}, \mathrm{S}-\mathrm{Y}$ & || $\begin{array}{l} \\
\end{array}$ \\
\hline & $\Gamma-\mathrm{Y}, \mathrm{T}-\mathrm{Y}, \mathrm{Y}-\mathrm{Z}, \mathrm{S}-\Gamma$ & $\begin{array}{c}\| \text { HoAlGe, TmAlGe, LuAlGe, ErAlGe, DyAlGe, YAlGe, ScAlGe, } \\
\text { TmAlSi, LuAlSi, YAlSi, } \mathrm{NaGa} \mathrm{YbGa}_{4} \mathrm{Ni}, \mathrm{LaSn}_{2} \mathrm{Rh}(\mathrm{ICSD}: \text { 410732), } \\
\mathrm{BaFe}_{2} \mathrm{~S}_{3}, \mathrm{EuIn}_{2} \mathrm{Au}\end{array}$ \\
\hline & $\Gamma-\mathrm{Y}, \mathrm{T}-\mathrm{Y}, \mathrm{Y}-\mathrm{Z}, \mathrm{S}-\mathrm{Y}$ & $\mathrm{CsLa}_{2} \mathrm{CuSe}_{4}, \| \mathrm{ReBiO}_{4}, \mathrm{~V}_{3} \mathrm{GeN}, \mathrm{Sr}_{3} \mathrm{Sn}_{5}, \mathrm{Ba}_{3} \mathrm{Sn}_{5}, \mathrm{CeFeSi}{ }_{2}$ \\
\hline & $\Gamma-\mathrm{Z}, \Gamma-\mathrm{T}, \mathrm{T}-\mathrm{Y}, \mathrm{Y}-\mathrm{Z}$ & $\begin{array}{c}\mathrm{Ba}_{5} \mathrm{Cd}_{2} \mathrm{Sb}_{5} \mathrm{~F}, \| \mathrm{BaGe}, \mathrm{Sr}_{5} \mathrm{Cd}_{2} \mathrm{Sb} \mathrm{Sb}_{5} \mathrm{~F}, \mathrm{BaPb}, \mathrm{BaSi}, \mathrm{SrGe}, \mathrm{BaSn}, \mathrm{SrSi} \\
\text { SrPb, SrSn, CaGe, } \mathrm{Tb}_{2} \mathrm{Si}_{3}\end{array}$ \\
\hline & $\Gamma-\mathrm{Y}, \Gamma-\mathrm{Z}, \Gamma-\mathrm{T}$ & $\| \mathrm{ZrCu}_{2}, \mathrm{TiCu}_{2}, \mathrm{Zr}_{3} \mathrm{NiO}$ \\
\hline & $\Gamma-\mathrm{Y}, \mathrm{S}-\Gamma, \mathrm{S}-\mathrm{Y}$ & $\begin{array}{c}\| \mathrm{Y}_{3} \mathrm{~B}_{7} \mathrm{~W}, \mathrm{YbSn}_{2} \mathrm{Pd}, \mathrm{CaSn}_{2} \mathrm{Pd}, \mathrm{SrSi}_{6}, \mathrm{Re}_{3} \mathrm{~B}, \mathrm{MgB}_{4} \mathrm{Os}_{3}, \mathrm{Ho}_{3} \mathrm{CrB}_{7}, \\
\mathrm{Tc}_{3} \mathrm{~B}, \mathrm{LaNi}_{5} \mathrm{P}_{3}, \mathrm{Cr}_{3} \mathrm{GeC}\end{array}$ \\
\hline & $\Gamma-\mathrm{Y}, \mathrm{T}-\mathrm{Y}, \mathrm{Y}-\mathrm{Z}$ & 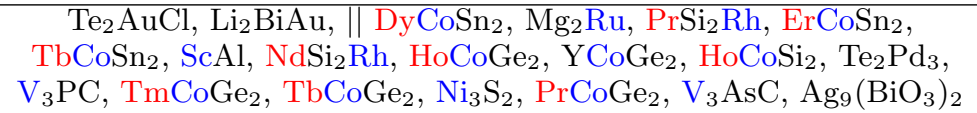 \\
\hline & $\Gamma-\mathrm{Z}, \Gamma-\mathrm{Y}, \Gamma-\mathrm{T}$ & $\| \mathrm{O}_{2}, \mathrm{LaAu}, \mathrm{SmAu}, \mathrm{PrAu}, \mathrm{NdAu}, \mathrm{SrCd}_{2} \mathrm{Pd}, \mathrm{SrMg}_{2} \mathrm{Pd}, \mathrm{CeNi}$ \\
\hline & S- $\Gamma, \mathrm{S}-\mathrm{Y}, \mathrm{R}-\mathrm{S}$ & $\| \mathrm{CaTi}_{2} \mathrm{O}_{4}$ \\
\hline & $\Gamma-\mathrm{Y}, \mathrm{S}-\Gamma$ & $\begin{array}{c}\text { C(ICSD : 252268) }[100], \| \mathrm{TaB}, \mathrm{NbB}, \mathrm{ZrSi}, \mathrm{VB}, \mathrm{HfPd}, \mathrm{TiNi}, \mathrm{BaIn}, \\
\text { HfNi, ZrIr, ZrRh, LiFeP, } \mathrm{Zr}_{3} \mathrm{Fe}\end{array}$ \\
\hline & $\Gamma-\mathrm{Y}, \mathrm{S}-\mathrm{Y}$ & $\begin{array}{c}\mathrm{ZrTe}_{5}[101], \mathrm{HfTe}_{5}[101], \mathrm{Na}_{15} \mathrm{SnGe}_{8} \mathrm{P}, \|_{\mathrm{P}_{2} \mathrm{PbAu}_{2}, \mathrm{SrSn}_{4}, \mathrm{Ti}_{3} \mathrm{Sn}} \\
\mathrm{Ca}_{3} \mathrm{Zn}, \mathrm{Ce}\left(\mathrm{Al}_{5} \mathrm{Ru}\right)_{2}, \mathrm{Mog}_{9} \mathrm{Se}_{11}, \mathrm{Nb}_{4} \mathrm{As}_{3}\end{array}$ \\
\hline & $\mathrm{S}-\Gamma, \mathrm{S}-\mathrm{Y}$ & $\begin{array}{c}\text { \|ा } \mathrm{ThTaN}_{3}, \mathrm{HgTe}, \mathrm{Ca}_{2} \mathrm{InN}, \mathrm{TmAu}, \mathrm{YAu}, \mathrm{InAs}, \mathrm{CaZn}, \mathrm{Li}_{4} \mathrm{H}_{5} \mathrm{Rh}, \mathrm{U}, \\
\text { InSb(ICSD : 156996), } \mathrm{Al}_{2} \mathrm{Fe}_{3} \mathrm{Si}_{4}, \mathrm{KHo}_{2} \mathrm{CuSe}_{4}\end{array}$ \\
\hline & $\mathrm{R}-\mathrm{S}$ & $\| \mathrm{K}_{3} \mathrm{Ga}_{13}$ \\
\hline & & Space Group 64: Cmce \\
\hline & $\begin{array}{l}\Gamma-\mathrm{Y}, \Gamma-\mathrm{Z}, \Gamma-\mathrm{T}, \mathrm{T}-\mathrm{Y}, \mathrm{T}-\mathrm{Z} \\
\mathrm{Y}-\mathrm{Z}, \mathrm{S}-\Gamma, \mathrm{S}-\mathrm{Y}\end{array}$ & $\| \mathrm{Zn}_{53} \mathrm{Ni}_{16}$ \\
\hline & $\begin{array}{l}\Gamma-\mathrm{Z}, \Gamma-\mathrm{Y}, \Gamma-\mathrm{T}, \mathrm{T}-\mathrm{Y}, \mathrm{T}-\mathrm{Z} \\
\mathrm{Y}-\mathrm{Z}, \mathrm{S}-\Gamma, \mathrm{S}-\mathrm{Y}\end{array}$ & $\| \mathrm{Ba}\left(\mathrm{Ni}_{5} \mathrm{P}_{3}\right)_{2}, \mathrm{Sr}\left(\mathrm{Ni}_{5} \mathrm{P}_{3}\right)_{2}$ \\
\hline & $\begin{array}{l}\text { Г-Y, Г-T, T-Z, Y-Z, T-Y, } \\
\text { S- } \Gamma, \text { S-Y }\end{array}$ & $\| \mathrm{NiGe}_{2}$ \\
\hline & $\begin{array}{l}\text { Г-Y, } \Gamma-Z, \Gamma-T, T-Y, T-Z \\
\text { Y-Z, S-Y }\end{array}$ & $\| \mathrm{Si}, \mathrm{ZnAu}_{3}$ \\
\hline & $\begin{array}{l}\Gamma-\mathrm{Y}, \Gamma-\mathrm{Z}, \Gamma-\mathrm{T}, \mathrm{T}-\mathrm{Y}, \mathrm{Y}-\mathrm{Z} \\
\mathrm{S}-\Gamma, \mathrm{S}-\mathrm{Y}\end{array}$ & $\| \mathrm{Hf}_{2} \mathrm{Si}_{4} \mathrm{Ni}_{3}, \mathrm{Ba}_{4} \mathrm{Ru}_{3} \mathrm{O}_{10}$ \\
\hline & $\begin{array}{l}\Gamma-\mathrm{Y}, \Gamma-\mathrm{Z}, \Gamma-\mathrm{T}, \mathrm{T}-\mathrm{Z}, \mathrm{Y}-\mathrm{Z} \\
\mathrm{S}-\Gamma, \mathrm{S}-\mathrm{Y}\end{array}$ & $\| \mathrm{SnBi}$ \\
\hline & $\begin{array}{l}\Gamma-\mathrm{Z}, \Gamma-\mathrm{Y}, \Gamma-\mathrm{T}, \mathrm{T}-\mathrm{Y}, \mathrm{Y}-\mathrm{Z} \\
\mathrm{S}-\Gamma, \mathrm{S}-\mathrm{Y}\end{array}$ & $\| \mathrm{FeGe}_{2}, \mathrm{Ba}_{2} \mathrm{Cd}_{3} \mathrm{Bi}_{4}, \mathrm{Ti}_{2} \mathrm{Sn}_{3}, \mathrm{Dy}_{2} \mathrm{Ni}_{7} \mathrm{Sn}_{3}$ \\
\hline & $\begin{array}{c}\Gamma-\mathrm{Y}, \Gamma-\mathrm{Z}, \Gamma-\mathrm{T}, \mathrm{T}-\mathrm{Y}, \mathrm{Y}-\mathrm{Z}, \\
\mathrm{S}-\mathrm{Y}\end{array}$ & $\| \mathrm{Ba}_{2} \mathrm{Ti}_{13} \mathrm{O}_{22}, \mathrm{Yb}_{2} \mathrm{Ge}_{6} \mathrm{Pd}$ \\
\hline
\end{tabular}


Table III - continued

\begin{tabular}{|c|c|c|}
\hline SOC or & HSL with Band & High Symmetry Line Semimetals \\
\hline & $\Gamma-Y, \Gamma-Z, \Gamma-T, T-Z, Y-Z$, & \\
\hline & S-Y & || Bi, MgIr \\
\hline & $\begin{array}{c}\Gamma-\mathrm{Y}, \Gamma-\mathrm{Z}, \mathrm{T}-\mathrm{Y}, \mathrm{Y}-\mathrm{Z}, \Gamma-\mathrm{T}, \\
\mathrm{S}-\Gamma\end{array}$ & $\| \mathrm{La}_{2} \mathrm{Ni}_{22} \mathrm{C}_{3}$ \\
\hline & $\begin{array}{c}\Gamma-Y, \Gamma-Z, T-Y, Y-Z, S-\Gamma, \\
\text { S-Y }\end{array}$ & $\| \mathrm{Ge}$ \\
\hline & $\begin{array}{c}\Gamma-\mathrm{Y}, \Gamma-\mathrm{Z}, \mathrm{T}-\mathrm{Z}, \mathrm{Y}-\mathrm{Z}, \Gamma-\mathrm{T}, \\
\text { S-Y }\end{array}$ & $\| \mathrm{Ho}_{2}\left(\mathrm{Ni}_{5} \mathrm{~B}_{3}\right)_{3}$ \\
\hline & $\begin{array}{c}\Gamma-\mathrm{Y}, \Gamma-\mathrm{Z}, \mathrm{T}-\mathrm{Z}, \mathrm{Y}-\mathrm{Z}, \mathrm{T}-\mathrm{Y} \\
\mathrm{S}-\Gamma\end{array}$ & $\| \mathrm{W}_{3} \mathrm{Br}_{8}$ \\
\hline & $\begin{array}{c}\Gamma-\mathrm{Z}, \Gamma-\mathrm{Y}, \Gamma-\mathrm{T}, \mathrm{T}-\mathrm{Y}, \mathrm{T}-\mathrm{Z}, \\
\mathrm{Y}-\mathrm{Z}\end{array}$ & |l Ga(ICSD : 43388), Ca, $\mathrm{Zr}_{7} \mathrm{Ni}_{10}$ \\
\hline & $\Gamma-\mathrm{Y}, \Gamma-\mathrm{T}, \mathrm{T}-\mathrm{Z}, \mathrm{Y}-\mathrm{Z}, \mathrm{S}-\Gamma$ & $\| \mathrm{SmSb}_{2}, \mathrm{TbSb}_{2}$ \\
\hline & $\Gamma-Y, \Gamma-Z, \Gamma-\mathrm{T}, \mathrm{S}-\Gamma, \mathrm{S}-\mathrm{Y}$ & $\| \mathrm{Ce}_{2} \mathrm{Ni}_{22} \mathrm{C}_{3}$ \\
\hline & $\Gamma-\mathrm{Y}, \Gamma-\mathrm{Z}, \Gamma-\mathrm{T}, \mathrm{T}-\mathrm{Z}, \mathrm{Y}-\mathrm{Z}$ & $\| \mathrm{CaO}$ \\
\hline & $\Gamma-Y, T-Y, Y-Z, S-\Gamma, S-Y$ & $\| \mathrm{Ge}, \mathrm{Sn}_{3} \mathrm{Pd}$ \\
\hline & $\Gamma-\mathrm{Z}, \Gamma-\mathrm{Y}, \Gamma-\mathrm{T}, \mathrm{S}-\Gamma, \mathrm{S}-\mathrm{Y}$ & $\| \mathrm{Pb}_{3} \mathrm{O}_{5}$ \\
\hline & $\Gamma-\mathrm{Y}, \Gamma-\mathrm{T}, \mathrm{T}-\mathrm{Z}, \mathrm{Y}-\mathrm{Z}$ & NdAsSe, $\|$ Ga(ICSD : 165978) \\
\hline & $\Gamma-\mathrm{Y}, \Gamma-\mathrm{Z}, \Gamma-\mathrm{T}, \mathrm{S}-\Gamma$ & $\| \mathrm{AsH}_{2} \mathrm{C}_{2} \mathrm{NCl}_{2} \mathrm{O}$ \\
\hline & $\Gamma-\mathrm{Y}, \Gamma-\mathrm{Z}, \Gamma-\mathrm{T}, \mathrm{S}-\mathrm{Y}$ & $\mathrm{BaYbSn}_{3}, \| \mathrm{V}_{2} \mathrm{GaSn}_{2}, \mathrm{Cs}, \mathrm{Fe}_{2} \mathrm{AgS}_{3}$ \\
\hline & $\Gamma-Y, T-Y, Y-Z, S-\Gamma$ & $\| \mathrm{La}_{2} \mathrm{Ni}_{3}$ \\
\hline & $\Gamma-Y, T-Y, Y-Z, S-Y$ & $\| \mathrm{CoGe}_{2}$ \\
\hline & $\Gamma-\mathrm{Z}, \Gamma-\mathrm{T}, \mathrm{T}-\mathrm{Y}, \mathrm{Y}-\mathrm{Z}$ & $\pi \mathrm{Ca}_{2} \mathrm{RuO}_{4}$ \\
\hline & $\Gamma-\mathrm{Y}, \Gamma-\mathrm{Z}, \mathrm{S}-\mathrm{Y}$ & $\| \mathrm{K}$ \\
\hline & $\Gamma-\mathrm{Y}, \mathrm{S}-\Gamma, \mathrm{S}-\mathrm{Y}$ & $\mathrm{Ca}_{5}\left(\mathrm{GaN}_{2}\right)_{2}, \| \mathrm{LaS}$ \\
\hline & $\Gamma-Y, T-Y, Y-Z$ & $\mathrm{SnO}, \mathrm{Sr}_{2} \mathrm{GeN}_{2} \|$ \\
\hline & $\Gamma-\mathrm{Z}, \Gamma-\mathrm{Y}, \Gamma-\mathrm{T}$ & $\mathrm{BaCaSn}_{3} \|$ \\
\hline & $\Gamma-Z, T-Z, Y-Z$ & $\mathrm{Yb}_{2}\left(\mathrm{Ni}_{5} \mathrm{~B}_{3}\right)_{3}$ \\
\hline & $\Gamma-\mathrm{Y}, \mathrm{S}-\Gamma$ & $\| \mathrm{AgN}$ \\
\hline & $\Gamma-\mathrm{Y}, \mathrm{S}-\mathrm{Y}$ & $\mathrm{P}, \mathrm{As} \|$ \\
\hline & $\Gamma-\mathrm{Z}$ & $\| \mathrm{NbCoTe}_{2}$ \\
\hline $\mathrm{SOC}$ & $\mathrm{R}-\mathrm{S}$ & $\| \mathrm{TbSb}_{2}, \mathrm{SmSb}_{2}, \mathrm{Bi}$ \\
\hline & & Space Group 65: $\mathrm{Cmmm}$ \\
\hline & $\begin{array}{c}-Z, \Gamma-Y, \Gamma-T, T-Y, Y-Z, \\
\text { S- } \Gamma, \text { S-Y, R-S, R-T, R-Z, } \\
\text { T-Z }\end{array}$ & $\| \mathrm{Lu}_{2} \mathrm{NiSn}_{6}$ \\
\hline & $\begin{array}{l}\text { Г-Y, Г-Z, Г-T, T-Y, T-Z, } \\
\text { Y-Z, S- } \Gamma, \text { S-Y, R-T, R-Z }\end{array}$ & $\| \mathrm{Ce}_{2} \mathrm{Sn}_{5}$ \\
\hline & $\begin{array}{l}\Gamma-\mathrm{Y}, \Gamma-\mathrm{Z}, \Gamma-\mathrm{T}, \mathrm{T}-\mathrm{Y}, \mathrm{T}-\mathrm{Z}, \\
\mathrm{Y}-\mathrm{Z}, \mathrm{S}-\mathrm{Y}, \mathrm{R}-\mathrm{S}, \mathrm{R}-\mathrm{T}, \mathrm{R}-\mathrm{Z}\end{array}$ & $\| \mathrm{Ce}_{3} \mathrm{Sn}_{7}$ \\
\hline & 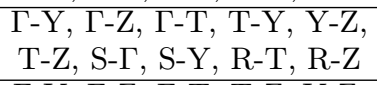 & $\| \mathrm{Nb}_{5} \mathrm{Ga}_{13}$ \\
\hline & $\begin{array}{l}\text { Г-Y, Г-Z, Г-T, T-Z, Y-Z, } \\
\text { T-Y, S- }, \text { S-Y, R-T, R-Z }\end{array}$ & $\| \mathrm{Ba}_{2} \mathrm{Yb}\left(\mathrm{CuO}_{2}\right)_{4}$ \\
\hline & $\begin{array}{c}\Gamma-Z, \Gamma-Y, \Gamma-T, T-Y, T-Z, \\
\text { Y-Z, S- } \Gamma, \text { S-Y, R-S, R-Z }\end{array}$ & $\| \mathrm{TiAl}_{2}$ \\
\hline & $\begin{array}{c}\text { Г-Z, Г-Y, }-\mathrm{T}, \mathrm{T}-\mathrm{Y}, \mathrm{T}-\mathrm{Z}, \\
\mathrm{Y}-\mathrm{Z}, \mathrm{S}-\Gamma, \mathrm{S}-\mathrm{Y}, \mathrm{R}-\mathrm{T}, \mathrm{R}-\mathrm{Z}\end{array}$ & $\| \mathrm{TbGe}_{2}$ \\
\hline & $\begin{array}{l}\text { T-Z, } \Gamma-Y, \Gamma-\mathrm{T}, \mathrm{T}-\mathrm{Y}, \mathrm{Y}-\mathrm{Z} \\
\mathrm{S}-\Gamma, \mathrm{S}-\mathrm{Y}, \mathrm{R}-\mathrm{S}, \mathrm{R}-\mathrm{Z}, \mathrm{T}-\mathrm{Z}\end{array}$ & $\| \mathrm{Er}_{2} \mathrm{NiSn}_{6}, \mathrm{Dy}_{2} \mathrm{NiSn}_{6}, \mathrm{Tm}_{2} \mathrm{NiSn}_{6}$ \\
\hline & $\begin{array}{c}\text { Г-Z, Г-Y, Г-T, T-Y, Y-Z, } \\
\text { T-Z, S- } \Gamma, \text { S-Y, R-S, R-Z }\end{array}$ & $\| \mathrm{ZrGa}_{2}$ \\
\hline & $\begin{array}{l}\Gamma-\mathrm{Y}, \Gamma-\mathrm{Z}, \Gamma-\mathrm{T}, \mathrm{T}-\mathrm{Y}, \mathrm{Y}-\mathrm{Z} \\
\mathrm{S}-\Gamma, \mathrm{S}-\mathrm{Y}, \mathrm{R}-\mathrm{T}, \mathrm{R}-\mathrm{Z}\end{array}$ & || VIr(ICSD : 169389) \\
\hline & $\begin{array}{c}\text { Г-Y, Г-Z, Г-T, T-Z, Y-Z, } \\
\text { T-Y, S-Y, R-S, R-T }\end{array}$ & $\| \mathrm{Er}_{4} \mathrm{Ni}_{13} \mathrm{C}_{4}$ \\
\hline & $\begin{array}{l}\text { Г-Y, }-\mathrm{Z}, \mathrm{T}-\mathrm{Y}, \mathrm{T}-\mathrm{Z}, \mathrm{\Gamma}-\mathrm{T}, \\
\quad \mathrm{Y}-\mathrm{Z}, \mathrm{R}-\mathrm{S}, \mathrm{R}-\mathrm{T}, \mathrm{R}-\mathrm{Z}\end{array}$ & $\| \mathrm{Y}_{11} \mathrm{In}_{9} \mathrm{Ni}_{4}$ \\
\hline
\end{tabular}


Table III - continued

\begin{tabular}{|c|c|c|}
\hline $\begin{array}{l}\text { SOC or } \\
\text { NSOC }\end{array}$ & $\begin{array}{l}\text { HSL with Band } \\
\text { Crossing }\end{array}$ & High Symmetry Line Semimetals \\
\hline & $\begin{array}{c}\Gamma-Z, \Gamma-Y, \Gamma-T, T-Y, T-Z, \\
\text { Y-Z, S- } \Gamma, \text { S-Y, R-T }\end{array}$ & $\| \mathrm{NdGa}_{2} \mathrm{Ni}$ \\
\hline & $\begin{array}{c}\text { Г-Z, Г-Y, Г-T, T-Y, T-Z, } \\
\text { Y-Z, S- } \Gamma, \text { S-Y, R-Z }\end{array}$ & $\| \mathrm{LuNiSn}_{4}$ \\
\hline & $\begin{array}{c}-Z, \Gamma-Y, \Gamma-T, T-Y, Y-Z \\
\text { T-Z, S- } \Gamma, S-Y, \text { R-S }\end{array}$ & $\| \mathrm{ThGe}_{2}$ \\
\hline & $\begin{array}{c}\Gamma-\mathrm{Z}, \Gamma-\mathrm{Y}, \Gamma-\mathrm{T}, \mathrm{T}-\mathrm{Y}, \mathrm{Y}-\mathrm{Z} \\
\mathrm{T}-\mathrm{Z}, \mathrm{S}-\Gamma, \mathrm{S}-\mathrm{Y}, \mathrm{R}-\mathrm{Z}\end{array}$ & $\| \mathrm{PrSn}_{2}$ \\
\hline & $\begin{array}{c}\text { Г-Z, Г-Y, T-Z, Y-Z, Г-T, } \\
\text { T-Y, S- } \Gamma, \text { S-Y, R-T }\end{array}$ & || $\mathrm{NdNiGe}_{3}$ \\
\hline & $\begin{array}{c}\Gamma-\mathrm{Y}, \Gamma-\mathrm{T}, \mathrm{T}-\mathrm{Y}, \mathrm{T}-\mathrm{Z}, \mathrm{S}-\Gamma, \\
\text { S-Y, R-T, R-Z }\end{array}$ & $\| \mathrm{SmGa}_{2} \mathrm{Ni}, \mathrm{PrGa}_{2} \mathrm{Ni}$ \\
\hline & 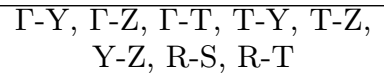 & $\| \operatorname{Pr}_{11} \mathrm{In}_{9} \mathrm{Ni}_{4}, \mathrm{Nd}_{11} \mathrm{In}_{9} \mathrm{Ni}_{4}$ \\
\hline & $\begin{array}{c}\Gamma-\mathrm{Y}, \Gamma-\mathrm{Z}, \Gamma-\mathrm{T}, \mathrm{T}-\mathrm{Y}, \mathrm{T}-\mathrm{Z} \\
\mathrm{Y}-\mathrm{Z}, \mathrm{R}-\mathrm{T}, \mathrm{R}-\mathrm{Z}\end{array}$ & $\| \mathrm{LaSn}_{2}, \mathrm{SmSn}_{2}$ \\
\hline & $\begin{array}{c}\text { Г-Y, Г-Z, Г-T, T-Y, T-Z, } \\
\text { Y-Z, S- } \Gamma, \text { S-Y }\end{array}$ & $\| \mathrm{NdSn}_{2}$ \\
\hline & $\begin{array}{c}\Gamma-\mathrm{Y}, \Gamma-\mathrm{Z}, \Gamma-\mathrm{T}, \mathrm{T}-\mathrm{Y}, \mathrm{Y}-\mathrm{Z} \\
\mathrm{S}-\mathrm{Y}, \mathrm{R}-\mathrm{T}, \mathrm{T}-\mathrm{Z}\end{array}$ & || VIr(ICSD : 104590) \\
\hline & $\begin{array}{c}\Gamma-Y, \Gamma-Z, \Gamma-\mathrm{T}, \mathrm{T}-\mathrm{Y}, \mathrm{Y}-\mathrm{Z}, \\
\mathrm{T}-\mathrm{Z}, \mathrm{S}-\Gamma, \mathrm{R}-\mathrm{S}\end{array}$ & $\|$ HoBC \\
\hline & $\begin{array}{c}\text { T-Y, } \Gamma-\mathrm{Z}, \Gamma-\mathrm{T}, \mathrm{T}-\mathrm{Z}, \mathrm{Y}-\mathrm{Z} \\
\mathrm{R}-\mathrm{S}, \mathrm{R}-\mathrm{T}, \mathrm{R}-\mathrm{Z}\end{array}$ & $\| \mathrm{Nd}_{2} \mathrm{CdCu}_{2}$ \\
\hline & $\begin{array}{c}\Gamma-\mathrm{Y}, \Gamma-\mathrm{Z}, \Gamma-\mathrm{T}, \mathrm{T}-\mathrm{Z}, \mathrm{Y}-\mathrm{Z} \\
\mathrm{S}-\Gamma, \mathrm{R}-\mathrm{S}, \mathrm{T}-\mathrm{Y}\end{array}$ & $\| \mathrm{Yb}_{4} \mathrm{Ni}_{13} \mathrm{C}_{4}$ \\
\hline & $\begin{array}{c}\Gamma-\mathrm{Y}, \Gamma-\mathrm{Z}, \Gamma-\mathrm{T}, \mathrm{T}-\mathrm{Z}, \mathrm{Y}-\mathrm{Z} \\
\mathrm{T}-\mathrm{Y}, \mathrm{S}-\Gamma, \mathrm{R}-\mathrm{T}\end{array}$ & $\| \mathrm{Lu}_{4} \mathrm{Ni}_{13} \mathrm{C}_{4}, \mathrm{Ho}_{4} \mathrm{Ni}_{13} \mathrm{C}_{4}$ \\
\hline & $\begin{array}{c}\Gamma-\mathrm{Y}, \Gamma-\mathrm{Z}, \mathrm{T}-\mathrm{Y}, \mathrm{T}-\mathrm{Z}, \mathrm{Y}-\mathrm{Z} \\
\mathrm{R}-\mathrm{S}, \mathrm{R}-\mathrm{T}, \mathrm{R}-\mathrm{Z}\end{array}$ & $\| \mathrm{Zn}_{5}\left(\mathrm{BRh}_{2}\right)_{4}$ \\
\hline & $\begin{array}{c}\Gamma-\mathrm{Y}, \mathrm{T}-\mathrm{Y}, \mathrm{Y}-\mathrm{Z}, \mathrm{S}-\mathrm{\Gamma}, \mathrm{S}-\mathrm{Y}, \\
\text { R-S, R-T, T-Z }\end{array}$ & $\| \mathrm{CdPt}_{3}, \mathrm{HgPt}_{3}$ \\
\hline & $\begin{array}{c}\text { T-Z, Г-T, T-Y, Y-Z, T-Z, } \\
\text { R-S, R-T, R-Z }\end{array}$ & $\| \mathrm{Zr}_{2} \mathrm{P}$ \\
\hline & $\begin{array}{c}-Z, \Gamma-Y, \Gamma-T, T-Y, Y-Z, \\
\text { T-Z, S- } \Gamma, S-Y\end{array}$ & $\| \mathrm{TlSbSe}_{2}$ \\
\hline & $\begin{array}{c}\Gamma-\mathrm{Z}, \Gamma-\mathrm{Y}, \mathrm{T}-\mathrm{Z}, \mathrm{Y}-\mathrm{Z}, \Gamma-\mathrm{T}, \\
\mathrm{R}-\mathrm{S}, \mathrm{R}-\mathrm{T}, \mathrm{R}-\mathrm{Z}\end{array}$ & $\| \mathrm{Ho}_{2} \mathrm{Ni}_{2} \mathrm{~Pb}$ \\
\hline & $\begin{array}{c}\text { ए-Z, T-Z, Y-Z, Г-T, T-Y, } \\
\text { S- } \Gamma, S-Y, R-Z\end{array}$ & $\| \mathrm{Ba}_{3} \mathrm{Nb}_{16} \mathrm{O}_{23}$ \\
\hline & $\begin{array}{c}\Gamma-\mathrm{Y}, \Gamma-\mathrm{T}, \mathrm{T}-\mathrm{Y}, \mathrm{T}-\mathrm{Z}, \mathrm{S}-\Gamma, \\
\text { R-S, R-T }\end{array}$ & $\| \mathrm{Dy}_{2} \mathrm{In}_{16} \mathrm{Pt}_{7}, \mathrm{~Tb}_{2} \mathrm{In}_{16} \mathrm{Pt}_{7}$ \\
\hline & $\begin{array}{c}\text { Г-Y, Г-T, T-Y, T-Z, Y-Z, } \\
\text { S- } \Gamma, \text { S-Y }\end{array}$ & $\| \mathrm{UGe}_{2}$ \\
\hline & $\begin{array}{c}\text { T-Y, }-\mathrm{T}, \mathrm{T}-\mathrm{Z}, \mathrm{Y}-\mathrm{Z}, \mathrm{S}-\mathrm{Y}, \\
\text { R-S, R-Z }\end{array}$ & || C(ICSD : 88812) \\
\hline & $\begin{array}{c}\Gamma-\mathrm{Y}, \Gamma-\mathrm{Z}, \Gamma-\mathrm{T}, \mathrm{T}-\mathrm{Y}, \mathrm{Y}-\mathrm{Z}, \\
\mathrm{T}-\mathrm{Z}, \mathrm{S}-\mathrm{Y}\end{array}$ & $\| \mathrm{ThB}_{4} \mathrm{Mo}$ \\
\hline & $\begin{array}{c}\Gamma-\mathrm{Y}, \Gamma-\mathrm{Z}, \Gamma-\mathrm{T}, \mathrm{T}-\mathrm{Z}, \mathrm{Y}-\mathrm{Z}, \\
\text { S- } \Gamma, \mathrm{R}-\mathrm{S}\end{array}$ & || VIr(ICSD : 169390) \\
\hline & $\begin{array}{c}\Gamma-\mathrm{Y}, \Gamma-\mathrm{Z}, \mathrm{T}-\mathrm{Y}, \mathrm{T}-\mathrm{Z}, \Gamma-\mathrm{T}, \\
\text { Y-Z, R-T }\end{array}$ & $\| \mathrm{YbAlB}_{4}$ \\
\hline & $\begin{array}{c}\Gamma-\mathrm{Y}, \Gamma-\mathrm{Z}, \mathrm{T}-\mathrm{Y}, \mathrm{T}-\mathrm{Z}, \mathrm{Y}-\mathrm{Z} \\
\text { S- } \Gamma, \mathrm{R}-\mathrm{Z}\end{array}$ & $\| \mathrm{Yb}_{2}\left(\mathrm{NiB}_{2}\right)_{3}$ \\
\hline & $\begin{array}{c}\text { T-Y, } \Gamma-Z, T-Y, Y-Z, \Gamma-T, \\
\text { T-Z, R-S }\end{array}$ & \| DyBC \\
\hline & $\begin{array}{c}\Gamma-\mathrm{Y}, \mathrm{T}-\mathrm{Y}, \mathrm{Y}-\mathrm{Z}, \Gamma-\mathrm{T}, \mathrm{T}-\mathrm{Z}, \\
\text { S- } \Gamma, \mathrm{R}-\mathrm{S}\end{array}$ & $\| \mathrm{YBC}$ \\
\hline & 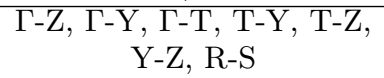 & $\| \mathrm{La}_{11} \mathrm{In}_{9} \mathrm{Ni}_{4}$ \\
\hline
\end{tabular}


Table III - continued

\begin{tabular}{|c|c|c|}
\hline $\begin{array}{l}\text { SOC or } \\
\text { NSOC }\end{array}$ & $\begin{array}{c}\text { HSL with Band } \\
\text { Crossing }\end{array}$ & High Symmetry Line Semimetals \\
\hline & $\begin{array}{c}-Z, \Gamma-Y, \Gamma-T, T-Y, T-Z, \\
\text { Y-Z, R-Z }\end{array}$ & $\| \mathrm{ErSi}_{3} \mathrm{Ni}, \mathrm{YNiGe}_{3}, \mathrm{YSi}_{3} \mathrm{Ni}, \mathrm{SmSi}_{3} \mathrm{Ni}$ \\
\hline & $\begin{array}{c}\text { T-Z, } \Gamma-Y, \Gamma-T, T-Y, Y-Z, \\
\text { T-Z, R-T }\end{array}$ & $\| \mathrm{La}_{4} \mathrm{Al}_{5} \mathrm{Br}_{2}$ \\
\hline & $\begin{array}{c}\text { I-Z, Г-Y, T-Z, Y-Z, T-Y, } \\
\text { R-T, R-Z }\end{array}$ & $\| \mathrm{Er}_{2}\left(\mathrm{NiB}_{2}\right)_{3}$ \\
\hline & $\begin{array}{c}\Gamma-\mathrm{Y}, \Gamma-\mathrm{T}, \mathrm{T}-\mathrm{Y}, \mathrm{T}-\mathrm{Z}, \mathrm{S}-\mathrm{Y}, \\
\mathrm{R}-\mathrm{T}\end{array}$ & $\| \mathrm{LaGa}_{2} \mathrm{Ni}$ \\
\hline & 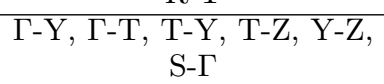 & $\| \mathrm{LuAlB}_{4}$ \\
\hline & $\begin{array}{c}\Gamma-\mathrm{Y}, \Gamma-\mathrm{T}, \mathrm{T}-\mathrm{Z}, \mathrm{Y}-\mathrm{Z}, \mathrm{R}-\mathrm{S}, \\
\mathrm{R}-\mathrm{Z}\end{array}$ & $\| \mathrm{LuMnGe}_{2}$ \\
\hline & $\begin{array}{c}-\mathrm{Y}, \Gamma-\mathrm{T}, \mathrm{T}-\mathrm{Z}, \mathrm{Y}-\mathrm{Z}, \mathrm{R}-\mathrm{T} \\
\mathrm{R}-\mathrm{Z}\end{array}$ & $\| \mathrm{GeH}_{4}$ \\
\hline & $\begin{array}{c}\Gamma-\mathrm{Y}, \Gamma-\mathrm{Z}, \Gamma-\mathrm{T}, \mathrm{T}-\mathrm{Z}, \mathrm{Y}-\mathrm{Z}, \\
\mathrm{R}-\mathrm{Z}\end{array}$ & $\| \mathrm{Ce}\left(\mathrm{Re}_{2} \mathrm{Si}\right)_{2}$ \\
\hline & $\begin{array}{c}\Gamma-\mathrm{Y}, \Gamma-\mathrm{Z}, \mathrm{T}-\mathrm{Y}, \mathrm{Y}-\mathrm{Z}, \Gamma-\mathrm{T}, \\
\mathrm{S}-\Gamma\end{array}$ & $\| \mathrm{SrCu}_{2} \mathrm{O}_{3}$ \\
\hline & $\begin{array}{c}\text {-Y, } \mathrm{Y}-\mathrm{Z}, \mathrm{T}-\mathrm{Y}, \mathrm{Y}-\mathrm{Z}, \Gamma-\mathrm{T}, \\
\mathrm{T}-\mathrm{Z}\end{array}$ & $\| \mathrm{CeSiNi}_{4}$ \\
\hline & $\begin{array}{c}\Gamma-Y, \Gamma-Z, T-Y, Y-Z, T-Z, \\
S-Y\end{array}$ & $\| \mathrm{Ce}_{2} \mathrm{Si}_{7}$ \\
\hline & $\begin{array}{c}\Gamma-\mathrm{Z}, \Gamma-\mathrm{Y}, \Gamma-\mathrm{T}, \mathrm{T}-\mathrm{Y}, \mathrm{T}-\mathrm{Z} \\
\mathrm{R}-\mathrm{T}\end{array}$ & $\| \mathrm{VRh}$ \\
\hline & $\begin{array}{c}\text { T-Z, } \Gamma-\mathrm{Y}, \mathrm{T}-\mathrm{Y}, \mathrm{T}-\mathrm{Z}, \mathrm{R}-\mathrm{S}, \\
\mathrm{R}-\mathrm{Z}\end{array}$ & $\| \mathrm{Ho}_{2}\left(\mathrm{NiB}_{2}\right)_{3}$ \\
\hline & $\begin{array}{c}\Gamma-\mathrm{Z}, \Gamma-\mathrm{Y}, \mathrm{T}-\mathrm{Z}, \mathrm{Y}-\mathrm{Z}, \Gamma-\mathrm{T} \\
\mathrm{T}-\mathrm{Y}\end{array}$ & $\| \mathrm{LuNiGe}_{3}, \mathrm{TbNiGe}_{3}, \mathrm{DyNiGe}_{3}, \mathrm{TmNiGe}_{3}, \mathrm{HoNiGe}_{3}, \mathrm{ErNiGe}_{3}$ \\
\hline & $\begin{array}{c}\Gamma-Z, \Gamma-Y, T-Z, Y-Z, R-T, \\
R-Z\end{array}$ & $\| \mathrm{Sr}_{2} \mathrm{Ge}_{2} \mathrm{~Pb}, \mathrm{SmNiGe}_{3}, \mathrm{PrNiGe}_{3}$ \\
\hline & $\Gamma-\mathrm{Y}, \Gamma-\mathrm{T}, \mathrm{T}-\mathrm{Y}, \mathrm{T}-\mathrm{Z}, \mathrm{R}-\mathrm{S}$ & $\| \mathrm{Ba}_{4} \mathrm{Nb}_{14} \mathrm{O}_{23}$ \\
\hline & $\Gamma-Y, \Gamma-\mathrm{T}, \mathrm{T}-\mathrm{Y}, \mathrm{T}-\mathrm{Z}, \mathrm{S}-\mathrm{Y}$ & $\| \mathrm{SmGa}_{2} \mathrm{Co}$ \\
\hline & Г-Y, Г-T, T-Z, Y-Z, R-S & $\mathrm{Al}_{2} \mathrm{~B}_{2} \mathrm{Ru}_{3}$ \\
\hline & $\Gamma-\mathrm{Y}, \Gamma-\mathrm{T}, \mathrm{T}-\mathrm{Z}, \mathrm{Y}-\mathrm{Z}, \mathrm{R}-\mathrm{Z}$ & $\mathrm{Tb}_{2} \mathrm{Mg}_{3} \mathrm{Ni}_{2}$ \\
\hline & $\Gamma-Y, \Gamma-T, T-Z, Y-Z, S-Y$ & $\| \mathrm{Rb}_{3} \mathrm{Au}_{7}$ \\
\hline & $\Gamma-\mathrm{Y}, \Gamma-\mathrm{Z}, \Gamma-\mathrm{T}, \mathrm{T}-\mathrm{Y}, \mathrm{Y}-\mathrm{Z}$ & $\pi \mathrm{Nd}_{5} \operatorname{In}_{11} \mathrm{Ni}_{6}, \operatorname{Pr}_{5} \operatorname{In}_{11} \mathrm{Ni}_{6}$ \\
\hline & Г-Y, Г-Z, Г-Т, T-Z, Y-Z & $\| \mathrm{Tb}_{2} \mathrm{~B}_{2} \mathrm{C}_{3}$ \\
\hline & $\Gamma-\mathrm{Y}, \Gamma-\mathrm{Z}, \mathrm{T}-\mathrm{Y}, \mathrm{Y}-\mathrm{Z}, \Gamma-\mathrm{T}$ & $\mathrm{TbMg}_{4} \mathrm{Cu}, \mathrm{YMg}_{4} \mathrm{Cu}$ \\
\hline & Г-Y, T-Y, Y-Z, R-T, R-Z & $\| \mathrm{TlAg}_{3} \mathrm{Te}_{2}$ \\
\hline & $\Gamma-\mathrm{Y}, \mathrm{T}-\mathrm{Y}, \mathrm{Y}-\mathrm{Z}, \mathrm{S}-\Gamma, \mathrm{S}-\mathrm{Y}$ & $\mathrm{Cu}_{2} \mathrm{SiNiS}_{4}$ \\
\hline & $\Gamma-\mathrm{Z}, \Gamma-\mathrm{T}, \mathrm{T}-\mathrm{Y}, \mathrm{Y}-\mathrm{Z}, \mathrm{R}-\mathrm{S}$ & $\| \mathrm{Fe}(\mathrm{NCl})_{2}$ \\
\hline & $\Gamma-\mathrm{Z}, \Gamma-\mathrm{Y}, \Gamma-\mathrm{T}, \mathrm{T}-\mathrm{Y}, \mathrm{Y}-\mathrm{Z}$ & $\mathrm{Ba}_{2} \mathrm{Nd}_{7} \mathrm{Si}_{11} \mathrm{~N}_{23}$ \\
\hline & Г-Z, Г-Y, T-Y, T-Z, R-Z & $\| \mathrm{Lu}_{2}\left(\mathrm{NiB}_{2}\right)_{3}$ \\
\hline & $\Gamma-Z, \Gamma-Y, T-Z, Y-Z, \Gamma-T$ & $\| \mathrm{ScSi}_{3} \mathrm{Ni}$ \\
\hline & $\Gamma-\mathrm{Y}, \mathrm{R}-\mathrm{Z}, \mathrm{T}-\mathrm{Z}$ & $\mathrm{ScGa}_{2}$ \\
\hline & $\Gamma-\mathrm{Z}, \mathrm{T}-\mathrm{Z}, \mathrm{Y}-\mathrm{Z}$ & $\mathrm{Ba}_{3} \mathrm{Ge}_{4} \|$ \\
\hline & $\Gamma-\mathrm{Y}, \mathrm{S}-\mathrm{Y}$ & $\| \mathrm{Li}_{7} \mathrm{Sn}_{2}$ \\
\hline & $\mathrm{R}-\mathrm{S}$ & $\| \mathrm{AgCNO}$ \\
\hline & & Space Group 66: $\mathrm{Cccm}$ \\
\hline NSOC & $\Gamma-\mathrm{Y}, \mathrm{S}-\Gamma$ & $\| \mathrm{ThI}_{3}$ \\
\hline & & Space Group 67: Cmme \\
\hline NTCOO & $\begin{array}{c}\Gamma-\mathrm{Y}, \Gamma-\mathrm{T}, \mathrm{T}-\mathrm{Z}, \mathrm{Y}-\mathrm{Z}, \mathrm{S}-\Gamma, \\
\mathrm{R}-\mathrm{Z}\end{array}$ & $\| \mathrm{FeSe}$ \\
\hline NSOC & $\Gamma-\mathrm{Y}, \Gamma-\mathrm{Z}, \Gamma-\mathrm{T}, \mathrm{T}-\mathrm{Z}, \mathrm{Y}-\mathrm{Z}$ & $\| \mathrm{C}(\mathrm{ICSD}: 88815)$ \\
\hline & $\mathrm{R}-\mathrm{Z}, \mathrm{T}-\mathrm{Z}$ & $\| \mathrm{Al}_{2} \mathrm{CuIr}$ \\
\hline & & Space Group 68: Ccce \\
\hline NSOC & $\begin{array}{c}\Gamma-\mathrm{Y}, \Gamma-\mathrm{Z}, \Gamma-\mathrm{T}, \mathrm{T}-\mathrm{Y}, \mathrm{Y}-\mathrm{Z} \\
\mathrm{S}-\Gamma, \mathrm{S}-\mathrm{Y} \\
\end{array}$ & $\| \mathrm{Sn}_{4} \mathrm{Pd}$ \\
\hline
\end{tabular}


Table III - continued

\begin{tabular}{|c|c|c|}
\hline \multirow[t]{2}{*}{$\begin{array}{l}\text { SOC or } \\
\text { NSOC }\end{array}$} & $\begin{array}{l}\text { HSL with Band } \\
\text { Crossing }\end{array}$ & High Symmetry Line Semimetals \\
\hline & $\begin{array}{c}\Gamma-\mathrm{Y}, \Gamma-\mathrm{Z}, \Gamma-\mathrm{T}, \mathrm{T}-\mathrm{Y}, \mathrm{Y}-\mathrm{Z} \\
\mathrm{S}-\mathrm{Y}\end{array}$ & $\| \mathrm{Sn}_{4} \mathrm{Pt}$ \\
\hline \multicolumn{3}{|r|}{ Space Group 69: Fmmm } \\
\hline \multirow{4}{*}{ NSOC } & $\begin{array}{c}\Gamma-Y, \Gamma-Z, \Gamma-T, T-Y, T-Z, \\
Y-Z\end{array}$ & $\| \mathrm{TiSi}_{2}$ \\
\hline & $\Gamma-\mathrm{T}, \Gamma-\mathrm{Y}, \mathrm{T}-\mathrm{Z}, \mathrm{Y}-\mathrm{Z}, \Gamma-\mathrm{Z}$ & $\mathrm{Sr}_{2} \mathrm{Cu}_{2} \mathrm{O}_{3}$ \\
\hline & $\Gamma-\mathrm{Y}, \Gamma-\mathrm{T}, \mathrm{T}-\mathrm{Z}, \mathrm{Y}-\mathrm{Z}$ & $\mathrm{Ca}_{2} \mathrm{Si}_{3} \mathrm{Ag}$ \\
\hline & $\Gamma-\mathrm{Y}, \Gamma-\mathrm{Z}, \Gamma-\mathrm{T}$ & $\| \mathrm{O}_{2}, \mathrm{NiGePt}_{2}$ \\
\hline \multicolumn{3}{|r|}{ Space Group 70: Fddd } \\
\hline \multirow{4}{*}{ NSOC } & $\Gamma-Y, \Gamma-\mathrm{T}, \Gamma-\mathrm{Z}$ & $\mathrm{Sr}(\mathrm{GaRh})_{2} \|$ \\
\hline & $\Gamma-Y, \Gamma-Z, \Gamma-\mathrm{T}$ & $\begin{array}{c}\| \text { LiBIr, } \mathrm{Ca}(\mathrm{BIr})_{2}, \mathrm{Ca}(\mathrm{BRh})_{2}, \mathrm{Sr}(\mathrm{BIr})_{2}, \mathrm{TiGe}_{2}, \mathrm{ZrSn}_{2}, \mathrm{TiSi}_{2}, \mathrm{In}_{2} \mathrm{Ir}, \\
\mathrm{Ce}(\mathrm{BRu})_{2}, \mathrm{Ce}(\mathrm{BIr})_{2}, \mathrm{Zn}(\mathrm{OF})_{2}, \mathrm{Gd}(\mathrm{BRu})_{2}, \mathrm{Ce}(\mathrm{BOs})_{2}\end{array}$ \\
\hline & $\Gamma-\mathrm{Z}, \Gamma-\mathrm{T}, \Gamma-\mathrm{Y}$ & $\| \mathrm{Sr}(\mathrm{BRh})_{2}, \mathrm{Ba}(\mathrm{GeRu})_{2} \mid \frac{5}{3}, \mathrm{Th}(\mathrm{BOs})_{2}, \mathrm{Th}(\mathrm{BRu})_{2}$ \\
\hline & $\Gamma-\mathrm{Y}, \Gamma-\mathrm{T}$ & $\| \mathrm{Gd}(\mathrm{BOs})_{2}$ \\
\hline \multicolumn{3}{|r|}{ Space Group 71: $\mathrm{Immm}$} \\
\hline \multirow{26}{*}{ NSOC } & $\begin{array}{l}\Gamma-\mathrm{X}, \mathrm{S}-\Gamma, \mathrm{R}-\Gamma, \mathrm{R}-\mathrm{X}, \mathrm{T}-\mathrm{X} \\
\text { W-R, W-S, W-T }\end{array}$ & $\| \mathrm{Ca}_{2} \mathrm{MgPt}_{2}, \mathrm{Sr}_{2} \mathrm{CdPt}_{2}$ \\
\hline & $\begin{array}{l}\Gamma-\mathrm{X}, \mathrm{S}-\Gamma, \mathrm{R}-\Gamma, \mathrm{R}-\mathrm{X}, \mathrm{T}-\mathrm{X} \\
\mathrm{W}-\mathrm{R}, \mathrm{W}-\mathrm{S}\end{array}$ & $\| \mathrm{Ca}_{2} \mathrm{CdPt}_{2}$ \\
\hline & $\begin{array}{l}\Gamma-\mathrm{X}, \mathrm{S}-\Gamma, \mathrm{S}-\mathrm{X}, \mathrm{R}-\Gamma, \mathrm{R}-\mathrm{X} \\
\mathrm{T}-\Gamma, \mathrm{T}-\mathrm{X}\end{array}$ & $\| \mathrm{LuNiGe}_{2}, \mathrm{ErNiGe}_{2}$ \\
\hline & $\begin{array}{c}\Gamma-\mathrm{X}, \mathrm{S}-\Gamma, \mathrm{S}-\mathrm{X}, \mathrm{R}-\mathrm{X}, \mathrm{T}-\Gamma \\
\text { W-S, W-T }\end{array}$ & || $\mathrm{TiMo}_{3}$ \\
\hline & $\begin{array}{c}\Gamma-\mathrm{X}, \mathrm{S}-\mathrm{X}, \mathrm{R}-\Gamma, \mathrm{R}-\mathrm{X}, \mathrm{T}-\mathrm{X} \\
\mathrm{W}-\mathrm{R}, \mathrm{W}-\mathrm{S}\end{array}$ & $\| \mathrm{Cu}_{3} \mathrm{As}_{4}$ \\
\hline & $\begin{array}{c}\Gamma-\mathrm{X}, \mathrm{R}-\Gamma, \mathrm{R}-\mathrm{X}, \mathrm{T}-\Gamma, \mathrm{T}-\mathrm{X} \\
\mathrm{W}-\mathrm{S}\end{array}$ & $\| \mathrm{ErGe}_{2} \mathrm{Pt}$ \\
\hline & $\begin{aligned} \Gamma-\mathrm{X}, \mathrm{R}-\Gamma, \mathrm{R}-\mathrm{X}, \mathrm{T}-\Gamma, \mathrm{T}-\mathrm{X} \\
\mathrm{W}-\mathrm{T}\end{aligned}$ & $\| \mathrm{Hf}_{54} \mathrm{Os}_{17}$ \\
\hline & $\begin{array}{c}\Gamma-\mathrm{X}, \mathrm{R}-\Gamma, \underset{\mathrm{R}}{\mathrm{R}-\mathrm{X}, \mathrm{T}}-\mathrm{T}, \mathrm{W}-\mathrm{R}, \\
\mathrm{W}\end{array}$ & $\| \mathrm{DyNiGe}_{2}$ \\
\hline & $\begin{array}{l}\Gamma-\mathrm{X}, \mathrm{R}-\Gamma, \mathrm{R}-\mathrm{X}, \mathrm{T}-\mathrm{X}, \mathrm{W}-\mathrm{R}, \\
\text { W-S }\end{array}$ & $\| \mathrm{Ba}_{3}(\mathrm{AlGe})_{2}, \mathrm{Ba}_{3}(\mathrm{AlSi})_{2}$ \\
\hline & $\begin{array}{c}\Gamma-\mathrm{X}, \mathrm{R}-\Gamma, \mathrm{T}-\Gamma, \mathrm{W}-\mathrm{R}, \mathrm{W}-\mathrm{S}, \\
\mathrm{W}-\mathrm{T}\end{array}$ & $\| \mathrm{Sc}_{4} \mathrm{Si}_{7} \mathrm{Ni}_{12}$ \\
\hline & $\begin{array}{l}\Gamma-\mathrm{X}, \mathrm{R}-\mathrm{X}, \mathrm{T}-\mathrm{X}, \mathrm{W}-\mathrm{R}, \mathrm{W}-\mathrm{S}, \\
\mathrm{W}-\mathrm{T}\end{array}$ & $\| \mathrm{Ca}_{2} \mathrm{CdPd}_{2}, \mathrm{Ca}_{2} \mathrm{MgPd}_{2}$ \\
\hline & $\begin{array}{l}\Gamma-\mathrm{X}, \mathrm{S}-\Gamma, \mathrm{R}-\Gamma, \mathrm{R}-\mathrm{X}, \mathrm{T}-\Gamma, \\
\mathrm{T}-\mathrm{X}\end{array}$ & $\| \mathrm{HoGe}_{2} \mathrm{Pt}, \mathrm{TbGe}_{2} \mathrm{Pt}, \mathrm{SmGe}_{2} \mathrm{Pt}$ \\
\hline & $\begin{array}{c}\Gamma-\mathrm{X}, \mathrm{S}-\Gamma, \mathrm{R}-\Gamma, \mathrm{T}-\Gamma, \mathrm{W}-\mathrm{S} \\
\mathrm{W}-\mathrm{T}\end{array}$ & $\| \mathrm{LaAl}_{5} \mathrm{Ni}_{2}$ \\
\hline & $\begin{array}{c}\Gamma-\mathrm{X}, \mathrm{S}-\Gamma, \mathrm{R}-\mathrm{X}, \mathrm{T}-\Gamma, \mathrm{T}-\mathrm{X} \\
\mathrm{W}-\mathrm{R}\end{array}$ & $\| \mathrm{TbGe}_{2} \mathrm{Pd}$ \\
\hline & $\begin{array}{c}\Gamma-\mathrm{X}, \mathrm{S}-\mathrm{\Gamma}, \mathrm{R}-\mathrm{X}, \mathrm{T}-\mathrm{X}, \mathrm{W}-\mathrm{R}, \\
\mathrm{W}-\mathrm{S}\end{array}$ & $\| \mathrm{Fe}_{2} \mathrm{~B}_{4} \mathrm{Mo}$ \\
\hline & $\begin{array}{c}\Gamma-\mathrm{X}, \mathrm{S}-\Gamma, \mathrm{S}-\mathrm{X}, \mathrm{R}-\Gamma, \mathrm{T}-\Gamma \\
\mathrm{W}-\mathrm{T}\end{array}$ & $\| \mathrm{Lu}_{6} \mathrm{Ni}_{2} \mathrm{Sn}$ \\
\hline & $\begin{array}{c}\Gamma-\mathrm{X}, \mathrm{S}-\mathrm{X}, \mathrm{R}-\Gamma, \mathrm{T}-\Gamma, \mathrm{T}-\mathrm{X} \\
\mathrm{W}-\mathrm{S}\end{array}$ & $\| \mathrm{La}_{2} \mathrm{ZnNi}_{2}$ \\
\hline & $\begin{array}{l}\mathrm{R}-\Gamma, \mathrm{R}-\mathrm{X}, \mathrm{T}-\mathrm{\Gamma}, \mathrm{T}-\mathrm{X}, \mathrm{W}-\mathrm{S}, \\
\mathrm{W}-\mathrm{T}\end{array}$ & $\| \mathrm{Ba}\left(\mathrm{NiP}_{2}\right)_{2}$ \\
\hline & $\Gamma-\mathrm{X}, \mathrm{R}-\Gamma, \mathrm{R}-\mathrm{X}, \mathrm{T}-\Gamma, \mathrm{W}-\mathrm{R}$ & $\| \mathrm{HoGe}_{2} \mathrm{Ir}$ \\
\hline & $\Gamma-\mathrm{X}, \mathrm{R}-\Gamma, \mathrm{R}-\mathrm{X}, \mathrm{T}-\mathrm{X}, \mathrm{W}-\mathrm{S}$ & $\mathrm{Hf}_{3}(\mathrm{NiGe})_{4}, \mathrm{Hf}_{27} \mathrm{P}_{16}$ \\
\hline & $\Gamma-\mathrm{X}, \mathrm{R}-\Gamma, \mathrm{T}-\Gamma, \mathrm{T}-\mathrm{X}, \mathrm{W}-\mathrm{S}$ & $\| \mathrm{Fe}(\mathrm{BW})_{2}$ \\
\hline & $\Gamma-\mathrm{X}, \mathrm{R}-\mathrm{X}, \mathrm{T}-\Gamma, \mathrm{T}-\mathrm{X}, \mathrm{W}-\mathrm{R}$ & $\mathrm{ErGe}_{2} \operatorname{Ir}, \mathrm{TbGe}_{2} \mathrm{Ir}$ \\
\hline & $\Gamma-\mathrm{X}, \mathrm{R}-\mathrm{X}, \mathrm{T}-\Gamma, \mathrm{T}-\mathrm{X}, \mathrm{W}-\mathrm{S}$ & $\mathrm{Sn} \|$ \\
\hline & $\Gamma-\mathrm{X}, \mathrm{S}-\Gamma, \mathrm{R}-\Gamma, \mathrm{R}-\mathrm{X}, \mathrm{W}-\mathrm{R}$ & $\| \mathrm{CeSi}_{5}, \mathrm{CeGe}_{5}$ \\
\hline & $\Gamma-\mathrm{X}, \mathrm{S}-\Gamma, \mathrm{R}-\Gamma, \mathrm{T}-\Gamma, \mathrm{T}-\mathrm{X}$ & $\| \mathrm{Tb}_{2} \mathrm{ZnNi}_{2}$ \\
\hline & $\Gamma-\mathrm{X}, \mathrm{S}-\Gamma, \mathrm{R}-\Gamma, \mathrm{T}-\Gamma, \mathrm{W}-\mathrm{S}$ & $\| \mathrm{PrAl}_{5} \mathrm{Ni}_{2}$ \\
\hline
\end{tabular}


Table III - continued

\begin{tabular}{|c|c|c|}
\hline $\begin{array}{l}\text { SOC or } \\
\text { NSOC }\end{array}$ & $\begin{array}{l}\text { HSL with Band } \\
\text { Crossing }\end{array}$ & High Symmetry Line Semimetals \\
\hline & $\Gamma-\mathrm{X}, \mathrm{S}-\Gamma, \mathrm{R}-\mathrm{X}, \mathrm{T}-\mathrm{X}, \mathrm{W}-\mathrm{T}$ & $\mathrm{Tb}_{2} \mathrm{Ni}_{2} \mathrm{Sn}$ \\
\hline & $\Gamma-\mathrm{X}, \mathrm{S}-\Gamma, \mathrm{S}-\mathrm{X}, \mathrm{T}-\Gamma, \mathrm{W}-\mathrm{S}$ & $\mathrm{Er}_{6} \mathrm{Ni}_{2} \mathrm{Sn}$ \\
\hline & $\Gamma-\mathrm{X}, \mathrm{S}-\Gamma, \mathrm{S}-\mathrm{X}, \mathrm{T}-\mathrm{X}, \mathrm{W}-\mathrm{S}$ & $\mathrm{Ce}_{3} \mathrm{Zn}_{11}$ \\
\hline & $\Gamma-\mathrm{X}, \mathrm{S}-\Gamma, \mathrm{T}-\Gamma, \mathrm{W}-\mathrm{R}, \mathrm{W}-\mathrm{S}$ & $\mathrm{Ce}_{2} \mathrm{Ni}_{2} \mathrm{Sn}$ \\
\hline & $\Gamma-\mathrm{X}, \mathrm{S}-\Gamma, \mathrm{T}-\mathrm{X}, \mathrm{W}-\mathrm{S}, \mathrm{W}-\mathrm{T}$ & $\mathrm{Ce}_{2} \mathrm{ZnNi}_{2}$ \\
\hline & $\Gamma-\mathrm{X}, \mathrm{S}-\mathrm{X}, \mathrm{R}-\Gamma, \mathrm{R}-\mathrm{X}, \mathrm{T}-\Gamma$ & $\mathrm{Ce}_{3}\left(\mathrm{BN}_{2}\right)_{2}$ \\
\hline & $\Gamma-\mathrm{X}, \mathrm{S}-\mathrm{X}, \mathrm{R}-\mathrm{X}, \mathrm{W}-\mathrm{S}, \mathrm{W}-\mathrm{T}$ & $\| \mathrm{La}_{3} \mathrm{Al}_{11}$ \\
\hline & $\Gamma-\mathrm{X}, \mathrm{S}-\mathrm{X}, \mathrm{T}-\Gamma, \mathrm{W}-\mathrm{R}, \mathrm{W}-\mathrm{T}$ & $\mathrm{Sm}_{6} \mathrm{InCo}_{2}$ \\
\hline & $\mathrm{S}-\Gamma, \mathrm{S}-\mathrm{X}, \mathrm{R}-\Gamma, \mathrm{R}-\mathrm{X}, \mathrm{W}-\mathrm{S}$ & || $\mathrm{Ti}_{3} \mathrm{~B}_{4}, \mathrm{BaYb}_{2} \mathrm{NiO}_{5}$ \\
\hline & $\Gamma-\mathrm{X}, \mathrm{R}-\Gamma, \mathrm{R}-\mathrm{X}, \mathrm{T}-\Gamma$ & $\| \mathrm{Ca}_{3}(\mathrm{AlSi})_{2}, \mathrm{Sr}_{3}(\mathrm{AlGe})_{2}, \underset{\mathrm{DyGe}}{\mathrm{Sr}_{3}(\mathrm{AlSn})_{2}, \mathrm{Sr}_{3}(\mathrm{AlSi})_{2}, \mathrm{Ba}_{3}(\mathrm{AlSn})_{2},}$ \\
\hline & $\Gamma-\mathrm{X}, \mathrm{R}-\Gamma, \mathrm{R}-\mathrm{X}, \mathrm{W}-\mathrm{T}$ & $\| \mathrm{Ti}_{6} \mathrm{Sn}_{5}$ \\
\hline & $\Gamma-\mathrm{X}, \mathrm{R}-\Gamma, \mathrm{T}-\Gamma, \mathrm{T}-\mathrm{X}$ & $\mathrm{Sc}_{3} \mathrm{RhC}_{4}, \mathrm{Sc}_{3} \mathrm{IrC}_{4}, \mathrm{Sc}_{3} \mathrm{CoC}_{4}$ \\
\hline & $\Gamma-\mathrm{X}, \mathrm{R}-\mathrm{X}, \mathrm{T}-\Gamma, \mathrm{W}-\mathrm{R}$ & $\| \mathrm{YGe}_{2} \mathrm{Ir}, \mathrm{NdGe} \mathrm{Ge}_{2} \mathrm{Ir}$ \\
\hline & $\Gamma-\mathrm{X}, \mathrm{R}-\mathrm{X}, \mathrm{T}-\Gamma, \mathrm{W}-\mathrm{S}$ & $\| \mathrm{YbNiGe}_{2}$ \\
\hline & $\Gamma-\mathrm{X}, \mathrm{R}-\mathrm{X}, \mathrm{T}-\mathrm{X}, \mathrm{W}-\mathrm{R}$ & $\mathrm{Th}_{2} \mathrm{MnN}_{3}$ \\
\hline & $\Gamma-\mathrm{X}, \mathrm{R}-\mathrm{X}, \mathrm{T}-\mathrm{X}, \mathrm{W}-\mathrm{S}$ & $\mathrm{Sc}_{6} \mathrm{Si}_{11} \mathrm{Ni}_{18}, \mathrm{Sc}_{6} \mathrm{Ni}_{18} \mathrm{Ge}_{11}$ \\
\hline & $\Gamma-\mathrm{X}, \mathrm{R}-\mathrm{X}, \mathrm{W}-\mathrm{R}, \mathrm{W}-\mathrm{S}$ & $\| \mathrm{Pt}_{2} \mathrm{~W}, \mathrm{MoPt}_{2}$ \\
\hline & $\Gamma-\mathrm{X}, \mathrm{S}-\Gamma, \mathrm{S}-\mathrm{X}, \mathrm{W}-\mathrm{S}$ & $\| \mathrm{Yb}_{3}(\mathrm{GePd})_{4}$ \\
\hline & $\Gamma-\mathrm{X}, \mathrm{S}-\Gamma, \mathrm{T}-\mathrm{X}, \mathrm{W}-\mathrm{R}$ & || NbPSe \\
\hline & $\Gamma-\mathrm{X}, \mathrm{S}-\mathrm{X}, \mathrm{R}-\Gamma, \mathrm{R}-\mathrm{X}$ & $\mathrm{Hf}_{3}(\mathrm{CuSi})_{4}, \mathrm{Zr}_{3}(\mathrm{CuSi})_{4}$ \\
\hline & $\Gamma-\mathrm{X}, \mathrm{S}-\mathrm{X}, \mathrm{R}-\mathrm{X}, \mathrm{W}-\mathrm{R}$ & $\mathrm{Tm}_{6} \mathrm{GaCo}_{2}, \mathrm{Ho}_{6} \mathrm{GaCo}_{2}$ \\
\hline & $\Gamma-\mathrm{X}, \mathrm{S}-\mathrm{X}, \mathrm{R}-\mathrm{X}, \mathrm{W}-\mathrm{S}$ & $\| \mathrm{Cr}_{3} \mathrm{~B}_{4}$ \\
\hline & $\Gamma-\mathrm{X}, \mathrm{T}-\Gamma, \mathrm{T}-\mathrm{X}, \mathrm{W}-\mathrm{R}$ & $\mathrm{Yb}_{3}(\mathrm{CuGe})_{4}$ \\
\hline & $\Gamma-\mathrm{X}, \mathrm{T}-\Gamma, \mathrm{T}-\mathrm{X}, \mathrm{W}-\mathrm{S}$ & $\| \mathrm{Ca}_{3} \mathrm{Ga}_{8}$ \\
\hline & $\Gamma-\mathrm{X}, \mathrm{T}-\Gamma, \mathrm{W}-\mathrm{R}, \mathrm{W}-\mathrm{S}$ & $\| \mathrm{LiCe}_{2} \mathrm{HO}_{3}$ \\
\hline & $\mathrm{R}-\Gamma, \mathrm{R}-\mathrm{X}, \mathrm{T}-\Gamma, \mathrm{T}-\mathrm{X}$ & $\mathrm{Sr}_{8} \mathrm{In}_{4} \mathrm{Cu}_{3} \mathrm{~N}_{5}, \mathrm{Pr}_{2} \mathrm{Co}_{2} \mathrm{SiC}, \mathrm{Nd}_{2} \mathrm{Co}_{2} \mathrm{SiC}$ \\
\hline & $\mathrm{S}-\Gamma, \mathrm{S}-\mathrm{X}, \mathrm{T}-\Gamma, \mathrm{T}-\mathrm{X}$ & $\| \mathrm{SrSi}, \mathrm{HfFeSi}_{2}$ \\
\hline & $\Gamma-\mathrm{X}, \mathrm{R}-\Gamma, \mathrm{T}-\mathrm{X}$ & $\| \mathrm{Yb}_{3} \mathrm{Cu}_{6} \mathrm{Sn}_{5}$ \\
\hline & $\Gamma-\mathrm{X}, \mathrm{R}-\Gamma, \mathrm{W}-\mathrm{S}$ & $\| \mathrm{Sr}(\mathrm{NiP})_{2}$ \\
\hline & $\Gamma-\mathrm{X}, \mathrm{R}-\mathrm{X}, \mathrm{T}-\Gamma$ & $\| \operatorname{LaTm}\left(\mathrm{Ge}_{2} \operatorname{Ir}\right)_{2}$ \\
\hline & $\Gamma-\mathrm{X}, \mathrm{R}-\mathrm{X}, \mathrm{W}-\mathrm{R}$ & $\mathrm{Ni}(\mathrm{BW})_{2}, \mathrm{Ni}(\mathrm{BMo})_{2}, \mathrm{Ce}\left(\mathrm{CrB}_{3}\right)_{2}$ \\
\hline & $\Gamma-\mathrm{X}, \mathrm{R}-\mathrm{X}, \mathrm{W}-\mathrm{S}$ & $\| \mathrm{Dy}_{2} \mathrm{~B}_{4} \mathrm{C}$ \\
\hline & $\Gamma-\mathrm{X}, \mathrm{S}-\Gamma, \mathrm{W}-\mathrm{R}$ & $\mathrm{Co}_{2} \mathrm{~B}_{4} \mathrm{Mo}$ \\
\hline & $\Gamma-\mathrm{X}, \mathrm{S}-\mathrm{X}, \mathrm{T}-\Gamma$ & $\mathrm{Sm}_{6} \mathrm{GaCo}_{2}$ \\
\hline & $\Gamma-\mathrm{X}, \mathrm{T}-\Gamma, \mathrm{W}-\mathrm{S}$ & $\| \mathrm{TiPd}_{2}$ \\
\hline & $\Gamma-\mathrm{X}, \mathrm{T}-\mathrm{X}, \mathrm{W}-\mathrm{S}$ & TiTeAs \| \\
\hline & $\Gamma-\mathrm{X}, \mathrm{T}-\mathrm{X}, \mathrm{W}-\mathrm{T}$ & $\mid \mathrm{Th}\left(\mathrm{CrB}_{3}\right)_{2}$ \\
\hline & $\mathrm{S}-\Gamma, \mathrm{S}-\mathrm{X}, \mathrm{W}-\mathrm{S}$ & $\| \mathrm{RuCl}_{2} \mathrm{O}$ \\
\hline & T- $\Gamma, T-X, W-R$ & $\|$ TaPS, NbPS \\
\hline & $\Gamma-\mathrm{X}, \mathrm{S}-\Gamma$ & $\mathrm{Ca}_{3} \mathrm{SiBr}_{2}, \| \mathrm{ZrMnSi}_{2}$ \\
\hline & $\Gamma-\mathrm{X}, \mathrm{S}-\mathrm{X}$ & $\mathrm{CrB}_{4} \|$ \\
\hline & $\Gamma-\mathrm{X}, \mathrm{T}-\mathrm{X}$ & $\| \mathrm{CaC}_{2}$ \\
\hline & $\mathrm{R}-\Gamma, \mathrm{R}-\mathrm{X}$ & $\| \mathrm{CaBi}_{2}\left(\mathrm{CO}_{4}\right)_{2}, \mathrm{Sr}_{10} \mathrm{Al}_{4} \mathrm{Si}_{6} \mathrm{O}$ \\
\hline & S- $\Gamma, S-X$ & $\mathrm{Ba}_{3}(\mathrm{LiSb})_{4}, \mathrm{Sr}_{3}(\mathrm{LiSb})_{4}, \mathrm{Ba}_{3}(\mathrm{LiBi})_{4}$ \\
\hline & W-R, W-S & $\| \mathrm{Ce}_{2} \mathrm{MnN}_{3}$ \\
\hline & $\mathrm{W}-\mathrm{R}$ & $\mathrm{Li}_{2} \mathrm{H}_{2} \mathrm{Pt}, \| \mathrm{PdN}_{2}, \mathrm{Ca}_{3}(\mathrm{AlGe})_{2}$ \\
\hline & $\mathrm{W}-\mathrm{S}$ & $\mathrm{CsFeS}_{2}, \| \mathrm{Pr}_{2} \mathrm{Ni}_{2} \mathrm{Sn}, \mathrm{Y}_{2} \mathrm{Ni}_{2} \mathrm{Sn}$ \\
\hline & & Space Group 72: Ibam \\
\hline & $\begin{array}{c}\Gamma-\mathrm{X}, \mathrm{S}-\Gamma, \mathrm{S}-\mathrm{X}, \mathrm{R}-\Gamma, \mathrm{R}-\mathrm{X} \\
\mathrm{T}-\Gamma, \mathrm{T}-\mathrm{X}\end{array}$ & $\| \mathrm{Sc}_{6} \mathrm{~Pb}_{5}$ \\
\hline & $\begin{array}{l}\Gamma-\mathrm{X}, \mathrm{S}-\Gamma, \mathrm{R}-\Gamma, \mathrm{R}-\mathrm{X}, \mathrm{T}-\mathrm{X} \\
\mathrm{W}-\mathrm{T}\end{array}$ & $\| \mathrm{Ti}_{6} \mathrm{Ge}_{5}$ \\
\hline & $\begin{array}{c}\Gamma-\mathrm{X}, \mathrm{S}-\Gamma, \mathrm{S}-\mathrm{X}, \mathrm{R}-\Gamma, \mathrm{R}-\mathrm{X} \\
\mathrm{W}-\mathrm{T}\end{array}$ & $\| \mathrm{Y}_{2} \mathrm{Si}_{5} \mathrm{Ni}_{3}, \mathrm{Ho}_{2} \mathrm{Si}_{5} \mathrm{Ni}_{3}, \mathrm{~Tb}_{2} \mathrm{Si}_{5} \mathrm{Ni}_{3}$ \\
\hline & $\Gamma-\mathrm{X}, \mathrm{R}-\Gamma, \mathrm{T}-\Gamma, \mathrm{T}-\mathrm{X}, \mathrm{W}-\mathrm{T}$ & $\| \mathrm{Sc}_{2} \mathrm{Cr}_{4} \mathrm{Si}_{5}$ \\
\hline & $\Gamma-\mathrm{X}, \mathrm{S}-\Gamma, \mathrm{S}-\mathrm{X}, \mathrm{R}-\mathrm{X}, \mathrm{W}-\mathrm{T}$ & $\| \mathrm{V}_{6} \mathrm{Si}_{5}, \mathrm{Ta}_{2} \mathrm{~V}_{4} \mathrm{Si}_{5}$ \\
\hline & $\Gamma-\mathrm{X}, \mathrm{S}-\mathrm{X}, \mathrm{R}-\Gamma, \mathrm{T}-\mathrm{X}, \mathrm{W}-\mathrm{T}$ & $\| \mathrm{Mn}_{4} \mathrm{Nb}_{2} \mathrm{Si}_{5}$ \\
\hline & $\Gamma-\mathrm{X}, \mathrm{S}-\mathrm{X}, \mathrm{T}-\Gamma, \mathrm{T}-\mathrm{X}, \mathrm{W}-\mathrm{T}$ & $\| \mathrm{Ni}(\mathrm{TePd})_{2}$ \\
\hline
\end{tabular}


Table III - continued

\begin{tabular}{|c|c|c|}
\hline \multirow[t]{15}{*}{$\begin{array}{l}\text { SOC or } \\
\text { NSOC }\end{array}$} & $\begin{array}{l}\text { HSL with Band } \\
\text { Crossing }\end{array}$ & High Symmetry Line Semimetals \\
\hline & $\Gamma-\mathrm{X}, \mathrm{S}-\Gamma, \mathrm{R}-\Gamma, \mathrm{T}-\mathrm{X}$ & $\mathrm{Mg}_{5} \mathrm{Ga}_{2}, \mathrm{Mg}_{5} \mathrm{In}_{2}$ \\
\hline & $\Gamma-\mathrm{X}, \mathrm{S}-\Gamma, \mathrm{R}-\mathrm{X}, \mathrm{W}-\mathrm{T}$ & $\| \mathrm{Dy}_{2} \mathrm{Si}_{5} \mathrm{Ni}_{3}$ \\
\hline & $\Gamma-\mathrm{X}, \mathrm{S}-\Gamma, \mathrm{S}-\mathrm{X}, \mathrm{W}-\mathrm{T}$ & $\| \mathrm{Y}_{2} \mathrm{Ge}_{5} \mathrm{Ru}_{3}, \mathrm{Sm}_{2} \mathrm{Ge}_{5} \mathrm{Ru}_{3}, \mathrm{~Tb}_{2} \mathrm{Si}_{5} \mathrm{Ru}_{3}, \mathrm{Er}_{2} \mathrm{Si}_{5} \mathrm{Ru}_{3}, \mathrm{La}_{2} \mathrm{Ge}_{5} \mathrm{Ru}_{3}$ \\
\hline & $\Gamma-\mathrm{X}, \mathrm{R}-\Gamma, \mathrm{R}-\mathrm{X}$ & $\| \mathrm{Sb}_{2} \mathrm{Pt}_{3}$ \\
\hline & $\Gamma-\mathrm{X}, \mathrm{R}-\Gamma, \mathrm{T}-\mathrm{X}$ & $\mathrm{Mg}_{5} \mathrm{Tl}_{2}$ \\
\hline & $\Gamma-\mathrm{X}, \mathrm{S}-\Gamma, \mathrm{T}-\Gamma$ & $\| \mathrm{Ti}_{3} \mathrm{Pt}_{5}$ \\
\hline & $\Gamma-\mathrm{X}, \mathrm{S}-\Gamma, \mathrm{W}-\mathrm{T}$ & $\| \mathrm{Nb}_{2} \mathrm{~V}_{4} \mathrm{Si}_{5}$ \\
\hline & $\Gamma-\mathrm{X}, \mathrm{S}-\mathrm{X}, \mathrm{R}-\mathrm{X}$ & $\mathrm{Ti}_{2} \mathrm{Mn}_{4} \mathrm{Si}_{5}$ \\
\hline & $\Gamma-\mathrm{X}, \mathrm{S}-\mathrm{X}, \mathrm{T}-\mathrm{X}$ & $\mathrm{Zr}_{2} \mathrm{Cr}_{4} \mathrm{Si}_{5}$ \\
\hline & $\mathrm{T}-\Gamma, \mathrm{T}-\mathrm{X}, \mathrm{W}-\mathrm{T}$ & $\| \mathrm{Tl}_{2} \mathrm{Fe}_{3} \mathrm{~S}_{4}$ \\
\hline & $\Gamma-\mathrm{X}, \mathrm{R}-\mathrm{X}$ & $\| \mathrm{Fe}(\mathrm{PdSe})_{2}, \mathrm{Nb}_{4} \mathrm{Cr}_{2} \mathrm{Si}_{5}$ \\
\hline & $\Gamma-\mathrm{X}, \mathrm{S}-\Gamma$ & $\mathrm{Hg}_{5}\left(\mathrm{BrO}_{2}\right)_{2} \|$ \\
\hline & $\Gamma-\mathrm{X}, \mathrm{T}-\Gamma$ & $\| \mathrm{Hg}_{5}\left(\mathrm{ClO}_{2}\right)_{2}$ \\
\hline & $\Gamma-\mathrm{X}, \mathrm{W}-\mathrm{T}$ & $\| \mathrm{Nb}_{2} \mathrm{Cr}_{4} \mathrm{Si}_{5}(\mathrm{ICSD}: 42905), \mathrm{Nb}_{2} \mathrm{Cr}_{4} \mathrm{Si}_{5}(\mathrm{ICSD}: 626410), \mathrm{Ta}_{2} \mathrm{Cr}_{4} \mathrm{Si}_{5}$ \\
\hline \multicolumn{3}{|r|}{ Space Group 74: Imma } \\
\hline \multirow{22}{*}{ NSOC } & $\begin{array}{c}-\mathrm{X}, \mathrm{S}-\Gamma, \mathrm{S}-\mathrm{X}, \mathrm{R}-\Gamma, \mathrm{R}-\mathrm{X} \\
\mathrm{T}-\Gamma, \mathrm{W}-\mathrm{R}, \mathrm{W}-\mathrm{S}\end{array}$ & $\| \mathrm{Te}$ \\
\hline & $\begin{array}{c}\Gamma-\mathrm{X}, \mathrm{R}-\Gamma, \mathrm{R}-\mathrm{X}, \mathrm{T}-\mathrm{X}, \mathrm{W}-\mathrm{R}, \\
\text { W-S }\end{array}$ & $\| \mathrm{Ca}_{2} \mathrm{Os}_{2} \mathrm{O}_{7}$ \\
\hline & $\begin{array}{c}\Gamma-\mathrm{X}, \mathrm{S}-\Gamma, \mathrm{S}-\mathrm{X}, \mathrm{R}-\Gamma, \mathrm{R}-\mathrm{X} \\
\mathrm{T}-\Gamma\end{array}$ & $\| \mathrm{Ge}, \mathrm{NaPO}$ \\
\hline & $\Gamma-\mathrm{X}, \mathrm{R}-\Gamma, \mathrm{T}-\Gamma, \mathrm{T}-\mathrm{X}, \mathrm{W}-\mathrm{S}$ & $\| \mathrm{BaGe}_{5}$ \\
\hline & $\Gamma-\mathrm{X}, \mathrm{S}-\Gamma, \mathrm{R}-\mathrm{X}, \mathrm{T}-\mathrm{X}, \mathrm{W}-\mathrm{R}$ & $\pi \mathrm{HoAlB}_{14}$ \\
\hline & $\Gamma-\mathrm{X}, \mathrm{S}-\Gamma, \mathrm{T}-\Gamma, \mathrm{W}-\mathrm{R}, \mathrm{W}-\mathrm{S}$ & TiMo \\
\hline & $\Gamma-\mathrm{X}, \mathrm{S}-\mathrm{X}, \mathrm{T}-\mathrm{X}, \mathrm{W}-\mathrm{R}, \mathrm{W}-\mathrm{S}$ & $\| \mathrm{K}_{3} \mathrm{PbAu}_{5}$ \\
\hline & $\Gamma-\mathrm{X}, \mathrm{R}-\mathrm{X}, \mathrm{T}-\Gamma, \mathrm{W}-\mathrm{S}$ & $\mathrm{PuAl}_{4}, \mathrm{PuGa}_{4}$ \\
\hline & $\Gamma-\mathrm{X}, \mathrm{R}-\mathrm{X}, \mathrm{W}-\mathrm{R}, \mathrm{W}-\mathrm{S}$ & $\| \mathrm{HfV}_{2}$ \\
\hline & $\Gamma-\mathrm{X}, \mathrm{S}-\mathrm{X}, \mathrm{T}-\Gamma, \mathrm{T}-\mathrm{X}$ & $\| \mathrm{CaAu}_{2}, \mathrm{YbAg}_{2}, \mathrm{YbCu}_{2}, \mathrm{CaAg}_{2}$ \\
\hline & $\Gamma-\mathrm{X}, \mathrm{R}-\Gamma, \mathrm{R}-\mathrm{X}$ & $\| \mathrm{BaZn}_{2}$ \\
\hline & Г-X, R-X, T-X & $\| \mathrm{CeZn}_{2}$ \\
\hline & $\Gamma-\mathrm{X}, \mathrm{S}-\Gamma, \mathrm{R}-\Gamma$ & $\mathrm{Ag}_{2} \mathrm{Te}_{2} \mathrm{O}_{7} \|$ \\
\hline & $\Gamma-\mathrm{X}, \mathrm{S}-\Gamma, \mathrm{S}-\mathrm{X}$ & $\| \mathrm{YbZn}_{2}, \mathrm{CaZn}_{2}, \mathrm{SrZn}_{2}, \mathrm{SrCd}_{2}, \mathrm{SrHg}_{2}, \mathrm{CaCd}_{2}$ \\
\hline & $\Gamma-\mathrm{X}, \mathrm{S}-\mathrm{X}, \mathrm{R}-\mathrm{X}$ & $\mathrm{NaB}_{15} \|$ \\
\hline & $\Gamma-\mathrm{X}, \mathrm{S}-\mathrm{X}, \mathrm{T}-\mathrm{X}$ & $\| \mathrm{SrAu}_{2}, \mathrm{SrAg}_{2}, \mathrm{BaAg}_{2}$ \\
\hline & $\Gamma-\mathrm{X}, \mathrm{T}-\mathrm{X}, \mathrm{W}-\mathrm{R}$ & $\| \mathrm{ErSi}_{2} \mathrm{Rh}_{3}$ \\
\hline & $\Gamma-\mathrm{X}, \mathrm{R}-\mathrm{X}$ & $\| \mathrm{SrAl}_{2}$ \\
\hline & $\Gamma-\mathrm{X}, \mathrm{S}-\Gamma$ & "II TlRe \\
\hline & $\Gamma-\mathrm{X}, \mathrm{S}-\mathrm{X}$ & $\| \mathrm{BaCd}_{2}$ \\
\hline & $\Gamma-\mathrm{X}, \mathrm{T}-\Gamma$ & $\mathrm{Si}, \mathrm{CuAu}, \mathrm{UMn}_{2}$ \\
\hline & $\Gamma-\mathrm{X}, \mathrm{T}-\mathrm{X}$ & $\| \mathrm{BaIn}_{2}$ \\
\hline \multicolumn{3}{|r|}{ Space Group 82: $I \overline{4}$} \\
\hline \multirow{2}{*}{ NSOC } & $\Gamma-\mathrm{M}, \mathrm{P}-\mathrm{X}$ & $\| \mathrm{Cr}_{3} \mathrm{P}$ \\
\hline & $\mathrm{P}-\mathrm{X}$ & $\| \mathrm{Ni}_{3} \mathrm{P}, \mathrm{Tl}_{5} \mathrm{Te}_{3}, \mathrm{Zr}_{3} \mathrm{Te}, \mathrm{Tc}_{3} \mathrm{P}$ \\
\hline \multicolumn{3}{|r|}{ Space Group 83: $P 4 / m$} \\
\hline \multirow{3}{*}{$\mathrm{SOC}$} & $\mathrm{A}-\mathrm{M}, \Gamma-\mathrm{Z}$ & $\| \mathrm{V}_{3} \mathrm{As}_{2}$ \\
\hline & $\mathrm{A}-\mathrm{M}$ & $\| \mathrm{Nd}_{5} \mathrm{Cu}_{5} \mathrm{O}_{13}$ \\
\hline & $\Gamma-\mathrm{Z}$ & $\| \mathrm{Ho}_{7} \mathrm{In}\left(\mathrm{CoGe}_{3}\right)_{4}$ \\
\hline \multicolumn{3}{|r|}{ Space Group 84: $P 4_{2} / m$} \\
\hline \multirow{2}{*}{ NSOC } & A-M, Г-Z, X-M, Г-M & $\mathrm{Sr}_{2} \mathrm{~B}_{8} \mathrm{Ru}_{7}$ \\
\hline & $\Gamma-\mathrm{Z}$ & PdSe $\|$ \\
\hline \multirow{2}{*}{$\mathrm{SOC}$} & A-M & $\| \mathrm{Sr}_{2} \mathrm{~B}_{8} \mathrm{Ru}_{7}$ \\
\hline & $\Gamma-\mathrm{Z}$ & PdSe $\|$ \\
\hline \multicolumn{3}{|r|}{ Space Group 85: $P 4 / n$} \\
\hline NSOC & $\Gamma-Z$ & $\| \mathrm{Ba}\left(\mathrm{RuO}_{2}\right)_{6}$ \\
\hline $\mathrm{SOC}$ & $\Gamma-\mathrm{Z}$ & $\mathrm{Ba}\left(\mathrm{RuO}_{2}\right)_{6}, \mathrm{Tl}_{5} \mathrm{Se}_{3}$ \\
\hline \multicolumn{3}{|r|}{ Space Group 86: $P 4_{2} / n$} \\
\hline & A-M, R-A, A-Z, X-Г, Г-M & $\| \mathrm{V}_{3} \mathrm{P}$ \\
\hline
\end{tabular}




\begin{tabular}{|c|c|c|}
\hline $\begin{array}{l}\text { SOC or } \\
\text { NSOC }\end{array}$ & $\begin{array}{l}\text { HSL with Band } \\
\text { Crossing }\end{array}$ & High Symmetry Line Semimetals \\
\hline & $\mathrm{A}-\mathrm{M}, \Gamma-\mathrm{Z}, \mathrm{X}-\Gamma, \Gamma-\mathrm{M}$ & $\| \mathrm{Hf}_{3} \mathrm{P}$ \\
\hline & $\Gamma-\mathrm{Z}, \mathrm{X}-\Gamma, \Gamma-\mathrm{M}$ & $\| \mathrm{Tm}_{3} \mathrm{Sb}$ \\
\hline & $\mathrm{R}-\mathrm{A}, \mathrm{A}-\mathrm{Z}, \Gamma-\mathrm{Z}$ & $\mathrm{Mg}_{15} \mathrm{Si}_{2} \operatorname{Ir}_{5}$ \\
\hline & $\mathrm{A}-\mathrm{M}$ & $\| \mathrm{Zr}_{3} \mathrm{P}$ \\
\hline \multirow{2}{*}{$\mathrm{SOC}$} & A-M & $\mathrm{Nb}_{3} \mathrm{As}, \mathrm{Nb}_{3} \mathrm{P}, \mathrm{Ta}_{3} \mathrm{P}, \mathrm{V}_{3} \mathrm{P}$ \\
\hline & $\Gamma-\mathrm{Z}$ & $\| \mathrm{Hf}_{3} \mathrm{P}$ \\
\hline \multicolumn{3}{|r|}{ Space Group 87: $I 4 / m$} \\
\hline \multirow{4}{*}{ NSOC } & $\Gamma-\mathrm{M}, \mathrm{X}-\Gamma, \mathrm{X}-\mathrm{M}$ & $\mathrm{TiAu}_{4}$ \\
\hline & $\Gamma-\mathrm{M}, \mathrm{X}-\mathrm{M}$ & $\| \mathrm{Ti}_{4} \mathrm{O}_{5}$ \\
\hline & $\Gamma-\mathrm{M}, \mathrm{X}-\mathrm{P}$ & $\mathrm{Zr}_{9} \mathrm{Pt}_{11}, \mathrm{Zr}_{9} \mathrm{Ni}_{11}, \mathrm{KTb}_{3} \mathrm{~F}_{12}$ \\
\hline & $\mathrm{X}-\mathrm{M}, \Gamma-\mathrm{M}$ & $\| \mathrm{Si}_{5} \mathrm{Pt}_{12}$ \\
\hline SOC & $\Gamma-\mathrm{M}$ & $\begin{array}{l}\| \mathrm{Te}(\mathrm{HO})_{6}, \mathrm{Ba}\left(\mathrm{Cu}_{2} \mathrm{P}\right)_{4}, \mathrm{Nd}\left(\mathrm{MoO}_{2}\right)_{6}, \mathrm{Pr}\left(\mathrm{MoO}_{2}\right)_{6}, \mathrm{Si}_{5} \mathrm{Pt}_{12}, \\
\mathrm{Li}_{4} \mathrm{Yb}_{5} \mathrm{Ge}_{4}, \mathrm{Zr}_{9} \mathrm{Pt}_{11}, \mathrm{Zr}_{9} \mathrm{Ni}_{11}, \mathrm{Fe}_{4} \mathrm{C}, \mathrm{Ba}_{6} \mathrm{Fe}_{8} \mathrm{~S}_{15}, \mathrm{KTb}_{3} \mathrm{~F}_{12}\end{array}$ \\
\hline \multicolumn{3}{|r|}{ Space Group 88: $I 4_{1} / a$} \\
\hline \multirow{2}{*}{ NSOC } & $\Gamma-\mathrm{M}, \mathrm{X}-\Gamma$ & $\| \mathrm{Ge}_{8} \mathrm{Pd}_{21}$ \\
\hline & $\Gamma-\mathrm{M}$ & || $\mathrm{ThBr}_{4}, \mathrm{NaPO}_{3}(\mathrm{ICSD}: 35197)$ \\
\hline $\mathrm{SOC}$ & $\Gamma-\mathrm{M}$ & $\| \mathrm{ThBr}_{4}, \mathrm{NaPO}_{3}(\mathrm{ICSD}: 35197), \mathrm{Ge}_{8} \mathrm{Pd}_{21}$ \\
\hline \multicolumn{3}{|r|}{ Space Group 91: $P 4_{1} 22$} \\
\hline $\mathrm{SOC}$ & $\mathrm{A}-\mathrm{M}, \Gamma-\mathrm{Z}$ & $\mathrm{Ag}_{3} \mathrm{RuO}_{4}$ \\
\hline \multicolumn{3}{|c|}{ Space Group 92: $P 4_{1} 2_{1} 2$} \\
\hline \multirow{3}{*}{ NSOC } & $\Gamma-\mathrm{Z}, \Gamma-\mathrm{M}$ & $\| \mathrm{Ca}, \mathrm{H}_{4} \mathrm{CN}_{2} \mathrm{O}$ \\
\hline & $\mathrm{X}-\Gamma, \Gamma-\mathrm{M}$ & $\mathrm{SiO}_{2}(\mathrm{ICSD}: 162626), \| \mathrm{Ni}_{11} \mathrm{As}_{8}$ \\
\hline & $\Gamma-\mathrm{Z}$ & $\| \mathrm{Er}_{2} \mathrm{Ti}_{3} \mathrm{Si}_{4}, \mathrm{Ho}_{2} \mathrm{Ti}_{3} \mathrm{Si}_{4}, \mathrm{Dy}_{2} \mathrm{Ti}_{3} \mathrm{Si}_{4}$ \\
\hline \multirow{2}{*}{ SOC } & $\mathrm{A}-\mathrm{M}, \Gamma-\mathrm{Z}$ & $\| \mathrm{Sc}_{2} \mathrm{Re}_{3} \mathrm{Si}_{4}$ \\
\hline & $\Gamma-\mathrm{Z}$ & $\| \mathrm{Ca}, \mathrm{Er}_{2} \mathrm{Ti}_{3} \mathrm{Si}_{4}, \mathrm{Ti}_{5} \mathrm{Si}_{4}, \mathrm{Ho}_{2} \mathrm{Ti}_{3} \mathrm{Si}_{4}, \mathrm{H}_{4} \mathrm{CN}_{2} \mathrm{O}, \mathrm{Dy}_{2} \mathrm{Ti}_{3} \mathrm{Si}_{4}$ \\
\hline \multicolumn{3}{|r|}{ Space Group 97: I422 } \\
\hline $\mathrm{SOC}$ & $\Gamma-\mathrm{M}$ & $\mathrm{Ta}_{2} \mathrm{Se}_{8} \mathrm{I} \|$ \\
\hline \multicolumn{3}{|r|}{ Space Group 99: $P 4 m m$} \\
\hline $\mathrm{SOC}$ & $\mathrm{Z}-\Gamma$ & $\| \mathrm{PrBPt}_{3}, \mathrm{NdBPt}_{3}$ \\
\hline \multicolumn{3}{|r|}{ Space Group 102: $\mathrm{P} 4_{2} \mathrm{~cm}$} \\
\hline NSOC & $\begin{array}{c}\text { M-A, Z-M, Г-M, X-A, R-A, } \\
\text { Z- } \Gamma, \mathrm{X}-\Gamma, \mathrm{R}-\Gamma\end{array}$ & 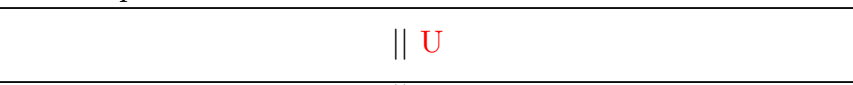 \\
\hline $\mathrm{SOC}$ & $\mathrm{Z}-\Gamma$ & $\| \mathrm{U}$ \\
\hline \multicolumn{3}{|c|}{ Space Group 107: $I 4 m m$} \\
\hline \multirow{3}{*}{ NSOC } & $\begin{array}{c}\mathrm{M}-\Gamma, \mathrm{X}-\mathrm{P}, \mathrm{X}-\mathrm{M}, \mathrm{X}-\Gamma, \mathrm{P}-\mathrm{M} \\
\mathrm{P}-\Gamma, \Gamma-\mathrm{N}\end{array}$ & $\| \operatorname{PrIn}_{2} \mathrm{Pd}$ \\
\hline & $\mathrm{X}-\mathrm{M}, \mathrm{X}-\Gamma, \mathrm{P}-\mathrm{M}, \mathrm{P}-\Gamma$ & $\| \mathrm{Sm}_{2} \mathrm{Cu}_{4} \mathrm{Sn}_{5}, \mathrm{Ba}_{2} \mathrm{TlCuHgO}_{5}$ \\
\hline & $\mathrm{M}-\Gamma, \mathrm{X}-\Gamma, \mathrm{P}-\Gamma$ & || $\mathrm{BaGe}_{3} \mathrm{Pd}, \mathrm{BaSn}_{3} \mathrm{Pd}, \mathrm{BaNiSn}_{3}$ \\
\hline $\mathrm{SOC}$ & $\mathrm{M}-\Gamma$ & $\mathrm{BaGe}_{3} \mathrm{Pd}, \mathrm{BaSn}_{3} \mathrm{Pd}, \mathrm{BaNiSn}_{3}, \mathrm{CeSi}_{3} \mathrm{Ru}, \mathrm{HfGa}_{2} \mathrm{Ni}, \mathrm{PrIn}_{2} \mathrm{Pd}$ \\
\hline \multicolumn{3}{|r|}{ Space Group 108: $I 4 \mathrm{~cm}$} \\
\hline $\mathrm{SOC}$ & $\mathrm{M}-\Gamma$ & $\mathrm{Bi}_{2} \mathrm{PdO}_{4}$ \\
\hline \multicolumn{3}{|r|}{ Space Group 109: $I 4_{1} m d$} \\
\hline NSOC & $\mathrm{M}-\mathrm{N}, \Gamma-\mathrm{N}$ & $\|$ CePIr, CeSiPt, CeSiNi \\
\hline SOC & $\mathrm{M}-\Gamma$ & || LaGeIr \\
\hline \multicolumn{3}{|r|}{ Space Group 110: $I 4_{1} c d$} \\
\hline NSOC & $\mathrm{M}-\Gamma, \mathrm{M}-\mathrm{N}, \Gamma-\mathrm{N}$ & $\| \mathrm{Li}_{2} \mathrm{~B}_{4} \mathrm{O}_{7}(\mathrm{ICSD}: 23876)$ \\
\hline $\mathrm{SOC}$ & $\mathrm{M}-\Gamma$ & $\| \mathrm{Li}_{2} \mathrm{~B}_{4} \mathrm{O}_{7}(\mathrm{ICSD}: 23876)$ \\
\hline \multicolumn{3}{|r|}{ Space Group 114: $P \overline{4} 2_{1} c$} \\
\hline NSOC & $\mathrm{R}-\mathrm{Z}$ & $\mathrm{Pd}_{4} \mathrm{~S}$ \\
\hline \multicolumn{3}{|r|}{ Space Group 116: $P \overline{4} c 2$} \\
\hline NSOC & $\Gamma-\mathrm{Z}, \Gamma-\mathrm{X}, \Gamma-\mathrm{R}, \Gamma-\mathrm{M}$ & $\mathrm{Sn}_{3} \mathrm{Ru}_{2}$ \\
\hline \multicolumn{3}{|r|}{ Space Group 117: $P \overline{4} b 2$} \\
\hline NSOC & $\Gamma-\mathrm{Z}, \Gamma-\mathrm{X}, \Gamma-\mathrm{R}, \Gamma-\mathrm{M}$ & $\mathrm{Bi}_{2} \mathrm{O}_{3}$ \\
\hline
\end{tabular}


Table III - continued

\begin{tabular}{|c|c|c|}
\hline $\begin{array}{l}\text { SOC or } \\
\text { NSOC }\end{array}$ & $\begin{array}{l}\text { HSL with Band } \\
\text { Crossing }\end{array}$ & High Symmetry Line Semimetals \\
\hline \multirow{3}{*}{ NSOC } & $\mathrm{A}-\mathrm{Z}, \Gamma-\mathrm{Z}, \Gamma-\mathrm{X}, \Gamma-\mathrm{R}, \Gamma-\mathrm{M}$ & $\| \mathrm{Ge}_{23} \mathrm{Mo}_{13}$ \\
\hline & $\mathrm{A}-\mathrm{Z}$ & $\mathrm{CuH}_{12} \mathrm{C}_{4}(\mathrm{NO})_{6} \|$ \\
\hline & $\Gamma-\mathrm{Z}$ & $\| \mathrm{Ba}_{4} \mathrm{Sm}_{2} \mathrm{Cu}_{2} \mathrm{O}_{9}$ \\
\hline \multicolumn{3}{|r|}{ Space Group 119: $I \overline{4} m 2$} \\
\hline $\mathrm{NSOC}$ & $\Gamma-\mathrm{N}, \mathrm{M}-\mathrm{N}, \mathrm{X}-\Gamma, \mathrm{X}-\mathrm{M}$ & $\| \mathrm{RbMnSe}_{2}, \mathrm{KMnTe}_{2}$ \\
\hline \multicolumn{3}{|r|}{ Space Group 120: $I \overline{4} c 2$} \\
\hline NSOC & $\Gamma-\mathrm{M}, \mathrm{M}-\mathrm{N}, \mathrm{X}-\Gamma$ & $\mathrm{Ce}\left(\mathrm{Ni}_{2} \mathrm{Sn}\right)_{2}$ \\
\hline $\mathrm{SOC}$ & $\Gamma-\mathrm{N}, \mathrm{M}-\mathrm{N}, \mathrm{X}-\mathrm{P}$ & $\mathrm{K}(\mathrm{SnAu})_{2}$ \\
\hline \multicolumn{3}{|r|}{ Space Group 121: $I \overline{4} 2 m$} \\
\hline \multirow{4}{*}{ NSOC } & $\begin{array}{c}\Gamma-\mathrm{M}, \Gamma-\mathrm{P}, \Gamma-\mathrm{X}, \mathrm{M}-\mathrm{P}, \mathrm{M}-\mathrm{X} \\
\mathrm{P}-\mathrm{X}\end{array}$ & $\| \mathrm{InFe}_{2} \mathrm{CuSe}_{4}$ \\
\hline & $\Gamma-\mathrm{M}, \Gamma-\mathrm{P}, \mathrm{M}-\mathrm{P}, \mathrm{P}-\mathrm{X}, \mathrm{M}-\mathrm{X}$ & \multirow{2}{*}{\begin{tabular}{||c|}
$\mathrm{Zr}_{3} \mathrm{Ir}$ \\
$\mathrm{V}_{3} \mathrm{~S}$
\end{tabular}} \\
\hline & $\Gamma-\mathrm{M}, \mathrm{M}-\mathrm{P}, \mathrm{M}-\mathrm{X}, \mathrm{P}-\mathrm{X}$ & \\
\hline & $\Gamma-\mathrm{M}$ & $\mathrm{Cu}_{2} \mathrm{HgGeSe}_{4}[62], \mathrm{Cu}_{2} \mathrm{SnHgSe}_{4}[62], \| \mathrm{Cu}_{3} \mathrm{SbSe}_{4}[62$ \\
\hline \multicolumn{3}{|r|}{ 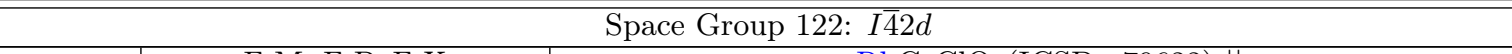 } \\
\hline \multirow{2}{*}{ NSOC } & $\Gamma-\mathrm{M}, \Gamma-\mathrm{P}, \Gamma-\mathrm{X}$ & $\mathrm{RhC}_{2} \mathrm{ClO}_{2}(\mathrm{ICSD}: 79622) \|$ \\
\hline & $\Gamma-\mathrm{M}$ & $\begin{array}{c}\mathrm{CdSnSb}_{2}[102], \mathrm{ZnSnSb}_{2}[103], \mathrm{CdGeAs}_{2}, \mathrm{CdSnAs}_{2}[103], \mathrm{InCuSe}_{2}, \\
\mathrm{InCuS}_{2}, \mathrm{TlCuSe}_{2}\end{array}$ \\
\hline \multicolumn{3}{|r|}{ Space Group 123: $P 4 / m m m$} \\
\hline \multirow{16}{*}{ NSOC } & $\begin{array}{c}\text { A-M, A-Z, R-A, A- }, \Gamma-\mathrm{M}, \\
\text { M-Z, R-X, X-A, X-M, Г-Z, } \\
\text { R-Z, X-Z }\end{array}$ & $\| \mathrm{VRh}$ \\
\hline & 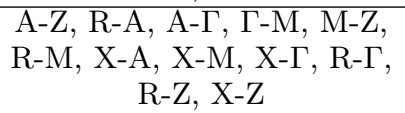 & $\| \mathrm{Ba}_{6} \mathrm{Ca}_{6} \mathrm{Tl}_{5} \mathrm{Cu}_{9} \mathrm{O}_{29}$ \\
\hline & $\begin{array}{c}\text { A-Z, R-A, A- }, \Gamma-\mathrm{M}, \mathrm{M}-\mathrm{Z}, \\
\mathrm{R}-\mathrm{Z}, \mathrm{R}-\mathrm{X}, \mathrm{R}-\mathrm{M}, \mathrm{X}-\mathrm{A}, \mathrm{X}-\mathrm{M}, \\
\mathrm{X}-\Gamma, \mathrm{X}-\mathrm{Z}\end{array}$ & $\| \mathrm{Ba}_{2} \mathrm{PrCu}_{3} \mathrm{O}_{8}$ \\
\hline & $\begin{array}{c}\text { A-M, A-Z, R-A, A-Г, Г-M, } \\
\Gamma-\mathrm{Z}, \mathrm{R}-\mathrm{Z}, \mathrm{R}-\mathrm{X}, \mathrm{X}-\mathrm{M}, \mathrm{X}-\Gamma \\
\mathrm{X}-\mathrm{Z}\end{array}$ & $\| \mathrm{CeSi}_{2} \mathrm{Mo}_{2} \mathrm{C}$ \\
\hline & 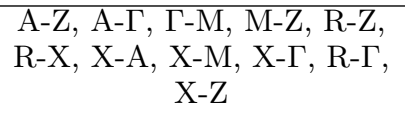 & $\| \mathrm{Ta}_{4} \mathrm{O}$ \\
\hline & $\begin{array}{c}\text { A-Z, R-A, A- }, \Gamma-\mathrm{M}, \mathrm{M}-\mathrm{Z}, \\
\mathrm{R}-\mathrm{X}, \mathrm{R}-\mathrm{M}, \mathrm{X}-\Gamma, \mathrm{R}-\Gamma, \mathrm{R}-\mathrm{Z} \\
\mathrm{X}-\mathrm{Z}\end{array}$ & $\| \mathrm{ZnCdPt}_{2}$ \\
\hline & $\begin{array}{l}\text { A-M, R-A, } \Gamma-\mathrm{M}, \mathrm{M}-\mathrm{Z}, \mathrm{R}-\mathrm{X} \\
\mathrm{R}-\mathrm{M}, \Gamma-\mathrm{Z}, \mathrm{X}-\Gamma, \mathrm{R}-\Gamma, \mathrm{R}-\mathrm{Z}\end{array}$ & $\| \mathrm{Mn}_{2} \mathrm{Co}_{2} \mathrm{C}$ \\
\hline & 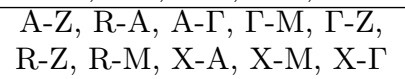 & $\| \mathrm{CeCr}_{2} \mathrm{Si}_{2} \mathrm{C}$ \\
\hline & $\begin{array}{l}\text { A-Z, R-A, Г-Z, M-Z, R-X, } \\
\text { R-M, X-Г, Г-M, R-Z, X-M }\end{array}$ & $\| \mathrm{FeNiPt}_{2}$ \\
\hline & $\begin{array}{l}\text { A-Z, R-A, R-X, R-M, Г-Z, } \\
\text { X- }, \text { }, \text {-M, R-Z, X-Z, X-M }\end{array}$ & $\| \mathrm{FePd}$ \\
\hline & $\begin{array}{l}\text { A-Z, R-A, A- }-\Gamma-\mathrm{M}, \mathrm{M}-\mathrm{Z} \\
\mathrm{R}-\mathrm{Z}, \mathrm{R}-\mathrm{M}, \mathrm{X}-\mathrm{A}, \mathrm{X}-\mathrm{M}\end{array}$ & $\| \mathrm{Hf}_{5} \mathrm{~Pb}$ \\
\hline & $\begin{array}{l}\text { A-Z, R-A, R-Z, R-X, R-M, } \\
\text { X- } \Gamma, \text { X-Z, X-M }\end{array}$ & $\| \mathrm{LaAl}_{2} \mathrm{BOs}_{2}$ \\
\hline & $\begin{array}{l}\text { A-M, R-A, R-X, R-M, Г-Z, } \\
\text { R-Z, X-Z }\end{array}$ & $\| \mathrm{CdPt}$ \\
\hline & $\begin{array}{l}\mathrm{A}-\mathrm{Z}, \mathrm{A}-\Gamma, \Gamma-\mathrm{M}, \mathrm{M}-\mathrm{Z}, \mathrm{R}-\mathrm{X} \\
\mathrm{X}-\mathrm{A}, \mathrm{X}-\mathrm{M}\end{array}$ & $\| \operatorname{Er}_{2} \mathrm{Mg}_{3} \mathrm{Ru}$ \\
\hline & $\begin{array}{l}\text { A-Z, R-A, A- }, \Gamma-\mathrm{M}, \Gamma-\mathrm{Z} \\
\text { R-Z, X-M }\end{array}$ & $\| \mathrm{YbPb}, \mathrm{YbSn}, \mathrm{CaPb}$ \\
\hline & $\begin{array}{l}\text { A-Z, R-A, A- } \Gamma, \Gamma-\mathrm{M}, \mathrm{M}-\mathrm{Z} \\
\mathrm{R}-\mathrm{Z}, \mathrm{X}-\Gamma\end{array}$ & $\begin{array}{c}\| \mathrm{Sr}_{2} \mathrm{ErCu}_{3}\left(\mathrm{PbO}_{4}\right)_{2}, \mathrm{Sr}_{2} \mathrm{DyCu}_{3}\left(\mathrm{PbO}_{4}\right)_{2}, \mathrm{Sr}_{2} \mathrm{SmCu}_{3}\left(\mathrm{PbO}_{4}\right)_{2}, \\
\mathrm{Sr}_{2} \mathrm{NdCu}_{3}\left(\mathrm{PbO}_{4}\right)_{2}\end{array}$ \\
\hline
\end{tabular}


Table III - continued

\begin{tabular}{|c|c|c|}
\hline SOC or & HSL with Band & High Symmetry Line Semimetals \\
\hline & Crossing & \\
\hline & $\begin{array}{l}\text { A-Z, R-A, A- } \Gamma, \Gamma-M, M-Z, \\
\text { R-Z, X-M }\end{array}$ & $\mathrm{O}_{2}(\mathrm{ICSD}: 28530) \|$ \\
\hline & $\begin{array}{l}\text { A-Z, R-A, R-Z, R-X, R-M, } \\
\text { X- } \Gamma, \text { X-M }\end{array}$ & $\| \mathrm{ZrHg}$ \\
\hline & $\begin{array}{c}\mathrm{A}-\mathrm{M}, \mathrm{A}-\mathrm{Z}, \mathrm{A}-\Gamma, \Gamma-\mathrm{Z}, \mathrm{M}-\mathrm{Z} \\
\mathrm{R}-\mathrm{Z}\end{array}$ & $\| \mathrm{Ba}\left(\mathrm{Cu}_{3} \mathrm{As}\right)_{2}$ \\
\hline & $\begin{array}{c}\mathrm{A}-\mathrm{M}, \mathrm{R}-\mathrm{A}, \underset{\mathrm{X}-\mathrm{C}-\mathrm{M}}{\mathrm{M}} \mathrm{M}-\mathrm{Z}, \mathrm{X}-\mathrm{A}, \\
\end{array}$ & $\| \mathrm{ZnCu}_{2} \mathrm{Ni}$ \\
\hline & $\begin{array}{c}\mathrm{A}-\mathrm{Z}, \mathrm{R}-\mathrm{A}, \mathrm{A}-\Gamma, \Gamma-\mathrm{M}, \Gamma-\mathrm{Z} \\
\mathrm{X}-\mathrm{M}\end{array}$ & $\| \mathrm{Al}_{3}\left(\mathrm{BRu}_{2}\right)_{2}$ \\
\hline & $\begin{array}{c}\text { A-Z, R-A, A- } \Gamma, \Gamma-M, M-Z, \\
\text { R-Z }\end{array}$ & $\| \mathrm{Ba}_{2} \mathrm{YTlCu}_{2} \mathrm{O}_{7}$ \\
\hline & $\begin{array}{c}\mathrm{A}-\mathrm{Z}, \mathrm{R}-\mathrm{A}, \underset{\mathrm{X}}{\mathrm{A}-\mathrm{\Gamma}, \mathrm{M}} \mathrm{\Gamma}-\mathrm{M}, \mathrm{M}-\mathrm{Z} \\
\end{array}$ & $\mathrm{FeSi}_{2}, \| \mathrm{UGa}_{5} \mathrm{Ir}, \mathrm{UGa}_{5} \mathrm{Co}, \mathrm{UGa}_{5} \mathrm{Rh}$ \\
\hline & $\mathrm{A}-\mathrm{M}, \mathrm{R}-\mathrm{A}, \mathrm{\Gamma}-\mathrm{Z}, \mathrm{R}-\mathrm{Z}, \mathrm{X}-\mathrm{Z}$ & $\mathrm{ZnPt}, \mathrm{HgPt}, \| \mathrm{ZnNi}$ \\
\hline & A-Z, A-Г, Г-M, M-Z, R-Z & $\begin{array}{c}\| \mathrm{Ba}_{2} \mathrm{Nd}_{2} \mathrm{Ti}_{2} \mathrm{Cu}_{2} \mathrm{O}_{11}, \mathrm{Ba}_{2} \mathrm{NdNb}\left(\mathrm{CuO}_{4}\right)_{2}, \mathrm{Sr}_{2} \mathrm{SmTa}\left(\mathrm{CuO}_{4}\right)_{2}, \\
\mathrm{Ba}_{2} \mathrm{LaTa}\left(\mathrm{CuO}_{4}\right)_{2}\end{array}$ \\
\hline & $\mathrm{A}-\mathrm{Z}, \mathrm{R}-\mathrm{A}, \mathrm{A}-\Gamma, \Gamma-\mathrm{M}, \mathrm{M}-\mathrm{Z}$ & $\| \mathrm{Sr}_{2} \mathrm{YTlCu}_{2} \mathrm{O}_{7}$ \\
\hline & A-M, R-A, X-A, Г-Z & $\mathrm{AlCuPt}_{2}, \mathrm{GaCuPt}_{2}, \mathrm{InCuPt}_{2}$ \\
\hline & $\mathrm{A}-\mathrm{Z}, \mathrm{R}-\mathrm{A}, \Gamma-\mathrm{Z}, \mathrm{M}-\mathrm{Z}$ & $\| \mathrm{MgNiH}_{2}$ \\
\hline & A-Z, R-Z, X-Г, X-M & $\| \mathrm{TiHg}$ \\
\hline & $\mathrm{R}-\mathrm{A}, \mathrm{R}-\mathrm{M}, \mathrm{X}-\mathrm{A}, \mathrm{X}-\mathrm{M}$ & $\| \mathrm{EuBi}_{2} \mathrm{IO}_{4}$ \\
\hline & $\mathrm{A}-\mathrm{M}, \mathrm{R}-\mathrm{A}$ & $\| \mathrm{NiHg}$ \\
\hline & $\mathrm{A}-\mathrm{Z}, \mathrm{R}-\mathrm{A}$ & $\| \mathrm{Na}_{2} \mathrm{Cl}$ \\
\hline & R-A, R-Z & $\| \mathrm{AgSbTe}_{2}[\underline{5}$ \\
\hline & $\mathrm{X}-\Gamma, \Gamma-\mathrm{M}$ & $\mathrm{CsCd}_{4} \mathrm{As}_{3}, \mathrm{NdTe}_{2} \mathrm{ClO}_{5} \|$ \\
\hline \multirow{3}{*}{$\mathrm{SOC}$} & A-M, Г-Z & 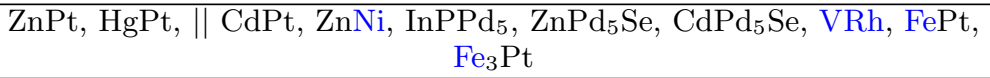 \\
\hline & A-M & $\begin{array}{c}\mathrm{SmBi}, \mathrm{ZnPd}, \mathrm{Si}_{2} \mathrm{Ru}, \| \mathrm{NdAs}, \mathrm{SmAs}, \mathrm{PrAs}, \mathrm{PrBi}, \mathrm{NdSb}, \mathrm{PrSb}, \mathrm{Na}{ }_{3} \mathrm{Cl}, \\
\mathrm{LaAs}, \mathrm{LaSb}_{\mathrm{Z}} \mathrm{ZnCdPt}_{2}, \mathrm{TlPPd}_{5}, \mathrm{TlAsPd}_{5}, \mathrm{InAsPd}_{5}, \mathrm{SmTl}_{\mathrm{A}}, \mathrm{ErTl}, \\
\mathrm{HgPd}_{5} \mathrm{Se}, \mathrm{RbN}_{3}, \mathrm{Sr}_{2} \mathrm{HoCu}_{3}\left(\mathrm{PbO}_{4}\right)_{2}, \mathrm{BaNb}_{4} \mathrm{O}_{6}, \mathrm{Er}_{2} \mathrm{Ga}_{8} \mathrm{Fe} \\
\mathrm{Tm}_{2} \mathrm{Ga}_{8} \mathrm{Fe}, \mathrm{MnAu}\end{array}$ \\
\hline & $\Gamma-\mathrm{Z}$ & 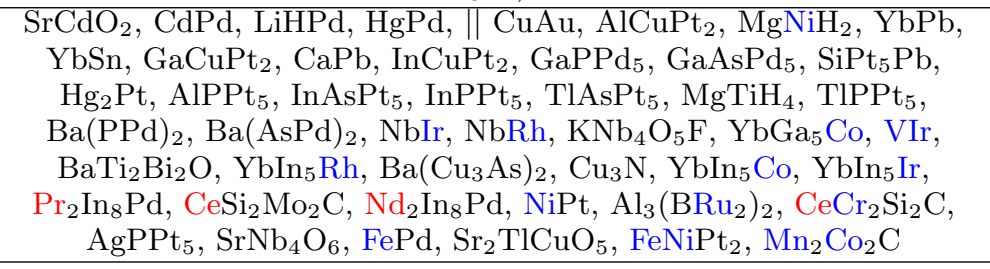 \\
\hline \multicolumn{3}{|r|}{ Space Group 124: $P 4 / m c c$} \\
\hline \multirow[t]{2}{*}{ NSOC } & 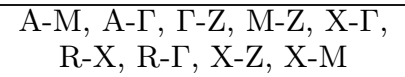 & $\| \mathrm{Zr}_{4} \mathrm{CuP}$ \\
\hline & $\begin{array}{l}\text { A-M, A- } \Gamma, \Gamma-Z, M-Z, X-\Gamma, \\
\text { R-X, X-Z }\end{array}$ & $\| \mathrm{Nb}_{4} \mathrm{CoP}$ \\
\hline \multirow{2}{*}{$\mathrm{SOC}$} & $\mathrm{A}-\mathrm{M}$ & $\| \mathrm{Zr}_{4} \mathrm{CuP}$ \\
\hline & $\Gamma-\mathrm{Z}$ & $\| \mathrm{Nb}_{4} \mathrm{FeSi}, \mathrm{Nb}_{4} \mathrm{CoP}$ \\
\hline \multicolumn{3}{|r|}{ Space Group 125: $P 4 / n b m$} \\
\hline \multirow{5}{*}{ NSOC } & $\begin{array}{l}\text { A-M, A-Z, Г-M, M-Z, R-Z, } \\
\Gamma-\mathrm{Z}, \mathrm{X}-\Gamma, \mathrm{R}-\Gamma, \mathrm{X}-\mathrm{Z}\end{array}$ & $\| \mathrm{Ce}_{2} \mathrm{Ga}_{12} \mathrm{Pd}$ \\
\hline & $\begin{array}{l}\mathrm{A}-\mathrm{Z}, \mathrm{A}-\Gamma, \Gamma-\mathrm{M}, \Gamma-\mathrm{Z}, \mathrm{R}-\mathrm{Z} \\
\mathrm{X}-\Gamma, \mathrm{R}-\Gamma, \mathrm{X}-\mathrm{Z}\end{array}$ & $\| \mathrm{CeGa}_{6}, \mathrm{PuGa}_{6}$ \\
\hline & $\begin{array}{l}\mathrm{A}-\mathrm{Z}, \mathrm{R}-\mathrm{Z}, \mathrm{X}-\Gamma, \Gamma-\mathrm{M}, \mathrm{R}-\Gamma \\
\mathrm{X}-\mathrm{Z}\end{array}$ & $\| \mathrm{Nd}_{2} \mathrm{Ga}_{12} \mathrm{Ni}$ \\
\hline & $\mathrm{A}-\Gamma, \Gamma-\mathrm{M}, \Gamma-\mathrm{Z}, \mathrm{X}-\Gamma$ & $\mathrm{SiO}_{2}(\mathrm{ICSD}: 170502)$ \\
\hline & $\mathrm{X}-\Gamma, \Gamma-\mathrm{M}$ & || $\mathrm{SiO}_{2}(\mathrm{ICSD}: 170538)$ \\
\hline $\mathrm{SOC}$ & $\Gamma-\mathrm{Z}$ & $\begin{array}{c}\mathrm{RbAg}_{5} \mathrm{Se}_{3}, \| \mathrm{La}_{2} \mathrm{Ga}_{12} \mathrm{Pd}, \mathrm{PuGa}_{6}, \mathrm{La}_{2} \mathrm{Ga}_{12} \mathrm{Ni}, \mathrm{SiO}_{2}(\mathrm{ICSD}: 170502), \\
\mathrm{Ce}_{2} \mathrm{Ga}_{12} \mathrm{Pd}\end{array}$ \\
\hline & & Space Group 126: $P 4 / n n c$ \\
\hline
\end{tabular}


Table III - continued

\begin{tabular}{|c|c|c|}
\hline $\begin{array}{l}\text { SOC or } \\
\text { NSOC }\end{array}$ & $\begin{array}{l}\text { HSL with Band } \\
\text { Crossing }\end{array}$ & High Symmetry Line Semimetals \\
\hline NSOC & $\mathrm{A}-\Gamma, \Gamma-\mathrm{M}, \Gamma-\mathrm{Z}, \mathrm{X}-\Gamma$ & $\mathrm{SiO}_{2}(\mathrm{ICSD}: 170503)$ \\
\hline SOC & $\Gamma-\mathrm{Z}$ & $\mathrm{SiO}_{2}(\mathrm{ICSD}: 170503)$ \\
\hline \multirow{32}{*}{ NSOC } & \multicolumn{2}{|r|}{ Space Group 127: $P 4 / m b m$} \\
\hline & $\begin{array}{c}\mathrm{A}-\mathrm{M}, \mathrm{A}-\mathrm{Z}, \mathrm{R}-\mathrm{A}, \mathrm{A}-\Gamma, \Gamma-\mathrm{M}, \\
\Gamma-\mathrm{Z}, \mathrm{M}-\mathrm{Z}, \mathrm{R}-\mathrm{Z}, \mathrm{X}-\Gamma, \mathrm{R}-\Gamma \\
\mathrm{X}-\mathrm{Z}\end{array}$ & $\| \mathrm{Th}_{3} \mathrm{Ga}_{2}$ \\
\hline & $\begin{array}{c}\text { A-M, A-Z, R-A, Г-M, M-Z, } \\
\text { R-Z, }-\mathrm{Z}, \mathrm{X}-\Gamma, \mathrm{R}-\Gamma, \mathrm{X}-\mathrm{Z} \\
\text { X-M }\end{array}$ & $\| \mathrm{Zr}_{3} \mathrm{Ga}_{2}$ \\
\hline & $\begin{array}{l}\text { A-Z, R-A, A- } \Gamma, \Gamma-\mathrm{M}, \Gamma-\mathrm{Z} \\
\mathrm{M}-\mathrm{Z}, \mathrm{R}-\mathrm{Z}, \mathrm{X}-\Gamma, \mathrm{R}-\Gamma, \mathrm{X}-\mathrm{Z}\end{array}$ & $\| \mathrm{Th}_{3} \mathrm{Al}_{2}$ \\
\hline & 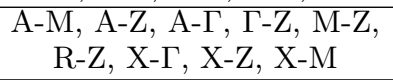 & $\| \mathrm{Hf}_{3} \mathrm{Ge}_{2}$ \\
\hline & $\begin{array}{c}\text { A-M, A-Z, R-A, A- }, \text { Г-M, } \\
\Gamma-\mathrm{Z}, \mathrm{M}-\mathrm{Z}, \mathrm{R}-\mathrm{Z}, \mathrm{X}-\mathrm{Z}\end{array}$ & $\| \mathrm{Pr}_{2} \mathrm{CdPd}_{2}, \mathrm{Sm}_{2} \mathrm{CdPd}_{2}$ \\
\hline & $\begin{array}{c}\text { A-M, A-Z, } \Gamma-\mathrm{M}, \Gamma-\mathrm{Z}, \mathrm{M}-\mathrm{Z} \\
\mathrm{R}-\mathrm{Z}, \mathrm{X}-\Gamma, \mathrm{X}-\mathrm{M}\end{array}$ & $\| \mathrm{Ta}_{2} \mathrm{FeB}_{2}$ \\
\hline & $\begin{array}{l}\mathrm{A}-\mathrm{M}, \Gamma-\mathrm{M}, \mathrm{M}-\mathrm{Z}, \Gamma-\mathrm{Z}, \mathrm{X}-\Gamma \\
\mathrm{R}-\mathrm{Z}, \mathrm{X}-\mathrm{Z}, \mathrm{X}-\mathrm{M}\end{array}$ & $\| \mathrm{Nb}_{2} \mathrm{FeB}_{2}$ \\
\hline & $\begin{array}{c}\text { A-Z, R-A, A- }, \text { }, \Gamma-\mathrm{M}, \mathrm{M}-\mathrm{Z} \\
\mathrm{R}-\mathrm{Z}, \Gamma-\mathrm{Z}, \mathrm{X}-\mathrm{M}\end{array}$ & $\| \mathrm{Ca}_{36} \mathrm{Sn}_{23}$ \\
\hline & $\begin{array}{c}\mathrm{A}-\mathrm{Z}, \mathrm{R}-\mathrm{A}, \mathrm{R}-\mathrm{Z}, \Gamma-\mathrm{Z}, \mathrm{X}-\Gamma, \\
\Gamma-\mathrm{M}, \mathrm{X}-\mathrm{Z}, \mathrm{X}-\mathrm{M}\end{array}$ & $\| \mathrm{B}_{2} \mathrm{Mo}_{3}$ \\
\hline & $\begin{array}{l}\text { A-Z, R-A, R-Z, X-Г, Г-M, } \\
\text { R- } \Gamma, \text { X-Z, X-M }\end{array}$ & $\| \mathrm{AlPt}_{3}(\mathrm{ICSD}: 107439)$ \\
\hline & $\begin{array}{c}\text { A-M, A-Z, A- } \Gamma, \Gamma-Z, M-Z, \\
\text { R-Z, X-Z }\end{array}$ & II $\mathrm{Th}_{3} \mathrm{Si}_{2}\left(\mathrm{ICSD}:\right.$ 652398), $\mathrm{Th}_{3} \mathrm{Ge}_{2}$ \\
\hline & $\begin{array}{c}\text { A-M, A-Z, R-A, A- }, \Gamma-\mathrm{M} \\
\text { M-Z, Г-Z }\end{array}$ & $\| \mathrm{Dy}_{2} \mathrm{CdPd}_{2}$ \\
\hline & $\begin{array}{c}\mathrm{A}-\mathrm{Z}, \mathrm{A}-\Gamma, \Gamma-\mathrm{M}, \mathrm{M}-\mathrm{Z}, \Gamma-\mathrm{Z}, \\
\mathrm{R}-\mathrm{Z}, \mathrm{X}-\mathrm{Z}\end{array}$ & $\| \mathrm{Nd}_{2} \mathrm{MgCu}_{2}, \mathrm{La}_{2} \mathrm{MgCu}_{2}, \mathrm{Nd}_{2} \mathrm{CdCu}_{2}$ \\
\hline & $\begin{array}{c}\text { A-Z, R-A, A- } \Gamma, \Gamma-M, \Gamma-Z, \\
\text { R-Z, X-Z }\end{array}$ & $\| \mathrm{Pr}_{2} \mathrm{CdNi}_{2}, \mathrm{Nd}_{2} \mathrm{CdNi}_{2}$ \\
\hline & $\begin{array}{c}\text { A-Z, R-A, R-Z, Г-Z, X- } \Gamma, \\
\Gamma-\mathrm{M}, \mathrm{X}-\mathrm{Z}\end{array}$ & $\| \mathrm{Mg}(\mathrm{ScGa})_{2}$ \\
\hline & $\begin{array}{c}\text { A-M, A- } \Gamma, \Gamma-\mathrm{M}, \Gamma-\mathrm{Z}, \mathrm{M}-\mathrm{Z}, \\
\mathrm{X}-\Gamma\end{array}$ & $\| \mathrm{Sn}\left(\mathrm{Mo}_{2} \mathrm{O}_{3}\right)_{2}$ \\
\hline & $\begin{array}{c}\text { A-M, A-Z, R-A, A- } \Gamma, \Gamma-Z, \\
\text { M-Z }\end{array}$ & $\| \mathrm{Sc}_{3} \mathrm{~B}_{2} \mathrm{Rh}_{5}$ \\
\hline & $\begin{array}{c}\mathrm{A}-\mathrm{Z}, \mathrm{A}-\Gamma, \Gamma-\mathrm{M}, \mathrm{M}-\mathrm{Z}, \mathrm{R}-\mathrm{Z}, \\
\mathrm{X}-\Gamma\end{array}$ & $\| \mathrm{Yb}_{2} \mathrm{CdGe}_{2}$ \\
\hline & $\mathrm{A}-\mathrm{M}, \mathrm{A}-\mathrm{Z}, \mathrm{A}-\Gamma, \mathrm{R}-\mathrm{Z}, \Gamma-\mathrm{Z}$ & $\mathrm{Ca}_{2} \mathrm{PbAu}_{2}$ \\
\hline & $\mathrm{A}-\mathrm{M}, \mathrm{A}-\mathrm{Z}, \mathrm{R}-\mathrm{A}, \mathrm{A}-\Gamma, \mathrm{R}-\mathrm{Z}$ & $\| \mathrm{Pr}_{7} \mathrm{Al}_{7} \mathrm{Co}_{6}$ \\
\hline & $\mathrm{A}-\mathrm{Z}, \mathrm{A}-\Gamma, \Gamma-\mathrm{M}, \mathrm{M}-\mathrm{Z}, \mathrm{X}-\Gamma$ & $\| \mathrm{Yb}_{2} \mathrm{SnGe}_{2}, \mathrm{Sr}_{2} \mathrm{~Pb}_{3}$ \\
\hline & A-Z, I-Z, M-Z, R-Z, X-Z & $\mathrm{La}_{2} \mathrm{CdRh}_{2}, \mathrm{Y}_{2} \mathrm{MgCu}_{2}, \mathrm{Ce}_{2} \mathrm{MgNi}_{2}$ \\
\hline & $\mathrm{A}-\mathrm{Z}, \Gamma-\mathrm{Z}, \mathrm{M}-\mathrm{Z}, \mathrm{X}-\Gamma, \Gamma-\mathrm{M}$ & $\| \mathrm{Ca}_{2} \mathrm{SnGe}_{2}$ \\
\hline & $\Gamma-\mathrm{Z}, \mathrm{X}-\Gamma, \Gamma-\mathrm{M}, \mathrm{R}-\mathrm{Z}, \mathrm{X}-\mathrm{Z}$ & $\| \mathrm{Nb}_{2} \mathrm{CrB}_{2}$ \\
\hline & $\mathrm{A}-\mathrm{M}, \mathrm{A}-\Gamma, \Gamma-\mathrm{Z}, \mathrm{M}-\mathrm{Z}$ & $\mathrm{Sc}_{2} \mathrm{GaB}_{2} \mathrm{Rh}_{5}$ \\
\hline & $\mathrm{A}-\mathrm{M}, \Gamma-\mathrm{M}, \mathrm{M}-\mathrm{Z}, \mathrm{X}-\mathrm{M}$ & $\mathrm{Tl}_{2} \mathrm{GeTe}_{5} \|$ \\
\hline & $\mathrm{A}-\Gamma, \Gamma-\mathrm{M}, \Gamma-\mathrm{Z}$ & $\| \mathrm{Dy}_{2} \mathrm{CdGe}_{2}, \mathrm{Sr}_{3} \mathrm{Hg}_{2}, \mathrm{Ca}_{3} \mathrm{Hg}_{2}, \mathrm{Li}_{2} \mathrm{Sn}_{5}$ \\
\hline & $\mathrm{A}-\mathrm{M}, \mathrm{A}-\mathrm{Z}, \mathrm{R}-\mathrm{Z}$ & $\| \mathrm{Nd}_{7} \mathrm{Al}_{7} \mathrm{Co}_{6}$ \\
\hline & A-Z, R-Z, Г-Z & $\mathrm{Lu}_{2} \mathrm{Pd}_{2} \mathrm{~Pb}, \mathrm{Tm}_{2} \mathrm{Pd}_{2} \mathrm{~Pb}, \mathrm{Er}_{2} \mathrm{Pd}_{2} \mathrm{~Pb}, \mathrm{Y}_{2} \mathrm{Pd}_{2} \mathrm{~Pb}, \mathrm{Sc}_{2} \mathrm{Ni}_{2} \mathrm{Sn}$ \\
\hline & $\mathrm{A}-\mathrm{Z}, \mathrm{R}-\mathrm{Z}$ & $\| \mathrm{Yb}_{2} \mathrm{CdPd}_{2}$ \\
\hline & $\Gamma-\mathrm{Z}$ & $\| \mathrm{Si}_{2} \mathrm{Mo}_{3}$ \\
\hline
\end{tabular}


Table III - continued

\begin{tabular}{|c|c|c|}
\hline $\begin{array}{l}\text { SOC or } \\
\text { NSOC }\end{array}$ & $\begin{array}{l}\text { HSL with Band } \\
\text { Crossing }\end{array}$ & High Symmetry Line Semimetals \\
\hline $\mathrm{SOC}$ & $\Gamma-\mathrm{Z}$ & 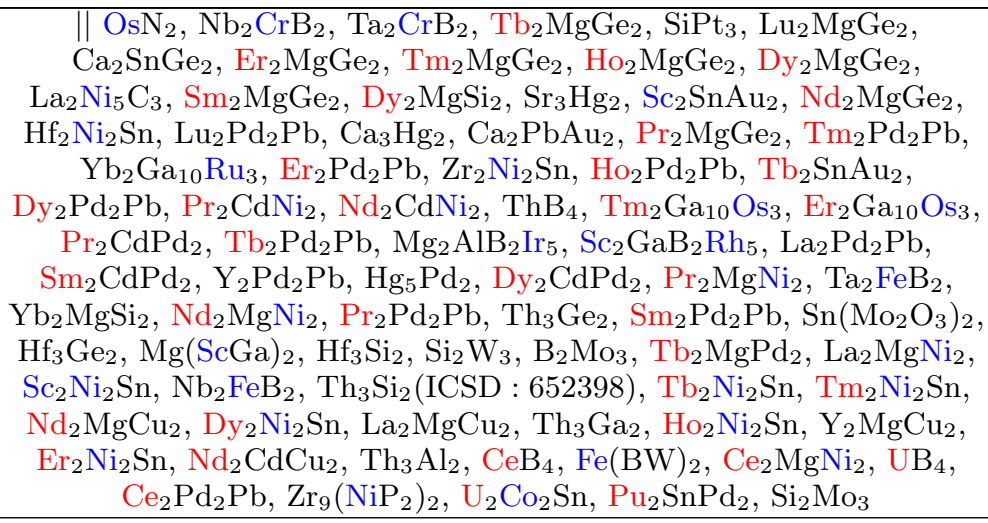 \\
\hline \multicolumn{3}{|r|}{ Space Group 128: P4/mnc } \\
\hline \multirow{5}{*}{ NSOC } & $\begin{array}{c}\text { A-M, Г-M, M-Z, Г-Z, X-Г, } \\
\text { R- } \Gamma, \mathrm{X}-\mathrm{M}\end{array}$ & \multirow{2}{*}{$\| \mathrm{Al}_{7} \mathrm{FeCu}_{2}$} \\
\hline & $\mathrm{A}-\mathrm{M}, \Gamma-\mathrm{M}, \mathrm{M}-\mathrm{Z}, \Gamma-\mathrm{Z}, \mathrm{X}-\Gamma$ & \\
\hline & $\mathrm{A}-\mathrm{M}, \Gamma-\mathrm{M}, \mathrm{M}-\mathrm{Z}, \mathrm{X}-\Gamma, \mathrm{X}-\mathrm{M}$ & $\| \mathrm{Ho}_{4} \mathrm{NiB}_{14}$ \\
\hline & A-M, Г-M, M-Z, Г-Z & $\| \mathrm{Nd}_{2} \mathrm{Re}_{3} \mathrm{Si}_{5}, \mathrm{Ho}_{2} \mathrm{Mn}_{3} \mathrm{Si}_{5}$ \\
\hline & $\mathrm{X}-\Gamma, \Gamma-\mathrm{M}$ & $\| \mathrm{U}_{2} \mathrm{Mn}_{3} \mathrm{Si}_{5}$ \\
\hline \multirow{2}{*}{$\mathrm{SOC}$} & R-A, R-X, R-M & $\| \mathrm{U}_{2} \mathrm{Mn}_{3} \mathrm{Si}_{5}$ \\
\hline & \multirow{2}{*}{$n-A, n-\Lambda, n-1 \mathrm{n}$} & $\begin{array}{c}\| \mathrm{Lu}_{2} \mathrm{Si}_{5} \mathrm{Ru}_{3}, \mathrm{Tm}_{2} \mathrm{Mn}_{3} \mathrm{Si}_{5}, \mathrm{Al}_{7} \mathrm{CoCu}_{2}, \mathrm{Dy}_{2} \mathrm{Fe}_{3} \mathrm{Si}_{5}, \mathrm{Y}_{4} \mathrm{CoB}_{13}, \\
\mathrm{Lu}_{4} \mathrm{CoB}_{13}, \mathrm{Al}_{7} \mathrm{FeCu}_{2}, \mathrm{~Tb}_{4} \mathrm{CoB}_{13}, \mathrm{Nb}_{3} \mathrm{InSe}_{12}, \mathrm{Nd}_{2} \mathrm{Re}_{3} \mathrm{Si}_{5}, \mathrm{Sm}_{2} \mathrm{Si}_{5} \mathrm{Ru}_{3}, \\
\mathrm{Ho}_{2} \mathrm{Mn}_{3} \mathrm{Si}_{5}\end{array}$ \\
\hline \multirow{15}{*}{ NSOC } & & Space Group 129: P4/nmm \\
\hline & $\begin{array}{l}\text { A-M, A-Z, A- } \Gamma, \Gamma-\mathrm{M}, \mathrm{M}-\mathrm{Z} \\
\mathrm{R}-\mathrm{Z}, \mathrm{X}-\Gamma, \mathrm{R}-\Gamma, \mathrm{X}-\mathrm{Z}\end{array}$ & $\| \mathrm{BiTeIO}_{3}$ \\
\hline & $\begin{array}{c}\mathrm{A}-\mathrm{Z}, \mathrm{A}-\Gamma, \Gamma-\mathrm{M}, \Gamma-\mathrm{Z}, \mathrm{M}-\mathrm{Z} \\
\mathrm{R}-\mathrm{Z}, \mathrm{X}-\Gamma, \mathrm{R}-\Gamma, \mathrm{X}-\mathrm{Z}\end{array}$ & $\|$ PrSbTe, PrAsSe, $\mathrm{ThAs}_{2}, \mathrm{ThSb}_{2}$ \\
\hline & $\begin{array}{l}\text { A-Z, A- } \Gamma, \Gamma-\mathrm{M}, \Gamma-\mathrm{Z}, \mathrm{R}-\mathrm{Z} \\
\mathrm{X}-\Gamma, \mathrm{R}-\mathrm{X}, \mathrm{R}-\Gamma, \mathrm{X}-\mathrm{Z}\end{array}$ & $\|$ TiSe, $\mathrm{Ho}_{10} \mathrm{In}_{20} \mathrm{Ni}_{9}$ \\
\hline & $\begin{array}{l}\text { A-Z, A- } \Gamma, \Gamma-\mathrm{M}, \mathrm{M}-\mathrm{Z}, \Gamma-\mathrm{Z} \\
\mathrm{X}-\Gamma, \mathrm{R}-\mathrm{X}, \mathrm{R}-\Gamma, \mathrm{R}-\mathrm{Z}\end{array}$ & $\| \mathrm{LaBi}_{2} \mathrm{Au}$ \\
\hline & $\begin{array}{l}\text { A-Z, A- } \Gamma, \Gamma-\mathrm{M}, \mathrm{M}-\mathrm{Z}, \mathrm{R}-\mathrm{Z} \\
\mathrm{X}-\Gamma, \mathrm{R}-\mathrm{X}, \mathrm{R}-\Gamma, \mathrm{X}-\mathrm{Z}\end{array}$ & $\| \mathrm{LiLaBi}_{2}$ \\
\hline & $\begin{array}{c}\mathrm{A}-\mathrm{Z}, \mathrm{A}-\Gamma, \Gamma-\mathrm{M}, \Gamma-\mathrm{Z}, \mathrm{R}-\mathrm{Z} \\
\mathrm{X}-\Gamma, \mathrm{R}-\Gamma, \mathrm{X}-\mathrm{Z}\end{array}$ & || LaNiPO, CaNiGe \\
\hline & $\begin{array}{c}\mathrm{A}-\mathrm{Z}, \mathrm{A}-\Gamma, \Gamma-\mathrm{M}, \mathrm{M}-\mathrm{Z}, \mathrm{R}-\mathrm{Z}, \\
\mathrm{X}-\Gamma, \mathrm{R}-\Gamma, \mathrm{X}-\mathrm{Z}\end{array}$ & 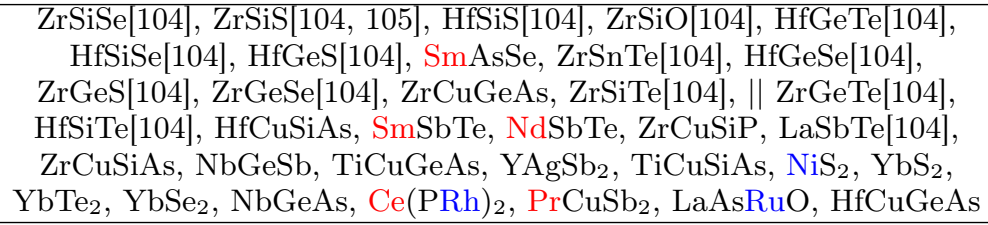 \\
\hline & $\begin{array}{l}\mathrm{A}-\mathrm{Z}, \mathrm{A}-\Gamma, \Gamma-\mathrm{M}, \mathrm{M}-\mathrm{Z}, \mathrm{R}-\mathrm{Z} \\
\mathrm{X}-\Gamma, \mathrm{R}-\mathrm{X}, \mathrm{X}-\mathrm{Z}\end{array}$ & $\| \mathrm{Sr}(\mathrm{AlAu})_{2}$ \\
\hline & $\begin{array}{l}\text { A-Z, A- } \Gamma, \Gamma-M, M-Z, X-\Gamma, \\
\text { R- } \Gamma, R-Z, X-Z\end{array}$ & $\| \mathrm{Ba}(\mathrm{ZnSn})_{2}, \mathrm{TbCoSi}, \mathrm{LaCoGe}, \mathrm{NdCoSi}, \mathrm{NdCoGe}$ \\
\hline & $\begin{array}{l}\text { A-M, A-Z, A- } \Gamma, \mathrm{R}-\mathrm{Z}, \mathrm{X}-\Gamma \\
\mathrm{R}-\Gamma, \mathrm{X}-\mathrm{Z}\end{array}$ & $\| \mathrm{ScCoC}_{2}$ \\
\hline & $\begin{array}{c}\mathrm{A}-\mathrm{Z}, \mathrm{A}-\Gamma, \Gamma-\mathrm{M}, \Gamma-\mathrm{Z}, \mathrm{M}-\mathrm{Z}, \\
\mathrm{R}-\mathrm{Z}, \mathrm{X}-\Gamma\end{array}$ & $\| \mathrm{Th}(\mathrm{GePt})_{2}, \mathrm{LaCoSb}_{2}$ \\
\hline & $\begin{array}{c}\mathrm{A}-\mathrm{Z}, \mathrm{A}-\Gamma, \Gamma-\mathrm{M}, \Gamma-\mathrm{Z}, \mathrm{M}-\mathrm{Z} \\
\mathrm{X}-\Gamma, \mathrm{R}-\Gamma\end{array}$ & $\| \mathrm{Ce}_{2} \mathrm{Zn}_{4} \mathrm{Ru}$ \\
\hline & $\begin{array}{c}\mathrm{A}-\mathrm{Z}, \mathrm{A}-\Gamma, \Gamma-\mathrm{M}, \mathrm{M}-\mathrm{Z}, \Gamma-\mathrm{Z} \\
\mathrm{X}-\Gamma, \mathrm{R}-\Gamma \\
\end{array}$ & $\| \mathrm{Ce}(\mathrm{SnPt})_{2}$ \\
\hline & $\begin{array}{c}\text { A-Z, A- } \Gamma, \Gamma-\mathrm{M}, \mathrm{M}-\mathrm{Z}, \mathrm{R}-\mathrm{Z} \\
\mathrm{R}-\mathrm{X}, \mathrm{R}-\Gamma\end{array}$ & $\| \mathrm{LaAgSb}_{2}$ \\
\hline
\end{tabular}


Table III - continued

\begin{tabular}{|c|c|c|}
\hline $\begin{array}{l}\text { SOC or } \\
\text { NSOC }\end{array}$ & $\begin{array}{c}\text { HSL with Band } \\
\text { Crossing }\end{array}$ & High Symmetry Line Semimetals \\
\hline & $\begin{array}{l}\mathrm{A}-\mathrm{Z}, \mathrm{A}-\Gamma, \Gamma-\mathrm{M}, \mathrm{M}-\mathrm{Z}, \mathrm{X}-\Gamma \\
\mathrm{R}-\mathrm{X}, \mathrm{X}-\mathrm{Z}\end{array}$ & $\| \mathrm{Sr}(\mathrm{GaAu})_{2}$ \\
\hline & $\begin{array}{c}\text { A-Z, } \Gamma-Z, \text { M-Z, R-Z, X- } \Gamma, \\
\text { R- } \Gamma, X-Z\end{array}$ & || CeTiGe \\
\hline & $\begin{array}{l}\text { A-Z, R-Z, X- } \Gamma, \Gamma-M, R-X, \\
\text { R- } \Gamma, X-Z\end{array}$ & \| LaNiAsO \\
\hline & $\begin{array}{c}\mathrm{A}-\mathrm{M}, \mathrm{A}-\mathrm{Z}, \Gamma-\mathrm{M}-\mathrm{M}, \Gamma-\mathrm{Z}, \mathrm{X}-\Gamma, \\
\mathrm{R}-\Gamma\end{array}$ & $\|$ FeSe \\
\hline & $\begin{array}{c}\mathrm{A}-\mathrm{M}, \mathrm{A}-\mathrm{Z}, \mathrm{\Gamma - \textrm {M }}, \mathrm{M}-\mathrm{Z}, \mathrm{R}-\mathrm{Z}, \\
\mathrm{X}-\Gamma\end{array}$ & $\| \operatorname{Li}_{2} \mathrm{Y}_{5} \operatorname{In}_{9}$ \\
\hline & $\begin{array}{c}\mathrm{A}-\mathrm{Z}, \mathrm{A}-\Gamma, \Gamma-\mathrm{M}, \Gamma-\mathrm{Z}, \mathrm{M}-\mathrm{Z}, \\
\mathrm{X}-\Gamma\end{array}$ & $\| \mathrm{Ba}(\mathrm{SbPd})_{2}$ \\
\hline & $\begin{array}{c}\mathrm{A}-\mathrm{Z}, \mathrm{A}-\Gamma, \underset{\mathrm{X}-\Gamma}{\Gamma-\mathrm{M}}, \mathrm{M}-\mathrm{Z}, \mathrm{R}-\mathrm{Z}, \\
\end{array}$ & $\| \mathrm{NbCrN}, \mathrm{Ta}_{2} \mathrm{Se}, \mathrm{BaMgPb}$ \\
\hline & $\begin{array}{l}\mathrm{A}-\mathrm{Z}, \Gamma-\mathrm{Z}, \mathrm{M}-\mathrm{Z}, \mathrm{X}-\Gamma, \Gamma-\mathrm{M} \\
\mathrm{R}-\Gamma\end{array}$ & || InBi(ICSD : 1253) \\
\hline & $\begin{array}{c}\mathrm{A}-\mathrm{Z}, \mathrm{R}-\mathrm{Z}, \mathrm{X}-\mathrm{\Gamma}, \Gamma-\mathrm{M}, \mathrm{R}-\Gamma, \\
\mathrm{X}-\mathrm{Z}\end{array}$ & BaAlGeF, $\|$ NiSe \\
\hline & $\mathrm{A}-\Gamma, \Gamma-\mathrm{M}, \Gamma-\mathrm{Z}, \mathrm{X}-\Gamma, \mathrm{R}-\Gamma$ & $\mathrm{Ce}(\mathrm{PIr})_{2}, \mathrm{U}(\mathrm{SiIr})_{2}$ \\
\hline & $\mathrm{A}-\mathrm{Z}, \mathrm{A}-\Gamma, \Gamma-\mathrm{M}, \Gamma-\mathrm{Z}, \mathrm{R}-\mathrm{Z}$ & $\| \mathrm{LiFeP}$ \\
\hline & $\mathrm{A}-\mathrm{Z}, \mathrm{A}-\Gamma, \Gamma-\mathrm{M}, \mathrm{M}-\mathrm{Z}, \Gamma-\mathrm{Z}$ & $\|$ NiAsRh \\
\hline & $\mathrm{A}-\mathrm{Z}, \mathrm{A}-\Gamma, \Gamma-\mathrm{M}, \mathrm{M}-\mathrm{Z}, \mathrm{R}-\mathrm{Z}$ & || $\mathrm{TiCd}, \mathrm{LaBiS}_{2} \mathrm{O}(\mathrm{ICSD}: 252466)$ \\
\hline & A-Z, A-Г, Г-M, M-Z, X-Г & 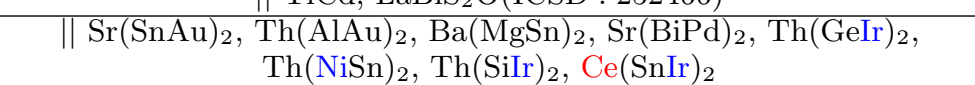 \\
\hline & $\mathrm{A}-\mathrm{Z}, \Gamma-\mathrm{Z}, \mathrm{M}-\mathrm{Z}, \mathrm{X}-\Gamma, \Gamma-\mathrm{M}$ & $\| \mathrm{NdMnSi}$, PrMnSi, PrMnGe \\
\hline & $\mathrm{A}-\mathrm{Z}, \mathrm{R}-\mathrm{Z}, \mathrm{X}-\Gamma, \mathrm{R}-\Gamma, \mathrm{X}-\mathrm{Z}$ & $\| \mathrm{YNiBC}$ \\
\hline & $\mathrm{A}-\mathrm{Z}, \mathrm{A}-\Gamma, \Gamma-\mathrm{M}, \Gamma-\mathrm{Z}$ & $\|$ ErNiBC, TbNiBC \\
\hline & $\mathrm{A}-\mathrm{Z}, \mathrm{A}-\Gamma, \Gamma-\mathrm{M}, \mathrm{M}-\mathrm{Z}$ & $\mathrm{ZrS}, \mathrm{PrPOsO}, \mathrm{YbFeSi}$ \\
\hline & $\mathrm{A}-\mathrm{Z}, \mathrm{R}-\mathrm{Z}, \Gamma-\mathrm{Z}, \mathrm{X}-\mathrm{Z}$ & \begin{tabular}{l|l}
$\mathrm{Np}$ \\
$\mathrm{n}$
\end{tabular} \\
\hline & $\mathrm{A}-\mathrm{Z}, \mathrm{R}-\mathrm{Z}, \mathrm{X}-\Gamma, \Gamma-\mathrm{M}$ & \# MnAlGe \\
\hline & $\Gamma-\mathrm{Z}, \mathrm{X}-\Gamma, \Gamma-\mathrm{M}, \mathrm{R}-\Gamma$ & $\mathrm{La}_{2} \mathrm{BiN} \|$ \\
\hline & $\mathrm{A}-\Gamma, \Gamma-\mathrm{M}, \Gamma-\mathrm{Z}$ & $\mathrm{CaGaN}$ \\
\hline & $\mathrm{A}-\mathrm{M}, \mathrm{A}-\mathrm{Z}, \mathrm{A}-\Gamma$ & $\| \mathrm{FeS}$ \\
\hline & $\mathrm{A}-\mathrm{Z}$ & $\|$ LuNiBC \\
\hline & $\Gamma-\mathrm{M}$ & ||| HfCd \\
\hline $\mathrm{SOC}$ & $\Gamma-\mathrm{Z}$ & 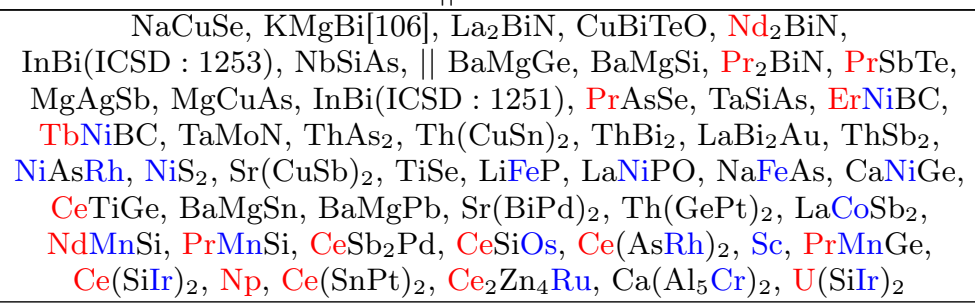 \\
\hline & & Space Group 130: P4/ncc \\
\hline & $\Gamma-\mathrm{Z}, \mathrm{R}-\mathrm{X}, \mathrm{R}-\Gamma, \mathrm{X}-\mathrm{Z}$ & $\mathrm{Nd}_{5} \mathrm{Ir}_{3}$ \\
\hline NSOC & $\mathrm{A}-\Gamma, \Gamma-\mathrm{M}, \Gamma-\mathrm{Z}$ & $\mathrm{La}_{5} \mathrm{Ga}_{3}$ \\
\hline NSUC & $\mathrm{A}-\mathrm{M}, \Gamma-\mathrm{M}, \mathrm{M}-\mathrm{Z}$ & $\| \mathrm{Ba}_{5} \mathrm{Ge}_{3}$ \\
\hline & $\Gamma-\mathrm{Z}, \Gamma-\mathrm{M}$ & $\mathrm{Pr}_{5} \mathrm{Ga}_{3}, \mathrm{Sm}_{5} \mathrm{Ga}_{3}$ \\
\hline $\mathrm{SOC}$ & $\Gamma-\mathrm{Z}$ & $\| \mathrm{Pr}_{5} \mathrm{Ga}_{3}, \mathrm{Sm}_{5} \mathrm{Ga}_{3}$ \\
\hline & & Space Group 131: $P 4_{2} / m m c$ \\
\hline & $\begin{array}{l}\text { A-M, A-Г, Г-M, Г-Z, M-Z, } \\
\text { R-M, X-M, X-Г, R-Z, X-Z }\end{array}$ & $\| \mathrm{ThC}_{2}$ \\
\hline NSOC & $\begin{array}{c}\mathrm{R}-\mathrm{A}, \mathrm{A}-\Gamma, \Gamma-\mathrm{M}, \Gamma-\mathrm{Z}, \mathrm{R}-\mathrm{M}, \\
\mathrm{X}-\mathrm{A}, \mathrm{X}-\mathrm{M}, \mathrm{X}-\Gamma\end{array}$ & $\| \mathrm{Ca}(\mathrm{BC})_{2}, \mathrm{Yb}(\mathrm{BC})_{2}$ \\
\hline & $\begin{array}{l}\text { R-A, R-Z, X- } \Gamma, \Gamma-\mathrm{M}, \mathrm{R}-\Gamma \\
\text { X-Z, X-M }\end{array}$ & $\| \mathrm{TcN}$ \\
\hline & $\begin{array}{c}\mathrm{R}-\mathrm{X}, \mathrm{X}-\mathrm{A}, \mathrm{X}-\mathrm{M}, \Gamma-\mathrm{Z}, \mathrm{X}-\Gamma, \\
\mathrm{R}-\Gamma, \mathrm{R}-\mathrm{Z}\end{array}$ & $\| \operatorname{IrN}$ \\
\hline soc & $\mathrm{A}-\mathrm{M}$ & $\mathrm{PtO}, \mathrm{PdO} \|$ \\
\hline
\end{tabular}


Table III - continued

\begin{tabular}{|c|c|c|}
\hline $\begin{array}{l}\text { SOC or } \\
\text { NSOC }\end{array}$ & $\begin{array}{l}\text { HSL with Band } \\
\text { Crossing }\end{array}$ & High Symmetry Line Semimetals \\
\hline & $\Gamma-Z$ & $\| \mathrm{Ca}(\mathrm{BC})_{2}, \mathrm{Yb}(\mathrm{BC})_{2}, \mathrm{TmCoC}$, ErCoC, $\mathrm{YCoC}$ \\
\hline & & Space Group 133: $P 4_{2} / n b c$ \\
\hline $\mathrm{SOC}$ & $\Gamma-\mathrm{Z}$ & $\| \mathrm{V}_{3} \mathrm{~S}$ \\
\hline \multirow{3}{*}{ NSOC } & \multicolumn{2}{|r|}{ Space Group 135: $P 4_{2} / m b c$} \\
\hline & $\begin{array}{c}\text { A-M, A-Г, Г-M, Г-Z, M-Z, } \\
\text { X- } \Gamma, \mathrm{R}-\Gamma\end{array}$ & $\| \mathrm{YB}_{2} \mathrm{C}, \mathrm{TbB}_{2} \mathrm{C}$ \\
\hline & $\begin{array}{c}\mathrm{A}-\Gamma, \Gamma-\mathrm{M}, \Gamma-\mathrm{Z}, \mathrm{X}-\Gamma, \mathrm{R}-\mathrm{Z} \\
\mathrm{X}-\mathrm{Z} \\
\end{array}$ & $\|$ Cs \\
\hline SOC & $\Gamma-\mathrm{Z}$ & \multirow{2}{*}{$\begin{array}{l}\mathrm{Sr}_{2} \mathrm{GeN}_{2}, \mathrm{Ca}_{2} \mathrm{GeN}_{2}, \| \mathrm{YB}_{2} \mathrm{C}, \mathrm{TbB}_{2} \mathrm{C} \\
\text { Space Group 136: } P 4_{2} / \text { mnm }\end{array}$} \\
\hline \multirow{28}{*}{ NSOC } & & \\
\hline & $\begin{array}{l}\text { A-M, A-Z, A-Г, Г-Z, M-Z, } \\
\quad \text { X- } \Gamma, \Gamma-\mathrm{M}, \mathrm{R}-\Gamma, \mathrm{X}-\mathrm{M}\end{array}$ & $\| \mathrm{Tb}_{2} \mathrm{Fe}_{14} \mathrm{~B}$ \\
\hline & 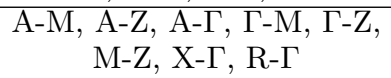 & $\| \mathrm{Er}_{2} \mathrm{Fe}_{14} \mathrm{~B}$ \\
\hline & $\begin{array}{l}\text { A-Z, A- } \Gamma, \Gamma-\mathrm{M}, \mathrm{M}-\mathrm{Z}, \Gamma-\mathrm{Z} \\
\text { X- } \Gamma, \mathrm{R}-\Gamma, \mathrm{X}-\mathrm{M}\end{array}$ & $\| \mathrm{Hf}_{2} \mathrm{InNi}_{2}, \mathrm{Zr}_{2} \mathrm{InPd}_{2}$ \\
\hline & $\begin{array}{c}\text { A-M, A- } \Gamma, \Gamma-\mathrm{M}, \Gamma-\mathrm{Z}, \mathrm{M}-\mathrm{Z}, \\
\text { X- } \Gamma, \mathrm{R}-\Gamma\end{array}$ & $\| \mathrm{Yb}_{2} \mathrm{PbAu}_{2}$ \\
\hline & $\begin{array}{l}\text { A-M, A-Z, A- }, \Gamma-\mathrm{Z}, \mathrm{M}-\mathrm{Z} \\
\Gamma-\mathrm{M}, \mathrm{X}-\mathrm{M}\end{array}$ & $\| \mathrm{Er}_{2} \mathrm{SnAu}_{2}$ \\
\hline & $\begin{array}{c}\text { A-M, A-Z, Г-M, Г-Z, M-Z, } \\
\text { X- } \Gamma, \text { R- } \Gamma\end{array}$ & $\| \mathrm{Na}_{3} \mathrm{Hg}_{2}(\mathrm{ICSD}: 172518), \mathrm{Na}_{3} \mathrm{Hg}_{2}(\mathrm{ICSD}: 104328)$ \\
\hline & $\begin{array}{c}\mathrm{A}-\mathrm{M}, \mathrm{A}-\mathrm{Z}, \mathrm{A}-\Gamma, \Gamma-\mathrm{Z}, \Gamma-\mathrm{M} \\
\mathrm{X}-\mathrm{M}\end{array}$ & $\| \mathrm{Yb}_{2} \mathrm{Pt}_{2} \mathrm{~Pb}$ \\
\hline & $\begin{array}{c}\mathrm{A}-\mathrm{M}, \mathrm{A}-\mathrm{Z}, \mathrm{A}-\Gamma, \Gamma-\mathrm{Z}, \mathrm{X}-\Gamma, \\
\mathrm{R}-\Gamma\end{array}$ & $\| \mathrm{Hf}_{3} \mathrm{Al}_{2}$ \\
\hline & $\begin{array}{l}\mathrm{A}-\mathrm{M}, \Gamma-\mathrm{M}, \mathrm{M}-\mathrm{Z}, \Gamma-\mathrm{Z}, \mathrm{X}-\Gamma, \\
\mathrm{R}-\Gamma\end{array}$ & $\| \mathrm{TiH}_{2}, \mathrm{RuO}_{2}, \mathrm{OsO}_{2}$ \\
\hline & $\begin{array}{c}\mathrm{A}-\mathrm{Z}, \mathrm{A}-\Gamma, \Gamma-\mathrm{M}, \mathrm{M}-\mathrm{Z}, \Gamma-\mathrm{Z} \\
\mathrm{X}-\Gamma\end{array}$ & $\| \mathrm{Nd}_{2} \mathrm{Fe}_{14} \mathrm{~B}$ \\
\hline & $\begin{array}{c}\mathrm{A}-\mathrm{Z}, \mathrm{A}-\Gamma, \Gamma-\mathrm{M}, \mathrm{M}-\mathrm{Z}, \Gamma-\mathrm{Z} \\
\mathrm{X}-\mathrm{M}\end{array}$ & $\| \mathrm{Y}_{2} \mathrm{Fe}_{14} \mathrm{~B}, \mathrm{Ho}_{2} \mathrm{Fe}_{14} \mathrm{~B}, \mathrm{Lu}_{2} \mathrm{Fe}_{14} \mathrm{~B}$ \\
\hline & $\mathrm{A}-\Gamma, \Gamma-\mathrm{M}, \Gamma-\mathrm{Z}, \mathrm{X}-\Gamma, \mathrm{R}-\Gamma$ & $\| \mathrm{Ga}_{3} \mathrm{Co}$ \\
\hline & $\mathrm{A}-\mathrm{M}, \mathrm{A}-\mathrm{Z}, \mathrm{A}-\Gamma, \Gamma-\mathrm{M}, \mathrm{M}-\mathrm{Z}$ & $\| \mathrm{Zr}\left(\mathrm{Fe}_{2} \mathrm{Si}\right)_{2}$ \\
\hline & $\mathrm{A}-\mathrm{M}, \mathrm{A}-\mathrm{Z}, \mathrm{A}-\Gamma, \Gamma-\mathrm{Z}, \mathrm{M}-\mathrm{Z}$ & $\| \mathrm{MoO}_{2}(\mathrm{ICSD}: 23722), \mathrm{Hf}_{2} \mathrm{Ni}_{2} \mathrm{Sn}$ \\
\hline & $\mathrm{A}-\mathrm{M}, \mathrm{A}-\mathrm{Z}, \mathrm{A}-\Gamma, \mathrm{X}-\Gamma, \Gamma-\mathrm{M}$ & $\| \mathrm{Sr}_{3} \mathrm{Li}_{2}$ \\
\hline & $\mathrm{A}-\mathrm{Z}, \mathrm{A}-\Gamma, \Gamma-\mathrm{M}, \Gamma-\mathrm{Z}, \mathrm{M}-\mathrm{Z}$ & $\mathrm{Zr}_{2} \mathrm{Ni}_{2} \mathrm{Sn}$ \\
\hline & $\mathrm{A}-\mathrm{Z}, \Gamma-\mathrm{Z}, \mathrm{M}-\mathrm{Z}, \mathrm{X}-\Gamma, \Gamma-\mathrm{M}$ & $\| \mathrm{Yb}\left(\mathrm{Ni}_{2} \mathrm{P}\right)_{2}$ \\
\hline & $\mathrm{A}-\Gamma, \Gamma-\mathrm{M}, \Gamma-\mathrm{Z}, \mathrm{X}-\Gamma$ & $\| \mathrm{In}_{3} \mathrm{Ir}, \mathrm{In}_{3} \mathrm{Co}, \mathrm{Nd}_{2} \mathrm{Co}_{14} \mathrm{~B}$ \\
\hline & $\mathrm{A}-\mathrm{M}, \mathrm{A}-\Gamma, \Gamma-\mathrm{Z}, \mathrm{M}-\mathrm{Z}$ & $\| \mathrm{MoO}_{2}(\mathrm{ICSD}: 108875), \mathrm{Tb}_{3} \mathrm{Al}_{2}$ \\
\hline & $\mathrm{A}-\mathrm{M}, \Gamma-\mathrm{M}, \mathrm{M}-\mathrm{Z}, \mathrm{X}-\Gamma$ & $\| \mathrm{Ca}_{3} \mathrm{Cd}_{2}$ \\
\hline & $\mathrm{A}-\mathrm{M}, \Gamma-\mathrm{M}, \mathrm{M}-\mathrm{Z}, \mathrm{X}-\mathrm{M}$ & $\| \mathrm{Lu}_{3} \mathrm{Al}_{2}$ \\
\hline & $\mathrm{A}-\mathrm{Z}, \mathrm{A}-\Gamma, \Gamma-\mathrm{M}, \mathrm{M}-\mathrm{Z}$ & $\| \mathrm{Pr}_{2} \mathrm{MoC}_{2}, \mathrm{Nd}_{2} \mathrm{MoC}_{2}, \mathrm{Nd}_{2} \mathrm{WC}_{2}, \mathrm{Pr}_{2} \mathrm{WC}_{2}, \mathrm{Ce}_{2} \mathrm{Fe}_{14} \mathrm{~B}$ \\
\hline & $\Gamma-\mathrm{Z}, \mathrm{X}-\Gamma, \Gamma-\mathrm{M}, \mathrm{R}-\Gamma$ & || $\mathrm{Ba}(\mathrm{TlHg})_{2}$ \\
\hline & $\mathrm{A}-\Gamma, \Gamma-\mathrm{M}, \Gamma-\mathrm{Z}$ & $\mathrm{PbO}_{2}, \mathrm{Mg}\left(\mathrm{BiO}_{3}\right)_{2}\left[57, \| \mathrm{PtO}_{2}\right.$ \\
\hline & $\mathrm{A}-\mathrm{M}, \mathrm{A}-\mathrm{Z}, \mathrm{A}-\Gamma$ & $\| \mathrm{WO}_{2}$ \\
\hline & $\mathrm{A}-\mathrm{M}, \Gamma-\mathrm{M}, \mathrm{M}-\mathrm{Z}$ & $\| \mathrm{Er}_{3} \mathrm{Al}_{2}, \mathrm{Tm}_{3} \mathrm{Al}_{2}, \mathrm{U}$ \\
\hline & $\mathrm{X}-\Gamma, \Gamma-\mathrm{M}$ & $\mathrm{CsAg}_{5} \mathrm{Se}_{3}, \| \mathrm{Ca}\left(\mathrm{Cu}_{2} \mathrm{P}\right)_{2}, \mathrm{Zr}_{2} \mathrm{In}_{5} \mathrm{Ni}$ \\
\hline $\mathrm{SOC}$ & $\Gamma-\mathrm{Z}$ & $\begin{array}{c}\mathrm{PbO}_{2}, \mathrm{Mg}\left(\mathrm{BiO}_{3}\right)_{2}[57], \| \mathrm{In}_{3} \mathrm{Ir}, \mathrm{In}_{3} \mathrm{Rh}, \mathrm{In}_{3} \mathrm{Co}, \mathrm{PtO}_{2}, \mathrm{Er}_{2} \mathrm{SnAu}_{2}, \\
\mathrm{Yb}_{2} \mathrm{PbAu}_{2}, \mathrm{BiPtPb}_{3}, \mathrm{Hf}_{2} \mathrm{Ni}_{2} \mathrm{Sn}, \mathrm{Yb}_{2} \mathrm{Pt}_{2} \mathrm{~Pb}, \mathrm{Hf}_{2} \mathrm{InNi}_{2}, \mathrm{Zr}_{2} \mathrm{Ni}_{2} \mathrm{Sn}, \\
\mathrm{Ti}_{2} \mathrm{InNi}_{2}, \mathrm{Zr}_{2} \mathrm{InPd}_{2}, \mathrm{~Tb}_{3} \mathrm{Al}_{2}, \mathrm{Zr}\left(\mathrm{Ni}_{2} \mathrm{P}\right)_{2}, \mathrm{Dy}_{3} \mathrm{Al}_{2}, \mathrm{Nd}_{2} \mathrm{Fe}_{14} \mathrm{~B}, \\
\mathrm{Ho}_{2} \mathrm{Fe}_{14} \mathrm{~B}, \mathrm{Lu}_{2} \mathrm{Fe}_{14} \mathrm{~B}, \mathrm{Y}_{2} \mathrm{Fe}_{14} \mathrm{~B}\end{array}$ \\
\hline & & Space Group 137: $P 4_{2} / n m c$ \\
\hline $\mathrm{NSOC}$ & $\begin{array}{c}\mathrm{A}-\Gamma, \Gamma-\mathrm{M}, \Gamma-\mathrm{Z}, \mathrm{X}-\Gamma, \mathrm{R}-\Gamma, \\
\mathrm{R}-\mathrm{Z}, \mathrm{X}-\mathrm{Z} \\
\end{array}$ & $\| \operatorname{Er}_{14}(\operatorname{InPd})_{3}$ \\
\hline DOS & $\mathrm{A}-\Gamma, \Gamma-\mathrm{M}, \Gamma-\mathrm{Z}, \mathrm{X}-\Gamma, \mathrm{R}-\Gamma$ & $\| \mathrm{ErAl}_{9}\left(\mathrm{Fe}_{2} \mathrm{Si}_{3}\right)_{2}, \mathrm{TbAl}_{9}\left(\mathrm{Fe}_{2} \mathrm{Si}_{3}\right)_{2}$ \\
\hline & $\mathrm{A}-\mathrm{M}, \Gamma-\mathrm{M}, \mathrm{M}-\mathrm{Z}, \mathrm{X}-\Gamma$ & $\| \mathrm{Y}_{14}(\mathrm{InRh})_{3}$ \\
\hline $\mathrm{SOC}$ & $\Gamma-\mathrm{Z}$ & $\mathrm{Cd}_{3} \mathrm{As}_{2}[63], \| \mathrm{Nb}_{4} \mathrm{O}_{5}, \mathrm{U}(\mathrm{CoB})_{4}, \mathrm{Ce}(\mathrm{CoB})_{4}$ \\
\hline
\end{tabular}


Table III - continued

\begin{tabular}{|c|c|c|}
\hline $\begin{array}{l}\text { SOC or } \\
\text { NSOC }\end{array}$ & $\begin{array}{l}\text { HSL with Band } \\
\text { Crossing }\end{array}$ & High Symmetry Line Semimetals \\
\hline \multicolumn{3}{|r|}{ Space Group 138: $P 4_{2} / \mathrm{ncm}$} \\
\hline NSOC & $\begin{array}{c}\text { A-M, A-Z, A- } \Gamma, \Gamma-\mathrm{M}, \mathrm{M}-\mathrm{Z}, \\
\Gamma-\mathrm{Z}, \mathrm{X}-\Gamma, \mathrm{R}-\Gamma\end{array}$ & $\| \mathrm{SmAu}_{6}$ \\
\hline $\mathrm{SOC}$ & $\Gamma-Z$ & $\mathrm{SmAu}_{6}$ \\
\hline \multirow{32}{*}{ NSOC } & \multicolumn{2}{|r|}{ Space Group 139: $I 4 / \mathrm{mmm}$} \\
\hline & $\begin{array}{l}\Gamma-\mathrm{M}, \mathrm{X}-\Gamma, \mathrm{X}-\mathrm{M}, \mathrm{P}-\Gamma, \mathrm{P}-\mathrm{M}, \\
\mathrm{N}-\Gamma, \mathrm{N}-\mathrm{M}, \mathrm{N}-\mathrm{P}\end{array}$ & $\| \mathrm{YbGa}_{5}$ \\
\hline & $\begin{array}{l}\Gamma-\mathrm{M}, \mathrm{X}-\Gamma, \mathrm{X}-\mathrm{P}, \mathrm{X}-\mathrm{M}, \mathrm{P}-\Gamma \\
\mathrm{N}-\Gamma, \mathrm{N}-\mathrm{M}, \mathrm{N}-\mathrm{P}\end{array}$ & $\| \mathrm{ThCo}_{2}$ \\
\hline & $\begin{array}{c}\mathrm{X}-\Gamma, \Gamma-\mathrm{M}, \mathrm{X}-\mathrm{M}, \mathrm{P}-\Gamma, \mathrm{P}-\mathrm{M}, \\
\mathrm{N}-\Gamma, \mathrm{N}-\mathrm{M}, \mathrm{X}-\mathrm{P}\end{array}$ & $\| \mathrm{Sr}(\mathrm{CoP})_{2}$ \\
\hline & $\begin{array}{c}\Gamma-\mathrm{M}, \mathrm{X}-\Gamma, \mathrm{P}-\Gamma, \mathrm{N}-\Gamma, \mathrm{N}-\mathrm{M}, \\
\text { X-M, X-P }\end{array}$ & $\| \mathrm{TiPt}_{8}$ \\
\hline & $\begin{array}{l}\Gamma-\mathrm{M}, \mathrm{X}-\Gamma, \mathrm{X}-\mathrm{M}, \mathrm{P}-\Gamma, \mathrm{P}-\mathrm{M} \\
\mathrm{N}-\mathrm{M}, \mathrm{X}-\mathrm{P}\end{array}$ & $\| \mathrm{U}(\mathrm{CuSi})_{2}$ \\
\hline & $\begin{array}{l}\Gamma-\mathrm{M}, \mathrm{X}-\Gamma, \mathrm{X}-\mathrm{M}, \mathrm{P}-\mathrm{M}, \mathrm{N}-\Gamma \\
\text { N-M, X-P }\end{array}$ & $\| \mathrm{NiS}_{2}$ \\
\hline & $\begin{array}{l}\Gamma-\mathrm{M}, \mathrm{X}-\Gamma, \mathrm{X}-\mathrm{P}, \mathrm{P}-\Gamma, \mathrm{P}-\mathrm{M} \\
\mathrm{N}-\mathrm{M}, \mathrm{X}-\mathrm{M}\end{array}$ & $\| \mathrm{Ca}\left(\mathrm{CdAu}_{2}\right)_{2}$ \\
\hline & $\begin{array}{c}\text { T-M, X-Г, X-P, P-M, N-M, } \\
\text { X-M, N-P }\end{array}$ & $\| \operatorname{InPd}_{3}(\mathrm{ICSD}: 247188)$ \\
\hline & $\begin{array}{c}\mathrm{X}-\Gamma, \Gamma-\mathrm{M}, \mathrm{X}-\mathrm{M}, \mathrm{P}-\Gamma, \mathrm{P}-\mathrm{M} \\
\mathrm{N}-\Gamma, \mathrm{N}-\mathrm{M}\end{array}$ & $\begin{aligned} \| \mathrm{ZrGa}_{3}(\mathrm{ICSD}: 635698), & \mathrm{ZrIn}_{3}(\mathrm{ICSD}: 640719), \mathrm{CaBi}_{2}(\mathrm{CO})_{2}, \\
& \mathrm{Hf}_{11} \mathrm{Ga}_{10}\end{aligned}$ \\
\hline & $\begin{array}{l}\mathrm{X}-\Gamma, \Gamma-\mathrm{M}, \mathrm{X}-\mathrm{M}, \mathrm{P}-\Gamma, \mathrm{P}-\mathrm{M} \\
\text { N-P, X-P }\end{array}$ & ( \\
\hline & $\begin{array}{l}\mathrm{X}-\Gamma, \mathrm{X}-\mathrm{P}, \mathrm{P}-\Gamma, \mathrm{P}-\mathrm{M}, \mathrm{N}-\Gamma \\
\mathrm{N}-\mathrm{M}, \mathrm{X}-\mathrm{M}\end{array}$ & $\| \mathrm{Sr}_{3} \mathrm{Ru}_{2} \mathrm{O}_{7}$ \\
\hline & $\begin{array}{c}\Gamma-\mathrm{M}, \mathrm{X}-\Gamma, \mathrm{P}-\Gamma, \mathrm{N}-\Gamma, \mathrm{N}-\mathrm{M} \\
\mathrm{X}-\mathrm{M}\end{array}$ & $\begin{array}{c}\| \mathrm{SrGa}_{4}, \mathrm{Ba}(\mathrm{ZnSi})_{2}, \mathrm{CaGa}_{4}, \mathrm{BaIn}_{4}, \mathrm{CaAl}_{4}, \mathrm{YbGa}_{4}, \mathrm{BaGa}_{4}, \mathrm{SrAl}_{4}, \\
\mathrm{BaAl}_{4}, \mathrm{Pr}_{2} \mathrm{Sb}, \mathrm{Nd}_{2} \mathrm{Bi}, \mathrm{Sm}_{2} \mathrm{Sb}\end{array}$ \\
\hline & $\begin{array}{c}\Gamma-\mathrm{M}, \mathrm{X}-\Gamma, \underset{\mathrm{X}}{\mathrm{P}-\mathrm{P}, \mathrm{N}} \mathrm{N}-\Gamma, \mathrm{X}-\mathrm{M}, \\
\end{array}$ & ( \\
\hline & $\begin{array}{c}\Gamma-\mathrm{M}, \mathrm{X}-\Gamma, \mathrm{P}-\Gamma, \mathrm{N}-\mathrm{M}, \mathrm{X}-\mathrm{M}, \\
\mathrm{X}-\mathrm{P}\end{array}$ & $\| \mathrm{Nb}_{4} \mathrm{Co}_{4} \mathrm{Si}_{7}$ \\
\hline & $\begin{array}{c}\Gamma-\mathrm{M}, \mathrm{X}-\Gamma, \mathrm{X}-\mathrm{M}, \mathrm{P}-\Gamma, \mathrm{P}-\mathrm{M} \\
\mathrm{N}-\mathrm{M}\end{array}$ & $\| \mathrm{U}(\mathrm{CuGe})_{2}$ \\
\hline & $\begin{array}{c}\Gamma-\mathrm{M}, \mathrm{X}-\Gamma, \mathrm{X}-\mathrm{M}, \mathrm{P}-\Gamma, \mathrm{P}-\mathrm{M} \\
\mathrm{N}-\mathrm{P}\end{array}$ & || ThGeSe, ThSiTe, ThSiSe, ThGeTe \\
\hline & $\begin{array}{c}\Gamma-\mathrm{M}, \mathrm{X}-\Gamma, \underset{\mathrm{X}}{\mathrm{X}-\mathrm{P}}, \mathrm{P} \\
\mathrm{C}-\mathrm{M}, \mathrm{N}-\mathrm{M},\end{array}$ & $\| \mathrm{Yb}(\mathrm{CoGe})_{2}, \mathrm{Ca}(\mathrm{CoGe})_{2}$ \\
\hline & $\begin{array}{c}\Gamma-\mathrm{M}, \mathrm{X}-\Gamma, \underset{\mathrm{X}-\mathrm{P}, \mathrm{X}-\mathrm{M}, \mathrm{N}-\Gamma}{\mathrm{N}-\mathrm{M}} \\
\end{array}$ & II $\operatorname{Th}\left(\mathrm{Al}_{2} \mathrm{Cr}\right)_{4}$ \\
\hline & 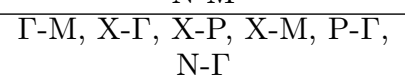 & $\| \mathrm{Mg}_{2} \mathrm{Ga}_{5}$ \\
\hline & $\begin{array}{c}\Gamma-\mathrm{M}, \mathrm{X}-\mathrm{P}, \mathrm{P}-\Gamma, \mathrm{P}-\mathrm{M}, \mathrm{N}-\mathrm{M}, \\
\text { X-M }\end{array}$ & $\| \mathrm{Ni}_{18} \mathrm{Bi}_{3} \mathrm{AsS}_{16}$ \\
\hline & $\begin{array}{c}\mathrm{X}-\Gamma, \Gamma-\mathrm{M}, \mathrm{X}-\mathrm{M}, \mathrm{P}-\Gamma, \mathrm{P}-\mathrm{M} \\
\mathrm{N}-\mathrm{P}\end{array}$ & $\| \mathrm{SrZnBi}_{2}$, ThSiS \\
\hline & $\begin{array}{c}\mathrm{X}-\Gamma, \Gamma-\mathrm{M}, \mathrm{X}-\mathrm{M}, \mathrm{P}-\Gamma, \mathrm{P}-\mathrm{M} \\
\mathrm{X}-\mathrm{P}\end{array}$ & $\| \mathrm{Ba}(\mathrm{FeP})_{2}$ \\
\hline & $\begin{array}{l}\mathrm{X}-\Gamma, \mathrm{X}-\mathrm{M}, \mathrm{P}-\Gamma, \mathrm{P}-\mathrm{M}, \mathrm{N}-\Gamma, \\
\mathrm{N}-\mathrm{M}\end{array}$ & $\| \mathrm{Ba}_{2} \mathrm{Ca}_{3} \mathrm{Tl}_{2}\left(\mathrm{CuO}_{3}\right)_{4}(\mathrm{ICSD}: 68585), \mathrm{UCuP}_{2}$ \\
\hline & $\Gamma-\mathrm{M}, \mathrm{N}-\Gamma, \mathrm{X}-\mathrm{M}, \mathrm{N}-\mathrm{P}, \mathrm{X}-\mathrm{P}$ & $\| \mathrm{Be}_{12} \mathrm{~W}, \mathrm{Be}_{12} \mathrm{Mo}$ \\
\hline & $\Gamma-\mathrm{M}, \mathrm{X}-\Gamma, \mathrm{P}-\Gamma, \mathrm{N}-\Gamma, \mathrm{N}-\mathrm{M}$ & $\| \mathrm{Ba}(\mathrm{CrAs})_{2}$ \\
\hline & $\Gamma-\mathrm{M}, \mathrm{X}-\Gamma, \mathrm{P}-\Gamma, \mathrm{N}-\mathrm{M}, \mathrm{N}-\mathrm{P}$ & $\| \mathrm{Sr}(\mathrm{CoAs})_{2}$ \\
\hline & $\Gamma-\mathrm{M}, \mathrm{X}-\Gamma, \mathrm{P}-\Gamma, \mathrm{N}-\mathrm{M}, \mathrm{X}-\mathrm{M}$ & $\| \mathrm{Sr}(\mathrm{AlPb})_{2}$ \\
\hline & $\Gamma-\mathrm{M}, \mathrm{X}-\Gamma, \mathrm{P}-\Gamma, \mathrm{X}-\mathrm{M}, \mathrm{N}-\mathrm{P}$ & $\| \mathrm{Ba}(\mathrm{NiAs})_{2}$ \\
\hline & $\Gamma-\mathrm{M}, \mathrm{X}-\Gamma, \mathrm{X}-\mathrm{M}, \mathrm{P}-\mathrm{M}, \mathrm{N}-\mathrm{M}$ & $\| \mathrm{Sr}(\mathrm{PRh})_{2}$ \\
\hline & $\Gamma-\mathrm{M}, \mathrm{X}-\Gamma, \mathrm{X}-\mathrm{M}, \mathrm{P}-\mathrm{M}, \mathrm{X}-\mathrm{P}$ & $\| \mathrm{Ca}\left(\mathrm{MnAl}_{2}\right)_{4}$ \\
\hline & $\Gamma-\mathrm{M}, \mathrm{X}-\Gamma, \mathrm{X}-\mathrm{P}, \mathrm{P}-\mathrm{M}, \mathrm{X}-\mathrm{M}$ & $\mathrm{Yb}(\mathrm{SiOs})_{2}$ \\
\hline
\end{tabular}


Table III - continued

\begin{tabular}{|c|c|c|}
\hline $\begin{array}{l}\text { SOC or } \\
\text { NSOC }\end{array}$ & $\begin{array}{l}\text { HSL with Band } \\
\text { Crossing }\end{array}$ & High Symmetry Line Semimetals \\
\hline & $\Gamma-\mathrm{M}, \mathrm{X}-\mathrm{M}, \mathrm{P}-\mathrm{M}, \mathrm{N}-\Gamma, \mathrm{X}-\mathrm{P}$ & $\| \mathrm{Zr}_{4} \mathrm{Fe}_{4} \mathrm{Si}_{7}$ \\
\hline & T-M, X-M, P-M, N-M, N-P & $\| \mathrm{YbPd}_{2}, \mathrm{CaPd}_{2}$ \\
\hline & $\Gamma-\mathrm{M}, \mathrm{X}-\mathrm{M}, \mathrm{P}-\mathrm{M}, \mathrm{N}-\mathrm{P}, \mathrm{X}-\mathrm{P}$ & $\| \mathrm{Ce}(\mathrm{SiOs})_{2}$ \\
\hline & $\Gamma-\mathrm{M}, \mathrm{X}-\mathrm{P}, \mathrm{P}-\Gamma, \mathrm{P}-\mathrm{M}, \mathrm{X}-\mathrm{M}$ & $\| \mathrm{BeAu}_{2}$ \\
\hline & $\mathrm{X}-\Gamma, \Gamma-\mathrm{M}, \mathrm{N}-\Gamma, \mathrm{N}-\mathrm{M}, \mathrm{N}-\mathrm{P}$ & $\| \mathrm{U}_{2} \mathrm{TeO}_{2}$ \\
\hline & $\mathrm{X}-\Gamma, \Gamma-\mathrm{M}, \mathrm{N}-\Gamma, \mathrm{N}-\mathrm{M}, \mathrm{X}-\mathrm{M}$ & $\| \mathrm{Yb}(\mathrm{AlGa})_{2}, \mathrm{Nd}_{2} \mathrm{Sb}, \mathrm{La}_{2} \mathrm{Sb}$ \\
\hline & $\mathrm{X}-\Gamma, \Gamma-\mathrm{M}, \mathrm{X}-\mathrm{M}, \mathrm{P}-\Gamma, \mathrm{P}-\mathrm{M}$ & $\mathrm{Ca}_{3} \mathrm{Cu}_{2}\left(\mathrm{BrO}_{2}\right)_{2}, \mathrm{Sr}(\mathrm{FeAs})_{2}, \mathrm{Ca}(\mathrm{FeAs})_{2}, \mathrm{ScSi}_{3} \mathrm{Ni}_{2}$ \\
\hline & $\mathrm{X}-\Gamma, \mathrm{X}-\mathrm{M}, \mathrm{P}-\Gamma, \mathrm{P}-\mathrm{M}, \mathrm{N}-\mathrm{P}$ & $\| \mathrm{BaCdSb}_{2}, \mathrm{SrCdBi}_{2}, \mathrm{ThGeS}$ \\
\hline & $\mathrm{X}-\Gamma, \mathrm{X}-\mathrm{P}, \mathrm{X}-\mathrm{M}, \mathrm{P}-\Gamma, \mathrm{P}-\mathrm{M}$ & $\| \mathrm{Er}_{2} \mathrm{Mg}_{2} \mathrm{Ru}$ \\
\hline & $\Gamma-\mathrm{M}, \mathrm{X}-\Gamma, \mathrm{N}-\Gamma, \mathrm{N}-\mathrm{P}$ & $\| \mathrm{CeB}_{2} \mathrm{Rh}_{2} \mathrm{C}, \mathrm{Dy}_{11} \mathrm{Sn}_{10}$ \\
\hline & $\Gamma-\mathrm{M}, \mathrm{X}-\Gamma, \mathrm{P}-\Gamma, \mathrm{X}-\mathrm{M}$ & $\| \mathrm{SrIn}_{4}, \mathrm{Th}(\mathrm{GeAu})_{2}, \mathrm{Th}(\mathrm{SiAu})_{2}, \mathrm{Ba}(\mathrm{NiP})_{2}$ \\
\hline & $\Gamma-\mathrm{M}, \mathrm{X}-\Gamma, \mathrm{X}-\mathrm{M}, \mathrm{N}-\mathrm{P}$ & $\| \mathrm{U}\left(\mathrm{Al}_{2} \mathrm{Cr}\right)_{4}$ \\
\hline & $\Gamma-\mathrm{M}, \mathrm{X}-\Gamma, \mathrm{X}-\mathrm{M}, \mathrm{P}-\mathrm{M}$ & \begin{tabular}{l||l}
$\mathrm{Hf}_{2} \mathrm{Pt}_{3}, \mathrm{Sr}(\mathrm{NiGe})_{2}$ \\
\end{tabular} \\
\hline & $\Gamma-\mathrm{M}, \mathrm{X}-\mathrm{M}, \mathrm{N}-\mathrm{P}, \mathrm{X}-\mathrm{P}$ & $\| \mathrm{Th}(\mathrm{SiOs})_{2}, \mathrm{Th}(\mathrm{GeOs})_{2}, \mathrm{Th}(\mathrm{GeRu})_{2}, \mathrm{Th}(\mathrm{FeSi})_{2}, \mathrm{Th}(\mathrm{FeGe})_{2}$ \\
\hline & $\Gamma-\mathrm{M}, \mathrm{X}-\mathrm{M}, \mathrm{P}-\mathrm{M}, \mathrm{N}-\mathrm{M}$ & $\| \mathrm{Ba}_{8} \mathrm{Zr}_{3}\left(\mathrm{SbO}_{6}\right)_{4}$ \\
\hline & $\mathrm{X}-\Gamma, \Gamma-\mathrm{M}, \mathrm{N}-\Gamma, \mathrm{N}-\mathrm{M}$ & $\| \mathrm{Ti}_{3}\left(\mathrm{BiO}_{3}\right)_{4}$ \\
\hline & $\mathrm{X}-\Gamma, \Gamma-\mathrm{M}, \mathrm{X}-\mathrm{M}, \mathrm{N}-\mathrm{P}$ & $\| \mathrm{U}(\mathrm{MnSi})_{2}, \mathrm{U}(\mathrm{MnGe})_{2}$ \\
\hline & $\mathrm{X}-\Gamma, \Gamma-\mathrm{M}, \mathrm{X}-\mathrm{M}, \mathrm{X}-\mathrm{P}$ & $\| \mathrm{DyAl}_{3}, \mathrm{ScAl}_{3}$ \\
\hline & $\mathrm{X}-\Gamma, \Gamma-\mathrm{M}, \mathrm{X}-\mathrm{P}, \mathrm{X}-\mathrm{M}$ & $\| \mathrm{Ca}(\mathrm{PRu})_{2}, \mathrm{Ca}(\mathrm{AsRu})_{2}, \mathrm{Ti}_{2} \mathrm{Pd}$ \\
\hline & $\mathrm{X}-\Gamma, \mathrm{N}-\Gamma, \mathrm{N}-\mathrm{M}, \mathrm{X}-\mathrm{M}$ & CuSnAu, ZrTiAs, HfVGe, ZrVGe, $\mathrm{Ca}_{3} \mathrm{SiBr}_{2}, \mathrm{Zr}_{2} \mathrm{Sb}$ \\
\hline & $\mathrm{X}-\Gamma, \mathrm{X}-\mathrm{M}, \mathrm{P}-\Gamma, \mathrm{P}-\mathrm{M}$ & $\mathrm{Zn}(\mathrm{BIr})_{2}, \| \mathrm{Zn}_{3} \mathrm{Ru}$ \\
\hline & $\mathrm{X}-\mathrm{P}, \mathrm{P}-\Gamma, \mathrm{P}-\mathrm{M}, \mathrm{N}-\mathrm{P}$ & $\| \mathrm{Al}_{2} \mathrm{Ru}$ \\
\hline & $\Gamma-\mathrm{M}, \mathrm{N}-\Gamma, \mathrm{X}-\mathrm{M}$ & $\begin{array}{c}\| \mathrm{Ca}(\mathrm{ZnSi})_{2}, \mathrm{Ca}(\mathrm{ZnGe})_{2}, \mathrm{Sr}(\mathrm{CdGe})_{2}, \mathrm{Be}_{12} \mathrm{Cr}, \mathrm{Zr}(\mathrm{CoSi})_{2}, \mathrm{Ca}\left(\mathrm{Al}_{2} \mathrm{Cu}\right)_{4}, \\
\mathrm{Hf}(\mathrm{CoSi})_{2}, \mathrm{Th}(\mathrm{SiRh})_{2}, \mathrm{Th}(\mathrm{GeRh})_{2}, \mathrm{Th}(\mathrm{CoSi})_{2}\end{array}$ \\
\hline & $\Gamma-\mathrm{M}, \mathrm{N}-\mathrm{M}, \mathrm{X}-\mathrm{M}$ & $\begin{aligned} \mathrm{C}(\mathrm{ICSD}: 190717), \| & \mathrm{Ba}(\mathrm{MgSi})_{2}, \mathrm{Ba}(\mathrm{MgGe})_{2}, \mathrm{Sr}(\mathrm{ZnGe})_{2}, \mathrm{Ba}(\mathrm{CuAs})_{2}, \\
& \mathrm{Ca}(\mathrm{CuP})_{2}, \mathrm{Sr}(\mathrm{CuAs})_{2}\end{aligned}$ \\
\hline & $\Gamma-\mathrm{M}, \mathrm{X}-\Gamma, \mathrm{N}-\Gamma$ & $\| \mathrm{Be}_{12} \mathrm{Pd}, \mathrm{Be}_{12} \mathrm{Pt}$ \\
\hline & $\Gamma-\mathrm{M}, \mathrm{X}-\Gamma, \mathrm{N}-\mathrm{M}$ & $\| \mathrm{TiH}_{2}$ \\
\hline & $\Gamma-\mathrm{M}, \mathrm{X}-\Gamma, \mathrm{P}-\Gamma$ & $\| \mathrm{CePd}_{2}$ \\
\hline & $\Gamma-\mathrm{M}, \mathrm{X}-\Gamma, \mathrm{X}-\mathrm{M}$ & $\| \mathrm{ThB}_{2} \mathrm{Rh}_{2} \mathrm{C}$ \\
\hline & $\Gamma-\mathrm{M}, \mathrm{X}-\mathrm{M}, \mathrm{N}-\mathrm{P}$ & $\pi \mathrm{Ti}_{2} \mathrm{H}_{4} \mathrm{Pd}, \mathrm{Sm}_{4} \mathrm{Bi}_{2} \mathrm{O}$ \\
\hline & $\Gamma-\mathrm{M}, \mathrm{X}-\mathrm{M}, \mathrm{X}-\mathrm{P}$ & $\| \mathrm{Ba}(\mathrm{SbRu})_{2}$ \\
\hline & $\mathrm{N}-\Gamma, \mathrm{N}-\mathrm{M}, \mathrm{N}-\mathrm{P}$ & $\mathrm{CaTa}_{2} \mathrm{Bi}_{2} \mathrm{O}_{9}$ \\
\hline & $\mathrm{X}-\Gamma, \Gamma-\mathrm{M}, \mathrm{N}-\mathrm{P}$ & $\mathrm{ZrAg}_{2}, \| \mathrm{BiSe}$ \\
\hline & $\mathrm{X}-\Gamma, \Gamma-\mathrm{M}, \mathrm{X}-\mathrm{M}$ & $\begin{array}{c}\| \mathrm{Ca}(\mathrm{PRh})_{2}, \mathrm{Yb}(\mathrm{SiPt})_{2}, \mathrm{Ca}(\mathrm{SiNi})_{2}, \mathrm{Yb}(\mathrm{SiNi})_{2}, \mathrm{Ca}(\mathrm{NiGe})_{2}, \\
\mathrm{Yb}(\mathrm{NiGe})_{2}, \mathrm{Ca}(\mathrm{CoP})_{2}, \mathrm{Sr}(\mathrm{AsRh})_{2}, \mathrm{Ca}(\mathrm{CoAs})_{2}\end{array}$ \\
\hline & $\mathrm{X}-\Gamma, \mathrm{X}-\mathrm{M}, \mathrm{N}-\mathrm{P}$ & $\| \mathrm{Sr}_{2} \mathrm{Bi}, \mathrm{Sr}_{2} \mathrm{Sb}, \mathrm{Sr}_{2} \mathrm{As}, \mathrm{Ba}_{2} \mathrm{Bi}, \mathrm{Ba}_{2} \mathrm{Sb}, \mathrm{Ba}_{2} \mathrm{As}$ \\
\hline & $\mathrm{X}-\Gamma, \mathrm{X}-\mathrm{P}, \mathrm{X}-\mathrm{M}$ & $\| \operatorname{LiTlPd}_{2}$ \\
\hline & $\Gamma-\mathrm{M}, \mathrm{N}-\Gamma$ & $\| \mathrm{Zr}\left(\mathrm{TiGa}_{2}\right)_{2}$ \\
\hline & $\Gamma-\mathrm{M}, \mathrm{X}-\Gamma$ & $\| \mathrm{SiPt}_{2}$ \\
\hline & $\Gamma-\mathrm{M}, \mathrm{X}-\mathrm{M}$ & $\| \mathrm{Hf}_{2} \mathrm{Pd}, \mathrm{Zr}_{2} \mathrm{Pd}$ \\
\hline & $\mathrm{N}-\Gamma, \mathrm{N}-\mathrm{M}$ & $\| \mathrm{Ce}(\mathrm{SiIr})_{2}(\mathrm{ICSD}: 621430), \mathrm{K}_{2} \mathrm{Mg}_{5} \mathrm{Sn}_{3}$ \\
\hline & $\mathrm{X}-\Gamma, \Gamma-\mathrm{M}$ & 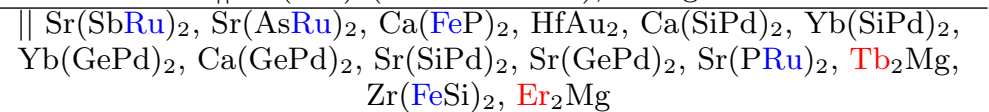 \\
\hline & $\mathrm{X}-\Gamma, \mathrm{X}-\mathrm{M}$ & $\| \mathrm{BaSi}_{2}, \mathrm{ThPt}_{2}$ \\
\hline & $\mathrm{X}-\mathrm{M}, \mathrm{X}-\mathrm{P}$ & $\mathrm{Li}_{2} \mathrm{H}_{2} \mathrm{Pd} \|$ \\
\hline & $\mathrm{N}-\mathrm{P}$ & $\mathrm{Ca}_{2} \mathrm{As}\left[\underline{56}, \mathrm{Hg}, \| \mathrm{Pu}(\mathrm{SiRh})_{2}\right.$ \\
\hline
\end{tabular}


Table III - continued

\begin{tabular}{|c|c|c|}
\hline $\begin{array}{l}\text { SOC or } \\
\text { NSOC }\end{array}$ & $\begin{array}{l}\text { HSL with Band } \\
\text { Crossing }\end{array}$ & High Symmetry Line Semimetals \\
\hline $\mathrm{SOC}$ & $\Gamma-\mathrm{M}$ & 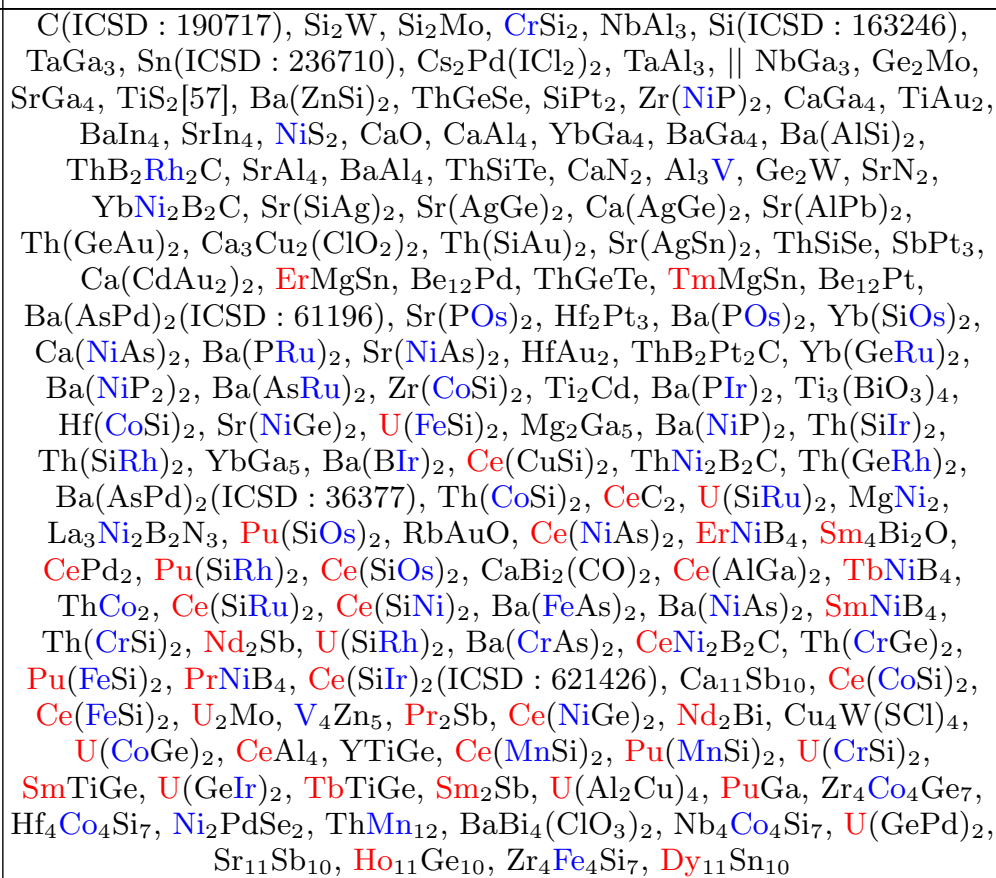 \\
\hline \multirow{18}{*}{ NSOC } & \multicolumn{2}{|r|}{ Space Group 140: $I 4 / \mathrm{mcm}$} \\
\hline & $\begin{array}{c}-\mathrm{M}, \mathrm{X}-\Gamma, \mathrm{X}-\mathrm{M}, \mathrm{P}-\Gamma, \mathrm{P}-\mathrm{M}, \\
\mathrm{N}-\Gamma, \mathrm{N}-\mathrm{M}, \mathrm{X}-\mathrm{P}\end{array}$ & $\| \mathrm{Nb}_{5} \mathrm{Ga}_{3}(\mathrm{ICSD}: 634764)$ \\
\hline & $\begin{array}{c}\Gamma-\mathrm{M}, \mathrm{X}-\Gamma, \mathrm{X}-\mathrm{M}, \mathrm{P}-\Gamma, \mathrm{P}-\mathrm{M}, \\
\text { N-M, X-P }\end{array}$ & $\| \mathrm{CeSi}_{4} \mathrm{Ni}_{9}$ \\
\hline & $\begin{array}{c}\Gamma-\mathrm{M}, \mathrm{X}-\Gamma, \mathrm{X}-\mathrm{P}, \mathrm{P}-\Gamma, \mathrm{P}-\mathrm{M} \\
\mathrm{N}-\Gamma, \mathrm{X}-\mathrm{M}\end{array}$ & $\| \mathrm{Ba}_{3} \mathrm{CrO}_{5}$ \\
\hline & $\begin{array}{c}\Gamma-\mathrm{M}, \mathrm{X}-\Gamma, \mathrm{X}-\mathrm{P}, \mathrm{P}-\mathrm{M}, \mathrm{N}-\Gamma, \\
\text { N-M, X-M }\end{array}$ & $\| \mathrm{Bi}$ \\
\hline & $\begin{array}{c}\Gamma-\mathrm{M}, \mathrm{X}-\Gamma, \mathrm{X}-\mathrm{P}, \mathrm{X}-\mathrm{M}, \mathrm{P}-\Gamma \\
\mathrm{N}-\Gamma, \mathrm{N}-\mathrm{M}\end{array}$ & || TlTe(ICSD : 653092) \\
\hline & $\begin{array}{c}\Gamma-\mathrm{M}, \mathrm{X}-\Gamma, \mathrm{X}-\mathrm{P}, \mathrm{X}-\mathrm{M}, \mathrm{P}-\mathrm{M}, \\
\mathrm{N}-\Gamma, \mathrm{N}-\mathrm{M}\end{array}$ & \| $\mathrm{Th}_{2} \mathrm{Zn}, \mathrm{Th}_{2} \mathrm{Hg}, \mathrm{Hf}_{2} \mathrm{Ge}(\mathrm{ICSD}: 636554)$ \\
\hline & $\begin{array}{c}\mathrm{X}-\Gamma, \Gamma-\mathrm{M}, \mathrm{X}-\mathrm{M}, \mathrm{P}-\Gamma, \mathrm{P}-\mathrm{M}, \\
\mathrm{N}-\Gamma, \mathrm{N}-\mathrm{M}\end{array}$ & $\| \mathrm{Sr}_{2} \mathrm{SmNb}\left(\mathrm{CuO}_{4}\right)_{2}$ \\
\hline & $\begin{array}{c}\Gamma-\mathrm{M}, \mathrm{X}-\Gamma, \underset{\mathrm{X}}{\mathrm{X}-\mathrm{P}, \mathrm{P}} \mathrm{N}-\Gamma, \mathrm{X}-\mathrm{M}, \\
\end{array}$ & $\| \mathrm{Sr}_{5} \mathrm{Si}_{3}(\mathrm{ICSD}: 160105)$ \\
\hline & $\begin{array}{c}\Gamma-\mathrm{M}, \mathrm{X}-\Gamma, \mathrm{X}-\mathrm{M}, \mathrm{P}-\Gamma, \mathrm{P}-\mathrm{M} \\
\mathrm{N}-\mathrm{M}\end{array}$ & $\| \mathrm{Ca}_{5} \mathrm{Ge}_{3}, \mathrm{Sn}_{2} \mathrm{Ru}, \mathrm{FeGe}_{2}, \mathrm{Ca}_{5} \mathrm{Si}_{3}, \mathrm{FeSn}_{2}$ \\
\hline & $\begin{array}{c}-\mathrm{M}, \mathrm{X}-\Gamma, \mathrm{X}-\mathrm{M}, \mathrm{P}-\mathrm{M}, \mathrm{N}-\mathrm{M}, \\
\mathrm{X}-\mathrm{P}\end{array}$ & $\| \mathrm{U}_{6} \mathrm{Ni}$ \\
\hline & $\begin{array}{c}\Gamma-\mathrm{M}, \mathrm{X}-\Gamma, \underset{\mathrm{X}-\mathrm{P}, \mathrm{P}-\mathrm{M}, \mathrm{N}-\mathrm{M},}{\mathrm{X}-\mathrm{M}} \\
\end{array}$ & $\| \mathrm{Ho}_{5} \mathrm{Co}_{2} \mathrm{Bi}$ \\
\hline & $\begin{array}{c}\Gamma-\mathrm{M}, \mathrm{X}-\Gamma, \mathrm{X}-\mathrm{P}, \mathrm{X}-\mathrm{M}, \mathrm{P}-\Gamma \\
\text { N-M }\end{array}$ & $\| \mathrm{U}_{6} \mathrm{Fe}$ \\
\hline & $\begin{array}{c}\Gamma-\mathrm{M}, \mathrm{X}-\Gamma, \underset{\mathrm{X}-\mathrm{P}, \mathrm{X}-\mathrm{M}, \mathrm{P}-\Gamma}{\mathrm{P}-\mathrm{M}} \\
\end{array}$ & $\| \mathrm{Ta}_{2} \mathrm{Si}$ \\
\hline & $\begin{array}{c}\Gamma-\mathrm{M}, \mathrm{X}-\Gamma, \mathrm{X}-\mathrm{P}, \mathrm{X}-\mathrm{M}, \mathrm{P}-\mathrm{M}, \\
\mathrm{N}-\Gamma\end{array}$ & $\| \mathrm{Ta}_{5} \mathrm{Ga}_{3}, \mathrm{Mn}_{5} \mathrm{~B}_{2} \mathrm{P}$ \\
\hline & $\begin{array}{c}\Gamma-\mathrm{M}, \mathrm{X}-\mathrm{M}, \mathrm{P}-\mathrm{M}, \mathrm{N}-\Gamma, \mathrm{N}-\mathrm{M}, \\
\text { X-P }\end{array}$ & \| TlTe(ICSD : 90900), $\mathrm{Th}_{2} \mathrm{Ge}, \mathrm{Zr}_{2} \mathrm{Si}$ \\
\hline & $\begin{array}{c}\text { T-M, X-P, P- } \Gamma, \text { P-M, N-M, } \\
\text { X-M }\end{array}$ & $\| \mathrm{Ca}_{5} \mathrm{Hg}_{3}, \mathrm{Ca}_{5} \mathrm{Zn}_{3}, \mathrm{Sr}_{5} \mathrm{Cd}_{3}$ \\
\hline & $\begin{array}{c}\Gamma-\mathrm{M}, \mathrm{X}-\mathrm{P}, \mathrm{X}-\mathrm{M}, \mathrm{P}-\Gamma, \mathrm{N}-\Gamma \\
\mathrm{N}-\mathrm{M}\end{array}$ & $\| \mathrm{Lu}_{7} \mathrm{Sb}_{3}$ \\
\hline
\end{tabular}


Table III - continued

\begin{tabular}{|c|c|c|}
\hline $\begin{array}{l}\text { SOC or } \\
\text { NSOC }\end{array}$ & $\begin{array}{l}\text { HSL with Band } \\
\text { Crossing }\end{array}$ & High Symmetry Line Semimetals \\
\hline & $\Gamma-\mathrm{M}, \mathrm{X}-\Gamma, \mathrm{P}-\Gamma, \mathrm{X}-\mathrm{M}, \mathrm{X}-\mathrm{P}$ & $\mathrm{PdPb}_{2}, \mathrm{PtPb}_{2}$ \\
\hline & $\Gamma-\mathrm{M}, \mathrm{X}-\Gamma, \mathrm{X}-\mathrm{M}, \mathrm{P}-\Gamma, \mathrm{P}-\mathrm{M}$ & $\| \mathrm{Ta}_{2} \mathrm{Ni}$ \\
\hline & $\Gamma-\mathrm{M}, \mathrm{X}-\Gamma, \mathrm{X}-\mathrm{P}, \mathrm{P}-\mathrm{M}, \mathrm{N}-\mathrm{M}$ & $\| \mathrm{Ti}_{4} \mathrm{NiBi}_{2}$ \\
\hline & $\Gamma-\mathrm{M}, \mathrm{X}-\Gamma, \mathrm{X}-\mathrm{P}, \mathrm{P}-\mathrm{M}, \mathrm{X}-\mathrm{M}$ & $\| \mathrm{SiPd}$ \\
\hline & $\Gamma-\mathrm{M}, \mathrm{X}-\Gamma, \mathrm{X}-\mathrm{P}, \mathrm{X}-\mathrm{M}, \mathrm{N}-\Gamma$ & $\mathrm{Ge}_{3} \mathrm{Mo}_{5}, \mathrm{Cr}_{5} \mathrm{Si}_{3}$ \\
\hline & $\Gamma-\mathrm{M}, \mathrm{X}-\Gamma, \mathrm{X}-\mathrm{P}, \mathrm{X}-\mathrm{M}, \mathrm{P}-\Gamma$ & $\| \mathrm{Cr}_{3} \mathrm{AsN}$ \\
\hline & $\Gamma-\mathrm{M}, \mathrm{X}-\Gamma, \mathrm{X}-\mathrm{P}, \mathrm{X}-\mathrm{M}, \mathrm{P}-\mathrm{M}$ & $\mathrm{Sc}_{3} \mathrm{Ga}_{2}$ \\
\hline & $\Gamma-\mathrm{M}, \mathrm{X}-\mathrm{M}, \mathrm{P}-\mathrm{M}, \mathrm{N}-\Gamma, \mathrm{N}-\mathrm{M}$ & $\| \mathrm{LiSn}_{4} \mathrm{Rh}$ \\
\hline & $\Gamma-\mathrm{M}, \mathrm{X}-\mathrm{M}, \mathrm{P}-\mathrm{M}, \mathrm{N}-\Gamma, \mathrm{X}-\mathrm{P}$ & $\| \mathrm{Hf}_{2} \mathrm{Si}, \mathrm{Hf}_{2} \mathrm{Ge}(\mathrm{ICSD}: 636548), \mathrm{Ta}_{2} \mathrm{Be}$ \\
\hline & Г-M, X-M, P-M, N-M, X-P & $\| \mathrm{Lu}_{5} \mathrm{SbPd}_{2}, \mathrm{~K}$ \\
\hline & $\Gamma-\mathrm{M}, \mathrm{X}-\mathrm{P}, \mathrm{X}-\mathrm{M}, \mathrm{P}-\Gamma, \mathrm{P}-\mathrm{M}$ & $\| \mathrm{V}_{5} \mathrm{As}_{3}$ \\
\hline & $\mathrm{X}-\Gamma, \Gamma-\mathrm{M}, \mathrm{X}-\mathrm{P}, \mathrm{P}-\Gamma, \mathrm{P}-\mathrm{M}$ & $\mathrm{Tl}_{2} \mathrm{Pt}$ \\
\hline & $\mathrm{X}-\Gamma, \mathrm{X}-\mathrm{P}, \mathrm{X}-\mathrm{M}, \mathrm{P}-\Gamma, \mathrm{P}-\mathrm{M}$ & $\mathrm{NdCo}_{9} \mathrm{Si}_{4}, \mathrm{TbCo}_{9} \mathrm{Si}_{4}$ \\
\hline & $\Gamma-\mathrm{M}, \mathrm{X}-\Gamma, \mathrm{P}-\Gamma, \mathrm{N}-\Gamma$ & $\mathrm{Tl}_{4} \mathrm{SnTe}_{3}, \mathrm{BaPbO}_{3} \|$ \\
\hline & $\Gamma-\mathrm{M}, \mathrm{X}-\Gamma, \mathrm{P}-\Gamma, \mathrm{X}-\mathrm{M}$ & $\| \mathrm{NdCu}_{9} \mathrm{Sn}_{4}$ \\
\hline & $\Gamma-\mathrm{M}, \mathrm{X}-\Gamma, \mathrm{X}-\mathrm{M}, \mathrm{P}-\mathrm{M}$ & $\| \mathrm{Pr}_{5} \mathrm{Tl}_{3}$ \\
\hline & $\Gamma-\mathrm{M}, \mathrm{X}-\Gamma, \mathrm{X}-\mathrm{P}, \mathrm{X}-\mathrm{M}$ & $\| \mathrm{Si}_{3} \mathrm{~W}_{5}$ \\
\hline & Г-M, X-M, P-M, N-M & $\mathrm{InTe}_{\mathrm{T}} \mathrm{TlInSe} \mathrm{I}_{2}\left(\mathrm{ICSD}:\right.$ 640528), InGaTe $_{2},|| \mathrm{InGaSe}_{2}$ \\
\hline & $\Gamma-\mathrm{M}, \mathrm{X}-\mathrm{P}, \mathrm{X}-\mathrm{M}, \mathrm{P}-\Gamma$ & || $\mathrm{Ti}_{5} \mathrm{FeSb}_{2}$ \\
\hline & $\mathrm{X}-\Gamma, \mathrm{X}-\mathrm{M}, \mathrm{P}-\Gamma, \mathrm{P}-\mathrm{M}$ & $\mathrm{Mg}_{2} \mathrm{Pt}, \mathrm{SrRuO}_{3}$ \\
\hline & $\Gamma-\mathrm{M}, \mathrm{N}-\Gamma, \mathrm{N}-\mathrm{M}$ & $\| \mathrm{MgSn}_{4} \mathrm{Ru}$ \\
\hline & $\Gamma-\mathrm{M}, \mathrm{X}-\Gamma, \mathrm{P}-\Gamma$ & $\mathrm{GePt}_{3}\left[58, \mathrm{Ca}, \mathrm{Sr}_{5} \mathrm{Ge}_{3}\right.$ \\
\hline & $\Gamma-\mathrm{M}, \mathrm{X}-\mathrm{M}, \mathrm{X}-\mathrm{P}$ & $\| \mathrm{Hf}_{2} \mathrm{Ni}, \mathrm{Zr}_{2} \mathrm{Ni}, \mathrm{U}_{3} \mathrm{Si}$ \\
\hline & $\mathrm{X}-\Gamma, \mathrm{X}-\mathrm{P}, \mathrm{X}-\mathrm{M}$ & $\| \mathrm{Ba}_{5} \mathrm{Sn}_{3}$ \\
\hline & $\mathrm{X}-\mathrm{M}, \mathrm{X}-\mathrm{P}$ & $\mathrm{Tl}_{2} \mathrm{SnTe}_{5} \|$ \\
\hline SOC & $\Gamma-\mathrm{M}$ & 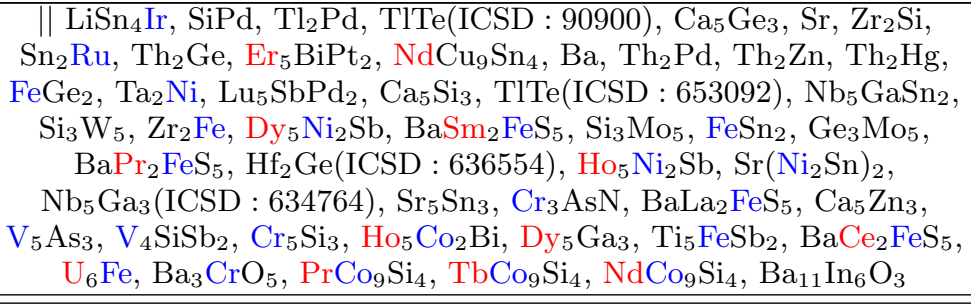 \\
\hline \multicolumn{3}{|r|}{ Space Group 141: I4 $/$ amd } \\
\hline \multirow{12}{*}{ NSOC } & $\begin{array}{c}\Gamma-\mathrm{M}, \mathrm{X}-\Gamma, \mathrm{P}-\Gamma, \mathrm{N}-\Gamma, \mathrm{N}-\mathrm{M} \\
\mathrm{N}-\mathrm{P}\end{array}$ & $\| \mathrm{ThGa}_{2}$ \\
\hline & $\Gamma-\mathrm{M}, \mathrm{X}-\Gamma, \mathrm{P}-\Gamma, \mathrm{N}-\Gamma, \mathrm{N}-\mathrm{M}$ & $\| \mathrm{V}_{2} \mathrm{H}$ \\
\hline & $\mathrm{X}-\Gamma, \Gamma-\mathrm{M}, \mathrm{N}-\Gamma, \mathrm{N}-\mathrm{M}, \mathrm{N}-\mathrm{P}$ & $\| \mathrm{TiAl}_{2}, \mathrm{HfV}_{2}$ \\
\hline & $\Gamma-\mathrm{M}, \mathrm{X}-\Gamma, \mathrm{P}-\Gamma, \mathrm{N}-\Gamma$ & $\| \mathrm{ZrSi}, \mathrm{BW}$ \\
\hline & $\Gamma-\mathrm{M}, \mathrm{X}-\Gamma, \mathrm{P}-\Gamma, \mathrm{N}-\mathrm{M}$ & $\| \mathrm{MoPb}$ \\
\hline & $\mathrm{X}-\Gamma, \Gamma-\mathrm{M}, \mathrm{N}-\Gamma, \mathrm{N}-\mathrm{M}$ & $\begin{array}{l}\| \text { Ge, Sn, NbP, TaP, } \mathrm{ZrIn}_{2}, \mathrm{ZrGa}, \mathrm{TiGa}_{2}, \mathrm{YbZn}_{11}, \mathrm{CaZn}_{11}, \mathrm{HfGa}_{2}, \\
\mathrm{SrZn}_{11}, \mathrm{SiAg}_{8}\left(\mathrm{SO}_{2}\right)_{2}, \mathrm{BaCd}_{11}, \mathrm{SrCd}_{11}, \mathrm{ScSn}_{2}\end{array}$ \\
\hline & $\Gamma-\mathrm{M}, \mathrm{N}-\Gamma, \mathrm{N}-\mathrm{P}$ & $\| \mathrm{ThGe}_{2}, \mathrm{ThSi}_{2}$ \\
\hline & $\Gamma-\mathrm{M}, \mathrm{X}-\Gamma, \mathrm{N}-\Gamma$ & $\mathrm{Ag}_{6} \mathrm{GeSO}_{8}, \mathrm{SiAg}_{6} \mathrm{SO}_{8}, \| \mathrm{NdAl}_{2} \mathrm{Cu}_{9}$ \\
\hline & $\Gamma-\mathrm{M}, \mathrm{X}-\Gamma, \mathrm{N}-\mathrm{M}$ & $\| \mathrm{ErNi}_{7} \mathrm{~B}_{3}$ \\
\hline & $\Gamma-\mathrm{M}, \mathrm{X}-\Gamma, \mathrm{P}-\Gamma$ & $\| \mathrm{Cu}_{2} \mathrm{Ag}_{2} \mathrm{O}_{3}, \mathrm{Yb}_{4} \mathrm{Si}_{2} \mathrm{~S}_{3} \mathrm{O}_{7}$ \\
\hline & $\mathrm{X}-\Gamma, \Gamma-\mathrm{M}$ & $\begin{array}{c}\mathrm{Mg}_{2} \mathrm{NF}, \| \mathrm{Si}, \mathrm{YbSi}_{2}, \mathrm{LiAg}, \underset{\mathrm{NdMgSi}}{2}, \mathrm{PrMgSi}_{2}, \mathrm{TiCu}_{2} \mathrm{P}, \mathrm{Cu}_{2} \mathrm{GeO}_{4}, \\
\mathrm{Zr}_{9} \mathrm{~S}_{2}\end{array}$ \\
\hline & $\Gamma-\mathrm{M}$ & $\| \mathrm{CaSi}_{2}, \mathrm{SrSi}_{2}, \mathrm{BaGe}_{2}, \mathrm{ThPb}$ \\
\hline \multirow{2}{*}{$\mathrm{SOC}$} & $\Gamma-\mathrm{M}, \mathrm{X}-\mathrm{P}$ & $\|$ ReB \\
\hline & $\Gamma-\mathrm{M}$ & $\begin{array}{c}\mathrm{Ag}_{6} \mathrm{GeSO}_{8}, \mathrm{SiAg}_{6} \mathrm{SO}_{8}, \mid \mathrm{Sr}, \mathrm{ThGa}_{2}, \mathrm{NbP}, \mathrm{ThPb}, \mathrm{LaMgSi}_{2}, \\
\mathrm{Cu}_{2} \mathrm{Ag}_{2} \mathrm{O}_{3}, \mathrm{SmCo}_{9} \mathrm{Si}_{2}\end{array}$ \\
\hline \multirow{6}{*}{ NSOC } & & Space Group 142: $I 4_{1} /$ acd \\
\hline & $\Gamma-\mathrm{M}, \mathrm{X}-\Gamma, \mathrm{P}-\Gamma, \mathrm{N}-\Gamma, \mathrm{N}-\mathrm{M}$ & $\| \mathrm{Ga}_{2} \mathrm{Pt}, \mathrm{Sn}_{2} \mathrm{Pd}$ \\
\hline & $\Gamma-\mathrm{M}, \mathrm{X}-\Gamma, \mathrm{P}-\Gamma, \mathrm{N}-\mathrm{M}$ & $\| \mathrm{U}(\mathrm{BRu})_{4}$ \\
\hline & $\Gamma-\mathrm{M}, \mathrm{X}-\Gamma, \mathrm{P}-\Gamma$ & $\| \mathrm{H}_{2} \mathrm{~S}, \mathrm{YbPS}_{4}, \mathrm{KSn}$ \\
\hline & $\Gamma-\mathrm{M}, \mathrm{N}-\Gamma$ & $\mathrm{Be}_{3} \mathrm{P}_{2} \|$ \\
\hline & $\Gamma-\mathrm{M}, \mathrm{X}-\mathrm{P}$ & $\| \mathrm{Sn}_{4} \mathrm{Ir}$ \\
\hline
\end{tabular}


Table III - continued

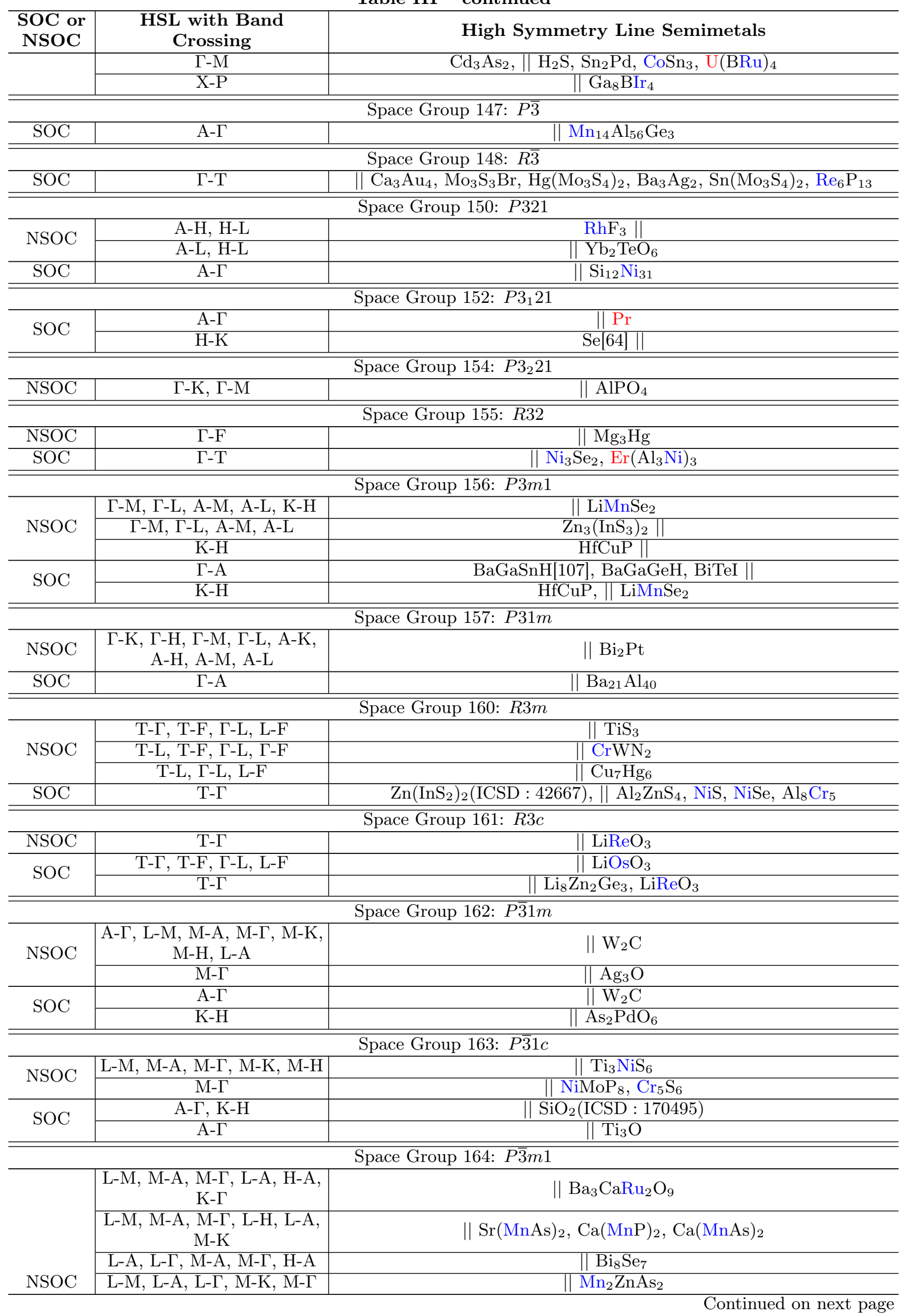


Table III - continued

\begin{tabular}{|c|c|c|}
\hline $\begin{array}{l}\text { SOC or } \\
\text { NSOC }\end{array}$ & $\begin{array}{l}\text { HSL with Band } \\
\text { Crossing }\end{array}$ & High Symmetry Line Semimetals \\
\hline & $\mathrm{L}-\mathrm{M}, \mathrm{M}-\mathrm{A}, \mathrm{M}-\Gamma, \mathrm{M}-\mathrm{K}, \mathrm{K}-\Gamma$ & $\mathrm{SiTe}_{2}$ \\
\hline & $\mathrm{L}-\mathrm{A}, \mathrm{H}-\mathrm{A}, \mathrm{M}-\mathrm{K}, \mathrm{M}-\mathrm{\Gamma}$ & || $\mathrm{BaSi}_{2}$ \\
\hline & $\mathrm{L}-\mathrm{A}, \mathrm{L}-\Gamma, \mathrm{M}-\mathrm{A}, \mathrm{M}-\Gamma$ & $\mathrm{CaZn}_{2}(\mathrm{PO})_{2}, \mathrm{~B}_{2} \mathrm{O}, \mathrm{Zn}\left(\mathrm{InS}_{2}\right)_{2}, \mathrm{Na}_{2} \mathrm{SO}_{4}$ \\
\hline & L-M, L-A, L- $\Gamma$ & $\| \mathrm{ZnNi}_{3} \mathrm{Sb}_{2}$ \\
\hline & L-A, H-A & $\mathrm{SbTe}, \mathrm{BaSn}_{2} 108, \mathrm{SrGe}_{2}, \mathrm{CaGe}_{2}$ \\
\hline & $\mathrm{M}-\Gamma, \mathrm{K}-\Gamma$ & $\mathrm{Sb}_{2} \mathrm{Te}, \mathrm{Mg}_{3} \mathrm{Bi}_{2} \mid 109$, , $\mid \mathrm{TiTe}_{2}, \mathrm{Zr}_{5} \mathrm{Te}_{6}$ \\
\hline \multirow{3}{*}{$\mathrm{SOC}$} & $\mathrm{A}-\Gamma, \mathrm{H}-\mathrm{K}$ & $\| \mathrm{LiVS}_{2}, \mathrm{Al}_{3} \mathrm{Pt}_{2}(\mathrm{ICSD}: 58134)$ \\
\hline & $\mathrm{A}-\Gamma$ & $\begin{array}{c}\mathrm{Sr}(\mathrm{MgBi})_{2}, \mathrm{Ba}(\mathrm{MgBi})_{2}, \mathrm{Ca}(\mathrm{ZnSb})_{2}, \mathrm{Sr}(\mathrm{ZnSb})_{2}, \| \mathrm{Zr}(\mathrm{CuP})_{2}, \mathrm{CaHg}_{2}, \\
\mathrm{CdInGaS} \\
\mathrm{TiSe}_{2}, \mathrm{Ti}_{2} \mathrm{O}, \mathrm{ZrN}_{2}, \mathrm{ZnNi}_{3} \mathrm{Sb}_{2}, \mathrm{AlCl}_{3}, \mathrm{Na}_{2} \mathrm{SO}_{4}, \mathrm{CeAlSi}_{2}, \\
\mathrm{Ba}_{3} \mathrm{CaIr}_{2} \mathrm{O}_{9}, \mathrm{Yb}_{2} \mathrm{SO}_{2}\end{array}$ \\
\hline & $\mathrm{H}-\mathrm{K}$ & $\| \mathrm{Rb}_{2} \mathrm{Hg}_{7}, \mathrm{Sc}_{2} \mathrm{C}, \mathrm{K}_{2} \mathrm{Hg}_{7}, \mathrm{Er}_{5} \mathrm{Si}_{3} \mathrm{C}$ \\
\hline \multicolumn{3}{|r|}{ Space Group 165: $P \overline{3} c 1$} \\
\hline \multirow{3}{*}{ NSOC } & $\mathrm{A}-\Gamma, \mathrm{L}-\Gamma, \mathrm{M}-\Gamma, \mathrm{K}-\Gamma$ & $\| \mathrm{Cu}_{3} \mathrm{As}(\mathrm{ICSD}: 16840)$ \\
\hline & $\mathrm{A}-\Gamma, \mathrm{L}-\Gamma, \mathrm{M}-\Gamma$ & $\mathrm{NdH}_{3}, \mathrm{YH}_{3}, \mathrm{TbH}_{3}, \mathrm{HoH}_{3}$ \\
\hline & $\mathrm{A}-\Gamma, \mathrm{M}-\Gamma, \mathrm{K}-\Gamma$ & $\| \mathrm{K}_{2} \mathrm{Ti}\left(\mathrm{GeO}_{3}\right)_{3}$ \\
\hline $\mathrm{SOC}$ & $\mathrm{A}-\Gamma$ & $\begin{array}{c}\| \mathrm{Cu}_{3} \mathrm{P}(\mathrm{ICSD}: 26775), \mathrm{Cu}_{3} \mathrm{P}(\mathrm{ICSD}: 16841), \mathrm{Cu}_{3} \mathrm{As}(\mathrm{ICSD}: 16840) \\
\mathrm{K}_{2} \mathrm{Sn}\left(\mathrm{GeO}_{3}\right)_{3}, \mathrm{Rb}_{2} \mathrm{Sn}\left(\mathrm{GeO}_{3}\right)_{3}\end{array}$ \\
\hline \multirow{17}{*}{ NSOC } & \multicolumn{2}{|r|}{ Space Group 166: $R \overline{3} m$} \\
\hline & F- $\Gamma$, F-T, L- $\Gamma$, L-T, $\Gamma-T$ & $\| \mathrm{Ba}\left(\mathrm{Ni}_{2} \mathrm{~B}\right)_{6}$ \\
\hline & F-L, F- $\Gamma, F-T, L-\Gamma$, L-T & $\mathrm{Yb}_{2} \mathrm{Zn}_{17}$ \\
\hline & F-L, F- $\Gamma, F-T, L-T, \Gamma-T$ & $\mathrm{Ce}_{2} \mathrm{Zn}_{17}$ \\
\hline & F-L, F-T, L- $\Gamma$, L-T, $\Gamma-\mathrm{T}$ & $\mathrm{TaCo}_{3}$ \\
\hline & $\mathrm{F}-\Gamma, \mathrm{F}-\mathrm{T}, \mathrm{L}-\Gamma, \mathrm{L}-\mathrm{T}$ & $\mathrm{Bi}_{8} \mathrm{Te}_{9}, \| \mathrm{Zr}_{3}\left(\mathrm{Al}_{2} \mathrm{C}_{3}\right)_{2}, \mathrm{Ti}_{5} \mathrm{Al}_{2} \mathrm{C}_{3}, \mathrm{MgNb}_{2}, \mathrm{Ba}_{4} \mathrm{Zr}\left(\mathrm{RuO}_{4}\right)_{3}$ \\
\hline & F-L, F- $\Gamma, F-T, L-T$ & $\| \mathrm{Te}_{4} \mathrm{Pt}_{3}$ \\
\hline & F-L, F- $\Gamma, \mathrm{L}-\mathrm{T}, \Gamma-\mathrm{T}$ & $\| \mathrm{Mg}_{2} \mathrm{SiNi}_{3}, \mathrm{UB}_{2} \mathrm{C}$ \\
\hline & F-L, L- $\Gamma, \mathrm{L}-\mathrm{T}, \mathrm{F}-\mathrm{\Gamma}$ & $\mathrm{Bi}_{2} \mathrm{Te}_{3}(\mathrm{ICSD}: 20289)\left[110112,\|\| \mathrm{Fe}_{7} \mathrm{Mo}_{6}\right.$ \\
\hline & F-L, L- $\Gamma, \mathrm{L}-\mathrm{T}, \Gamma-\mathrm{T}$ & $\| \mathrm{Sr}\left(\mathrm{Ni}_{2} \mathrm{~B}\right)_{6}$ \\
\hline & F-T, L-T, $\Gamma-\mathrm{T}, \mathrm{F}-\Gamma$ & $\| \mathrm{Ga}$ \\
\hline & F- $\Gamma, \mathrm{L}-\Gamma, \Gamma-\mathrm{T}$ & $\| \mathrm{ThB}_{2} \mathrm{C}, \mathrm{CeB}_{2} \mathrm{C}, \mathrm{CaMg}_{2} \mathrm{Ni}_{9}, \mathrm{Th}_{2} \mathrm{Fe}_{17}$ \\
\hline & F-L, F- $\Gamma$, F-T & $\| \mathrm{Hf}_{3}\left(\mathrm{Al}_{2} \mathrm{C}_{3}\right)_{2}, \mathrm{Ti}_{8} \mathrm{C}_{5}$ \\
\hline & F-L, L- $\Gamma, \mathrm{L}-\mathrm{T}$ & $\mathrm{K}_{5} \mathrm{InPb}_{8}, \| \mathrm{In}_{2} \mathrm{Te}_{3}, \mathrm{NiTe}, \mathrm{Tl}_{2} \mathrm{Pd}_{3} \mathrm{~S}_{2}, \mathrm{Ce}_{2} \mathrm{Zn}_{2} \mathrm{Ni}_{5}$ \\
\hline & $\mathrm{F}-\Gamma, \mathrm{L}-\mathrm{T}$ & $\begin{array}{c}\text { C(ICSD : 29123), } \mathrm{Hg}(\mathrm{ICSD}: 174005), \| \mathrm{In}_{2} \mathrm{Ni}_{3} \mathrm{~S}_{2}, \mathrm{Te}_{3} \mathrm{Pt}_{2}, \mathrm{Tl}_{2} \mathrm{Ni}_{3} \mathrm{~S}_{2}, \\
\mathrm{In}_{2} \mathrm{Ni}_{3} \mathrm{Se}_{2}\end{array}$ \\
\hline & $\mathrm{F}-\Gamma$ & $\begin{array}{c}\mathrm{Sb}_{2} \mathrm{Te}_{3}[111] 113, \mathrm{Te}_{3} \mathrm{As}_{2}, \mathrm{Sr}(\mathrm{SnAs})_{2}, \mathrm{Ba}_{7} \mathrm{Al}_{10}, \| \\
\mathrm{CaGe}_{2}(\mathrm{ICSD}: 110107), \mathrm{CaSi}_{2}(\mathrm{ICSD}: 32006), \mathrm{GaP}, \mathrm{Pd}_{3}(\mathrm{PbS})_{2}, \\
\mathrm{Pd}_{3}(\mathrm{PbSe})_{2}, \mathrm{Fe}_{7} \mathrm{~W}_{6}\end{array}$ \\
\hline & L-T & $\begin{array}{c}\mathrm{Bi}_{4} \mathrm{Te}_{3}, \mathrm{Ca}(\mathrm{GaAs})_{2}, \mathrm{Bi}[114], \mathrm{Sb}, \mathrm{As}[115], \mathrm{Bi}_{4} \mathrm{Se}_{3}, \mathrm{P}[57], \mathrm{Sb}_{8} \mathrm{Te}_{3}, \| \\
\mathrm{CaGe}_{2}(\mathrm{ICSD}: 185658), \mathrm{CaSi}_{2}(\mathrm{ICSD}: 193539), \mathrm{Sb}_{16} \mathrm{Te}_{3}, \mathrm{TePt}, \mathrm{Tb}_{2} \mathrm{C}, \\
\mathrm{Ho}_{2} \mathrm{C}, \mathrm{Dy}_{2} \mathrm{C}, \mathrm{Y}_{2} \mathrm{C}, \mathrm{TiS}, \mathrm{BaRuO}_{3}, \mathrm{Ba}_{7}(\mathrm{BIr})_{12}\end{array}$ \\
\hline $\mathrm{SOC}$ & $\Gamma-\mathrm{T}$ & 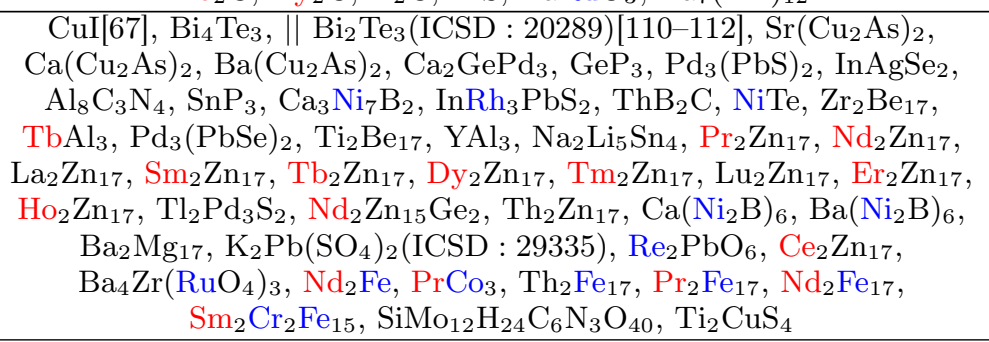 \\
\hline & & Space Group 167: $R \overline{3} c$ \\
\hline & F-L, F- $\Gamma, F-T, \Gamma-T$ & $\mathrm{Ba}_{2}\left(\mathrm{Ni}_{3} \mathrm{~B}_{2}\right)_{3}$ \\
\hline & $\mathrm{F}-\Gamma, \mathrm{L}-\Gamma, \Gamma-\mathrm{T}$ & $\| \mathrm{Mg}_{21} \mathrm{Zn}_{25}$ \\
\hline NSOC & F-L, F- $\Gamma, F-T$ & $\| \mathrm{Mo}_{15} \mathrm{Se}_{19}$ \\
\hline & $\mathrm{F}-\Gamma, \Gamma-\mathrm{T}$ & $\mathrm{Zr}_{3} \mathrm{O}, \mathrm{Ba}_{6} \mathrm{Ga}_{5} \mathrm{~N}, \mathrm{Sr}_{6} \mathrm{Ga}_{5} \mathrm{~N}, \mathrm{Ba}_{6} \mathrm{In}_{5} \mathrm{~N}, \mathrm{Fe}_{2} \mathrm{O}_{3}$ \\
\hline & $\mathrm{F}-\Gamma$ & $\| \mathrm{YbGa}_{7} \mathrm{Au}_{3}, \mathrm{Ni}_{8} \mathrm{P}_{3}, \mathrm{Rb}_{4} \mathrm{CdBr}_{6}$ \\
\hline $\mathrm{SOC}$ & $\Gamma-\mathrm{T}$ & $\begin{array}{c}\| \mathrm{SiO}_{2}(\mathrm{ICSD}: 170515), \mathrm{Ti}_{2} \mathrm{O}_{3}, \mathrm{Zr}_{3} \mathrm{O}, \mathrm{Ba}_{6} \mathrm{Ga}_{5} \mathrm{~N}, \mathrm{Hf}\left(\mathrm{TeCl}_{6}\right)_{2}, \mathrm{Ni}_{3} \mathrm{C}, \\
\mathrm{Al}_{3} \mathrm{Au}_{8}, \mathrm{YbAl}_{7} \mathrm{Au}_{3}, \mathrm{Sr}_{6} \mathrm{Ga}_{5} \mathrm{~N}, \mathrm{Yb}_{8} \mathrm{In}_{3}, \mathrm{Ba}_{6} \mathrm{In}_{5} \mathrm{~N}, \mathrm{Cr}_{2} \mathrm{O}_{3}, \mathrm{~K}_{2} \mathrm{Mog}_{9} \mathrm{~S}_{11}, \\
\mathrm{Rb}_{2} \mathrm{Mog}_{11} \mathrm{~S}_{11}, \mathrm{Ba}_{2}\left(\mathrm{Ni}_{3} \mathrm{~B}_{2}\right)_{3}, \mathrm{Na}_{8} \mathrm{Hg}_{3}, \mathrm{Mg}_{21} \mathrm{Zn}_{25}, \mathrm{Rb}_{4} \mathrm{Mo}_{21} \mathrm{~S}_{25}, \\
\mathrm{Cs}_{2} \mathrm{Mo}_{15} \mathrm{~S}_{19}, \mathrm{~K}_{2} \mathrm{Mo}_{15} \mathrm{~S}_{19}, \mathrm{LaCuO}_{3}\end{array}$ \\
\hline
\end{tabular}


Table III - continued

\begin{tabular}{|c|c|c|}
\hline $\begin{array}{l}\text { SOC or } \\
\text { NSOC }\end{array}$ & $\begin{array}{l}\text { HSL with Band } \\
\text { Crossing }\end{array}$ & High Symmetry Line Semimetals \\
\hline \multicolumn{3}{|r|}{ Space Group 174: $P \overline{6}$} \\
\hline $\mathrm{NSOC}$ & $\mathrm{A}-\Gamma, \Gamma-\mathrm{K}, \mathrm{K}-\mathrm{M}$ & $\mathrm{Nb}_{9} \mathrm{As}_{7} \mathrm{Pd}$ \\
\hline SOC & $\mathrm{A}-\Gamma, \Gamma-\mathrm{K}, \mathrm{K}-\mathrm{M}, \mathrm{H}-\mathrm{K}$ & $\mathrm{Nb}_{9} \mathrm{As}_{7} \mathrm{Pd}$ \\
\hline \multicolumn{3}{|r|}{ Space Group 176: $P 6_{3} / m$} \\
\hline \multirow{4}{*}{ NSOC } & $\mathrm{A}-\Gamma, \mathrm{M}-\mathrm{K}, \mathrm{M}-\Gamma, \mathrm{H}-\mathrm{K}, \mathrm{L}-\mathrm{M}$ & $\| \mathrm{Yb}_{5} \mathrm{Si}_{3} \mathrm{SO}_{12}$ \\
\hline & $\mathrm{M}-\mathrm{K}, \mathrm{K}-\Gamma, \mathrm{H}-\mathrm{K}, \mathrm{L}-\mathrm{M}$ & $\| \mathrm{ScB}_{4} \operatorname{Ir}_{3}$ \\
\hline & $\mathrm{M}-\Gamma, \mathrm{K}-\Gamma$ & $\mathrm{Mo}_{15} \mathrm{Se}_{19}, \| \mathrm{Mo}_{15} \mathrm{~S}_{19}, \mathrm{DySi}_{3} \mathrm{Rh}_{5}, \mathrm{ErSi}_{3} \mathrm{Rh}_{5}, \mathrm{~Tb}_{15}\left(\mathrm{Ni}_{4} \mathrm{P}_{3}\right)_{7}$ \\
\hline & $\mathrm{M}-\mathrm{K}, \mathrm{K}-\Gamma$ & $\| \mathrm{Mog}_{9} \mathrm{Se}_{11}, \mathrm{TbSi}_{3} \mathrm{Rh}_{5}$ \\
\hline \multirow{2}{*}{ SOC } & $\mathrm{A}-\Gamma$ & $\mathrm{Nb}_{3} \mathrm{Te}_{3} \mathrm{As}, \mathrm{Ti}_{3} \mathrm{Se}_{4}, \mathrm{LiCaAl}_{3}, \mathrm{Ba}_{3} \mathrm{MnN}_{3}, \mathrm{Sn}_{4} \mathrm{~B}_{3} \mathrm{Ir}_{7}, \mathrm{Na}_{2} \mathrm{Zn}_{2}\left(\mathrm{TeO}_{3}\right)_{3}$ \\
\hline & $\mathrm{H}-\mathrm{K}$ & $\mathrm{Mo}_{15} \mathrm{Se}_{19}, \| \mathrm{ScB}_{4} \mathrm{Ir}_{3}, \mathrm{Mg}_{3} \mathrm{Sn}$ \\
\hline \multicolumn{3}{|r|}{ Space Group 178: $P 6_{1} 22$} \\
\hline $\mathrm{SOC}$ & A- $\Gamma, \mathrm{L}-\mathrm{M}$ & $\mathrm{Sc}, \mathrm{Zr}_{5} \mathrm{Ir}_{3}$ \\
\hline \multicolumn{3}{|r|}{ Space Group 180: $P 6_{2} 22$} \\
\hline \multirow{2}{*}{ NSOC } & L-A, H-A, M-, K-Г, L-M & $\mathrm{Sn}_{2} \mathrm{Mo}$ \\
\hline & $\mathrm{M}-\mathrm{K}, \mathrm{M}-\Gamma$ & $\| \mathrm{Mg}_{2} \mathrm{Ni}$ \\
\hline SOC & $\mathrm{A}-\Gamma$ & $\| \mathrm{LuBPt}_{2}, \mathrm{YBPt}_{2}, \mathrm{MgBIr}, \mathrm{HfSn}_{2}, \mathrm{NdBPt}_{2}$ \\
\hline \multicolumn{3}{|r|}{ Space Group 181: $P 6_{4} 22$} \\
\hline NSOC & $\mathrm{L}-\mathrm{H}, \mathrm{L}-\mathrm{A}, \mathrm{M}-\Gamma, \mathrm{K}-\Gamma$ & $\| \mathrm{AlPO}_{4}(\mathrm{ICSD}: 66999)$ \\
\hline SOC & $\mathrm{A}-\Gamma$ & $\| \mathrm{MgNi}_{3} \mathrm{~B}_{2}, \mathrm{Al}_{2} \mathrm{~W}$ \\
\hline \multicolumn{3}{|r|}{ Space Group 182: $P 6_{3} 22$} \\
\hline \multirow{2}{*}{ NSOC } & $\mathrm{A}-\Gamma, \mathrm{M}-\mathrm{K}, \mathrm{M}-\Gamma, \mathrm{K}-\Gamma, \mathrm{L}-\mathrm{M}$ & $\| \mathrm{Ta}$ \\
\hline & $\mathrm{M}-\Gamma, \mathrm{K}-\Gamma$ & $\| \mathrm{Mn}\left(\mathrm{NbS}_{2}\right)_{3}$ \\
\hline \multirow{4}{*}{$\mathrm{SOC}$} & A- $\Gamma, \mathrm{H}-\mathrm{K}, \mathrm{L}-\mathrm{M}$ & $\mathrm{Pd}_{3} \mathrm{~N}, \mathrm{Ni}_{3} \mathrm{~N}, \mathrm{LiSmAlF}_{6}$ \\
\hline & $\mathrm{A}-\Gamma, \mathrm{H}-\mathrm{K}$ & $\| \mathrm{RbPbIO}_{6}, \mathrm{Nb}_{3} \mathrm{VS}_{6}$ \\
\hline & $\mathrm{A}-\Gamma, \mathrm{L}-\mathrm{M}$ & $\| \mathrm{Al}_{5} \mathrm{~W}, \mathrm{Al}_{5} \mathrm{Mo}, \mathrm{ReO}_{3}$ \\
\hline & $\mathrm{A}-\Gamma$ & $\| \mathrm{Zr}_{3} \mathrm{O}, \mathrm{Ta}$ \\
\hline \multicolumn{3}{|r|}{ Space Group 183: $P 6 m m$} \\
\hline $\mathrm{SOC}$ & $\Gamma-\mathrm{A}$ & $\mathrm{Ta}_{21} \mathrm{Te}_{13}$ \\
\hline \multicolumn{3}{|r|}{ Space Group 185: $\mathrm{P}_{3} \mathrm{~cm}$} \\
\hline NSOC & $\begin{array}{l}\text { M-L, M-H, M- }, \text { M-A, L-K, } \\
\text { L- } \Gamma, \text { L-A, K-H, K- }, \text { K-A, } \\
\text { H- } \Gamma, H-A\end{array}$ & $\| \mathrm{Sb}_{2} \mathrm{Pd}_{5}, \mathrm{Ni}_{5} \mathrm{As}_{2}$ \\
\hline $\mathrm{SOC}$ & $\Gamma-\mathrm{A}$ & $\mathrm{K}_{3} \mathrm{Bi}, \| \mathrm{Cu}_{3} \mathrm{P}, \mathrm{Mg}_{3} \mathrm{Pt}, \mathrm{Mg}_{3} \mathrm{Pd}, \mathrm{Yb}_{2} \mathrm{~S}_{3}, \mathrm{RbTiI}_{3}$ \\
\hline \multicolumn{3}{|r|}{ Space Group 186: $P 6_{3} m c$} \\
\hline \multirow{10}{*}{ NSOC } & $\begin{array}{c}\text { Г-A, M-L, M-K, M-H, M- }, \\
\text { M-A, L- } \Gamma, \text { K- } \Gamma, H-\Gamma\end{array}$ & $\| \mathrm{Th}_{7} \mathrm{Ni}_{3}$ \\
\hline & $\begin{array}{c}\Gamma-\mathrm{A}, \mathrm{M}-\mathrm{K}, \mathrm{L}-\mathrm{K}, \mathrm{K}-\mathrm{H}, \mathrm{K}-\Gamma \\
\mathrm{K}-\mathrm{A}, \mathrm{M}-\Gamma, \mathrm{L}-\Gamma\end{array}$ & $\| \mathrm{Ca}_{3}\left(\mathrm{Cu}_{2} \mathrm{Sn}\right)_{4}$ \\
\hline & $\begin{array}{c}\text { M-K, L-K, K-H, K- } \Gamma, \mathrm{K}-\mathrm{A}, \\
\text { M-L, M- } \Gamma, \mathrm{M}-\mathrm{A}\end{array}$ & $\| \mathrm{Nd}_{23} \mathrm{Mg}_{4} \mathrm{Rh}_{7}$ \\
\hline & $\begin{array}{c}\Gamma-\mathrm{A}, \mathrm{M}-\Gamma, \mathrm{L}-\Gamma, \mathrm{K}-\Gamma, \mathrm{H}-\Gamma \\
\mathrm{M}-\mathrm{L}, \mathrm{M}-\mathrm{A}\end{array}$ & $\| \mathrm{FeS}$ \\
\hline & $\Gamma-\mathrm{A}, \mathrm{M}-\Gamma, \mathrm{L}-\Gamma, \mathrm{K}-\Gamma, \mathrm{H}-\Gamma$ & LiGaGe[74] || \\
\hline & $\mathrm{M}-\mathrm{K}, \mathrm{L}-\mathrm{K}, \mathrm{K}-\mathrm{H}, \mathrm{K}-\mathrm{\Gamma}, \mathrm{K}-\mathrm{A}$ & $\| \mathrm{Nd}_{23} \mathrm{Cd}_{4} \mathrm{Rh}_{7}$ \\
\hline & M-L, M-K, M-H, M-Г, M-A & $\mathrm{SrHgPb}[116], \mathrm{DyGeAu}$ \\
\hline & $\Gamma-\mathrm{A}, \mathrm{M}-\mathrm{L}, \mathrm{M}-\mathrm{A}, \mathrm{L}-\Gamma$ & $\| \mathrm{Sm}_{4} \mathrm{Cl}_{6} \mathrm{O}$ \\
\hline & $\Gamma-\mathrm{A}, \mathrm{M}-\Gamma, \mathrm{L}-\Gamma$ & $\begin{array}{c}\text { YbSbAu, YbBiAu, YbCuBi, } \| \text { HoGeAu, CaSnHg, LuGeAu, YGeAu, } \\
\text { TbGeAu, SmGeAu, YbZnPb, LaGeAu, NdGeAu, PrSbPt, PrGeAu, } \\
\text { TmGeAu, NdSbPt, YSiAu }\end{array}$ \\
\hline & $\Gamma-\mathrm{A}$ & $\begin{array}{l}\text { Er }_{8} \mathrm{Ga}_{3} \mathrm{Co}, \mathrm{Tm}_{8} \mathrm{Ga}_{3} \mathrm{Co}, \| \text { ErCuGe, TbAgSn, HoAgSn, ErCuPb, } \\
\text { HoSnAu, DyAgSn, DyCuSn, SmCuSn, LuSnAu, LuCuPb, HoCuPb, } \\
\text { TmCuPb, LuCuSn, YCuSn, NdAgSn, TbCuPb, YCuPb, ErAgSn, } \\
\text { DyCuPb, YAgSn, HoAgPb, PrAgSn, YSnAu, TmAgSn, DyAgPb, } \\
\text { LuAgSn, TbAgPb, NdCuSn, PrAgPb, DyCuGe, SmAgPb, ErZnGa, } \\
\text { HoCuGe, NdAgPb, ScSnAu, ScCuSn, SmCuPb, TbCuGe, ScGeAu, } \\
\text { LiYSn, LiErSn, LiTmSn }\end{array}$ \\
\hline
\end{tabular}


Table III - continued

\begin{tabular}{|c|c|c|}
\hline $\begin{array}{l}\text { SOC or } \\
\text { NSOC }\end{array}$ & $\begin{array}{l}\text { HSL with Band } \\
\text { Crossing }\end{array}$ & High Symmetry Line Semimetals \\
\hline \multirow[b]{2}{*}{$\mathrm{SOC}$} & $\Gamma-\mathrm{A}, \mathrm{K}-\mathrm{H}$ & $\| \mathrm{Tc}_{7} \mathrm{~B}_{3}, \mathrm{LuMn}_{5}$ \\
\hline & $\Gamma-\mathrm{A}$ & 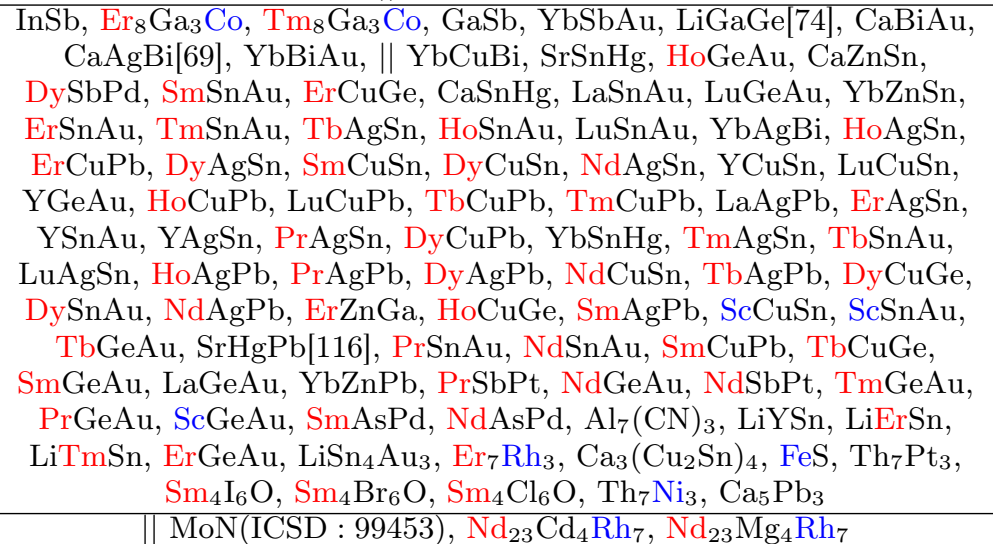 \\
\hline \multicolumn{3}{|r|}{ Space Group 187: $P \overline{6} m 2$} \\
\hline \multirow{6}{*}{ NSOC } & $\begin{array}{l}\text { A-M, Г-M, L-M, H-A, H-L, } \\
\text { K- } \Gamma, H-K\end{array}$ & $\| \mathrm{Mo}_{2} \mathrm{C}$ \\
\hline & $\begin{array}{l}\mathrm{A}-\mathrm{L}, \mathrm{A}-\mathrm{M}, \mathrm{\Gamma}-\mathrm{L}, \Gamma-\mathrm{M}, \mathrm{H}-\mathrm{A} \\
\mathrm{K}-\mathrm{M}\end{array}$ & $\| \mathrm{NbSe}_{2}$ \\
\hline & A-L, H-A, H-L, $\Gamma-\mathrm{M}, \mathrm{K}-\Gamma$ & $\|$ LiIr \\
\hline & $\mathrm{K}-\mathrm{\Gamma}, \mathrm{K}-\mathrm{M}, \mathrm{H}-\mathrm{K}$ & $\| \mathrm{Sr}_{4} \operatorname{In}_{13} \mathrm{Au}_{9}$ \\
\hline & $\mathrm{H}-\mathrm{A}, \mathrm{H}-\mathrm{L}$ & $\| \mathrm{TaInS}_{2}, \mathrm{NbInSe}_{2}(\mathrm{ICSD}: 53102), \mathrm{NbInS}_{2}(\mathrm{ICSD}: 74702)$ \\
\hline & $\mathrm{H}-\mathrm{K}$ & $\| \mathrm{RuC}$ \\
\hline \multirow{4}{*}{$\mathrm{SOC}$} & $\mathrm{K}-\Gamma, \mathrm{K}-\mathrm{M}, \mathrm{H}-\mathrm{K}$ & $\| \operatorname{IrN}, \mathrm{RhN}$ \\
\hline & $\mathrm{A}-\Gamma, \mathrm{H}-\mathrm{K}$ & $\begin{aligned} \| \text { OsC, LiNiN, AgN, } & \mathrm{TcN}, \mathrm{Yb}\left(\mathrm{As}_{2} \mathrm{Rh}_{3}\right)_{2}, \mathrm{Sr}\left(\mathrm{As}_{2} \mathrm{Rh}_{3}\right)_{2}, \mathrm{Ca}\left(\mathrm{As}_{2} \mathrm{Rh}_{3}\right)_{2}, \\
& \mathrm{Mg}\left(\mathrm{Co}_{3} \mathrm{P}_{2}\right)_{2}, \mathrm{~Tb}_{8} \mathrm{Ni}_{18} \mathrm{P}_{11}\end{aligned}$ \\
\hline & A- $\Gamma$ & $\begin{array}{c}\mathrm{TaN}[70], \mathrm{ZrTe}[71], \mathrm{VN}, \mathrm{NbN}, \mathrm{TiS}[72], \mathrm{WC}, \mathrm{MoC}[73], \| \mathrm{CrC}, \mathrm{TcB}, \\
\text { YPPt, } \mathrm{Re}_{3} \mathrm{~N}, \mathrm{ZrTaNO}\end{array}$ \\
\hline & $\mathrm{H}-\mathrm{K}$ & $\begin{array}{l}\mathrm{OsC}_{2}, \| \mathrm{PtN}_{2}, \mathrm{PtC}, \mathrm{TaInSe} \mathrm{Ta}_{2}, \mathrm{NbInS}_{2}(\mathrm{ICSD}: 640054), \mathrm{Tc}_{3} \mathrm{~N}, \mathrm{RuC} \\
\quad \mathrm{Mg}\left(\mathrm{As}_{2} \mathrm{Rh}_{3}\right)_{2}, \mathrm{Mg}\left(\mathrm{P}_{2} \mathrm{Rh}_{3}\right)_{2}, \mathrm{Mo}_{2} \mathrm{C}, \mathrm{La}_{2} \mathrm{~B}_{3} \mathrm{Br}_{2}, \mathrm{Sr}_{4} \mathrm{In}_{13} \mathrm{Au}_{9} \\
\end{array}$ \\
\hline \multicolumn{3}{|r|}{ Space Group 189: $P \overline{6} 2 m$} \\
\hline \multirow{9}{*}{ NSOC } & 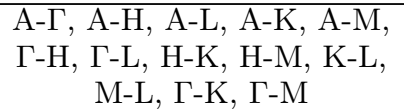 & || HoAlNi, ErAlPd \\
\hline & $\begin{array}{c}\mathrm{A}-\Gamma, \mathrm{A}-\mathrm{H}, \mathrm{A}-\mathrm{L}, \mathrm{A}-\mathrm{K}, \mathrm{A}-\mathrm{M}, \\
\Gamma-\mathrm{H}, \Gamma-\mathrm{L}, \mathrm{H}-\mathrm{K}, \mathrm{H}-\mathrm{M}, \mathrm{K}-\mathrm{L} \\
\mathrm{M}-\mathrm{L}\end{array}$ & $\begin{array}{l}\text { "| LuInPt, TmSnRh, ErSnRh, HoSnIr, HoSnRh, ErTlPd, LuSnRh, } \\
\text { TbSnRh, TmSnIr, HoTlPd, LuSnIr, YTlPd, DyTlPd, HoInPd, } \\
\text { LuAlPd, TbTlPd, TmInPt, ErInPt, YInPd, ErInNi, DySnRh, YInNi, } \\
\text { YAlPd, NdInPt, HoInNi, ErInPd, LuInPd, DyInNi, TbInPt, SmInPd, } \\
\text { TmInPd, NdInPd(ICSD : 640080), TbInPd, PrInNi, NdTlPd, } \\
\text { SmTlPd, ScInPt, LaTlPd, PrInPd, HoInPt, LaInNi, YInPt, DyInPt, } \\
\text { NdInNi, SmInNi, TbInNi, YSnRh }\end{array}$ \\
\hline & $\begin{array}{c}\text { A- } \Gamma, \text { A-H, H-L, A-K, A-M, } \\
\Gamma-\mathrm{H}, \Gamma-\mathrm{L}, \mathrm{H}-\mathrm{K}, \mathrm{H}-\mathrm{M}, \mathrm{K}-\mathrm{L} \\
\text { M-L }\end{array}$ & || MgGaAu \\
\hline & $\begin{array}{c}\text { A-H, A-L, A-K, A-M, Г-H, } \\
\Gamma-\mathrm{K}, \Gamma-\mathrm{M}, \Gamma-\mathrm{L}, \mathrm{H}-\mathrm{K}, \mathrm{K}-\mathrm{M} \\
\text { K-L }\end{array}$ & $\| \operatorname{In}_{5} \mathrm{~B}_{4} \operatorname{Ir}_{9}$ \\
\hline & $\begin{array}{l}\text { A- } \Gamma, \text { A-H, A-L, A-K, A-M, } \\
\Gamma-\mathrm{H}, \Gamma-\mathrm{K}, \Gamma-\mathrm{M}, \Gamma-\mathrm{L}, \mathrm{K}-\mathrm{M}\end{array}$ & $\| \mathrm{LiBPt}_{3}$ \\
\hline & $\begin{array}{l}\mathrm{A}-\Gamma, \mathrm{A}-\mathrm{H}, \mathrm{A}-\mathrm{L}, \mathrm{A}-\mathrm{K}, \Gamma-\mathrm{H} \\
\Gamma-\mathrm{M}, \Gamma-\mathrm{L}, \mathrm{H}-\mathrm{K}, \mathrm{K}-\mathrm{M}, \mathrm{K}-\mathrm{L}\end{array}$ & $\| \mathrm{LaInPt}$ \\
\hline & 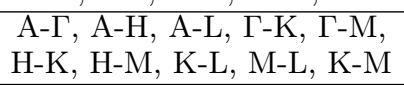 & $\| \mathrm{LaMg}_{4} \mathrm{Cu}$ \\
\hline & $\begin{array}{l}\mathrm{A}-\Gamma, \mathrm{A}-\mathrm{H}, \mathrm{A}-\mathrm{L}, \mathrm{H}-\mathrm{L}, \mathrm{A}-\mathrm{K} \\
\Gamma-\mathrm{M}, \Gamma-\mathrm{L}, \mathrm{H}-\mathrm{M}, \mathrm{K}-\mathrm{M}, \mathrm{K}-\mathrm{L}\end{array}$ & || ScMnSi \\
\hline & $\begin{array}{l}\mathrm{A}-\mathrm{\Gamma}, \mathrm{A}-\mathrm{H}, \mathrm{H}-\mathrm{L}, \mathrm{A}-\mathrm{M}, \Gamma-\mathrm{H} \\
\Gamma-\mathrm{M}, \mathrm{H}-\mathrm{K}, \mathrm{H}-\mathrm{M}, \mathrm{K}-\mathrm{M}, \mathrm{M}-\mathrm{L}\end{array}$ & $\| \mathrm{Zr}_{6} \mathrm{Al}_{2} \mathrm{Ni}$ \\
\hline
\end{tabular}


Table III - continued

\begin{tabular}{|c|c|c|}
\hline $\begin{array}{l}\text { SOC or } \\
\text { NSOC }\end{array}$ & $\begin{array}{l}\text { HSL with Band } \\
\text { Crossing }\end{array}$ & High Symmetry Line Semimetals \\
\hline & $\begin{array}{l}\text { A- } \Gamma, \text { A-L, H-L, A-H, A-K, } \\
\Gamma-\mathrm{M}, \Gamma-\mathrm{L}, \mathrm{H}-\mathrm{M}, \mathrm{K}-\mathrm{M}, \mathrm{K}-\mathrm{L}\end{array}$ & $\| \mathrm{Sr}_{2} \mathrm{In}_{4} \mathrm{Pt}_{3}$ \\
\hline & $\begin{array}{l}\text { A-H, A-L, A-K, A-M, Г-K, } \\
\Gamma-\mathrm{M}, \mathrm{H}-\mathrm{K}, \mathrm{H}-\mathrm{M}, \mathrm{K}-\mathrm{L}, \mathrm{M}-\mathrm{L}\end{array}$ & || CeInIr, CeInRh \\
\hline & $\begin{array}{l}\text { A- } \Gamma, \text { A-H, A-L, A-M, Г-M, } \\
\text { H-M, K-M, M-L, Г-K }\end{array}$ & $\| \mathrm{V}_{4} \mathrm{P}_{2} \mathrm{C}$ \\
\hline & $\begin{array}{c}\text { A- } \Gamma, \text { A-H, A-L, H-L, A-K, } \\
\text { A-M, Г-H, H-K, H-M }\end{array}$ & || HfGaRh, HfGaCo \\
\hline & $\begin{array}{l}\mathrm{A}-\Gamma, \mathrm{A}-\mathrm{H}, \mathrm{H}-\mathrm{L}, \mathrm{A}-\mathrm{L}, \Gamma-\mathrm{H} \\
\Gamma-\mathrm{K}, \Gamma-\mathrm{M}, \mathrm{K}-\mathrm{L}, \mathrm{M}-\mathrm{L}\end{array}$ & \| ThAlIr, ThGaIr, ThAlRh, ThGaRh \\
\hline & $\begin{array}{l}\mathrm{A}-\Gamma, \mathrm{A}-\mathrm{H}, \mathrm{A}-\mathrm{L}, \Gamma-\mathrm{H}, \Gamma-\mathrm{K} \\
\Gamma-\mathrm{M}, \Gamma-\mathrm{L}\end{array}$ & $\begin{array}{l}\text { 1| NdAlPd, DyAlNi, NdInPd(ICSD : 157627), LaInPd, PrSnRh, } \\
\text { NdSnRh, LaSnRh, YAlNi }\end{array}$ \\
\hline & $\begin{array}{l}\text { A-H, A-L, A-M, Г-M, H-M, } \\
\text { K-M, M-L }\end{array}$ & e \\
\hline & $\begin{array}{c}\text { A-H, H-L, A-L, } \text { - }-\mathrm{L}, \mathrm{K}-\mathrm{L}, \\
\text { M-L }\end{array}$ & $\| \mathrm{Sn}_{5}\left(\mathrm{BRh}_{3}\right)_{2}$ \\
\hline & $\begin{array}{c}\text { A-M, Г-M, H-M, K-M, } \\
\text { M-L, Г-K }\end{array}$ & |l TbAgGe, CeInCu \\
\hline & A-L, H-L, Г-L, K-L, M-L & $\mathrm{Sn}_{5}\left(\mathrm{BIr}_{3}\right)_{2}$ \\
\hline & $\mathrm{A}-\Gamma, \Gamma-\mathrm{K}, \mathrm{K}-\mathrm{M}, \Gamma-\mathrm{M}$ & $\| \mathrm{Co}_{2} \mathrm{As}$ \\
\hline & $\mathrm{A}-\mathrm{H}, \mathrm{A}-\mathrm{L}, \Gamma-\mathrm{K}, \Gamma-\mathrm{M}$ & $\|$ CoNiAs \\
\hline & $\mathrm{A}-\mathrm{H}, \mathrm{H}-\mathrm{L}, \Gamma-\mathrm{K}, \Gamma-\mathrm{M}$ & $\| \mathrm{Sc}_{6} \mathrm{NiTe}_{2}$ \\
\hline & $\mathrm{A}-\Gamma, \Gamma-\mathrm{K}, \Gamma-\mathrm{M}$ & NiPRh, MnNbSi, NbReSi \\
\hline & $\mathrm{A}-\mathrm{H}, \mathrm{A}-\mathrm{L}$ & $\|$ YbAlCu, ErAlNi \\
\hline & $\Gamma-\mathrm{K}, \Gamma-\mathrm{M}$ & 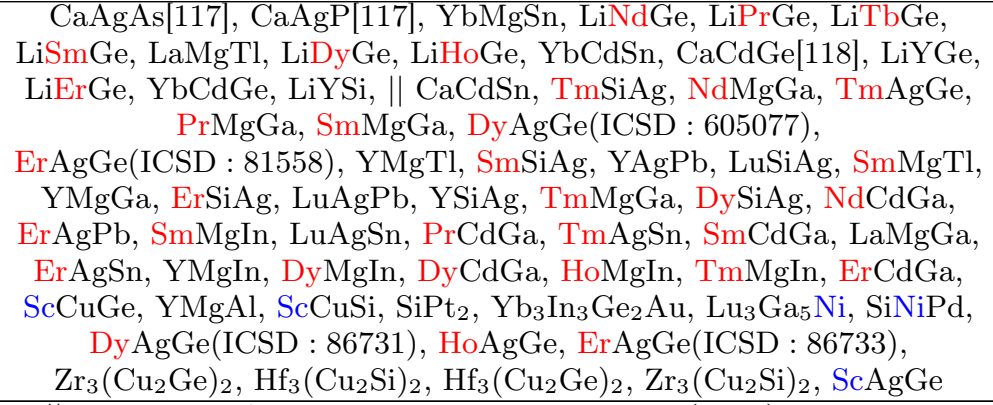 \\
\hline \multirow{3}{*}{$\mathrm{SOC}$} & $\mathrm{A}-\Gamma, \mathrm{H}-\mathrm{K}$ & $\begin{array}{c}\| \mathrm{Yb}_{3} \mathrm{Si}_{5}, \mathrm{Er}_{3} \mathrm{Al}_{3} \mathrm{NiGe}_{2}, \mathrm{HfGeRu}, \mathrm{ZrSiRu}, \mathrm{K}_{5}\left(\mathrm{DyI}_{4}\right)_{3}, \mathrm{Zr}_{6} \mathrm{Sb}_{2} \mathrm{Pt} \\
\mathrm{Zr}_{6} \mathrm{Ga}_{2} \mathrm{Ni}\end{array}$ \\
\hline & $\mathrm{A}-\Gamma$ & 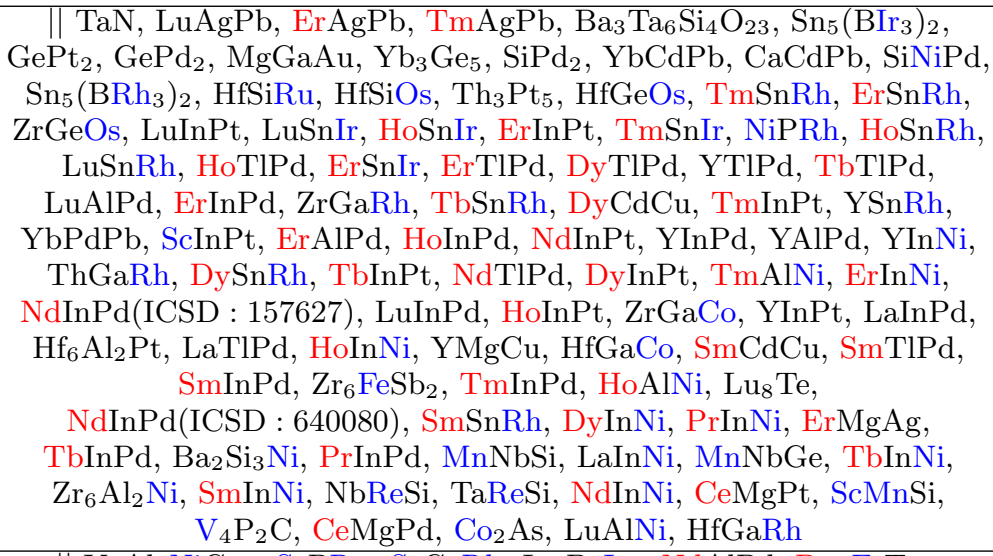 \\
\hline & $\mathrm{H}-\mathrm{K}$ & $\begin{array}{c}\mathrm{Y}_{3} \mathrm{Al}_{3} \mathrm{NiGe}_{2}, \mathrm{ScPRu}, \mathrm{ScGeRh}, \mathrm{In}_{5} \mathrm{~B}_{4} \mathrm{Ir}_{9}, \mathrm{NdAlPd}, \mathrm{Dy}_{6} \mathrm{FeTe}_{2}, \\
\mathrm{Sc}_{6} \mathrm{Te}_{2} \mathrm{Os}, \mathrm{TbAlPd}, \mathrm{TbAlNi}, \mathrm{PrAlPd}, \mathrm{Zr}_{6} \mathrm{FeTe}_{2}, \mathrm{Lu}_{6} \mathrm{FeSb}_{2}, \mathrm{PrAlNi} \\
\mathrm{Sc}_{6} \mathrm{FeTe}_{2}, \mathrm{NdAlNi}_{2} \mathrm{Ho}_{6} \mathrm{FeBi}_{2}, \mathrm{Sc}_{6} \mathrm{NiTe}_{2}, \mathrm{Tm}_{6} \mathrm{FeSb}_{2}, \mathrm{Ho}_{6} \mathrm{FeSb}_{2}, \\
\mathrm{Y}_{6} \mathrm{FeSb}_{2}, \mathrm{Dy}_{6} \mathrm{FeSb}_{2}, \mathrm{Zr}_{6} \mathrm{Al}_{2} \mathrm{Fe}, \mathrm{Tb}_{6} \mathrm{FeSb}_{2}, \mathrm{~Tb}_{6} \mathrm{FeBi}_{2}, \mathrm{~Tb}_{12}\left(\mathrm{OsC}_{3}\right)_{5} \\
\mathrm{Dy}_{3} \mathrm{Mn}_{3} \mathrm{Ga}_{2} \mathrm{Si}, \mathrm{Tb}_{3} \mathrm{Mr}_{3} \mathrm{Ga}_{2} \mathrm{Si}, \mathrm{Hf}_{2} \mathrm{Co}_{4} \mathrm{P}_{3}\end{array}$ \\
\hline & & Space Group 190: $P \overline{6} 2 c$ \\
\hline
\end{tabular}


Table III - continued

\begin{tabular}{|c|c|c|}
\hline & & \\
\hline $\begin{array}{l}\text { SOC or } \\
\text { NSOC }\end{array}$ & $\begin{array}{c}\text { HSL with Band } \\
\text { Crossing }\end{array}$ & High Symmetry Line Semimetals \\
\hline NSOC & $\begin{array}{c}\mathrm{A}-\Gamma, \Gamma-\mathrm{H}, \Gamma-\mathrm{K}, \Gamma-\mathrm{M}, \Gamma-\mathrm{L}, \\
\mathrm{K}-\mathrm{M}\end{array}$ & || FeS(ICSD : 291010), FeS(ICSD : 291010) \\
\hline & $\mathrm{A}-\Gamma$ & FeS(ICSD : 291010), FeS(ICSD : 291010) \\
\hline SUC & H-K & || $\mathrm{Zr}_{6} \mathrm{Al}_{2} \mathrm{NiH}_{10}$ \\
\hline & & Space Group 191: $P 6 / \mathrm{mmm}$ \\
\hline & $\begin{array}{c}\text { A- } \Gamma, \mathrm{L}-\mathrm{A}, \mathrm{H}-\mathrm{A}, \mathrm{L}-\mathrm{H}, \mathrm{L}-\mathrm{M}, \\
\mathrm{L}-\mathrm{K}, \mathrm{L}-\Gamma, \mathrm{M}-\mathrm{H}, \mathrm{M}-\mathrm{A}, \mathrm{H}-\mathrm{K}, \\
\mathrm{H}-\Gamma, \mathrm{K}-\mathrm{A}, \mathrm{M}-\Gamma, \mathrm{K}-\Gamma\end{array}$ & $\| \mathrm{NdIr}_{5}, \mathrm{NdCo}_{5}$ \\
\hline & $\begin{array}{c}\text { A- } \Gamma, \mathrm{L}-\mathrm{A}, \mathrm{H}-\mathrm{A}, \mathrm{L}-\mathrm{M}, \mathrm{L}-\mathrm{K} \\
\mathrm{L}-\Gamma, \mathrm{M}-\mathrm{H}, \mathrm{M}-\mathrm{A}, \mathrm{H}-\mathrm{K}, \mathrm{H}-\Gamma \\
\mathrm{K}-\mathrm{A}, \mathrm{M}-\Gamma, \mathrm{K}-\Gamma\end{array}$ & $\| \mathrm{LaCo}_{5}$ \\
\hline & $\begin{array}{c}\text { A- }, \text { L-A, H-A, L-H, L-M, } \\
\text { L-K, L- } \Gamma, \text { M-H, M-A, H-K, } \\
\text { H- } \Gamma, \text { K-A }\end{array}$ & $\| \mathrm{CePt}_{5}$ \\
\hline & $\begin{array}{c}\text { A- } \Gamma, \mathrm{L}-\mathrm{A}, \mathrm{H}-\mathrm{A}, \mathrm{L}-\mathrm{H}, \mathrm{L}-\mathrm{M} \\
\mathrm{L}-\mathrm{K}, \mathrm{L}-\Gamma, \mathrm{M}-\mathrm{H}, \mathrm{H}-\mathrm{K}, \mathrm{H}-\Gamma \\
\mathrm{M}-\Gamma\end{array}$ & $\| \mathrm{CePd}_{5}$ \\
\hline NSOC & $\begin{array}{c}\text { A- } \Gamma, \text { L-A, H-A, L-H, M-H, } \\
\text { M-A, H-K, H- }, \text { K-A, M- }, \\
\text { K- } \Gamma\end{array}$ & $\| \operatorname{LaIr}_{5}$ \\
\hline & $\begin{array}{c}\text { A- } \Gamma, \mathrm{L}-\mathrm{A}, \mathrm{H}-\mathrm{A}, \mathrm{L}-\mathrm{M}, \mathrm{L}-\mathrm{K} \\
\mathrm{L}-\Gamma, \mathrm{M}-\mathrm{H}, \mathrm{H}-\mathrm{K}, \mathrm{H}-\Gamma, \mathrm{M}-\Gamma \\
\mathrm{K}-\Gamma\end{array}$ & $\begin{array}{c}\| \mathrm{HoSi}_{2} \mathrm{Rh}_{3}, \mathrm{DySi}_{2} \mathrm{Rh}_{3}, \mathrm{SmSi}_{2} \mathrm{Rh}_{3}, \mathrm{NdSi}_{2} \mathrm{Rh}_{3}, \mathrm{ErSi}_{2} \mathrm{Rh}_{3}, \mathrm{YSi}_{2} \mathrm{Rh}_{3}, \\
\mathrm{LaSi}_{2} \mathrm{Rh}_{3}\end{array}$ \\
\hline & $\begin{array}{c}\text { A- } \Gamma, \mathrm{L}-\mathrm{H}, \mathrm{H}-\mathrm{A}, \mathrm{L}-\mathrm{A}, \mathrm{L}-\mathrm{K}, \\
\mathrm{L}-\Gamma, \mathrm{M}-\mathrm{H}, \mathrm{M}-\mathrm{K}, \mathrm{M}-\Gamma, \mathrm{K}-\mathrm{A}, \\
\mathrm{K}-\Gamma\end{array}$ & $\| \mathrm{TiS}_{2}$ \\
\hline & $\begin{array}{c}\text { A- } \Gamma, \mathrm{L}-\mathrm{H}, \mathrm{L}-\mathrm{A}, \mathrm{H}-\mathrm{A}, \mathrm{L}-\mathrm{M}, \\
\mathrm{L}-\mathrm{K}, \mathrm{L}-\Gamma, \mathrm{M}-\mathrm{A}, \mathrm{K}-\mathrm{A}, \mathrm{M}-\Gamma \\
\mathrm{K}-\Gamma\end{array}$ & $\| \mathrm{ZnI}_{2}$ \\
\hline & $\begin{array}{c}\text { L-H, L-A, H-A, L-M, L-K, } \\
\text { L- } \Gamma, \text { M-H, H-K, H- } \Gamma, M-\Gamma, \\
\text { K- } \Gamma\end{array}$ & $\| \operatorname{Zr}(\mathrm{CoGe})_{6}$ \\
\hline & $\begin{array}{l}\text { A- } \Gamma, \mathrm{L}-\mathrm{A}, \mathrm{H}-\mathrm{A}, \mathrm{L}-\mathrm{M}, \mathrm{L}-\mathrm{K} \\
\mathrm{M}-\mathrm{H}, \mathrm{M}-\Gamma, \mathrm{H}-\mathrm{K}, \mathrm{K}-\Gamma, \mathrm{L}-\Gamma\end{array}$ & $\| \mathrm{TbCu}_{5}, \mathrm{HoCu}_{5}, \mathrm{YCu}_{5}, \mathrm{SmCu}_{5}, \mathrm{PrCu}_{5}, \mathrm{NdCu}_{5}, \mathrm{LaCu}_{5}$ \\
\hline & $\begin{array}{l}\text { A- } \Gamma, \mathrm{L}-\mathrm{H}, \mathrm{H}-\mathrm{A}, \mathrm{L}-\mathrm{A}, \mathrm{L}-\Gamma \\
\mathrm{M}-\mathrm{A}, \mathrm{M}-\Gamma, \mathrm{H}-\Gamma, \mathrm{K}-\mathrm{A}, \mathrm{K}-\Gamma\end{array}$ & $\| \mathrm{NiS}_{2}$ \\
\hline & $\begin{array}{c}\text { A- } \Gamma, \mathrm{L}-\mathrm{H}, \mathrm{H}-\mathrm{A}, \mathrm{L}-\mathrm{A}, \mathrm{L}-\Gamma \\
\mathrm{M}-\mathrm{H}, \mathrm{M}-\Gamma, \mathrm{H}-\mathrm{K}, \mathrm{K}-\Gamma, \mathrm{L}-\mathrm{M}\end{array}$ & $\| \mathrm{KIn}_{9} \mathrm{Co}_{2}$ \\
\hline & $\begin{array}{c}\mathrm{L}-\mathrm{A}, \mathrm{H}-\mathrm{A}, \mathrm{L}-\Gamma, \mathrm{M}-\mathrm{A}, \mathrm{M}-\Gamma, \\
\mathrm{H}-\Gamma, \mathrm{K}-\mathrm{A}, \mathrm{K}-\Gamma, \mathrm{M}-\mathrm{K}\end{array}$ & $\| \mathrm{CaC}_{2}$ \\
\hline & $\begin{array}{c}\text { L-H, H-A, L-A, L-M, L-K, } \\
\text { L- } \Gamma, \text { M-H, H-K, H- } \Gamma\end{array}$ & $\| \mathrm{YB}_{2} \operatorname{Ir}_{3}, \operatorname{ErB}_{2} \operatorname{Ir}_{3}$ \\
\hline & $\begin{array}{c}\text { L-H, L-A, H-A, L-M, L-K, } \\
\text { L- } \Gamma, \text { M-H, H-K, H- }\end{array}$ & $\| \mathrm{LuB}_{2} \operatorname{Ir}_{3}, \mathrm{DyB}_{2} \mathrm{Ir}_{3}$ \\
\hline & $\begin{array}{c}\text { L-H, L-A, L-K, L- } \Gamma, \text { M-H, } \\
\text { M-K, M-A, M- }, \text { L-M }\end{array}$ & $\| \mathrm{Li}_{2} \mathrm{Pt}\left[74, \mathrm{Li}_{2} \mathrm{Pd}\right.$ \\
\hline & $\begin{array}{l}\mathrm{A}-\Gamma, \mathrm{L}-\mathrm{H}, \mathrm{H}-\mathrm{A}, \mathrm{L}-\Gamma, \mathrm{M}-\mathrm{H} \\
\mathrm{M}-\Gamma, \mathrm{H}-\mathrm{K}, \mathrm{K}-\Gamma\end{array}$ & $\| \mathrm{YbGa}_{2}$ \\
\hline & $\begin{array}{l}\text { L-A, H-A, L-M, L-K, L- } \Gamma, \\
\text { M-H, H-K, H- }\end{array}$ & $\| \mathrm{SmCo}_{3} \mathrm{~B}_{2}$ \\
\hline & $\begin{array}{c}\mathrm{A}-\Gamma, \mathrm{L}-\Gamma, \mathrm{M}-\Gamma, \mathrm{H}-\Gamma, \mathrm{K}-\Gamma, \\
\mathrm{M}-\mathrm{K}\end{array}$ & $\| \mathrm{CeGa}_{2}$ \\
\hline & $\begin{array}{c}\mathrm{A}-\Gamma, \mathrm{L}-\mathrm{H}, \mathrm{H}-\mathrm{A}, \mathrm{M}-\mathrm{H}, \mathrm{H}-\mathrm{K}, \\
\mathrm{H}-\Gamma\end{array}$ & $\| \mathrm{HfBe}_{2}, \mathrm{ThCd}_{2}$ \\
\hline & $\begin{array}{c}\text { L-A, H-A, L-H, L-M, L-K, } \\
\text { L- } \Gamma\end{array}$ & $\| \mathrm{Mg}$ \\
\hline & L-A, H-A, M-K, M- $\Gamma, \mathrm{K}-\Gamma$ & $\mathrm{BaSi}_{2}, \mathrm{CaSi}_{2}$ \\
\hline & L-M, M-H, M-K, M-A, M- $\Gamma$ & $\| \mathrm{CaNi}_{4} \mathrm{~B}$ \\
\hline & $\mathrm{A}-\Gamma, \mathrm{L}-\mathrm{A}, \mathrm{H}-\mathrm{A}, \mathrm{M}-\mathrm{A}$ & $\| \mathrm{SrHg}_{2}$ \\
\hline
\end{tabular}


Table III - continued

\begin{tabular}{|c|c|c|}
\hline $\begin{array}{l}\text { SOC or } \\
\text { NSOC }\end{array}$ & $\begin{array}{l}\text { HSL with Band } \\
\text { Crossing }\end{array}$ & High Symmetry Line Semimetals \\
\hline & L-H, H-A, M- $\Gamma, \mathrm{K}-\Gamma$ & $\| \mathrm{UB}_{2}$ \\
\hline & L-M, M-A, M- & $\| \mathrm{Ti}, \mathrm{ZrTi}_{2}$ \\
\hline & L-A, H-A & $\| \mathrm{U}$ \\
\hline \multirow[t]{3}{*}{$\mathrm{SOC}$} & $\mathrm{A}-\Gamma, \mathrm{H}-\mathrm{K}$ & $\begin{array}{c}\| \mathrm{B}_{2} \mathrm{~W}, \mathrm{RbAu}_{5}, \mathrm{YbGa}_{2}, \mathrm{HoSi}_{2} \mathrm{Rh}_{3}, \mathrm{DySi}_{2} \mathrm{Rh}_{3}, \mathrm{SmSi}_{2} \mathrm{Rh}_{3}, \mathrm{NdSi}_{2} \mathrm{Rh}_{3}, \\
\mathrm{ThSi}_{2}, \mathrm{~B}_{2} \mathrm{Mo}_{\mathrm{O}}, \mathrm{YSi}_{2} \mathrm{Rh}_{3}, \mathrm{LaSi}_{2} \mathrm{Rh}_{3}, \mathrm{ThAu}_{2}, \mathrm{HfBe}_{2}, \mathrm{ErSi}_{2} \mathrm{Rh}_{3}, \mathrm{ZrBe}_{2}, \\
\mathrm{SrZn}_{5}, \mathrm{TbCu}_{5}, \mathrm{CrB}_{2}, \mathrm{Zr}_{2} \mathrm{TaN}_{3}, \mathrm{HoCu}_{5}, \mathrm{YCu}_{5}, \mathrm{LuCo}_{3} \mathrm{~B}_{2}, \mathrm{TmCo}_{3} \mathrm{~B}_{2}, \\
\mathrm{ErCo}_{3} \mathrm{~B}_{2}, \mathrm{TaTi}_{2} \mathrm{~N}_{3}, \mathrm{DyCo}_{3} \mathrm{~B}_{2}, \mathrm{HoCo}_{3} \mathrm{~B}_{2}, \mathrm{TbCo}_{3} \mathrm{~B}_{2}, \mathrm{YCo}_{3} \mathrm{~B}_{2}, \mathrm{SmCu}_{5} \text {, } \\
\mathrm{PrCu}_{5}, \mathrm{NdCu}_{5}, \mathrm{LaCu}_{5}, \mathrm{ThSi}_{2} \mathrm{Ru}_{3}, \mathrm{ThCd}_{2}, \mathrm{Hf}_{2} \mathrm{TaN}_{3}, \mathrm{PrBPt}_{4}, \\
\mathrm{LaGa}_{2} \mathrm{Rh}_{3}, \mathrm{CeAl}_{2} \mathrm{Pt}_{3}, \mathrm{U}_{2} \mathrm{Ti}_{3}, \mathrm{LaIr}_{5}, \mathrm{TaNO}_{2} \mathrm{UV}_{2}, \mathrm{Pr}_{2} \mathrm{Co}_{7} \mathrm{~B}_{3}, \mathrm{FeSn}, \\
\mathrm{Hf}(\mathrm{MnGe})_{6}, \mathrm{Hf}(\mathrm{FeGe})_{6} \\
\end{array}$ \\
\hline & $\mathrm{A}-\Gamma$ & 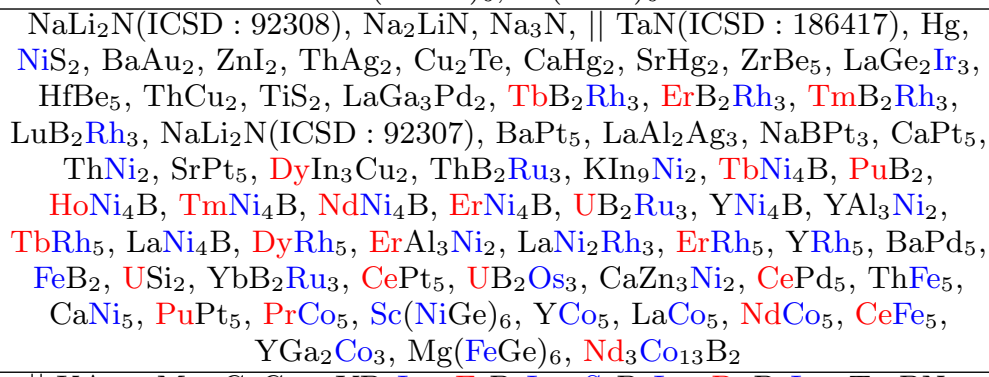 \\
\hline & \multirow[t]{2}{*}{$\mathrm{H}-\mathrm{K}$} & $\begin{array}{c}\| \mathrm{KAu}_{5}, \mathrm{Mo}, \mathrm{CaGa}_{2}, \mathrm{YB}_{2} \mathrm{Ir}_{3}, \mathrm{ErB}_{2} \mathrm{Ir}_{3}, \mathrm{ScB}_{2} \mathrm{Ir}_{3}, \mathrm{DyB}_{2} \mathrm{Ir}_{3}, \mathrm{Ta}_{2} \mathrm{BN}_{3}, \\
\mathrm{ScCo}_{3} \mathrm{~B}_{2}, \mathrm{SmCo}_{3} \mathrm{~B}_{2}, \mathrm{ThZn}_{2}, \mathrm{Zr}(\mathrm{CoGe})_{6}, \mathrm{Mg}(\mathrm{CoGe})_{6}, \mathrm{CeSi}_{2} \mathrm{Ru}_{3} \\
\mathrm{CeCo}_{4} \mathrm{~B}\end{array}$ \\
\hline \multirow{10}{*}{ NSOC } & & Space Group 193: $P 6_{3} / m c m$ \\
\hline & \multirow{2}{*}{\begin{tabular}{|c|} 
A- $\Gamma$, L-M, L-K, M-H, M-A, \\
M- $\Gamma$, H-K, K-A, K- $\Gamma$ \\
A- $\Gamma$, L-M, M-H, M-K, M-A, \\
M- $\Gamma$, L- $\Gamma$, K- $\Gamma$, H-K \\
\end{tabular}} & $\| \mathrm{Tb}_{5} \mathrm{Sb}_{3}, \mathrm{Sm}_{5} \mathrm{Sb}_{3}$ \\
\hline & & $\| \mathrm{La}_{3} \mathrm{MgBi}_{5}$ \\
\hline & $\begin{array}{c}\mathrm{A}-\Gamma, \mathrm{L}-\mathrm{A}, \mathrm{H}-\mathrm{A}, \mathrm{M}-\mathrm{A}, \mathrm{K}-\mathrm{A}, \\
\mathrm{M}-\Gamma, \mathrm{K}-\Gamma\end{array}$ & $\| \mathrm{Hf}_{5} \mathrm{Sn}_{3}, \mathrm{Ti}_{5} \mathrm{Si}_{3}, \mathrm{Ti}_{5} \mathrm{Ge}_{3}$ \\
\hline & $\begin{array}{l}\text { A- } \Gamma, \text { L-K, M-K, H-K, K-A, } \\
\text { K- } \Gamma\end{array}$ & $\| \mathrm{Tm}_{5} \mathrm{Sb}_{3}, \mathrm{Er}_{5} \mathrm{Sb}_{3}$ \\
\hline & $\mathrm{A}-\Gamma, \mathrm{L}-\Gamma, \mathrm{M}-\Gamma, \mathrm{H}-\Gamma, \mathrm{K}-\Gamma$ & $\| \mathrm{WO}_{3}$ \\
\hline & $\mathrm{A}-\Gamma, \mathrm{L}-\Gamma, \mathrm{M}-\Gamma, \mathrm{M}-\mathrm{K}, \mathrm{K}-\Gamma$ & $\| \mathrm{Y}_{5} \mathrm{Ga}_{3}$ \\
\hline & $\mathrm{M}-\mathrm{K}, \mathrm{K}-\Gamma, \mathrm{M}-\Gamma$ & || $\mathrm{Rh}_{7}\left(\mathrm{PbO}_{5}\right)_{3}$ \\
\hline & $\mathrm{M}-\Gamma, \mathrm{K}-\Gamma$ & $\| \mathrm{Hf}_{5} \mathrm{Ge}_{3}, \mathrm{Zr}_{5} \mathrm{Sn}_{3}, \mathrm{Zr}_{5} \mathrm{Ge}_{3}, \mathrm{Zr}_{5} \mathrm{~Pb}_{3}, \mathrm{Ti}_{5} \mathrm{Sn}_{3}$ \\
\hline & $\mathrm{M}-\mathrm{K}, \mathrm{K}-\Gamma$ & $\| \mathrm{K}_{3} \mathrm{~N}$ \\
\hline \multirow[t]{3}{*}{$\mathrm{SOC}$} & $\mathrm{A}-\Gamma, \mathrm{H}-\mathrm{K}$ & $\begin{array}{c}\| \mathrm{La}_{3} \mathrm{HfSb}_{5}, \mathrm{La}_{3} \mathrm{TiSb}_{5}, \mathrm{La}_{3} \mathrm{ZrSb}_{5}, \mathrm{Hg}_{5} \mathrm{Au}_{6}, \mathrm{Mg}_{5} \mathrm{Hg}_{3}, \mathrm{La}_{3} \mathrm{MgBi}_{5} \\
\mathrm{Zr}_{5} \mathrm{Sn}_{4}, \mathrm{Hf}_{5} \mathrm{Al}_{3} \mathrm{~N}, \mathrm{Yb}_{5} \mathrm{Ge}_{3}, \mathrm{Ho}_{5} \mathrm{Sb}_{3}, \mathrm{Zr}_{5} \mathrm{ZnPb}_{3}, \mathrm{Th}_{5} \mathrm{Sn}_{4}, \mathrm{Tm}_{5} \mathrm{Sb}_{3}, \\
\mathrm{~Tb}_{5} \mathrm{Sb}_{3}, \mathrm{Er}_{5} \mathrm{Sb}_{3}, \mathrm{Y}_{5} \mathrm{CuPb}_{3}, \mathrm{Ge}_{3} \mathrm{Mo}_{5} \mathrm{C}, \mathrm{Ta}_{5} \mathrm{Ga}_{3}, \mathrm{Zr}_{5} \mathrm{Sb}_{4}, \mathrm{Fe}_{5} \mathrm{Si}_{3} \\
\mathrm{Sm}_{5} \mathrm{Sb}_{3}, \mathrm{TlAgSe}_{2}, \mathrm{Yb}_{5} \mathrm{~Pb}_{3}, \mathrm{Zr}_{5} \mathrm{Al}_{4}\end{array}$ \\
\hline & $\mathrm{A}-\Gamma$ & $\begin{array}{r}\mathrm{WO}_{3}, \mathrm{Y}_{5} \mathrm{Ga}_{3}, \mathrm{Ti}_{3} \mathrm{O}, \mathrm{Hf}_{5} \mathrm{Si}_{3}, \mathrm{Zr}_{5} \mathrm{Si}_{3}, \mathrm{Zr}_{5} \mathrm{Ge}_{3}, \mathrm{Th}_{5} \mathrm{Sn}_{3}, \mathrm{Lu}_{5} \mathrm{Rh}_{3}, \\
\mathrm{Er}_{5} \mathrm{Rh}_{3}, \mathrm{Lu}_{5} \mathrm{Si}_{3} \mathrm{~B}, \mathrm{Sc}_{5} \mathrm{Ga}_{3}, \mathrm{Ho}_{5} \mathrm{Rh}_{3}, \mathrm{~Tb}_{5} \mathrm{Tl}_{3}, \mathrm{Dy}_{5} \mathrm{Si}_{3} \mathrm{~B}, \mathrm{Ho}_{5} \mathrm{Si}_{3} \mathrm{~B} \\
\mathrm{Ho}_{5} \mathrm{Ga}_{3}, \mathrm{LaSiNO}_{2}, \mathrm{Er}_{5} \mathrm{Ga}_{3}, \mathrm{Ce}_{5} \mathrm{Ge}_{3} \\
\end{array}$ \\
\hline & $\mathrm{H}-\mathrm{K}$ & $\| \mathrm{Hf}_{5} \mathrm{Sn}_{4}, \mathrm{Yb}_{5} \mathrm{Sn}_{3}, \mathrm{Ti}_{5} \mathrm{Ga}_{4}$ \\
\hline \multicolumn{3}{|r|}{ Space Group 194: $P 6_{3} / m m c$} \\
\hline & 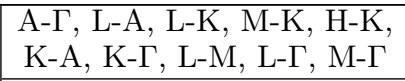 & $\| \mathrm{Er}_{2} \mathrm{Fe}_{17}$ \\
\hline & $\begin{array}{c}\text { A- } \Gamma, \mathrm{L}-\mathrm{A}, \mathrm{L}-\Gamma, \mathrm{M}-\Gamma, \mathrm{H}-\Gamma \\
\text { K- } \Gamma, \mathrm{L}-\mathrm{M}, \mathrm{M}-\mathrm{A}, \mathrm{M}-\mathrm{K}\end{array}$ & $\| \mathrm{Sr}_{2} \mathrm{Mg}_{17}$ \\
\hline & $\begin{array}{l}\mathrm{A}-\Gamma, \mathrm{L}-\mathrm{A}, \mathrm{L}-\mathrm{M}, \mathrm{M}-\mathrm{H}, \mathrm{M}-\mathrm{K} \\
\mathrm{M}-\mathrm{A}, \mathrm{M}-\Gamma, \mathrm{L}-\Gamma, \mathrm{H}-\mathrm{K}\end{array}$ & $\| \mathrm{Li}_{2} \mathrm{Ca}$ \\
\hline & $\begin{array}{l}\mathrm{A}-\Gamma, \mathrm{L}-\mathrm{A}, \mathrm{L}-\mathrm{M}, \mathrm{M}-\mathrm{H}, \mathrm{M}-\mathrm{K} \\
\mathrm{M}-\mathrm{A}, \mathrm{M}-\Gamma, \mathrm{L}-\Gamma, \mathrm{K}-\Gamma\end{array}$ & $\| \mathrm{LiIO}_{3}$ \\
\hline & $\begin{array}{l}\mathrm{A}-\Gamma, \mathrm{L}-\mathrm{M}, \mathrm{L}-\Gamma, \mathrm{M}-\mathrm{H}, \mathrm{M}-\mathrm{K} \\
\mathrm{M}-\mathrm{A}, \mathrm{M}-\Gamma, \mathrm{H}-\Gamma, \mathrm{K}-\Gamma\end{array}$ & \| $\mathrm{Mg}_{2} \mathrm{Ge}, \mathrm{Mg}_{2} \mathrm{Sn}, \mathrm{MgGa}_{2}, \mathrm{CaIn} 2$ (ICSD : 58906), $\mathrm{Ni}_{2} \mathrm{Ge}$ \\
\hline & $\begin{array}{c}\text { A- } \Gamma, \text { L-A, L-M, L- } \Gamma, \text { M-A, } \\
\text { M- } \Gamma, \mathrm{K}-\Gamma, \mathrm{M}-\mathrm{K}\end{array}$ & $\| \mathrm{ThMg}_{2}$ \\
\hline
\end{tabular}


Table III - continued

\begin{tabular}{|c|c|c|}
\hline $\begin{array}{l}\text { SOC or } \\
\text { NSOC }\end{array}$ & $\begin{array}{c}\text { HSL with Band } \\
\text { Crossing }\end{array}$ & High Symmetry Line Semimetals \\
\hline & $\begin{array}{l}\text { A- } \Gamma, \mathrm{L}-\mathrm{M}, \mathrm{M}-\mathrm{H}, \mathrm{M}-\mathrm{K}, \mathrm{M}-\mathrm{A} \\
\mathrm{M}-\Gamma, \mathrm{L}-\Gamma, \mathrm{K}-\Gamma\end{array}$ & $\| \mathrm{Nb}_{4} \mathrm{CrSe}_{8}$ \\
\hline & $\begin{array}{l}\text { L-A, L-M, M-H, M-K, M-A, } \\
\text { M- } \Gamma, L-\Gamma, K-\Gamma\end{array}$ & \| MnSb, MnBi \\
\hline & 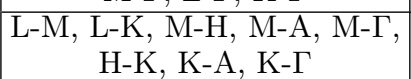 & $\| \mathrm{Mg}_{3} \mathrm{Cd}, \mathrm{BaRuO}_{3}(\mathrm{ICSD}: 246019)$ \\
\hline & $\begin{array}{c}\mathrm{A}-\Gamma, \mathrm{L}-\mathrm{A}, \mathrm{L}-\Gamma, \mathrm{M}-\mathrm{A}, \mathrm{M}-\Gamma \\
\mathrm{M}-\mathrm{K}, \mathrm{K}-\Gamma\end{array}$ & $\| \mathrm{TmAsPt}, \mathrm{Nb}_{4} \mathrm{CrS}_{8}, \mathrm{VCo}_{3}$ \\
\hline & $\begin{array}{l}\mathrm{A}-\Gamma, \mathrm{L}-\mathrm{A}, \mathrm{L}-\Gamma, \mathrm{M}-\Gamma, \mathrm{H}-\Gamma \\
\mathrm{K}-\Gamma, \mathrm{M}-\mathrm{A}\end{array}$ & $\| \mathrm{SrNi}_{2} \mathrm{Ge}$ \\
\hline & $\begin{array}{l}\mathrm{A}-\Gamma, \mathrm{L}-\mathrm{A}, \mathrm{L}-\mathrm{M}, \mathrm{L}-\Gamma, \mathrm{M}-\mathrm{A} \\
\mathrm{M}-\Gamma, \mathrm{K}-\Gamma\end{array}$ & $\| \mathrm{Pr}_{3} \mathrm{Al}, \mathrm{Nd}_{3} \mathrm{Al}$ \\
\hline & $\begin{array}{c}\mathrm{A}-\Gamma, \mathrm{L}-\mathrm{M}, \mathrm{L}-\Gamma, \mathrm{M}-\mathrm{A}, \mathrm{M}-\mathrm{K} \\
\mathrm{M}-\Gamma, \mathrm{K}-\Gamma\end{array}$ & $\| \mathrm{TaZn}_{2}, \mathrm{NbZn}_{2}$ \\
\hline & $\begin{array}{c}\text { L-M, M-H, M-K, M-A, } \\
\text { M- } \Gamma, \mathrm{K}-\Gamma, \mathrm{H}-\mathrm{K}\end{array}$ & $\| \mathrm{Ti}_{3} \mathrm{Sn}$ \\
\hline & $\begin{array}{c}\mathrm{A}-\Gamma, \mathrm{L}-\mathrm{A}, \mathrm{L}-\Gamma, \mathrm{M}-\mathrm{A}, \mathrm{M}-\Gamma, \\
\mathrm{K}-\Gamma\end{array}$ & $\|$ YAsPt \\
\hline & $\begin{array}{c}\mathrm{A}-\Gamma, \mathrm{L}-\Gamma, \mathrm{M}-\Gamma, \mathrm{H}-\Gamma, \mathrm{K}-\Gamma, \\
\mathrm{M}-\mathrm{K}\end{array}$ & $\| \mathrm{NiP}_{2} \mathrm{~W}, \mathrm{BaTiSe}_{3}, \mathrm{Sm}(\mathrm{ICSD}:$ 652632), $\mathrm{Pr}(\mathrm{ICSD}: 649176), \mathrm{Nd}, \mathrm{La}$ \\
\hline & $\begin{array}{c}\mathrm{A}-\Gamma, \mathrm{L}-\mathrm{M}, \mathrm{L}-\Gamma, \mathrm{M}-\mathrm{A}, \mathrm{M}-\Gamma, \\
\mathrm{K}-\Gamma\end{array}$ & $\| \mathrm{Ce}_{3}\left(\mathrm{Al}_{3} \mathrm{Ru}\right)_{4}$ \\
\hline & $\begin{array}{c}\mathrm{A}-\Gamma, \mathrm{L}-\mathrm{M}, \mathrm{M}-\mathrm{A}, \mathrm{M}-\Gamma, \mathrm{M}-\mathrm{K}, \\
\mathrm{K}-\Gamma\end{array}$ & $\| \mathrm{Na}_{4} \mathrm{CaSn}_{6}$ \\
\hline & $\begin{array}{c}\mathrm{L}-\mathrm{A}, \mathrm{L}-\Gamma, \mathrm{M}-\mathrm{A}, \mathrm{M}-\Gamma, \mathrm{K}-\Gamma, \\
\mathrm{M}-\mathrm{K}\end{array}$ & $\| \mathrm{Ti}_{2} \mathrm{PbC}, \mathrm{Ti}_{3} \mathrm{SnC}_{2}$ \\
\hline & $\begin{array}{c}\mathrm{L}-\mathrm{A}, \mathrm{L}-\mathrm{M}, \mathrm{L}-\Gamma, \mathrm{M}-\mathrm{A}, \mathrm{M}-\Gamma \\
\mathrm{K}-\Gamma\end{array}$ & || BaBiAu, BaSbAu(ICSD : 106270) \\
\hline & $\begin{array}{c}\mathrm{L}-\mathrm{A}, \mathrm{L}-\mathrm{M}, \mathrm{L}-\Gamma, \mathrm{M}-\Gamma, \mathrm{K}-\Gamma, \\
\mathrm{M}-\mathrm{K}\end{array}$ & $\| \mathrm{SrBiAu}$ \\
\hline & $\begin{array}{l}\text { L-M, M-H, M-K, M-A, } \\
\text { M- } \Gamma, \text { K- }\end{array}$ & $\| \mathrm{Nb}_{2} \mathrm{SnC}, \mathrm{V}_{2} \mathrm{GeC}, \mathrm{FeSn}, \mathrm{Ba}_{3} \mathrm{MgRu}_{2} \mathrm{O}_{9}$ \\
\hline & $\mathrm{A}-\Gamma, \mathrm{L}-\Gamma, \mathrm{M}-\Gamma, \mathrm{H}-\Gamma, \mathrm{K}-\Gamma$ & $\mathrm{BaTiS}_{3},|| \mathrm{BaZrSe}_{3}, \mathrm{YH}_{3}(\mathrm{ICSD}: 180316)$, CaZnSn, $\mathrm{SrTiS}_{3}$ \\
\hline & $\mathrm{A}-\Gamma, \mathrm{L}-\Gamma, \mathrm{M}-\Gamma, \mathrm{M}-\mathrm{K}, \mathrm{K}-\Gamma$ & || $\mathrm{Si}_{3} \mathrm{Pt}_{2}, \mathrm{Nd}_{2} \mathrm{Ni}_{17}$ \\
\hline & $\mathrm{A}-\Gamma, \mathrm{L}-\mathrm{M}, \mathrm{M}-\mathrm{A}, \mathrm{M}-\Gamma, \mathrm{K}-\Gamma$ & $\begin{array}{c}\text { \I PrZnGa, ErZnGa, TmZnGa, YZnGa, DyZnGa, HfGaCu, ErZnIn, } \\
\text { HoZnIn, YAlZn, DyZnIn, TmAlZn, ErAlZn, ZrGaCu, DyAlZn, } \\
\text { PrZnIn, NdZnIn, SmZnGa, SmZnIn }\end{array}$ \\
\hline & $\mathrm{L}-\mathrm{A}, \mathrm{L}-\Gamma, \mathrm{M}-\mathrm{A}, \mathrm{M}-\Gamma, \mathrm{K}-\Gamma$ & $\mathrm{Ta}_{3} \mathrm{AlC}_{2}[58], \| \mathrm{Ti}_{4} \mathrm{AlN}_{3}, \mathrm{Pr}_{2} \mathrm{~B}_{3} \mathrm{Cl}$ \\
\hline & L-K, M-K, H-K, K-A, K- $\Gamma$ & $\|$ BRh(ICSD : 193833) \\
\hline & L-M, M-A, M-, M-K, H-K & $\|$ MoN(ICSD : 251629) \\
\hline & $\mathrm{L}-\mathrm{M}, \mathrm{M}-\mathrm{A}, \mathrm{M}-\Gamma, \mathrm{M}-\mathrm{K}, \mathrm{K}-\Gamma$ & $\mathrm{ZrAl}_{2}, \mathrm{HfAl}_{2}, \mathrm{Be}_{2} \mathrm{~W}, \mathrm{Be}_{2} \mathrm{Cr}, \mathrm{Be}_{2} \mathrm{Mo}, \mathrm{USnPt}_{2}, \mathrm{MgNi}_{2}$ \\
\hline & L-M, M-H, M-K, M-A, M- & $\| \mathrm{Be}_{2} \mathrm{~V}, \mathrm{Mn}_{3} \mathrm{Al}_{9} \mathrm{Si}, \mathrm{CrTe}, \mathrm{Ba}_{3} \mathrm{ZnRu}_{2} \mathrm{O}_{9}, \mathrm{Ba}_{3} \mathrm{CaRu}_{2} \mathrm{O}_{9}$ \\
\hline & $\mathrm{A}-\Gamma, \mathrm{L}-\Gamma, \mathrm{M}-\Gamma, \mathrm{H}-\mathrm{K}$ & $\| \mathrm{Ba}_{3} \mathrm{LiN}, \mathrm{Ba}_{3} \mathrm{NaN}$ \\
\hline & $\mathrm{A}-\Gamma, \mathrm{L}-\Gamma, \mathrm{M}-\Gamma, \mathrm{K}-\Gamma$ & $\begin{array}{c}\| \mathrm{BaTl}_{2}, \mathrm{SrIn}_{2}, \mathrm{CaGa}_{2}, \mathrm{HfPt}_{3}, \mathrm{Zr}, \mathrm{NdPbAu}, \mathrm{SmCuPb}, \mathrm{HfPd}_{3}, \\
\mathrm{ErCuPb}, \mathrm{HoCuPb}, \mathrm{DyCuPb}, \mathrm{ZrPd}_{3}, \mathrm{TiNi}_{3}\end{array}$ \\
\hline & $\mathrm{A}-\Gamma, \mathrm{L}-\mathrm{M}, \mathrm{L}-\Gamma, \mathrm{M}-\mathrm{A}$ & $\| \mathrm{NdVGe}_{3}, \mathrm{PrVGe}_{3}, \mathrm{LaVGe}_{3}$ \\
\hline & $\mathrm{A}-\Gamma, \mathrm{M}-\Gamma, \mathrm{K}-\Gamma, \mathrm{M}-\mathrm{K}$ & $\| \mathrm{ZrOs}_{2}$ \\
\hline & $\mathrm{A}-\Gamma, \mathrm{M}-\mathrm{K}, \mathrm{K}-\Gamma, \mathrm{M}-\Gamma$ & || \begin{tabular}{l|l} 
& EuPPt
\end{tabular} \\
\hline & $\mathrm{A}-\Gamma, \mathrm{L}-\Gamma, \mathrm{M}-\Gamma$ & $\begin{array}{c}\text { SrAgBi } 74] \text {, SrCuBi, CaSbAu, BaCuBi }[119], \mathrm{CaAgBi}[120], \mathrm{CaCuBi} \\
\text { YbCuBi, } / \mid \text { BaCdGe, BaAsAu, SrZnPb, BaZnSn, SbIr, } \mathrm{Al}_{3} \mathrm{Ir} \\
\text { LaPbAu, FeTe }\end{array}$ \\
\hline & $\mathrm{A}-\Gamma, \mathrm{M}-\Gamma, \mathrm{K}-\Gamma$ & $\begin{array}{c}\| \text { TmCuGe, HoCuGe, YbAlGa, PrCuPb, PrCuSn, NdCuSn, HoCuSn, } \\
\text { ErCuSn, SmCuSn, TbCuSn, DyCuSn, TmCuSn, LuCuSn, TiAlAu, } \\
\text { ZrMn } 2 \text { (ICSD : 246493) }\end{array}$ \\
\hline & $\mathrm{A}-\Gamma, \mathrm{M}-\mathrm{K}, \mathrm{K}-\Gamma$ & $\| \mathrm{HfOs}_{2}$ \\
\hline & $\mathrm{M}-\Gamma, \mathrm{K}-\Gamma, \mathrm{M}-\mathrm{K}$ & SrAgSb, BaAgSb, BaAgBi[119], $\mathrm{NiMoP}_{2}$ \\
\hline & $\mathrm{M}-\mathrm{K}, \mathrm{K}-\Gamma, \mathrm{M}-\Gamma$ & $\| \mathrm{K}, \mathrm{Rb}, \mathrm{Cs}(\mathrm{ICSD}: 109023)$ \\
\hline & $\mathrm{M}-\mathrm{K}, \mathrm{M}-\Gamma, \mathrm{H}-\mathrm{K}$ & $\mathrm{ErMg}_{2}, \mathrm{TbMg}_{2}, \mathrm{YMg}_{2}, \mathrm{LaMg}_{2}$ \\
\hline & $\mathrm{M}-\mathrm{K}, \mathrm{M}-\Gamma, \mathrm{K}-\Gamma$ & $\| \mathrm{Nb}_{2} \mathrm{GeC}, \mathrm{PtN}_{2}, \mathrm{Ti}_{2} \mathrm{CdC}$ \\
\hline
\end{tabular}


Table III - continued

\begin{tabular}{|c|c|c|}
\hline $\begin{array}{l}\text { SOC or } \\
\text { NSOC }\end{array}$ & $\begin{array}{l}\text { HSL with Band } \\
\text { Crossing }\end{array}$ & High Symmetry Line Semimetals \\
\hline & $\mathrm{M}-\Gamma, \mathrm{K}-\Gamma$ & $\begin{array}{c}\mathrm{Ca}(\mathrm{InP})_{2} 121, \mathrm{Sr}(\mathrm{InP})_{2} 121, \mathrm{Rb}_{3} \mathrm{Bi} 78, \mathrm{InSe}, \mathrm{Cd}, \mathrm{Zn}, \| \mathrm{Sr}_{2} \mathrm{Si}, \\
\mathrm{BRh}(\mathrm{ICSD}: 150732), \mathrm{Yb}_{2} \mathrm{Sn}, \mathrm{TiS}_{2}(\mathrm{ICSD}: 181503), \mathrm{PtPb}, \mathrm{HoMg}_{2} \mathrm{Cu}_{9}, \\
\mathrm{HfCr}_{2}(\mathrm{ICSD}: 109213), \mathrm{ZrRe}_{2}\end{array}$ \\
\hline & $\mathrm{M}-\mathrm{K}, \mathrm{K}-\Gamma$ & $\mathrm{C}(\mathrm{ICSD}: 28419)\left[58,\|\| \mathrm{LaI}, \mathrm{BaCu}, \mathrm{ThI}_{2}\right.$ \\
\hline & $\mathrm{M}-\mathrm{K}, \mathrm{M}-\Gamma$ & $\| \mathrm{Er}_{2} \mathrm{Al}_{3} \mathrm{Co}, \mathrm{HfTc}_{2}, \mathrm{ScZn}_{2}, \mathrm{YZn}_{2}, \mathrm{ZrTc}_{2}$ \\
\hline & $\mathrm{H}-\mathrm{K}$ & $\| \mathrm{Sr}, \mathrm{Ca}, \mathrm{YbHg}_{3}, \mathrm{SrHg}_{3}$ \\
\hline & L-M & $\mathrm{Ti}_{2} \mathrm{Sn}[58] \|$ \\
\hline \multirow{4}{*}{$\mathrm{SOC}$} & $\mathrm{A}-\Gamma, \mathrm{L}-\mathrm{H}, \mathrm{H}-\mathrm{A}$ & $\begin{array}{l}\| \mathrm{Ag}(\mathrm{ICSD}: \text { 64707), ReN, Sm(ICSD : 652632), Nd, } \\
\operatorname{Pr}\left(\mathrm{ICSD}: \text { 649176), } \mathrm{Mg}_{5} \mathrm{Pd}_{2}, \mathrm{La}, \mathrm{CeAlAu}, \mathrm{Th}_{2} \mathrm{Ni}_{17}\right.\end{array}$ \\
\hline & L-H, H-A, H-K & $\| \operatorname{ErTc}_{2}$ \\
\hline & $\mathrm{A}-\Gamma, \mathrm{H}-\mathrm{K}$ & 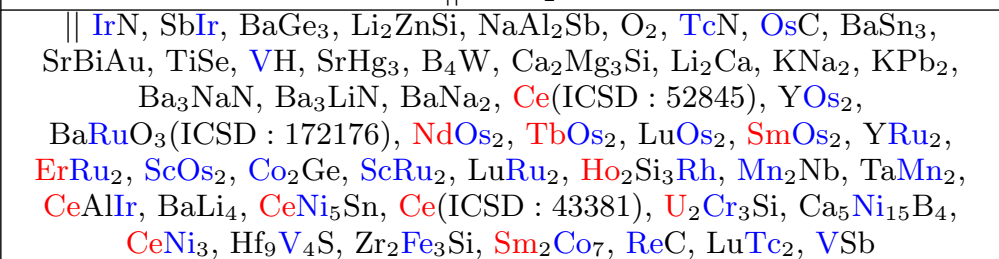 \\
\hline & L-H, H-A & $\| \mathrm{Yb}, \mathrm{ScRe}_{2}, \mathrm{Nb}_{2} \mathrm{CrSe}_{4}$ \\
\hline
\end{tabular}


Table III - continued

\begin{tabular}{|c|c|c|}
\hline $\begin{array}{l}\text { SOC or } \\
\text { NSOC }\end{array}$ & $\begin{array}{l}\text { HSL with Band } \\
\text { Crossing }\end{array}$ & High Symmetry Line Semimetals \\
\hline & $\mathrm{A}-\Gamma$ & 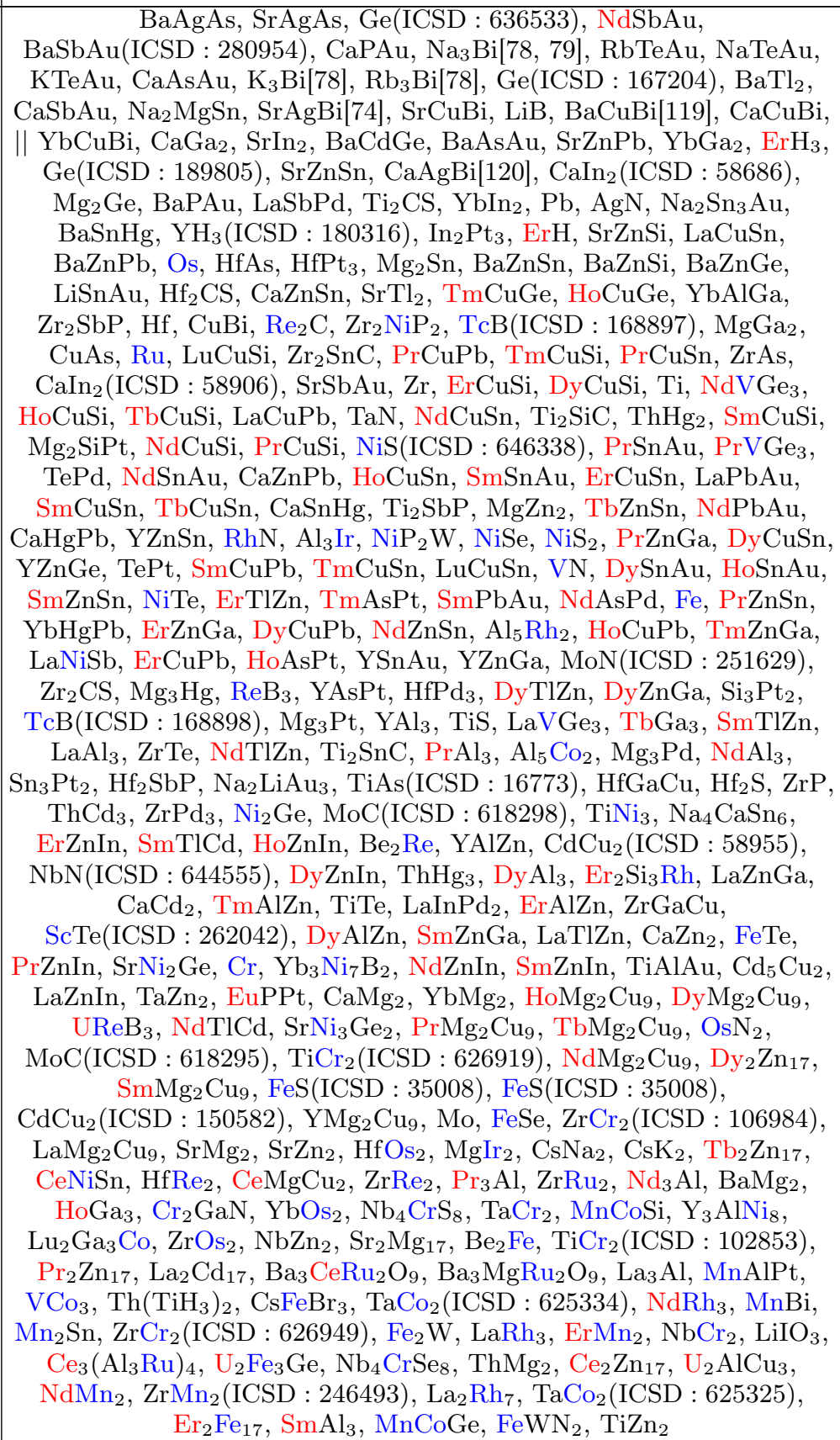 \\
\hline & $\mathrm{H}-\mathrm{K}$ & 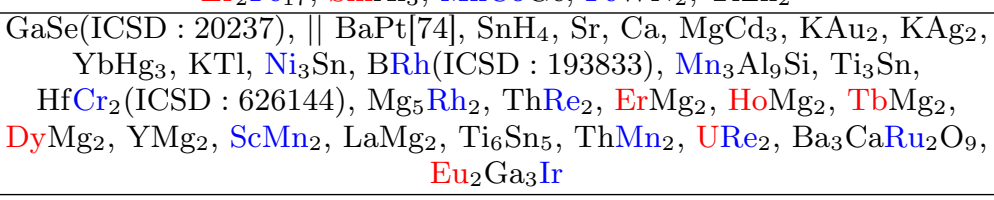 \\
\hline \multicolumn{3}{|r|}{ Space Group 197: I23 } \\
\hline $\mathrm{SOC}$ & $\Gamma-\mathrm{H}, \mathrm{H}-\mathrm{P}, \Gamma-\mathrm{P}$ & $\mathrm{Nd}_{4} \mathrm{Os}_{6} \mathrm{O}_{19}$ \\
\hline \multicolumn{3}{|r|}{ Space Group 198: $P 2_{1} 3$} \\
\hline $\mathrm{SOC}$ & $\overline{\Gamma-\mathrm{R}}$ & $\mathrm{CrSi}$ \\
\hline \multicolumn{3}{|r|}{ Space Group 200: $P m \overline{3}$} \\
\hline \multirow[b]{2}{*}{ NSOC } & $\mathrm{X}-\Gamma, \mathrm{M}-\Gamma, \mathrm{M}-\mathrm{R}, \mathrm{M}-\mathrm{X}$ & $\mathrm{Mg}_{30} \mathrm{Al}_{24} \mathrm{Ag}_{19}$ \\
\hline & $\mathrm{X}-\Gamma, \mathrm{M}-\Gamma, \mathrm{M}-\mathrm{X}, \mathrm{X}-\mathrm{R}$ & $\| \mathrm{Al}_{17}\left(\mathrm{Re}_{2} \mathrm{Si}\right)_{2}$ \\
\hline
\end{tabular}


Table III - continued

\begin{tabular}{|c|c|c|}
\hline $\begin{array}{l}\text { SOC or } \\
\text { NSOC }\end{array}$ & $\begin{array}{l}\text { HSL with Band } \\
\text { Crossing }\end{array}$ & High Symmetry Line Semimetals \\
\hline & $\mathrm{X}-\Gamma, \mathrm{M}-\mathrm{X}, \mathrm{M}-\Gamma$ & $\mathrm{NaCl}_{7}$ \\
\hline \multicolumn{3}{|r|}{ Space Group 204: $\operatorname{Im} \overline{3}$} \\
\hline \multirow{3}{*}{ NSOC } & $\Gamma-\mathrm{H}, \mathrm{N}-\Gamma, \mathrm{N}-\mathrm{H}, \mathrm{N}-\mathrm{P}$ & $\mathrm{LuO}_{3}$ \\
\hline & $\Gamma-\mathrm{H}, \mathrm{N}-\mathrm{H}$ & $\| \mathrm{SrC}_{10}$ \\
\hline & $\Gamma-\mathrm{H}, \mathrm{N}-\mathrm{P}$ & $\mathrm{Ho}_{8} \mathrm{Mg}_{12} \mathrm{Zn}_{61}$ \\
\hline \multicolumn{3}{|r|}{ Space Group 205: $P a \overline{3}$} \\
\hline \multirow{2}{*}{ NSOC } & $\mathrm{X}-\Gamma, \mathrm{M}-\Gamma$ & $\| \mathrm{Sb}_{2} \mathrm{Pd}, \mathrm{NiSe}_{2}, \mathrm{NiS}_{2}(\mathrm{ICSD}: 40328), \mathrm{NiS}_{2}(\mathrm{ICSD}: 68169)$ \\
\hline & $\mathrm{X}-\Gamma, \mathrm{M}-\mathrm{X}$ & $\| \mathrm{CaGaAu}_{3}$ \\
\hline $\mathrm{SOC}$ & $\Gamma-\mathrm{R}$ & $\| \mathrm{Bi}_{2} \mathrm{Pt}$ \\
\hline \multicolumn{3}{|r|}{ Space Group 208: $P 4_{2} 32$} \\
\hline NSOC & $\mathrm{M}-\mathrm{X}, \mathrm{X}-\mathrm{R}$ & $\mathrm{AsH}_{3}$ \\
\hline \multicolumn{3}{|r|}{ Space Group 212: $P 4_{3} 32$} \\
\hline NSOC & $\mathrm{X}-\Gamma$ & $\mathrm{V}_{8} \mathrm{C}_{7}$ \\
\hline SOC & $\mathrm{X}-\Gamma$ & $\mathrm{V}_{8} \mathrm{C}_{7}$ \\
\hline \multicolumn{3}{|r|}{ Space Group 213: $P 4_{1} 32$} \\
\hline \multirow{2}{*}{ NSOC } & $\mathrm{X}-\Gamma, \mathrm{M}-\Gamma$ & $\mathrm{Ag}_{3} \mathrm{AuS}_{2}$ \\
\hline & $\Gamma-\mathrm{R}$ & $\| \mathrm{Ba}_{6} \mathrm{Si}_{25}$ \\
\hline \multirow{3}{*}{ SOC } & $\mathrm{X}-\Gamma, \Gamma-\mathrm{R}$ & $\mathrm{Re}_{2} \mathrm{~W}_{3} \mathrm{C}, \mathrm{Ba}_{6} \mathrm{Ge}_{25}$ \\
\hline & $\Gamma-\mathrm{R}$ & $\| \mathrm{Ba}_{6} \mathrm{Si}_{25}$ \\
\hline & $\mathrm{X}-\Gamma$ & $\mathrm{Ag}_{3} \mathrm{AuS}_{2}$ \\
\hline \multicolumn{3}{|r|}{ Space Group 214: $I 4_{1} 32$} \\
\hline NSOC & $\Gamma-\mathrm{H}, \mathrm{N}-\Gamma$ & $\| \mathrm{Ca}_{3} \mathrm{Zn}_{3}\left(\mathrm{TeO}_{6}\right)_{2}$ \\
\hline $\mathrm{SOC}$ & $\Gamma-\mathrm{H}$ & $\mathrm{Ca}_{3} \mathrm{Zn}_{3}\left(\mathrm{TeO}_{6}\right)_{2}$ \\
\hline \multicolumn{3}{|r|}{ Space Group 215: $P \overline{4} 3 m$} \\
\hline $\mathrm{SOC}$ & $\Gamma-\mathrm{R}$ & LiMgIr \\
\hline \multicolumn{3}{|r|}{ Space Group 216: $F \overline{4} 3 m$} \\
\hline \multirow{2}{*}{ NSOC } & $\Gamma-\mathrm{L}, \mathrm{X}-\Gamma$ & $\| \mathrm{Sm}_{11} \mathrm{Cd}_{45}$ \\
\hline & $\mathrm{W}-\mathrm{X}$ & $\| \mathrm{SnS}, \mathrm{Mn}_{2} \mathrm{Ge}, \mathrm{ZnNi}$ \\
\hline \multirow[t]{2}{*}{ SOC } & $\Gamma-\mathrm{L}$ & $\begin{array}{c}\mathrm{LiMgSbPt}, \| \mathrm{LiMgSnAu}_{\mathrm{M}} \mathrm{CdC}, \mathrm{TmCu}_{5}, \mathrm{HoCu}_{4} \mathrm{Au}, \mathrm{DyCu}_{5}, \mathrm{HoCu}_{5}, \\
\mathrm{SmCu}_{4} \mathrm{Ag}, \mathrm{ErCu}_{5}, \mathrm{HoCu}_{4} \mathrm{Ag}, \mathrm{TmCu}_{4} \mathrm{Ag}, \mathrm{LuCu}_{5}, \mathrm{YbCdCu}_{4}, \\
\mathrm{DyInPt}_{4}, \mathrm{Dy}_{4} \mathrm{CdCo}_{\mathrm{CH}} \mathrm{Ho}_{4} \mathrm{CdCo}, \mathrm{Tb}_{4} \mathrm{CdCo}_{\mathrm{CbCu}} \mathrm{TbCu}_{4} \mathrm{Ag}, \mathrm{ErCu}_{4} \mathrm{Ag}, \\
\mathrm{ErCu}_{4} \mathrm{Au}, \mathrm{HoInPt}_{4}, \mathrm{TbCu}_{4} \mathrm{Au}, \mathrm{TbInPt}{ }_{4}, \mathrm{TbCu}_{5}, \mathrm{DyCu}_{4} \mathrm{Ag}, \mathrm{MnSbPt}\end{array}$ \\
\hline & $\mathrm{W}-\mathrm{X}$ & $\| \mathrm{UNiSn}, \mathrm{Sm}_{11} \mathrm{Cd}_{45}, \mathrm{UCu}_{4} \mathrm{Pd}, \mathrm{Mn}_{2} \mathrm{GaCo}, \mathrm{Mn}_{5}\left(\mathrm{NiBi}_{2}\right)_{2}$ \\
\hline \multicolumn{3}{|r|}{ Space Group 217: $I \overline{4} 3 m$} \\
\hline NSOC & $\Gamma-\mathrm{P}, \mathrm{H}-\mathrm{P}, \mathrm{P}-\mathrm{N}, \mathrm{H}-\mathrm{N}$ & $\mathrm{Zn}_{11} \mathrm{Ni}_{2}$ \\
\hline SOC & $\Gamma-\mathrm{H}, \Gamma-\mathrm{P}$ & $\mathrm{Ti}_{5} \mathrm{Re}_{24}$ \\
\hline \multicolumn{3}{|r|}{ 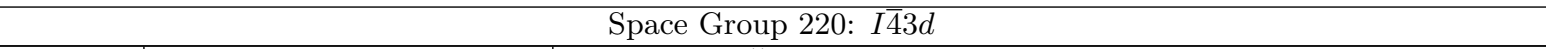 } \\
\hline \multirow{2}{*}{ NSOC } & $\Gamma-\mathrm{H}, \Gamma-\mathrm{P}, \Gamma-\mathrm{N}$ & $\| \mathrm{La}_{2} \mathrm{C}_{3}, \mathrm{Nd}_{2} \mathrm{C}_{3}, \mathrm{Pr}_{2} \mathrm{C}_{3}, \mathrm{Sm}_{2} \mathrm{C}_{3}, \mathrm{U}_{3} \mathrm{Ni}_{3} \mathrm{Sb}_{4}$ \\
\hline & $\Gamma-\mathrm{H}$ & $\begin{array}{c}\| \mathrm{Sc}_{2} \mathrm{C}_{3}, \mathrm{Cu}_{3} \mathrm{As}, \mathrm{Tm}_{2} \mathrm{C}_{3}, \mathrm{Er}_{2} \mathrm{C}_{3}, \mathrm{Ho}_{2} \mathrm{C}_{3}, \mathrm{Y}_{2} \mathrm{C}_{3}, \mathrm{Lu}_{2} \mathrm{C}_{3}, \mathrm{Ga}_{8} \mathrm{Cu}_{3} \mathrm{~W}_{2}, \\
\mathrm{Ga}_{8} \mathrm{Cu}_{3} \mathrm{Mo}_{2}, \mathrm{U}_{2} \mathrm{C}_{3}\end{array}$ \\
\hline \multicolumn{3}{|r|}{ Space Group 221: $P m \overline{3} m$} \\
\hline & $\Gamma-\mathrm{R}, \mathrm{M}-\mathrm{R}, \mathrm{X}-\mathrm{R}, \mathrm{M}-\mathrm{X}$ & $\| \mathrm{ZrRu}_{3} \mathrm{C}$ \\
\hline & $\mathrm{X}-\Gamma, \Gamma-\mathrm{R}, \mathrm{M}-\mathrm{X}, \mathrm{M}-\mathrm{R}$ & $\| \mathrm{HgPt}_{3}, \mathrm{ZnPt}_{3}, \mathrm{MgPt}_{3}$ \\
\hline & $\mathrm{X}-\Gamma, \mathrm{M}-\Gamma, \mathrm{M}-\mathrm{R}, \mathrm{X}-\mathrm{R}$ & $\| \mathrm{TaRh}_{3}, \mathrm{NbRh}_{3}, \mathrm{TaCo}_{3}, \mathrm{VCo}_{3}, \mathrm{PaRh}_{3}$ \\
\hline & $\mathrm{X}-\Gamma, \mathrm{M}-\Gamma, \mathrm{M}-\mathrm{X}, \mathrm{M}-\mathrm{R}$ & || $\mathrm{W}_{3} \mathrm{~N}_{4}, \mathrm{PuSn}_{3}$ \\
\hline & $\mathrm{X}-\Gamma, \mathrm{M}-\Gamma, \mathrm{M}-\mathrm{X}, \mathrm{X}-\mathrm{R}$ & $\| \mathrm{YbPb}_{3}, \mathrm{CaPb}_{3}$ \\
\hline & $\mathrm{X}-\Gamma, \mathrm{M}-\mathrm{X}, \mathrm{X}-\mathrm{R}, \mathrm{M}-\mathrm{R}$ & HfOs, TiFe, HfRu, ZrOs, TiRu, MnV, $\| \mathrm{ZrRu}$ \\
\hline & $\Gamma-\mathrm{R}, \mathrm{M}-\mathrm{R}, \mathrm{X}-\mathrm{R}$ & $\| \mathrm{PaIr}_{3}, \mathrm{ThRu}_{3} \mathrm{C}$ \\
\hline NSOC & $\mathrm{M}-\Gamma, \mathrm{M}-\mathrm{X}, \mathrm{M}-\mathrm{R}$ & SrTe, CaTe[122], $\|$ InSb, HgTe \\
\hline & $\mathrm{M}-\Gamma, \mathrm{M}-\mathrm{X}, \mathrm{X}-\mathrm{R}$ & $\| \mathrm{InNi}_{3} \mathrm{~N}, \mathrm{Pt}_{3} \mathrm{PbC}, \mathrm{SnPt}_{3} \mathrm{C}, \mathrm{Pd}_{3} \mathrm{PbC}, \mathrm{SnPd}_{3} \mathrm{C}, \mathrm{Ni}_{3} \mathrm{GeC}$ \\
\hline & $\mathrm{X}-\Gamma, \Gamma-\mathrm{R}, \mathrm{M}-\Gamma$ & \begin{tabular}{l||l|} 
& TaIr $_{3}, \mathrm{NbIr}_{3}$ \\
\end{tabular} \\
\hline & $\mathrm{X}-\Gamma, \mathrm{M}-\Gamma, \mathrm{M}-\mathrm{R}$ & 开 OsC \\
\hline & $\mathrm{X}-\Gamma, \mathrm{M}-\mathrm{X}, \mathrm{X}-\mathrm{R}$ & $\mathrm{BaB}_{6}[58], \mathrm{TiOs}, \| \mathrm{YbPd}_{3}, \mathrm{YbPt}_{3}$ \\
\hline & M-R, M-X & $\begin{array}{c}\text { ScIr, \| LuIr, HoIr, YIr, TmIr, ScRh, ErIr, ScCo, LuRh, TmRh, ErRh, } \\
\text { HoRh, DyRh, YRh, TbRh, SmRh }\end{array}$ \\
\hline
\end{tabular}


Table III - continued

\begin{tabular}{|c|c|c|}
\hline $\begin{array}{l}\text { SOC or } \\
\text { NSOC }\end{array}$ & $\begin{array}{l}\text { HSL with Band } \\
\text { Crossing }\end{array}$ & High Symmetry Line Semimetals \\
\hline & M-X, X-R & $\begin{array}{l}\text { TiBe, } \| \text { TbAl, PrAl, SmAl, DyAl, ScAl, YAl, SnTe, NdAl, PbSe, PbS, } \\
\text { ErIn, TmIn, CeZn, CeCd(ICSD : 186646), CeCd(ICSD : 182832) }\end{array}$ \\
\hline & $\mathrm{X}-\Gamma, \mathrm{M}-\Gamma$ & $\begin{array}{c}\mathrm{Tm}_{3} \mathrm{TlC}, \mathrm{Er}_{3} \mathrm{TlC}, \| \mathrm{Pr}_{3} \mathrm{TlC}, \mathrm{Ho}_{3} \mathrm{TlC}, \mathrm{Dy}_{3} \mathrm{TlC}, \mathrm{Tb}_{3} \mathrm{TlC}, \mathrm{Nd}_{3} \mathrm{TlC} \\
\mathrm{Ho}_{3} \mathrm{InC}, \mathrm{Er}_{3} \mathrm{InC}, \mathrm{Tb}_{3} \mathrm{InC}, \mathrm{Tm}_{3} \mathrm{InC}, \mathrm{Tm}_{3} \mathrm{GaC}, \mathrm{Er}_{3} \mathrm{GaC}, \mathrm{Ho}_{3} \mathrm{GaC} \\
\mathrm{Dy}_{3} \mathrm{GaC}\end{array}$ \\
\hline & $\mathrm{M}-\mathrm{R}$ & 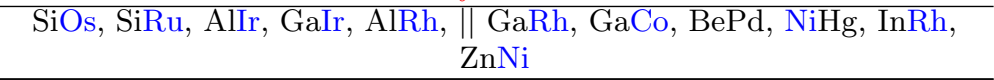 \\
\hline \multirow{6}{*}{$\mathrm{SOC}$} & $\mathrm{X}-\Gamma, \Gamma-\mathrm{R}, \mathrm{M}-\mathrm{R}$ & $\| \mathrm{ThSn}_{3}$ \\
\hline & $\Gamma-\mathrm{R}, \mathrm{M}-\mathrm{R}$ & $\|$ AlRe, $\mathrm{TmAg}_{3}, \mathrm{HoBPd}_{3}, \mathrm{DyBPd}_{3}, \mathrm{TbBPd}_{3}, \mathrm{Ba}_{6} \mathrm{Ni}_{25} \mathrm{~S}_{27}$ \\
\hline & $\mathrm{X}-\Gamma, \mathrm{M}-\mathrm{R}$ & $\begin{array}{c}\text { II OsC, } \mathrm{AlCu}_{3}, \mathrm{ReN}(\mathrm{ICSD}: 187714), \mathrm{TcN}(\mathrm{ICSD}: 187710), \mathrm{ZrRu}_{3} \mathrm{C} \text {, } \\
\mathrm{ScGa}_{3}, \mathrm{ScIr}_{3} \mathrm{C}, \mathrm{YIn}_{3}, \mathrm{VIr}_{3}, \mathrm{PrTl}_{3}, \mathrm{NdTl}_{3}, \mathrm{SmTl}_{3}, \mathrm{ScIn}_{3}, \mathrm{LuTl}_{3}, \\
\mathrm{TmTl}_{3}, \mathrm{ErTl}_{3}, \mathrm{HoTl}_{3}, \mathrm{DyTl}_{3}, \mathrm{TbTl}_{3}, \mathrm{YTl}_{3}, \mathrm{LaTl}_{3}, \mathrm{ZrRu}_{3}, \mathrm{HfRu}_{3}, \\
\mathrm{URu}_{3}, \mathrm{Ca}_{3} \mathrm{Hg}, \mathrm{CaHg}_{11}, \mathrm{Ce}_{3} \mathrm{Sn}, \mathrm{Cr}_{3} \mathrm{GaN}\end{array}$ \\
\hline & $\Gamma-\mathrm{R}$ & $\| \mathrm{ThPb}_{3}, \mathrm{NaN}_{3}, \mathrm{Nd}_{3} \mathrm{PbN}, \mathrm{Ni}_{3} \mathrm{Pt}, \mathrm{CePb}_{3}$ \\
\hline & M-R & 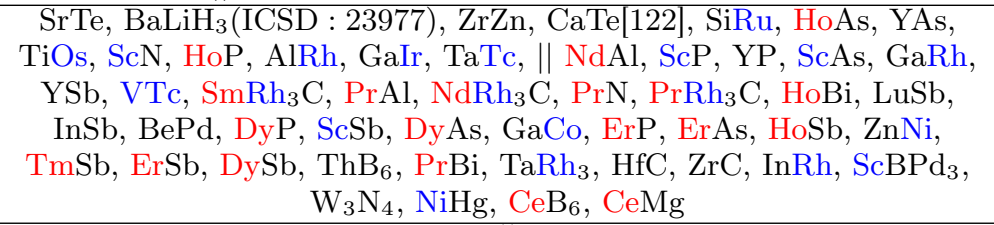 \\
\hline & $\mathrm{X}-\Gamma$ & $\begin{array}{c}\mathrm{Ba}_{3} \mathrm{SnO}, \mathrm{Y}_{3} \mathrm{GaC}, \mathrm{Y}_{3} \mathrm{AlC}, \mathrm{Sc}_{3} \mathrm{AlC}, \| \mathrm{CaSn}_{3}, \mathrm{YbSn}_{3}, \mathrm{Pr}_{3} \mathrm{TlC}, \mathrm{Tb}_{3} \mathrm{InC}, \\
\mathrm{YbPb}_{3}, \mathrm{Nd}_{3} \mathrm{TlC}, \mathrm{CaPb}_{3}, \mathrm{Dy}_{3} \mathrm{GaC}, \mathrm{Ho}_{3} \mathrm{GaC}, \mathrm{ThRu}_{3} \mathrm{C}, \mathrm{YbBRh}_{3}, \\
\mathrm{Nb}_{4} \mathrm{C}_{3}, \mathrm{TmGa}_{3} \mathrm{Ru}, \mathrm{TbGa} \mathrm{Oa}_{3} \mathrm{Os}, \mathrm{BaHg}_{11}, \mathrm{NpAs}, \mathrm{GeTe}, \mathrm{Fe}_{3} \mathrm{SnC}\end{array}$ \\
\hline \multicolumn{3}{|r|}{ Space Group 223: $\operatorname{Pm} \overline{3} n$} \\
\hline \multirow{5}{*}{ NSOC } & $\mathrm{X}-\Gamma, \Gamma-\mathrm{R}, \mathrm{M}-\Gamma, \mathrm{M}-\mathrm{X}$ & $\mathrm{Tl}_{3} \mathrm{Ir} \|$ \\
\hline & M- $\Gamma, \mathrm{M}-\mathrm{X}, \mathrm{M}-\mathrm{R}$ & \multirow{2}{*}{$\frac{\mathrm{Nb}_{3} \mathrm{Bi}}{\mathrm{BaC}_{20} \|}$} \\
\hline & $\mathrm{X}-\Gamma, \Gamma-\mathrm{R}, \mathrm{M}-\Gamma$ & \\
\hline & $\mathrm{M}-\Gamma, \mathrm{M}-\mathrm{X}$ & $\| \mathrm{Ti}_{3} \mathrm{Hg}, \mathrm{Zr}_{3} \mathrm{Hg}$ \\
\hline & $\Gamma-\mathrm{R}$ & $\mathrm{Nb}_{3} \mathrm{Al}, \mathrm{Nb}_{3} \mathrm{Ga}, \mathrm{Nb}_{3} \mathrm{In}$ \\
\hline \multirow{6}{*}{$\mathrm{SOC}$} & $\mathrm{X}-\Gamma, \Gamma-\mathrm{R}, \mathrm{M}-\mathrm{R}$ & $\| \mathrm{Zn}_{3} \mathrm{Au}$ \\
\hline & $\Gamma-\mathrm{R}, \mathrm{M}-\mathrm{R}$ & \multirow{2}{*}{$\frac{\mathrm{Ti}_{3} \mathrm{Pt}, \mathrm{K}_{4} \mathrm{Ge}_{23}, \mathrm{LaSn}_{3} \mathrm{Ru}, \mathrm{Cs}_{4} \mathrm{Sn}_{23}}{\| \mathrm{Pt}_{3} \mathrm{O}_{4}, \mathrm{Yb}_{3} \mathrm{Sn}_{13} \mathrm{Rh}_{4}}$} \\
\hline & $\mathrm{X}-\Gamma, \Gamma-\mathrm{R}$ & \\
\hline & $\Gamma-\mathrm{R}$ & \multirow{2}{*}{$\frac{\mathrm{K}_{4} \mathrm{Sn}_{23}, \mathrm{~W}_{3} \mathrm{O}, \mathrm{Ag}_{3} \mathrm{PO}_{4}, \mathrm{Nb}_{3} \mathrm{Al}, \mathrm{Th}_{3} \mathrm{Sn}_{13} \mathrm{Rh}_{4}, \mathrm{Nb}_{3} \mathrm{Ga}, \mathrm{Nb}_{3} \mathrm{In}}{\| \mathrm{Cr}, \mathrm{BeMo}_{3}, \mathrm{Ba}_{4} \mathrm{Si}_{23}}$} \\
\hline & $\mathrm{M}-\mathrm{R}$ & \\
\hline & $\mathrm{X}-\Gamma$ & $\mathrm{CaPd}_{3} \mathrm{O}_{4}, \mathrm{SrPd}_{3} \mathrm{O}_{4}, \| \mathrm{Nb}_{3} \mathrm{Rh}$ \\
\hline \multicolumn{3}{|r|}{ Space Group 224: $P n \overline{3} m$} \\
\hline \multirow{2}{*}{ NSOC } & $\mathrm{X}-\Gamma, \mathrm{M}-\Gamma, \mathrm{M}-\mathrm{R}, \mathrm{X}-\mathrm{R}$ & $\mathrm{Zn}_{3} \mathrm{As}_{2}, \| \mathrm{Cd}_{3} \mathrm{As}_{2}$ \\
\hline & $\mathrm{X}-\Gamma, \mathrm{M}-\Gamma$ & $\mathrm{Be}_{3} \mathrm{P}_{2}, \mathrm{Cd}_{3} \mathrm{P}_{2} \|$ \\
\hline \multirow{2}{*}{$\mathrm{SOC}$} & M-R & $\| \mathrm{Zn}_{3} \mathrm{As}_{2}, \mathrm{Cd}_{3} \mathrm{As}_{2}$ \\
\hline & $\mathrm{X}-\Gamma$ & $\mathrm{Ag}_{2} \mathrm{O}, \| \mathrm{Bi}_{2} \mathrm{O}_{3}, \mathrm{Be}_{3} \mathrm{P}_{2}$ \\
\hline \multicolumn{3}{|r|}{ Space Group 225: $F m \overline{3} m$} \\
\hline \multirow{11}{*}{ NSOC } & $\mathrm{X}-\Gamma, \mathrm{L}-\Gamma, \mathrm{W}-\mathrm{L}, \mathrm{W}-\mathrm{X}$ & \\
\hline & $\mathrm{X}-\Gamma, \mathrm{W}-\Gamma, \mathrm{L}-\mathrm{X}, \mathrm{W}-\mathrm{X}$ & $\mathrm{MnGaCo}_{2}, \mathrm{MnAlCo}_{2}$ \\
\hline & $\mathrm{X}-\Gamma, \mathrm{W}-\Gamma, \mathrm{W}-\mathrm{L}$ & $\| \mathrm{AlCuSe}_{2}, \mathrm{AlCuS}_{2}$ \\
\hline & $\mathrm{X}-\Gamma, \mathrm{W}-\Gamma, \mathrm{W}-\mathrm{X}$ & \multirow{2}{*}{$\frac{\| \mathrm{Hf}_{6} \mathrm{Ni}_{16} \mathrm{Ge}_{7}}{\| \mathrm{Mn}_{2} \mathrm{SnW}}$} \\
\hline & $\mathrm{X}-\Gamma, \mathrm{W}-\mathrm{X}, \mathrm{L}-\mathrm{X}$ & \\
\hline & $\mathrm{X}-\Gamma, \mathrm{W}-\mathrm{X}, \mathrm{W}-\Gamma$ & $\mathrm{ZnSiP}_{2}, \mathrm{CdGeP}_{2}, \mathrm{AlCuTe}_{2}$ \\
\hline & $\mathrm{X}-\Gamma, \mathrm{L}-\Gamma$ & $\| \mathrm{Eu}_{2} \mathrm{H}_{6} \mathrm{Ru}$ \\
\hline & $\mathrm{X}-\Gamma, \mathrm{L}-\mathrm{X}$ & $\| \mathrm{Tl}_{3} \mathrm{Ir}\left(\mathrm{NO}_{2}\right)_{6}, \mathrm{La}_{21} \mathrm{Fe}_{8} \mathrm{Bi}_{7} \mathrm{C}_{12}$ \\
\hline & $\mathrm{X}-\Gamma, \mathrm{W}-\Gamma$ & $\begin{array}{c}\mathrm{Be}_{2} \mathrm{Si}, \mathrm{ScSb}, \mathrm{ScBi}, \| \mathrm{Os}, \mathrm{Li}_{2} \mathrm{MgSi}, \mathrm{ScGaCu}_{2}, \mathrm{ScAlCu}_{2}, \mathrm{ScInAg}_{2}, \\
\mathrm{ScInCu}_{2}, \mathrm{ScAlAg}_{2}, \mathrm{HfNi} \mathrm{Na}_{2} \mathrm{Sn}, \mathrm{TaAlNi}_{2}, \mathrm{TaGaNi}_{2}, \mathrm{NbAlNi}_{2}, \mathrm{NbGaNi}_{2} \text {, } \\
\mathrm{ZrNi}_{2} \mathrm{Sn}, \mathrm{Zn}_{7} \mathrm{Mo}, \mathrm{TiNi}_{2} \mathrm{Sn}, \mathrm{VGaNi}_{2}, \mathrm{Ta}_{6} \mathrm{Be}_{15} \mathrm{Cu}_{8}, \mathrm{AlVNi}_{2}\end{array}$ \\
\hline & $\mathrm{X}-\Gamma, \mathrm{W}-\mathrm{X}$ & 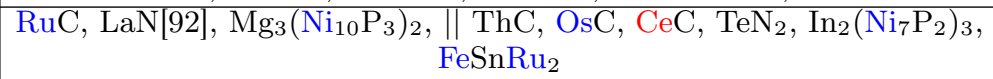 \\
\hline & W-L & SnTe[20], SnSe[123], SnS[123], Yb, Sr, $\| \mathrm{Ca}, \mathrm{Ba}$ \\
\hline
\end{tabular}


Table III - continued

\begin{tabular}{|c|c|c|}
\hline $\begin{array}{l}\text { SOC or } \\
\text { NSOC }\end{array}$ & $\begin{array}{l}\text { HSL with Band } \\
\text { Crossing }\end{array}$ & High Symmetry Line Semimetals \\
\hline \multirow[t]{3}{*}{$\mathrm{SOC}$} & $\mathrm{X}-\Gamma, \mathrm{L}-\Gamma$ & $\begin{array}{c}\| \mathrm{UC}, \mathrm{Mg}_{6} \mathrm{Cu}_{16} \mathrm{Si}_{7}, \mathrm{Hf}_{6} \mathrm{Si}_{7} \mathrm{Ni}_{16}, \mathrm{Hf}_{6} \mathrm{Ni}_{16} \mathrm{Ge}_{7}, \mathrm{Zr}_{6} \mathrm{Si}_{7} \mathrm{Ni}_{16}, \mathrm{Zr}_{6} \mathrm{Ni}_{16} \mathrm{Ge}_{7}, \\
\mathrm{Ti}_{6} \mathrm{Ni}_{16} \mathrm{Ge}_{7}, \mathrm{Sc}_{6} \mathrm{Ga}_{16} \mathrm{Ru}_{7}, \mathrm{Zr}_{6} \mathrm{Co}_{6} \mathrm{Si}_{7}, \mathrm{Sc}_{6} \mathrm{Si}_{7} \mathrm{Ni}_{16}, \mathrm{U}_{2}\left(\mathrm{Ni}_{7} \mathrm{~B}_{2}\right)_{3}, \\
\mathrm{Ti}_{6} \mathrm{Co}_{16} \mathrm{Si}_{7}, \mathrm{Zr}_{6} \mathrm{Al}_{16} \mathrm{Ru}_{7}, \mathrm{U}_{3} \mathrm{Si}_{11} \mathrm{Pt}_{23}, \mathrm{Nb}_{6} \mathrm{Co}_{16} \mathrm{Si}_{7}, \mathrm{Ta}_{6} \mathrm{Co}_{16} \mathrm{Ge}_{7}, \\
\mathrm{Nb}_{6} \mathrm{Co}_{16} \mathrm{Ge}_{7}, \mathrm{Ta}_{6} \mathrm{Co}_{16} \mathrm{Si}_{7}, \mathrm{BrN}\end{array}$ \\
\hline & $\mathrm{L}-\Gamma$ & $\begin{array}{c}\| \mathrm{PtO}, \mathrm{Ti}_{6} \mathrm{Al}_{16} \mathrm{Ni}_{7}, \mathrm{Zr}_{6} \mathrm{Co}_{16} \mathrm{Ge}_{7}, \mathrm{Zr}_{2}\left(\mathrm{Ni}_{7} \mathrm{~B}_{2}\right)_{3}, \mathrm{Ce}_{2}\left(\mathrm{Ni}_{7} \mathrm{~B}_{2}\right)_{3}, \mathrm{Ho}_{6} \mathrm{Fe}_{23}, \\
\mathrm{Fe}_{23} \mathrm{~B}_{6}, \mathrm{La}_{2} \mathrm{VReO}_{6}, \mathrm{Pt}\left(\mathrm{Cl}_{3} \mathrm{O}\right)_{2}\end{array}$ \\
\hline & $\mathrm{X}-\Gamma$ & 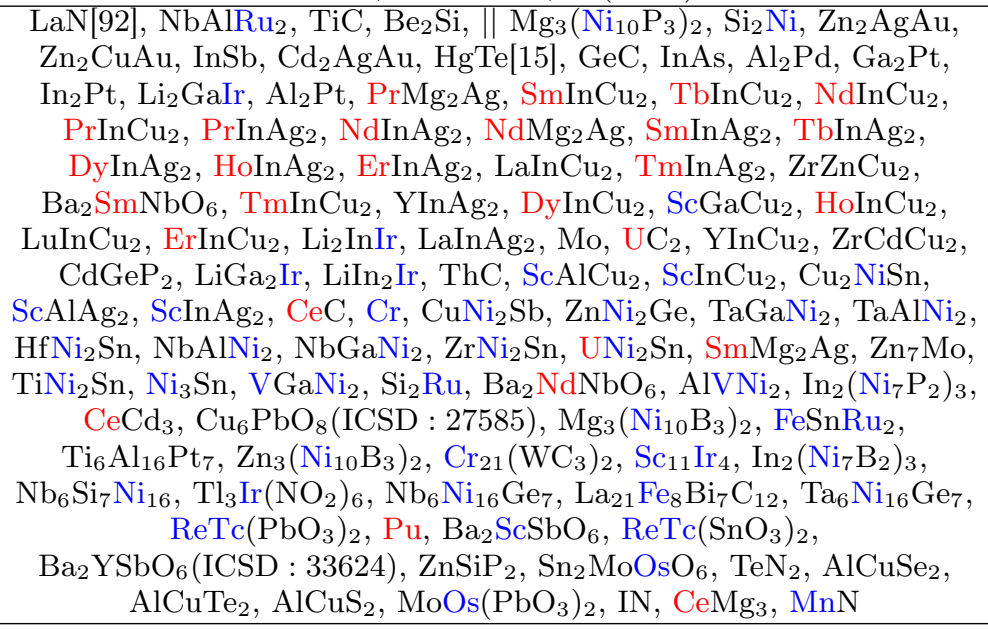 \\
\hline \multicolumn{3}{|r|}{ Space Group 226: $F m \overline{3} c$} \\
\hline \multirow{2}{*}{ NSOC } & $\mathrm{X}-\Gamma, \mathrm{L}-\Gamma, \mathrm{W}-\mathrm{X}$ & $\mathrm{UBe}_{13}$ \\
\hline & $\mathrm{X}-\Gamma$ & $\mathrm{PuBe}_{13}$ \\
\hline \multirow{3}{*}{$\mathrm{SOC}$} & $\mathrm{X}-\Gamma, \mathrm{L}-\Gamma$ & $\| \mathrm{LaCO}_{13}$ \\
\hline & $\mathrm{L}-\Gamma$ & $\mathrm{BaBe}_{13}, \mathrm{SrBe}_{13}, \mathrm{YbBe}_{13}$ \\
\hline & $\mathrm{X}-\Gamma$ & $\| \mathrm{UBe}_{13}$ \\
\hline \multicolumn{3}{|r|}{ Space Group 227: $F d \overline{3} m$} \\
\hline \multirow{15}{*}{ NSOC } & $\mathrm{X}-\Gamma, \mathrm{L}-\Gamma, \mathrm{L}-\mathrm{X}, \mathrm{W}-\mathrm{L}$ & $\| \mathrm{PuNi}_{2}, \mathrm{Fe}_{3} \mathrm{Mo}_{3} \mathrm{~N}$ \\
\hline & $\mathrm{X}-\Gamma, \mathrm{L}-\Gamma, \mathrm{W}-\Gamma, \mathrm{L}-\mathrm{X}$ & $\| \mathrm{Ti}_{2} \mathrm{Ni}$ \\
\hline & $\mathrm{X}-\Gamma, \mathrm{L}-\Gamma, \mathrm{W}-\Gamma, \mathrm{W}-\mathrm{L}$ & $\| \mathrm{Hg}_{2} \mathrm{Os}_{2} \mathrm{O}_{7}, \mathrm{Cd}_{2} \mathrm{Ru}_{2} \mathrm{O}_{7}, \mathrm{Cd}_{2} \mathrm{Os}_{2} \mathrm{O}_{7}$ \\
\hline & $\mathrm{X}-\Gamma, \mathrm{L}-\Gamma, \mathrm{L}-\mathrm{X}$ & $\| \mathrm{PuMn}_{2}$ \\
\hline & $\mathrm{X}-\Gamma, \mathrm{L}-\Gamma, \mathrm{W}-\Gamma$ & $\| \mathrm{PuRu}_{2}, \mathrm{UIr}_{2}, \mathrm{Co}_{2} \mathrm{Mo}_{4} \mathrm{C}$ \\
\hline & $\mathrm{X}-\Gamma, \mathrm{L}-\mathrm{X}, \mathrm{W}-\mathrm{L}$ & $\| \mathrm{Ce}\left(\mathrm{TiAl}_{10}\right)_{2}, \mathrm{Nb}_{2} \mathrm{Fe}, \mathrm{Co}_{3} \mathrm{~W}_{3} \mathrm{~N}$ \\
\hline & $\mathrm{X}-\Gamma, \mathrm{W}-\Gamma, \mathrm{L}-\mathrm{X}$ & $\begin{array}{c}\| \mathrm{Ti}_{2} \mathrm{C}, \mathrm{Li}_{2} \mathrm{Ca}, \mathrm{LiTiO}_{2}, \mathrm{CeNi}_{2}, \mathrm{Mg}_{3}\left(\mathrm{Al}_{9} \mathrm{Cr}\right)_{2}, \mathrm{CeAl}_{2}, \mathrm{Cr}_{2} \mathrm{HgS}_{4}, \\
\mathrm{Ni}_{6} \mathrm{Mo}_{6} \mathrm{C}\end{array}$ \\
\hline & $\mathrm{X}-\Gamma, \mathrm{W}-\Gamma, \mathrm{W}-\mathrm{L}$ & $\| \mathrm{Zr}_{3} \mathrm{Zn}_{3} \mathrm{~N}, \mathrm{Hf}_{3} \mathrm{Zn}_{3} \mathrm{~N}$ \\
\hline & $\mathrm{L}-\Gamma, \mathrm{L}-\mathrm{X}$ & $\| \mathrm{YbS}_{2}, \mathrm{Zn}_{6} \mathrm{Ni}_{7} \mathrm{Ge}_{2}$ \\
\hline & $\mathrm{X}-\Gamma, \mathrm{L}-\Gamma$ & || $\mathrm{Zr}_{3} \mathrm{~V}_{3} \mathrm{C}, \mathrm{Te}_{3} \mathrm{Pd}_{13}, \mathrm{Ni}_{3} \mathrm{Mo}_{3} \mathrm{~N}$ \\
\hline & $\mathrm{X}-\Gamma, \mathrm{L}-\mathrm{X}$ & $\| \mathrm{MgIn}_{2}, \mathrm{YbAl}_{2}, \mathrm{CaAl}_{2}, \mathrm{Si}(\mathrm{ICSD}: 56721), \mathrm{Sm}_{2} \mathrm{Ru}_{2} \mathrm{O}_{7}$ \\
\hline & $\mathrm{X}-\Gamma, \mathrm{W}-\Gamma$ & $\begin{array}{c}\mathrm{TiS}_{2}[57], \mathrm{PbAu}_{2}, \| \mathrm{ThAl}_{2}, \mathrm{Be}_{22} \mathrm{Mo}, \mathrm{Be}_{22} \mathrm{~W}, \mathrm{Ni}_{2} \mathrm{~W}_{4} \mathrm{C}, \mathrm{Nb}_{3} \mathrm{Zn}_{3} \mathrm{~N}, \\
\mathrm{~V}_{2} \mathrm{FeO}_{4}\end{array}$ \\
\hline & $\mathrm{X}-\Gamma, \mathrm{W}-\mathrm{L}$ & \begin{tabular}{l||l|l} 
& $\mathrm{ZrMn}_{2}$ \\
\end{tabular} \\
\hline & $\mathrm{L}-\Gamma$ & $\| \mathrm{Ti}_{3} \mathrm{Ni}_{3} \mathrm{O}$ \\
\hline & $\mathrm{W}-\mathrm{L}$ & $\mathrm{Zr}\left(\mathrm{TiH}_{2}\right)_{2} \|$ \\
\hline \multirow{4}{*}{$\mathrm{SOC}$} & $\mathrm{X}-\Gamma, \mathrm{L}-\Gamma, \mathrm{W}-\mathrm{X}$ & $\| \mathrm{Mn}_{3} \mathrm{~W}_{3} \mathrm{C}$ \\
\hline & $\mathrm{X}-\Gamma, \mathrm{L}-\Gamma$ & $\| \mathrm{Al}_{2} \mathrm{Re}_{3} \mathrm{~B}, \mathrm{PuNi}_{2}, \mathrm{Co}_{2} \mathrm{Mo}_{4} \mathrm{~N}, \mathrm{~K}_{17} \mathrm{In}_{41}, \mathrm{Co}_{2} \mathrm{Mo}_{4} \mathrm{C}$ \\
\hline & $\mathrm{L}-\Gamma$ & $\begin{array}{c}\| \mathrm{CeOs}_{2}, \mathrm{YbIr}_{2}, \mathrm{ZrIr}_{2}, \mathrm{CaIr}_{2}, \mathrm{SrIr}_{2}, \mathrm{Zn}_{2} \mathrm{PdO}_{4}, \mathrm{ThRu}_{2}, \mathrm{ZrCo}_{2}, \mathrm{CeRu}_{2}, \\
\mathrm{YbRh}_{2}, \mathrm{TiCo}_{2}, \mathrm{SrRh}_{2}, \mathrm{PuOs}_{2}, \mathrm{PuFe}_{2}, \mathrm{Ce}\left(\mathrm{Al}_{10} \mathrm{Co}\right)_{2}, \mathrm{Ti}_{3} \mathrm{Ni}_{3} \mathrm{O}, \\
\mathrm{Zr}_{4} \mathrm{Pd}_{2} \mathrm{O}, \mathrm{Fe}_{2} \mathrm{~W}_{2} \mathrm{C}, \mathrm{Zr}_{2} \mathrm{Fe}, \mathrm{Ba}_{10} \mathrm{Ga}_{2}, \mathrm{BaRh}_{2}, \mathrm{CaRh}_{2}, \mathrm{Cr}_{2} \mathrm{Ni}_{2}, \mathrm{Lu}_{2} \mathrm{FeS}_{4}, \\
\mathrm{Sc}_{2} \mathrm{FeS}_{4}, \mathrm{ZrFe}_{2}, \mathrm{YbCo}_{2}, \mathrm{HfFe}_{2}, \mathrm{CeFe}_{2}, \mathrm{Ho}_{2} \mathrm{Ru}_{2} \mathrm{O}_{7}\end{array}$ \\
\hline & $\mathrm{X}-\Gamma$ & $\begin{array}{c}\mathrm{PbAu}_{2}, \mid \mathrm{Sb}_{2} \mathrm{O}_{5}, \mathrm{HfNi}_{2}, \mathrm{Li}_{2} \mathrm{Ca}, \mathrm{Hf}_{3} \mathrm{Zn}_{3} \mathrm{~N}, \mathrm{CaPt}_{2}, \mathrm{Be}_{22} \mathrm{~W}, \mathrm{Be}_{22} \mathrm{Mo} \\
\mathrm{ZrNi}_{2}, \mathrm{Mg}_{2} \mathrm{Pd}, \mathrm{ZrZn}_{22}, \mathrm{Na}_{3} \mathrm{In}_{2} \mathrm{Au}, \mathrm{CdNi}, \mathrm{Al}_{10} \mathrm{~V}, \mathrm{Nb}_{3} \mathrm{Zn}_{3} \mathrm{~N} \\
\mathrm{Na}_{17} \mathrm{In}_{12} \mathrm{Ga}_{29}, \mathrm{Co}_{2} \mathrm{~W}_{4} \mathrm{C}, \mathrm{Ni}_{6} \mathrm{Mo}_{6} \mathrm{C}, \mathrm{Fe}_{3} \mathrm{~W}_{3} \mathrm{C}, \mathrm{Mn}_{3} \mathrm{Mo}_{3} \mathrm{C}, \mathrm{Zr}_{3} \mathrm{Zn}_{3} \mathrm{~N}\end{array}$ \\
\hline \multicolumn{3}{|r|}{ Space Group 229: $\operatorname{Im} \overline{3} m$} \\
\hline & $\Gamma-\mathrm{H}, \mathrm{P}-\Gamma, \mathrm{N}-\Gamma$ & $\mathrm{Ge}_{7} \mathrm{Ir}_{3}$ \\
\hline
\end{tabular}


Table III - continued

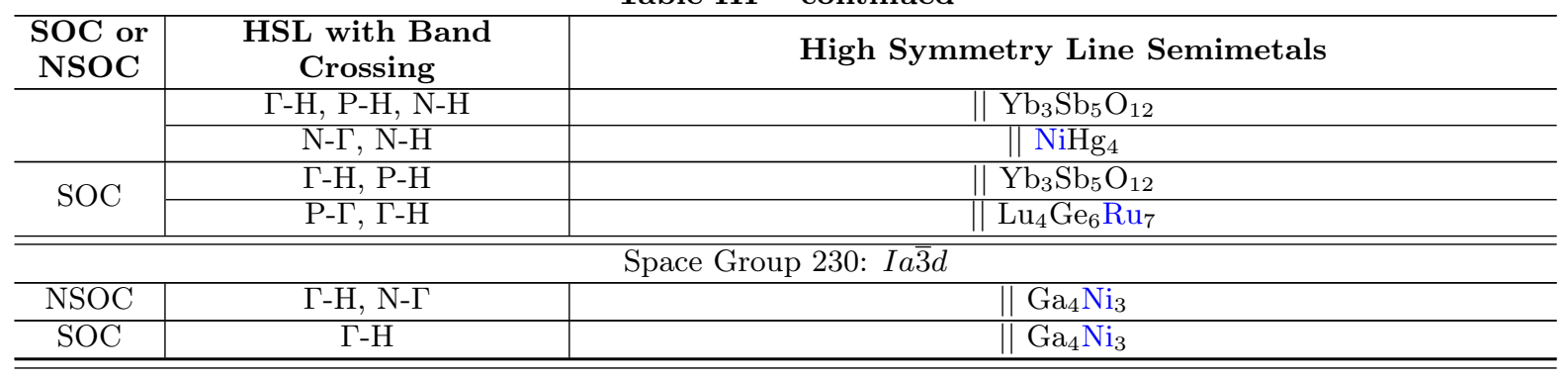

Table IV: List of Generic Momenta Semimetals

\begin{tabular}{|c|c|}
\hline \multicolumn{2}{|r|}{ List of Generic Momenta Semimetals } \\
\hline \multicolumn{2}{|r|}{ Space Group 2: $P \overline{\overline{1}}$} \\
\hline $\mathbb{Z}_{2,2,2,4}$ & Materials \\
\hline 0001 & $\| \mathrm{Tc}_{2} \mathrm{As}_{3}, \mathrm{Hf}_{8} \mathrm{Ni}_{21}, \mathrm{Ti}_{5} \mathrm{O}_{9}$ \\
\hline 0002 & $\| \mathrm{NaCuF}_{3}, \mathrm{In}_{3} \mathrm{Cu}_{7}$ \\
\hline 0003 & 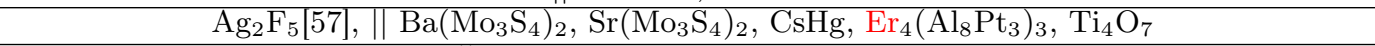 \\
\hline 0010 & $\| \mathrm{SbS}, \mathrm{CuMoO}_{4}, \mathrm{~K}_{3} \mathrm{HRhC}_{5} \mathrm{~N}_{5} \mathrm{O}$ \\
\hline 0011 & $\|$ TiNi, $\mathrm{NaV}_{2} \mathrm{P}_{2}\left(\mathrm{H}_{4} \mathrm{O}_{7}\right)_{2}, \mathrm{CuAg}_{5} \mathrm{As}_{3} \mathrm{O}_{11}$ \\
\hline 0012 & $\| \mathrm{SrAs}_{3}[94], \mathrm{Ta}_{6} \mathrm{~S}$ \\
\hline 0013 & $\| \mathrm{Yb}_{8} \mathrm{Tl}_{3}$ \\
\hline 0100 & $\mathrm{Ni}_{13} \mathrm{Sn}_{8} \mathrm{P}_{3}$ \\
\hline 0101 & $\mathrm{CaP}_{3}[94 \|$ \\
\hline 0102 & $\mathrm{KHg}, \mathrm{Zr}_{8} \mathrm{Ni}_{21}, \mathrm{~K}_{3} \mathrm{Nb}_{7} \mathrm{O}_{19}, \mathrm{CuH}_{18} \mathrm{C}_{4} \mathrm{~N}_{10}(\mathrm{ClO})_{2}$ \\
\hline 0103 & $\| \operatorname{Pr}_{8}(\mathrm{BCl})_{7}, \mathrm{NaYb}\left(\mathrm{PS}_{3}\right)_{2}$ \\
\hline 0110 & $\| \mathrm{Pt}_{2} \mathrm{PbO}_{4}, \mathrm{CsCuP}_{3} \mathrm{H}_{8} \mathrm{O}_{13}, \mathrm{Ni}_{10} \mathrm{Sn}_{5} \mathrm{P}_{3}, \mathrm{SrTi}_{11} \mathrm{O}_{20}$ \\
\hline 0111 & $\| \mathrm{NbCuO}_{3} \mathrm{~F}, \mathrm{Rb}_{2} \mathrm{CuH}_{4}\left(\mathrm{C}_{2} \mathrm{O}_{5}\right)_{2}$ \\
\hline 0112 & \\
\hline 0113 & $\| \mathrm{BC}_{5}$ \\
\hline 1000 & $\| \mathrm{Ga}_{5} \mathrm{Rh}_{3}, \mathrm{Nd}_{2} \mathrm{Sn}_{3}, \mathrm{Hf}_{3} \mathrm{Ni}_{7}, \mathrm{CuH}_{10} \mathrm{SeO}_{9}$ \\
\hline 1001 & $\mathrm{CaAs}_{3}\left[94, \| \mathrm{Cu}_{3} \mathrm{Se}_{2}\left(\mathrm{ClO}_{3}\right)_{2}, \mathrm{FeMo}_{3} \mathrm{~S}_{4}, \mathrm{~K}_{2} \mathrm{U}\left(\mathrm{PSe}_{3}\right)_{3}\right.$ \\
\hline 1002 & $\mathrm{CuH}_{9} \mathrm{CSN}_{4} \mathrm{Cl}_{3} \mathrm{O}, \| \mathrm{Yb}_{3} \mathrm{Ge}_{8}$ \\
\hline 1003 & || $\mathrm{AgSO}_{4}$ \\
\hline 1010 & $\|$ UIr, $\mathrm{EuBO}_{3}$ \\
\hline 1101 & $\| \mathrm{Sr}_{2} \mathrm{YCu}_{3}\left(\mathrm{PbO}_{4}\right)_{2}$ \\
\hline 1103 & $\| \mathrm{K}\left(\mathrm{OsO}_{3}\right)_{2}, \mathrm{Nb}_{6} \mathrm{SI}_{9}, \mathrm{C}_{2} \mathrm{NClO}$ \\
\hline 1111 & $\mathrm{Y}_{16} \mathrm{~B}_{4} \mathrm{C}_{8} \mathrm{I}_{19}, \| \mathrm{Cu}_{2} \mathrm{Mo}_{3} \mathrm{Se}_{4}, \mathrm{Bi}_{3}\left(\mathrm{TeCl}_{5}\right)_{2}$ \\
\hline 1112 & $\| \mathrm{Sr}_{2} \mathrm{FeN}_{2}, \mathrm{MnH}_{10} \mathrm{SeO}_{9}$ \\
\hline 1113 & $\| \mathrm{WSCl}_{4}, \mathrm{Yb}_{2} \mathrm{Te}_{5} \mathrm{O}_{13}$ \\
\hline \multicolumn{2}{|r|}{ Space Group 11: $P 2_{1} / m$} \\
\hline $\mathbb{Z}_{2}$ & Materials \\
\hline 1 & $\| \mathrm{FeH}_{4}, \mathrm{Sr}(\mathrm{BiPd})_{2}, \mathrm{Ba}(\mathrm{BiPd})_{2}$ \\
\hline \multicolumn{2}{|r|}{ Space Group 12: $C 2 / m$} \\
\hline $\mathbb{Z}_{2,2,2,4}$ & Materials \\
\hline 0002 & $\| \mathrm{Sc}_{3} \mathrm{Si}_{3} \mathrm{Ni}$ \\
\hline 1100 & $\| \mathrm{Ho}_{2} \mathrm{Ge}_{2} \mathrm{Rh}, \mathrm{BaTl}_{4}$ \\
\hline 1102 & $\mathrm{~K}_{6} \mathrm{CdPb}_{8}, \mathrm{Sc}_{5} \mathrm{NCl}_{8}, \mathrm{La}_{4} \mathrm{Si}_{4} \mathrm{I}_{3}$ \\
\hline \multicolumn{2}{|r|}{ Space Group 14: $P 2_{1} / c$} \\
\hline $\mathbb{Z}_{2}$ & Materials \\
\hline 1 & $\begin{array}{c}\| \mathrm{CuClF}_{10}(\mathrm{ICSD}: 200807), \mathrm{BiSe}, \mathrm{CuTeO}_{4}(\mathrm{ICSD}: 1671), \mathrm{CuTeO}_{4}(\mathrm{ICSD}: 1672), \mathrm{KPH}_{2} \mathrm{O}_{3}, \mathrm{Y}_{8} \mathrm{Co}_{5}, \\
\operatorname{Pr}_{5} \mathrm{O}_{9} \\
\end{array}$ \\
\hline \multicolumn{2}{|r|}{ Space Group 15: $C 2 / c$} \\
\hline $\mathbb{Z}_{2,2,2,4}$ & Materials \\
\hline 0002 & $\mathrm{Ba}_{3} \mathrm{CaIr}_{2} \mathrm{O}_{9}, \mathrm{Pd}_{13} \mathrm{~Pb}_{9}$ \\
\hline 1100 & $\| \mathrm{P}_{2} \mathrm{Pt}_{5}, \mathrm{Ca}_{5} \mathrm{Au}_{2}$ \\
\hline 1102 & $\mathrm{Ho}_{2}\left(\mathrm{GePt}_{3}\right)_{3}, \mathrm{~Tb}_{2}\left(\mathrm{GePt}_{3}\right)_{3}, \mathrm{Ni}_{4} \mathrm{P}_{16} \mathrm{~W}$ \\
\hline
\end{tabular}


Table IV - continued

\begin{tabular}{|c|c|}
\hline \multicolumn{2}{|r|}{ Generic Momenta Semimetals } \\
\hline \multicolumn{2}{|r|}{ Materials } \\
\hline \multicolumn{2}{|r|}{$\| \mathrm{NiS}_{2}$} \\
\hline \multicolumn{2}{|r|}{ Space Group 82: $I \overline{4}$} \\
\hline \multicolumn{2}{|r|}{ Materials } \\
\hline 1 & $\mathrm{ZnCu}_{2} \mathrm{SnSe}_{4}, \| \mathrm{ZnCu}_{2} \mathrm{GeTe}_{4}$ \\
\hline \\
\hline \multicolumn{2}{|r|}{$\frac{\text { Space Group 85: } P 4 / n}{\text { Materials }}$} \\
\hline 1 & $\mathrm{Sm}_{9}(\mathrm{SbO})_{5}, \mathrm{Pr}_{9}(\mathrm{SbO})_{5}, \mathrm{Dy}_{9}(\mathrm{SbO})_{5}, \mathrm{~Tb}_{9}(\mathrm{SbO})_{5}, \mathrm{La}_{9}(\mathrm{SbO})_{5}, \mathrm{Si}_{5} \mathrm{Pt}_{12}$ \\
\hline \\
\hline \multicolumn{2}{|r|}{$\begin{array}{c}\text { Space Group 87: } I 4 / m \\
\text { Materials }\end{array}$} \\
\hline \multicolumn{2}{|r|}{$\| \mathrm{Sr}_{2} \mathrm{NiOsO}_{6}$} \\
\hline \\
\hline \multicolumn{2}{|r|}{$\frac{\text { Space Group 88: } I 4_{1} / a}{\text { Materials }}$} \\
\hline 1 & $\| \mathrm{MgGa}, \mathrm{Hf}\left(\mathrm{VH}_{2}\right)_{2}$ \\
\hline \multicolumn{2}{|r|}{ Space Group 147: $P \overline{3}$} \\
\hline \multicolumn{2}{|r|}{ Materials } \\
\hline \multicolumn{2}{|r|}{$\| \mathrm{Ge}_{9} \mathrm{Pd}_{25}$} \\
\hline \multicolumn{2}{|r|}{ Space Group 148: $R \overline{3}$} \\
\hline \multicolumn{2}{|r|}{ Materials } \\
\hline \multicolumn{2}{|r|}{$\mathrm{Pr}_{7} \mathrm{CoI}_{12} \|$} \\
\hline \multicolumn{2}{|r|}{$\| \mathrm{Th}_{3} \mathrm{Au}_{4}$} \\
\hline \multicolumn{2}{|r|}{$\mathrm{Tl}_{6} \mathrm{TeO}_{12}, \| \mathrm{Cr}_{2} \mathrm{Se}_{3}$} \\
\hline 1110 & $\| \mathrm{Ca}_{3} \mathrm{Au}_{4}, \mathrm{Tl}_{2} \mathrm{Mog}_{9} \mathrm{~S}_{11}$ \\
\hline \multicolumn{2}{|r|}{$\mathrm{Ti}_{3} \mathrm{NiS}_{6}, \mathrm{CrGeTe}_{3}, \mathrm{Mo}_{3} \mathrm{Se}_{4}$} \\
\hline \multicolumn{2}{|r|}{$\mathrm{Mo}_{3} \mathrm{~S}_{4}, \mathrm{Ca}_{13} \mathrm{Si}_{10} \mathrm{H}_{12}\left(\mathrm{O}_{17} \mathrm{~F}_{5}\right)_{2}$} \\
\hline 1113 & \begin{tabular}{r||l}
$\mathrm{Sc}_{7} \mathrm{NCl}_{12}, \| \mathrm{Te}_{4} \mathrm{Mo}_{3}$ \\
\end{tabular} \\
\hline \multicolumn{2}{|r|}{ Space Group 162: $P \overline{3} 1 m$} \\
\hline \multicolumn{2}{|r|}{ Materials } \\
\hline 1 & $\| \mathrm{SiO}_{2}(\mathrm{ICSD}: 170552)$ \\
\hline \multicolumn{2}{|r|}{ Space Group 164: $P \overline{3} m 1$} \\
\hline \multicolumn{2}{|r|}{ Materials } \\
\hline 1 & $\| \mathrm{TiSe}_{2}$ \\
\hline \multicolumn{2}{|r|}{ Space Group 166: $R \overline{3} m$} \\
\hline $\mathbb{Z}_{2}$ & Materials \\
\hline 1 & $\| \mathrm{V}_{3} \mathrm{Sb}_{2}$ \\
\hline
\end{tabular}

Table V: List of Topological Insulators

\begin{tabular}{|c|c|}
\hline \multicolumn{2}{|r|}{ List of Topological Insulators } \\
\hline \\
\hline \multicolumn{2}{|r|}{$\begin{array}{r}\text { Space Group 2: } P \overline{\overline{1}} \\
\text { Materials }\end{array}$} \\
\hline 0001 & $\| \mathrm{Tc}_{2} \mathrm{As}_{3}, \mathrm{Hf}_{8} \mathrm{Ni}_{21}, \mathrm{Ti}_{5} \mathrm{O}_{9}$ \\
\hline 0003 & $\mathrm{Ag}_{2} \mathrm{~F}_{5}[57], \mathrm{K}_{11}\left(\mathrm{In}_{2} \mathrm{Bi}_{3}\right)_{3}, \mathrm{Ba}\left(\mathrm{Mo}_{3} \mathrm{~S}_{4}\right)_{2}, \mathrm{Sr}\left(\mathrm{Mo}_{3} \mathrm{~S}_{4}\right)_{2}, \mathrm{CsHg}, \mathrm{Er}_{4}\left(\mathrm{Al}_{8} \mathrm{Pt}_{3}\right)_{3}, \mathrm{Ti}_{4} \mathrm{O}_{7}$ \\
\hline 0011 & $\| \mathrm{TiNi}, \mathrm{NaV}_{2} \mathrm{P}_{2}\left(\mathrm{H}_{4} \mathrm{O}_{7}\right)_{2}$ \\
\hline 0101 & $\mathrm{CaP}_{3} 94, \| \mathrm{Bi}_{3}\left(\mathrm{TeCl}_{5}\right)_{2}, \mathrm{Yb}_{8} \mathrm{Tl}_{3}$ \\
\hline 0103 & $\| \mathrm{Pr}_{8}(\mathrm{BCl})_{7}, \mathrm{NaYb}\left(\mathrm{PS}_{3}\right)_{2}$ \\
\hline 0111 & $\| \mathrm{NbCuO}_{3} \mathrm{~F}$ \\
\hline 0113 & || $\mathrm{BC}_{5}$ \\
\hline 1001 & $\mathrm{CaAs}_{3} 94, \mathrm{Cu}_{3} \mathrm{Se}_{2}\left(\mathrm{ClO}_{3}\right)_{2}, \mathrm{Nd}_{2} \mathrm{Sn}_{3}$ \\
\hline 1003 & $\| \mathrm{AgSO}_{4}$ \\
\hline 1101 & $\| \mathrm{Sr}_{2} \mathrm{YCu}_{3}\left(\mathrm{PbO}_{4}\right)_{2}$ \\
\hline 1103 & $\mathrm{Nb}_{6} \mathrm{SI}_{9}, \mathrm{~K}\left(\mathrm{OsO}_{3}\right)_{2}, \mathrm{C}_{2} \mathrm{NClO}$ \\
\hline 1111 & $\| \mathrm{Y}_{16} \mathrm{~B}_{4} \mathrm{C}_{8} \mathrm{I}_{19}, \mathrm{Cu}_{2} \mathrm{Mo}_{3} \mathrm{Se}_{4}$ \\
\hline 1113 & $\| \mathrm{WSCl}_{4}, \mathrm{Yb}_{2} \mathrm{Te}_{5} \mathrm{O}_{13}$ \\
\hline & Space Group 10: $P 2 / m$ \\
\hline
\end{tabular}


Table V - continued

\begin{tabular}{|c|c|}
\hline SI & \multirow{2}{*}{$\begin{array}{c}\text { Topological Insulators } \\
\text { Materials }\end{array}$} \\
\hline $\mathbb{Z}_{2,2,2,4}$ & \\
\hline 1001 & \multirow{2}{*}{$\begin{array}{l}\mathrm{ZrTiTe}_{4}, \mathrm{Na}_{7}\left(\mathrm{Cu}_{6} \mathrm{~S}_{5}\right)_{2} \\
\quad \| \mathrm{Th}_{3} \mathrm{~B}_{2} \mathrm{C}_{3}, \mathrm{Bi}\end{array}$} \\
\hline 1111 & \\
\hline \multicolumn{2}{|r|}{ Space Group 11: $P 2_{1} / m$} \\
\hline $\mathbb{Z}_{2,2,2,4}$ & Materials \\
\hline 0001 & $\|$ TiNi, HfGePd, $\mathrm{Zr}_{2} \mathrm{CoP}$ \\
\hline 0003 & 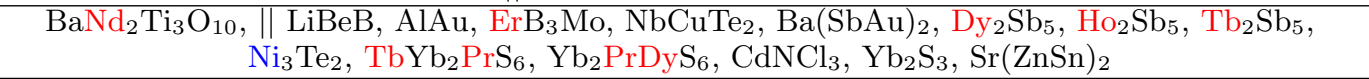 \\
\hline 0011 & $\| \mathrm{SnH}_{4}, \mathrm{Mo}_{2} \mathrm{~S}_{3}$ \\
\hline 1001 & $\mathrm{Ta}_{2} \mathrm{Se}_{3}, \mathrm{ZrTe}_{3}, \mathrm{Nb}_{2} \mathrm{Se}_{3}$ \\
\hline 1003 & ||l TbNi \\
\hline 1011 & $\| \mathrm{CaSb}_{2}, \mathrm{Si}_{5} \mathrm{Rh}_{4}$ \\
\hline 1013 & $\mathrm{Bi}(\mathrm{ICSD}: 42679), \mathrm{CeSi}_{2} \mathrm{Ru}$ \\
\hline \multicolumn{2}{|r|}{ Space Group 12: $C 2 / m$} \\
\hline $\mathbb{Z}_{2,2,2,4}$ & Materials \\
\hline 0001 & 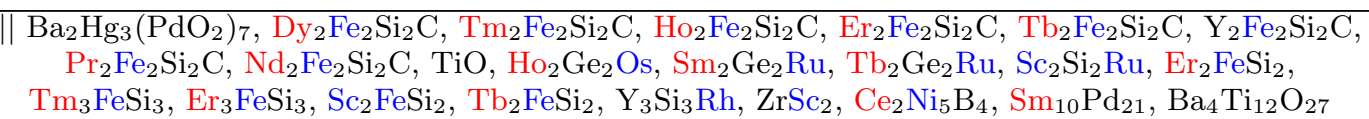 \\
\hline 0003 & $\begin{array}{c}\| \mathrm{Ba}_{11}\left(\mathrm{CdSb}_{2}\right)_{6}, \mathrm{KAg}_{3} \mathrm{Te}_{2}, \mathrm{~K}_{2} \mathrm{Ag}_{4} \mathrm{Se}_{3}, \mathrm{RbAg}_{3} \mathrm{Se}_{2}, \mathrm{KAg}_{3} \mathrm{Se}_{2}, \mathrm{Ba}_{11}\left(\mathrm{Cd}_{4} \mathrm{Bi}_{7}\right)_{2}, \mathrm{Hg}, \mathrm{CaGa}_{4}, \mathrm{TaSb}_{2}, \\
\mathrm{YbGa}_{4}, \mathrm{Ta}_{2} \mathrm{PdSe}_{6}, \mathrm{Ca}(\mathrm{CuSn})_{2}, \mathrm{Nb}_{2} \mathrm{PdS}_{6}, \mathrm{BaThTe}_{4}, \mathrm{Te}, \mathrm{Sr}(\mathrm{CuSn})_{2}, \mathrm{Bi}_{2} \mathrm{Pd}, \mathrm{Sr}(\mathrm{SnHg})_{2}, \\
\mathrm{Ba}_{3}\left(\mathrm{LiSn}_{2}\right)_{4}, \mathrm{Rb}_{2} \mathrm{TeO}_{3}, \mathrm{Bi}_{2} \mathrm{Pd}_{5}, \mathrm{Ba}\left(\mathrm{Cu}_{5} \mathrm{P}_{2}\right)_{2}, \mathrm{Nb}_{2} \mathrm{Te}_{3}, \mathrm{Hf}_{7} \mathrm{P}_{4}, \mathrm{Sn}_{4} \mathrm{Sb}_{6} \mathrm{~S}_{13}, \mathrm{~K}_{3} \mathrm{Rh}_{\mathrm{Wh}} \mathrm{WBr}_{5}\end{array}$ \\
\hline 0011 & $\begin{array}{c}\left.\| \mathrm{SrAs}_{3}, \mathrm{BaAs}_{3} 94\right], \mathrm{Er}_{2} \mathrm{Mo}_{2} \mathrm{C}_{3}(\mathrm{ICSD}: 88515), \mathrm{Te}_{3} \mathrm{Pt}_{2}, \mathrm{La}_{3} \mathrm{Ge}_{3} \mathrm{Br}_{2}, \mathrm{Nd}_{2} \mathrm{BC}, \mathrm{Ta}_{4} \mathrm{Te}_{16} \mathrm{Pd}_{3} \\
\mathrm{~K}_{2} \mathrm{Ca}\left(\mathrm{NO}_{2}\right)_{4}, \mathrm{Pr}_{2} \mathrm{CdCu}_{2}, \mathrm{Nb}_{2} \mathrm{CrSe}_{4}, \mathrm{~K}_{2} \mathrm{PtS}_{6}, \mathrm{Ga}_{5} \mathrm{Fe}_{6}, \mathrm{Sc}_{8} \mathrm{Te}_{3}\end{array}$ \\
\hline 0013 & $\begin{array}{r}\mathrm{SrP}_{3}(\mathrm{ICSD}: 96543)[94], \mathrm{P}_{3} \mathrm{Au}_{2}, \mathrm{BaSb}_{3}, \mathrm{CaC}_{2}(\mathrm{ICSD}: 252716), \mathrm{Ni}_{3} \mathrm{Sn}_{4}, \mathrm{Ca}_{4} \mathrm{Ni}_{3} \mathrm{C}_{5} \\
\mathrm{Mg}_{5} \mathrm{Si}_{6}(\mathrm{ICSD}: \text { : } 5529), \mathrm{Te}_{4} \mathrm{Pt}_{3}, \mathrm{NiP}_{3} \mathrm{~W}_{2}, \mathrm{La}_{3} \mathrm{Si}_{3} \mathrm{Cl}_{2}, \mathrm{La}_{3} \mathrm{Ge}_{3} \mathrm{Cl}_{2}, \mathrm{Bi}_{4} \mathrm{I}, \mathrm{Ti}_{3} \mathrm{O}_{5}, \mathrm{Sr}_{13} \mathrm{Al}_{6} \mathrm{Si}_{8} \mathrm{O} \\
\mathrm{NaN}_{3}(\mathrm{ICSD}: 29375), \mathrm{Cu}_{4} \mathrm{Bi}_{5} \mathrm{PbS}_{11}\end{array}$ \\
\hline 1101 & $\begin{array}{c}\mathrm{Cs}_{2} \mathrm{Pd}\left(\mathrm{IBr}_{2}\right)_{2}, \mathrm{Yb}_{2}(\mathrm{ZnGe})_{3}, \mathrm{Al}_{3} \mathrm{Mo}, \mathrm{KNp}\left(\mathrm{CO}_{3}\right)_{2}, \mathrm{~V}_{2} \mathrm{NiS}_{4}, \mathrm{~V}_{2} \mathrm{NiSe}_{4}, \mathrm{~K}_{4} \mathrm{GaAu}_{8}, \mathrm{Ba}_{5} \mathrm{Al}_{2} \mathrm{Ge}_{7}, \\
\mathrm{LiO}_{2}, \mathrm{FeGe}, \mathrm{Cr}_{3} \mathrm{Ga}_{4}, \mathrm{CeGaCo}, \mathrm{BaSi}\end{array}$ \\
\hline 1103 & $\operatorname{BiI}(\mathrm{ICSD}: 1559), \| \mathrm{Sr}_{3}\left(\mathrm{AlGe}_{2}\right)_{2}, \mathrm{Pr}, \mathrm{Nd}_{2} \mathrm{Ni}_{5} \mathrm{~B}_{4}, \mathrm{La}_{2} \mathrm{Ni}_{5} \mathrm{~B}_{4}, \mathrm{Mn}_{3} \mathrm{As}_{2}, \mathrm{MoCl}_{5}$ \\
\hline 1111 & $\| \mathrm{Ba}_{5} \mathrm{P}_{5} \mathrm{I}_{3}, \mathrm{Sr}_{11}\left(\mathrm{MgSi}_{5}\right)_{2}, \mathrm{Ni}_{3}(\mathrm{BiSe})_{2}, \mathrm{Cr}_{2} \mathrm{NiS}_{4}, \mathrm{Bi}_{2} \mathrm{Rh}_{3} \mathrm{~S}_{2}$ \\
\hline 1113 & $\mathrm{FeBi}_{4} \mathrm{~S}_{7}, \| \mathrm{Sb}_{2} \mathrm{Te}_{3}(\mathrm{ICSD}: 187496), \mathrm{Zr}_{7} \mathrm{P}_{4}, \mathrm{~Tb}_{4} \mathrm{In}_{20} \mathrm{Ni}_{11}, \mathrm{Ba}(\mathrm{NiAs})_{2}$ \\
\hline \multicolumn{2}{|r|}{$\begin{array}{c}\text { Space Group 14: } P 2_{1} / c \\
\end{array}$} \\
\hline $\mathbb{Z}_{2,2,2,4}$ & Materials \\
\hline 0001 & $\|$ CaSiPt, CaSiPd, S(ICSD : 50338), $\mathrm{Cs}_{11} \mathrm{O}_{3}, \mathrm{CuH}_{8} \mathrm{CN}_{5} \mathrm{Cl}_{3}$ \\
\hline 0003 & $\begin{array}{c}\mathrm{K}_{5} \mathrm{SnBi}_{3}, \| \mathrm{Ag}_{2} \mathrm{Te}[97], \mathrm{BaCu}_{2}\left(\mathrm{AsO}_{4}\right)_{2}, \mathrm{CaSiO}_{3}(\mathrm{ICSD}: 36311), \mathrm{CuTeO}_{3}, \mathrm{Y}_{2} \mathrm{Si}_{4} \mathrm{Mo}_{3}, \\
\mathrm{Bi}_{2} \mathrm{O}_{3}\left(\mathrm{ICSD}: \text { 15752), } \mathrm{LaGaNi}_{2} \mathrm{Na}_{3} \mathrm{InBi}_{2}, \mathrm{PrSiPd}_{2} \mathrm{H}_{8} \mathrm{~N}_{2} \mathrm{O}, \mathrm{Ba}_{2} \mathrm{P}_{3} \mathrm{Pd}, \mathrm{Te}_{4} \mathrm{Pd}_{9}, \mathrm{U}_{2} \mathrm{Ge}_{4} \mathrm{Mo}_{3},\right. \\
\mathrm{U}_{2} \mathrm{Si}_{4} \mathrm{Mo}_{3}, \mathrm{KSm}_{2} \mathrm{Cl}_{5}, \mathrm{HgH}_{4} \mathrm{C}_{4}\left(\mathrm{NCl}_{2}\right)_{2}\end{array}$ \\
\hline 1001 & $\begin{array}{c}\| \mathrm{Hg}, \mathrm{CuTe}_{2} \mathrm{O}_{5}, \mathrm{Ba}(\mathrm{SiRh})_{2}, \mathrm{CuSeO}_{3}, \mathrm{Ba}(\mathrm{GaPt})_{2}, \mathrm{NaCuPO}_{4}, \mathrm{As}_{5} \mathrm{~Pb}_{3} \mathrm{~S}_{10} \\
\mathrm{CuClF}_{10}(\mathrm{ICSD}: 200807), \mathrm{ReO}_{2}\end{array}$ \\
\hline 1003 & $\begin{array}{c}\| \mathrm{Bi}_{2} \mathrm{Rh}, \mathrm{Y}_{4} \mathrm{C}_{7}, \mathrm{Ho}_{4} \mathrm{C}_{7}(\mathrm{ICSD}: 83381), \mathrm{ZnP}_{2}(\mathrm{ICSD}: 43334), \mathrm{TcO}_{2}, \mathrm{Be}\left(\mathrm{B}_{3} \mathrm{H}_{8}\right)_{2}, \mathrm{Sn}_{3} \mathrm{PO}_{6}, \mathrm{Pd}_{7} \mathrm{Se}_{2}, \\
\mathrm{Ca}_{5} \mathrm{Au}_{4}, \mathrm{Cu}_{5} \mathrm{Sn}_{4}, \mathrm{SnHg}_{2}(\mathrm{SBr})_{2}(\mathrm{ICSD}: 85584), \mathrm{NbCoTe}_{2}, \mathrm{Hg}_{2} \mathrm{~Pb}(\mathrm{SBr})_{2}, \mathrm{Er}_{5} \mathrm{Si}_{4}, \mathrm{OsF}_{5}, \mathrm{UMo}, \\
\mathrm{Ho}_{2}\left(\mathrm{Ni}_{5} \mathrm{~B}_{2}\right)_{3}\end{array}$ \\
\hline \multicolumn{2}{|r|}{$\begin{array}{l}\text { Space Group } 15: C 2 / c \\
\end{array}$} \\
\hline $\mathbb{Z}_{2,2,2,4}$ & \\
\hline 0001 & $\begin{array}{c}\mathrm{MoP}_{4}, \mathrm{CrP}_{4}, \mathrm{Pr}_{2} \mathrm{~B}_{5}, \mathrm{Nd}_{2} \mathrm{~B}_{5}, \mathrm{Ti}_{3} \mathrm{O}_{5}, \mathrm{Ho}_{2}\left(\mathrm{GePt}_{3}\right)_{3}, \mathrm{Dy}_{2}\left(\mathrm{GePt}_{3}\right)_{3}, \mathrm{Zr}_{2} \mathrm{Si}_{4} \mathrm{Ru}_{3}, \mathrm{Nd}_{4} \mathrm{Ge}_{3} \mathrm{Rh}_{4}, \\
\mathrm{Ba}_{2} \mathrm{LiFe}_{2} \mathrm{~N}_{3}, \mathrm{Sr}_{2} \mathrm{LiFe}_{2} \mathrm{~N}_{3}, \mathrm{Hf}_{3} \mathrm{As}\end{array}$ \\
\hline 0003 & $\begin{array}{c}\| \mathrm{Ca}_{3}\left(\mathrm{AlAs}_{2}\right)_{2}, \mathrm{BaH}_{8} \mathrm{PtC}_{4}(\mathrm{NO})_{4}(\mathrm{ICSD}: 4412), \mathrm{V}_{2} \mathrm{Cu}_{2} \mathrm{O}_{7}, \mathrm{~B}_{2} \mathrm{Pd}_{5}, \mathrm{Yb}_{2} \mathrm{Te}_{4} \mathrm{O}_{11}, \mathrm{Ta}_{4} \mathrm{Te}_{9} \mathrm{I}_{4} \mathrm{O} \\
\mathrm{Sc}_{3}\left(\mathrm{ReSi}_{2}\right)_{2}, \mathrm{Na}_{2} \mathrm{TbO}_{3}, \mathrm{Ta}_{6} \mathrm{~S}, \mathrm{Nb}_{4} \mathrm{Te}_{9} \mathrm{I}_{4} \mathrm{O}, \mathrm{RbB}_{6} \mathrm{H}_{8} \mathrm{O}_{7}\end{array}$ \\
\hline 1101 & || $\mathrm{Sb}_{2} \mathrm{Te}_{3}$ \\
\hline 1103 & $\mathrm{BaCuN}, \mathrm{Hf}_{2} \mathrm{Si}_{4} \mathrm{Ru}_{3}, \mathrm{Ta}_{3} \mathrm{As}, \mathrm{LiDy}_{2} \mathrm{Cl}_{5}, \mathrm{Pd}_{13} \mathrm{~Pb}_{9}, \mathrm{RbCuCl}_{3}, \mathrm{LaNdI}_{4}$ \\
\hline \multicolumn{2}{|r|}{ Space Group 47: Pmmm } \\
\hline $\mathbb{Z}_{2,2,2,4}$ & Materials \\
\hline 0013 & $\mathrm{Sr}_{2}(\mathrm{PPd})_{3}$ \\
\hline 0103 & \multirow{2}{*}{$\frac{\mathrm{Ba}_{2} \mathrm{TmCu}_{3} \mathrm{O}_{7}, \mathrm{Ba}_{2} \mathrm{ErCu}_{3} \mathrm{O}_{7}, \mathrm{Ba}_{2} \mathrm{HoCu}_{3} \mathrm{O}_{7}, \mathrm{Ba}_{2} \mathrm{DyCu}_{3} \mathrm{O}_{7}, \mathrm{Ba}_{2} \mathrm{LaCu}_{3} \mathrm{O}_{7}, \mathrm{Ba}_{2} \mathrm{NdCu}_{3} \mathrm{O}_{7}}{\| \mathrm{Ba}_{2} \mathrm{YCu}_{3} \mathrm{O}_{7}, \mathrm{Ba}_{2} \mathrm{LuCu}_{3} \mathrm{O}_{7}}$} \\
\hline 1011 & \\
\hline \multicolumn{2}{|r|}{ Space Group 51: Pmma } \\
\hline $\mathbb{Z}_{2,2,2,4}$ & Materials \\
\hline 0001 & || $\mathrm{TbInCO} 2$ \\
\hline
\end{tabular}


Table V - continued

\begin{tabular}{|c|c|}
\hline SI & Topological Insulators \\
\hline 0003 & $\| \mathrm{Rb}_{2} \mathrm{TlAu}_{3}, \mathrm{WO}_{3}$ \\
\hline 0011 & $\| \mathrm{Te}_{2} \mathrm{AuI}, \mathrm{TiPd}, \mathrm{TiPt}$ \\
\hline 0101 & $\| \mathrm{Fe}_{4} \mathrm{Cu}_{2} \mathrm{~S}_{7}$ \\
\hline 0103 & $\| \mathrm{LaAl}_{4} \mathrm{Co}, \mathrm{PrAl}_{4} \mathrm{Co}, \mathrm{NdAl}_{4} \mathrm{Co}$ \\
\hline 0111 & $\| \mathrm{AlPt}_{2}, \mathrm{Y}_{5} \mathrm{Mg}_{8} \mathrm{Cu}_{5}$ \\
\hline & Space Group 52: Pnna \\
\hline $\mathbb{Z}_{4}$ & Materials \\
\hline 3 & $\| \mathrm{TaSbO}_{4}$ \\
\hline & Space Group 53: Pmna \\
\hline $\mathbb{Z}_{2,2,2,4}$ & Materials \\
\hline 0101 & $\mathrm{NbNiTe}_{2}, \mathrm{TaNiTe}_{2}$ \\
\hline & Space Group 54: Pcca \\
\hline $\mathbb{Z}_{2,2,2,4}$ & Materials \\
\hline 0101 & $\| \mathrm{La}_{2} \mathrm{Re}_{3} \mathrm{~B}_{7}$ \\
\hline 0103 & $\mathrm{C}(\mathrm{ICSD}: 88813), \mathrm{FeS}_{2}$ \\
\hline & Space Group 55: Pbam \\
\hline $\mathbb{Z}_{2,2,2,4}$ & Materials \\
\hline 0001 & $\begin{array}{r}\| \mathrm{ErVB}_{4}, \mathrm{HoVB}_{4}, \mathrm{TbVB}_{4}, \mathrm{Nd}_{2} \mathrm{NiB}_{10}, \mathrm{Sc}(\mathrm{BC})_{2}, \mathrm{Lu}_{2} \mathrm{FeB}_{6}, \mathrm{ErB}_{4} \mathrm{Rh}, \mathrm{Sr}_{5}\left(\mathrm{InBi}_{3}\right)_{2}, \mathrm{TmB}_{4} \mathrm{Rh}, \\
\mathrm{YB}_{4} \mathrm{Rh}, \mathrm{Ca}_{5}\left(\mathrm{AlBi}_{3}\right)_{2}, \mathrm{La}_{2} \mathrm{Cu}_{2} \mathrm{O}_{5}, \mathrm{YbB}_{4} \mathrm{Ru}, \mathrm{Nd}_{2} \mathrm{Cu}_{2} \mathrm{O}_{5}, \mathrm{YbB}_{4} \mathrm{Os}, \mathrm{ScCoGe}_{2}, \mathrm{Nd}\left(\mathrm{Al}_{4} \mathrm{Co}\right)_{2}, \\
\operatorname{Pr}\left(\mathrm{Al}_{4} \mathrm{Co}\right)_{2}, \mathrm{Sc}_{2} \mathrm{CrB}_{6}, \mathrm{Sr}_{13}\left(\mathrm{MgSi}_{10}\right)_{2}\end{array}$ \\
\hline 0003 & $\| \mathrm{Zr}_{5}\left(\mathrm{In}_{2} \mathrm{Rh}\right)_{2}, \mathrm{Sr}_{2} \mathrm{Cu}_{2} \mathrm{O}_{5}, \mathrm{Sc}_{2} \mathrm{~B}_{6} \mathrm{Rh}, \mathrm{Ga}_{2} \mathrm{AsRh}_{5}, \mathrm{HfReSi}{ }_{2}, \mathrm{~K}_{2} \mathrm{U}_{7} \mathrm{O}_{22}, \mathrm{TiMnSi}_{2}$ \\
\hline 0011 & $\| \mathrm{Yb}\left(\mathrm{Al}_{4} \mathrm{Co}\right)_{2}, \mathrm{Th}_{2} \mathrm{NiB}_{10}, \mathrm{Mg}_{2} \mathrm{~B}_{4} \mathrm{Ru}_{5}, \mathrm{~Pb}_{3} \mathrm{O}_{4}(\mathrm{ICSD}: 97282), \mathrm{SmMnB}_{4}, \mathrm{Ti}_{9} \mathrm{Se}_{2}, \mathrm{~B}_{8} \mathrm{Ru}_{11}$ \\
\hline 0013 & $\| \mathrm{LuAlB}_{4}, \mathrm{YbB}_{4} \mathrm{Rh}, \mathrm{YbCoB}_{4}, \operatorname{Sr}\left(\operatorname{In}_{4} \mathrm{Rh}\right)_{2}$ \\
\hline & Space Group 57: Pbcm \\
\hline $\mathbb{Z}_{2,2,2,4}$ & Materials \\
\hline 0003 & $\| \mathrm{LiSn}_{5} \mathrm{Rh}_{3}$ \\
\hline 1001 & $\| \mathrm{Hf}(\mathrm{FeSi})_{2}, \mathrm{PrNiSb}_{3}, \mathrm{NdNiSb}_{3}, \mathrm{SmNiSb}_{3}$ \\
\hline 1003 & \begin{tabular}{l||l}
$\mathrm{LaNiSb}_{3}, \mathrm{Ta}_{2} \mathrm{~S}$ \\
\end{tabular} \\
\hline & Space Group 58: Pnnm \\
\hline $\mathbb{Z}_{4}$ & Materials \\
\hline 1 & $\mathrm{CrB}_{4}, \mathrm{NiAs}_{2}, \mathrm{NiSb}_{2}, \mathrm{Th}_{2} \mathrm{Al}_{2} \mathrm{C}_{3}, \mathrm{NiSe}_{2}, \mathrm{NiS}_{2}, \mathrm{~B}_{2} \mathrm{Mo}_{2} \mathrm{Ir}, \mathrm{Zr}_{2} \mathrm{Se}, \mathrm{Ti}_{2} \mathrm{Se}, \mathrm{Th}\left(\mathrm{Fe}_{2} \mathrm{P}\right)_{2}$ \\
\hline 3 & 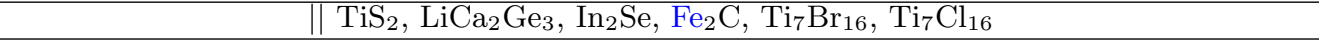 \\
\hline & Space Group 59: Pmmn \\
\hline $\mathbb{Z}_{2,2,2,4}$ & Materials \\
\hline 0003 & $\begin{array}{l}\text { TiIN, } \mathrm{SiNi}, \mathrm{Cu}_{3} \mathrm{Sb}, \mathrm{Ag}_{3} \mathrm{Sb}, \mathrm{K}_{3} \mathrm{Sn}_{4} \mathrm{Au}, \mathrm{SmAu} \mathrm{Su}_{3}, \mathrm{TmAu}_{3}, \mathrm{Zn}_{2} \mathrm{CuAu}, \mathrm{YAu}_{3}, \mathrm{ErAu}_{3}, \mathrm{Rb}_{3} \mathrm{Sn}_{4} \mathrm{Au} \text {, } \\
\mathrm{InAu}_{3}, \mathrm{LuAu}_{3}, \mathrm{AlCu}_{3}, \mathrm{SrCu}_{2} \mathrm{O}_{3}, \mathrm{Sr}(\mathrm{InAu})_{3}, \mathrm{~K}(\mathrm{SnAu})_{3}, \mathrm{Rb}_{3} \mathrm{~Pb}_{4} \mathrm{Au}, \mathrm{Ni}_{3} \mathrm{Mo}\end{array}$ \\
\hline 0011 & $\| \mathrm{Hf}_{8} \mathrm{FeTe}_{6}$ \\
\hline 0013 & $\| \mathrm{Er}_{5} \mathrm{Al}_{3} \mathrm{Ni}_{3} \mathrm{Ge}_{4}, \mathrm{Nd}_{3} \mathrm{Sb}_{5} \mathrm{Pd}_{6}$ \\
\hline & Space Group 60: Pbcn \\
\hline $\mathbb{Z}_{4}$ & Materials \\
\hline 1 & CuAsSe $\|$ \\
\hline 3 & $\| \mathrm{PbO}_{2}, \mathrm{~W}_{2} \mathrm{C}, \mathrm{Mo}_{2} \mathrm{C}, \mathrm{GeTe}$ \\
\hline & Space Group 61: Pbca \\
\hline $\mathbb{Z}_{4}$ & Materials \\
\hline 1 & $\mathrm{Sn}_{2} \mathrm{Au}, \mathrm{DyBr}_{2}, \mathrm{TmCl}_{2}, \mathrm{Zr}_{7} \mathrm{Ni}_{10}$ \\
\hline 3 & $\| \mathrm{SmCuTeS}, \mathrm{SnTePd}, \mathrm{Bi}_{2} \mathrm{Pt}$ \\
\hline & Space Group 62: Pnma \\
\hline $\mathbb{Z}_{4}$ & Materials \\
\hline
\end{tabular}


Table V - continued

\begin{tabular}{|c|c|}
\hline SI & Topological Insulators \\
\hline 1 & 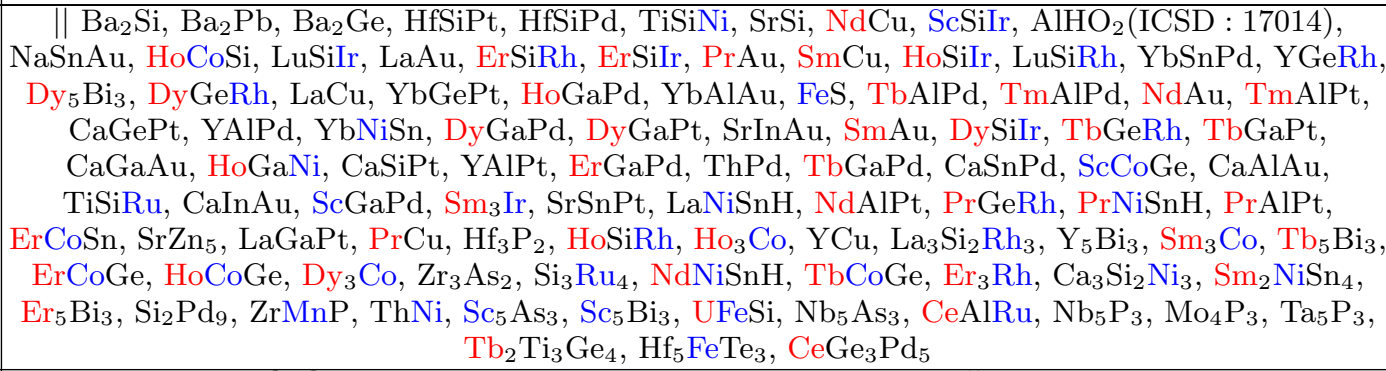 \\
\hline 3 & 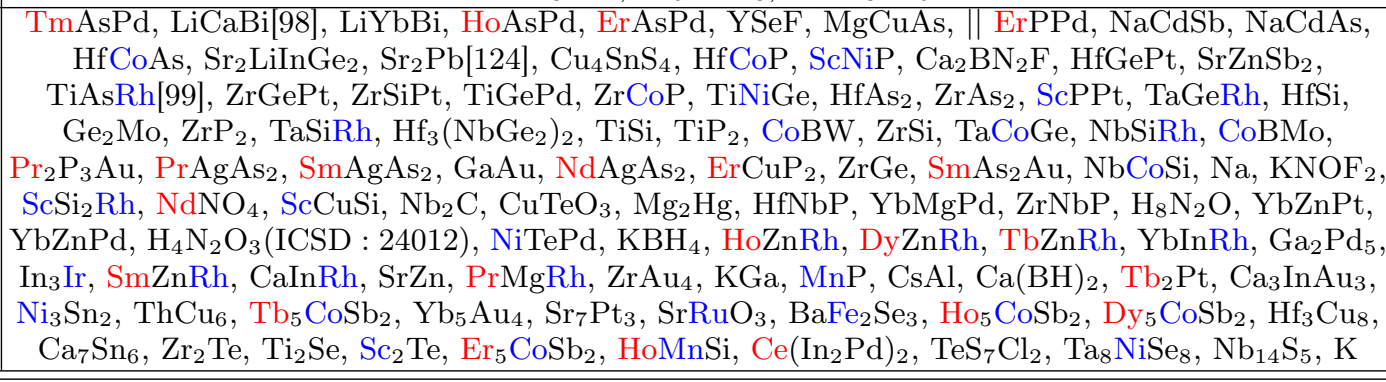 \\
\hline \multicolumn{2}{|r|}{ Space Group 63: $\mathrm{Cmcm}$} \\
\hline $\mathbb{Z}_{2,2,2,4}$ & Materials \\
\hline 0001 & 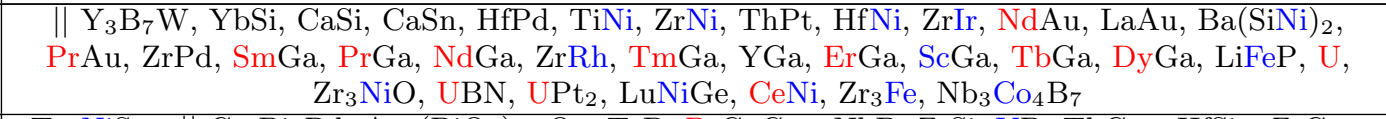 \\
\hline 0003 & 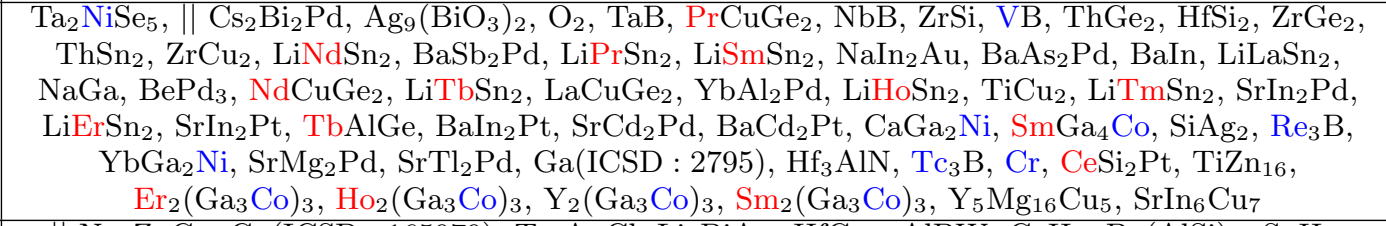 \\
\hline 1101 & 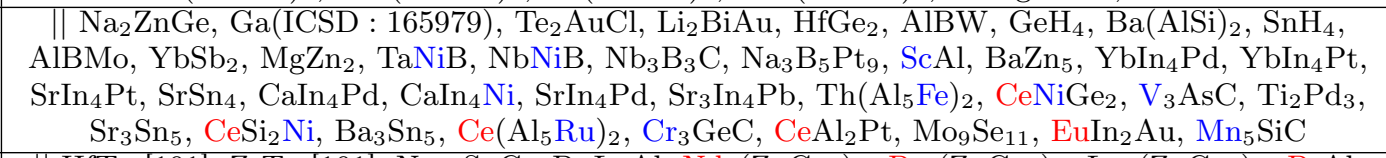 \\
\hline 1103 & 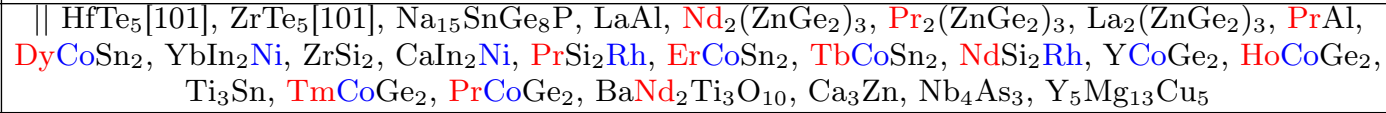 \\
\hline
\end{tabular}

\begin{tabular}{|c|c|}
\hline \multicolumn{2}{|r|}{ Space Group 64: Cmce } \\
\hline $\mathbb{Z}_{2,2,2,4}$ & Materials \\
\hline 0001 & $\mathrm{KTl}, \mathrm{Sn}_{3} \mathrm{Pd}, \mathrm{La}_{2} \mathrm{Ni}_{3}, \mathrm{Zn}_{53} \mathrm{Ni}_{16}$ \\
\hline 0003 & $\| \mathrm{Ge}, \mathrm{Ge}, \mathrm{Si}, \mathrm{Hf}_{2} \mathrm{Si}_{4} \mathrm{Ni}_{3}, \mathrm{AgN}, \mathrm{Ba}_{4} \mathrm{Ru}_{3} \mathrm{O}_{10}, \mathrm{Dy}_{2} \mathrm{Ni}_{7} \mathrm{Sn}_{3}, \mathrm{AsH}_{2} \mathrm{C}_{2} \mathrm{NCl}_{2} \mathrm{O}$ \\
\hline 1101 & $\mathrm{P}, \mathrm{As}, \mathrm{Ga}(\mathrm{ICSD}: 43388), \mathrm{FeGe}_{2}, \mathrm{SnBi}, \mathrm{V}_{2} \mathrm{GaSn}_{2}, \mathrm{Ba}_{2} \mathrm{Cd}_{3} \mathrm{Bi}_{4}, \mathrm{Zr}_{7} \mathrm{Ni}_{10}, \mathrm{~W}_{3} \mathrm{Br}_{8}, \mathrm{La}_{2} \mathrm{Ni}_{22} \mathrm{C}_{3}$ \\
\hline 1103 & $\| \mathrm{SnO}, \mathrm{K}, \mathrm{Cs}, \mathrm{Ti}_{2} \mathrm{Sn}_{3}, \mathrm{Fe}_{2} \mathrm{AgS}_{3}, \mathrm{~Pb}_{3} \mathrm{O}_{5}$ \\
\hline \multicolumn{2}{|r|}{ Space Group 65: $\mathrm{Cmmm}$} \\
\hline $\mathbb{Z}_{2,2,2,4}$ & Materials \\
\hline 0001 & $\mathrm{Zn}_{5}\left(\mathrm{BRh}_{2}\right)_{4}, \mathrm{YMg}_{4} \mathrm{Cu}, \mathrm{UGe}_{2}$ \\
\hline 0003 & $\begin{array}{c}\text { II } \mathrm{ThGe}_{2}, \mathrm{ScSi}_{3} \mathrm{Ni}, \mathrm{LaSn}_{2}, \mathrm{Ho}_{2} \mathrm{Ni}_{2} \mathrm{~Pb}, \mathrm{TlAg}_{3} \mathrm{Te}_{2}, \mathrm{NdSn}_{2}, \mathrm{Lu}_{2} \mathrm{NiSn}_{6}, \mathrm{SmSn}_{2}, \mathrm{TbGe}_{2}, \mathrm{Yb}_{2}\left(\mathrm{NiB}_{2}\right)_{3}, \\
\mathrm{~Tb}_{2} \mathrm{Mg}_{3} \mathrm{Ni}_{2}, \mathrm{Ho}_{2}\left(\mathrm{NiB}_{2}\right)_{3}, \mathrm{Lu}_{2}\left(\mathrm{NiB}_{2}\right)_{3}, \mathrm{Er}_{4} \mathrm{Ni}_{13} \mathrm{C}_{4}, \mathrm{Lu}_{4} \mathrm{Ni}_{13} \mathrm{C}_{4}, \mathrm{Ho}_{4} \mathrm{Ni}_{13} \mathrm{C}_{4}, \mathrm{CeSiNi}_{4}, \\
\mathrm{Ba}_{2} \mathrm{Nd}_{7} \mathrm{Si}_{11} \mathrm{~N}_{23}\end{array}$ \\
\hline 0011 & || $\mathrm{Ba}_{3} \mathrm{Ge}_{4}, \mathrm{~Tb}_{2} \mathrm{~B}_{2} \mathrm{C}_{3}, \mathrm{ScGa}_{2}$ \\
\hline 0013 & || $\mathrm{LuAlB}_{4}, \mathrm{YbAlB}_{4}, \mathrm{Nd}_{2} \mathrm{CdCu}_{2}$ \\
\hline 1101 & $\begin{array}{c}\| \mathrm{ZrGa}_{2}, \mathrm{TiAl}_{2}, \mathrm{Li}_{7} \mathrm{Sn}_{2}, \mathrm{YBC}, \mathrm{Er}_{2} \mathrm{NiSn}_{6}, \mathrm{Tm}_{2} \mathrm{NiSn}_{6}, \mathrm{LuNiSn}_{4}, \mathrm{VIr}(\mathrm{ICSD}: 169389), \mathrm{SmGa}_{2} \mathrm{Co}, \\
\mathrm{Ce}_{2} \mathrm{Si}_{7}, \mathrm{LuMnGe}_{2}\end{array}$ \\
\hline 1103 & $\| \mathrm{La}_{4} \mathrm{Al}_{5} \mathrm{Br}_{2}, \mathrm{Dy}_{2} \mathrm{In}_{16} \mathrm{Pt}_{7}, \mathrm{~Tb}_{2} \mathrm{In}_{16} \mathrm{Pt}_{7}$ \\
\hline 1111 & $\| \mathrm{Sr}_{2} \mathrm{Ge}_{2} \mathrm{~Pb}, \mathrm{ThB}_{4} \mathrm{Mo}, \mathrm{Pr}_{11} \mathrm{In}_{9} \mathrm{Ni}_{4}, \mathrm{Zr}_{2} \mathrm{P}$ \\
\hline 1113 & $\begin{array}{c}\| \mathrm{LuNiGe}_{3}, \mathrm{Rb}_{3} \mathrm{Au}_{7}, \mathrm{TbNiGe}_{3}, \mathrm{Al}_{2} \mathrm{~B}_{2} \mathrm{Ru}_{3}, \mathrm{DyNiGe}_{3}, \mathrm{HoNiGe}_{3}, \mathrm{ErNiGe}_{3}, \operatorname{VIr}(\mathrm{ICSD}: 169390), \\
\mathrm{Nd}_{11} \mathrm{In}_{9} \mathrm{Ni}_{4}\end{array}$ \\
\hline
\end{tabular}

Space Group 66: $\mathrm{Cccm}$ 
Table V - continued

\begin{tabular}{|c|c|}
\hline \multicolumn{2}{|r|}{ Topological Insulators } \\
\hline $\mathbb{Z}_{2,2,2,4}$ & Materials \\
\hline 0001 & $\| \mathrm{ThI}_{3}$ \\
\hline \multicolumn{2}{|r|}{ Space Group 67: Cmme } \\
\hline $\mathbb{Z}_{2,2,2,4}$ & Materials \\
\hline 0013 & $\| \mathrm{Al}_{2} \mathrm{CuIr}$ \\
\hline \multicolumn{2}{|r|}{ Space Group 69: Fmmm } \\
\hline $\mathbb{Z}_{2,2,2,4}$ & Materials \\
\hline 0003 & $\mathrm{Be}_{2} \mathrm{Zn}, \mathrm{TiSi}_{2}, \mathrm{Ca}_{3}\left(\mathrm{~B}_{3} \mathrm{Rh}_{4}\right)_{2}, \mathrm{Sr}_{2} \mathrm{Cu}_{2} \mathrm{O}_{3}$ \\
\hline 1103 & $\| \mathrm{Ca}_{7}\left(\mathrm{~B}_{7} \mathrm{Rh}_{10}\right)_{2}$ \\
\hline \multicolumn{2}{|r|}{ Space Group 70: Fddd } \\
\hline $\mathbb{Z}_{4}$ & Materials \\
\hline 1 & $\begin{array}{c}\mathrm{Sr}(\mathrm{GaRh})_{2}, \| \mathrm{LiBIr}, \mathrm{Ca}(\mathrm{BIr})_{2}, \mathrm{Ca}(\mathrm{BRh})_{2}, \mathrm{Sr}(\mathrm{BRh})_{2}, \mathrm{Sr}(\mathrm{BIr})_{2}, \mathrm{Ba}(\mathrm{GeRu})_{2}\left[\underline{5}, \mathrm{Th}(\mathrm{BRu})_{2},\right. \\
\mathrm{Ce}(\mathrm{BOs})_{2}, \mathrm{Ce}(\mathrm{BRu})_{2}, \mathrm{Ce}(\mathrm{BIr})_{2}, \mathrm{Gd}(\mathrm{BOs})_{2}, \mathrm{Gd}(\mathrm{BRu})_{2}\end{array}$ \\
\hline 3 & $\| \mathrm{TiGe}_{2}, \mathrm{ZrSn}_{2}, \mathrm{TiSi}_{2}, \mathrm{Th}(\mathrm{BOs})_{2}$ \\
\hline \multicolumn{2}{|r|}{ Space Group 71: $I m m m$} \\
\hline $\mathbb{Z}_{2,2,2,4}$ & Materials \\
\hline 0001 & $\| \mathrm{CeSi}_{5}, \mathrm{Ba}_{3}(\mathrm{AlGe})_{2}, \mathrm{Ba}_{3}(\mathrm{AlSi})_{2}, \mathrm{CeGe}_{5}, \mathrm{La}_{2} \mathrm{ZnNi}_{2}, \mathrm{Fe}_{2} \mathrm{~B}_{4} \mathrm{Mo}, \mathrm{Lu}_{6} \mathrm{Ni}_{2} \mathrm{Sn}, \mathrm{LiCe}_{2} \mathrm{HO}_{3}$ \\
\hline 0003 & $\begin{array}{c}\mathrm{Ca}_{3} \mathrm{SiBr}_{2}, \mid \mathrm{TiTeAs} \mathrm{Ni}(\mathrm{BW})_{2}, \mathrm{Dy}_{2} \mathrm{~B}_{4} \mathrm{C}, \mathrm{Ni}(\mathrm{BMo})_{2}, \mathrm{Th}\left(\mathrm{CrB}_{3}\right)_{2}, \mathrm{Fe}(\mathrm{BW})_{2}, \mathrm{TiMo}_{3}, \mathrm{Ce}\left(\mathrm{CrB}_{3}\right)_{2}, \\
\mathrm{LaAl}_{5} \mathrm{Ni}_{2}, \mathrm{Ca}_{3} \mathrm{Ga}_{8}, \mathrm{HoGe}_{2} \mathrm{Ir}, \mathrm{ErGe}_{2} \mathrm{Ir}, \mathrm{Er}_{6} \mathrm{Ni}_{2} \mathrm{Sn}_{2} \mathrm{Ce}_{3} \mathrm{Zn}_{11}, \mathrm{ZrMnSi}_{2}\end{array}$ \\
\hline 1111 & $\begin{array}{c}\| \mathrm{CrB}_{4}, \mathrm{Sn}, \mathrm{Pt}_{2} \mathrm{~W}, \mathrm{TiPd}_{2}, \mathrm{MoPt}_{2}, \mathrm{Sc}_{3} \mathrm{RhC}_{4}, \mathrm{Sc}_{3} \mathrm{IrC}_{4}, \mathrm{PrAl}_{5} \mathrm{Ni}_{2}, \mathrm{Sc}_{3} \mathrm{CoC}_{4}, \mathrm{DyGe}_{2} \mathrm{Pt}, \mathrm{TbGe}_{2} \mathrm{Ir}, \\
\mathrm{Cu}_{3} \mathrm{As}_{4}, \mathrm{ReN}_{2}\end{array}$ \\
\hline 1113 & $\begin{array}{c}\| \mathrm{Ca}_{3}(\mathrm{AlSi})_{2}, \mathrm{Sr}_{3}(\mathrm{AlGe})_{2}, \mathrm{Sr}_{3}(\mathrm{AlSi})_{2}, \mathrm{Sr}_{3}(\mathrm{AlSn})_{2}, \mathrm{CaC}_{2}, \mathrm{Ba}_{3}(\mathrm{AlSn})_{2}, \mathrm{Hf}_{3}(\mathrm{NiGe})_{4}, \mathrm{Co}_{2} \mathrm{~B}_{4} \mathrm{Mo}, \\
\mathrm{Tb}_{2} \mathrm{Ni}_{2} \mathrm{Sn}, \mathrm{Sr}(\mathrm{NiP})_{2}, \mathrm{Hf}_{27} \mathrm{P}_{16}\end{array}$ \\
\hline \multicolumn{2}{|r|}{ Space Group 72: Ibam } \\
\hline $\mathbb{Z}_{2,2,2,4}$ & Materials \\
\hline 0001 & $\| \mathrm{Sc}_{6} \mathrm{~Pb}_{5}$ \\
\hline 0003 & $\| \mathrm{Hg}_{5}\left(\mathrm{BrO}_{2}\right)_{2}, \mathrm{Hg}_{5}\left(\mathrm{ClO}_{2}\right)_{2}, \mathrm{Sc}_{2} \mathrm{Cr}_{4} \mathrm{Si}_{5}$ \\
\hline 1111 & $\mathrm{Mn}_{4} \mathrm{Nb}_{2} \mathrm{Si}_{5}, \mathrm{~V}_{6} \mathrm{Si}_{5}, \mathrm{Ta}_{2} \mathrm{~V}_{4} \mathrm{Si}_{5}, \mathrm{Nb}_{2} \mathrm{~V}_{4} \mathrm{Si}_{5}$ \\
\hline 1113 & $\| \mathrm{Fe}(\mathrm{PdSe})_{2}, \mathrm{Ni}(\mathrm{TePd})_{2}, \mathrm{Mg}_{5} \mathrm{Ga}_{2}, \mathrm{Mg}_{5} \mathrm{In}_{2}$ \\
\hline \multicolumn{2}{|r|}{ Space Group 73: Ibca } \\
\hline $\mathbb{Z}_{2,2,2,4}$ & Materials \\
\hline 0003 & $\| \mathrm{K}_{3} \mathrm{Na}_{2} \mathrm{SnBi}_{3}$ \\
\hline \multicolumn{2}{|r|}{ Space Group 74: Imma } \\
\hline $\mathbb{Z}_{2,2,2,4}$ & Materials \\
\hline 0001 & $\| \mathrm{Si}, \mathrm{ErSi}_{2} \mathrm{Rh}_{3}, \mathrm{TlRe}, \mathrm{PuGa}_{4}$ \\
\hline 0003 & $\mathrm{Ge}, \mathrm{CaAu}_{2}, \mathrm{YbAg}_{2}, \mathrm{CaAg}_{2}, \mathrm{BaGe}_{5}, \mathrm{HfV}_{2}, \mathrm{CuAu}$ \\
\hline 1111 & $\| \mathrm{SrAl}_{2}, \mathrm{BaCd}_{2}, \mathrm{HoAlB}_{14}$ \\
\hline 1113 & $\| \mathrm{BaIn}_{2}, \mathrm{Ca}_{2} \mathrm{Os}_{2} \mathrm{O}_{7}$ \\
\hline \multicolumn{2}{|r|}{ Space Group 82: $I \overline{4}$} \\
\hline $\mathbb{Z}_{2}$ & Materials \\
\hline 1 & $\mathrm{ZnCu}_{2} \mathrm{SnSe}_{4}, \mathrm{ZnCu}_{2} \mathrm{GeTe}_{4}, \mathrm{Ta}_{3} \mathrm{Ge}, \mathrm{Ni}_{3} \mathrm{P}, \mathrm{Tl}_{5} \mathrm{Te}_{3}, \mathrm{Cr}_{3} \mathrm{P}, \mathrm{Zr}_{3} \mathrm{Te}, \mathrm{CdHgS}{ }_{4}, \mathrm{Fe}_{3} \mathrm{P}$ \\
\hline \multicolumn{2}{|r|}{ Space Group 83: $P 4 / m$} \\
\hline $\mathbb{Z}_{2,4,8}$ & Materials \\
\hline 111 & $\| \mathrm{Ti}_{2} \mathrm{Ga}_{3}$ \\
\hline 127 & $\| \mathrm{Ca}_{7} \mathrm{Ni}_{4} \mathrm{Sn}_{13}$ \\
\hline \multicolumn{2}{|r|}{ Space Group 85: $P 4 / n$} \\
\hline $\mathbb{Z}_{2,2,2,4}$ & Materials \\
\hline 0001 & $\| \mathrm{SrBr}_{2}, \mathrm{Si}_{5} \mathrm{Pt}_{12}$ \\
\hline \multicolumn{2}{|r|}{ Space Group 86: $P 4_{2} / n$} \\
\hline $\mathbb{Z}_{2,2,2,4}$ & Materials \\
\hline 0001 & $\| \mathrm{Ta}_{3} \mathrm{Ge}$ \\
\hline 0003 & || $\mathrm{Nb}_{3} \mathrm{Si}, \mathrm{Tm}_{3} \mathrm{Sb}$ \\
\hline \multicolumn{2}{|r|}{ Space Group 87: $I 4 / m$} \\
\hline $\mathbb{Z}_{2,8}$ & Materials \\
\hline 07 & $\mid \mathrm{Mo}_{5} \mathrm{As}_{4}(\mathrm{ICSD}: 43186), \mathrm{Ni}_{4} \mathrm{~W}, \mathrm{Ni}_{4} \mathrm{Mo}$ \\
\hline 11 & $\mathrm{Zr}_{5} \mathrm{Te}_{4}, \mathrm{Ti}_{5} \mathrm{Te}_{4}, \mathrm{Mo}_{5} \mathrm{As}_{4}(\mathrm{ICSD}: 610956)$ \\
\hline
\end{tabular}


Table V - continued

\begin{tabular}{|c|c|}
\hline SI & Topological Insulators \\
\hline 13 & \begin{tabular}{l||l}
$\mathrm{U}(\mathrm{CrC})_{4}$ \\
\end{tabular} \\
\hline 15 & $\mathrm{YbAu}_{4}, \mathrm{Ba}\left(\mathrm{Cu}_{2} \mathrm{As}\right)_{4}, \mathrm{Ti}_{4} \mathrm{O}_{5}, \mathrm{Ti}_{5} \mathrm{Se}_{4}, \mathrm{Hf}_{5} \mathrm{Te}_{4}$ \\
\hline & Space Group 114: $P \overline{4} 2_{1} c$ \\
\hline $\mathbb{Z}_{2}$ & Materials \\
\hline 1 & $\| \mathrm{Pd}_{4} \mathrm{~S}$ \\
\hline & Space Group 115: $P \overline{4} m 2$ \\
\hline $\mathbb{Z}_{2}$ & Materials \\
\hline 1 & $\mathrm{Li}_{2} \mathrm{~B}_{2} \mathrm{C}, \mathrm{Zr}_{2} \mathrm{CuSb}_{3}, \mathrm{Hf}_{2} \mathrm{CuSb}_{3}, \mathrm{Ti}_{2} \mathrm{CuSb}_{3}$ \\
\hline & Space Group 116: $P \overline{4} c 2$ \\
\hline $\mathbb{Z}_{2}$ & Materials \\
\hline 1 & $\mathrm{Ga}_{17} \mathrm{Rh}_{10}$ \\
\hline & Space Group 118: $P \overline{4} n 2$ \\
\hline $\mathbb{Z}_{2}$ & Materials \\
\hline 1 & $\mathrm{CuH}_{12} \mathrm{C}_{4}(\mathrm{NO})_{6}, \| \mathrm{Ba}_{4} \mathrm{Sm}_{2} \mathrm{Cu}_{2} \mathrm{O}_{9}, \mathrm{Ge}_{23} \mathrm{Mo}_{13}$ \\
\hline & Space Group 119: $I \overline{4} m 2$ \\
\hline $\mathbb{Z}_{2}$ & Materials \\
\hline 1 & $\| \mathrm{TlAgTe}_{2}$ \\
\hline & Space Group 121: $I \overline{4} 2 m$ \\
\hline $\mathbb{Z}_{2}$ & Materials \\
\hline 1 & 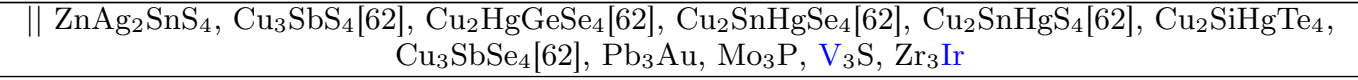 \\
\hline & Space Group 122: $I \overline{4} 2 d$ \\
\hline $\mathbb{Z}_{2}$ & Materials \\
\hline 1 & $\begin{array}{r}\mathrm{ZnSnSb}_{2}[103], \mathrm{CdSnSb}_{2}[102], \| \mathrm{CdGeAs}_{2}, \mathrm{ZnSnAs}_{2}, \mathrm{CdSnAs}_{2}[103], \mathrm{InCuS}_{2}, \mathrm{InCuSe}_{2}, \mathrm{TlCuSe}_{2}, \\
\mathrm{TlCuS}_{2}, \mathrm{UFeC}_{2}, \mathrm{Zr}_{11} \mathrm{Sb}_{18}\end{array}$ \\
\hline & Space Group 123: $P 4 / \mathrm{mmm}$ \\
\hline $\mathbb{Z}_{2,4,8}$ & Materials \\
\hline 003 & $\| \mathrm{BaMg}_{4} \mathrm{Si}_{3}, \mathrm{Ba}_{6} \mathrm{Ca}_{6} \mathrm{Tl}_{5} \mathrm{Cu}_{9} \mathrm{O}_{29}$ \\
\hline 005 & $\mathrm{Ba}_{2} \mathrm{Sm}\left(\mathrm{CuO}_{2}\right)_{3}, \mathrm{Ba}_{2} \mathrm{Ho}\left(\mathrm{CuO}_{2}\right)_{3}, \mathrm{Ba}_{2} \mathrm{Y}\left(\mathrm{CuO}_{2}\right)_{3}, \mathrm{Ba}_{2} \mathrm{Lu}\left(\mathrm{CuO}_{2}\right)_{3}, \mathrm{Ba}_{2} \mathrm{Nd}\left(\mathrm{CuO}_{2}\right)_{3}, \mathrm{Er}_{2} \mathrm{Mg}_{3} \mathrm{Ru}$ \\
\hline 007 & $\| \mathrm{NdTe}_{2} \mathrm{ClO}_{5}, \mathrm{MgNi}$ \\
\hline 013 & || $\mathrm{Ti}_{3} \mathrm{Pd}_{5}(\mathrm{ICSD}: 105726)$ \\
\hline 017 & $\mathrm{Ba}_{2} \mathrm{NdNb}\left(\mathrm{CuO}_{4}\right)_{2}, \mathrm{Sr}_{2} \mathrm{SmTa}\left(\mathrm{CuO}_{4}\right)_{2}, \mathrm{Ba}_{2} \mathrm{LaTa}\left(\mathrm{CuO}_{4}\right)_{2}$ \\
\hline 021 & \begin{tabular}{l||l}
$\mathrm{ZnCu}_{2} \mathrm{Ni}$ \\
\end{tabular} \\
\hline 023 & $\mathrm{O}_{2}(\mathrm{ICSD}: 28530), \mathrm{NaBi}, \mathrm{Hf}_{5} \mathrm{~Pb}$ \\
\hline 033 & $\| \mathrm{LaAl}_{2} \mathrm{BRu}_{2}$ \\
\hline 035 & $\mathrm{CsZn}_{4} \mathrm{As}_{3}, \mathrm{Ba}_{2} \mathrm{Nd}_{2} \mathrm{Ti}_{2} \mathrm{Cu}_{2} \mathrm{O}_{11}, \mathrm{MgSc}_{2} \mathrm{Ru}, \mathrm{ZrHg}, \mathrm{BaTi}_{2} \mathrm{As}_{2} \mathrm{O}$ \\
\hline 101 & $\| \mathrm{Ti}_{3} \mathrm{Pd}_{5}(\mathrm{ICSD}: 167651)$ \\
\hline 105 & II NiHg \\
\hline 107 & $\mathrm{Sr}_{2} \mathrm{NdCu}_{3}\left(\mathrm{PbO}_{4}\right)_{2}$ \\
\hline 111 & $\| \mathrm{Na}_{2} \mathrm{Cl}$ \\
\hline 113 & $\| \mathrm{TiHg}$ \\
\hline 117 & $\| \mathrm{Sr}_{2} \mathrm{YTlCu}_{2} \mathrm{O}_{7}, \mathrm{CeIn}_{5} \mathrm{Co}$ \\
\hline 121 & || $\mathrm{U}_{2} \mathrm{Ga}_{8} \mathrm{Ru}$ \\
\hline 123 & $\| \mathrm{Sr}_{2} \mathrm{ErCu}_{3}\left(\mathrm{PbO}_{4}\right)_{2}, \mathrm{Sr}_{2} \mathrm{DyCu}_{3}\left(\mathrm{PbO}_{4}\right)_{2}, \mathrm{Sr}_{2} \mathrm{SmCu}_{3}\left(\mathrm{PbO}_{4}\right)_{2}$ \\
\hline 125 & || $\mathrm{Ba}_{2} \mathrm{CaCu}_{2} \mathrm{HgO}_{6}$ \\
\hline 133 & $\| \mathrm{Ba}_{2} \mathrm{~Tb}_{2} \mathrm{Ti}_{2} \mathrm{Cu}_{2} \mathrm{O}_{11}$ \\
\hline & Space Group 124: P4/mcc \\
\hline $\mathbb{Z}_{2,8}$ & Materials \\
\hline 17 & $\| \mathrm{Nb}_{4} \mathrm{SiNi}$ \\
\hline & Space Group 125: $P 4 / n b m$ \\
\hline $\mathbb{Z}_{2,2,2,4}$ & Materials \\
\hline 0001 & $\mathrm{SiO}_{2}(\mathrm{ICSD}: 170538)$ \\
\hline 0003 & $\| \mathrm{PtPb}_{4}$ \\
\hline & Space Group 127: $P 4 / m b m$ \\
\hline $\mathbb{Z}_{4,8}$ & Materials \\
\hline 01 & $\| \mathrm{Y}_{2} \mathrm{MgGe}_{2}, \mathrm{Tm}_{2} \mathrm{CdGe}_{2}, \mathrm{Y}_{2} \mathrm{CdGe}_{2}, \mathrm{Sm}_{2} \mathrm{CdGe}_{2}, \mathrm{Nd}_{2} \mathrm{CdGe}_{2}, \mathrm{Ti}_{3} \mathrm{In}_{4}$ \\
\hline
\end{tabular}


Table V - continued

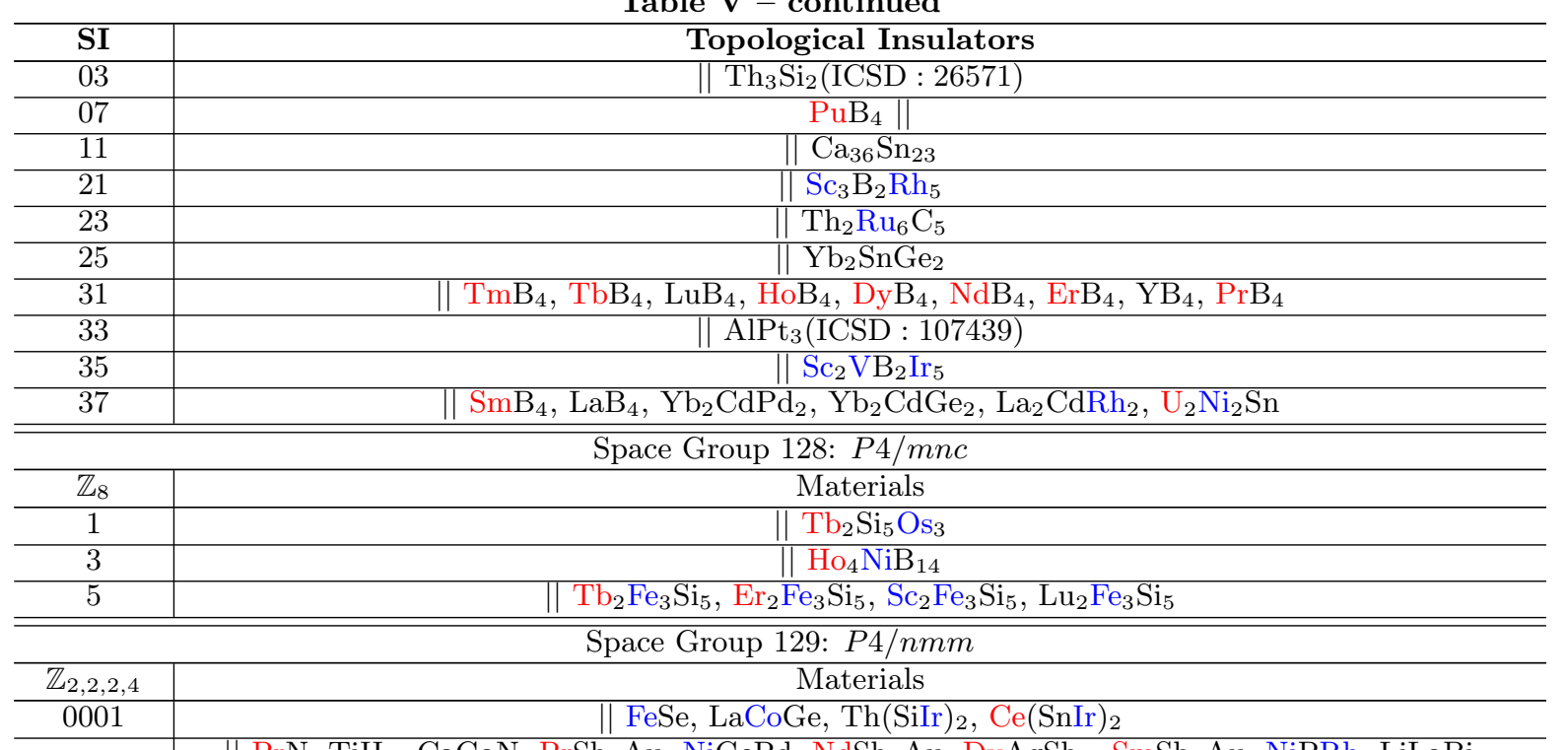

|| PrN, $\mathrm{TiH}_{2}, \mathrm{CaGaN} \mathrm{PrSb}_{2} \mathrm{Au}, \mathrm{NiGePd}, \mathrm{NdSb}_{2} \mathrm{Au}, \mathrm{DyAgSb}_{2}, \mathrm{SmSb}_{2} \mathrm{Au}, \mathrm{NiPRh}, \mathrm{LiLaBi}_{2}$,

$\mathrm{LuCuAs}_{2}, \mathrm{YAgSb}_{2}, \mathrm{PrAgSb}_{2}, \mathrm{LaSb}_{2} \mathrm{Au}, \mathrm{HoCuP}_{2}, \mathrm{NdAgAs}_{2}, \mathrm{TmCuAs}_{2}, \mathrm{ErCuAs}_{2}, \mathrm{HoCuAs}_{2}$,

$0003 \mathrm{DyCuAs}_{2}, \mathrm{TbCuAs}_{2}, \mathrm{YCuAs}_{2}, \mathrm{YCuP}_{2}, \mathrm{HoCuSb}_{2}, \mathrm{SmCuSb}_{2}, \mathrm{LaCuSb}_{2}, \mathrm{DyCuSb}_{2}, \mathrm{YCuSb}_{2}$,

$\mathrm{PrBi}_{2} \mathrm{Au}, \mathrm{TbCuSb}_{2}, \mathrm{Ca}(\mathrm{BeGe})_{2}, \mathrm{SmBi}_{2} \mathrm{Au}, \mathrm{NdCuSb}_{2}, \mathrm{ErCuSb}_{2}, \mathrm{TmCuSb}_{2}, \mathrm{HfSb}_{2}, \mathrm{Ba}(\mathrm{ZnSn})_{2}$,

$\mathrm{LuCuSb}_{2}, \mathrm{CoPPd}, \mathrm{Sr}(\mathrm{SnAu})_{2}, \mathrm{Sr}(\mathrm{AsPt})_{2}, \mathrm{Th}(\mathrm{NiP})_{2}$

$0011 \quad \quad|| \mathrm{YNiBC}, \mathrm{Ce}(\mathrm{PRh})_{2}, \mathrm{LaBiS}_{2} \mathrm{O}(\mathrm{ICSD}: 252466)$

$0013 \quad \|$ ThCuPO, $\mathrm{ScCoC}_{2}, \mathrm{TiCd}, \mathrm{Sr}(\mathrm{AlAu})_{2}, \mathrm{PrCuSb}_{2}, \mathrm{NdAsRuO}, \mathrm{SmAsRuO}, \mathrm{LaAgSb}_{2}, \mathrm{SrCuSeF}$ $\mathrm{CeFeSb}_{2}$

\begin{tabular}{|c|c|}
\hline \multicolumn{2}{|r|}{ Space Group 130: P4/ncc } \\
\hline $\mathbb{Z}_{4}$ & Materials \\
\hline 1 & $\| \mathrm{Y}_{5} \mathrm{Ga}_{3}$ \\
\hline \multicolumn{2}{|r|}{ Space Group 131: $P 4_{2} / m m c$} \\
\hline $\mathbb{Z}_{2,2,2,4}$ & Materials \\
\hline 0003 & HoCoC, DyCoC, TbCoC, $\mathrm{Ca}\left(\mathrm{PtO}_{2}\right)_{2}, \mathrm{SrPbF}_{6}$ \\
\hline 1103 & \begin{tabular}{l||l} 
& TePdI \\
\end{tabular} \\
\hline \multicolumn{2}{|r|}{ Space Group 133: $P 4_{2} / n b c$} \\
\hline $\mathbb{Z}_{4}$ & Materials \\
\hline 3 & $\| \mathrm{Ta}_{3} \mathrm{P}$ \\
\hline \multicolumn{2}{|r|}{ Space Group 135: $P 4_{2} / m b c$} \\
\hline $\mathbb{Z}_{4}$ & Materials \\
\hline 1 & $\| \mathrm{YbB}_{2} \mathrm{C}, \mathrm{Cs}$ \\
\hline 3 & $\mathrm{LuB}_{2} \mathrm{C}, \mathrm{TmB}_{2} \mathrm{C}, \mathrm{HoB}_{2} \mathrm{C}, \mathrm{DyB}_{2} \mathrm{C}, \mathrm{ScB}_{2} \mathrm{C}$ \\
\hline \multicolumn{2}{|r|}{ Space Group 136: $P 4_{2} / m n m$} \\
\hline $\mathbb{Z}_{4}$ & Materials \\
\hline 1 & $\| \mathrm{Ga}_{3} \mathrm{Co}, \mathrm{Zr}_{2} \mathrm{In}_{5} \mathrm{Ni}, \mathrm{Nd}_{2} \mathrm{Co}_{14} \mathrm{~B}, \mathrm{~Tb}_{2} \mathrm{Fe}_{14} \mathrm{~B}$ \\
\hline 3 & $\begin{array}{c}\text { II } \mathrm{CsAg}_{5} \mathrm{Te}_{3}, \mathrm{CsAg}_{5} \mathrm{Se}_{3}, \mathrm{Ca}\left(\mathrm{Cu}_{2} \mathrm{P}\right)_{2}, \mathrm{Na}_{10}\left(\mathrm{Ga}_{2} \mathrm{Sn}\right)_{3}, \mathrm{Ba}(\mathrm{TlHg})_{2}, \mathrm{Yb}\left(\mathrm{Ni}_{2} \mathrm{P}\right)_{2}, \mathrm{Ca}_{3} \mathrm{Cd}_{2}, \mathrm{Y}_{3} \mathrm{Al}_{2}, \\
\mathrm{Sr}_{3} \mathrm{Li}_{2}, \mathrm{Zr}_{3} \mathrm{Al}_{2}, \mathrm{Tm}_{2} \mathrm{Fe}_{14} \mathrm{~B}\end{array}$ \\
\hline \multicolumn{2}{|r|}{ Space Group 137: $P 4_{2} / n m c$} \\
\hline $\mathbb{Z}_{4}$ & Materials \\
\hline 1 & $\mathrm{Y}_{14}(\mathrm{InRh})_{3}$ \\
\hline \multicolumn{2}{|r|}{ Space Group 139: $I 4 / \mathrm{mmm}$} \\
\hline $\mathbb{Z}_{2,8}$ & Materials \\
\hline 01 & $\begin{array}{c}\mathrm{Ba}(\mathrm{SbRu})_{2}, \mathrm{Yb}(\mathrm{SiRu})_{2}, \mathrm{Sr}(\mathrm{GeRu})_{2}, \mathrm{Ca}(\mathrm{GeRu})_{2}, \mathrm{Th}(\mathrm{SiOs})_{2}, \mathrm{CeB}_{2} \mathrm{Ir}_{2} \mathrm{C}, \mathrm{Th}(\mathrm{GeOs})_{2}, \mathrm{CeB}_{2} \mathrm{Rh}_{2} \mathrm{C}, \\
\mathrm{Sr}(\mathrm{PRu})_{2}, \mathrm{Th}(\mathrm{GeRu})_{2}, \mathrm{Ba}(\mathrm{AsRh})_{2}, \mathrm{Th}(\mathrm{FeGe})_{2}, \mathrm{La}_{2} \mathrm{Sb}, \mathrm{U}(\mathrm{NiGe})_{2}\end{array}$ \\
\hline 03 & $\begin{array}{c}\| \mathrm{CdHg}_{2}, \mathrm{MgHg}_{2}, \mathrm{SmCuP}_{2}, \mathrm{Ca}(\mathrm{AlZn})_{2}, \mathrm{La}_{2} \mathrm{PdO}_{4}, \mathrm{Yb}(\mathrm{MnSi})_{2}, \mathrm{Sr}_{2} \mathrm{Cu}_{2} \mathrm{Ni}(\mathrm{SO})_{2}, \mathrm{MgPd}_{3}, \\
\mathrm{Ni}_{18} \mathrm{Bi}_{3} \mathrm{AsS}_{16}\end{array}$ \\
\hline 05 & $\begin{array}{c}\| \mathrm{Ba}(\mathrm{GeRh})_{2}, \mathrm{Sr}(\mathrm{SbRu})_{2}, \mathrm{Ti}_{2} \mathrm{Pd}, \mathrm{Sr}(\mathrm{AsRu})_{2}, \mathrm{Sr}(\mathrm{CoSi})_{2}, \mathrm{Ca}(\mathrm{FeP})_{2}, \mathrm{DyMgSn}, \mathrm{HoMgSn}, \\
\mathrm{Sr}(\mathrm{CoGe})_{2}, \mathrm{Ba}(\mathrm{CoGe})_{2}, \mathrm{BiSe}, \mathrm{Th}(\mathrm{SiRu})_{2}, \mathrm{Ba}_{2} \mathrm{Bi}, \mathrm{U}(\mathrm{SiOs})_{2}, \mathrm{Sr}(\mathrm{PRh})_{2}, \mathrm{Ba}(\mathrm{PRh})_{2}, \mathrm{U}(\mathrm{FeGe})_{2}, \\
\mathrm{Th}(\mathrm{FeSi})_{2}, \mathrm{Sr}(\mathrm{CoAs})_{2}, \mathrm{~Tb}_{2} \mathrm{Mg}, \mathrm{Zr}(\mathrm{FeSi})_{2}, \mathrm{Er}_{2} \mathrm{Mg}, \mathrm{ScSi}_{3} \mathrm{Ni}_{2}\end{array}$ \\
\hline
\end{tabular}


Table V - continued

\begin{tabular}{|c|c|}
\hline SI & Topological Insulators \\
\hline 07 & $\begin{array}{c}\| \mathrm{ZrAg}_{2}, \mathrm{Cd}_{2} \mathrm{Hg}, \mathrm{BeAu}_{2}, \mathrm{Zr}\left(\mathrm{VGa}_{2}\right)_{2}, \mathrm{Be}_{12} \mathrm{~W}, \mathrm{TiH}_{2}, \mathrm{Hf}\left(\mathrm{VGa}_{2}\right)_{2}, \mathrm{InPd}_{3}(\mathrm{ICSD}: 247188), \mathrm{Bi}_{2} \mathrm{Pd}, \\
\mathrm{Be}_{12} \mathrm{Mo}, \mathrm{Ca}(\mathrm{SiAu})_{2}, \mathrm{Sr}(\mathrm{SiAu})_{2}, \mathrm{ThSiS}, \mathrm{Ca}(\mathrm{PPd})_{2}, \mathrm{Sr}(\mathrm{PPd})_{2}, \mathrm{ZrGa}(\mathrm{ICSD}: 635698), \\
\mathrm{HfAl}_{3}(\mathrm{ICSD}: 608082), \mathrm{ZrAl}_{3}(\mathrm{ICSD}: 107130), \mathrm{ZrIn}_{3}(\mathrm{ICSD}: 640719), \mathrm{Sr}(\mathrm{InHg})_{2}, \mathrm{Sr}(\mathrm{CrAs})_{2}, \\
\mathrm{Sm}\left(\mathrm{NiGe}_{3}, \mathrm{Pt}(\mathrm{NCl})_{4}, \mathrm{U}_{2} \mathrm{TeO}_{2}, \mathrm{Ce}_{2} \mathrm{Ga}_{10} \mathrm{Ni}\right.\end{array}$ \\
\hline 11 & $\begin{array}{c}\| \mathrm{Mg}, \mathrm{Ba}(\mathrm{MgGe})_{2}, \mathrm{Sr}(\mathrm{ZnGe})_{2}, \mathrm{Ba}(\mathrm{CuAs})_{2}, \mathrm{Ca}(\mathrm{CuP})_{2}, \mathrm{Sr}(\mathrm{CuAs})_{2}, \mathrm{HfCuGe}, \mathrm{Ca}\left(\mathrm{Al}_{2} \mathrm{Cu}\right)_{4}, \\
\mathrm{Ti}_{2} \mathrm{H}_{4} \mathrm{Pd}, \mathrm{BaBi}_{4}\left(\mathrm{BrO}_{3}\right)_{2}\end{array}$ \\
\hline 13 & $\begin{array}{c}\| \mathrm{Yb}(\mathrm{PRu})_{2}, \mathrm{Sr}(\mathrm{GeIr})_{2}, \mathrm{Ca}(\mathrm{PRu})_{2}, \mathrm{Ca}(\mathrm{GeIr})_{2}, \mathrm{Yb}(\mathrm{GeRh})_{2}, \mathrm{Ca}(\mathrm{SiRh})_{2}, \mathrm{Yb}(\mathrm{SiRh})_{2}, \mathrm{Sr}(\mathrm{GeRh})_{2}, \\
\mathrm{Ca}(\mathrm{GeRh})_{2}, \mathrm{Ca}(\mathrm{AsRu})_{2}, \mathrm{Yb}(\mathrm{CoGe})_{2}, \mathrm{Ca}(\mathrm{CoSi})_{2}, \mathrm{Ca}(\mathrm{CoGe})_{2}, \mathrm{Yb}(\mathrm{CoSi})_{2}, \mathrm{TmZrSb}, \mathrm{ErZrSb}, \\
\mathrm{HoZrSb}, \mathrm{DyZrSb}, \mathrm{TbZrSb}, \mathrm{LuZrSb}, \mathrm{Ca}_{11} \mathrm{Bi}_{10}, \mathrm{SmS}, \mathrm{YS}, \mathrm{Yb}_{11} \mathrm{Sb}_{10}\end{array}$ \\
\hline 15 & $\begin{aligned} \| \mathrm{TlPd}_{3}(\mathrm{ICSD}: & 247272), \mathrm{Hg}, \mathrm{YbAu}_{2}, \mathrm{YbPd}_{2}, \mathrm{CaPd}_{2}, \mathrm{YbAg}_{2}, \mathrm{Ca}_{3} \mathrm{Cu}_{2}\left(\mathrm{BrO}_{2}\right)_{2}, \mathrm{Ca}(\mathrm{ZnGe})_{2}, \\
& \mathrm{ZrPd}_{2}, \mathrm{Sr}(\mathrm{CdGe})_{2}, \mathrm{TiPd}_{2}, \mathrm{Be}_{12} \mathrm{Cr}, \mathrm{Yb}\left(\mathrm{Al}_{2} \mathrm{Mo}_{2}, \mathrm{U}\left(\mathrm{MnSi}_{2}\right)_{2}\right.\end{aligned}$ \\
\hline 17 & $\| \mathrm{Hf}_{2} \mathrm{Pd}, \mathrm{Zr}_{2} \mathrm{Pd}, \mathrm{Ba}(\mathrm{FeP})_{2}, \mathrm{Sr}(\mathrm{FeAs})_{2}, \mathrm{Ca}(\mathrm{FeAs})_{2}, \mathrm{Ce}(\mathrm{CoP})_{2}$ \\
\hline & Space Group 140: $I 4 / \mathrm{mcm}$ \\
\hline $\mathbb{Z}_{2,8}$ & Materials \\
\hline 01 & $\| \mathrm{Hf}_{2} \mathrm{Si}, \mathrm{Hf}_{2} \mathrm{Ge}(\mathrm{ICSD}: 636548), \mathrm{Ta}_{2} \mathrm{Be}, \mathrm{Ta}_{5} \mathrm{Ga}_{3}, \mathrm{U}_{3} \mathrm{Si}$ \\
\hline 03 & \begin{tabular}{l||l}
$\mathrm{SiB}_{2} \mathrm{Mo}_{5}$ \\
\end{tabular} \\
\hline 05 & $\| \mathrm{Sr}_{5} \mathrm{Ge}_{3}, \mathrm{~Tb}_{5} \mathrm{SbAu}_{2}, \mathrm{~Tb}_{6} \mathrm{Al}_{3} \mathrm{Si}$ \\
\hline 07 & $\mathrm{TiSb}_{2}, \mathrm{Ta}_{2} \mathrm{Si}, \mathrm{Tl}_{2} \mathrm{Pt}, \mathrm{In}_{5} \mathrm{Bi}_{3}, \mathrm{Sr}_{5} \mathrm{Si}_{3}(\mathrm{ICSD}: 160105)$ \\
\hline 11 & $\| \mathrm{Hf}_{2} \mathrm{Ni}, \mathrm{PdPb}_{2}, \mathrm{PtPb}_{2}, \mathrm{Zr}_{2} \mathrm{Ni}$ \\
\hline 13 & $\| \mathrm{Ti}_{4} \mathrm{FeBi}_{2}, \mathrm{Mn}_{5} \mathrm{~B}_{2} \mathrm{P}$ \\
\hline 15 & $\| \mathrm{Sb}, \mathrm{Ca}\left(\mathrm{Ni}_{2} \mathrm{Sn}\right)_{2}$ \\
\hline 17 & || $\mathrm{Sc}$ \\
\hline & Space Group 142: I4 $1 /$ acd \\
\hline $\mathbb{Z}_{4}$ & Materials \\
\hline 3 & $\| \mathrm{ZnAu}_{3}$ \\
\hline & Space Group 147: $P \overline{3}$ \\
\hline $\mathbb{Z}_{2,2,2,4}$ & Materials \\
\hline 0011 & $\| \mathrm{In}_{3} \mathrm{Au}_{7}$ \\
\hline 0013 & $\| \mathrm{Ge}_{9} \mathrm{Pd}_{25}$ \\
\hline & "Space Group 148: $R \overline{3}$ \\
\hline $\mathbb{Z}_{2,2,2,4}$ & Materials \\
\hline 0001 & $\| \mathrm{Zr}_{3} \mathrm{Pd}_{4}, \mathrm{Ti}_{3} \mathrm{Ni}_{4}$ \\
\hline 0003 & $\begin{array}{r}\| \mathrm{Bi}_{14} \mathrm{Te}_{13} \mathrm{~S}_{8}, \mathrm{Tl}_{6} \mathrm{TeO}_{12}, \mathrm{AgBiO}_{3}, \mathrm{Mo}_{6} \mathrm{PbSe}_{8}, \mathrm{Yb}\left(\mathrm{Mo}_{3} \mathrm{~S}_{4}\right)_{2}, \mathrm{Ca}\left(\mathrm{Mo}_{3} \mathrm{~S}_{4}\right)_{2}, \mathrm{Te}_{7} \mathrm{Pd}_{20}, \mathrm{Ba}\left(\mathrm{Mo}_{3} \mathrm{~S}_{4}\right)_{2}, \\
\mathrm{Sr}_{3} \mathrm{Ag}_{2}, \mathrm{Sr}\left(\mathrm{Mo}_{3} \mathrm{~S}_{4}\right)_{2}, \mathrm{Mo}_{6} \mathrm{PbS}_{8}, \mathrm{TiCl}_{3}, \mathrm{Cr}_{2} \mathrm{Se}_{3}\end{array}$ \\
\hline 1111 & || $\mathrm{CrGeTe}_{3}, \mathrm{Mo}_{3} \mathrm{Se}_{4}, \mathrm{Pr}_{3} \mathrm{Pt}_{4}, \mathrm{RbMo}_{6} \mathrm{Se}_{7}$ \\
\hline 1113 & $\| \mathrm{Sc}_{7} \mathrm{NCl}_{12}, \mathrm{Ti}_{3} \mathrm{NiS}_{6}, \mathrm{Te}_{4} \mathrm{Mo}_{3}, \mathrm{CsTe}_{7} \mathrm{Mo}_{6}$ \\
\hline & Space Group 162: $P \overline{3} 1 \mathrm{~m}$ \\
\hline $\mathbb{Z}_{2,2,2,4}$ & Materials \\
\hline 0003 & $\| \mathrm{Ag}_{3} \mathrm{O}$ \\
\hline 0013 & $\| \mathrm{Ti}_{6} \mathrm{O}$ \\
\hline & Space Group 163: $P \overline{3} 1 c$ \\
\hline $\mathbb{Z}_{4}$ & Materials \\
\hline 1 & $\| \mathrm{NiMoP}_{8}, \mathrm{Ti}_{3} \mathrm{NiS}_{6}, \mathrm{Ti}_{6} \mathrm{O}$ \\
\hline & Space Group 164: $P \overline{3} m 1$ \\
\hline $\mathbb{Z}_{2,2,2,4}$ & Materials \\
\hline 0001 & $\| \mathrm{Zr}_{5} \mathrm{Te}_{6}$ \\
\hline 0003 & $\begin{array}{c}\mathrm{Bi}_{2} \mathrm{~Pb}_{2} \mathrm{Se}_{5}, \mathrm{Ca}(\mathrm{MgBi})_{2}, \mathrm{Yb}(\mathrm{MgBi})_{2}, \| \mathrm{Sb}_{2} \mathrm{Te}, \mathrm{Ge}_{2} \mathrm{Sb}_{2} \mathrm{Te}_{5}, \mathrm{Mg}_{3} \mathrm{Bi}_{2}[109], \mathrm{Li}_{3} \mathrm{YBi}_{2}, \mathrm{Li}_{3} \mathrm{SmBi}_{2}, \\
\mathrm{Li}_{3} \mathrm{PrBi}_{2}, \mathrm{Ta}_{2} \mathrm{C}, \mathrm{V}_{2} \mathrm{C}, \mathrm{Nb}_{2} \mathrm{C}, \mathrm{SiTe}_{2}, \mathrm{Te}_{2} \mathrm{Pd}, \mathrm{Te}_{2} \mathrm{Pt}, \mathrm{W}_{2} \mathrm{C}, \mathrm{NiTe}_{2}, \mathrm{Li}_{2} \mathrm{ZnSi}(\mathrm{ICSD}: 642380), \mathrm{Cu}_{3} \mathrm{P}, \\
\mathrm{PrAlSi}_{2}, \mathrm{Ag}_{2} \mathrm{O}, \mathrm{LaAlSi}_{2}, \mathrm{Bi}_{8} \mathrm{Se}_{7}, \mathrm{Cr}_{3} \mathrm{Te}_{4}\end{array}$ \\
\hline 0011 & $\begin{array}{c}\mathrm{Bi}_{4} \mathrm{Te}_{7} \mathrm{~Pb}, \| \mathrm{BaSn}_{2}[108], \mathrm{GeBi}_{4} \mathrm{Te}_{7}, \mathrm{Ge}_{5}\left(\mathrm{Te}_{4} \mathrm{As}\right)_{2}, \mathrm{Ta}_{2} \mathrm{CS}_{2}, \mathrm{Nb}_{2} \mathrm{CS}_{2}, \mathrm{Sr}(\mathrm{MnAs})_{2}, \mathrm{Ba}_{3} \mathrm{CaRu}_{2} \mathrm{O}_{9}, \\
\mathrm{Ca}(\mathrm{MnP})_{2}, \mathrm{Ca}(\mathrm{MnAs})_{2}\end{array}$ \\
\hline 0013 & $\mathrm{GeSb}_{4} \mathrm{Te}_{7}, \| \mathrm{SbTe}, \mathrm{SrGe}_{2}, \mathrm{CaGe}_{2}, \mathrm{CaSi}_{2}, \mathrm{CoO}_{2}(\mathrm{ICSD}: 53994), \mathrm{CoO}_{2}(\mathrm{ICSD}: 26763)$ \\
\hline & $\begin{array}{l}\text { Space Group 165: } P \overline{3} c 1 \\
\end{array}$ \\
\hline $\mathbb{Z}_{4}$ & Materials \\
\hline 3 & $\mathrm{NdH}_{3}, \mathrm{YH}_{3}, \mathrm{TbH}_{3}, \mathrm{HoH}_{3}, \| \mathrm{Cu}_{3} \mathrm{As}(\mathrm{ICSD}: 26776)$ \\
\hline & Space Group 166: $R \overline{3} m$ \\
\hline $\mathbb{Z}_{2,2,2,4}$ & Materials \\
\hline
\end{tabular}


Table V - continued

\begin{tabular}{|c|c|}
\hline \multicolumn{2}{|r|}{ Topological Insulators } \\
\hline 0001 & $\begin{array}{c}\mathrm{TlSbTe}_{2}\left[125,126, \mathrm{TlBiS}_{2} \underset{125}{[126]}, \| \mathrm{Ba}_{7} \mathrm{Al}_{10}, \mathrm{CaGe}_{2}(\mathrm{ICSD}: 110107), \mathrm{CaSi}_{2}(\mathrm{ICSD}: 32006),\right. \\
\mathrm{Ti}_{8} \mathrm{C}_{5}, \mathrm{BaPb}_{3}, \mathrm{Fe}_{7} \mathrm{Mo}_{6}, \mathrm{Fe}_{7} \mathrm{~W}_{6}, \mathrm{Ni}_{17} \mathrm{~S}_{18}\end{array}$ \\
\hline 0003 & 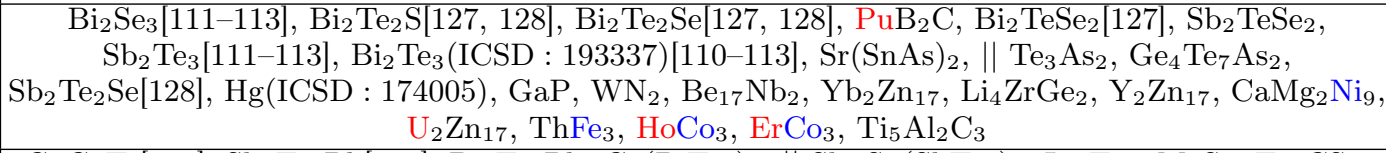 \\
\hline 1111 & $\begin{array}{c}\mathrm{GaGeTe}[129], \mathrm{Sb}_{2} \mathrm{Te}_{4} \mathrm{~Pb}[130], \mathrm{Bi}_{2} \mathrm{Te}_{4} \mathrm{~Pb}, \mathrm{Ge}\left(\mathrm{BiTe}_{2}\right)_{2}, \| \mathrm{Sb}, \mathrm{Sn}\left(\mathrm{SbTe}_{2}\right)_{2}, \mathrm{In}_{2} \mathrm{Te}_{3}, \mathrm{MoS}_{2}, \mathrm{Ta}_{2} \mathrm{CS}_{2}, \\
\mathrm{Mg}_{2} \mathrm{SiNi}_{3}, \mathrm{Li}_{2} \mathrm{Si}, \mathrm{B}_{2} \mathrm{Mo}, \mathrm{TePt}, \mathrm{Hf}_{3}\left(\mathrm{Al}_{2} \mathrm{C}_{3}\right)_{2}, \mathrm{Li}_{8} \mathrm{~Pb}_{3}, \mathrm{~Tb}_{2} \mathrm{C}, \mathrm{Dy}_{2} \mathrm{C}, \mathrm{Ho}_{2} \mathrm{C}, \mathrm{Te}_{3} \mathrm{Pt}_{2}, \mathrm{Y}_{2} \mathrm{C}, \mathrm{BaRuO}_{3}, \\
\mathrm{Ba}_{7}(\mathrm{BIr})_{12}, \mathrm{UB}_{2} \mathrm{C}\end{array}$ \\
\hline 1113 & $\begin{array}{c}\mathrm{Ca}(\mathrm{GaAs})_{2}, \| \mathrm{As} 115, \mathrm{Sb}_{8} \mathrm{Te}_{3}, \mathrm{CaGe}_{2}(\mathrm{ICSD}: 185658), \mathrm{Sb}_{16} \mathrm{Te}_{3}, \mathrm{CaSi}_{2}(\mathrm{ICSD}: 193539) \\
\mathrm{ErAl}_{4} \mathrm{Ge}_{2} \mathrm{Au}, \mathrm{TaCuN}_{2}(\mathrm{ICSD}: 190938), \mathrm{TiS}, \mathrm{CsGa}_{7}, \mathrm{RbGa}_{7}, \mathrm{TaCo}_{3}, \mathrm{SrSn}_{3}\end{array}$ \\
\hline \multicolumn{2}{|r|}{ Space Group 167: $R \overline{3} c$} \\
\hline $\mathbb{Z}_{4}$ & Materials \\
\hline 1 & \multirow{2}{*}{$\frac{\| \mathrm{YbGa}_{7} \mathrm{Au}_{3}, \mathrm{~V}_{2} \mathrm{O}_{3}}{\mathrm{Mo}_{15} \mathrm{Se}_{19}, \mathrm{Ni}_{8} \mathrm{P}_{3}, \mathrm{Fe}_{2} \mathrm{O}_{3}, \mathrm{Rb}_{4} \mathrm{CdBr}_{6}}$} \\
\hline 3 & \\
\hline \multicolumn{2}{|r|}{ Space Group 176: $P 6_{3} / m$} \\
\hline $\mathbb{Z}_{12}$ & Materials \\
\hline 11 & $\| \mathrm{DySi}_{3} \mathrm{Rh}_{5}, \mathrm{ErSi}_{3} \mathrm{Rh}_{5}$ \\
\hline \multicolumn{2}{|r|}{ Space Group 191: $P 6 / \mathrm{mmm}$} \\
\hline $\mathbb{Z}_{6,12}$ & Materials \\
\hline 03 & $\mathrm{Li}_{2} \mathrm{Pd}, \mathrm{Li}_{2} \mathrm{Pt}[74], \mathrm{CaNi}_{4} \mathrm{~B}$ \\
\hline 09 & $\| \mathrm{Ti}, \mathrm{Hf}, \mathrm{ZrTi}_{2}, \mathrm{YCo}_{4} \mathrm{~B}$ \\
\hline 19 & $\| \mathrm{BaSi}_{2}, \mathrm{CaSi}_{2}$ \\
\hline 111 & $\mathrm{OsN}_{2}, \mathrm{CaPd}_{5}$ \\
\hline 27 & $\| \mathrm{DyGa}_{2} \mathrm{Cu}_{3}$ \\
\hline 29 & $\mathrm{SrB}_{2}, \mathrm{CaB}_{2}, \mathrm{YbB}_{2}, \mathrm{BaGa}_{2}\left[4, \mathrm{La}_{10} \mathrm{Si}_{8} \mathrm{O}_{3}, \mathrm{CeZn}_{3} \mathrm{Cu}_{2}\right.$ \\
\hline 211 & $\| \mathrm{NdIr}_{5}, \mathrm{Sm}_{2} \mathrm{Co}_{7} \mathrm{~B}_{3}$ \\
\hline 33 & $\| \mathrm{LuB}_{2} \mathrm{Ir}_{3}, \mathrm{TbB}_{2} \mathrm{Ir}_{3}, \mathrm{NdB}_{2} \operatorname{Ir}_{3}$ \\
\hline 35 & $\| \mathrm{BaSn}_{5}$ \\
\hline 39 & $\mathrm{LaB}_{2} \mathrm{Ir}_{3}, \mathrm{PrB}_{2} \mathrm{Ir}_{3}$ \\
\hline 41 & $\| \mathrm{CeB}_{2} \mathrm{Ru}_{3}$ \\
\hline 45 & $\| \mathrm{UGa}_{2}$ \\
\hline 47 & $\| \mathrm{CaZn}_{5}$ \\
\hline 49 & $\mathrm{UB}_{2}, \mathrm{CeGa}_{2}$ \\
\hline 511 & $\| \mathrm{Mg}$ \\
\hline \multicolumn{2}{|r|}{ Space Group 193: $P 6_{3} / \mathrm{mcm}$} \\
\hline $\mathbb{Z}_{12}$ & Materials \\
\hline 1 & $\mathrm{Hf}_{5} \mathrm{Ge}_{3}, \mathrm{Zr}_{5} \mathrm{Sn}_{3}, \mathrm{Zr}_{5} \mathrm{~Pb}_{3}, \mathrm{Hf}_{5} \mathrm{Sn}_{3}, \mathrm{Ti}_{5} \mathrm{Sn}_{3}, \mathrm{Ti}_{5} \mathrm{Si}_{3}, \mathrm{Ti}_{5} \mathrm{Ge}_{3}$ \\
\hline 5 & $\| \mathrm{Rh}_{7}\left(\mathrm{PbO}_{5}\right)_{3}$ \\
\hline & Space Group 194: $P 6_{3} / m m c$ \\
\hline $\mathbb{Z}_{12}$ & Materials \\
\hline 1 & $\mathrm{Ta}_{3} \mathrm{AlC}_{2}[58], \mathrm{Zr}_{2} \mathrm{NiAs}_{2}, \mathrm{Ti}_{4} \mathrm{AlN}_{3}, \mathrm{~K}, \mathrm{Rb}, \mathrm{Cs}(\mathrm{ICSD}: 109023), \mathrm{PtN}_{2}, \mathrm{HfCr}_{2}(\mathrm{ICSD}: 109213)$ \\
\hline 3 & $\| \mathrm{Nb}_{2} \mathrm{CS}, \mathrm{YAlPd}_{2}, \mathrm{CaSiNi}_{2}, \mathrm{Ca}_{2} \mathrm{IN}, \mathrm{DyInPt}_{2}, \mathrm{TbInPt}_{2}, \mathrm{YInPt}_{2}, \mathrm{Be}_{2} \mathrm{~V}, \mathrm{Ba}_{3} \mathrm{ZnRu}_{2} \mathrm{O}_{9}$ \\
\hline 5 & $\|$ PbS, FeSn, CeNiGe, MnSb \\
\hline 7 & 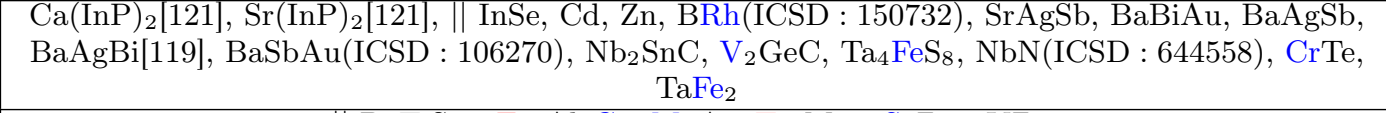 \\
\hline 9 & $\| \mathrm{BaTiSe}_{3}, \mathrm{Er}_{2} \mathrm{Al}_{3} \mathrm{Co}, \mathrm{MnAs}, \mathrm{TmMg}_{2}, \mathrm{ScZn}_{2}, \mathrm{YZn}_{2}$ \\
\hline 11 & 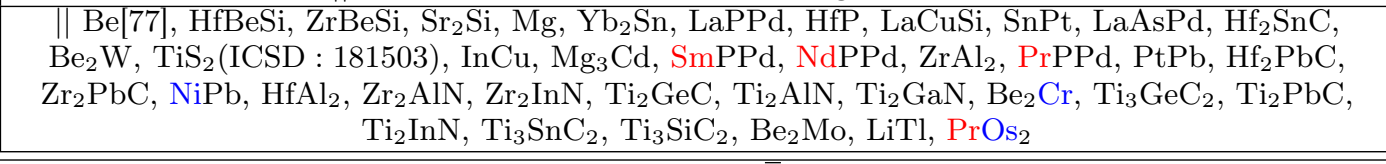 \\
\hline & Space Group 200: $P m \overline{3}$ \\
\hline $\mathbb{Z}_{2,2,2,4}$ & Materials \\
\hline 0003 & $\| \mathrm{Mg}_{2} \mathrm{Zn}_{11}$ \\
\hline 1111 & $\| \mathrm{Mg}_{30} \mathrm{Al}_{24} \mathrm{Ag}_{19}$ \\
\hline & Space Group 204: Im $\overline{3}$ \\
\hline $\mathbb{Z}_{2,2,2,4}$ & Materials \\
\hline
\end{tabular}


Table V - continued

\begin{tabular}{|c|c|}
\hline \multicolumn{2}{|r|}{ Topological Insulators } \\
\hline 0001 & $\| \mathrm{Ba}\left(\mathrm{Ge}_{3} \mathrm{Pt}\right)_{4}, \mathrm{Cs}_{2} \mathrm{Hg}_{27}$ \\
\hline 1113 & $\| \mathrm{SrC}_{10}$ \\
\hline \multicolumn{2}{|r|}{ Space Group 205: $P a \overline{3}$} \\
\hline $\mathbb{Z}_{4}$ & Materials \\
\hline 1 & $\mathrm{OsS}_{2}, \mathrm{Sb}_{2} \mathrm{Pd}, \mathrm{NiSe}_{2}, \mathrm{NiS}_{2}(\mathrm{ICSD}: 40328), \mathrm{NiS}_{2}(\mathrm{ICSD}: 68169)$ \\
\hline \multicolumn{2}{|r|}{ 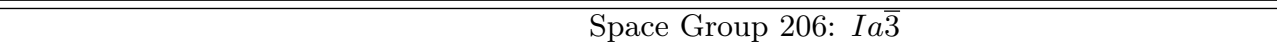 } \\
\hline \multicolumn{2}{|r|}{ Materials } \\
\hline 0003 & $\mathrm{Zn}_{3} \mathrm{~N}_{2}, \mathrm{Cd}_{3} \mathrm{~N}_{2}$ \\
\hline \multicolumn{2}{|r|}{ "Space Group 215: $P \overline{4} 3 m$} \\
\hline \multicolumn{2}{|r|}{ Materials } \\
\hline 1 & $\mathrm{Cu}_{3} \mathrm{AsS}_{4}, \mathrm{In}_{4} \mathrm{Au}_{9}$ \\
\hline \multicolumn{2}{|r|}{ Space Group 216: $F \overline{4} 3 m$} \\
\hline $\mathbb{Z}_{2}$ & Materials \\
\hline
\end{tabular}

$\mathrm{NaCuO}, \mathrm{NaAgO}$ [57], || InN, HgS[86], RbCuO[57], RbAgO, KAgO, KCuO[57], LiCuO, TlN [87],

LiPrSn, LiNdSn, CuF, UNi, ReN, $\mathrm{Mn}_{2} \mathrm{Ge}, \mathrm{Li}_{2} \mathrm{GaAu}, \mathrm{ZnNi}, \mathrm{Li}_{2} \mathrm{GaRh}, \mathrm{Li}_{2} \mathrm{AlRh}, \mathrm{Li}_{2} \mathrm{GaIr}_{\text {, }}$

$1 \quad$ ThNiSn(ICSD : 646771), Li $\mathrm{LnRh}_{2} \mathrm{Li}_{2} \mathrm{InIr}, \mathrm{AgN}, \mathrm{HfNiSn}(\mathrm{ICSD}: 104249), \mathrm{ErNi}_{4} \mathrm{Au}, \mathrm{Er}_{4} \mathrm{InRh}_{\text {, }}$ $\mathrm{La}_{4} \mathrm{MgRu}, \mathrm{UNi}_{4} \mathrm{Sn}, \mathrm{Tb}_{4} \mathrm{MgIr}_{\mathrm{LaInPt}}, \mathrm{DyNi}_{4} \mathrm{Au}, \mathrm{UPt}_{5}, \mathrm{HfNi}_{5}, \mathrm{TbNi}_{4} \mathrm{Au}, \mathrm{YbCdNi}_{4}, \mathrm{HoNi}_{4} \mathrm{Au}_{\text {, }}$ $\mathrm{MnNiP}, \mathrm{Li}_{21} \mathrm{Si}_{5}, \mathrm{NdCu}_{4} \mathrm{Ag}$

\begin{tabular}{|c|c|}
\hline \multicolumn{2}{|r|}{ Space Group 217: $I \overline{4} 3 m$} \\
\hline $\mathbb{Z}_{2}$ & Materials \\
\hline 1 & $\mathrm{Cu}_{3} \mathrm{SbS}_{3}, \mathrm{Cu}_{3} \mathrm{AsS}_{3}$ \\
\hline \multicolumn{2}{|r|}{ Space Group 220: $I \overline{4} 3 d$} \\
\hline $\mathbb{Z}_{2}$ & Materials \\
\hline 1 & $\begin{array}{c}\| \mathrm{Sc}_{2} \mathrm{C}_{3}, \mathrm{Tm}_{2} \mathrm{C}_{3}, \mathrm{Dy}_{2} \mathrm{C}_{3}, \mathrm{Ho}_{2} \mathrm{C}_{3}, \mathrm{Cu}_{3} \mathrm{As}, \mathrm{La}_{2} \mathrm{C}_{3}, \mathrm{Er}_{2} \mathrm{C}_{3}, \mathrm{~Tb}_{2} \mathrm{C}_{3}, \mathrm{Sm}_{2} \mathrm{C}_{3}, \mathrm{Lu}_{2} \mathrm{C}_{3}, \mathrm{Nd}_{2} \mathrm{C}_{3}, \mathrm{Pr}_{2} \mathrm{C}_{3}, \\
\mathrm{Y}_{2} \mathrm{C}_{3}, \mathrm{Ga}_{8} \mathrm{Cu}_{3} \mathrm{~W}_{2}, \mathrm{Ga}_{8} \mathrm{Cu}_{3} \mathrm{Mo}_{2}, \mathrm{U}_{3} \mathrm{Ni}_{3} \mathrm{Sb}_{4}, \mathrm{U}_{2} \mathrm{C}_{3}\end{array}$ \\
\hline \multicolumn{2}{|r|}{ Space Group 221: $P m \overline{3} m$} \\
\hline $\mathbb{Z}_{4,8}$ & Materials \\
\hline 01 & Dy ${ }_{3} \mathrm{TlC}, \mathrm{Ho}_{3} \mathrm{TlC}, \mathrm{Tb}_{3} \mathrm{TlC}, \mathrm{Tm}_{3} \mathrm{TlC}, \mathrm{Er}_{3} \mathrm{TlC}, \mathrm{Tm}_{3} \mathrm{InC}, \mathrm{Er}_{3} \mathrm{InC}, \mathrm{TaIr}_{3}, \mathrm{NbIr}_{3}, \mathrm{Tm}_{3} \mathrm{GaC}$ \\
\hline 07 & $\| \mathrm{MgPd}, \mathrm{MgNi}$ \\
\hline 11 & $\| \mathrm{CsSnI}_{3}[58], \mathrm{ZrOs}, \mathrm{ZrRu}, \mathrm{MnV}, \mathrm{CsPbI}_{3}$ \\
\hline 13 & \multirow{2}{*}{\begin{tabular}{|l|l|} 
TbAl, SmAl, DyAl, YAl, CeCd(ICSD : 186646), CeCd(ICSD : 182832) \\
|| $\mathrm{Nd}_{3} \mathrm{GaC}, \mathrm{SnTe}, \mathrm{PbSe}, \mathrm{PbS}, \mathrm{PuSn}_{3}, \mathrm{MoPt}_{3}, \mathrm{USn}_{3}$
\end{tabular}} \\
\hline 17 & \\
\hline 25 & $\mid$\begin{tabular}{l|l} 
& $\mathrm{Ti}_{3} \mathrm{Hg}$ \\
\end{tabular} \\
\hline 31 & $\mathrm{Er}_{3} \mathrm{AlC}\left(\mathrm{ICSD}:\right.$ 606192), $\mathrm{Tb}_{3} \mathrm{AlC}\left(\mathrm{ICSD}:\right.$ 606265), $\mathrm{Pr}_{3} \mathrm{InC}, \mathrm{Pr}_{3} \mathrm{GaC}, \mathrm{Sm}_{3} \mathrm{InC}, \mathrm{YbPd}_{3}, \mathrm{YbPt}_{3}$ \\
\hline 33 & \multirow{2}{*}{$\begin{array}{c}\mathrm{CsGeBr}_{3}[58], \mathrm{KIO}_{3}, \mathrm{TlIO}_{3}, \| \mathrm{HfOs}, \mathrm{HfRu}, \mathrm{TiFe}, \mathrm{TiRu} \\
\| \mathrm{BaB}_{6}[58], \mathrm{TiBe}, \mathrm{ScAl}\end{array}$} \\
\hline 35 & \\
\hline \multicolumn{2}{|r|}{ Space Group 223: $\operatorname{Pm} \overline{3} n$} \\
\hline $\mathbb{Z}_{4}$ & Materials \\
\hline 1 & $\| \mathrm{BaC}_{20}, \mathrm{Ti}_{3} \mathrm{Hg}, \mathrm{Zr}_{3} \mathrm{Hg}, \mathrm{Tl}_{3} \mathrm{Ir}$ \\
\hline 3 & $\mathrm{Ta}_{3} \mathrm{Sb}, \mathrm{Nb}_{3} \mathrm{Sb}, \mathrm{Nb}_{3} \mathrm{Bi}, \mathrm{V}_{3} \mathrm{As}, \mathrm{V}_{3} \mathrm{Sb}$ \\
\hline \multicolumn{2}{|r|}{ Space Group 224: $P n \overline{3} m$} \\
\hline $\mathbb{Z}_{2,2,2,4}$ & Materials \\
\hline 0001 & $\| \mathrm{Cd}_{3} \mathrm{P}_{2}$ \\
\hline \multicolumn{2}{|r|}{ Space Group 225: $F m \overline{3} m$} \\
\hline $\mathbb{Z}_{8}$ & Materials \\
\hline 1 & $\| \mathrm{PaN}, \mathrm{CeO}, \mathrm{Ni}_{21}\left(\mathrm{SnP}_{3}\right)_{2}, \mathrm{CeSe}, \mathrm{Li}_{2} \mathrm{MgSi}, \mathrm{CeS}, \mathrm{Ca}_{7} \mathrm{Ge}, \mathrm{Ce}_{3}\left(\mathrm{Si}_{3} \mathrm{Pd}_{10}\right)_{2}, \mathrm{Cr}_{21}\left(\mathrm{MoC}_{3}\right)_{2}, \mathrm{Cr}_{6} \mathrm{Si}_{7} \mathrm{Ni}_{16}$ \\
\hline 3 & $\| \mathrm{Os}, \operatorname{LiInAg}_{2}, \mathrm{Y}_{6} \mathrm{Fe}_{23}$ \\
\hline 5 & 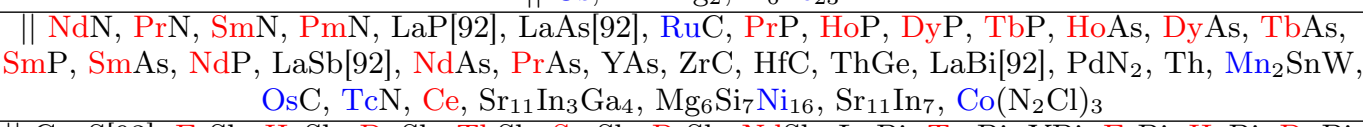 \\
\hline 7 & 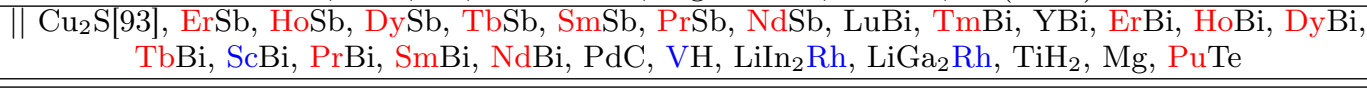 \\
\hline \multicolumn{2}{|r|}{ Space Group 227: $F d \overline{3} m$} \\
\hline $\mathbb{Z}_{4}$ & Materials \\
\hline
\end{tabular}


Table V - continued

\begin{tabular}{|c|c|}
\hline SI & Topological Insulators \\
\hline 1 & $\begin{array}{c}\text { II } \mathrm{TiS}_{2}[57], \mathrm{Ti}_{2} \mathrm{C}, \mathrm{YbPt}_{2}, \mathrm{UOs}_{2}, \mathrm{ThIr}_{2}, \mathrm{LiTiO}_{2}, \mathrm{Ti}_{3} \mathrm{Al}_{2} \mathrm{NiN}, \mathrm{Co}_{2} \mathrm{NiS}_{4}, \mathrm{Mg}_{3}\left(\mathrm{Al}_{9} \mathrm{Cr}_{2}, \mathrm{Hg}_{2} \mathrm{Os}_{2} \mathrm{O}_{7},\right. \\
\mathrm{Yb}_{2} \mathrm{CdS}_{4}, \mathrm{Yb}_{2} \mathrm{MgS}_{4}, \mathrm{Yb}_{2} \mathrm{MgSe}_{4}, \mathrm{Cd}_{2} \mathrm{Ru}_{2} \mathrm{O}_{7}, \mathrm{Yb}_{2} \mathrm{CdSe}_{4}, \mathrm{Ti}_{2} \mathrm{Ni}_{2}, \mathrm{~V}_{2} \mathrm{FeO}_{4}, \mathrm{Yb}_{2} \mathrm{Ti}_{2} \mathrm{O}_{7}, \mathrm{Zr}_{2} \mathrm{Cu}, \\
\mathrm{Ti}_{4} \mathrm{Cu}_{2} \mathrm{O}, \mathrm{Fe}\left(\mathrm{NiS}_{2}\right)_{2}, \mathrm{Cd}_{2} \mathrm{Os}_{2} \mathrm{O}_{7}, \mathrm{Cr}_{2} \mathrm{HgS}_{4}\end{array}$ \\
\hline 3 & $\begin{array}{c}\| \mathrm{Se}, \mathrm{ThAl}_{2}, \mathrm{Nb}_{3} \mathrm{Cr}_{3} \mathrm{C}, \mathrm{CeNi}_{2}, \mathrm{UCo}_{2}, \mathrm{Nb}_{8} \mathrm{Zn}_{4} \mathrm{C}_{3}, \mathrm{Ni}_{2} \mathrm{~W}_{4} \mathrm{C}, \mathrm{Nb}_{3} \mathrm{Co}_{2} \mathrm{Si}, \mathrm{U}\left(\mathrm{Al}_{10} \mathrm{~V}\right)_{2}, \mathrm{Nb}_{4} \mathrm{Zn}_{2} \mathrm{C} \\
\mathrm{PuRu}_{2}, \mathrm{Ta}_{4} \mathrm{Ni}_{2} \mathrm{C}, \mathrm{Nb}_{4} \mathrm{Ni}_{2} \mathrm{C}, \mathrm{CeAl}_{2}\end{array}$ \\
\hline \multicolumn{2}{|r|}{ Space Group 229: Im $\overline{3} m$} \\
\hline $\mathbb{Z}_{2,8}$ & Materials \\
\hline 07 & $\| \mathrm{Be}$ \\
\hline
\end{tabular}

Table VI: List of Topological Crystalline Insulators

\begin{tabular}{|c|c|}
\hline \multicolumn{2}{|r|}{ List of Topological Crystalline Insulators } \\
\hline \\
\hline \multicolumn{2}{|r|}{$\frac{\text { Space Group 2: } P \overline{1}}{\text { Materials }}$} \\
\hline 0002 & $\mathrm{NaCuF}_{3}, \mathrm{In}_{3} \mathrm{Cu}_{7}$ \\
\hline 0010 & | $\mathrm{SbS}, \mathrm{CuMoO}_{4}$ \\
\hline 0012 & $\mathrm{SrAs}_{3}\left[94, \mathrm{Ta}_{6} \mathrm{~S}\right.$ \\
\hline 0100 & $\| \mathrm{Ni}_{13} \mathrm{Sn}_{8} \mathrm{P}_{3}$ \\
\hline 0102 & $\| \mathrm{KHg}, \mathrm{Zr}_{8} \mathrm{Ni}_{21}, \mathrm{CuH}_{18} \mathrm{C}_{4} \mathrm{~N}_{10}(\mathrm{ClO})_{2}$ \\
\hline 0110 & $\mathrm{Pt}_{2} \mathrm{PbO}_{4}, \mathrm{CsCuP}_{3} \mathrm{H}_{8} \mathrm{O}_{13}, \mathrm{Ni}_{10} \mathrm{Sn}_{5} \mathrm{P}_{3}, \mathrm{SrTi}_{11} \mathrm{O}_{20}$ \\
\hline 0112 & $\| \mathrm{CuH}_{2} \mathrm{SO}_{5}$ \\
\hline 1000 & $\| \mathrm{Ga}_{5} \mathrm{Rh}_{3}, \mathrm{CuH}_{10} \mathrm{SeO}_{9}$ \\
\hline 1002 & $\mathrm{CuH}_{9} \mathrm{CSN}_{4} \mathrm{Cl}_{3} \mathrm{O}, \mathrm{Yb}_{3} \mathrm{Ge}_{8}$ \\
\hline 1102 & $\| \mathrm{Hf}_{3} \mathrm{Ni}_{7}, \mathrm{Rb}_{2} \mathrm{CuH}_{4}\left(\mathrm{C}_{2} \mathrm{O}_{5}\right)_{2}$ \\
\hline \multicolumn{2}{|r|}{ Space Group 10: $P 2 / m$} \\
\hline $\mathbb{Z}_{2,2,2,4}$ & Materials \\
\hline 0110 & $\mathrm{~Tb}_{4} \mathrm{Mg}_{3} \mathrm{Co}_{2}$ \\
\hline 0112 & $\| \mathrm{TiBrO}$ \\
\hline 1002 & $\| \mathrm{ZrTiSe}_{4}$ \\
\hline 1112 & $\|$ NbRh \\
\hline \multicolumn{2}{|r|}{ Space Group 11: $P 2_{1} / m$} \\
\hline $\mathbb{Z}_{2,2,2,4}$ & Materials \\
\hline 0002 & \multirow{2}{*}{ 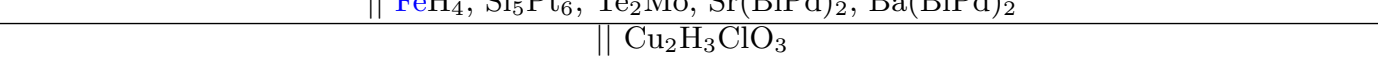 } \\
\hline 0010 & \\
\hline 0012 & $\| \mathrm{La}_{2} \mathrm{CuSnO}_{6}, \mathrm{Ce}(\mathrm{InPt})_{2}$ \\
\hline 1000 & $\mathrm{BaSb}_{2}(\mathrm{ICSD}: 409517), \mathrm{Ba}_{3}(\mathrm{GeN})_{2}, \mathrm{TiPd}$ \\
\hline 1002 & $\mathrm{SrSb}_{2}, \mathrm{BaSb}_{2}(\mathrm{ICSD}: 413519), \mathrm{NbPt}_{3}, \mathrm{SiS}_{4}$ \\
\hline 1010 & \begin{tabular}{l||l|l|l}
$\mathrm{TaSe}_{3}$ \\
\end{tabular} \\
\hline 1012 & $\mathrm{TaPt}_{3}$ \\
\hline \multicolumn{2}{|r|}{ Space Group 12: $C 2 / m$} \\
\hline $\mathbb{Z}_{2,2,2,4}$ & Materials \\
\hline 0002 & $\mathrm{Zr}_{2} \mathrm{Ni}_{7}, \mathrm{Mg}_{4} \mathrm{Zn}_{7}$ \\
\hline 0010 & $\begin{array}{c}\mathrm{NaSr}_{2} \mathrm{AlP}_{3}, \| \mathrm{BiS}, \mathrm{BiSe}(\mathrm{ICSD}: 251720), \mathrm{BiSe}(\mathrm{ICSD}: 251717), \mathrm{Tc}_{2} \mathrm{P}_{3}, \mathrm{Mg}_{5} \mathrm{Si}_{6}(\mathrm{ICSD}: 54780) \\
\mathrm{Si}_{2} \mathrm{NiSe}_{4}, \mathrm{MgNb}_{2}, \mathrm{Ce}_{2} \mathrm{Mo}_{2} \mathrm{C}_{3}, \mathrm{Ti}_{4} \mathrm{FeS}_{8}, \mathrm{LaSe}\end{array}$ \\
\hline 0012 & $\mathrm{Cs}\left(\mathrm{MoO}_{3}\right)_{3}, \mathrm{~K}\left(\mathrm{MoO}_{3}\right)_{3}, \| \mathrm{Bi}, \mathrm{Li}_{2} \mathrm{RuO}_{3}, \mathrm{Yb}_{3}\left(\mathrm{Ga}_{2} \mathrm{Ge}_{3}\right)_{2}, \mathrm{Fe}_{3} \mathrm{Se}_{4}, \mathrm{Bi}_{2} \mathrm{Pt}$ \\
\hline 1100 & $\| \mathrm{Nb}_{6} \mathrm{C}_{5}, \mathrm{Ho}_{2} \mathrm{Ge}_{2} \mathrm{Rh}, \mathrm{Ti}_{3} \mathrm{Te}_{4}, \mathrm{Y}_{2} \mathrm{Si}_{2} \mathrm{Rh}, \mathrm{Sc}_{2} \mathrm{CoSi}_{2}, \mathrm{BaTl}_{4}, \mathrm{Nd}_{6} \mathrm{In}_{22} \mathrm{Pt}_{13}, \mathrm{LaIO}_{3}, \mathrm{Cr}_{4} \mathrm{As}_{3}$ \\
\hline 1102 & $\begin{array}{c}\| \mathrm{K}_{6} \mathrm{CdPb}_{8}, \mathrm{Ba}_{3}\left(\mathrm{ZnAs}_{2}\right)_{2}, \mathrm{Ba}_{3}\left(\mathrm{CdAs}_{2}\right)_{2}, \mathrm{Ba}_{3}\left(\mathrm{CdSb}_{2}\right)_{2}, \mathrm{Sc}_{5} \mathrm{NCl}_{8}, \mathrm{AlCu}, \mathrm{IrC}_{2}, \mathrm{Hf}_{2} \mathrm{Bi}_{1} \mathrm{MgZn}_{5} \\
\mathrm{Zr}_{2} \mathrm{CoSi}_{2}, \mathrm{La}_{4} \mathrm{Si}_{4} \mathrm{I}_{3}, \mathrm{AsPd}_{5}, \mathrm{Zr}_{2} \mathrm{Cu}, \mathrm{Ni}_{3}(\mathrm{BiS})_{2}, \mathrm{Nb}_{7} \mathrm{P}_{4}, \mathrm{PtN}_{4}(\mathrm{ClO})_{2}, \mathrm{Ba}_{7} \mathrm{Ir}_{6} \mathrm{O}_{19}\end{array}$ \\
\hline 1110 & $\begin{array}{c}\| \mathrm{VP}_{2}, \mathrm{La}_{4} \mathrm{~B}_{4} \mathrm{Br}_{5}, \mathrm{Lu}_{2} \mathrm{Mo}_{2} \mathrm{C}_{3}, \mathrm{Zr}_{2} \mathrm{HBr}_{2}, \mathrm{Zn}_{13} \mathrm{Fe}, \mathrm{Dy}_{2} \mathrm{Cr}_{2} \mathrm{C}_{3}, \mathrm{~Tb}_{2} \mathrm{Cr}_{2} \mathrm{C}_{3}, \mathrm{Tm}_{2} \mathrm{Cr}_{2} \mathrm{C}_{3}, \mathrm{Lu}_{2} \mathrm{Cr}_{2} \mathrm{C}_{3}, \\
\mathrm{Nb}_{2} \mathrm{Se}, \mathrm{Ti}_{2} \mathrm{NiSe}_{4}, \mathrm{La}_{4} \mathrm{C}_{5} \mathrm{Cl}_{2}, \mathrm{La}_{5} \mathrm{Si}_{5} \mathrm{I}_{3}, \mathrm{Pr}_{2} \mathrm{Al}_{19} \mathrm{Co}_{6}, \mathrm{U}_{2} \mathrm{~W}_{2} \mathrm{C}_{3}, \mathrm{U}_{4} \mathrm{Ga}_{20} \mathrm{Ni}_{11}\end{array}$ \\
\hline 1112 & $\begin{array}{c}\left.\| \mathrm{NbP}_{2}[95], \mathrm{NbAs}_{2}[95], \mathrm{TaAs}_{2} 96\right], \mathrm{BC}_{2} \mathrm{~N}, \mathrm{~K}_{4} \mathrm{Cu}_{8} \mathrm{Te}_{11}, \mathrm{VAs}_{2}, \mathrm{NbSb}_{2} \text { 95], } \\
\mathrm{Er}_{2} \mathrm{Mo}_{2} \mathrm{C}_{3}\left(\mathrm{ICSD}_{2}: 617669\right), \mathrm{Cs}, \mathrm{Tm}_{2} \mathrm{Mo}_{2} \mathrm{C}_{3}, \mathrm{ReGe}_{2}, \mathrm{Sm}_{2} \mathrm{Mo}_{2} \mathrm{C}_{3}, \mathrm{NaN}_{3}(\mathrm{ICSD}: 29375), \mathrm{Mo}_{2} \mathrm{As}_{3}, \\
\mathrm{Yb}_{2} \mathrm{Ge}_{2} \mathrm{Ir}, \mathrm{Ta}_{2} \mathrm{Te}_{3}, \mathrm{Ni}\left(\mathrm{RhSe}_{2}\right)_{2}, \mathrm{Ca}_{6} \mathrm{Cu}_{2} \mathrm{Sn}_{7}, \mathrm{As}_{3} \mathrm{~W}_{2}, \mathrm{Sm}_{2} \mathrm{Cr}_{2} \mathrm{C}_{3}, \mathrm{Ho}_{2} \mathrm{Cr}_{2} \mathrm{C}_{3}, \mathrm{Ti}_{2} \mathrm{NiS}_{4}, \mathrm{Er}_{2} \mathrm{Cr}_{2} \mathrm{C}_{3}, \\
\mathrm{Co}_{2} \mathrm{NiSe}_{4}, \mathrm{Ba}_{2} \mathrm{Ni}_{5} \mathrm{Ge}_{4}, \mathrm{In}_{7} \mathrm{Cu}_{10}\end{array}$ \\
\hline & Space Group 14: $P 2_{1} / c$ \\
\hline $\mathbb{Z}_{2,2,2,4}$ & Materials \\
\hline
\end{tabular}


Table VI - continued

\begin{tabular}{|c|c|}
\hline SI & Topological Crystalline Insulators \\
\hline 0002 & $\mathrm{OsN}_{2}, \mathrm{CuTeO}_{4}(\mathrm{ICSD}: 1672), \mathrm{CuTeO}_{4}(\mathrm{ICSD}: 1671), \mathrm{KPH}_{2} \mathrm{O}_{3}, \mathrm{Re}_{2} \mathrm{Si}, \mathrm{CoH}_{2}(\mathrm{SN})_{4}, \mathrm{Pr}_{5} \mathrm{O}_{9}$ \\
\hline 1000 & $\mathrm{CsNb}_{3} \mathrm{SBr}_{7}, \| \mathrm{Sm}_{2} \mathrm{~B}_{5}, \mathrm{PrCu}_{6}, \mathrm{NdCu}_{6}, \mathrm{Pr}_{2} \mathrm{Mo}_{2} \mathrm{C}_{3}, \mathrm{LaCu}_{6}, \mathrm{La}_{11} \mathrm{C}_{11} \mathrm{Cl}_{7}, \mathrm{HgH}_{4} \mathrm{C}_{4}\left(\mathrm{Br}_{2} \mathrm{~N}\right)_{2}$ \\
\hline 1002 & $\begin{array}{c}\mathrm{Sr}_{2} \mathrm{Cu}_{2} \mathrm{Te}\left(\mathrm{BrO}_{3}\right)_{2}, \| \mathrm{CaCuAs}_{2} \mathrm{O}_{7}, \mathrm{SN}(\mathrm{ICSD}: 41967), \mathrm{CuHClO}, \mathrm{La}(\mathrm{GePt})_{2}, \mathrm{CuHOF}, \mathrm{La}_{3} \mathrm{C}_{3} \mathrm{Cl}_{2}, \\
\mathrm{La}_{2} \mathrm{RuO}_{5}, \mathrm{~K}\left(\mathrm{GeSe}_{2}\right)_{2}, \mathrm{Pd}_{34} \mathrm{Se}_{11}\end{array}$ \\
\hline \multicolumn{2}{|r|}{ Space Group 15: $C 2 / c$} \\
\hline $\mathbb{Z}_{2,2,2,4}$ & Materials \\
\hline 0002 & $\| \mathrm{Cu}_{6} \mathrm{Sn}_{5}$ \\
\hline 1100 & $\| \mathrm{P}_{2} \mathrm{Pt}_{5}, \mathrm{Ca}_{5} \mathrm{Au}_{2}, \mathrm{Sm}_{4} \mathrm{Si}_{3} \mathrm{Pd}_{4}, \mathrm{Na}_{6} \mathrm{TlSb}_{4}, \mathrm{~Tb}_{10} \mathrm{~B}_{7} \mathrm{C}_{10}, \mathrm{Mo}_{9} \mathrm{O}_{26}$ \\
\hline 1102 & $\mathrm{Ca}_{2} \mathrm{SiIr}_{2}, \mathrm{~Tb}_{2}\left(\mathrm{GePt}_{3}\right)_{3}, \mathrm{Ni}_{4} \mathrm{P}_{16} \mathrm{~W}, \mathrm{Cu}_{2} \mathrm{P}_{2} \mathrm{O}_{7}, \mathrm{Sc}_{3} \mathrm{Re}_{2} \mathrm{Si}_{3}, \mathrm{~K}_{3} \mathrm{Na}\left(\mathrm{RuO}_{4}\right)_{2}, \mathrm{YbP}_{5} \mathrm{O}_{14}, \mathrm{Na}_{2} \mathrm{PrO}_{3}$ \\
\hline \multicolumn{2}{|r|}{ Space Group 47: Pmmm } \\
\hline $\mathbb{Z}_{2,2,2,4}$ & Materials \\
\hline 0102 & $\| \mathrm{ZnB}_{3} \operatorname{Ir}_{4}$ \\
\hline \multicolumn{2}{|r|}{ Space Group 51: Pmma } \\
\hline $\mathbb{Z}_{2,2,2,4}$ & Materials \\
\hline 0010 & $\| \mathrm{NbRh}, \mathrm{Ca}_{5}(\mathrm{PPd})_{6}, \mathrm{Li}_{2} \mathrm{Ca}_{3} \mathrm{~N}_{6}$ \\
\hline 0012 & $\mathrm{NdInNi}_{2}, \mathrm{LaInNi}_{2}, \mathrm{PrInNi}_{2}, \mathrm{BiPd}_{3}, \mathrm{Zn}_{5} \mathrm{~B}_{3} \mathrm{Ir}_{7}$ \\
\hline 0100 & $\| \mathrm{GeH}_{4}, \mathrm{TiNi}, \mathrm{PrGaCo}_{2}, \mathrm{TaIr}, \mathrm{NbIr}$ \\
\hline 0102 & $\| \mathrm{MgCd}$ \\
\hline 0110 & $\|$ Te, $\mathrm{NdSbPd}, \mathrm{Zn}_{5} \mathrm{~B}_{3} \mathrm{Rh}_{7}$ \\
\hline 0112 & $\| \mathrm{MoPt}, \mathrm{GaPt}_{2}$ \\
\hline \multicolumn{2}{|r|}{ Space Group 55: Pbam } \\
\hline $\mathbb{Z}_{2,2,2,4}$ & \\
\hline 0002 & $\begin{array}{c}\mathrm{Ca}_{5}\left(\mathrm{GaSb}_{3}\right)_{2}, \mathrm{Yb}_{5}\left(\mathrm{AlSb}_{3}\right)_{2}, \mathrm{Yb}_{5}\left(\mathrm{InSb}_{3}\right)_{2}, \mathrm{SmFeB}_{4}, \mathrm{LuNiB}_{4}, \mathrm{Yb}\left(\mathrm{Ga}_{4} \mathrm{Co}_{2}\right)_{2}, \mathrm{Yb}_{2} \mathrm{AlB}_{6}, \mathrm{Y}_{2} \mathrm{ReB}_{6}, \\
\mathrm{Th}_{6} \mathrm{Cd}_{7}, \mathrm{Ce}_{2} \mathrm{NiB}_{10}, \mathrm{HfGe}_{2} \mathrm{Rh}\end{array}$ \\
\hline 0010 & $\| \mathrm{Tb}_{4} \mathrm{C}_{5}, \mathrm{HoReB}_{4}, \mathrm{YReB}_{4}, \mathrm{Th}_{2} \mathrm{FeB}_{10}, \underset{\mathrm{Th}_{5}\left(\mathrm{In}_{2} \mathrm{Rh}\right)_{2}, \mathrm{Ho}_{4} \mathrm{C}_{5}, \mathrm{Y}_{4} \mathrm{C}_{5}, \mathrm{ZrCrSi}_{2}, \mathrm{Ca}_{3}\left(\mathrm{Bi}_{2} \mathrm{Pd}\right)_{4}}{ }$ \\
\hline 0012 & || $\mathrm{MgGa}_{2}, \mathrm{ZrGe}_{2} \mathrm{Rh}, \mathrm{TiFeSi}_{2}$ \\
\hline \multicolumn{2}{|r|}{ Space Group 56: Pccn } \\
\hline $\mathbb{Z}_{4}$ & Materials \\
\hline 2 & $\mathrm{ZnCuTeCl}_{2} \mathrm{O}_{3}$ \\
\hline & Space Group 57: Pbcm \\
\hline $\mathbb{Z}_{2,2,2,4}$ & Materials \\
\hline 0002 & $\| \mathrm{Ca}_{3}(\mathrm{GaPt})_{2}, \mathrm{Ca}_{3}(\mathrm{GaPd})_{2}$ \\
\hline 1000 & W, NdAl, SmAl, TbAl, DyAl, TmAl, LuAl, LaCoSb $3, \mathrm{SmCoSb}_{3}, \mathrm{NdCoSb}_{3}, \mathrm{PrCoSb}_{3}$ \\
\hline & $\begin{array}{l}\text { Space Group 58: Pnnm } \\
\end{array}$ \\
\hline $\mathbb{Z}_{4}$ & Materials \\
\hline 2 & $\mathrm{OsN}_{2}, \mathrm{Ge}_{2} \mathrm{Pt}, \mathrm{RuN}{ }_{2}, \mathrm{Co}_{2} \mathrm{C}, \mathrm{RbBi}_{3} \mathrm{~S}_{5}, \mathrm{Ti}_{2} \mathrm{~S}, \mathrm{Zr}_{14} \mathrm{P}_{9}$ \\
\hline & Space Group 59: Pmmn \\
\hline $\mathbb{Z}_{2,2,2,4}$ & Materials \\
\hline 0010 & $\| \mathrm{Er}_{2} \mathrm{Ge}_{5}, \mathrm{Ni}_{3} \mathrm{Sn}, \mathrm{SmGePt}, \mathrm{ErSiPd}, \mathrm{YSiPd}, \mathrm{PrGePt}$ \\
\hline 0012 & $\| \mathrm{B}_{2} \mathrm{Os}, \mathrm{B}_{2} \mathrm{Ru}, \mathrm{TbAu}_{3}, \mathrm{DyAu}_{3}, \mathrm{HoAu}_{3}, \mathrm{Sr}_{6} \mathrm{Ge}_{5} \mathrm{~N}_{2}, \mathrm{Ba}_{6} \mathrm{Ge}_{5} \mathrm{~N}_{2}, \mathrm{Dy}_{2} \mathrm{Sn}_{5}, \mathrm{Pr}_{3} \mathrm{Sb}_{5} \mathrm{Pd}_{6}$ \\
\hline & Space Group 60: Pbcn \\
\hline $\mathbb{Z}_{4}$ & Materials \\
\hline 2 & $\| \mathrm{NiS}_{2}$ \\
\hline & Space Group 61: Pbca \\
\hline $\mathbb{Z}_{4}$ & Materials \\
\hline 2 & $\| \mathrm{SmI}_{2}, \mathrm{Ho}_{2}\left(\mathrm{Ni}_{2} \mathrm{~B}\right)_{5}$ \\
\hline & Space Group 62: Pnma \\
\hline $\mathbb{Z}_{4}$ & Materials \\
\hline 2 & 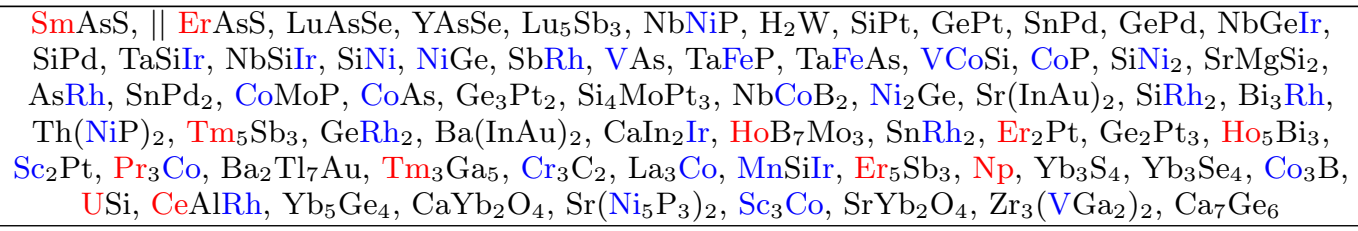 \\
\hline & Space Group 63: $\mathrm{Cmcm}$ \\
\hline
\end{tabular}


Table VI - continued

\begin{tabular}{|c|c|}
\hline SI & Topological Crystalline Insulators \\
\hline $\mathbb{Z}_{2,2,2,4}$ & Materials \\
\hline 0002 & 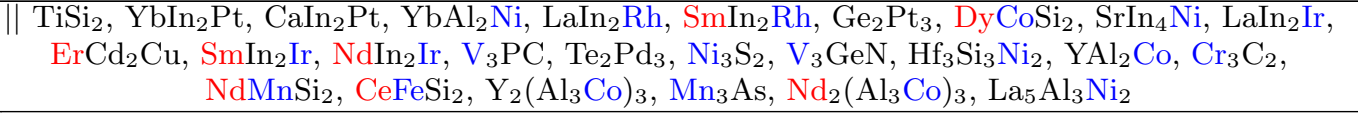 \\
\hline 1100 & 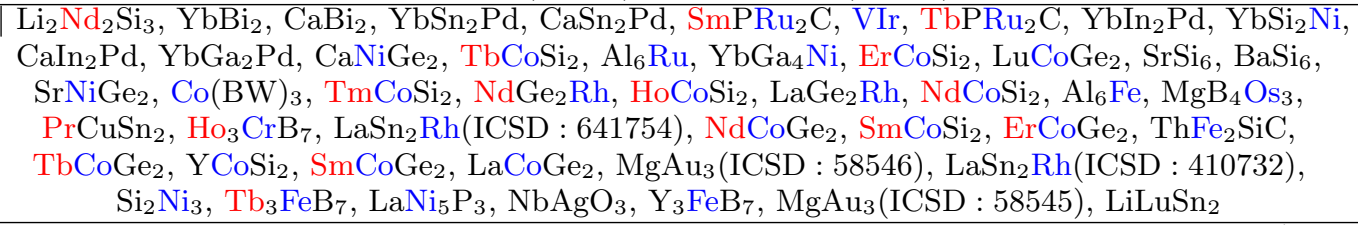 \\
\hline
\end{tabular}

1102 HoAlGe, LuAlGe, ErAlGe, DyAlGe, SrSi, YAlGe, ScAlGe, LuAlSi, TmAlSi, SrSn, SrPb, YAlSi,

1102 CaGe, LaGa, DyAu, Ca $\mathrm{InN}_{2}$ TmAu, ErAu, YAu, InAs, CaZn, $\mathrm{Li}_{4} \mathrm{H}_{5} \mathrm{Rh}, \mathrm{InSb}(\mathrm{ICSD}: 156996)$, $\mathrm{Ba}_{3} \mathrm{~Pb}_{5}, \mathrm{BaFe}_{2} \mathrm{~S}_{3}, \mathrm{~K}_{3} \mathrm{Ga}_{13}$

\begin{tabular}{|c|c|}
\hline \multicolumn{2}{|r|}{ Space Group 64: Cmce } \\
\hline $\mathbb{Z}_{2,2,2,4}$ & Materials \\
\hline 0002 & NdAsSe, Ga(ICSD : 165978), $\mathrm{Yb}_{2}\left(\mathrm{Ni}_{5} \mathrm{~B}_{3}\right)_{3}, \mathrm{NbCoTe}_{2}, \mathrm{Ba}\left(\mathrm{Ni}_{5} \mathrm{P}_{3}\right)_{2}, \mathrm{Sr}\left(\mathrm{Ni}_{5} \mathrm{P}_{3}\right)_{2}$ \\
\hline 1100 & $\| \mathrm{BaCaSn}_{3}, \mathrm{BaYbSn}_{3}, \mathrm{Ho}_{2}\left(\mathrm{Ni}_{5} \mathrm{~B}_{3}\right)_{3}, \mathrm{MgIr}, \mathrm{Ce}_{2} \mathrm{Ni}_{22} \mathrm{C}_{3}$ \\
\hline 1102 & $\| \mathrm{Ca}_{5}\left(\mathrm{GaN}_{2}\right)_{2}, \mathrm{LaS}, \mathrm{NiGe}_{2}, \mathrm{Yb}_{2} \mathrm{Ge}_{6} \mathrm{Pd}$ \\
\hline \multicolumn{2}{|r|}{ Space Group 65: $\mathrm{Cmmm}$} \\
\hline $\mathbb{Z}_{2,2,2,4}$ & Materials \\
\hline 0010 & $\| \mathrm{Y}_{11} \mathrm{In}_{9} \mathrm{Ni}_{4}, \mathrm{Ce}_{3} \mathrm{Sn}_{7}$ \\
\hline 0012 & $\| \mathrm{ErSi}_{3} \mathrm{Ni}, \mathrm{YSi}_{3} \mathrm{Ni}, \mathrm{SmSi}_{3} \mathrm{Ni}, \mathrm{ReN}_{2}$ \\
\hline 1100 & $\| \mathrm{Cu}_{2} \mathrm{SiNiS}_{4}, \mathrm{HoBC}, \mathrm{DyBC}, \mathrm{Ba}_{3} \mathrm{Nb}_{16} \mathrm{O}_{23}$ \\
\hline 1102 & $\mathrm{AgCNO}, \mathrm{YNiGe}_{3}, \mathrm{SmNiGe}_{3}, \mathrm{PrNiGe}_{3}, \mathrm{PrSn}_{2}, \mathrm{Ba}_{2} \mathrm{Yb}\left(\mathrm{CuO}_{2}\right)_{4}, \mathrm{SmGa}_{2} \mathrm{Ni}, \mathrm{NdNiGe}_{3}$ \\
\hline 1110 & || $\mathrm{TlSbSe}_{2}, \mathrm{Nb}_{5} \mathrm{Ga}_{13}, \mathrm{Ba}_{4} \mathrm{Nb}_{14} \mathrm{O}_{23}$ \\
\hline 1112 & 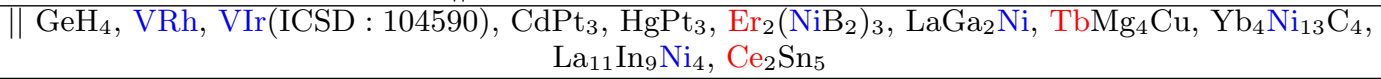 \\
\hline \multicolumn{2}{|r|}{ Space Group 67: Cmme } \\
\hline $\mathbb{Z}_{2,2,2,4}$ & Materials \\
\hline 0012 & C(ICSD : 88815) \\
\hline \multicolumn{2}{|r|}{ Space Group 69: Fmmm } \\
\hline \multicolumn{2}{|r|}{$\frac{\text { Space Group 69: Fmmm }}{\text { Materials }}$} \\
\hline 0002 & $\mathrm{Sr}_{3}\left(\mathrm{~B}_{3} \mathrm{Rh}_{4}\right)_{2}$ \\
\hline 1010 & $\| \mathrm{Rh}_{4} \mathrm{~Pb}_{5}$ \\
\hline 1102 & $\| \mathrm{Ca}_{2} \mathrm{Si}_{3} \mathrm{Ag}$ \\
\hline \multicolumn{2}{|r|}{ Space Group 71: Immm } \\
\hline $\mathbb{Z}_{2,2,2,4}$ & Materials \\
\hline 0002 & $\| \mathrm{ErGe}_{2} \mathrm{Pt}, \mathrm{YbNiGe}_{2}, \mathrm{Yb}_{3}(\mathrm{CuGe})_{4}, \mathrm{Yb}_{3}(\mathrm{GePd})_{4}, \mathrm{Cr}_{3} \mathrm{~B}_{4}, \mathrm{YGe}_{2} \mathrm{Ir}, \mathrm{Hf}_{54} \mathrm{Os}_{17}$ \\
\hline 1110 & $\begin{array}{l}\| \mathrm{HoGe}_{2} \mathrm{Pt}, \mathrm{TbGe}_{2} \mathrm{Pt}, \mathrm{SmGe}_{2} \mathrm{Pt}, \mathrm{Hf}_{3}(\mathrm{CuSi})_{4}, \mathrm{Zr}_{3}(\mathrm{CuSi})_{4}, \mathrm{TbGe}_{2} \mathrm{Pd}, \mathrm{Yb}_{3} \mathrm{Cu}_{6} \mathrm{Sn}_{5}, \mathrm{LuNiGe}_{2}, \\
\quad \mathrm{LaTm}\left(\mathrm{Ge}_{2} \mathrm{Ir}\right)_{2}, \mathrm{ErNiGe}_{2}, \mathrm{NdGe}_{2} \mathrm{Ir}, \mathrm{Tm}_{6} \mathrm{GaCo}_{2}, \mathrm{Ho}_{6} \mathrm{GaCo}_{2}, \mathrm{Sm}_{6} \mathrm{GaCo}_{2}, \mathrm{Sm}_{6} \mathrm{InCo}_{2}\end{array}$ \\
\hline 1112 & $\begin{array}{c}\quad \mathrm{CsFeS}_{2}, \mathrm{Li}_{2} \mathrm{H}_{2} \mathrm{Pt}, \mathrm{PdN}_{2}, \mathrm{Sr}_{3}\left(\mathrm{LiSb}_{4}, \mathrm{Ba}_{3}(\mathrm{LiSb})_{4}, \mathrm{Ca}_{3}(\mathrm{AlGe})_{2}, \mathrm{Ba}_{3}(\mathrm{LiBi})_{4}, \mathrm{Ca}_{2} \mathrm{CdPd}_{2},\right. \\
\mathrm{Ca}_{2} \mathrm{MgPd}_{2}, \mathrm{Ti}_{3} \mathrm{~B}_{4}, \mathrm{Ca}_{2} \mathrm{MgPt}_{2}, \mathrm{Sr}_{2} \mathrm{CdPt}_{2}, \mathrm{Pr}_{2} \mathrm{Ni}_{2} \mathrm{Sn}, \mathrm{BaY}_{2} \mathrm{NiO}_{5}, \mathrm{~Tb}_{2} \mathrm{ZnNi}_{2}, \mathrm{Sr}_{10} \mathrm{Al}_{4} \mathrm{Si}_{6} \mathrm{O} \\
\mathrm{CaBi}_{2}\left(\mathrm{CO}_{4}\right)_{2}, \mathrm{Y}_{2} \mathrm{Ni}_{2} \mathrm{Sn}, \mathrm{Th}_{2} \mathrm{MnN}_{3}, \mathrm{Ce}_{2} \mathrm{ZnNi}_{2}, \mathrm{DrNiGe}_{2}, \mathrm{Ce}_{2} \mathrm{Ni}_{2} \mathrm{Sn}_{2} \mathrm{Ti}_{6} \mathrm{Sn}_{5}, \mathrm{Sc}_{6} \mathrm{Si}_{11} \mathrm{Ni}_{18} \\
\mathrm{Sc}_{6} \mathrm{Ni}_{18} \mathrm{Ge}_{11}, \mathrm{Sc}_{4} \mathrm{Si}_{7} \mathrm{Ni}_{12}\end{array}$ \\
\hline \multicolumn{2}{|r|}{$\begin{array}{ll}\text { Space Group 72: Ibam }\end{array}$} \\
\hline $\mathbb{Z}_{2,2,2,4}$ & Materials \\
\hline 0002 & $\| \mathrm{Ti}_{3} \mathrm{Pt}_{5}$ \\
\hline 1110 & $\| \mathrm{Nb}_{2} \mathrm{Cr}_{4} \mathrm{Si}_{5}(\mathrm{ICSD}: 42905), \mathrm{Ti}_{6} \mathrm{Ge}_{5}, \mathrm{Nb}_{2} \mathrm{Cr}_{4} \mathrm{Si}_{5}(\mathrm{ICSD}: 626410), \mathrm{Ta}_{2} \mathrm{Cr}_{4} \mathrm{Si}_{5}$ \\
\hline 1112 & $\| \mathrm{Sb}_{2} \mathrm{Pt}_{3}, \mathrm{Y}_{2} \mathrm{Si}_{5} \mathrm{Ni}_{3}, \mathrm{Ho}_{2} \mathrm{Si}_{5} \mathrm{Ni}_{3}, \mathrm{Mg}_{5} \mathrm{Tl}_{2}, \mathrm{~Tb}_{2} \mathrm{Si}_{5} \mathrm{Ni}_{3}$ \\
\hline \multicolumn{2}{|r|}{ Space Group 74: Imma } \\
\hline $\mathbb{Z}_{2,2,2,4}$ & Materials \\
\hline 0002 & $\| \mathrm{Ca}_{3} \mathrm{GeO}$ \\
\hline 1110 & || $\mathrm{BaZn}_{2}, \mathrm{UMn}_{2}$ \\
\hline 1112 & $\mathrm{YbZn}_{2}, \mathrm{CaZn}_{2}, \mathrm{SrZn}_{2}, \mathrm{SrCd}_{2}, \mathrm{SrHg}_{2}, \mathrm{CaCd}_{2}, \mathrm{NdNi}_{4} \mathrm{~B}$ \\
\hline \multicolumn{2}{|r|}{ Space Group 86: $P 4_{2} / n$} \\
\hline $\mathbb{Z}_{2,2,2,4}$ & Materials \\
\hline
\end{tabular}


Table VI - continued

\begin{tabular}{|c|c|}
\hline SI & Topological Crystalline Insulators \\
\hline 1110 & $\| \mathrm{Ta}_{3} \mathrm{Si}, \mathrm{Mg}_{15} \mathrm{Si}_{2} \mathrm{Ir}_{5}$ \\
\hline \multicolumn{2}{|r|}{ Space Group 87: $I 4 / m$} \\
\hline $\mathbb{Z}_{2,8}$ & Materials \\
\hline 10 & $\mathrm{Sr}_{2} \mathrm{CuCO}_{5}$ \\
\hline 14 & $\| \mathrm{TiAu}_{4}$ \\
\hline \multicolumn{2}{|r|}{ Space Group 88: $I 4_{1} / a$} \\
\hline $\mathbb{Z}_{4}$ & Materials \\
\hline 2 & $\mathrm{MgGa}, \mathrm{Hf}\left(\mathrm{VH}_{2}\right)_{2}$ \\
\hline \multicolumn{2}{|r|}{ Space Group 123: $P 4 / \mathrm{mmm}$} \\
\hline $\mathbb{Z}_{2,4,8}$ & Materials \\
\hline 002 & $\mathrm{CsCd}_{4} \mathrm{Ass}_{3}$ \\
\hline 010 & $\| \mathrm{SiSnPd}_{5}$ \\
\hline 012 & $\| \mathrm{FeSi}_{2}$ \\
\hline 014 & $\mathrm{BaMg}_{4} \mathrm{Ge}_{3}$ \\
\hline 020 & \| NaAs \\
\hline 024 & $\| \mathrm{CeIn}_{5} \mathrm{Ir}$ \\
\hline 026 & $\mathrm{TiCdHg}_{2}$ \\
\hline 034 & $\| \mathrm{GaPPt}_{5}$ \\
\hline 036 & $\mathrm{RbZn}_{4} \mathrm{As}_{3}, \mathrm{UGa}_{5} \mathrm{Ir}, \mathrm{UGa}_{5} \mathrm{Co}, \mathrm{UGa}_{5} \mathrm{Rh}, \mathrm{HoS}$ \\
\hline 102 & $\| \mathrm{Ba}_{2} \mathrm{PrCu}_{3} \mathrm{O}_{8}$ \\
\hline 112 & $\| \mathrm{Ti}_{2} \mathrm{HPd}$ \\
\hline 116 & $\| \mathrm{AgPPd}_{5}$ \\
\hline 126 & $\| \mathrm{AgSbTe}_{2}[5], \mathrm{Ba}_{2} \mathrm{YTlCu}_{2} \mathrm{O}_{7}$ \\
\hline 132 & $\| \mathrm{BaTi}_{2} \mathrm{Sb}_{2} \mathrm{O}$ \\
\hline 136 & $\| \mathrm{Ta}_{4} \mathrm{O}$ \\
\hline \multicolumn{2}{|r|}{ Space Group 125: $P 4 / n b m$} \\
\hline $\mathbb{Z}_{2,2,2,4}$ & Materials \\
\hline 0010 & $\| \mathrm{CeGa}_{6}$ \\
\hline 0012 & $\| \mathrm{Nd}_{2} \mathrm{Ga}_{12} \mathrm{Ni}$ \\
\hline \multicolumn{2}{|r|}{ Space Group 127: $P 4 / m b m$} \\
\hline $\mathbb{Z}_{4,8}$ & Materials \\
\hline 06 & $\| \mathrm{Er}_{2} \mathrm{CdGe}_{2}, \mathrm{Dy}_{2} \mathrm{CdGe}_{2}, \mathrm{Li}_{2} \mathrm{Sn}_{5}$ \\
\hline 14 & $\| \mathrm{Yb}_{3} \mathrm{Ag}_{2}$ \\
\hline 22 & $\| \mathrm{Sr}_{2} \mathrm{~Pb}_{3}$ \\
\hline 24 & $\| \mathrm{Fe}(\mathrm{BMo})_{2}$ \\
\hline 26 & $\| \mathrm{Nd}_{7} \mathrm{Al}_{7} \mathrm{Co}_{6}, \mathrm{Pr}_{7} \mathrm{Al}_{7} \mathrm{Co}_{6}$ \\
\hline 34 & $\| \mathrm{Zr}_{3} \mathrm{Ga}_{2}, \mathrm{Pu}_{2} \mathrm{SnPt}_{2}$ \\
\hline \multicolumn{2}{|r|}{ Space Group 129: P4/nmm } \\
\hline $\mathbb{Z}_{2,2,2,4}$ & Materials \\
\hline 0002 & 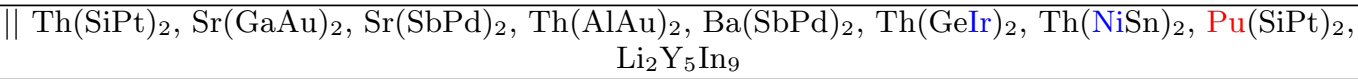 \\
\hline 0010 & $\begin{array}{c}\text { || ZrSiTe[104], } \mathrm{BaNiS}_{2}, \mathrm{ZrNiP}_{2}, \mathrm{NbCrN} \mathrm{Ba}(\mathrm{MgPb})_{2}, \mathrm{Ba}(\mathrm{CuSb})_{2}, \mathrm{LaNiAsO}, \mathrm{Ba}(\mathrm{MgSn})_{2}, \\
\mathrm{BiTeIO}_{3}, \mathrm{Ce}(\mathrm{GeIr})_{2}, \mathrm{Ho}_{10} \mathrm{In}_{20} \mathrm{Ni}_{9}\end{array}$ \\
\hline 0012 & 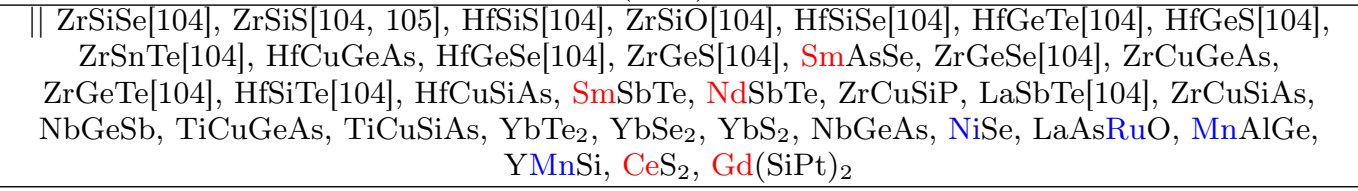 \\
\hline \multicolumn{2}{|r|}{$\begin{array}{l}\text { Space Group 130: P4/ncc } \\
\end{array}$} \\
\hline $\mathbb{Z}_{4}$ & Materials \\
\hline 2 & $\| \mathrm{La}_{5} \mathrm{Ga}_{3}$ \\
\hline \multicolumn{2}{|r|}{ Space Group 131: $P 4_{2} / m m c$} \\
\hline $\mathbb{Z}_{2,2,2,4}$ & Materials \\
\hline 1100 & $\| \mathrm{TcN}$ \\
\hline \multicolumn{2}{|r|}{ Space Group 136: $P 4_{2} / m n m$} \\
\hline $\mathbb{Z}_{4}$ & Materials \\
\hline
\end{tabular}


Table VI - continued

\begin{tabular}{|c|c|}
\hline SI & Topological Crystalline Insulators \\
\hline 2 & $\begin{array}{c}\mathrm{Pr}_{2} \mathrm{MoC}_{2}, \mathrm{Er}_{3} \mathrm{Al}_{2}, \mathrm{Pr}_{2} \mathrm{WC}_{2}, \mathrm{Nd}_{2} \mathrm{MoC}_{2}, \mathrm{Nd}_{2} \mathrm{WC}_{2}, \mathrm{Lu}_{3} \mathrm{Al}_{2}, \mathrm{Hf}_{3} \mathrm{Al}_{2}, \mathrm{Tm}_{3} \mathrm{Al}_{2}, \mathrm{Ce}_{2} \mathrm{Fe}_{14} \mathrm{~B}, \\
\mathrm{Er}_{2} \mathrm{Fe}_{14} \mathrm{~B}, \mathrm{U}\end{array}$ \\
\hline \multicolumn{2}{|r|}{$\begin{array}{ll}\text { Space Group 139: } I 4 / \mathrm{mmm} \\
\end{array}$} \\
\hline $\mathbb{Z}_{2,8}$ & Materials \\
\hline 02 & $\begin{array}{c}\| \mathrm{BePd}_{2}, \mathrm{Yb}(\mathrm{CuSi})_{2}, \mathrm{Yb}(\mathrm{SiAu})_{2}, \mathrm{Ca}(\mathrm{GeAu})_{2}, \mathrm{Ca}(\mathrm{CuGe})_{2}, \mathrm{Sr}(\mathrm{GeAu})_{2}, \mathrm{Ca}(\mathrm{CuSi})_{2}, \mathrm{Sr}(\mathrm{SbPd})_{2}, \\
\mathrm{Ba}(\mathrm{SbPd})_{2}, \mathrm{Yb}(\mathrm{NiP})_{2}, \mathrm{Ca}(\mathrm{NiP})_{2}, \mathrm{Ca}(\mathrm{SiPd})_{2}, \mathrm{Hf}(\mathrm{SiNi})_{2}, \mathrm{Yb}(\mathrm{GePd})_{2}, \mathrm{Sr}(\mathrm{NiSb})_{2}, \mathrm{Ca}(\mathrm{GePd})_{2}, \\
\mathrm{Sr}(\mathrm{SiPd})_{2}, \mathrm{Sr}(\mathrm{GePd})_{2}, \mathrm{Zr}(\mathrm{SiNi})_{2}, \mathrm{Ti}_{2} \mathrm{Bi}, \mathrm{PrScGe}, \mathrm{Yb}_{11} \mathrm{Ge}_{10}\end{array}$ \\
\hline 04 & $\begin{array}{c}\| \mathrm{Ca}_{2} \mathrm{As}[56], \mathrm{HfH}_{2}, \mathrm{ZrH}_{2}, \mathrm{ThH}_{2}, \mathrm{~K}_{2} \mathrm{Mg}_{5} \mathrm{Sn}_{3}, \mathrm{Ca}(\mathrm{ZnSi})_{2}, \mathrm{Ba}_{2} \mathrm{CaTl}_{2}\left(\mathrm{CuO}_{4}\right)_{2}(\mathrm{ICSD}: 68198) \\
\mathrm{Tb}_{2} \mathrm{Al}_{4} \mathrm{NiGe}_{2}, \mathrm{Na}_{2} \mathrm{Ti}_{2} \mathrm{Sb}_{2} \mathrm{O}, \mathrm{Ce}(\mathrm{SiIr})_{2}(\mathrm{ICSD}: 621430), \mathrm{Ce}(\mathrm{GePd})_{2}, \mathrm{Ba}_{11} \mathrm{Bi}_{10}, \mathrm{Ti}_{3} \mathrm{Cu}_{4}\end{array}$ \\
\hline 06 & $\begin{array}{c}\| \mathrm{Yb}(\mathrm{CuGe})_{2}, \mathrm{Sr}(\mathrm{CuGe})_{2}, \mathrm{Sr}(\mathrm{CuSi})_{2}, \mathrm{Yb}(\mathrm{SiAg})_{2}, \mathrm{Ba}(\mathrm{AgGe})_{2}, \mathrm{Ba}(\mathrm{AgSn})_{2}, \mathrm{Sr}(\mathrm{CdGa})_{2}, \mathrm{DyAl}_{3}, \\
\mathrm{ScAl}_{3}, \mathrm{Zr}\left(\mathrm{TiGa}_{2}\right)_{2}, \mathrm{Yb}_{8} \mathrm{Ge}_{3} \mathrm{Sb}_{5}, \mathrm{TiPt}_{8}, \mathrm{Ca}\left(\mathrm{MnAl}_{2}\right)_{4}, \mathrm{Sc}_{11}\left(\mathrm{AlGe}_{4}\right)_{2}\end{array}$ \\
\hline 10 & $\begin{array}{c}\| \mathrm{Th}(\mathrm{CuSi})_{2}, \mathrm{Th}(\mathrm{CuGe})_{2}, \mathrm{Ca}(\mathrm{AsPd})_{2}, \mathrm{Ba}(\mathrm{CdGe})_{2}, \mathrm{Sr}(\mathrm{AsPd})_{2}, \mathrm{Th}(\mathrm{GePd})_{2}, \mathrm{Th}(\mathrm{SiPd})_{2}, \\
\mathrm{Th}(\mathrm{SiPt})_{2}, \mathrm{Th}(\mathrm{GePt})_{2}, \mathrm{Th}(\mathrm{NiGe})_{2}, \mathrm{Th}(\mathrm{SiNi})_{2}, \mathrm{Ce}(\mathrm{NiP})_{2}, \mathrm{Sr}(\mathrm{CoP})_{2}, \mathrm{Th}(\mathrm{SiTc})_{2}, \mathrm{Yb}(\mathrm{FeSi})_{2}, \\
\mathrm{Ca}(\mathrm{MnGe})_{2}, \mathrm{NdScGe}, \mathrm{Ce}(\mathrm{SiAu})_{2}, \mathrm{U}(\mathrm{CuSi})_{2}, \mathrm{U}(\mathrm{CuGe})_{2}, \mathrm{Ti}_{4} \mathrm{Si}_{7} \mathrm{Ni}_{4}, \mathrm{CaU}_{2}\left(\mathrm{PO}_{2}\right)_{2}\end{array}$ \\
\hline 12 & $\begin{array}{c}\mathrm{Li}_{2} \mathrm{H}_{2} \mathrm{Pd}, \mathrm{Sr}_{2} \mathrm{Bi}, \mathrm{Sr}_{2} \mathrm{Sb}, \mathrm{Sr}_{2} \mathrm{As}, \mathrm{BaSi}_{2}, \mathrm{ThPt}_{2}, \mathrm{TbMgSn}, \mathrm{Hf} \mathrm{Z}_{2} \mathrm{Zn}, \mathrm{NdMgSn}, \mathrm{PrMgSn}_{2} \mathrm{Hf}_{2} \mathrm{Cd} \\
\mathrm{Er}_{2} \mathrm{Mg}_{2} \mathrm{Ru}, \mathrm{Zr}_{3}\left(\mathrm{Cu}_{2} \mathrm{Si}_{3}\right)_{2}, \mathrm{Hf}_{2} \mathrm{Hg}, \mathrm{Ba}_{2} \mathrm{Sb}, \mathrm{Ca}_{3} \mathrm{SiBr}_{2}, \mathrm{Ba}_{2} \mathrm{Hg}, \mathrm{Ba}_{2} \mathrm{Cd}, \mathrm{Zr}_{2} \mathrm{Zn}, \mathrm{Ba}_{2} \mathrm{As}, \mathrm{Hf}_{2} \mathrm{H}_{2} \mathrm{Pd} \\
\mathrm{Th}\left(\mathrm{Al}_{2} \mathrm{Cr}\right)_{4}, \mathrm{Sr}_{3} \mathrm{Ru}_{2} \mathrm{O}_{7}\end{array}$ \\
\hline 14 & $\begin{array}{c}\| \mathrm{Ba}(\mathrm{BRh})_{2}, \mathrm{Ca}(\mathrm{PRh})_{2}, \mathrm{Yb}(\mathrm{SiPd})_{2}, \mathrm{Yb}(\mathrm{SiPt})_{2}, \mathrm{ThZn}_{4}, \mathrm{Ca}(\mathrm{SiNi})_{2}, \mathrm{Yb}(\mathrm{SiNi})_{2}, \mathrm{Ca}(\mathrm{NiGe})_{2}, \\
\mathrm{Yb}(\mathrm{NiGe})_{2}, \mathrm{CaIn}, \mathrm{Ca}(\mathrm{CoP})_{2}, \mathrm{Sr}(\mathrm{AsRh})_{2}, \mathrm{Ca}(\mathrm{CoAs})_{2}, \mathrm{Ca}_{2} \mathrm{Sb}_{4} \mathrm{Pd}_{3}, \mathrm{SmScSi}, \mathrm{TbScGe}, \mathrm{Ba}(\mathrm{MnAs})_{2}, \\
\mathrm{Th}(\mathrm{MnSi})_{2}, \mathrm{U}(\mathrm{NiP})_{2}, \mathrm{Hf}_{11} \mathrm{Ga}_{10}, \mathrm{U}(\mathrm{MnGe})_{2}\end{array}$ \\
\hline 16 & $\begin{array}{c}\| \mathrm{Zn}(\mathrm{BIr})_{2}, \mathrm{Ti}_{2} \mathrm{H}_{2} \mathrm{Pd}, \mathrm{ZrTiAs}, \mathrm{HfVGe}, \mathrm{ZrVGe} \mathrm{Sr}_{2} \mathrm{Ti}_{2} \mathrm{As}_{2} \mathrm{OF}_{2}, \mathrm{Ti}_{2} \mathrm{Zn}, \mathrm{Zr}_{2} \mathrm{Cd}, \mathrm{Ba}_{2} \mathrm{Zn}, \mathrm{Zn}_{3} \mathrm{Ru} \\
\mathrm{Zr}_{2} \mathrm{H}_{2} \mathrm{Pd}, \mathrm{Zr}_{2} \mathrm{Sb}, \mathrm{YZrSb}, \mathrm{UCuP}_{2}\end{array}$ \\
\hline \multicolumn{2}{|r|}{ Space Group 140: $I 4 / \mathrm{mcm}$} \\
\hline $\mathbb{Z}_{2,8}$ & \multirow{2}{*}{$\begin{array}{c}\text { Materials } \\
\| \mathrm{Ca}\end{array}$} \\
\hline 02 & \\
\hline 04 & $\mathrm{GePt}_{3}[58], \mathrm{CrSb}_{2}, \mathrm{SrRuO}_{3}, \mathrm{~Tb}_{5} \mathrm{BiAu}_{2}, \mathrm{Lu}_{7} \mathrm{Sb}_{3}$ \\
\hline 06 & $\| \mathrm{Sc}_{3} \mathrm{Ga}_{2}, \mathrm{U}_{6} \mathrm{Ni}$ \\
\hline 10 & $\mathrm{Bi}, \mathrm{Pr}_{5} \mathrm{Tl}_{3}, \mathrm{CeSi}_{4} \mathrm{Ni}_{9}$ \\
\hline 12 & $\| \mathrm{SrCu}_{2}\left(\mathrm{BO}_{3}\right)_{2}, \mathrm{Ba}_{5} \mathrm{Sn}_{3}$ \\
\hline 14 & $\| \mathrm{La}_{3} \mathrm{Ga}_{4} \mathrm{Cl}$ \\
\hline 16 & $\| \mathrm{Mg}_{2} \mathrm{Pt}, \mathrm{Ca}_{5} \mathrm{Hg}_{3}, \mathrm{Sr}_{5} \mathrm{Cd}_{3}$ \\
\hline \multicolumn{2}{|r|}{ Space Group 142: I41/acd } \\
\hline $\mathbb{Z}_{4}$ & Materials \\
\hline 2 & $\| \mathrm{Be}_{3} \mathrm{P}_{2}, \mathrm{YbPS}_{4}, \mathrm{KSn}$ \\
\hline \multicolumn{2}{|r|}{ Space Group 148: $R \overline{3}$} \\
\hline $\mathbb{Z}_{2,2,2,4}$ & Materials \\
\hline 0002 & $\| \mathrm{P}_{2} \mathrm{Pd}_{15}, \mathrm{Rb}_{4}\left(\mathrm{Mo}_{7} \mathrm{Se}_{8}\right)_{3}$ \\
\hline 1110 & $\| \mathrm{Th}_{3} \mathrm{Au}_{4}, \mathrm{Cs}_{2} \mathrm{Te}_{10} \mathrm{Mo}_{9}, \mathrm{Tl}_{2} \mathrm{Mog}_{9} \mathrm{~S}_{11}, \mathrm{Nd}_{3} \mathrm{Au}_{4}, \mathrm{Rb}_{2} \mathrm{Mo}_{9} \mathrm{Se}_{10}$ \\
\hline 1112 & $\| \mathrm{Sr}_{7} \mathrm{Nb}_{6} \mathrm{O}_{21}, \mathrm{Mo}_{3} \mathrm{~S}_{4}, \mathrm{CuSiO}_{3}, \mathrm{Rb}_{5} \mathrm{Mo}_{18} \mathrm{~S}_{19}$ \\
\hline \multicolumn{2}{|r|}{ Space Group 162: $P \overline{3} 1 m$} \\
\hline $\mathbb{Z}_{2,2,2,4}$ & Materials \\
\hline 0002 & $\mathrm{SiO}_{2}(\mathrm{ICSD}: 170552)$ \\
\hline \multicolumn{2}{|r|}{ Space Group 163: $P \overline{\overline{3}} 1 c$} \\
\hline $\mathbb{Z}_{4}$ & Materials \\
\hline 2 & $\| \mathrm{Cr}_{5} \mathrm{~S}_{6}$ \\
\hline \multicolumn{2}{|r|}{ Space Group 164: $P \overline{3} m 1$} \\
\hline $\mathbb{Z}_{2,2,2,4}$ & Materials \\
\hline 0002 & $\| \mathrm{Hf}(\mathrm{CuP})_{2}, \mathrm{ZrTe}_{2}, \mathrm{TiPt}_{3}$ \\
\hline 0010 & \multirow{2}{*}{ 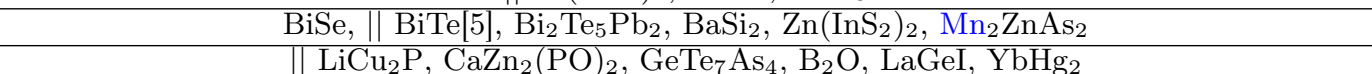 } \\
\hline 0012 & \\
\hline \multicolumn{2}{|r|}{ Space Group 166: $R \overline{3} m$} \\
\hline $\mathbb{Z}_{2,2,2,4}$ & Materials \\
\hline 0002 & $\| \mathrm{Bi}[114], \mathrm{P}[57], \mathrm{HoAl}_{3}, \mathrm{Sr}\left(\mathrm{Ni}_{2} \mathrm{~B}\right)_{6}, \mathrm{~V}_{3} \mathrm{Sb}_{2}, \mathrm{Ce}_{2} \mathrm{Zn}_{2} \mathrm{Ni}_{5}$ \\
\hline 1110 & $\begin{array}{c}\mathrm{C}(\mathrm{ICSD}: 29123), \| \mathrm{Bi}_{4} \mathrm{Se}_{3}, \mathrm{~B}_{3} \mathrm{Mo}, \mathrm{LuHCl}, \mathrm{ErHCl}, \mathrm{ZrBr}, \mathrm{ScHCl}, \mathrm{Tl}_{2} \mathrm{Ni}_{3} \mathrm{~S}_{2}, \mathrm{MgNb}_{2}, \mathrm{Ni}_{3}(\mathrm{PbSe})_{2}, \\
\text { B(ICSD : 43431) }\end{array}$ \\
\hline 1112 & $\begin{array}{c}\mathrm{Bi}_{2} \mathrm{TeI}\left[66, \| \mathrm{RbCd}_{4} \mathrm{As}_{3}, \mathrm{KCd}_{4} \mathrm{As}_{3}, \mathrm{NaCd}_{4} \mathrm{As}_{3}, \mathrm{Sr}\left(\mathrm{Ag}_{2} \mathrm{Sb}\right)_{2}, \mathrm{ZrCl}, \mathrm{Nb}_{2} \mathrm{CS}_{2}, \mathrm{In}_{2} \mathrm{Ni}_{3} \mathrm{~S}_{2}, \mathrm{Ni}_{3}(\mathrm{PbS})_{2},\right. \\
\mathrm{In}_{2} \mathrm{Ni}_{3} \mathrm{Se}_{2}, \mathrm{Te}_{4} \mathrm{Pt}_{3}, \mathrm{Ni}_{3}(\mathrm{SnS})_{2}, \mathrm{HfNi}_{3}, \mathrm{Tl}_{2} \mathrm{Pd}_{3} \mathrm{Se}_{2}, \mathrm{Ga}, \mathrm{ZrTi}_{2} \mathrm{Ni}_{9}, \mathrm{LaRh}_{3}, \mathrm{Zr}_{3}\left(\mathrm{Al}_{2} \mathrm{C}_{3}\right)_{2}\end{array}$ \\
\hline
\end{tabular}


Table VI - continued

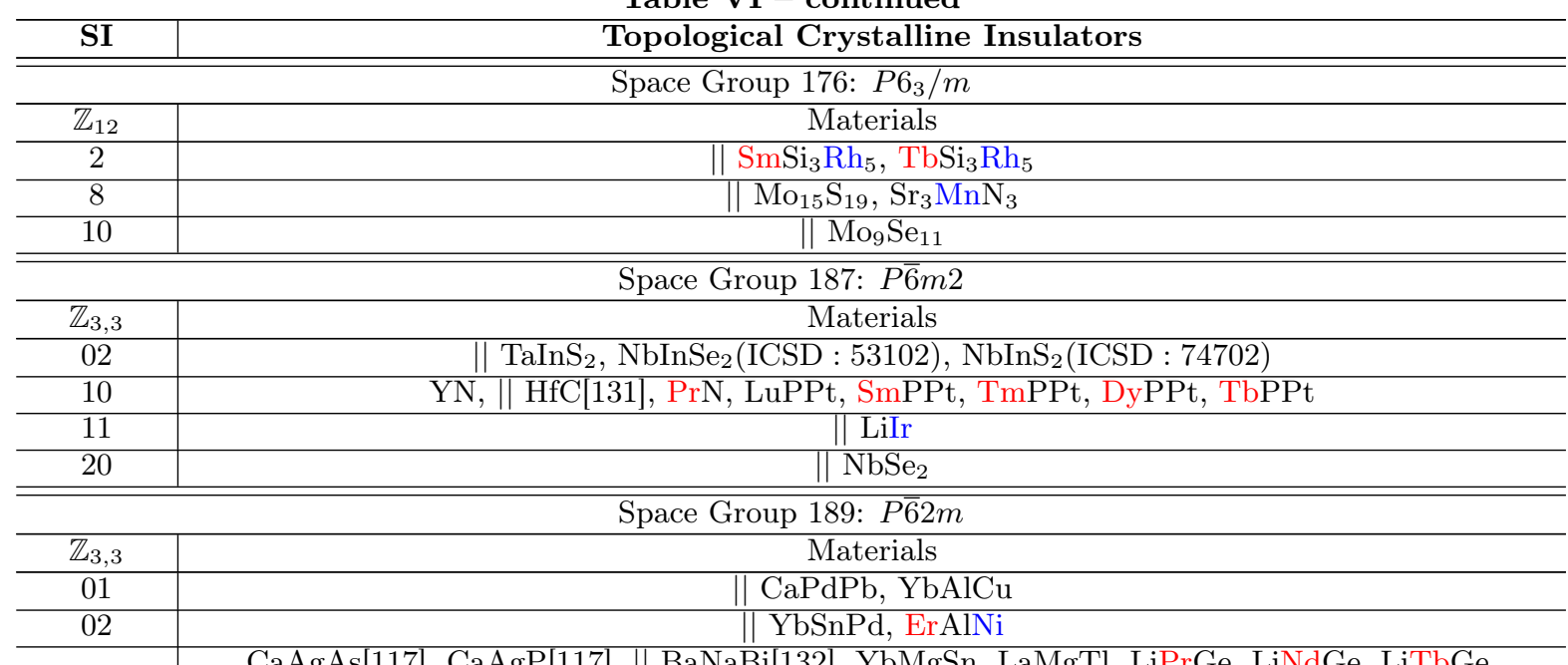

CaAgAs [17], CaAgP 117], || BaNaBi[132, YbMgSn, LaMgTl, LiPrGe, LiNdGe, LiTbGe,

LiSmGe, LiHoGe, YbCdSn, LiYGe, CaCdGe 118], LiErGe, YbCdGe, LiYSi, CaCdSn, NdMgGa,

TmAgGe, TiO, TmSiAg, SmMgGa, PrMgGa, DyAgGe(ICSD : 605077), ErAgGe(ICSD : 81558),

SmMgTl, SmSiAg, YAgPb, ScAgGe, LuSiAg, YMgGa, ErSiAg, YMgTl, YSiAg, TmMgGa,

10 DySiAg, SmMgIn, NdCdGa, LuAgSn, TmAgSn, ErAgSn, PrCdGa, SmCdGa, LaMgGa, YMgIn,

DyMgIn, $\operatorname{Pr}_{2}\left(\mathrm{Zn}_{2} \mathrm{Ge}\right)_{3}$, DyCdGa, TmMgIn, HoMgIn, ErCdGa, Nd ${ }_{2}\left(\mathrm{Zn}_{2} \mathrm{Ge}\right)_{3}$, ScCuGe, ScCuSi, $\mathrm{Sm}_{2}\left(\mathrm{Zn}_{2} \mathrm{Ge}\right)_{3}, \mathrm{SiPt}_{2}, \mathrm{Yb}_{3} \mathrm{In}_{3} \mathrm{Ge}_{2} \mathrm{Au}, \mathrm{TiGePd}, \mathrm{Lu}_{3} \mathrm{Ga}_{5} \mathrm{Ni}, \mathrm{Zr}_{3}\left(\mathrm{Cu}_{2} \mathrm{Ge}\right)_{2}, \mathrm{Hf}_{3}\left(\mathrm{Cu}_{2} \mathrm{Si}\right)_{2}$,

$\mathrm{Hf}_{3}\left(\mathrm{Cu}_{2} \mathrm{Ge}\right)_{2}, \mathrm{TbAgGe}, \mathrm{Zr}_{3}\left(\mathrm{Cu}_{2} \mathrm{Si}\right)_{2}, \mathrm{Mg}_{3} \mathrm{Al}_{8} \mathrm{FeSi}_{6}, \operatorname{PrTlPd}, \mathrm{CeInCu}$

\begin{tabular}{|c|c|}
\hline 11 & $\| \mathrm{YbSnPt}, \mathrm{LaCdAu}, \mathrm{LaMg}_{4} \mathrm{Cu}, \mathrm{Hf}_{6} \mathrm{Ga}_{2} \mathrm{Fe}$ \\
\hline 12 & $\| \mathrm{YbInAu}, \mathrm{LiBPt}_{3}, \mathrm{LaInPt}$ \\
\hline 20 & $\begin{array}{c}\text { II YMgAl, HoAgGe, DyAgGe(ICSD : 86731), ErAgGe(ICSD : 86733), } \mathrm{Li}_{5}\left(\mathrm{NiN}_{3}, \mathrm{Hf}_{6} \mathrm{NiSb}_{2},\right. \\
\text { HfFeGe }\end{array}$ \\
\hline 21 & $\begin{array}{l}\text { \|SmInPt, ThAlIr, ThAlRh, PrSnRh, LaSnRh, NdSnRh, YAlNi, Th}{ }_{3} \mathrm{Pd}_{5}, \mathrm{SmMgAg}_{\text {CeInIr }} \\
\text { CrMgAg, }\end{array}$ \\
\hline 22 & || YbMgPb, ThGaIr, DyAlNi, YMgAg, PrCdCu, DyMgAg, CoNiAs, LaMgAg, CeInRh \\
\hline & Space Group 191: $P 6 / \mathrm{mmm}$ \\
\hline $\mathbb{Z}_{6,12}$ & Materials \\
\hline 06 & $\mathrm{DyB}_{2} \mathrm{Rh}_{3}, \mathrm{HoB}_{2} \mathrm{Rh}_{3}, \mathrm{YB}_{2} \mathrm{Rh}_{3}, \mathrm{SmB}_{2} \mathrm{Rh}_{3}, \mathrm{NdB}_{2} \mathrm{Rh}_{3}, \mathrm{LaB}_{2} \mathrm{Rh}_{3}, \mathrm{PrB}_{2} \mathrm{Rh}_{3}$ \\
\hline 08 & $\| \mathrm{Ba}$ \\
\hline 10 & TaN(ICSD : 76457) \\
\hline 20 & $\|$ MoN(ICSD : 60168) \\
\hline 22 & || $\mathrm{TmB}_{2} \mathrm{Ir}_{3}, \mathrm{HoB}_{2} \mathrm{Ir}_{3}, \mathrm{SmB}_{2} \operatorname{Ir}_{3}$ \\
\hline 26 & $\| \mathrm{NdAl}_{3} \mathrm{Pd}_{2}, \mathrm{SmAl}_{3} \mathrm{Pd}_{2}, \mathrm{LaAl}_{3} \mathrm{Pd}_{2}, \mathrm{PrAl}_{3} \mathrm{Ni}_{2}, \mathrm{NdAl}_{3} \mathrm{Ni}_{2}, \mathrm{TiBe}_{12}$ \\
\hline 36 & $\| \mathrm{NdCo}_{4} \mathrm{~B}, \mathrm{PrCo}_{4} \mathrm{~B}, \mathrm{Dy}_{3} \mathrm{Co}_{11} \mathrm{~B}_{4}, \mathrm{Nd}_{3} \mathrm{Co}_{11} \mathrm{~B}_{4}, \mathrm{Pr}_{3} \mathrm{Co}_{11} \mathrm{~B}_{4}$ \\
\hline 38 & $\| \mathrm{ThAl}_{2}, \mathrm{ZrB}_{2}\left[75, \mathrm{TiB}_{2}\left[75, \mathrm{HfB}_{2}, \mathrm{ThGa}_{2}, \mathrm{CaC}_{2}, \mathrm{YbSi}_{2}\right.\right.$ \\
\hline 42 & $\| \mathrm{Nd}_{5} \mathrm{Co}_{21} \mathrm{~B}_{4}$ \\
\hline 44 & $\mathrm{~K}_{2} \mathrm{Si}_{2} \mathrm{~Pb}_{2} \mathrm{O}, \mathrm{UFe}_{3} \mathrm{~B}_{2}$ \\
\hline 48 & $\| \mathrm{SrPd}_{5}$ \\
\hline 50 & $\mathrm{MgB}_{2}[58], \mathrm{SrGa}_{2}$ \\
\hline 56 & $\| \mathrm{ThNi}_{5}$ \\
\hline 58 & $\| \mathrm{U}$ \\
\hline 510 & $\| \mathrm{Si}, \mathrm{KIn}_{9} \mathrm{Co}_{2}, \mathrm{CeNi}_{5}$ \\
\hline & Space Group 193: $P 6_{3} / \mathrm{mcm}$ \\
\hline $\mathbb{Z}_{12}$ & Materials \\
\hline 8 & $\| \mathrm{K}_{3} \mathrm{~N}$ \\
\hline & Space Group 194: $P 6_{3} / m m c$ \\
\hline $\mathbb{Z}_{12}$ & Materials \\
\hline 2 & $\begin{array}{c}\| \mathrm{CeO}_{2}, \mathrm{NdSbPd}, \mathrm{PrSbPd}, \mathrm{SmSbPd}, \mathrm{TbSbPd}, \mathrm{NiMoP}_{2}, \mathrm{YCuSi}, \mathrm{CoAs}, \mathrm{CoSb}, \mathrm{BiRh}, \mathrm{SiNi}_{2}, \\
\mathrm{Tm}_{2} \mathrm{Fe}_{17}\end{array}$ \\
\hline 4 & $\begin{array}{c}\mathrm{C}(\mathrm{ICSD}: 28419)[58], \mathrm{C}(\mathrm{ICSD}: 193439)\left[\frac{58]}{\mathrm{YRe}_{2}, \mathrm{UPt}_{3}} \mathrm{Cu}_{2} \mathrm{~S}, \mathrm{Ti}_{2} \mathrm{CdC}, \mathrm{Pr}_{2} \mathrm{~B}_{3} \mathrm{Cl}, \mathrm{TaSe}_{2}(\mathrm{ICSD}: 651948) \text {, }\right.\end{array}$ \\
\hline 6 & || $\mathrm{Ti}_{2} \mathrm{Sn}[58], \mathrm{ThPd}_{3}, \mathrm{Co}_{2} \mathrm{Sn}, \mathrm{USnPt}_{2}, \mathrm{BaRuO}_{3}(\mathrm{ICSD}: 246019), \mathrm{Ce}_{2} \mathrm{Co}_{5} \mathrm{~B}_{2}$ \\
\hline
\end{tabular}


Table VI - continued

\begin{tabular}{|c|c|}
\hline \multicolumn{2}{|r|}{$\begin{array}{l}\text { Topological Crystalline Insulators } \\
\end{array}$} \\
\hline 8 & $\begin{array}{l}\mathrm{KHgSb}[80,81], \mid \mathrm{Na}_{2} \mathrm{MgPb}, \mathrm{KHgAs}[80], \mathrm{Na}_{2} \mathrm{CdSn}[58], \text { LaI, NiS(ICSD : 646352), } \\
\mathrm{TiS}_{2} \text { (ICSD : 181507), BaCu, CrS(ICSD : 16723), CrS(ICSD : 626613), } \mathrm{MgNi}_{2}\end{array}$ \\
\hline 10 & $\| \mathrm{VP}, \mathrm{Nb}_{2} \mathrm{GeC}, \mathrm{NaTl}$ \\
\hline \multicolumn{2}{|r|}{ Space Group 200: $P m \overline{3}$} \\
\hline $\mathbb{Z}_{2,2,2,4}$ & Materials \\
\hline 1110 & $\| \mathrm{NaCl}_{7}$ \\
\hline 1112 & $\mathrm{Al}_{17}\left(\mathrm{Re}_{2} \mathrm{Si}\right)_{2}$ \\
\hline \multicolumn{2}{|r|}{ Space Group 204: Im $\overline{3}$} \\
\hline $\mathbb{Z}_{2,2,2,4}$ & Materials \\
\hline 0002 & $\| \mathrm{Al}_{12} \mathrm{~W}, \mathrm{Al}_{12} \mathrm{Mo}, \mathrm{SmC}_{10}$ \\
\hline 1110 & $\| \mathrm{Ho}_{8} \mathrm{Mg}_{12} \mathrm{Zn}_{61}, \mathrm{NaCu}_{3}\left(\mathrm{RuO}_{3}\right)_{4}$ \\
\hline \multicolumn{2}{|r|}{ Space Group 205: $P a \overline{3}$} \\
\hline $\mathbb{Z}_{4}$ & Materials \\
\hline 2 & $\mathrm{Cu}_{3} \mathrm{WO}_{6}, \mathrm{In}_{5} \mathrm{~S}_{4}$ \\
\hline \multicolumn{2}{|r|}{ Space Group 221: $P m \overline{3} m$} \\
\hline $\mathbb{Z}_{4,8}$ & Materials \\
\hline 02 & $\begin{array}{c}\mathrm{Yb}_{3} \mathrm{PbO}, \mathrm{ThTaN}_{3}, \mathrm{Ca}_{3} \mathrm{SnO}[90], \mathrm{Ca}_{3} \mathrm{GeO}[90], \mathrm{Yb}_{3} \mathrm{SnO}, \mathrm{Sr}_{3} \mathrm{PbO}[90], \mathrm{Sr}_{3} \mathrm{SnO}[90], \mathrm{Ba}_{3} \mathrm{PbO}[90] \\
\mathrm{Ca}_{3} \mathrm{PbO}[58,90], \mathrm{BaTe}, \| \mathrm{Sr}_{3} \mathrm{BiN}[90], \mathrm{LaN}, \mathrm{HoN}, \mathrm{ErN}, \mathrm{YN}, \mathrm{NdN}, \mathrm{ThC}, \mathrm{Ca}_{3} \mathrm{BiN}\end{array}$ \\
\hline 06 & $\begin{array}{l}\text { ScIr, LuIr, TmIr, ErIr, HoIr, YIr, ScRh, LuRh, ScCo, TmRh, ErRh, HoRh, YRh, DyRh, TbRh, } \\
\text { SmRh }\end{array}$ \\
\hline 10 & $\| \mathrm{HfBRh}_{3}, \mathrm{LuRh}_{3} \mathrm{C}, \mathrm{TmRh}_{3} \mathrm{C}, \mathrm{ErRh}_{3} \mathrm{C}, \mathrm{HoRh}_{3} \mathrm{C}, \mathrm{DyRh}{ }_{3} \mathrm{C}, \mathrm{TbRh}_{3} \mathrm{C}, \mathrm{PaIr}_{3}$ \\
\hline 12 & || $\mathrm{HfPd}_{3}, \mathrm{NbRh}_{3}, \mathrm{PaRh}_{3}, \mathrm{VCo}_{3}, \mathrm{TlHPd}_{3}$ \\
\hline 14 & $\| \mathrm{InAg}, \mathrm{GaAg}, \mathrm{Cu}_{3} \mathrm{Au}$ \\
\hline 20 & || $\mathrm{YbPd}, \mathrm{MgPt}_{3}$ \\
\hline 24 & || CaPd, MnRh, CaNi \\
\hline 26 & $\|$ AlIr, AlCo, BeNi, $\mathrm{ZnPt}_{3}$ \\
\hline 30 & $\mathrm{LiAg}, \mathrm{Ni}_{3} \mathrm{GeC}, \mathrm{SnPd}_{3} \mathrm{C}, \mathrm{Pd}_{3} \mathrm{PbC}, \mathrm{InNi}_{3} \mathrm{~N}$ \\
\hline 32 & $\| \mathrm{Ti}_{3} \mathrm{TIN}, \mathrm{TcN}(\mathrm{ICSD}: 187707)$ \\
\hline 36 & $\| \mathrm{AlAu}, \mathrm{Pt}_{3} \mathrm{~Pb}, \mathrm{SnPt}_{3}, \mathrm{SiNi}_{3}, \mathrm{Ni}_{3} \mathrm{Ge}, \mathrm{Ni}_{3} \mathrm{Sn}, \mathrm{Mn}_{3} \mathrm{PtN}$ \\
\hline \multicolumn{2}{|r|}{ Space Group 224: $P n \overline{3} m$} \\
\hline $\mathbb{Z}_{2,2,2,4}$ & Materials \\
\hline 0002 & $\| \mathrm{Zn}_{3} \mathrm{P}_{2}$ \\
\hline \multicolumn{2}{|r|}{ Space Group 225: $F m \overline{3} m$} \\
\hline $\mathbb{Z}_{8}$ & Materials \\
\hline 2 & $\| \mathrm{ScAs}, \mathrm{ScSb}, \mathrm{MgZnAg}_{2}, \mathrm{PtC}, \mathrm{NiC}, \mathrm{ThB}_{12}, \mathrm{TiCo}_{2} \mathrm{Sn}, \mathrm{NbGaCo}_{2}, \mathrm{VGaCo}_{2}, \mathrm{TaGaCo}_{2}, \mathrm{HfCo}_{2} \mathrm{Sn}$ \\
\hline 4 & $\begin{array}{c}\text { SnSe 123], SnS [123], PbSe, } \mathrm{SnTe}_{20]}, \mathrm{TePb}[20], \mathrm{Yb}, \| \mathrm{BiI}_{3}, \mathrm{Sr}, \mathrm{W}, \mathrm{Ru}, \mathrm{Ca}, \mathrm{Ba}, \mathrm{Al}_{2} \mathrm{O}, \mathrm{Cu}_{3} \mathrm{SbS}_{4}, \\
\mathrm{Sc}_{2}\left(\mathrm{Ni}_{7} \mathrm{~B}_{2}\right)_{3}, \mathrm{Tm}_{2}\left(\mathrm{Ni}_{7} \mathrm{~B}_{2}\right)_{3}, \mathrm{NaB}, \mathrm{KB}\end{array}$ \\
\hline 6 & $\begin{array}{c}\text { WC, } \mathrm{ErInAu}_{2}, \mathrm{HoInAu}_{2}, \mathrm{YInAu}_{2}, \mathrm{TmInAu}_{2}, \mathrm{DyInAu}_{2}, \mathrm{SmInAu}_{2}, \mathrm{LuInAu}_{2}, \mathrm{TbInAu}_{2} \\
\mathrm{PrInAu}_{2}, \mathrm{NdInAu}_{2}, \mathrm{ScInAu}_{2}, \mathrm{ScAlAu} \mathrm{Su}_{2}, \mathrm{Cu}_{6} \mathrm{PbO}_{8}(\mathrm{ICSD}: 280596), \mathrm{InCu}_{6} \mathrm{ClO}_{8}, \mathrm{MnGaCo}_{2} \\
\mathrm{FeCu}_{2} \mathrm{Sn}, \mathrm{MnAlCo}_{2}\end{array}$ \\
\hline \multicolumn{2}{|r|}{ Space Group 227: $F d \overline{3} m$} \\
\hline $\mathbb{Z}_{4}$ & Materials \\
\hline 2 & $\begin{array}{c}\mathrm{Zr}\left(\mathrm{TiH}_{2}\right)_{2}, \mathrm{La}_{2} \mathrm{Hf}_{2} \mathrm{O}_{7}(\mathrm{ICSD}: 173790), \| \mathrm{La}_{2} \mathrm{Zr}_{2} \mathrm{O}_{7}(\mathrm{ICSD}: 184089), \mathrm{YbS}_{2}, \mathrm{SrAl}_{2}, \mathrm{BaPt}_{2}, \mathrm{BaAl}_{2}, \\
\mathrm{SrPt}_{2}, \mathrm{Zn}_{6} \mathrm{Ni}_{7} \mathrm{Ge}_{2}, \mathrm{Ce}\left(\mathrm{TiAl}_{10}\right)_{2}, \mathrm{Zr}_{3} \mathrm{~V}_{3} \mathrm{C}, \mathrm{Te}_{3} \mathrm{Pd}_{13}, \mathrm{Nb}_{2} \mathrm{Fe}, \mathrm{ZrMn}_{2}, \mathrm{HfZn}_{2}, \mathrm{Ba}_{21} \mathrm{Si}_{2} \mathrm{O}_{5}, \mathrm{PuMn}_{2}, \\
\mathrm{Ni}_{3} \mathrm{Mo}_{3} \mathrm{~N}, \mathrm{Cd}_{2} \mathrm{Re}_{2} \mathrm{O}_{7}\end{array}$ \\
\hline \multicolumn{2}{|r|}{ Space Group 229: $\operatorname{Im} \overline{3} m$} \\
\hline $\mathbb{Z}_{2,8}$ & Materials \\
\hline 16 & $\| \mathrm{W}$ \\
\hline \multicolumn{2}{|r|}{ Space Group 230: $I a \overline{3} d$} \\
\hline $\mathbb{Z}_{4}$ & Materials \\
\hline 2 & $\| \mathrm{Al}_{4} \mathrm{Ni}_{3}$ \\
\hline
\end{tabular}

